University of Nebraska - Lincoln

DigitalCommons@University of Nebraska - Lincoln

Faculty Publications in the Biological Sciences

Papers in the Biological Sciences

4-20-2018

\title{
Body size downgrading of mammals over the late Quaternary
}

\author{
Felisa A. Smith \\ University of New Mexico, fasmith@unm.edu \\ Rosemary E. Elliott Smith \\ University of California, San Diego \\ S. Kathleen Lyons \\ University of Nebraska - Lincoln, katelyons@unl.edu \\ Jonathan L. Payne \\ Stanford University, jlpayne@stanford.edu
}

Follow this and additional works at: https://digitalcommons.unl.edu/bioscifacpub

Part of the Animal Sciences Commons, Biology Commons, Paleobiology Commons, Paleontology

Commons, and the Population Biology Commons

Smith, Felisa A.; Elliott Smith, Rosemary E.; Lyons, S. Kathleen; and Payne, Jonathan L., "Body size downgrading of mammals over the late Quaternary" (2018). Faculty Publications in the Biological Sciences. 752.

https://digitalcommons.unl.edu/bioscifacpub/752

This Article is brought to you for free and open access by the Papers in the Biological Sciences at DigitalCommons@University of Nebraska - Lincoln. It has been accepted for inclusion in Faculty Publications in the Biological Sciences by an authorized administrator of DigitalCommons@University of Nebraska - Lincoln. 


\title{
Body size downgrading of mammals over the late Quaternary
}

\author{
Felisa A. Smith, ${ }^{1}$ Rosemary E. Elliott Smith, ${ }^{2}$ \\ S. Kathleen Lyons, ${ }^{3}$ and Jonathan L. Payne ${ }^{4}$
}

\begin{abstract}
1 Department of Biology, University of New Mexico, Albuquerque, NM 87131, USA 2 Department of Mathematics, University of California, San Diego, La Jolla, CA 92093, USA 3 School of Biological Sciences, University of Nebraska- Lincoln, Lincoln, NE 68588, USA 4 Department of Geological Sciences, Stanford University, Stanford, CA 94305, USA
\end{abstract}

Corresponding author — F. A. Smith, email fasmith@unm.edu

\begin{abstract}
Since the late Pleistocene, large-bodied mammals have been extirpated from much of Earth. Although all habitable continents once harbored giant mammals, the few remaining species are largely confined to Africa. This decline is coincident with the global expansion of hominins over the late Quaternary. Here, we quantify mammalian extinction selectivity, continental body size distributions, and taxonomic diversity over five time periods spanning the past 125,000 years and stretching approximately 200 years into the future. We demonstrate that sizeselective extinction was already under way in the oldest interval and occurred on all continents, within all trophic modes, and across all time intervals. Moreover, the degree of selectivity was unprecedented in 65 million years of mammalian evolution. The distinctive selectivity signature implicates hominin activity as a primary driver of taxonomic losses and ecosystem homogenization. Because megafauna have a disproportionate influence on ecosystem structure and function, past and present body size downgrading is reshaping Earth's biosphere.
\end{abstract}

Published in Science 360 (2018), pp 310-313 + supplement.

doi 10.1126/science.aao5987

Copyright 2018 AAAS. Used by permission.

Submitted 7 August 2017; accepted 7 March 2018; published 20 April 2018. 
W ild mammals are in decline globally because of a lethal combination of human-mediated threats, including hunting, introduced predators, and habitat modification (1-5). Extinction risk is particularly acute for the largest mammals, which are more frequently in conflict with humans $(1,6)$. The ongoing extirpation of large-bodied mammals is a major conservation concern because their decline can lead to the loss of ecological function within communities $(3,5$, 7). Megafauna have crucial direct and indirect impacts on vegetation structure, biogeochemical cycling, ecological interactions, and climate (7-10). Although the current extinction rate is higher than earlier in the Cenozoic (4), the ongoing biodiversity crisis may be an acceleration of a long-term trend over the late Quaternary. For example, a striking feature of the Pleistocene was the abundance and diversity of extremely large mammals such as the mammoth, giant ground sloth, wooly rhinoceros, and sabretooth tiger on all habitable continents. The debate about the causes of the terminal Pleistocene megafauna extinction has been long and acrimonious, with particular controversy surrounding the role of humans (11-13).

Multiple hominins - including at a minimum Neandertals, Denisovans, and archaic/modern humans-have been part of ecosystems throughout the late Pleistocene. Genetic analyses reveal a complicated history, with substantial admixture between populations (14). Anthropologists remain divided about the routes, exact timing, and number of early migrations from Africa (14-18), but several hominin species were probably widespread across Africa and Eurasia around 80 thousand to 60 thousand years (ka) ago (15-17). Further expansion followed, with modern Homo sapiens reaching Australia $~ 60$ to $50 \mathrm{ka}$ ago and crossing into the Americas 15 to $13 \mathrm{ka}$ ago (15). Migrations were likely driven or facilitated by climatic factors $(17,18)$ and were followed by rapid increases in population sizes $(17,19)$. For example, hominin populations in western Europe increased 10-fold by the Neandertal-to-Modern human transition 40 ka ago (19). Middle to Upper Paleolithic hominins were hunters who lived in groups and used both tools and fire (20); thus, it is plausible that their activities and rapid population growth influenced mammal biodiversity well before the terminal Pleistocene.

We investigated the influence of these emerging and increasingly sophisticated hominin predators on continental and global 
mammalian biodiversity over the late Quaternary (21). Ongoing biodiversity loss is robustly linked to human activities (1-5); and previous work linked extinction risk over the Holocene, terminal Pleistocene, and end- Pleistocene to human activities $(4,6,11-13,22-25)$; but earlier influences remain poorly characterized. Although recent work on paleodemography exists for $\mathrm{H}$. sapiens over the late Pleistocene and Holocene (17), a lack of data for other hominins precludes direct comparison of mammalian extinction risk over time against hominin population density. However, should we find significant differences between the pattern of late Quaternary extinction selectivity and the rest of the Cenozoic mammal record, this would strongly suggest a role of hominin activity $(13,24,25)$.

We used two data sets to test the potential role of hominin activity on extinction selectivity, mammalian body size distributions, and patterns of biodiversity over time and into the future (21). First, we updated a spatially explicit global record of body size and trophic mode for nonvolant, terrestrial mammals for the late Quaternary (MOM). Second, we constructed a global data set of Cenozoic mammals with associated stratigraphic duration, body mass, and trophic mode. We categorized late Quaternary extinctions into five temporal bins: late Pleistocene (125 to $70 \mathrm{ka}$ ago), which corresponded with the initial waves of migration of hominins out of Africa; end Pleistocene (70 to $20 \mathrm{ka}$ ago), which represented the continued expansion of hominins into Eurasia and the colonization of Australasia; terminal Pleistocene (20 to $10 \mathrm{ka}$ ago), which encompassed the migration of humans into the Americas; Holocene (10 to 0 ka ago), which represented further expansion of humans throughout the globe; and future $(\sim+0.2 \mathrm{ka})$, where we assumed that all currently threatened mammals become extinct (21). We binned the Cenozoic fossil data set into intervals of 1 million years $(\mathrm{Ma})$ as a reference standard and computed temperature metrics for each bin (21). For each time interval, we characterized the size selectivity of extinction using logistic regression and examined overall body size distribution and trophic guild structure (tables S1 to S7) (21). For the late Quaternary, we also characterized size selectivity by continent and trophic level.

Our analyses demonstrated a striking and significantly size-biased pattern of mammalian extinction over the late Quaternary, distinct in the Cenozoic record (Figs. 1 to 3 and fig. S1). We found a mass 


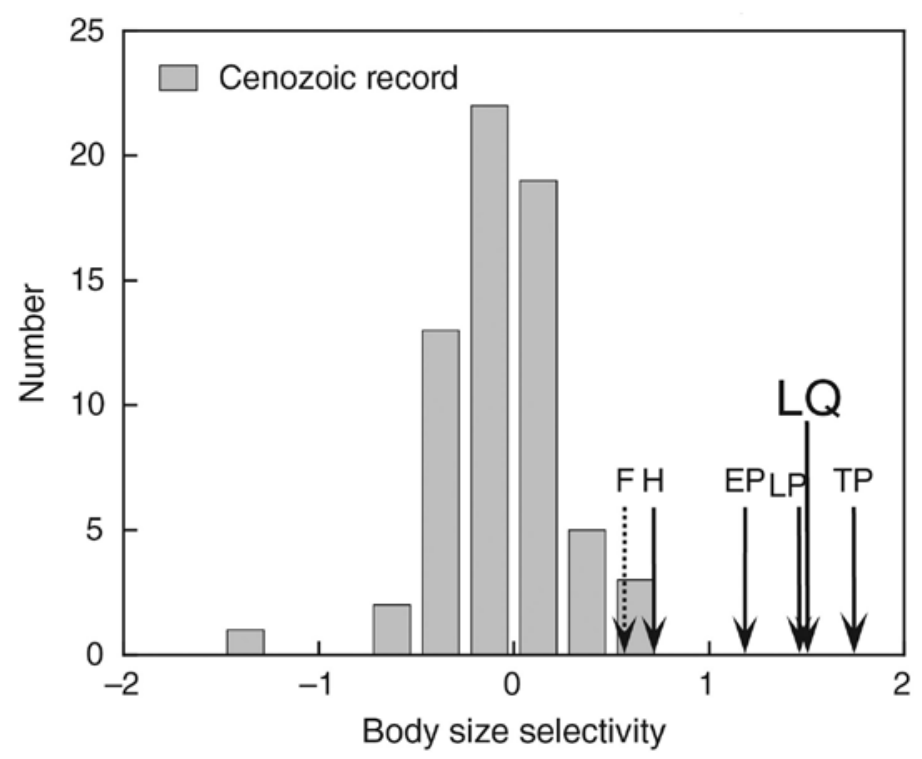

Fig. 1. Distribution of body size selectivity coefficients over the Cenozoic mammal record. All selectivity coefficients reflect change in the natural logarithm of the odds of extinction associated with a one-log10-unit change in body mass. Values of zero indicate no bias, positive values indicate bias against larger size, and negative values indicate bias against smaller size. $L Q$, average of all late Quaternary (LP to $H$ ) extinctions; LP, late Pleistocene; EP, end Pleistocene; TP, terminal Pleistocene; $\mathrm{H}$, Holocene; and $\mathrm{F}$, future extinctions.

difference of two to three orders of magnitude between victims and survivors of late Quaternary extinction intervals (Fig. 2A and table S1), reflecting a significant association between size and extinction probability (Fig. 2B and table S5). This size bias occurred on each continent (Fig. 2, C and D) and within each major trophic group (Fig. 2, E and F), with the magnitude of the size difference and the statistical measure of size selectivity decreasing between the Pleistocene and Holocene (Fig. 2, A to F). The reduced selectivity of the Holocene and future extinctions likely reflects changes in the nature of threats. Today, many smaller-bodied animals are vulnerable because of habitat alteration, introduced predators, or urbanization $(5-7,11,26)$.

Comparison of extinctions across the entirety of the Cenozoic demonstrated that body mass was rarely significantly associated with the probability of extinction before the late Pleistocene (Figs. 1 and 3, $E$ and $F$ ), and further, size differences between victims and survivors never approached those observed in the Pleistocene (tables S1 and S3). There was a preferential loss of small-bodied species in the 


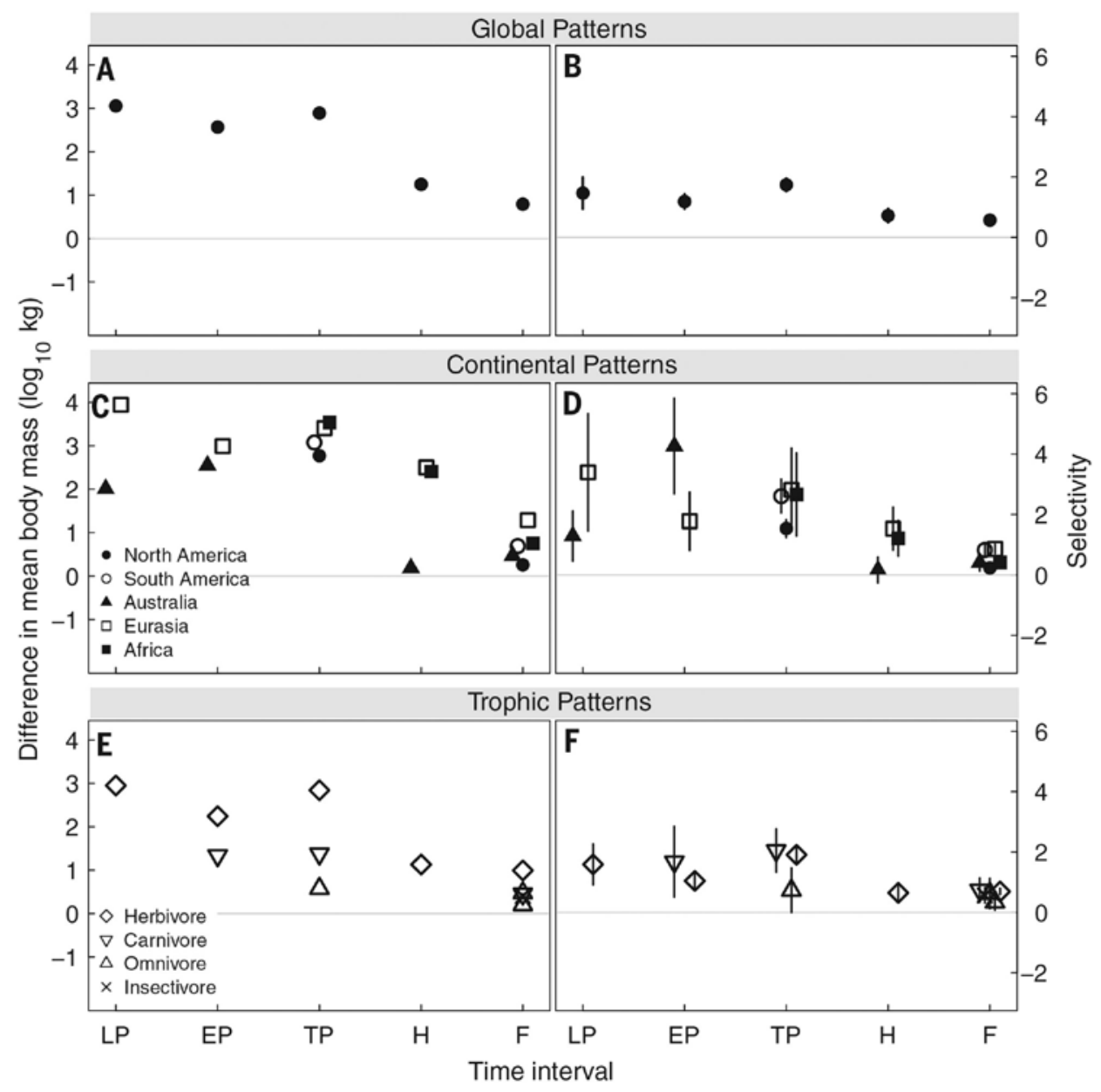

Fig. 2. Analyses of size bias in the mammalian fossil record. (A and B) Global patterns of extinction. (A) Difference in the mean of log-transformed sizes of victims versus survivors for intervals across the late Quaternary. (B) Selectivity coefficients measuring the association between body size and extinction probability derived from logistic regression of extinction status as a function of body mass. Multiple regressions controlling for the additive contributions of continental location and trophic guild yield even stronger associations between extinction status and body mass (table S5) (21). (C and D) Extinction patterns on each continent. (C) Size differences. (D) Size selectivity coefficients. ( $E$ and F) Influence of trophic guild on extinction risk. (E) Size differences. (F) Size selectivity coefficients. Bars indicate 95\% confidence interval $(\mathrm{Cl})$.

Oligocene that is perhaps linked to expansion of grasslands and prairies ( 29 Ma ago) (Fig. 3E), although this value had high uncertainty. However, no interval over the past $65 \mathrm{Ma}$ was as selective as the late Quaternary. Moreover, climate change did not increase extinction risk 


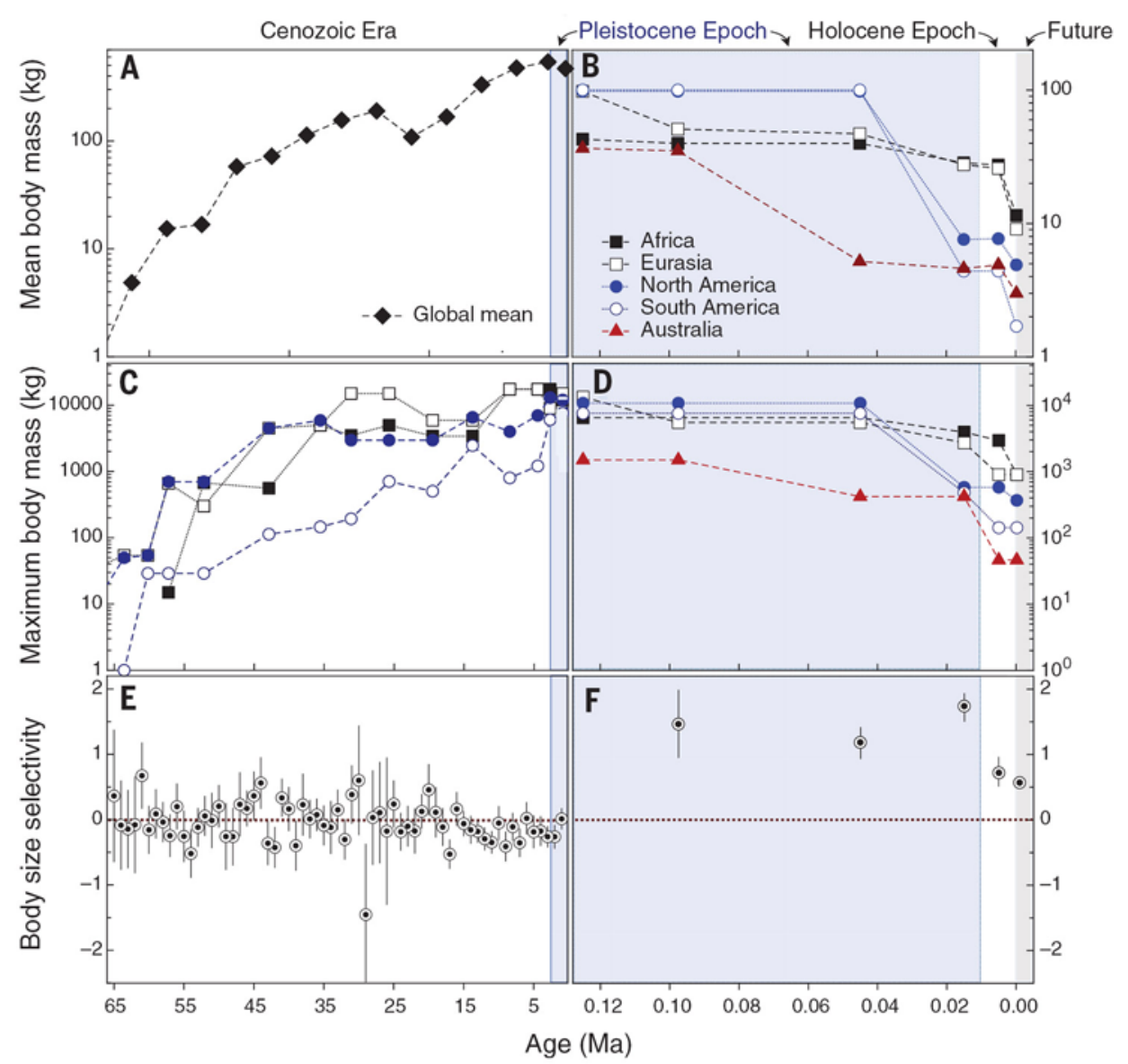

Fig. 3. Body size and its influence on extinction risk. (A) Global mean body size over the Cenozoic (65 to $1 \mathrm{Ma}$ ago). (B) Mean body size by continent over the late Quaternary (past $125 \mathrm{ka}$ ). (C) Maximum body size across the Cenozoic by continent. (D) Maximum body size over the late Quaternary and into the future. (E) Size selectivity coefficients across the entire Cenozoic fossil record. (F) Size selectivity of late Quaternary extinctions. Bars indicate $95 \% \mathrm{Cl}$. All masses are in kilograms. Light blue shading indicates late Pleistocene, white shading indicates Holocene, and gray shading indicates the future (+200 years). Ages here and elsewhere are plotted as midpoint of time interval.

for large-bodied mammals before the spread of hominins. We found no relationship between temperature change over the Cenozoic and size bias of extinction; neither small nor large mammals were more vulnerable to extinction during times of high climate variability (table S3 and fig. S4). The probability that the late Pleistocene and Cenozoic selectivity coefficients came from the same distribution was very low $(P<0.001)$, given either log likelihood or nonparametric tests (fig. S3). 


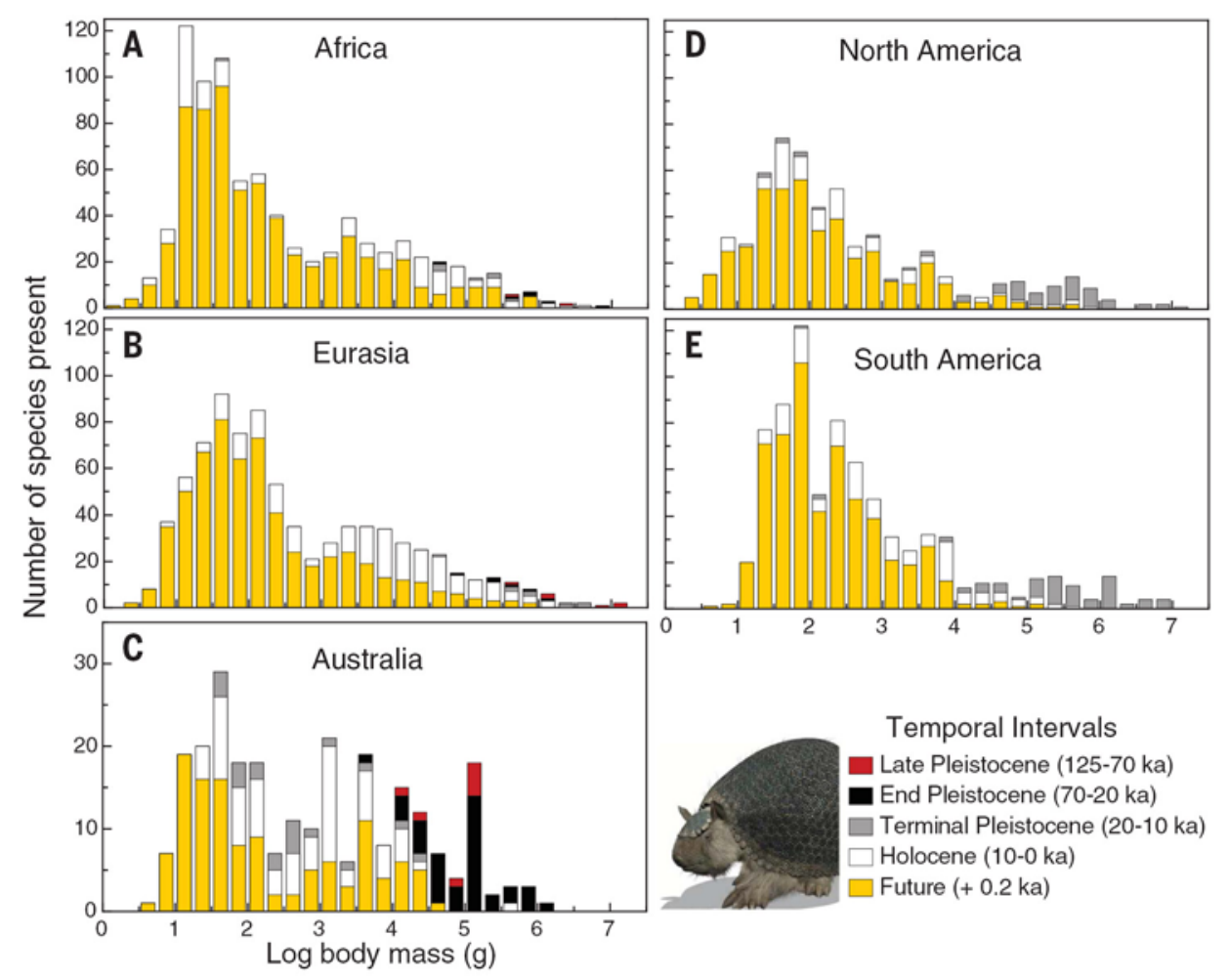

Fig. 4. The body size distribution of terrestrial, nonvolant mammals on each continent over the late Quaternary. (A) Africa. (B) Eurasia. (C) Australia. (D) North America. (E) South America. Body sizes for each temporal interval are plotted; distributions are overlaid from oldest to youngest. Yellow shading indicates the predicted distribution in the future, if vulnerable species go extinct.

Moreover, grouped as a single extinction event (as they would appear to a future paleontologist), the Quaternary extinction pulse was by far the most selective episode of extinction in the Cenozoic (Fig. 1). Such pronounced size selectivity is highly unusual in other fossil records; larger-bodied vertebrates and mollusks did not experience increased extinction risk over the Cenozoic or during the five mass extinction events (27). Because a reported signature of human hunting is size selectivity $(24,25)$, our results are consistent with the hypothesis that hominin activities contributed to extinctions long before the terminal Pleistocene.

The late Quaternary biodiversity losses led to dramatic, time-transgressive shifts in both mean and maximum body mass on each continent (Fig. 3), which followed hominin dispersal patterns (15) and began much earlier than previously suspected. Because body size 
distributions are related to the size of the landmass (28), the largest average or maximum body mass would be expected on Eurasia, followed by Africa, then North and South America, and the smallest on Australia. This expectation was largely met in the late Pleistocene (Fig. 3 ), but Africa was a notable outlier, with a mean body mass $\sim 50 \%$ less than that of Eurasia or the Americas before $125 \mathrm{ka}$ ago (table S1). We hypothesize that the late Pleistocene size distribution in Africa reflects the long prehistory of hominin-mammal interactions (29). This finding suggests that the homogenization of natural ecosystems was a consequence of hominin behavior in general and not specific to $\mathrm{H}$. sapiens. Over the following $\sim 100 \mathrm{ka}$, mean body mass dropped dramatically - first by $50 \%$ in Eurasia, and then by an order of magnitude in Australia-while remaining largely unchanged in the Americas until the terminal Pleistocene. Thus, for most of the late Quaternary, mean and maximum body masses were larger in the Americas than elsewhere-a pattern largely exceptional in the mammalian fossil record (Fig. 3 and table S1) (28). By the terminal Pleistocene, other hominin species were extinct, and the remaining $\mathrm{H}$. sapiens had developed efficient long-range weapons (11). The latter likely contributed to the severity of the extinction in the New World (Fig. 1), with 11.5 and 9.7\% of nonvolant terrestrial species lost in North and South America, respectively (tables S1 and S2). The loss of biodiversity resulted in a greater than 10 -fold drop in both mean and maximum body mass, which was a steeper decline than elsewhere (Fig. 3, B and D). For example, mean mass of nonvolant terrestrial mammals in North America fell from 98.0 to $7.6 \mathrm{~kg}$ (table S1).

Future extinctions will continue the pattern of biodiversity loss and body size downgrading (fig. S1). If all species currently at risk are eventually driven extinct, 22.4 to $53.7 \%$ of mammals will be lost relative to $125 \mathrm{ka}$ ago (table S2). This will further decrease mean body mass in North America from 7.7 to $4.9 \mathrm{~kg}$ (Fig. 3B and table S1); similar declines are predicted for other continents. Thus, the largest mammal on earth in a few hundred years may well be a domestic cow (Bos taurus) at $\sim 900 \mathrm{~kg}$. Furthermore, the loss of currently endangered species would reduce terrestrial mammal body mass to the lowest values in the past $45 \mathrm{Ma}$ (Fig.3, A and C, as compared with Fig. 3, B and D). The last time the body size distribution of terrestrial vertebrates was similarly disrupted was $\sim 66 \mathrm{Ma}$ ago, during the end-Cretaceous mass extinction. 
Because body size is strongly linked to most biological rates and processes (30), the extirpation of large mammals led to a fundamental restructuring of energy flow through mammal communities over the late Quaternary. The severe body size downgrading - a truncation of more than two orders of magnitude-resulted in substantial shifts from bimodal toward unimodal size distributions (Fig. 4 and fig. S1). Homogenization of distributions continued through the Holocene and is predicted to continue into the future (Fig. 4 and table S4). Extinctions also led to changes in the proportional representation of trophic guilds, especially herbivores (fig. S2). In the future, continental distributions will be severely skewed toward smaller mammals (Fig. 4) -in particular, rodents (fig. S2). Ecological principles suggest that changes in energy flow over the Pleistocene likely led to compensatory changes, potentially numerical responses by surviving smallerbodied mammals to maintain ecosystem homeostasis (31). By the Holocene, however, humans were a strong influence on energy flow within ecosystems. Global expansion was accompanied by increased human densities (17) and animal domestication (10). By historical time, the terrestrial biosphere was transformed from one dominated by wild animals into one dominated by humans and their livestock, many provisioned with domesticated crops $(2,5,10)$. Today, the biomass of the $>4.5$ billion domesticated animals on Earth exceeds estimates for wild mammals at the terminal Pleistocene (10).

Our study highlights the long and sustained influence of humans and other hominins on terrestrial ecosystems. As Neandertals, Denisovans, and humans spread across the globe over the late Quaternary, a highly size-biased extinction followed, a pattern distinct in the Cenozoic mammal record. The subsequent downgrading of body size was severe and differentially targeted herbivores. Thus, contemporary biodiversity loss is part of a trend spanning more than $125 \mathrm{ka}$, with expected future extinctions of greater magnitude, but reduced size selectivity, than in the past. The homogenization of ecosystems has dramatically influenced the past, present, and future role of wild mammals in the terrestrial biosphere.

Acknowledgments - We thank the Smith laboratory for its enthusiasm and assistance and P. J. Wagner for comparing distributions of selectivity coefficients. 
Funding: Funding was provided by NSF grants DEB-1555525, DEB-1257625, and EAR-1151022.

Author contributions: F.A.S., R.E.E.S., and S.K.L. compiled and analyzed data; J.L.P. performed additional analyses; F.A.S. wrote the first draft; and all coauthors provided comments.

Competing interests: None

Data and materials: Data needed to evaluate the conclusions are present in the paper or the supplementary materials.

\section{References}

1. M. Cardillo et al., Science 309, 1239-1241 (2005).

2. A. D. Barnosky et al., Nature 471, 51-57 (2011).

3. J. A. Estes et al., Science 333, 301-306 (2011).

4. G. Ceballos et al., Sci. Adv. 1, e1400253 (2015).

5. R. Dirzo et al., Science 345, 401-406 (2014).

6. World Wildlife Fund, Living Planet Report 2016. Risk and Resilience in a New Era (Gland, 2016).

7. F. A. Smith, C. E. Doughty, Y. Malhi, J.-C. Svenning, J. Terborgh, Ecography 39, 99-108 (2016).

8. C. N. Johnson, Proc. Biol. Sci. 276, 2509-2519 (2009).

9. C. E. Doughty et al., Proc. Natl. Acad. Sci. U.S.A. 113, 868-873 (2016).

10. F. A. Smith et al., Proc. Natl. Acad. Sci. U.S.A. 113, 874-879 (2016).

11. S. K. Lyons et al., Evol. Ecol. Res. 6, 339-358 (2004).

12. P. L. Koch, A. D. Barnosky, Annu. Rev. Ecol. Syst. 37, 215-250 (2006).

13. W. Zuo, F. A. Smith, E. L. Charnov, Am. Nat. 182, 524-531 (2013).

14. R. Nielsen et al., Nature 541, 302-310 (2017).

15. S. Oppenheimer, Philos. Trans. R. Soc. London B Biol. Sci. 367, 770-784 (2012).

16. Q. D. Atkinson, R. D. Gray, A. J. Drummond, Mol. Biol. Evol. 25, 468-474 (2008).

17. A. Timmermann, T. Friedrich, Nature 538, 92-95 (2016).

18. S. L. Carto, A. J. Weaver, R. Hetherington, Y. Lam, E. C. Wiebe, J. Hum. Evol. 56, 139-151 (2009).

19. P. Mellars, J. C. French, Science 333, 623-627 (2011).

20. W. Roebroeks, P. Villa, Proc. Natl. Acad. Sci. U.S.A. 108, 5209-5214 (2011).

21. Materials and methods are available as supplementary materials.

22. D. A. Burney, T. F. Flannery, Trends Ecol. Evol. 20, 395-401 (2005).

23. S. T. Turvey, Ed., Holocene Extinctions (Oxford Univ. Press, 2009).

24. T. Surovell, N. M. Waguespack, in American Megafaunal Extinctions at the End of the Pleistocene, G. Hayes, Ed. (Springer, 2009), pp. 77-105.

25. J. Alroy, Science 292, 1893-1896 (2001).

26. R. E. Plotnick, F. A. Smith, S. K. Lyons, Ecol. Lett. 19, 546-553 (2016). 
27. J. L. Payne, A. M. Bush, N. A. Heim, M. L. Knope, D. J. McCauley, Science 353, 1284-1286 (2016).

28. F. A. Smith et al., Science 330, 1216-1219 (2010).

29. T. Surovell, N. Waguespack, P. J. Brantingham, Proc. Natl. Acad. Sci. U.S.A. 102, 6231-6236 (2005).

30. R. H. Peters, The Ecological Implications of Body Size (Cambridge Univ. Press, 1983).

31. S. K. M. Ernest, J. H. Brown, Ecology 82, 2118-2132 (2001).

\section{Supplementary Materials}

- Materials and Methods

- $\quad$ Figures S1 to S4

- $\quad$ Tables S1 to S7

- References (32-197) 


\section{Smith et al. Body size downgrading of mammals over the late Quaternary}

\section{SUPPLEMENTARY MATERIALS}

Materials and Methods

Supplementary References 32-197

Figures S1 to S4

Tables S1 to S7

\section{MATERIALS AND METHODS}

\section{A. Data compilation and treatment}

Data for the analyses were obtained from an updated version of MOM v10 (32), which included taxonomy, continental distribution, body mass, conservation status, and trophic affiliation for late Quaternary mammals (table S6). Additional species of extinct mammals were added from (33-160). Taxonomy was updated to agree with Mammal Species of the World v3.0 (MSW3; 34) as of September 2017. Current conservation status for each species was extracted from the IUCN Red List of Threatened Species (35). Because of differences or synonymies in the taxonomy recognized by the IUCN and that employed by Mammal Species of the World, the conservation status of 11 modern species could not be readily determined. Because our focus was on nonvolant, terrestrial mammals, we eliminated all bats, marine or oceanic species, insular mammals, and introductions. Missing last occurrence dates for fossil species were obtained from the literature (36-159) and the Paleobiology database (160). Species were coded as 'extant' or 'extinct'. Extinct species were further binned into one of four time intervals representing the approximate date of their extinction - late Pleistocene (125-70 ka), end Pleistocene (70-20 ka), terminal Pleistocene (20-10 ka), and Holocene (10-0 ka). Our analysis ignores speciation; we assume that extant mammal species were also present at the late Pleistocene. Because the average species 'lifespan' of a mammal is 1-2 million years (163167), this assumption likely introduces minimal error into our computations. This assumption also minimizes the effects of taphonomy and makes estimates of extinction selectivity for the late Pleistocene comparable to the estimates for the future. We also note that although the Late Quaternary fossil mammal record is quite good, there likely remain a small number of animals not yet described, and for some animals, affiliations and/or species status are unclear. 
Additionally, any mammal species listed on the IUCN Red List (36) as near threatened, vulnerable, endangered, critically endangered, or extinct in the wild, was coded into a $5^{\text {th }}$ category as a 'Future' (+200 years) extinction. We choose to include these because the conservation status of virtually all mammals in those threat categories has deteriorated over time; and current studies suggest their extinction is highly likely (1-6). The Red List is, of course, not a perfect predictor of the future state of the biosphere, but it represents our best and most comprehensive assessment of the probability that any given species will go extinct in the near future. Our analysis is somewhat conservative in that we do not include those mammals falling into the 'data deficient' category. These are mammals that are so poorly studied or rare that their conservation status is unclear; many likely fall into a threat category. We hypothesize that the 'Future' scenario represents a time approximately two centuries in the future, but the precise timing does not influence the outcome of the analysis. Setting a different threat level classification as the threshold for placing species into the extinct versus surviving categories for the future scenario does not substantially alter the outcome of the selectivity analysis. Our final data set for the late Quaternary included 3301 mammalian species (table S6). Of these, 37 were missing body mass information and so could not be used in analyses requiring a mass estimate. We computed statistical moments for extant and extinct species at each time interval using the 'moments' package in R $(161,162)$; summary statistics and analyses are presented in tables S1- S4 and fig. S1. All analyses for both the late Quaternary and Cenozoic datasets were conducted on log10 transformed masses.

Trophic groups from MOM were collapsed into four general categories: herbivore, carnivore, insectivore and omnivore. Any species whose primary dietary designation was listed as a browser or grazer, irrespective of the type of forage (e.g., bamboo, roots and tubers, etc.), was considered an herbivore. Those whose primary designation indicated that were eating meat of any kind, including fish, but excluding insects and earthworms, was assigned as a carnivore. Species that exclusively eat insects were assigned as insectivores, and species whose dietary assignations were ambiguous or that mixed major dietary modes was considered an omnivore. Of the 3301 mammals in our dataset (table S6), we were unable to determine trophic classification for 150 . These were mostly small, poorly described extant species ( $N=145)$, and 23 of these were also missing body mass information. Only five of these mammals were extinct, and these were equally spread among three continents. Ten animals with diet information were missing a body mass estimate. We computed the percentage of nonvolant terrestrial mammals of each guild over time on each continent (fig. S2).

The Cenozoic mammal database was compiled by co-author Lyons. Initial species lists were downloaded from the Paleobiology Database (160). Body masses were compiled from a variety of literature sources $(32,168-194)$. When 
no mass was available, it was estimated from measurements on teeth using taxon-specific (usually order or familylevel) regressions taken from Damuth and MacFadden (195). The M1 length or area was used preferentially, since regressions computed with that tooth have higher precision (195). The current database contains estimates on the body size of 3246 mammal species beginning in the late Jurassic through the late Pleistocene. We used the last 65 Ma for our analyses because Mesozoic data were too sparse to calculate robust measures of extinction selectivity at $1 \mathrm{Ma}$ intervals. This left 2786 species for analysis (table S7). To obtain information on tropic affiliations, diet information was downloaded from the Paleobiology Database (PBDB). The Paleobiology Database often included information on diet at a finer scale than needed for our analyses. Therefore, diet was reclassified into carnivore, omnivore, herbivore, and insectivore as done for the modern/late Quaternary mammals. For example, a species labeled as a browser or grazer was coded as an herbivore in our analysis. If a species was classified in the PBDB as both meat eating and plant eating, we classified it as an omnivore. If a species was only classified to the coarse levels we needed, we accepted the PBDB classification. Table S7 includes the original PBDB information as well as our reclassifications. We also performed analyses at the guild level for rodents, herbivores, and carnivores. Rodents were defined based on membership in the order Rodentia. Herbivores were classified as described above. Carnivores were classified based on taxonomic identity (e.g., membership in the orders Carnivora or Creodontia). In this instance, mixed feeders such as Ursus (bears) were classified as being in the carnivore guild.

\section{B. Analysis of selectivity}

Extinction selectivity was quantified using logistic regression analysis. Survival status was treated as a binary outcome variable ('survived into the next time interval' versus 'did not survive') and $\log _{10}$-transformed body size was treated as the continuous predictor variable. The selectivity coefficients (table S3; Figs. 1,2) reflect the amount of change in the odds of extinction associated with a one $\log _{10}$-unit change in body mass. Analyses were conducted both as single regressions with log-transformed body mass as the only predictor of extinction and as multiple regressions (for the Late Pleistocene through Future intervals) in which extinction was predicted by body mass, trophic guild, and continent (table S5). Interactive effects were not considered due to the many potential interactions among trophic categories and continents; the consistency of selectivity among continents and trophic guilds (Fig. 2) suggests that interactive effects do not dominate the signal in this dataset. The associations between extinction and body mass obtained from the multiple regression analysis were larger than those obtained from the single regression, but the single regression results are reported in the main text because the fossil record dataset lacks sufficient information to conduct the same multiple regression analysis (table S5). In addition, the late Pleistocene and end-Pleistocene

intervals contain too few victims in some trophic and continent categories to yield stable coefficients for all categories of the trophic and continent predictors. All analyses were performed using the R programming language 
(version 3.2.1; 161) within the RStudio platform (version $0.99 .451 ; 162)$, using the $g \operatorname{lm}()$ function. For the analysis of Cenozoic fossil mammals, survival was assessed across million-year intervals.

We evaluated whether the distribution of late Pleistocene selectivity coefficients was significantly different from the distribution of Cenozoic coefficients in two ways. Both assess whether the distribution of selectivity coefficients come from the same overall distribution. Because the distributions of coefficients are fairly symmetrical around zero (Fig. 1; Fig. S3), we fit the selection coefficients to normal distributions: one for all the coefficients, one for Cenozoic only, and a third for late Pleistocene coefficients only. We calculated the exact probability of getting the Cenozoic coefficients and late Pleistocene coefficients from the overall normal distribution, and then separately for the Cenozoic and late Pleistocene distributions. We applied a standard log likelihood ratio test given that the null hypothesis (i.e., the late Pleistocene and Cenozoic share the same normal distribution) is a special case of the test hypothesis, that the Cenozoic and late Pleistocene have independent distributions. The second test was a Mann Whitney test, a non-parametric analog of the t-test, which assessed the probability of ranked coefficients being distributed as evenly as they were in the actual data set given a null assumption of an even distribution of ranks in both partitions. Both tests rejected the null hypothesis of a single distribution with extremely high levels of confidence (fig. S3). While many authors have noted differences between survivors and victims of the terminal Pleistocene and Holocene events, and noted the size bias in contemporary extinction risk (e.g., 1-7,11-13, 22-25,27), rarely have patterns been examined within the context of the entire Cenozoic mammal record. Alroy (196) concluded that the magnitude of terminal Pleistocene extinctions was higher than during five climatic 'crisis' events in North America with substantially more bias against large-bodied mammals, but he did not examine patterns on other continents. Thus, our work represents a unique and comprehensive characterization of extinction across the entire Cenozoic record.

\section{Analysis of the effect of climate on extinction selectivity.}

We examined the potential influence of temperature and temperature fluctuations on the body size selectivity of extinction using linear regression analysis. Climate data were extracted from a global compilation of Cenozoic oceanic oxygen and carbon isotopic data derived from fossil foraminifera (197) and available at ftp://ftp.ncdc.noaa.gov/pub/data/paleo/contributions by author/zachos2001/zachos2001.txt. We calculated mean temperature for the last 65 million years by averaging all values within one-million-year intervals (i.e., for 1 million years, temperatures were averaged from 2-1 million years; table S3). We ran linear regressions using the 'Im' function in $\mathrm{R}$ using mean temperature and standard deviation of temperature within bins, and the difference between the mean temperatures of adjacent time bins (fig. S4). When analyzing the relationship between standard 
deviation of temperature and selectivity, selection against large size was analyzed separately from selection against small size. This is because we wanted to know if larger climate fluctuations were associated with larger selectivity. We found no significant relationship between body size selectivity and any of the measures of climate: neither warmer, nor cooler temperatures led to preferential extinction of large (or small-bodied) mammals (fig. S4). Similarly, fluctuations in climate also did not lead to body size bias in extinction risk.

\section{Tests of body size distributions}

For each time step, we wanted to know if the extinctions that occurred resulted in significant changes to the shape of the body size distribution for that continent. Changes to body size distributions over the last 125 ka were assessed using Kolomorgorov-Smirnov tests in R studio (162). For each continent and time period, body size distributions were compared to the preceding time period to determine if there were significant differences associated with the extinctions that occurred at that time (Table S4). 


\section{Global Mammals}

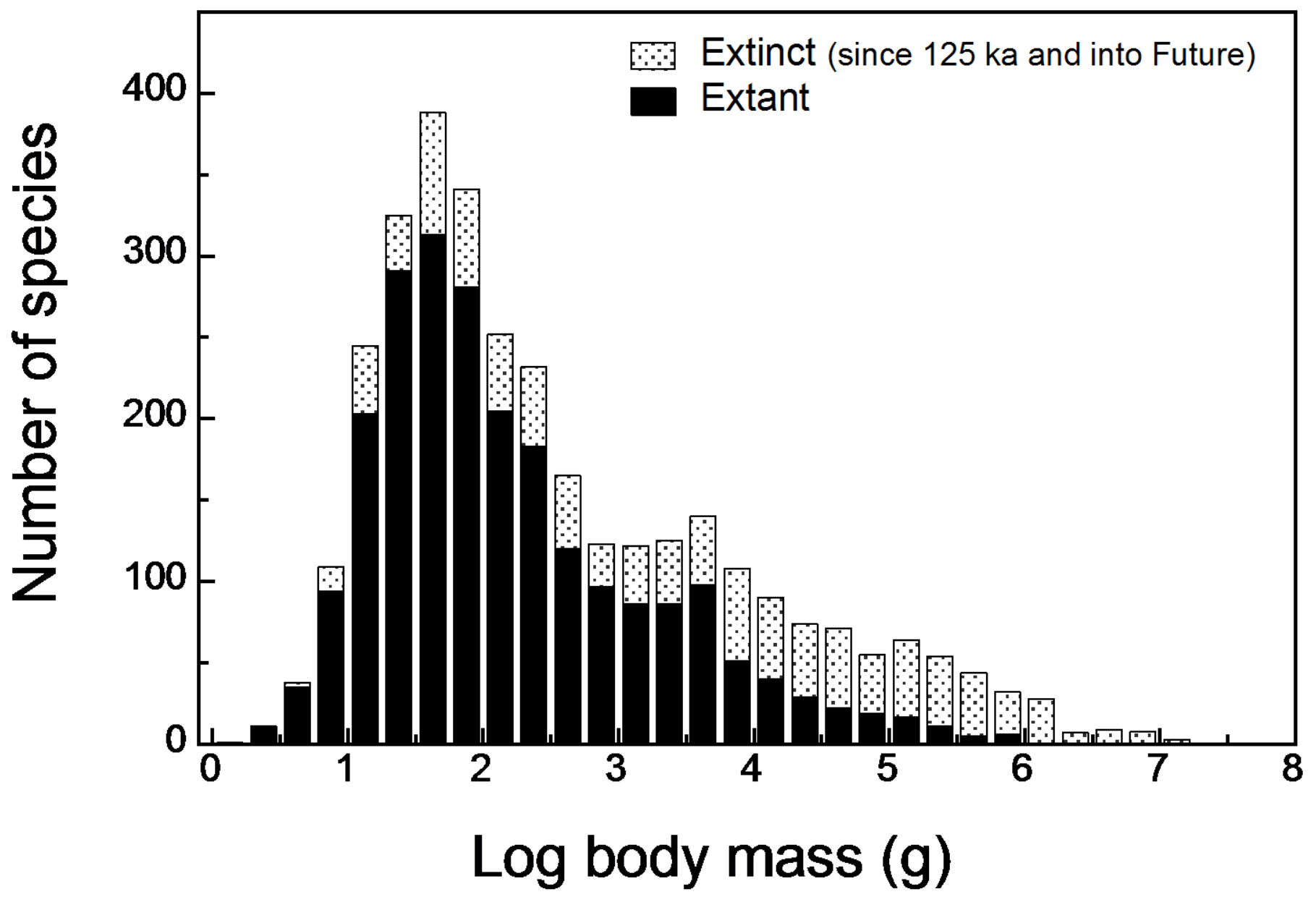

Fig. S1. The global body size distribution of terrestrial, nonvolant mammals. Black stipling is what the global distribution of mammals would look like without human-mediated extinctions over the late Quaternary; it includes extinct mammals from the late Pleistocene through present as well as those projected to go extinct in the near Future (e.g., LP-F). The black distribution represents the remaining mammals, and is what the global distribution of mammal body mass will look like in ca. 200 years if further extinctions occur as described in the text. 


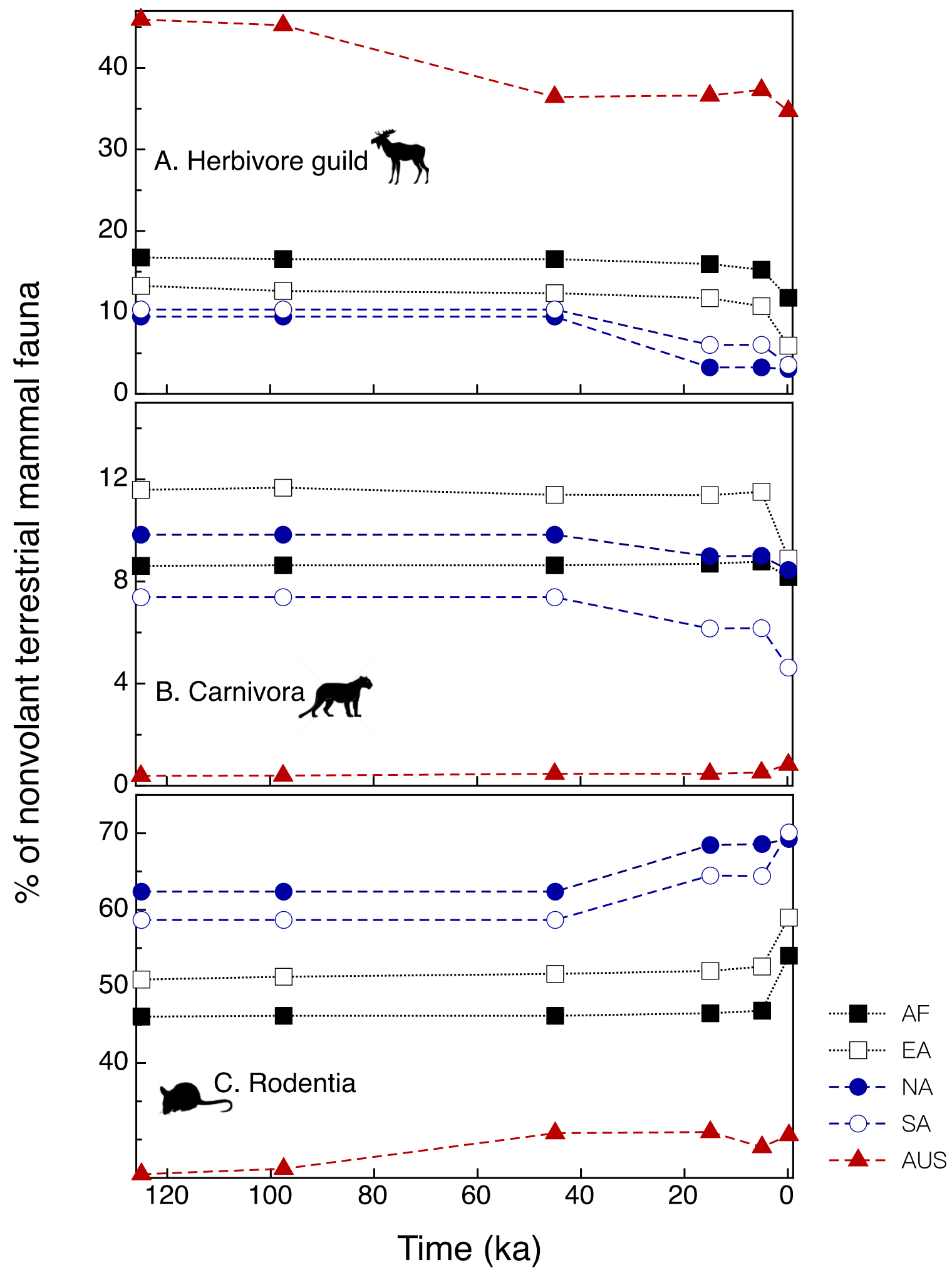

Fig. S2. Representation of herbivores, carnivores and rodents in the terrestrial, nonvolant mammalian continental fauna. Legend: $\mathrm{AF}=$ Africa, $\mathrm{EA}=$ Eurasia, $\mathrm{NA}=$ North America, $\mathrm{SA}=$ South America. 


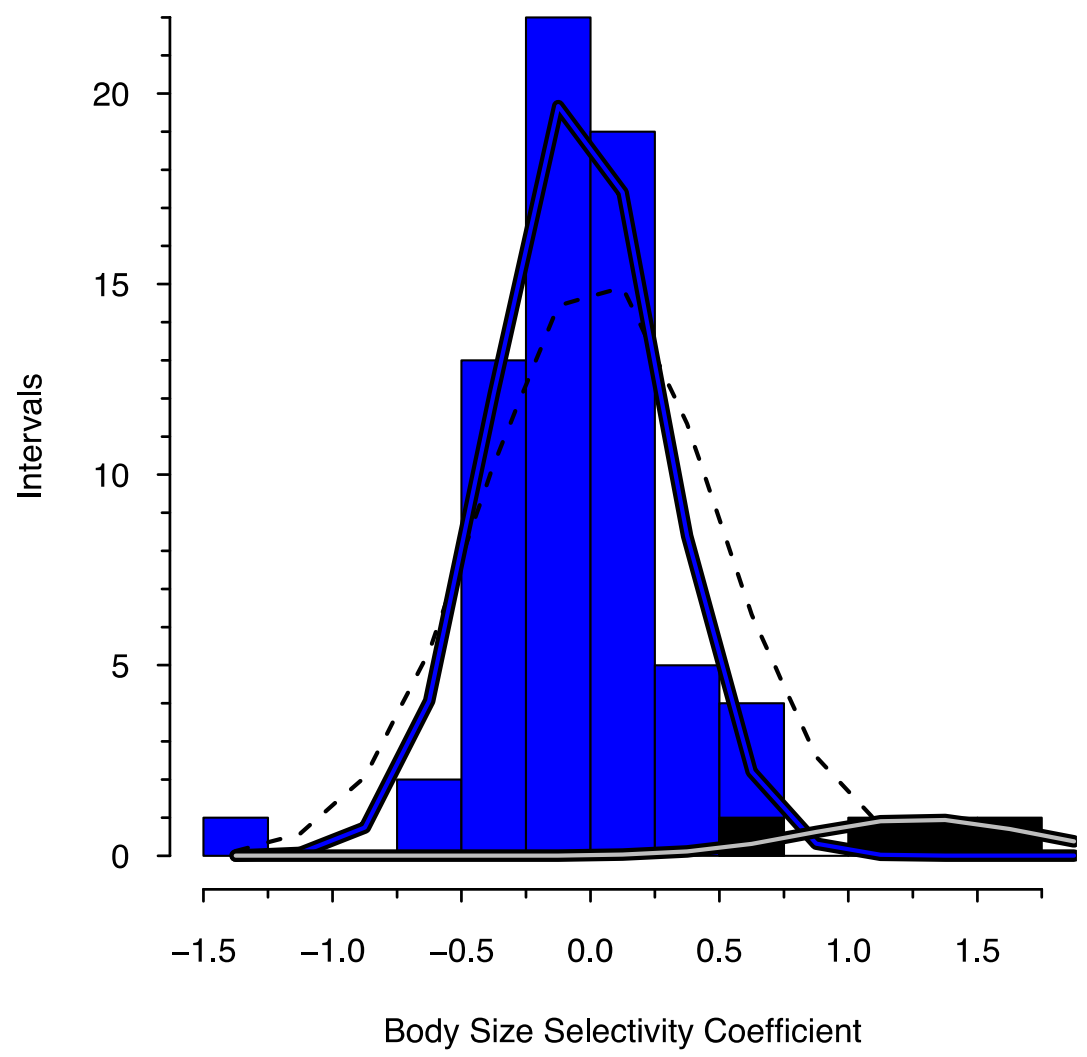

Fig. S3. Stacked histogram of body size selectivity coefficients. Cenozoic indicated by blue shading and late Pleistocene by black shading. Lines represent the separate best fit normal distributions: all coefficients (dashed line); Cenozoic (solid blue line with black outline); late Pleistocene (solid white line with black outlines). Mann-Whitney tests indicate that the Cenozoic and late Pleistocene selectivity coefficients came from different distributions $(U=0, p=0.0008)$. The log likelihood of the null hypothesis was -136.6 , and the log likelihood of the test hypothesis was -116.0 (i.e., the distributions of selectivity coefficients were $8.4 \times 10^{8}$ times more probable given the test hypothesis of two different distributions). This is significant at $p=1.5 \times 10^{-10}$ using a log likelihood ratio test. 

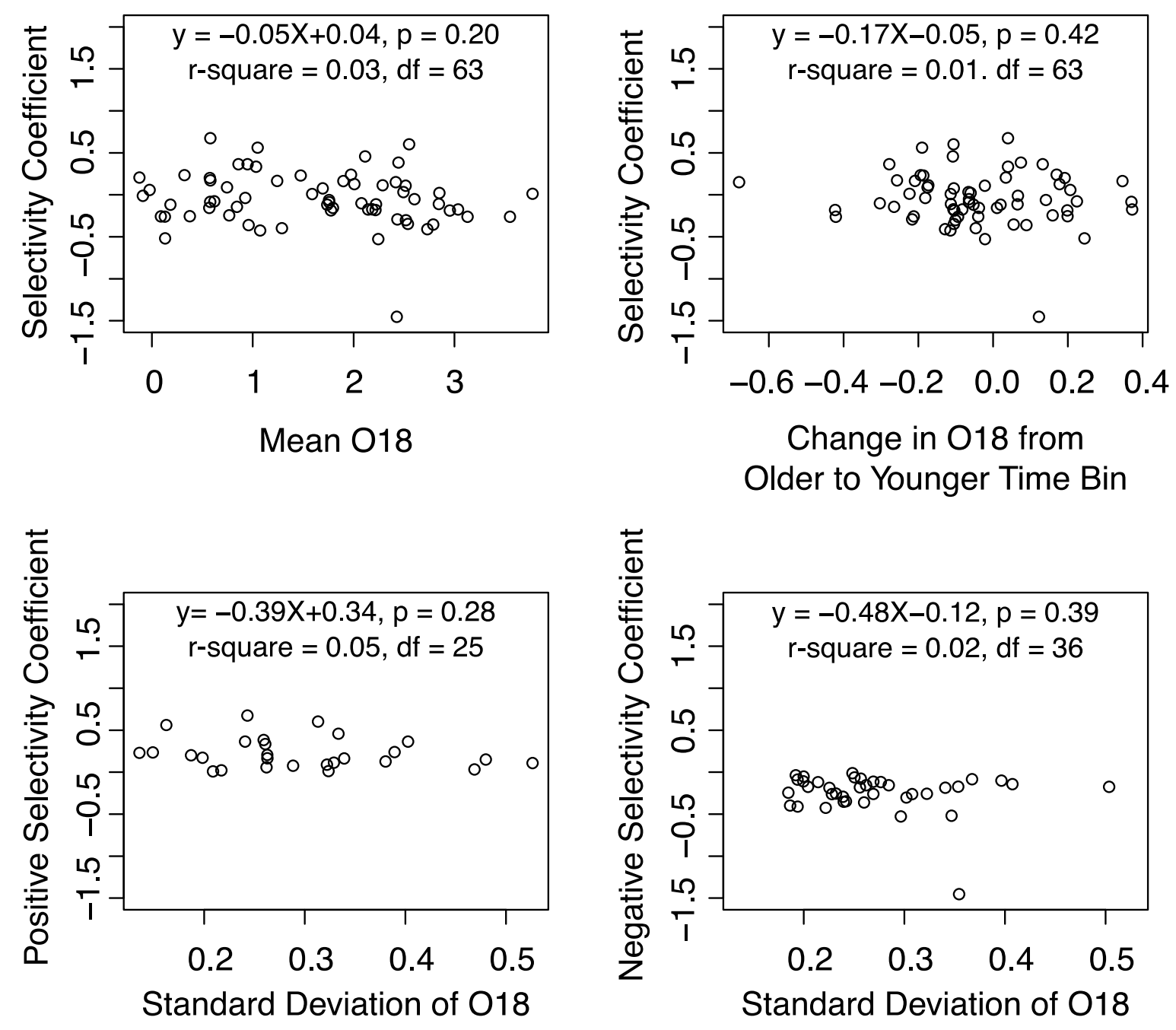

Fig. S4. Results of linear regressions between temperature and body size selectivity. OLS regressions were used to determine the if body size selectivity was related to mean temperate (upper left), first differences of temperature from an older to younger temporal bin (upper right), or standard deviation of temperature (lower panels). The relationship between positive (lower left) and negative (lower right) selectivity coefficients and climate variability within a temporal bin were analyzed separately (See SI part C). Body size selectivity was never significantly related to any of the measures of climate and climate variability we used. Climate data are from (197) and body size coefficients are from table S3. Results of regression equations are presented in equation form with each panel. $p=p$-value, $r$-square $=r^{2}, d f=$ degrees of freedom. 
Table S1. Summary statistics of extinct and extant terrestrial, non-volant, native mammals on each continent for each time period. More LP, EP and TP extinctions are known, but mass estimates are unavailable. These are without exception large-bodied forms, which would likely increase the degree of selectivity for these events. Mammal species without body mass estimates are excluded from statistical analyses.

SURVIVING SPECIES

EXTINCT SPECIES

\begin{tabular}{|c|c|c|c|c|c|c|c|c|c|c|c|c|c|c|c|c|c|}
\hline Data Set & Count & Mean (g) & Median (g) & $\operatorname{Min}(\mathrm{g})$ & $\operatorname{Max}(\mathrm{g})$ & Std. Deviation & Skew & Kurtosis & Data Set & Count & Mean (g) & Median (g) & $\operatorname{Min}(\mathrm{g})$ & $\operatorname{Max}(g)$ & Std. Deviation & Skew & Kurtosis \\
\hline \multicolumn{18}{|c|}{ Prior to extinctions } \\
\hline GLOBAL & 3301 & 80711 & 145 & 2 & 13300000 & 593631.3 & 13.91 & 236.06 & & & & & & & & & \\
\hline AF & 844 & 47409 & 81 & 2 & 6500000 & 327543.9 & 13.48 & 223.06 & & & & & & & & & \\
\hline AUS & 259 & 36567 & 525 & 4 & 1500000 & 138935.5 & 6.92 & 60.35 & & & & & & & & & \\
\hline EA & 837 & 97798 & 157 & 2 & 13300000 & 785549.9 & 13.46 & 203.62 & & & & & & & & & \\
\hline NA & 590 & 97998 & 124 & 2 & 10800000 & 703126.3 & 11.67 & 151.27 & & & & & & & & & \\
\hline SA & 771 & 100324 & 225 & 5 & 7580000 & 582939.7 & 8.94 & 92.58 & & & & & & & & & \\
\hline \multicolumn{18}{|c|}{ LATE PLEISTOCENE EXTINCTIONS (LP) -- 125-70 ka } \\
\hline GLOBAL & 3288 & 69019 & 143 & 2 & 10800000 & 475297.5 & 12.95 & 205.16 & GLOBAL & 13 & 3004538 & 173000 & 10000 & 13300000 & 5034024.0 & 1.37 & 3.06 \\
\hline AUS & 252 & 35034 & 500 & 4 & 1500000 & 140270.1 & 6.94 & 60.01 & AUS & 7 & 91771 & 100000 & 10000 & 173000 & 57247.1 & -0.16 & 1.91 \\
\hline EA & 831 & 51037 & 154 & 2 & 5500000 & 327429.3 & 12.10 & 174.92 & EA & 6 & 6402765 & 5224779 & 549541 & 13300000 & 5922836.7 & 0.17 & 1.21 \\
\hline NA & 590 & 97998 & 124 & 2 & 10800000 & 703126.3 & 11.67 & 151.27 & NA & 0 & & & & & & & \\
\hline SA & 771 & 100324 & 225 & 5 & 7580000 & 582939.7 & 8.94 & 92.58 & SA & 0 & & & & & & & \\
\hline \multicolumn{18}{|c|}{ END PLEISTOCENE EXTINCTIONS (EP) -- 70-20 ka } \\
\hline GLOBAL & 3237 & 65728 & 136 & 2 & 10800000 & 475095.8 & 13.14 & 208.74 & GLOBAL & 51 & 293366 & 118000 & 5000 & 2000000 & 438160.2 & 2.39 & 8.37 \\
\hline AF & 838 & 44514 & 80 & 2 & 6500000 & 320460.3 & 14.18 & 242.88 & AF & 6 & 1250000 & 1250000 & 500000 & 2000000 & 1060660.2 & 0.00 & 1.00 \\
\hline AUS & 213 & 4646 & 133 & 4 & 420000 & 29251.6 & 13.56 & 192.69 & AUS & 39 & 201000 & 100000 & 5000 & 1500000 & 302892.5 & 2.72 & 10.58 \\
\hline EA & 825 & 47123 & 150 & 2 & 5500000 & 322446.6 & 12.64 & 187.29 & EA & 6 & 574870 & 400000 & 69984 & 1599558 & 562409.7 & 1.08 & 2.87 \\
\hline \multicolumn{18}{|c|}{ TERMINAL PLEISTOCENE EXTINCTIONS (TP) -- 20-10 ka } \\
\hline GLOBAL & 3080 & 17814 & 119 & 2 & 3940034 & 132754.2 & 17.84 & 413.80 & GLOBAL & 157 & 1021194 & 317000 & 20 & 10800000 & 1854113.5 & 2.86 & 11.29 \\
\hline AF & 830 & 29547 & 79 & 2 & 3940034 & 198113.8 & 14.36 & 248.16 & $\mathrm{AF}$ & 8 & 1806356 & 899995 & 44500 & 6500000 & 2273818.6 & 1.45 & 3.66 \\
\hline AUS & 213 & 4646 & 133 & 4 & 420000 & 29251.6 & 13.56 & 192.69 & AUS & 0 & & & & & & & \\
\hline EA & 817 & 27608 & 147 & 2 & 2720000 & 155848.9 & 11.03 & 153.32 & EA & 8 & 1986371 & 774921 & 39994 & 5500000 & 2194532.8 & 0.77 & 1.87 \\
\hline NA & 523 & 7646 & 84 & 2 & 579255 & 45906.0 & 8.64 & 84.76 & NA & 67 & 812597 & 236500 & 20 & 10800000 & 1965638.1 & 3.71 & 16.41 \\
\hline SA & 697 & 4409 & 164 & 5 & 480000 & 26252.9 & 12.25 & 187.83 & SA & 74 & 1028830 & 350000 & 144 & 7580000 & 1644521.8 & 2.45 & 8.40 \\
\hline \multicolumn{18}{|c|}{ HOLOCENE EXTINCTIONS (H) -- 10-0 ka } \\
\hline GLOBAL & 3039 & 16840 & 115 & 2 & 3940034 & 131824.5 & 18.35 & 431.05 & GLOBAL & 41 & 91102 & 3250 & 38 & 900000 & 177419.7 & 2.85 & 12.19 \\
\hline AF & 820 & 27464 & 77 & 2 & 3940034 & 196185.3 & 14.86 & 261.05 & AF & 10 & 218228 & 150000 & 55 & 900000 & 284658.5 & 1.69 & 4.77 \\
\hline AUS & 193 & 4859 & 127 & 4 & 420000 & 30653.9 & 12.99 & 176.14 & AUS & 20 & 2591 & 277 & 38 & 30000 & 6848.4 & 3.50 & 14.30 \\
\hline EA & 808 & 25854 & 147 & 2 & 2720000 & 155101.0 & 11.31 & 158.51 & EA & 9 & 180796 & 149000 & 12000 & 431250 & 153112.1 & 0.39 & 1.76 \\
\hline NA & 522 & 7659 & 84 & 2 & 579255 & 45949.2 & 8.63 & 84.59 & NA & 1 & 945 & 945 & 945 & 945 & & & \\
\hline SA & 696 & 4416 & 165 & 5 & 480000 & 26271.3 & 12.24 & 187.57 & SA & 1 & 97 & 97 & 97 & 97 & & & \\
\hline \multicolumn{18}{|c|}{ FUTURE EXTINCTIONS (F) -- ca. +0.2 ka } \\
\hline GLOBAL & 2328 & 6944 & 83 & 2 & 900000 & 46621.9 & 12.81 & 201.95 & GLOBAL & 711 & 49151 & 758 & 4 & 3940034 & 256368.8 & 10.04 & 122.95 \\
\hline AF & 649 & 11570 & 62 & 2 & 900000 & 62178.9 & 9.41 & 108.57 & AF & 171 & 87766 & 2109 & 4 & 3940034 & 407466.0 & 7.44 & 63.13 \\
\hline AUS & 120 & 2963 & 62 & 4 & 46250 & 7022.8 & 3.45 & 16.72 & AUS & 73 & 7975 & 525 & 20 & 420000 & 49075.6 & 8.27 & 69.97 \\
\hline
\end{tabular}


Table S2. Alpha diversity over the late Quaternary of nonvolant, terrestrial native mammals on each continent. Introduced, volant, and marine species removed from analysis.

\begin{tabular}{|c|c|c|c|c|c|}
\hline Time (ka) & Africa & Australia & Eurasia & $\begin{array}{l}\text { North } \\
\text { America }\end{array}$ & $\begin{array}{l}\text { South } \\
\text { America }\end{array}$ \\
\hline$\sim 125 \mathrm{ka}$ & 836 & 259 & 837 & 590 & 772 \\
\hline Late Pleistocene & 834 & 252 & 831 & 590 & 772 \\
\hline End Pleistocene & 834 & 214 & 825 & 590 & 772 \\
\hline Terminal Pleistocene & 828 & 213 & 817 & 523 & 698 \\
\hline Holocene & 820 & 193 & 808 & 522 & 697 \\
\hline Future & 649 & 120 & 599 & 412 & 548 \\
\hline $\begin{array}{l}\text { \% biodiversity loss from } \\
125 \text { ka to modern }\end{array}$ & 1.9 & 25.5 & 3.5 & 11.5 & 9.7 \\
\hline $\begin{array}{l}\% \text { loss from } 125 \mathrm{ka} \text { to } \\
\text { future }(+0.2 \mathrm{ka})\end{array}$ & 22.4 & 53.7 & 28.4 & 30.2 & 29.0 \\
\hline
\end{tabular}


Table S3. Body size selectivity coefficients and climate data for the Cenozoic

\begin{tabular}{|c|c|c|c|c|c|}
\hline $\begin{array}{c}\text { Time (Ma, unless } \\
\text { otherwise } \\
\text { specified) }\end{array}$ & Coefficient & $\begin{array}{l}\text { Length of } \\
95 \% \\
\text { confidenc } \\
\text { e interval }\end{array}$ & Mean 180 & $\begin{array}{c}\text { Standar } \\
\text { d } \\
\text { Deviatio } \\
\text { n } 180 \\
\end{array}$ & $\begin{array}{l}\text { Difference in } \\
180 \text { between } \\
\text { adjacent } \\
\text { temporal bins }\end{array}$ \\
\hline $\begin{array}{c}\text { Future } \\
\text { (ca. }+200 \text { years) }\end{array}$ & 0.570 & 0.074 & NA & NA & NA \\
\hline $\begin{array}{l}\text { Holocene } \\
\text { (10 to } 0 \text { ka) }\end{array}$ & 0.721 & 0.227 & NA & NA & NA \\
\hline $\begin{array}{c}\text { Terminal } \\
\text { Pleistocene } \\
\text { (20-10 ka) }\end{array}$ & 1.741 & 0.210 & NA & NA & NA \\
\hline $\begin{array}{l}\text { End Pleistocene } \\
\qquad(70-20 \mathrm{ka})\end{array}$ & 1.186 & 0.240 & NA & NA & NA \\
\hline $\begin{array}{l}\text { Late Pleistocene } \\
\qquad(125-70 \mathrm{ka})\end{array}$ & 1.466 & 0.523 & NA & NA & NA \\
\hline 1 & 0.012 & 0.162 & 3.78 & 0.32 & -0.22 \\
\hline 2 & -0.262 & 0.183 & 3.55 & 0.31 & -0.42 \\
\hline 3 & -0.261 & 0.163 & 3.13 & 0.23 & -0.09 \\
\hline 4 & -0.174 & 0.228 & 3.04 & 0.20 & -0.08 \\
\hline 5 & -0.187 & 0.252 & 2.95 & 0.23 & -0.10 \\
\hline 6 & 0.022 & 0.249 & 2.85 & 0.22 & -0.06 \\
\hline 7 & -0.353 & 0.220 & 2.79 & 0.24 & 0.06 \\
\hline 8 & -0.110 & 0.213 & 2.84 & 0.20 & -0.11 \\
\hline 9 & -0.410 & 0.230 & 2.73 & 0.19 & -0.13 \\
\hline 10 & -0.052 & 0.261 & 2.60 & 0.20 & -0.06 \\
\hline 11 & -0.347 & 0.172 & 2.54 & 0.24 & -0.10 \\
\hline 12 & -0.293 & 0.177 & 2.43 & 0.24 & -0.22 \\
\hline 13 & -0.181 & 0.172 & 2.22 & 0.26 & -0.42 \\
\hline 14 & -0.156 & 0.214 & 1.79 & 0.26 & -0.04 \\
\hline 15 & -0.062 & 0.193 & 1.76 & 0.25 & 0.14 \\
\hline 16 & 0.164 & 0.261 & 1.90 & 0.34 & 0.35 \\
\hline 17 & -0.528 & 0.225 & 2.24 & 0.30 & -0.02 \\
\hline
\end{tabular}




\begin{tabular}{|c|c|c|c|c|c|}
\hline 18 & -0.113 & 0.290 & 2.22 & 0.27 & 0.07 \\
\hline 19 & 0.114 & 0.387 & 2.29 & 0.33 & -0.17 \\
\hline 20 & 0.458 & 0.394 & 2.12 & 0.33 & -0.11 \\
\hline 21 & 0.127 & 0.263 & 2.01 & 0.38 & 0.18 \\
\hline 22 & -0.173 & 0.346 & 2.18 & 0.35 & -0.11 \\
\hline 23 & -0.101 & 0.311 & 2.08 & 0.40 & -0.30 \\
\hline 24 & -0.186 & 0.290 & 1.77 & 0.34 & 0.20 \\
\hline 25 & 0.240 & 0.363 & 1.97 & 0.39 & 0.17 \\
\hline 26 & -0.175 & 1.129 & 2.14 & 0.50 & 0.37 \\
\hline 27 & 0.108 & 0.776 & 2.52 & 0.53 & -0.02 \\
\hline 28 & 0.033 & 0.724 & 2.49 & 0.47 & -0.07 \\
\hline 29 & -1.453 & 1.084 & 2.43 & 0.35 & 0.12 \\
\hline 30 & 0.602 & 0.839 & 2.55 & 0.31 & -0.11 \\
\hline 31 & 0.384 & 0.449 & 2.44 & 0.26 & 0.07 \\
\hline 32 & -0.302 & 0.309 & 2.52 & 0.30 & -0.10 \\
\hline 33 & 0.150 & 0.310 & 2.42 & 0.48 & -0.68 \\
\hline 34 & -0.118 & 0.406 & 1.74 & 0.21 & 0.02 \\
\hline 35 & -0.088 & 0.306 & 1.76 & 0.19 & -0.06 \\
\hline 36 & 0.076 & 0.247 & 1.69 & 0.28 & -0.11 \\
\hline 37 & 0.010 & 0.297 & 1.59 & 0.21 & -0.11 \\
\hline 38 & 0.230 & 0.474 & 1.48 & 0.14 & -0.19 \\
\hline 39 & -0.398 & 0.384 & 1.29 & 0.19 & -0.05 \\
\hline 40 & 0.165 & 0.339 & 1.24 & 0.26 & -0.21 \\
\hline 41 & 0.335 & 0.293 & 1.03 & 0.26 & 0.04 \\
\hline 42 & -0.425 & 0.314 & 1.07 & 0.22 & -0.11 \\
\hline 43 & -0.361 & 0.337 & 0.96 & 0.26 & 0.09 \\
\hline 44 & 0.562 & 0.394 & 1.05 & 0.16 & -0.19 \\
\hline 45 & 0.364 & 0.375 & 0.86 & 0.24 & -0.28 \\
\hline 46 & 0.172 & 0.275 & 0.58 & 0.20 & -0.26 \\
\hline 47 & 0.234 & 0.492 & 0.32 & 0.15 & -0.19 \\
\hline 48 & -0.260 & 0.442 & 0.13 & 0.27 & -0.04 \\
\hline 49 & -0.258 & 0.512 & 0.09 & 0.32 & -0.21 \\
\hline 50 & 0.206 & 0.326 & -0.12 & 0.26 & 0.03 \\
\hline
\end{tabular}




\begin{tabular}{|c|c|c|c|c|c|}
\hline 51 & -0.012 & 0.426 & -0.09 & 0.25 & 0.07 \\
\hline 52 & 0.058 & 0.305 & -0.02 & 0.26 & 0.21 \\
\hline 53 & -0.117 & 0.296 & 0.18 & 0.28 & -0.05 \\
\hline 54 & -0.518 & 0.370 & 0.13 & 0.35 & 0.24 \\
\hline 55 & -0.255 & 0.394 & 0.36 & 0.23 & 0.20 \\
\hline 56 & 0.201 & 0.353 & 0.57 & 0.19 & 0.19 \\
\hline 57 & -0.245 & 0.328 & 0.76 & 0.18 & 0.16 \\
\hline 58 & -0.037 & 0.308 & 0.93 & 0.19 & -0.18 \\
\hline 59 & 0.089 & 0.378 & 0.74 & 0.32 & -0.18 \\
\hline 60 & -0.155 & 0.368 & 0.57 & 0.28 & 0.01 \\
\hline 61 & 0.674 & 0.509 & 0.58 & 0.24 & 0.04 \\
\hline 62 & -0.078 & 0.741 & 0.62 & 0.26 & 0.22 \\
\hline 63 & -0.143 & 0.600 & 0.84 & 0.41 & -0.26 \\
\hline 64 & -0.084 & 0.684 & 0.58 & 0.37 & 0.37 \\
\hline 65 & 0.364 & 1.014 & 0.95 & 0.40 & 0.13 \\
\hline
\end{tabular}


Table S4. Statistics on continental distributions over time. Tests were formed to examine the change in distribution on each continent from one time period to the next. D is the K-S test statistic; $p$, the p-value.

\begin{tabular}{|c|c|c|c|c|c|c|c|c|c|c|}
\hline \multirow[t]{2}{*}{ Time comparison (ka) } & \multicolumn{2}{|c|}{ Africa } & \multicolumn{2}{|c|}{ Australia } & \multicolumn{2}{|c|}{ Eurasia } & \multicolumn{2}{|c|}{$\begin{array}{c}\text { North } \\
\text { America }\end{array}$} & \multicolumn{2}{|c|}{$\begin{array}{c}\text { South } \\
\text { America }\end{array}$} \\
\hline & D & $p$ & $D$ & $p$ & $\mathrm{D}$ & $p$ & $\mathrm{D}$ & $p$ & D & $p$ \\
\hline 125 to 70 & 0.00 & 1.00 & 0.00 & 1.00 & 0.02 & 1.00 & 0.00 & 1.00 & 0.00 & 1.00 \\
\hline 70 to 20 & 0.002 & 1.00 & 0.13 & 0.04 & 0.01 & 1.00 & 0.00 & 1.00 & 0.00 & 1.00 \\
\hline 20 to 10 & 0.01 & 1.00 & 0.03 & 1.00 & 0.00 & 1.00 & 0.88 & 0.03 & 0.08 & 0.01 \\
\hline 10 to 0 & 0.01 & 1.00 & 0.03 & 1.00 & 0.01 & 1.00 & 0.002 & 1.00 & 0.01 & 1.00 \\
\hline 0 to -0.2 & 0.05 & 0.23 & 0.12 & 0.22 & 0.11 & $<0.001$ & 0.04 & 0.94 & 0.06 & 0.16 \\
\hline
\end{tabular}


Table S5. Comparison of results from single logistic regression of extinction as a function of body mass to results from a multiple logistic regression analysis of extinction as a function of body mass, continent, and trophic guild.

\begin{tabular}{|c|c|c|c|c|c|c|c|c|c|}
\hline \multirow[b]{2}{*}{ Time Interval } & \multirow{2}{*}{$\begin{array}{l}\text { Single regression } \\
\text { analysis (extinction } \\
\sim \text { body mass) } \\
\text { Body size } \\
\text { coefficient } \\
(95 \% \mathrm{Cl})\end{array}$} & \multicolumn{8}{|c|}{$\begin{array}{c}\text { Multiple regression analysis } \\
\text { (extinction body mass + continent }+ \text { trophic guild) }\end{array}$} \\
\hline & & $\begin{array}{l}\text { Body size } \\
\text { coefficient } \\
(95 \% \mathrm{Cl})\end{array}$ & $\begin{array}{c}\text { Herbivore } \\
\text { coefficient } \\
(95 \% \mathrm{Cl})\end{array}$ & $\begin{array}{c}\text { Omnivore } \\
\text { Coefficient } \\
(95 \% \mathrm{Cl})\end{array}$ & $\begin{array}{l}\text { Insectivore } \\
\text { coefficient } \\
(95 \% \mathrm{Cl})\end{array}$ & $\begin{array}{c}\text { Australia } \\
\text { coefficient } \\
(95 \% \mathrm{Cl})\end{array}$ & $\begin{array}{c}\text { North } \\
\text { America } \\
\text { coefficient } \\
(95 \% \mathrm{Cl})\end{array}$ & $\begin{array}{c}\text { Eurasia } \\
\text { coefficient } \\
(95 \% \mathrm{Cl})\end{array}$ & $\begin{array}{c}\text { South } \\
\text { America } \\
\text { coefficient } \\
(95 \% \mathrm{Cl})\end{array}$ \\
\hline $\begin{array}{c}\text { Future } \\
\text { (ca. }+200 \text { years) }\end{array}$ & $\begin{array}{c}0.57 \\
(0.07)\end{array}$ & $\begin{array}{c}0.65 \\
(0.09)\end{array}$ & $\begin{array}{l}-0.24 \\
(0.17)\end{array}$ & $\begin{array}{l}-0.17 \\
(0.20)\end{array}$ & $\begin{array}{c}0.54 \\
(0.21)\end{array}$ & $\begin{array}{c}0.94 \\
(0.18)\end{array}$ & $\begin{array}{c}0.27 \\
(0.15)\end{array}$ & $\begin{array}{c}0.24 \\
(0.13)\end{array}$ & $\begin{array}{c}0.18 \\
(0.14)\end{array}$ \\
\hline $\begin{array}{l}\text { Holocene } \\
\text { (10 to } 0 \text { ka) }\end{array}$ & $\begin{array}{c}0.72 \\
(0.23)\end{array}$ & $\begin{array}{c}0.71 \\
(0.29)\end{array}$ & $\begin{array}{c}0.71 \\
(0.75)\end{array}$ & $\begin{array}{l}-0.93 \\
(1.26)\end{array}$ & $\begin{array}{l}-0.07 \\
(1.06)\end{array}$ & $\begin{array}{c}2.45 \\
(0.44)\end{array}$ & $\begin{array}{l}-1.35 \\
(1.07)\end{array}$ & $\begin{array}{c}0.04 \\
(0.48)\end{array}$ & $\begin{array}{l}-1.64 \\
(1.07)\end{array}$ \\
\hline $\begin{array}{l}\text { Terminal } \\
\text { Pleistocene } \\
\text { (20-10 ka) }\end{array}$ & $\begin{array}{c}1.74 \\
(0.21)\end{array}$ & $\begin{array}{c}2.05 \\
(0.28)\end{array}$ & $\begin{array}{l}-0.18 \\
(0.36)\end{array}$ & $\begin{array}{c}0.44 \\
(0.56)\end{array}$ & $\begin{array}{l}-0.35 \\
(0.79)\end{array}$ & $\begin{array}{c}-12.82 \\
(623.52)\end{array}$ & $\begin{array}{c}3.97 \\
(0.50)\end{array}$ & $\begin{array}{c}0.13 \\
(0.58)\end{array}$ & $\begin{array}{c}3.55 \\
(0.49)\end{array}$ \\
\hline $\begin{array}{l}\text { End Pleistocene } \\
\text { (70-20 ka) }\end{array}$ & $\begin{array}{c}1.19 \\
(0.24)\end{array}$ & $\begin{array}{c}3.07 \\
(0.88)\end{array}$ & $\begin{array}{l}-1.68 \\
(0.78)\end{array}$ & $\begin{array}{c}-16.11 \\
(2283.63)\end{array}$ & $\begin{array}{c}-16.00 \\
(2419.97)\end{array}$ & $\begin{array}{c}7.42 \\
(1.13)\end{array}$ & $\begin{array}{c}-18.03 \\
(2198.96)\end{array}$ & $\begin{array}{c}1.22 \\
(0.92)\end{array}$ & $\begin{array}{c}-17.97 \\
(1959.24)\end{array}$ \\
\hline $\begin{array}{l}\text { Late Pleistocene } \\
\text { (125-70 ka) }\end{array}$ & $\begin{array}{c}1.47 \\
(0.52)\end{array}$ & $\begin{array}{c}2.55 \\
(1.02)\end{array}$ & $\begin{array}{c}17.49 \\
(6590.96)\end{array}$ & $\begin{array}{c}3.19 \\
(7519.34)\end{array}$ & $\begin{array}{c}22.46 \\
(6590.96)\end{array}$ & $\begin{array}{c}21.12 \\
(3392.52)\end{array}$ & $\begin{array}{c}-0.44 \\
(5077.90)\end{array}$ & $\begin{array}{c}19.02 \\
(3392.52)\end{array}$ & $\begin{array}{c}-0.92 \\
(4728.32)\end{array}$ \\
\hline $\begin{array}{l}\text { Late Quaternary } \\
\qquad(125-0 \text { ka) }\end{array}$ & $\begin{array}{c}1.49 \\
(0.15)\end{array}$ & $\begin{array}{c}1.75 \\
(0.19)\end{array}$ & $\begin{array}{c}1.74 \\
(0.10)\end{array}$ & $\begin{array}{c}0.05 \\
(0.54)\end{array}$ & $\begin{array}{l}-0.09 \\
(0.28)\end{array}$ & $\begin{array}{l}-0.17 \\
(0.47)\end{array}$ & $\begin{array}{c}0.53 \\
(0.37)\end{array}$ & $\begin{array}{c}3.91 \\
(0.38)\end{array}$ & $\begin{array}{c}2.74 \\
(0.36)\end{array}$ \\
\hline $\begin{array}{l}\text { Late Quaternary } \\
\text { plus Future (125ka } \\
\text { to ca. }+200 \text { years) }\end{array}$ & $\begin{array}{c}0.76 \\
(0.07)\end{array}$ & $\begin{array}{c}0.84 \\
(0.08)\end{array}$ & $\begin{array}{c}0.83 \\
(0.04)\end{array}$ & $\begin{array}{c}0.29 \\
(0.13)\end{array}$ & $\begin{array}{c}1.44 \\
(0.17)\end{array}$ & $\begin{array}{c}0.50 \\
(0.14)\end{array}$ & $\begin{array}{l}-0.29 \\
(0.16)\end{array}$ & $\begin{array}{c}0.34 \\
(0.13)\end{array}$ & $\begin{array}{c}0.59 \\
(0.20)\end{array}$ \\
\hline
\end{tabular}




\begin{tabular}{|c|c|c|c|c|c|c|c|c|c|c|c|c|}
\hline Continent & Order & FAMILY & Genus & Species & Common & Log(Mass) & $\begin{array}{l}\text { Combined } \\
\text { Mass (g) }\end{array}$ & Status & $\begin{array}{l}\text { Time of } \\
\text { extinction }\end{array}$ & $\begin{array}{l}\text { Last.Occura } \\
\text { nce.kybp. }\end{array}$ & $\begin{array}{l}\text { IUCN.Redlist. } \\
\text { status }\end{array}$ & trophic \\
\hline $\mathrm{AF}$ & Afrosoricida & Chrysochloridae & Amblysomus & hottentotus & $\begin{array}{l}\text { Hottentot Golden Mole, } \\
\text { Zulu Golden Mole }\end{array}$ & 1.72 & 53 & extant & 0 & NA & LC & ginsect \\
\hline $\mathrm{AF}$ & Afrosoricida & Chrysochloridae & Calcochloris & obtusirostris & Yellow Golden Mole & 1.38 & 24 & extant & 0 & NA & LC & ginsect \\
\hline $\mathrm{AF}$ & Afrosoricida & Chrysochloridae & Calcochloris & tytonis & Somali Golden Mole & 1.38 & 24 & extant & 0 & NA & $\mathrm{DD}$ & ginsect \\
\hline $\mathrm{AF}$ & Afrosoricida & Chrysochloridae & Carpitalpa & arendsi & Arend's Golden Mole & 1.72 & 52 & extant & Future & NA & vu & ginsect \\
\hline $\mathrm{AF}$ & Afrosoricida & Chrysochloridae & Chlorotalpa & duthieae & Duthie's Golden Mole & 1.61 & 41 & extant & Future & NA & vu & ginsect \\
\hline $\mathrm{AF}$ & Afrosoricida & Chrysochloridae & Chlorotalpa & sclateri & Sclater's Golden Mole & 1.61 & 41 & extant & 0 & NA & LC & ginsect \\
\hline $\mathrm{AF}$ & Afrosoricida & Chrysochloridae & Chrysochloris & asiatica & Cape Golden Mole & 1.69 & 49 & extant & 0 & NA & LC & ginsect \\
\hline $\mathrm{AF}$ & Afrosoricida & Chrysochloridae & Chrysochloris & stuhlmanni & $\begin{array}{l}\text { Stuhlmann's Golden } \\
\text { Mole }\end{array}$ & 1.60 & 40 & extant & 0 & NA & LC & ginsect \\
\hline $\mathrm{AF}$ & Afrosoricida & Chrysochloridae & Chrysochloris & visagiei & Visagie's Golden Mole & 1.48 & 30 & extant & 0 & NA & DD & ginsect \\
\hline $\mathrm{AF}$ & Afrosoricida & Chrysochloridae & Chrysospalax & trevelyani & Giant Golden Mole & 2.63 & 427 & extant & Future & NA & EN & ginsect \\
\hline AF & Afrosoricida & Chrysochloridae & Chrysospalax & villosus & $\begin{array}{l}\text { Rough-haired Golden } \\
\text { Mole }\end{array}$ & 2.08 & 120 & extant & Future & NA & VU & ginsect \\
\hline AF & Afrosoricida & Chrysochloridae & Cryptochloris & wintoni & $\begin{array}{l}\text { De Winton's Golden } \\
\text { Mole }\end{array}$ & 1.40 & 25 & extant & Future & NA & $C R$ & ginsect \\
\hline $\mathrm{AF}$ & Afrosoricida & Chrysochloridae & Cryptochloris & zyli & Van Zyl's Golden Mole & 1.40 & 25 & extant & Future & NA & EN & ginsect \\
\hline $\mathrm{AF}$ & Afrosoricida & Chrysochloridae & Eremitalpa & granti & Grant's Golden Mole & 1.42 & 26 & extant & 0 & NA & LC & ginsect \\
\hline $\mathrm{AF}$ & Afrosoricida & Chrysochloridae & Neamblysomus & gunningi & Gunning's Golden Mole & 1.45 & 28 & extant & Future & NA & EN & ginsect \\
\hline $\mathrm{AF}$ & Afrosoricida & Chrysochloridae & Neamblysomus & julianae & Juliana's Golden Mole & 1.34 & 22 & extant & Future & NA & EN & ginsect \\
\hline AF & Afrosoricida & Tenrecidae & Micropotamogale & lamottei & $\begin{array}{l}\text { Nimba Otter Shrew, } \\
\text { Pygmy Otter-shrew }\end{array}$ & 1.84 & 70 & extant & Future & NA & NT & piscivore \\
\hline AF & Afrosoricida & Tenrecidae & Micropotamogale & ruwenzorii & $\begin{array}{l}\text { Rwenzori Otter Shrew, } \\
\text { Ruwenzori Otter Shrew }\end{array}$ & 1.99 & 99 & extant & 0 & NA & LC & invert \\
\hline AF & Afrosoricida & Tenrecidae & Potamogale & velox & $\begin{array}{l}\text { Giant Otter Shrew, } \\
\text { Giant Otter-shrew }\end{array}$ & 2.82 & 660 & extant & 0 & NA & LC & piscivore \\
\hline AF & Artiodactyla & Bovidae & Addax & nasomaculatus & $\begin{array}{l}\text { Addax } \\
\text { Impala, Black-faced }\end{array}$ & 4.85 & 70000 & extant & Future & NA & $C R$ & graze \\
\hline AF & Artiodactyla & Bovidae & Aepyceros & melampus & $\begin{array}{l}\text { Impala, Common } \\
\text { Impala }\end{array}$ & 4.72 & 52500 & extant & 0 & NA & LC & zraze/browse \\
\hline AF & Artiodactyla & Bovidae & Alcelaphus & buselaphus & Hartebeest & 5.23 & 171002 & extant & 0 & NA & LC & graze \\
\hline AF & Artiodactyla & Bovidae & Alcelaphus & lichtensteinii & $\begin{array}{l}\text { Lichtenstein's } \\
\text { hartebeest }\end{array}$ & 5.23 & 171498 & extant & 0 & NA & LC & Graze \\
\hline AF & Artiodactyla & Bovidae & Ammodorcas & clarkei & $\begin{array}{l}\text { Dibatag, Clarke's } \\
\text { Gazelle }\end{array}$ & 4.45 & 28050 & extant & Future & NA & VU & Browse \\
\hline AF & Artiodactyla & Bovidae & Ammotragus & lervia & $\begin{array}{l}\text { Aoudad, Uaddan, } \\
\text { Barbary Sheep }\end{array}$ & 4.68 & 48000 & extant & Future & NA & Vu & zraze/browse \\
\hline $\mathrm{AF}$ & Artiodactyla & Bovidae & Antidorcas & australis & southern springobk & 4.60 & 40000 & extinct & Holocene & 7 & EX & э̧raze/browse \\
\hline $\mathrm{AF}$ & Artiodactyla & Bovidae & Antidorcas & bondi & Bond's springbok & 4.53 & 34000 & extinct & Holocene & 7 & EX & zraze/browse \\
\hline $\mathrm{AF}$ & Artiodactyla & Bovidae & Antidorcas & marsupialis & $\begin{array}{l}\text { Springbok } \\
\text { Hirola, Hunter's }\end{array}$ & 4.50 & 31500 & extant & 0 & NA & LC & graze/browse \\
\hline $\mathrm{AF}$ & Artiodactyla & Bovidae & Beatragus & hunteri & $\begin{array}{l}\text { Antelope, Herola, } \\
\text { Hunter's Hartebeest }\end{array}$ & 4.90 & 80000 & extant & Future & NA & $C R$ & graze \\
\hline AF & Artiodactyla & Bovidae & Bos & primigenius & & 5.95 & 900000 & extinct & Holocene & 6 & EX & graze \\
\hline $\mathrm{AF}$ & Artiodactyla & Bovidae & Bos & taurus & cattle & 5.95 & 900000 & extant & 0 & NA & LC & Graze \\
\hline $\mathrm{AF}$ & Artiodactyla & Bovidae & Capra & ibex & Alpine Ibex, Ibex & 5.00 & 101000 & extant & 0 & NA & LC & orowse/graze \\
\hline $\mathrm{AF}$ & Artiodactyla & Bovidae & Capra & nubiana & Nubian Ibex & 4.90 & 79000 & extant & Future & NA & Vu & orowse/graze \\
\hline $\mathrm{AF}$ & Artiodactyla & Bovidae & Capra & walie & Walia Ibex, Walia & 5.00 & 100000 & extant & Future & NA & EN & orowse/graze \\
\hline
\end{tabular}




\begin{tabular}{|c|c|c|c|c|c|c|c|c|c|c|c|c|}
\hline $\mathrm{AF}$ & Artiodactyla & Bovidae & Capricornis & rubidus & Red Serow & 4.18 & 15000 & extant & Future & NA & NT & Browse \\
\hline $\mathrm{AF}$ & Artiodactyla & Bovidae & Cephalophus & adersi & Aders' Duiker & 3.97 & 9250 & extant & Future & NA & $C R$ & frug/browse \\
\hline $\mathrm{AF}$ & Artiodactyla & Bovidae & Cephalophus & callipygus & Peters' Duiker & 4.26 & 18250 & extant & 0 & NA & LC & frug/browse \\
\hline AF & Artiodactyla & Bovidae & Cephalophus & dorsalis & $\begin{array}{l}\text { Bay Duiker } \\
\text { Harvey's Duiker, }\end{array}$ & 4.30 & 20000 & extant & Future & NA & NT & frug/browse \\
\hline $\mathrm{AF}$ & Artiodactyla & Bovidae & Cephalophus & harveyi & $\begin{array}{l}\text { Harvey's Red Duiker, } \\
\text { East African Red Duiker }\end{array}$ & 4.16 & 14500 & extant & 0 & NA & LC & frug/browse \\
\hline AF & Artiodactyla & Bovidae & Cephalophus & jentinki & Jentink's Duiker & 4.85 & 70000 & extant & Future & NA & EN & frug/browse \\
\hline $\mathrm{AF}$ & Artiodactyla & Bovidae & Cephalophus & leucogaster & White-bellied Duiker & 4.10 & 12700 & extant & Future & NA & NT & frug/browse \\
\hline AF & Artiodactyla & Bovidae & Cephalophus & monticola & Natal Red Duiker, Red & 3.80 & 6250 & extant & 0 & NA & $\mathrm{LC}$ & frug/browse \\
\hline $\mathrm{AF}$ & Artiodactyla & Bovidae & Cephalophus & natalensis & $\begin{array}{l}\text { Forest Duiker, Natal } \\
\text { Duiker }\end{array}$ & 4.08 & 12000 & extant & 0 & NA & LC & frug/browse \\
\hline $\mathrm{AF}$ & Artiodactyla & Bovidae & Cephalophus & niger & Black Duiker & 4.31 & 20400 & extant & 0 & NA & LC & frug/browse \\
\hline $\mathrm{AF}$ & Artiodactyla & Bovidae & Cephalophus & nigrifrons & Black-fronted Duiker & 4.14 & 13900 & extant & 0 & NA & LC & frug/browse \\
\hline $\mathrm{AF}$ & Artiodactyla & Bovidae & Cephalophus & ogilbyi & Ogilby's Duiker & 4.30 & 20000 & extant & 0 & NA & $\mathrm{LC}$ & frug/browse \\
\hline $\mathrm{AF}$ & Artiodactyla & Bovidae & Cephalophus & rufilatus & Red-flanked Duiker & 4.08 & 12000 & extant & 0 & NA & LC & frug/browse \\
\hline $\mathrm{AF}$ & Artiodactyla & Bovidae & Cephalophus & silvicultor & Yellow-backed Duiker & 4.86 & 72500 & extant & Future & NA & NT & frug/browse \\
\hline $\mathrm{AF}$ & Artiodactyla & Bovidae & Cephalophus & spadix & Abbott's Duiker, Minde & 4.75 & 56000 & extant & Future & NA & EN & frug/browse \\
\hline $\mathrm{AF}$ & Artiodactyla & Bovidae & Cephalophus & weynsi & Weyns's Duiker & 4.23 & 17000 & extant & 0 & NA & $\mathrm{LC}$ & frug/browse \\
\hline $\mathrm{AF}$ & Artiodactyla & Bovidae & Cephalophus & zebra & $\begin{array}{l}\text { Zebra Duiker, Banded } \\
\text { Duiker, Zebra Antelope }\end{array}$ & 4.24 & 17500 & extant & Future & NA & vu & frug/browse \\
\hline $\mathrm{AF}$ & Artiodactyla & Bovidae & Connochaetes & gnou & $\begin{array}{l}\text { Black Wildebeest, } \\
\text { White-tailed Gnu } \\
\text { Common Wildebeest, }\end{array}$ & 5.26 & 179999 & extant & 0 & NA & $\mathrm{LC}$ & zraze/browse \\
\hline $\mathrm{AF}$ & Artiodactyla & Bovidae & Connochaetes & taurinus & $\begin{array}{l}\text { White-bearded } \\
\text { Wildebeest, Blue } \\
\text { Wildebeest }\end{array}$ & 5.26 & 179999 & extant & 0 & NA & LC & graze \\
\hline $\mathrm{AF}$ & Artiodactyla & Bovidae & Damaliscus & hypsodon & $\begin{array}{l}\text { related to wildebeest; } \\
\text { was D. dorcas }\end{array}$ & & & extinct & TP & 12.3 & EX & graze \\
\hline $\mathrm{AF}$ & Artiodactyla & Bovidae & Damaliscus & lunatus & Topi, Tsessebe, Tiang & 5.13 & 136000 & extant & 0 & NA & LC & graze \\
\hline $\mathrm{AF}$ & Artiodactyla & Bovidae & Damaliscus & niro & & & & extinct & EP & 63 & EX & graze \\
\hline $\mathrm{AF}$ & Artiodactyla & Bovidae & Damaliscus & pygargus & Blesbok, Bontebok & 5.01 & 102000 & extant & 0 & NA & LC & Graze \\
\hline $\mathrm{AF}$ & Artiodactyla & Bovidae & Dorcatragus & megalotis & Beira, Beira Antelope & 4.01 & 10250 & extant & Future & NA & vu & Browse \\
\hline $\mathrm{AF}$ & Artiodactyla & Bovidae & Eudorcas & rufifrons & Red-fronted Gazelle & 4.43 & 27000 & extant & Future & NA & vu & „̧raze/browse \\
\hline $\mathrm{AF}$ & Artiodactyla & Bovidae & Eudorcas & rufina & Red Gazelle & 4.60 & 40000 & extinct & Holocene & NA & EX & browse \\
\hline $\mathrm{AF}$ & Artiodactyla & Bovidae & Eudorcas & thomsonii & Thomson's Gazelle & 4.31 & 20500 & extant & Future & NA & NT & Browse \\
\hline $\mathrm{AF}$ & Artiodactyla & Bovidae & Gazella & atlantica & & 4.65 & 44500 & extinct & TP & 11 & EX & Browse \\
\hline $\mathrm{AF}$ & Artiodactyla & Bovidae & Gazella & cuvieri & Cuvier's Gazelle, Edmi & 4.40 & 25000 & extant & Future & NA & EN & „̧raze/browse \\
\hline $\mathrm{AF}$ & Artiodactyla & Bovidae & Gazella & dorcas & Dorcas Gazelle & 4.36 & 23000 & extant & Future & NA & Vu & Browse \\
\hline $\mathrm{AF}$ & Artiodactyla & Bovidae & Gazella & gazella & Mountain Gazelle, Idmi & 4.35 & 22500 & extant & Future & NA & vu & Browse \\
\hline $\mathrm{AF}$ & Artiodactyla & Bovidae & Gazella & granti & Grant's gazelle & 4.74 & 55000 & extant & 0 & NA & LC & orowse/graze \\
\hline $\mathrm{AF}$ & Artiodactyla & Bovidae & Gazella & leptoceros & Slender-horned Gazelle & 4.35 & 22200 & extant & Future & NA & EN & э̧raze/browse \\
\hline $\mathrm{AF}$ & Artiodactyla & Bovidae & Gazella & spekei & *Speke's Gazelle & 4.30 & 20000 & extant & Future & NA & EN & эraze/browse \\
\hline $\mathrm{AF}$ & Artiodactyla & Bovidae & Gazella & tingitana & & & & extinct & EP & 37 & EX & zraze/browse \\
\hline $\mathrm{AF}$ & Artiodactyla & Bovidae & Hippotragus & equinus & Roan Antelope & 5.43 & 269998 & extant & 0 & NA & LC & graze \\
\hline $\mathrm{AF}$ & Artiodactyla & Bovidae & Hippotragus & leucophaeus & Bluebuck, Blue Buck & 5.18 & 150000 & extinct & Holocene & 0.2 & EX & Graze \\
\hline $\mathrm{AF}$ & Artiodactyla & Bovidae & Hippotragus & niger & $\begin{array}{l}\text { Sable Antelope, Giant } \\
\text { Sable Antelope }\end{array}$ & 5.36 & 227499 & extant & 0 & NA & LC & graze \\
\hline $\mathrm{AF}$ & Artiodactyla & Bovidae & Kobus & ellipsiprymnus & Waterbuck & 5.32 & 210000 & extant & 0 & NA & LC & graze \\
\hline $\mathrm{AF}$ & Artiodactyla & Bovidae & Kobus & $k o b$ & Kob & 4.89 & 78500 & extant & 0 & NA & LC & graze \\
\hline $\mathrm{AF}$ & Artiodactyla & Bovidae & Kobus & leche & Southern Lechwe & 5.04 & 110749 & extant & 0 & NA & LC & graze \\
\hline
\end{tabular}




\begin{tabular}{|c|c|c|c|c|c|c|c|c|c|c|c|c|}
\hline $\mathrm{AF}$ & Artiodactyla & Bovidae & Kobus & megaceros & Nile Lechwe & 4.94 & 87500 & extant & Future & NA & EN & „̧raze/browse \\
\hline $\mathrm{AF}$ & Artiodactyla & Bovidae & Kobus & vardonii & Puku & 4.85 & 71501 & extant & Future & NA & NT & graze \\
\hline $\mathrm{AF}$ & Artiodactyla & Bovidae & Litocranius & walleri & Gerenuk & 4.58 & 38000 & extant & Future & NA & NT & Browse \\
\hline $\mathrm{AF}$ & Artiodactyla & Bovidae & Madoqua & guentheri & $\begin{array}{l}\text { Guenther's Dik-dik, } \\
\text { Gônther's Dik-dik }\end{array}$ & 3.88 & 7500 & extant & 0 & NA & LC & Browse \\
\hline $\mathrm{AF}$ & Artiodactyla & Bovidae & Madoqua & kirkii & Kirk's Dik-dik & 3.72 & 5300 & extant & 0 & NA & LC & Browse \\
\hline $\mathrm{AF}$ & Artiodactyla & Bovidae & Madoqua & piacentinii & Silver Dik-dik & 3.40 & 2500 & extant & 0 & NA & DD & Browse \\
\hline $\mathrm{AF}$ & Artiodactyla & Bovidae & Madoqua & saltiana & Salt's Dik-dik & 3.51 & 3250 & extant & 0 & NA & LC & Browse \\
\hline $\mathrm{AF}$ & Artiodactyla & Bovidae & Megalotragus & priscus & giant wildebeest & 5.30 & 200000 & extinct & Holocene & 7.5 & EX & Graze \\
\hline $\mathrm{AF}$ & Artiodactyla & Bovidae & Nanger & dama & $\begin{array}{l}\text { Dama Gazelle, Mhorr } \\
\text { Gazelle, Addra Gazelle } \\
\text { Gazelle de }\end{array}$ & 4.86 & 73000 & extant & Future & NA & $C R$ & Browse \\
\hline $\mathrm{AF}$ & Artiodactyla & Bovidae & Nanger & soemmerringii & $\begin{array}{l}\text { Soemmerring, } \\
\text { *Soemmerring's } \\
\text { Gazelle }\end{array}$ & 4.60 & 40000 & extant & Future & NA & vu & orowse/graze \\
\hline $\mathrm{AF}$ & Artiodactyla & Bovidae & Neotragus & batesi & $\begin{array}{l}\text { Bates' Pygmy Antelope, } \\
\text { Dwarf Antelope }\end{array}$ & 3.48 & 3000 & extant & 0 & NA & LC & Browse \\
\hline $\mathrm{AF}$ & Artiodactyla & Bovidae & Neotragus & moschatus & suni & 3.81 & 6500 & extant & 0 & NA & LC & Browse \\
\hline $\mathrm{AF}$ & Artiodactyla & Bovidae & Neotragus & pygmaeus & Royal Antelope & 3.60 & 4000 & extant & 0 & NA & LC & browse/frug \\
\hline $\mathrm{AF}$ & Artiodactyla & Bovidae & Oreotragus & oreotragus & Klipspringer & 4.11 & 13000 & extant & 0 & NA & LC & orowse/graze \\
\hline $\mathrm{AF}$ & Artiodactyla & Bovidae & Oryx & dammah & Scimitar-horned Oryx & 5.30 & 200000 & extinct & Holocene & NA & EW & эraze/browse \\
\hline $\mathrm{AF}$ & Artiodactyla & Bovidae & Oryx & gazella & Gemsbok & 5.23 & 169001 & extant & 0 & NA & LC & эraze/browse \\
\hline $\mathrm{AF}$ & Artiodactyla & Bovidae & Ourebia & ourebi & $\begin{array}{l}\text { Oribi } \\
\text { Grey Rhebok, Rhebok, }\end{array}$ & 4.24 & 17250 & extant & 0 & NA & LC & graze \\
\hline $\mathrm{AF}$ & Artiodactyla & Bovidae & Pelea & capreolus & $\begin{array}{l}\text { Common Rhebok, Vaal } \\
\text { Rhebok }\end{array}$ & 4.30 & 20000 & extant & 0 & NA & LC & graze \\
\hline $\mathrm{AF}$ & Artiodactyla & Bovidae & Pelorovis & antiquus & giant buffalo & 6.00 & 1000000 & extinct & TP & 11 & EX & Graze \\
\hline $\mathrm{AF}$ & Artiodactyla & Bovidae & Philantomba & maxwellii & Maxwell's Duiker & 4.08 & 12000 & extant & 0 & NA & LC & frug/browse \\
\hline $\mathrm{AF}$ & Artiodactyla & Bovidae & Raphicerus & campestris & Steenbok & 4.02 & 10500 & extant & 0 & NA & LC & jowse/graze \\
\hline $\mathrm{AF}$ & Artiodactyla & Bovidae & Raphicerus & melanotis & Cape Grysbok & 4.01 & 10250 & extant & 0 & NA & LC & Browse \\
\hline $\mathrm{AF}$ & Artiodactyla & Bovidae & Raphicerus & sharpei & Sharpe's Grysbok & 3.98 & 9500 & extant & 0 & NA & LC & orowse/graze \\
\hline $\mathrm{AF}$ & Artiodactyla & Bovidae & Redunca & arundinum & Southern Reedbuck & 4.76 & 58000 & extant & 0 & NA & LC & graze \\
\hline $\mathrm{AF}$ & Artiodactyla & Bovidae & Redunca & fulvorufula & Mountain Reedbuck & 4.47 & 29500 & extant & 0 & NA & LC & graze \\
\hline $\mathrm{AF}$ & Artiodactyla & Bovidae & Redunca & redunca & $\begin{array}{l}\text { Bohor Reedbuck, } \\
\text { Common Reedbuck }\end{array}$ & 4.64 & 44050 & extant & 0 & NA & $\mathrm{LC}$ & graze \\
\hline $\mathrm{AF}$ & Artiodactyla & Bovidae & Sylvicapra & grimmia & $\begin{array}{l}\text { Common Duiker, Grey } \\
\text { Duiker, Bush Duiker }\end{array}$ & 4.29 & 19500 & extant & 0 & NA & LC & browse/frug \\
\hline $\mathrm{AF}$ & Artiodactyla & Bovidae & Syncerus & caffer & African Buffalo & 5.76 & 580003 & extant & 0 & NA & LC & graze \\
\hline $\mathrm{AF}$ & Artiodactyla & Bovidae & Taurotragus & derbianus & giant eland & 5.83 & 680000 & extant & 0 & NA & LC & orowse/graze \\
\hline $\mathrm{AF}$ & Artiodactyla & Bovidae & Taurotragus & oryx & common eland & 5.76 & 569994 & extant & 0 & NA & LC & Browse \\
\hline $\mathrm{AF}$ & Artiodactyla & Bovidae & Tragelaphus & angasii & Nyala & 4.94 & 86600 & extant & 0 & NA & LC & jrowse/graze \\
\hline $\mathrm{AF}$ & Artiodactyla & Bovidae & Tragelaphus & buxtoni & Mountain Nyala & 5.33 & 215001 & extant & Future & NA & EN & orowse/graze \\
\hline $\mathrm{AF}$ & Artiodactyla & Bovidae & Tragelaphus & eurycerus & Bongo & 5.52 & 329003 & extant & Future & NA & NT & jrowse/graze \\
\hline $\mathrm{AF}$ & Artiodactyla & Bovidae & Tragelaphus & imberbis & Lesser Kudu & 4.91 & 81576 & extant & Future & NA & NT & Browse \\
\hline $\mathrm{AF}$ & Artiodactyla & Bovidae & Tragelaphus & scriptus & Bushbuck & 4.64 & 43250 & extant & 0 & NA & LC & orowse/graze \\
\hline $\mathrm{AF}$ & Artiodactyla & Bovidae & Tragelaphus & spekii & Sitatunga, Marshbuck & 4.89 & 77999 & extant & 0 & NA & LC & jowse/graze \\
\hline $\mathrm{AF}$ & Artiodactyla & Bovidae & Tragelaphus & strepsiceros & Greater Kudu & 5.33 & 213501 & extant & 0 & NA & LC & Browse \\
\hline $\mathrm{AF}$ & Artiodactyla & Camelidae & Camelus & thomasi & & 5.70 & 500000 & extinct & EP & 24 & EX & J̄raze/browse \\
\hline
\end{tabular}




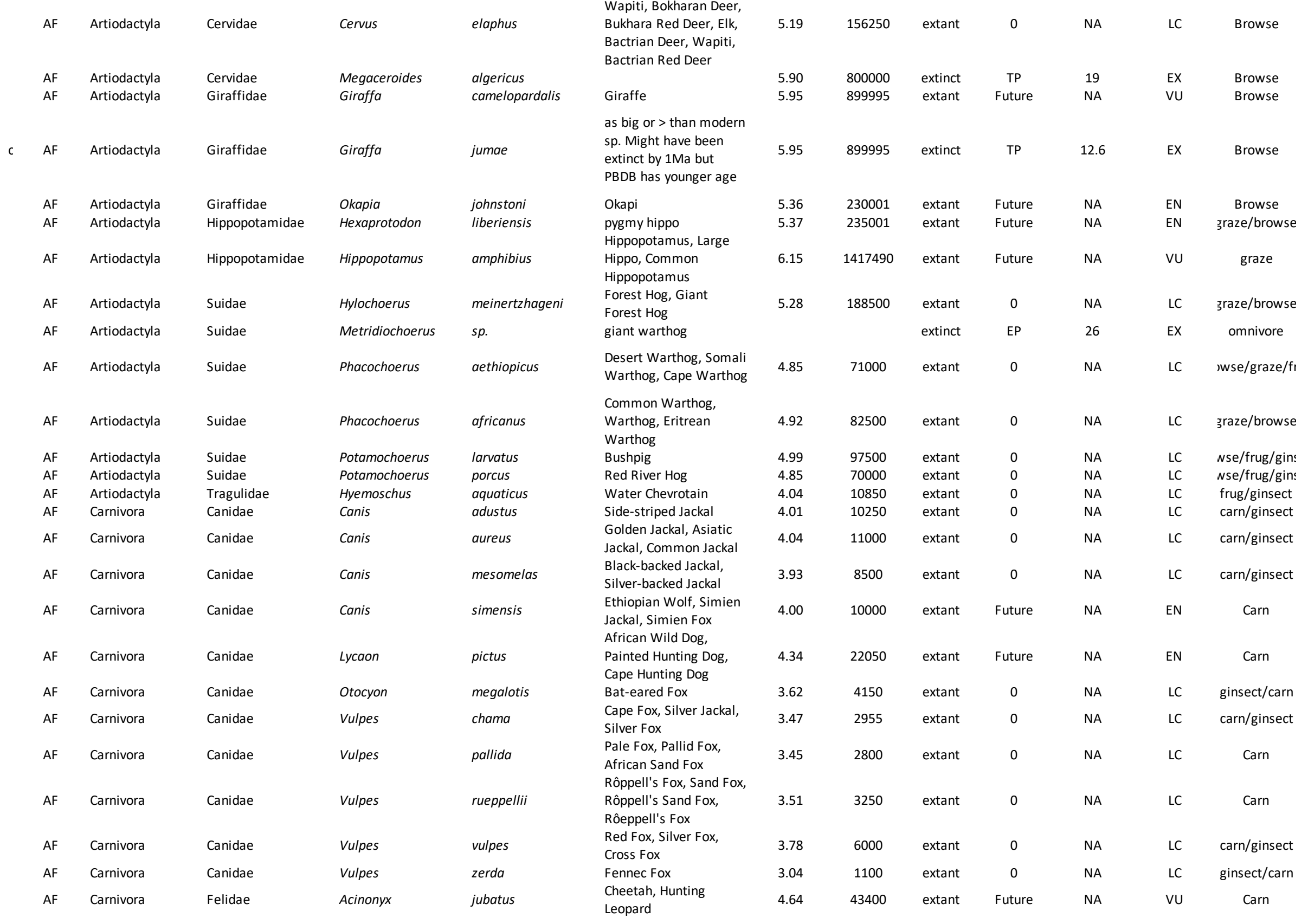




\begin{tabular}{|c|c|c|c|c|c|c|c|c|c|c|c|c|}
\hline $\mathrm{AF}$ & Carnivora & Felidae & Caracal & caracal & $\begin{array}{l}\text { Caracal, Desert Lynx, } \\
\text { African Caracal, Asian } \\
\text { Caracal }\end{array}$ & 4.14 & 13750 & extant & 0 & NA & LC & Carn \\
\hline $\mathrm{AF}$ & Carnivora & Felidae & Felis & chaus & $\begin{array}{l}\text { Jungle Cat, Swamp Cat, } \\
\text { Reed Cat }\end{array}$ & 3.83 & 6750 & extant & 0 & NA & LC & Carn \\
\hline $\mathrm{AF}$ & Carnivora & Felidae & Felis & margarita & $\begin{array}{l}\text { Sand Cat, Sand Dune } \\
\text { Cat }\end{array}$ & 3.40 & 2525 & extant & Future & NA & NT & Carn \\
\hline $\mathrm{AF}$ & Carnivora & Felidae & Felis & nigripes & $\begin{array}{l}\text { Black-footed Cat, Small- } \\
\text { spotted Cat }\end{array}$ & 3.11 & 1300 & extant & Future & NA & Vu & Carn \\
\hline AF & Carnivora & Felidae & Felis & silvestris & Wild Cat, Wildcat & 3.67 & 4650 & extant & 0 & NA & LC & Carn \\
\hline $\mathrm{AF}$ & Carnivora & Felidae & Leptailurus & serval & Serval & 4.08 & 12000 & extant & 0 & NA & LC & Carn \\
\hline $\mathrm{AF}$ & Carnivora & Felidae & Panthera & leo & Lion, African Lion & 5.21 & 161499 & extant & Future & NA & VU & Carn \\
\hline AF & Carnivora & Felidae & Panthera & pardus & $\begin{array}{l}\text { Leopard } \\
\text { African Golden Cat, }\end{array}$ & 4.74 & 55000 & extant & Future & NA & VU & Carn \\
\hline $\mathrm{AF}$ & Carnivora & Felidae & Profelis & aurata & Golden Cat & 4.03 & 10650 & extant & Future & NA & VU & Carn \\
\hline $\mathrm{AF}$ & Carnivora & Herpestidae & Atilax & paludinosus & $\begin{array}{l}\text { Marsh Mongoose, } \\
\text { Water Mongoose }\end{array}$ & 3.52 & 3300 & extant & 0 & NA & LC & ivert/piscivor \\
\hline $\mathrm{AF}$ & Carnivora & Herpestidae & Bdeogale & crassicauda & Bushy-tailed Mongoose & 3.19 & 1550 & extant & 0 & NA & LC & ginsect \\
\hline AF & Carnivora & Herpestidae & Bdeogale & jacksoni & $\begin{array}{l}\text { Jackson's Mongoose } \\
\text { Black-legged }\end{array}$ & 3.40 & 2500 & extant & Future & NA & NT & ginsect/carn \\
\hline $\mathrm{AF}$ & Carnivora & Herpestidae & Bdeogale & nigripes & $\begin{array}{l}\text { Mongoose, Black-footed } \\
\text { Mogoose }\end{array}$ & 3.40 & 2500 & extant & 0 & NA & LC & ginsect/carn \\
\hline $\mathrm{AF}$ & Carnivora & Herpestidae & Crossarchus & alexandri & Alexander's Cusimanse & 3.18 & 1500 & extant & 0 & NA & LC & ginsect/carn \\
\hline $\mathrm{AF}$ & Carnivora & Herpestidae & Crossarchus & ansorgei & $\begin{array}{l}\text { Ansorge's Cusimanse, } \\
\text { Angolan Cusimanse } \\
\text { Common Cusimanse, }\end{array}$ & 2.85 & 700 & extant & 0 & NA & LC & ginsect/carn \\
\hline AF & Carnivora & Herpestidae & Crossarchus & obscurus & $\begin{array}{l}\text { Long-nosed Cusimanse, } \\
\text { Cusimanse }\end{array}$ & 3.10 & 1250 & extant & 0 & NA & LC & ginsect/carn \\
\hline $\mathrm{AF}$ & Carnivora & Herpestidae & Cynictis & penicillata & $\begin{array}{l}\text { Yellow Mongoose } \\
\text { Pousargues's }\end{array}$ & 2.92 & 836 & extant & 0 & NA & LC & ginsect/carn \\
\hline $\mathrm{AF}$ & Carnivora & Herpestidae & Dologale & dybowskii & $\begin{array}{l}\text { Mongoose, Savanna } \\
\text { Mongoose } \\
\text { Kaokoveld Slender } \\
\text { Mongoose, Black }\end{array}$ & 2.54 & 350 & extant & 0 & NA & DD & ginsect/carn \\
\hline $\mathrm{AF}$ & Carnivora & Herpestidae & Galerella & flavescens & $\begin{array}{l}\text { Slender Mongoose, } \\
\text { Black Mongoose, } \\
\text { Angolan Slender } \\
\text { Mongoose }\end{array}$ & 2.88 & 750 & extant & 0 & NA & LC & ginsect/carn \\
\hline $\mathrm{AF}$ & Carnivora & Herpestidae & Galerella & pulverulenta & $\begin{array}{l}\text { Cape Grey Mongoose, } \\
\text { Small Grey Mongoose } \\
\text { Common Slender }\end{array}$ & 2.90 & 797 & extant & 0 & NA & LC & ginsect/carn \\
\hline $\mathrm{AF}$ & Carnivora & Herpestidae & Galerella & sanguinea & $\begin{array}{l}\text { Mongoose, Slender } \\
\text { Mongoose }\end{array}$ & 2.74 & 550 & extant & 0 & NA & LC & carn/ginsect \\
\hline $\mathrm{AF}$ & Carnivora & Herpestidae & Galerella & swalius & $\begin{array}{l}\text { Namibian slender } \\
\text { mongoose } \\
\text { Somali Dwarf } \\
\text { Mongoose, Desert }\end{array}$ & 2.88 & 750 & extant & 0 & NA & LC & ginsect/carn \\
\hline $\mathrm{AF}$ & Carnivora & Herpestidae & Helogale & hirtula & $\begin{array}{l}\text { Dwarf Mongoose, } \\
\text { Ethiopian Dwarf } \\
\text { Mongoose }\end{array}$ & 2.46 & 289 & extant & 0 & NA & LC & ginsect/carn \\
\hline
\end{tabular}




\begin{tabular}{|c|c|c|c|c|c|c|c|c|c|c|c|c|}
\hline AF & Carnivora & Herpestidae & Helogale & parvula & $\begin{array}{l}\text { Common Dwarf } \\
\text { Mongoose, Dwarf } \\
\text { Mongoose }\end{array}$ & 2.48 & 300 & extant & 0 & NA & LC & ginsect/carn \\
\hline $\mathrm{AF}$ & Carnivora & Herpestidae & Herpestes & ichneumon & $\begin{array}{l}\text { Egyptian Mongoose, } \\
\text { Large Grey Mongoose }\end{array}$ & 3.45 & 2850 & extant & 0 & NA & $\mathrm{LC}$ & Carn \\
\hline AF & Carnivora & Herpestidae & Herpestes & naso & Long-nosed Mongoose & 3.48 & 3000 & extant & 0 & NA & LC & Carn \\
\hline AF & Carnivora & Herpestidae & Ichneumia & albicauda & White-tailed Mongoose & 3.54 & 3500 & extant & 0 & NA & LC & ginsect/carn \\
\hline AF & Carnivora & Herpestidae & Liberiictis & kuhni & Liberian Mongoose & 3.33 & 2150 & extant & Future & NA & Vu & ginsect \\
\hline AF & Carnivora & Herpestidae & Mungos & gambianus & Gambian Mongoose & 3.18 & 1500 & extant & 0 & NA & LC & ginsect/carn \\
\hline $\mathrm{AF}$ & Carnivora & Herpestidae & Mungos & mungo & Banded Mongoose & 3.28 & 1925 & extant & 0 & NA & LC & ginsect/carn \\
\hline $\mathrm{AF}$ & Carnivora & Herpestidae & Paracynictis & selousi & Selous's Mongoose & 3.21 & 1640 & extant & 0 & NA & LC & ginsect/carn \\
\hline AF & Carnivora & Herpestidae & Rhynchogale & melleri & Meller's Mongoose & 3.40 & 2500 & extant & 0 & NA & LC & ginsect \\
\hline $\mathrm{AF}$ & Carnivora & Herpestidae & Suricata & suricatta & $\begin{array}{l}\text { Meerkat, Suricate, } \\
\text { Slender-tailed Meerkat }\end{array}$ & 2.86 & 726 & extant & 0 & NA & LC & ginsect \\
\hline $\mathrm{AF}$ & Carnivora & Hyaenidae & Crocuta & crocuta & Spotted Hyaena & 4.80 & 63000 & extant & 0 & NA & LC & Carn \\
\hline AF & Carnivora & Hyaenidae & Hyaena & brunnea & brown hyaena & 4.51 & 32200 & extant & Future & NA & NT & Carn \\
\hline $\mathrm{AF}$ & Carnivora & Hyaenidae & Hyaena & hyaena & Striped Hyaena & 4.66 & 46000 & extant & Future & NA & NT & Carn \\
\hline AF & Carnivora & Hyaenidae & Proteles & cristata & Aardwolf & 4.00 & 10000 & extant & 0 & NA & LC & ginsect \\
\hline $\mathrm{AF}$ & Carnivora & Mustelidae & Aonyx & capensis & $\begin{array}{l}\text { African Clawless Otter, } \\
\text { Cape Clawless Otter } \\
\text { Congo Clawless Otter, } \\
\text { Zaire Clawless Otter, }\end{array}$ & 4.28 & 19000 & extant & Future & NA & NT & ivert/piscivor \\
\hline $\mathrm{AF}$ & Carnivora & Mustelidae & Aonyx & congicus & $\begin{array}{l}\text { Small-clawed Otter, } \\
\text { Small-toothed Clawless } \\
\text { Otter, Cameroon } \\
\text { Clawless Otter }\end{array}$ & 4.38 & 23800 & extant & Future & NA & NT & piscivore \\
\hline $\mathrm{AF}$ & Carnivora & Mustelidae & Hydrictis & maculicollis & $\begin{array}{l}\text { Spotted-necked Otter, } \\
\text { Speckle-throated Otter, } \\
\text { Spot-necked Otter }\end{array}$ & 3.60 & 4000 & extant & Future & NA & NT & piscivore \\
\hline $\mathrm{AF}$ & Carnivora & Mustelidae & Ictonyx & libycus & $\begin{array}{l}\text { Libyan Striped Weasel, } \\
\text { Saharan Striped } \\
\text { Weasel, }\end{array}$ & 2.80 & 625 & extant & 0 & NA & LC & carn/ginsect \\
\hline $\mathrm{AF}$ & Carnivora & Mustelidae & Ictonyx & striatus & $\begin{array}{l}\text { Zorilla, Striped Polecat, } \\
\text { Striped Weasel } \\
\text { Eurasian Otter, } \\
\text { European Otter, }\end{array}$ & 3.11 & 1300 & extant & 0 & NA & LC & ginsect/carn \\
\hline $\mathrm{AF}$ & Carnivora & Mustelidae & Lutra & lutra & $\begin{array}{l}\text { European River Otter, } \\
\text { Old World Otter, } \\
\text { Common Otter }\end{array}$ & 4.04 & 11000 & extant & Future & NA & NT & piscivore \\
\hline $\mathrm{AF}$ & Carnivora & Mustelidae & Mellivora & capensis & Honey Badger, Ratel & 3.90 & 8000 & extant & 0 & NA & LC & ginsect/carn \\
\hline $\mathrm{AF}$ & Carnivora & Mustelidae & Mustela & nivalis & Least Weasel, Weasel & 2.27 & 187 & extant & 0 & NA & LC & carn/ginsect \\
\hline $\mathrm{AF}$ & Carnivora & Mustelidae & Mustela & putorius & $\begin{array}{l}\text { Western Polecat, } \\
\text { European Polecat }\end{array}$ & 3.04 & 1100 & extant & 0 & NA & LC & carn/ginsect \\
\hline $\mathrm{AF}$ & Carnivora & Mustelidae & Poecilogale & albinucha & $\begin{array}{l}\text { African Striped Weasel } \\
\text { African Palm Civet, Two- }\end{array}$ & 2.53 & 340 & extant & 0 & NA & LC & Carn \\
\hline AF & Carnivora & Nandiniidae & Nandinia & binotata & $\begin{array}{l}\text { spotted Palm Civet, } \\
\text { Tree Civet }\end{array}$ & 3.30 & 2000 & extant & 0 & NA & $\mathrm{LC}$ & frug/carn \\
\hline $\mathrm{AF}$ & Carnivora & Viverridae & Civettictis & civetta & African Civet & 4.08 & 12000 & extant & 0 & NA & LC & carn/frug \\
\hline $\mathrm{AF}$ & Carnivora & Viverridae & Genetta & abyssinica & $\begin{array}{l}\text { Ethiopian Genet, } \\
\text { Abyssinian Genet }\end{array}$ & 3.22 & 1650 & extant & 0 & NA & DD & carn/ginsect \\
\hline $\mathrm{AF}$ & Carnivora & Viverridae & Genetta & angolensis & $\begin{array}{l}\text { Miombo Genet, } \\
\text { Angolan Genet }\end{array}$ & 3.22 & 1650 & extant & 0 & NA & LC & Carn \\
\hline
\end{tabular}




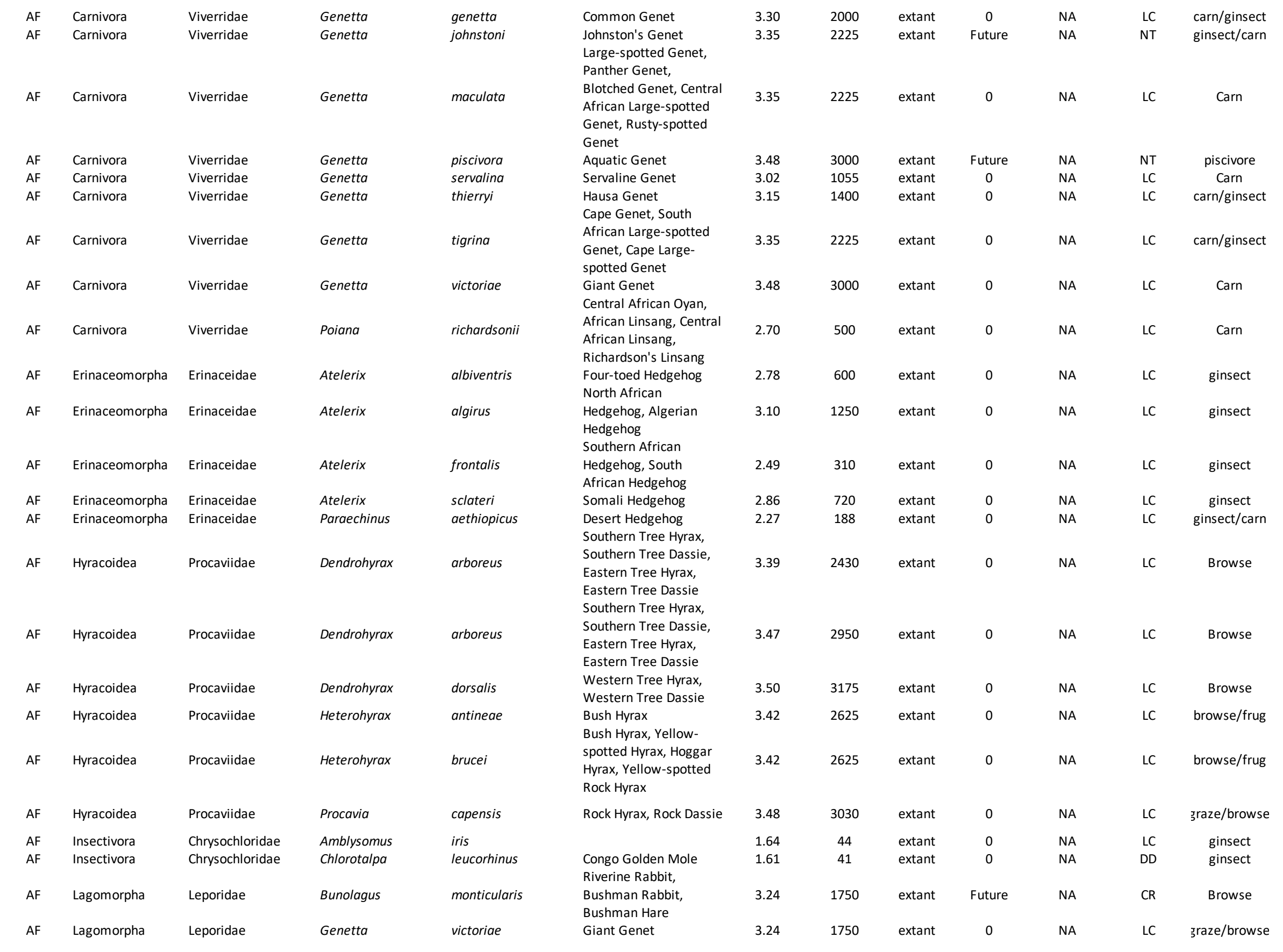




\begin{tabular}{|c|c|c|c|c|c|c|c|c|c|c|c|c|}
\hline AF & Lagomorpha & Leporidae & Lepus & capensis & $\begin{array}{l}\text { Cape Hare, Desert Hare, } \\
\text { Arabian Hare, Brown } \\
\text { Hare }\end{array}$ & 3.25 & 1775 & extant & 0 & NA & LC & Jraze/browse \\
\hline AF & Lagomorpha & Leporidae & Lepus & fagani & Ethiopian Hare & 3.44 & 2764 & extant & 0 & NA & DD & కraze/browse \\
\hline $\mathrm{AF}$ & Lagomorpha & Leporidae & Lepus & saxatilis & $\begin{array}{l}\text { Scrub Hare, Savannah } \\
\text { Hare }\end{array}$ & 3.37 & 2363 & extant & 0 & NA & $\mathrm{LC}$ & Jraze/browse \\
\hline $\mathrm{AF}$ & Lagomorpha & Leporidae & Lepus & starcki & Ethiopian Highland Hare & 3.44 & 2750 & extant & 0 & NA & $\mathrm{LC}$ & Jraze/browse \\
\hline AF & Lagomorpha & Leporidae & Oryctolagus & cuniculus & European Rabbit & 3.33 & 2150 & extant & Future & NA & NT & Jraze/browse \\
\hline $\mathrm{AF}$ & Lagomorpha & Leporidae & Poelagus & marjorita & $\begin{array}{l}\text { Bunyoro Rabbit } \\
\text { Natal Red Rock Hare, }\end{array}$ & 3.40 & 2500 & extant & 0 & NA & LC & graze \\
\hline AF & Lagomorpha & Leporidae & Pronolagus & crassicaudatus & $\begin{array}{l}\text { Natal Red Rockhare, } \\
\text { Greater Red Rockhare } \\
\text { Jameson's Red Rock }\end{array}$ & 3.32 & 2089 & extant & 0 & NA & $\mathrm{LC}$ & graze \\
\hline AF & Lagomorpha & Leporidae & Pronolagus & randensis & $\begin{array}{l}\text { Hare, Jameson's Red } \\
\text { Rockhare }\end{array}$ & 3.37 & 2335 & extant & 0 & NA & LC & graze \\
\hline AF & Lagomorpha & Leporidae & Pronolagus & rupestris & $\begin{array}{l}\text { Smith's Red Rock Hare, } \\
\text { Smith's Red Rockhare } \\
\text { Short-snouted Sengi, }\end{array}$ & 3.24 & 1754 & extant & 0 & NA & LC & graze \\
\hline $\mathrm{AF}$ & Macroscelidea & Macroscelididae & Elephantulus & brachyrhynchus & $\begin{array}{l}\text { Short-snouted Elephant- } \\
\text { shrew }\end{array}$ & 1.68 & 48 & extant & 0 & NA & $\mathrm{LC}$ & ginsect \\
\hline AF & Macroscelidea & Macroscelididae & Elephantulus & edwardii & $\begin{array}{l}\text { Cape Rock Sengi, Cape } \\
\text { Rock Elephant-shrew }\end{array}$ & 1.68 & 48 & extant & 0 & NA & $\mathrm{LC}$ & ginsect \\
\hline AF & Macroscelidea & Macroscelididae & Elephantulus & fuscipes & $\begin{array}{l}\text { Dusky-footed Sengi, } \\
\text { Dusky-footed Elephant- } \\
\text { shrew }\end{array}$ & 1.76 & 57 & extant & 0 & NA & DD & ginsect \\
\hline AF & Macroscelidea & Macroscelididae & Elephantulus & fuscus & $\begin{array}{l}\text { Dusky Sengi, Dusky } \\
\text { Elephant-shrew }\end{array}$ & 1.69 & 49 & extant & 0 & NA & DD & ginsect \\
\hline $\mathrm{AF}$ & Macroscelidea & Macroscelididae & Elephantulus & intufi & $\begin{array}{l}\text { Bushveld Sengi, } \\
\text { Bushveld Elephant- } \\
\text { shrew }\end{array}$ & 1.69 & 50 & extant & 0 & NA & $\mathrm{LC}$ & ginsect \\
\hline$A F$ & Macroscelidea & Macroscelididae & Elephantulus & myurus & $\begin{array}{l}\text { Eastern Rock Sengi, } \\
\text { Eastern Elephant-shrew }\end{array}$ & 1.72 & 52 & extant & 0 & NA & $\mathrm{LC}$ & ginsect \\
\hline$A F$ & Macroscelidea & Macroscelididae & Elephantulus & revoili & Somali Elephant-shrew & 1.51 & 33 & extant & 0 & NA & DD & ginsect \\
\hline$A F$ & Macroscelidea & Macroscelididae & Elephantulus & rozeti & $\begin{array}{l}\text { North African Sengi, } \\
\text { North African Elephant- } \\
\text { shrew }\end{array}$ & 1.68 & 48 & extant & 0 & NA & $\mathrm{LC}$ & ginsect \\
\hline$A F$ & Macroscelidea & Macroscelididae & Elephantulus & rufescens & $\begin{array}{l}\text { Rufous Sengi, Rufous } \\
\text { Elephant-shrew }\end{array}$ & 1.57 & 38 & extant & 0 & NA & $\mathrm{LC}$ & ginsect \\
\hline AF & Macroscelidea & Macroscelididae & Elephantulus & rupestris & $\begin{array}{l}\text { Western Rock Sengi, } \\
\text { Western Rock Elephant- } \\
\text { shrew } \\
\text { Karoo Round-eared }\end{array}$ & 1.82 & 66 & extant & 0 & NA & LC & ginsect \\
\hline$A F$ & Macroscelidea & Macroscelididae & Macroscelides & proboscideus & $\begin{array}{l}\text { Sengi, Karoo Round- } \\
\text { eared Elephant-shrew }\end{array}$ & 1.58 & 38 & extant & 0 & NA & LC & ginsect \\
\hline AF & Macroscelidea & Macroscelididae & Petrodromus & tetradactylus & $\begin{array}{l}\text { Four-toed Sengi, Four- } \\
\text { toed Elephant-shrew } \\
\text { Golden-rumped Sengi, }\end{array}$ & 2.27 & 187 & extant & 0 & NA & LC & ginsect \\
\hline AF & Macroscelidea & Macroscelididae & Rhynchocyon & chrysopygus & $\begin{array}{l}\text { Golden-rumped } \\
\text { Elephant-shrew }\end{array}$ & 2.73 & 540 & extant & Future & NA & EN & ginsect \\
\hline
\end{tabular}




\begin{tabular}{|c|c|c|c|c|c|c|c|c|c|c|c|c|}
\hline $\mathrm{AF}$ & Macroscelidea & Macroscelididae & Rhynchocyon & cirnei & $\begin{array}{l}\text { Chequered Sengi, } \\
\text { Chequered Elephant- } \\
\text { shrew }\end{array}$ & 2.69 & 490 & extant & Future & NA & NT & ginsect \\
\hline AF & Macroscelidea & Macroscelididae & Rhynchocyon & petersi & Black and Rufous Sengi & 2.63 & 424 & extant & 0 & NA & LC & ginsect \\
\hline AF & Perissodactyla & Equidae & Equus & algericus & large horse & & & extinct & Holocene & 6.6 & EX & Graze \\
\hline AF & Perissodactyla & Equidae & Equus & asinus & & 5.22 & 164999 & extant & 0 & NA & LC & graze \\
\hline AF & Perissodactyla & Equidae & Equus & burchellii & & 5.44 & 276000 & extant & Future & NA & NT & graze \\
\hline AF & Perissodactyla & Equidae & Equus & caballus & & 5.40 & 250000 & extant & 0 & NA & -999 & graze \\
\hline AF & Perissodactyla & Equidae & Equus & capensis & & 5.60 & 400000 & extinct & TP & 11 & EX & graze \\
\hline AF & Perissodactyla & Equidae & Equus & grevyi & Grevy's Zebra & 5.61 & 408000 & extant & Future & NA & EN & graze \\
\hline AF & Perissodactyla & Equidae & Equus & melkiensis & $\begin{array}{l}\text { related to wild ass } \\
\text { Plains Zebra, Painted }\end{array}$ & & & extinct & EP & 37 & EX & graze \\
\hline $\mathrm{AF}$ & Perissodactyla & Equidae & Equus & quagga & $\begin{array}{l}\text { Zebra, Common Zebra, } \\
\text { Burchell's Zebra } \\
\text { Mountain Zebra, }\end{array}$ & 5.60 & 400000 & extinct & Holocene & NA & EX & graze \\
\hline AF & Perissodactyla & Equidae & Equus & zebra & $\begin{array}{l}\text { Hartmann's Mountain } \\
\text { Zebra }\end{array}$ & 5.46 & 286999 & extant & Future & NA & VU & graze \\
\hline $\mathrm{AF}$ & Perissodactyla & Rhinocerotidae & Ceratotherium & simum & $\begin{array}{l}\text { White Rhinoceros, } \\
\text { Northern White } \\
\text { Rhinoceros, Square- } \\
\text { lipped Rhinoceros }\end{array}$ & 6.47 & 2949986 & extant & Future & NA & NT & graze \\
\hline $\mathrm{AF}$ & Perissodactyla & Rhinocerotidae & Diceros & bicornis & $\begin{array}{l}\text { Black Rhinoceros, Hook- } \\
\text { lipped Rhinoceros }\end{array}$ & 6.07 & 1180511 & extant & Future & NA & CR & Browse \\
\hline AF & Perissodactyla & Rhinocerotidae & Stephanorhinus & hemitoechus & narrow-nosed rhino & 6.30 & 2000000 & extinct & EP & 41 & EX & zraze/browse \\
\hline AF & Perissodactyla & Rhinocerotidae & Stephanorhinus & kirchbergensis & Merck's rhinoceros & 6.48 & 3000000 & extinct & TP & 19 & EX & 3rowse/graze \\
\hline AF & Pholidota & Manidae & Manis & gigantea & Giant Ground Pangolin & 4.52 & 33000 & extant & Future & NA & VU & ginsect \\
\hline $\mathrm{AF}$ & Pholidota & Manidae & Manis & temminckii & $\begin{array}{l}\text { Temminck's Ground } \\
\text { Pangolin }\end{array}$ & 3.86 & 7180 & extant & Future & NA & VU & ginsect \\
\hline AF & Pholidota & Manidae & Manis & tetradactyla & Black-bellied Pangolin & 3.44 & 2750 & extant & Future & NA & VU & ginsect \\
\hline AF & Pholidota & Manidae & Manis & tricuspis & White-bellied Pangolin & 3.32 & 2067 & extant & Future & NA & VU & ginsect \\
\hline $\mathrm{AF}$ & Primates & Cercopithecidae & Allenopithecus & nigroviridis & $\begin{array}{l}\text { Allen's Swamp Monkey, } \\
\text { Blackish-green Guenon }\end{array}$ & 3.68 & 4750 & extant & 0 & NA & LC & frug/ginsect \\
\hline $\mathrm{AF}$ & Primates & Cercopithecidae & Cercocebus & agilis & $\begin{array}{l}\text { Agile Mangabey } \\
\text { Tana River Mangabey, }\end{array}$ & 3.90 & 8000 & extant & 0 & NA & LC & frug \\
\hline $\mathrm{AF}$ & Primates & Cercopithecidae & Cercocebus & galeritus & $\begin{array}{l}\text { Tana River Crested } \\
\text { Mangabey } \\
\text { Red-capped Mangabey, } \\
\text { White-collared }\end{array}$ & 3.89 & 7835 & extant & Future & NA & EN & frug \\
\hline $\mathrm{AF}$ & Primates & Cercopithecidae & Cercocebus & torquatus & $\begin{array}{l}\text { Mangabey, Red- } \\
\text { crowned Mangabey, } \\
\text { Sooty Mangabey, } \\
\text { Collared Mangabey } \\
\text { Red-tailed Monkey, } \\
\text { Schmidt's Guenon, }\end{array}$ & 3.89 & 7750 & extant & Future & NA & VU & frug \\
\hline $\mathrm{AF}$ & Primates & Cercopithecidae & Cercopithecus & ascanius & $\begin{array}{l}\text { Black-cheeked White- } \\
\text { nosed Monkey, Redtail } \\
\text { Monkey, Red-tailed } \\
\text { Guenon }\end{array}$ & 3.55 & 3550 & extant & 0 & NA & LC & frug/ginsect \\
\hline
\end{tabular}




\begin{tabular}{|c|c|c|c|c|c|c|c|c|c|c|c|c|}
\hline $\mathrm{AF}$ & Primates & Cercopithecidae & Cercopithecus & campbelli & $\begin{array}{l}\text { Campbell's Monkey, } \\
\text { Campbell's Guenon, } \\
\text { Campbell's Mona } \\
\text { Monkey } \\
\text { Moustached Monkey, }\end{array}$ & 3.61 & 4100 & extant & 0 & NA & LC & frug \\
\hline $\mathrm{AF}$ & Primates & Cercopithecidae & Cercopithecus & cephus & $\begin{array}{l}\text { Mustached Monkey, } \\
\text { Moustached Guenon }\end{array}$ & 3.54 & 3485 & extant & 0 & NA & LC & frug \\
\hline $\mathrm{AF}$ & Primates & Cercopithecidae & Cercopithecus & diana & $\begin{array}{l}\text { Diana Monkey, Diana } \\
\text { Guenon } \\
\text { Dryas Monkey, Salonga }\end{array}$ & 3.62 & 4175 & extant & Future & NA & VU & frug \\
\hline $\mathrm{AF}$ & Primates & Cercopithecidae & Cercopithecus & dryas & $\begin{array}{l}\text { Guenon, Dryad Monkey, } \\
\text { Dryas Guenon } \\
\text { Red-bellied Monkey, } \\
\text { White-throated }\end{array}$ & 3.49 & 3075 & extant & Future & NA & CR & frug/browse \\
\hline AF & Primates & Cercopithecidae & Cercopithecus & erythrogaster & $\begin{array}{l}\text { Monkey, White- } \\
\text { throated Guenon, Red- } \\
\text { bellied Guenon }\end{array}$ & 3.40 & 2500 & extant & Future & NA & VU & frug/ginsect \\
\hline $\mathrm{AF}$ & Primates & Cercopithecidae & Cercopithecus & erythrotis & $\begin{array}{l}\text { Red-eared Monkey, } \\
\text { Russet-eared Guenon, } \\
\text { Red-eared Guenon }\end{array}$ & 3.59 & 3918 & extant & Future & NA & VU & frug \\
\hline $\mathrm{AF}$ & Primates & Cercopithecidae & Cercopithecus & hamlyni & $\begin{array}{l}\text { Owl-faced Monkey, } \\
\text { Hamlyn's Monkey, Owl- } \\
\text { faced Guenon }\end{array}$ & 3.84 & 6875 & extant & Future & NA & Vu & browse/frug \\
\hline $\mathrm{AF}$ & Primates & Cercopithecidae & Cercopithecus & Ihoesti & $\begin{array}{l}\text { L'Hoest's Monkey } \\
\text { Blue Monkey, Samango, }\end{array}$ & 3.73 & 5375 & extant & Future & NA & VU & frug \\
\hline $\mathrm{AF}$ & Primates & Cercopithecidae & Cercopithecus & mitis & $\begin{array}{l}\text { Golden Monkey, } \\
\text { Diademed Monkey, } \\
\text { Sykes' Monkey }\end{array}$ & 3.70 & 5000 & extant & 0 & NA & LC & frug/browse \\
\hline $\mathrm{AF}$ & Primates & Cercopithecidae & Cercopithecus & mona & $\begin{array}{l}\text { Mona Monkey, Mona } \\
\text { Guenon }\end{array}$ & 3.62 & 4208 & extant & 0 & NA & $\mathrm{LC}$ & frug \\
\hline $\mathrm{AF}$ & Primates & Cercopithecidae & Cercopithecus & neglectus & $\begin{array}{l}\text { De Brazza's Monkey, De } \\
\text { Brazza's Guenon }\end{array}$ & 3.74 & 5480 & extant & 0 & NA & LC & frug/browse \\
\hline AF & Primates & Cercopithecidae & Cercopithecus & nictitans & Putty-nosed Monkey & 3.76 & 5803 & extant & 0 & NA & $\mathrm{LC}$ & frug/browse \\
\hline $\mathrm{AF}$ & Primates & Cercopithecidae & Cercopithecus & petaurista & Spot-nosed Monkey & 3.44 & 2775 & extant & 0 & NA & LC & frug \\
\hline AF & Primates & Cercopithecidae & Cercopithecus & pogonias & crested mona monkey, & 3.58 & 3765 & extant & 0 & NA & LC & frug \\
\hline $\mathrm{AF}$ & Primates & Cercopithecidae & Cercopithecus & preussi & Preuss's monkey & 3.72 & 5250 & extant & Future & NA & EN & frug/browse \\
\hline AF & Primates & Cercopithecidae & Cercopithecus & sclateri & Sclater's Monkey & 3.53 & 3375 & extant & Future & NA & Vu & frug \\
\hline $\mathrm{AF}$ & Primates & Cercopithecidae & Cercopithecus & solatus & Sun-tailed Monkey & 3.81 & 6500 & extant & Future & NA & VU & frug \\
\hline AF & Primates & Cercopithecidae & Cercopithecus & wolfi & Wolf's mona monkey & 3.60 & 4000 & extant & 0 & NA & LC & frug \\
\hline AF & Primates & Cercopithecidae & Chlorocebus & aethiops & $\begin{array}{l}\text { Grivet Monkey } \\
\text { Angolan Colobus, }\end{array}$ & 3.62 & 4155 & extant & 0 & NA & LC & frug \\
\hline $\mathrm{AF}$ & Primates & Cercopithecidae & Colobus & angolensis & $\begin{array}{l}\text { Angola Colobus, } \\
\text { Angolan Black-and- } \\
\text { white Colobus } \\
\text { Guereza, Magistrate }\end{array}$ & 3.99 & 9850 & extant & 0 & NA & LC & Browse \\
\hline $\mathrm{AF}$ & Primates & Cercopithecidae & Colobus & guereza & $\begin{array}{l}\text { Colobus, Eastern Black- } \\
\text { and-white Colobus } \\
\text { King Colobus, Western } \\
\text { Black-and-White }\end{array}$ & 4.03 & 10600 & extant & 0 & NA & LC & Browse \\
\hline $\mathrm{AF}$ & Primates & Cercopithecidae & Colobus & polykomos & $\begin{array}{l}\text { Colobus, Western Pied } \\
\text { Colobus, Ursine Black- } \\
\text { and-White Colobus }\end{array}$ & 4.00 & 9917 & extant & Future & NA & Vu & browse/frug \\
\hline
\end{tabular}




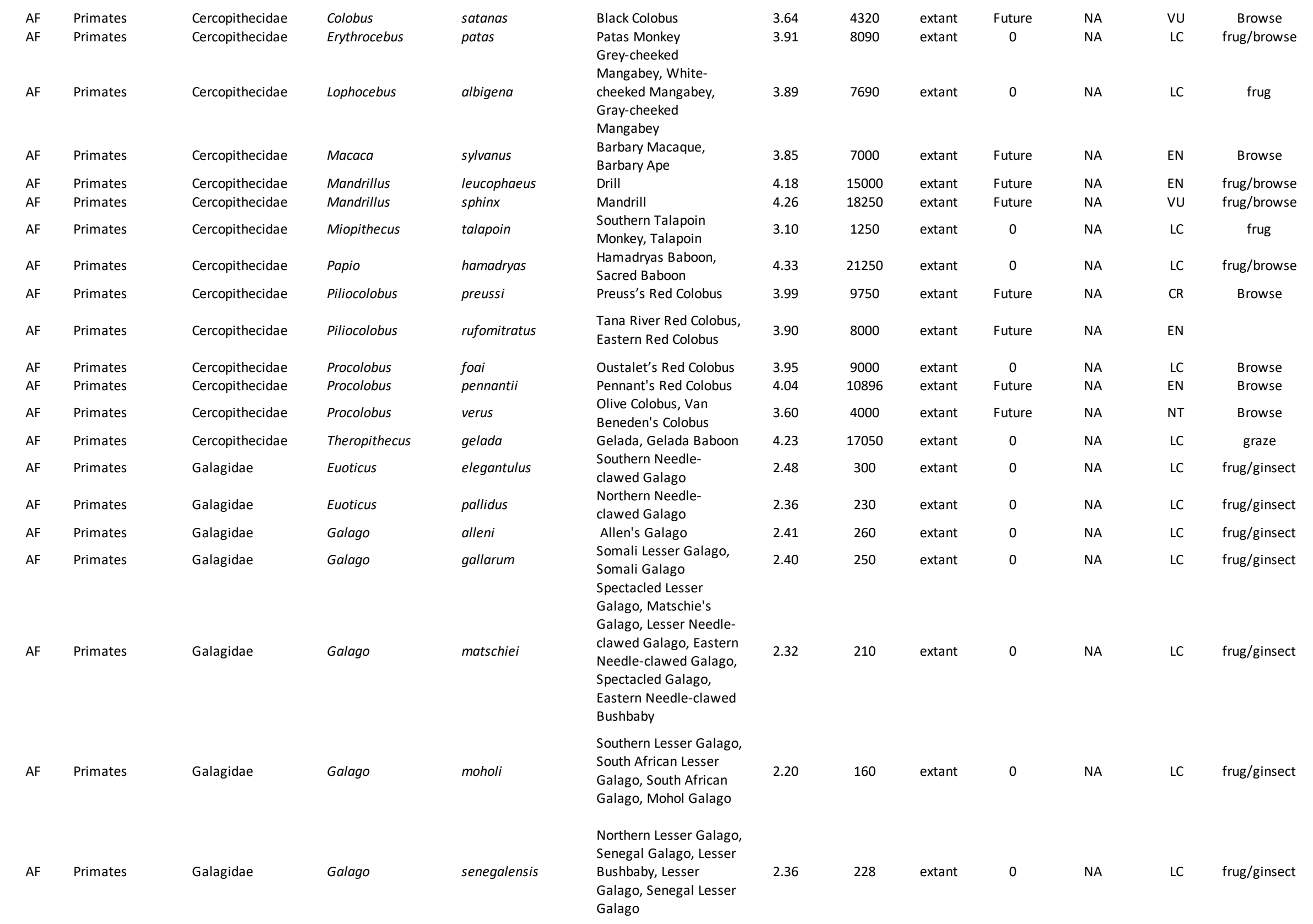


Galago, Large-eared

Greater Galago,

3.18

1500

extant

NA

LC frug/ginsect

Garnett's Greater

Galago

\begin{tabular}{|c|c|c|c|c|}
\hline AF & Primates & Galagidae & Otolemur & garnettii \\
\hline$A F$ & Primates & Galagonidae & Galago & demidoff \\
\hline $\mathrm{AF}$ & Primates & Galagonidae & Galago & zanzibaric \\
\hline$A F$ & Primates & Hominidae & Gorilla & gorilla \\
\hline AF & Primates & Hominidae & Pan & paniscus \\
\hline$A F$ & Primates & Hominidae & Pan & troglodytes \\
\hline AF & Primates & Lorisidae & Arctocebus & aureus \\
\hline$A F$ & Primates & Lorisidae & Arctocebus & calabarens \\
\hline $\mathrm{AF}$ & Primates & Lorisidae & Perodicticus & potto \\
\hline$A F$ & Proboscidea & Elephantidae & Elephas & iolensis \\
\hline AF & Proboscidea & Elephantidae & Loxodonta & africana \\
\hline$A F$ & Rodentia & Anomaluridae & Anomalurus & beecrofti \\
\hline $\mathrm{AF}$ & Rodentia & Anomaluridae & Anomalurus & derbianus \\
\hline$A F$ & Rodentia & Anomaluridae & Anomalurus & pelii \\
\hline$A F$ & Rodentia & Anomaluridae & Anomalurus & pusillus \\
\hline AF & Rodentia & Anomaluridae & Idiurus & macrotis \\
\hline$A F$ & Rodentia & Anomaluridae & Idiurus & zenkeri \\
\hline
\end{tabular}

Garnett's Greater

Galago, Small-eared

Galago, Small-eared

2.88

760

reater Galago

Demidoff's Dwarf

Galago

\begin{tabular}{|c|c|c|c|c|c|c|}
\hline 1.79 & 61 & extant & 0 & NA & LC & ginsect \\
\hline 2.00 & 101 & extant & 0 & NA & LC & ginsect/frug \\
\hline 5.12 & 131626 & extant & Future & NA & CR & frug/browse \\
\hline 4.53 & 34000 & extant & Future & NA & EN & dg/browse/ca \\
\hline 4.63 & 42500 & extant & Future & NA & EN & ıg/browse/ca \\
\hline 2.37 & 235 & extant & 0 & NA & LC & ginsect \\
\hline 2.32 & 210 & extant & 0 & NA & LC & ginsect/frug \\
\hline 3.04 & 1100 & extant & 0 & NA & LC & frug/ginsect \\
\hline 6.81 & 6500000 & extinct & TP & 12 & EX & graze \\
\hline 6.60 & 3940034 & extant & Future & NA & VU & క̧raze/browse \\
\hline 2.59 & 389 & extant & 0 & NA & LC & frug/browse \\
\hline 2.81 & 650 & extant & 0 & NA & LC & browse/frug \\
\hline 3.25 & 1770 & extant & 0 & NA & DD & browse/frug \\
\hline 2.40 & 250 & extant & 0 & NA & LC & browse/frug \\
\hline 1.48 & 30 & extant & 0 & NA & LC & frug \\
\hline 2.00 & 100 & extant & 0 & NA & LC & frug \\
\hline
\end{tabular}

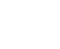

Tanzania Coast Dwarf

Galago

Western Gorilla,

Lowland Gorilla

Bonobo, Pygmy

Chimpanzee

Chimpanzee, Robust

Chimpanzee, Common

Chimpanzee

Golden Angwantibo,

Golden Potto

Calabar Angwantibo,

Golden Potto,

West African Potto,

Western Potto

African Elephant

Beecroft's Scaly-tailed

Squirrel, Beecroft's

Flying Squirrel

Lord Derby's Scaly-

tailed Squirrel, Derby's

Flying Squirrel, Lord

Derby's Flying Squirre

Pel's Flying Squirrel,

Pel's Scaly-tailed

Squirrel

Dwarf Scaly-tailed

Squirrel

Long-eared Flying

Squirrel, Long-eared

Scaly-tailed Flying

Squirrel

Pygmy Scaly-tailed

Flying Squirrel 


\begin{tabular}{|c|c|c|c|c|c|c|c|c|c|c|c|c|}
\hline AF & Rodentia & Anomaluridae & Zenkerella & insignis & $\begin{array}{l}\text { Cameroon Scaly-tail, } \\
\text { Flightless Scaly-tailed } \\
\text { Squirrel }\end{array}$ & 2.30 & 200 & extant & 0 & NA & LC & frug \\
\hline AF & Rodentia & Bathyergidae & Bathyergus & janetta & $\begin{array}{l}\text { Namaqua Dune Mole } \\
\text { Rat }\end{array}$ & 2.52 & 332 & extant & 0 & NA & LC & Browse \\
\hline AF & Rodentia & Bathyergidae & Bathyergus & suillus & Cape Dune Mole Rat & 2.80 & 625 & extant & 0 & NA & LC & Browse \\
\hline AF & Rodentia & Bathyergidae & Cryptomys & bocagei & Bocage's Mole Rat & 1.97 & 94 & extant & 0 & NA & LC & jrowse/graze \\
\hline $\mathrm{AF}$ & Rodentia & Bathyergidae & Cryptomys & damarensis & & 2.19 & 153 & extant & 0 & NA & LC & orowse/graze \\
\hline AF & Rodentia & Bathyergidae & Cryptomys & foxi & & 2.24 & 174 & extant & 0 & NA & $\mathrm{DD}$ & Jrowse/graze \\
\hline AF & Rodentia & Bathyergidae & Cryptomys & hottentotus & African Mole Rat & 2.01 & 102 & extant & 0 & NA & LC & orowse/graze \\
\hline AF & Rodentia & Bathyergidae & Cryptomys & mechowi & & 2.43 & 272 & extant & 0 & NA & LC & jrowse/graze \\
\hline $\mathrm{AF}$ & Rodentia & Bathyergidae & Cryptomys & ochraceocinereus & & 2.30 & 200 & extant & 0 & NA & LC & orowse/graze \\
\hline $\mathrm{AF}$ & Rodentia & Bathyergidae & Cryptomys & zechi & & 2.35 & 223 & extant & 0 & NA & LC & orowse/graze \\
\hline AF & Rodentia & Bathyergidae & Georychus & capensis & Cape Mole Rat & 2.37 & 233 & extant & 0 & NA & LC & Browse \\
\hline AF & Rodentia & Bathyergidae & Heliophobius & argenteocinereus & Silvery Mole Rat & 2.26 & 181 & extant & 0 & NA & LC & Browse \\
\hline AF & Rodentia & Bathyergidae & Heterocephalus & glaber & Naked Mole Rat & 1.74 & 55 & extant & 0 & NA & LC & Browse \\
\hline$A F$ & Rodentia & Cricetidae & Lophiomys & imhausi & Crested Rat & 2.88 & 755 & extant & 0 & NA & LC & frug/browse \\
\hline AF & Rodentia & Ctenodactylidae & Ctenodactylus & gundi & $\begin{array}{l}\text { Gundi, North African } \\
\text { Gundi }\end{array}$ & 2.46 & 289 & extant & 0 & NA & LC & э̧raze/browse \\
\hline $\mathrm{AF}$ & Rodentia & Ctenodactylidae & Ctenodactylus & vali & Val's Gundi & 2.24 & 175 & extant & 0 & NA & DD & эraze/browse \\
\hline AF & Rodentia & Ctenodactylidae & Felovia & vae & Felou Gundi & 2.31 & 205 & extant & 0 & NA & DD & эraze/browse \\
\hline AF & Rodentia & Ctenodactylidae & Pectinator & spekei & Speke's Pectinator & 2.20 & 160 & extant & 0 & NA & LC & Browse \\
\hline $\mathrm{AF}$ & Rodentia & Dipodidae & Allactaga & tetradactyla & Four-toed Jerboa & 1.72 & 52 & extant & Future & NA & Vu & graze \\
\hline$A F$ & Rodentia & Dipodidae & Jaculus & jaculus & Lesser Egyptian Jerboa & 1.75 & 57 & extant & 0 & NA & LC & frug/graze \\
\hline AF & Rodentia & Dipodidae & Jaculus & orientalis & Greater Egyptian Jerboa & 2.14 & 138 & extant & 0 & NA & LC & frug/graze \\
\hline AF & Rodentia & Gliridae & Eliomys & melanurus & Asian Garden Dormouse & 2.09 & 123 & extant & 0 & NA & LC & frug/ginsect \\
\hline $\mathrm{AF}$ & Rodentia & Gliridae & Graphiurus & christyi & Christy's Dormouse & 1.45 & 28 & extant & 0 & NA & LC & ginsect/carn \\
\hline AF & Rodentia & Gliridae & Graphiurus & crassicaudatus & $\begin{array}{l}\text { Jentink's Dormouse } \\
\text { Kellen's Dormouse, }\end{array}$ & 1.45 & 28 & extant & 0 & NA & DD & ginsect/carn \\
\hline AF & Rodentia & Gliridae & Graphiurus & kelleni & $\begin{array}{l}\text { Kellen's African } \\
\text { Dormouse }\end{array}$ & 1.45 & 28 & extant & 0 & NA & LC & ginsect/carn \\
\hline AF & Rodentia & Gliridae & Graphiurus & lorraineus & Lorrain Dormouse & 1.43 & 27 & extant & 0 & NA & LC & ginsect/carn \\
\hline AF & Rodentia & Gliridae & Graphiurus & microtis & Small-eared Dormouse & 1.45 & 28 & extant & 0 & NA & LC & ginsect/carn \\
\hline AF & Rodentia & Gliridae & Graphiurus & monardi & Monard's Dormouse & 1.45 & 28 & extant & 0 & NA & DD & ginsect/carn \\
\hline AF & Rodentia & Gliridae & Graphiurus & murinus & Woodland Dormouse & 1.28 & 19 & extant & 0 & NA & LC & ginsect/carn \\
\hline $\mathrm{AF}$ & Rodentia & Gliridae & Graphiurus & ocularis & $\begin{array}{l}\text { Spectacled Dormouse, } \\
\text { Namtap }\end{array}$ & 1.48 & 30 & extant & 0 & NA & LC & ginsect/carn \\
\hline AF & Rodentia & Gliridae & Graphiurus & platyops & Rock Dormouse & 1.66 & 46 & extant & 0 & NA & LC & ginsect/carn \\
\hline AF & Rodentia & Gliridae & Graphiurus & rupicola & Stone Dormouse & 1.45 & 28 & extant & 0 & NA & LC & ginsect/carn \\
\hline AF & Rodentia & Gliridae & Graphiurus & surdus & Silent Dormouse & 1.30 & 20 & extant & 0 & NA & DD & ginsect/carn \\
\hline AF & Rodentia & Hystricidae & Atherurus & africanus & $\begin{array}{l}\text { African Brush-tailed } \\
\text { Porcupine }\end{array}$ & 3.29 & 1934 & extant & 0 & NA & LC & frug/browse \\
\hline AF & Rodentia & Hystricidae & Hystrix & africaeaustralis & $\begin{array}{l}\text { Cape Porcupine } \\
\text { Crested Porcupine, }\end{array}$ & 4.17 & 14936 & extant & 0 & NA & LC & Browse \\
\hline AF & Rodentia & Hystricidae & Hystrix & cristata & $\begin{array}{l}\text { North African Crested } \\
\text { Porcupine }\end{array}$ & 4.30 & 20000 & extant & 0 & NA & LC & browse/frug \\
\hline AF & Rodentia & Muridae & Acomys & cahirinus & $\begin{array}{l}\text { Cairo Spiny Mouse, } \\
\text { Greater Wilfred's } \\
\text { Mouse, Northeast } \\
\text { African Spiny Mouse }\end{array}$ & 1.59 & 39 & extant & 0 & NA & LC & frug/browse \\
\hline
\end{tabular}




\begin{tabular}{|c|c|c|c|c|c|c|c|c|c|c|c|c|}
\hline $\mathrm{AF}$ & Rodentia & Muridae & Acomys & cineraceus & $\begin{array}{l}\text { Gray Spiny Mouse, Grey } \\
\text { Spiny Mouse }\end{array}$ & 1.51 & 33 & extant & 0 & NA & LC & frug/browse \\
\hline AF & Rodentia & Muridae & Acomys & ignitus & Fiery Spiny Mouse & 1.38 & 24 & extant & 0 & NA & LC & frug/browse \\
\hline AF & Rodentia & Muridae & Acomys & kempi & Kemp's Spiny Mouse & 1.34 & 22 & extant & 0 & NA & LC & frug/browse \\
\hline AF & Rodentia & Muridae & Acomys & louisae & Louise's Spiny Mouse & 1.31 & 20 & extant & 0 & NA & LC & frug/browse \\
\hline AF & Rodentia & Muridae & Acomys & mullah & Mullah Spiny Mouse & 1.51 & 33 & extant & 0 & NA & LC & frug/browse \\
\hline AF & Rodentia & Muridae & Acomys & percivali & Percival's Spiny Mouse & 1.34 & 22 & extant & 0 & NA & LC & frug/browse \\
\hline AF & Rodentia & Muridae & Acomys & russatus & Golden Spiny Mouse & 1.70 & 50 & extant & 0 & NA & LC & frug/browse \\
\hline $\mathrm{AF}$ & Rodentia & Muridae & Acomys & spinosissimus & $\begin{array}{l}\text { Spiny Mouse, Southern } \\
\text { African Spiny Mouse }\end{array}$ & 1.42 & 27 & extant & 0 & NA & LC & frug/browse \\
\hline AF & Rodentia & Muridae & Acomys & subspinosus & Cape Spiny Mouse & 1.32 & 21 & extant & 0 & NA & LC & frug/browse \\
\hline AF & Rodentia & Muridae & Acomys & wilsoni & Wilson's Spiny Mouse & 1.27 & 19 & extant & 0 & NA & LC & frug/browse \\
\hline $\mathrm{AF}$ & Rodentia & Muridae & Aethomys & bocagei & $\begin{array}{l}\text { Bocage's Rock Rat, } \\
\text { Bocage's Aethomys }\end{array}$ & 1.89 & 77 & extant & 0 & NA & LC & క̧raze/browse \\
\hline AF & Rodentia & Muridae & Aethomys & chrysophilus & $\begin{array}{l}\text { Red Rock Rat, Red Veld } \\
\text { Aethomys }\end{array}$ & 1.87 & 75 & extant & 0 & NA & LC & క̧raze/browse \\
\hline $\mathrm{AF}$ & Rodentia & Muridae & Aethomys & granti & $\begin{array}{l}\text { Grant's Rock Rat, } \\
\text { Grant's Rock Mouse, } \\
\text { Grant's Aethomys }\end{array}$ & 1.60 & 40 & extant & 0 & NA & LC & క̧raze/browse \\
\hline $\mathrm{AF}$ & Rodentia & Muridae & Aethomys & hindei & $\begin{array}{l}\text { Hinde's Rock Rat, } \\
\text { Hinde's Aethomys }\end{array}$ & 2.01 & 104 & extant & 0 & NA & LC & క̧raze/browse \\
\hline AF & Rodentia & Muridae & Aethomys & kaiseri & $\begin{array}{l}\text { Kaiser's Rock Rat, } \\
\text { Kaiser's Aethomys }\end{array}$ & 1.95 & 90 & extant & 0 & NA & LC & эraze/browse \\
\hline AF & Rodentia & Muridae & Aethomys & nyikae & $\begin{array}{l}\text { Nyika Rock Rat, Nyika } \\
\text { Aethomys }\end{array}$ & 1.89 & 77 & extant & 0 & NA & LC & э̧raze/browse \\
\hline $\mathrm{AF}$ & Rodentia & Muridae & Aethomys & silindensis & $\begin{array}{l}\text { Selinda Veld Rat, } \\
\text { Seilinda Aethomys, } \\
\text { Silinda Rock Rat }\end{array}$ & 1.89 & 77 & extant & 0 & NA & DD & క̧raze/browse \\
\hline $\mathrm{AF}$ & Rodentia & Muridae & Aethomys & stannarius & $\begin{array}{l}\text { Tinfields Rock Rat, } \\
\text { West African Aethomys }\end{array}$ & 1.89 & 77 & extant & 0 & NA & DD & эraze/browse \\
\hline AF & Rodentia & Muridae & Aethomys & thomasi & $\begin{array}{l}\text { Thomas's Rock Rat, } \\
\text { Thomas's Aethomys }\end{array}$ & 1.89 & 77 & extant & 0 & NA & LC & క̧raze/browse \\
\hline AF & Rodentia & Muridae & Ammodillus & imbellis & $\begin{array}{l}\text { Ammodile, Somali } \\
\text { Gerbil, Walo } \\
\text { Long-tailed Field }\end{array}$ & 1.70 & 50 & extant & 0 & NA & DD & frug/ginsect \\
\hline AF & Rodentia & Muridae & Apodemus & sylvaticus & $\begin{array}{l}\text { Mouse, Wood Mouse, } \\
\text { Small Wood Mouse }\end{array}$ & 1.33 & 22 & extant & 0 & NA & LC & frug/ginsect \\
\hline AF & Rodentia & Muridae & Arvicanthis & abyssinicus & $\begin{array}{l}\text { Abyssinian Grass Rat, } \\
\text { Ethiopian Arvicanthis }\end{array}$ & 1.89 & 77 & extant & 0 & NA & LC & frug/graze \\
\hline $\mathrm{AF}$ & Rodentia & Muridae & Arvicanthis & blicki & $\begin{array}{l}\text { Blick's Grass Rat, Blick's } \\
\text { Arvicanthis }\end{array}$ & 2.11 & 128 & extant & Future & NA & NT & frug/graze \\
\hline AF & Rodentia & Muridae & Arvicanthis & nairobae & $\begin{array}{l}\text { Nairobi Grass Rat, East } \\
\text { African Arvicanthis }\end{array}$ & 2.02 & 104 & extant & 0 & NA & LC & frug/graze \\
\hline AF & Rodentia & Muridae & Arvicanthis & niloticus & $\begin{array}{l}\text { African Grass Rat, } \\
\text { African Arvicanthis }\end{array}$ & 2.03 & 107 & extant & 0 & NA & LC & frug/graze \\
\hline AF & Rodentia & Muridae & Arvicanthis & somalicus & Neumann's Arvicanthi & 2.02 & 105 & extant & 0 & NA & LC & frug/graze \\
\hline $\mathrm{AF}$ & Rodentia & Muridae & Colomys & goslingi & $\begin{array}{l}\text { African Wading Rat, } \\
\text { African Water Rat }\end{array}$ & 1.80 & 63 & extant & 0 & NA & LC & ginsect \\
\hline AF & Rodentia & Muridae & Dasymys & foxi & Fox's Shaggy Rat & 2.14 & 138 & extant & 0 & NA & DD & Browse \\
\hline $\mathrm{AF}$ & Rodentia & Muridae & Dasymys & incomtus & $\begin{array}{l}\text { African Marsh Rat, } \\
\text { Common Dasymys }\end{array}$ & 2.14 & 138 & extant & 0 & NA & LC & Browse \\
\hline AF & Rodentia & Muridae & Dasymys & montanus & Montane Shaggy Rat & 2.14 & 138 & extant & Future & NA & EN & Browse \\
\hline
\end{tabular}




\begin{tabular}{|c|c|c|c|c|c|c|c|c|c|c|c|c|}
\hline $\mathrm{AF}$ & Rodentia & Muridae & Dasymys & nudipes & Angolan Marsh Rat & 2.14 & 138 & extant & 0 & NA & DD & Browse \\
\hline $\mathrm{AF}$ & Rodentia & Muridae & Dasymys & rufulus & $\begin{array}{l}\text { West African Shaggy } \\
\text { Rat } \\
\text { Kivu African Climbing }\end{array}$ & 2.14 & 138 & extant & 0 & NA & LC & Browse \\
\hline AF & Rodentia & Muridae & Dendromus & kivu & $\begin{array}{l}\text { Mouse, Kivu Climbing } \\
\text { Mouse }\end{array}$ & & & extant & 0 & NA & LC & frug \\
\hline $\mathrm{AF}$ & Rodentia & Muridae & Deomys & ferrugineus & Congo Forest Mouse & 1.79 & 62 & extant & 0 & NA & LC & ginsect \\
\hline AF & Rodentia & Muridae & Dephomys & defua & Defua Rat & 1.63 & 43 & extant & 0 & NA & LC & frug/ginsect \\
\hline AF & Rodentia & Muridae & Dephomys & eburnea & & 1.72 & 53 & extant & 0 & NA & & frug/ginsect \\
\hline AF & Rodentia & Muridae & Desmodilliscus & braueri & Pouched Gerbil & 1.00 & 10 & extant & 0 & NA & LC & frug \\
\hline AF & Rodentia & Muridae & Desmodillus & auricularis & Cape Short-eared Gerbil & 1.73 & 53 & extant & 0 & NA & LC & frug \\
\hline AF & Rodentia & Muridae & Desmomys & harringtoni & Harrington's Rat & 1.95 & 90 & extant & 0 & NA & LC & graze \\
\hline AF & Rodentia & Muridae & Dipodillus & campestris & North African Gerbil & 1.45 & 28 & extant & 0 & NA & LC & frug/browse \\
\hline $\mathrm{AF}$ & Rodentia & Muridae & Dipodillus & dasyurus & Wagner's Gerbil & 1.37 & 24 & extant & 0 & NA & $\mathrm{LC}$ & frug/browse \\
\hline AF & Rodentia & Muridae & Dipodillus & harwoodi & Harwood's Gerbil & 1.05 & 11 & extant & 0 & NA & LC & \\
\hline $\mathrm{AF}$ & Rodentia & Muridae & Dipodillus & jamesi & James's Gerbil & 1.32 & 21 & extant & 0 & NA & DD & frug/browse \\
\hline AF & Rodentia & Muridae & Dipodillus & maghrebi & $\begin{array}{l}\text { Greater Short-tailed } \\
\text { Gerbil }\end{array}$ & 1.32 & 21 & extant & 0 & NA & LC & frug/browse \\
\hline AF & Rodentia & Muridae & Dipodillus & quadrimaculatus & North African Gerbil & 1.32 & 21 & extant & 0 & NA & LC & \\
\hline $\mathrm{AF}$ & Rodentia & Muridae & Dipodillus & simoni & $\begin{array}{l}\text { Lesser Short-tailed } \\
\text { Gerbil }\end{array}$ & 1.24 & 17 & extant & 0 & NA & LC & frug/browse \\
\hline $\mathrm{AF}$ & Rodentia & Muridae & Dipodillus & somalicus & Somalian Gerbil & 1.32 & 21 & extant & 0 & NA & DD & frug/browse \\
\hline $\mathrm{AF}$ & Rodentia & Muridae & Dipodillus & stigmonyx & Khartoum Gerbil & 1.32 & 21 & extant & 0 & NA & DD & frug/browse \\
\hline AF & Rodentia & Muridae & Gerbilliscus & boehmi & Boehm's Gerbil & 1.99 & 97 & extant & 0 & NA & LC & frug/graze \\
\hline $\mathrm{AF}$ & Rodentia & Muridae & Gerbilliscus & brantsii & Highveld Gerbil & 2.06 & 114 & extant & 0 & NA & LC & \\
\hline AF & Rodentia & Muridae & Gerbilliscus & guineae & Guinea Gerbil & 2.01 & 102 & extant & 0 & NA & $\mathrm{LC}$ & frug/graze \\
\hline AF & Rodentia & Muridae & Gerbilliscus & kempi & Kemp's Gerbil & 2.00 & 101 & extant & 0 & NA & LC & frug/graze \\
\hline AF & Rodentia & Muridae & Gerbilliscus & leucogaster & Bushveld Gerbil & 1.86 & 73 & extant & 0 & NA & LC & frug/graze \\
\hline AF & Rodentia & Muridae & Gerbilliscus & phillipsi & Phillip's Gerbil & 1.99 & 97 & extant & 0 & NA & LC & frug/graze \\
\hline AF & Rodentia & Muridae & Gerbilliscus & robustus & Fringe-tailed Gerbil & 1.98 & 96 & extant & 0 & NA & LC & frug/graze \\
\hline $\mathrm{AF}$ & Rodentia & Muridae & Gerbillurus & paeba & $\begin{array}{l}\text { Hairy-footed Gerbil, } \\
\text { Pigmy Gerbil } \\
\text { Namib Brush-tailed }\end{array}$ & 1.37 & 23 & extant & 0 & NA & LC & frug \\
\hline $\mathrm{AF}$ & Rodentia & Muridae & Gerbillurus & setzeri & $\begin{array}{l}\text { Gerbil, Setzer's Hairy- } \\
\text { footed Gerbil }\end{array}$ & 1.58 & 38 & extant & 0 & NA & LC & frug \\
\hline $\mathrm{AF}$ & Rodentia & Muridae & Gerbillurus & tytonis & $\begin{array}{l}\text { Dune Hairy-footed } \\
\text { Gerbil, Namib Dune } \\
\text { Gerbil, Nimib Dune } \\
\text { Gerbil }\end{array}$ & 1.49 & 31 & extant & 0 & NA & LC & frug \\
\hline $\mathrm{AF}$ & Rodentia & Muridae & Gerbillurus & vallinus & $\begin{array}{l}\text { Bushy-tailed Hairy- } \\
\text { footed Gerbil }\end{array}$ & 1.55 & 35 & extant & 0 & NA & LC & frug \\
\hline $\mathrm{AF}$ & Rodentia & Muridae & Gerbillus & acticola & Berbera Gerbil & 1.45 & 28 & extant & 0 & NA & DD & frug/browse \\
\hline $\mathrm{AF}$ & Rodentia & Muridae & Gerbillus & afra & & 1.99 & 97 & extant & 0 & NA & & frug/graze \\
\hline $\mathrm{AF}$ & Rodentia & Muridae & Gerbillus & agag & Agag Gerbil & 1.45 & 28 & extant & 0 & NA & $\mathrm{DD}$ & frug/browse \\
\hline $\mathrm{AF}$ & Rodentia & Muridae & Gerbillus & agag & Agag Gerbil & 1.45 & 28 & extant & 0 & NA & DD & frug/browse \\
\hline $\mathrm{AF}$ & Rodentia & Muridae & Gerbillus & agag & Agag Gerbil & 1.45 & 28 & extant & 0 & NA & DD & frug/browse \\
\hline $\mathrm{AF}$ & Rodentia & Muridae & Gerbillus & amoenus & Pleasant Gerbil & 1.12 & 13 & extant & 0 & NA & LC & frug/browse \\
\hline $\mathrm{AF}$ & Rodentia & Muridae & Gerbillus & andersoni & Anderson's Gerbil & 1.43 & 27 & extant & 0 & NA & LC & frug/browse \\
\hline AF & Rodentia & Muridae & Gerbillus & andersoni & Anderson's Gerbil & 1.45 & 28 & extant & 0 & NA & LC & frug/browse \\
\hline AF & Rodentia & Muridae & Gerbillus & bottai & Botta's Gerbil & 1.45 & 28 & extant & 0 & NA & DD & frug/browse \\
\hline AF & Rodentia & Muridae & Gerbillus & brockmani & Brockman's Gerbil & 1.45 & 28 & extant & 0 & NA & DD & frug/browse \\
\hline
\end{tabular}




\begin{tabular}{|c|c|c|c|c|c|c|c|c|c|c|c|c|}
\hline $\mathrm{AF}$ & Rodentia & Muridae & Gerbillus & burtoni & Burton's Gerbil & 1.45 & 28 & extant & 0 & NA & DD & frug/browse \\
\hline AF & Rodentia & Muridae & Gerbillus & diminutus & Least Gerbil & 1.24 & 17 & extant & 0 & NA & LC & frug/browse \\
\hline AF & Rodentia & Muridae & Gerbillus & dongolanus & Greater Egyptian Gerbil & 1.45 & 28 & extant & 0 & NA & LC & frug/browse \\
\hline AF & Rodentia & Muridae & Gerbillus & dunni & Somalia Gerbil & 1.45 & 28 & extant & 0 & NA & LC & frug/browse \\
\hline AF & Rodentia & Muridae & Gerbillus & floweri & Flower's Gerbil & 1.45 & 28 & extant & 0 & NA & LC & frug/browse \\
\hline $\mathrm{AF}$ & Rodentia & Muridae & Gerbillus & garamantis & Dwarf Gerbil & 1.45 & 28 & extant & 0 & NA & LC & frug/browse \\
\hline $\mathrm{AF}$ & Rodentia & Muridae & Gerbillus & gerbillus & Lesser Egyptian Gerbil & 1.40 & 25 & extant & 0 & NA & LC & frug/browse \\
\hline $\mathrm{AF}$ & Rodentia & Muridae & Gerbillus & grobbeni & Grobben's Gerbil & 1.45 & 28 & extant & 0 & NA & DD & frug/browse \\
\hline AF & Rodentia & Muridae & Gerbillus & henleyi & Pygmy Gerbil & 0.90 & 8 & extant & 0 & NA & $\mathrm{LC}$ & frug/browse \\
\hline AF & Rodentia & Muridae & Gerbillus & hesperinus & Western Gerbil & 1.45 & 28 & extant & Future & NA & EN & frug/browse \\
\hline $\mathrm{AF}$ & Rodentia & Muridae & Gerbillus & hoogstraali & Hoogstraal's Gerbil & 1.45 & 28 & extant & Future & NA & Vu & frug/browse \\
\hline $\mathrm{AF}$ & Rodentia & Muridae & Gerbillus & latastei & Lataste's Gerbil & 1.60 & 40 & extant & 0 & NA & LC & frug/browse \\
\hline AF & Rodentia & Muridae & Gerbillus & lowei & Lowe's Gerbil & 1.45 & 28 & extant & 0 & NA & DD & frug/browse \\
\hline $\mathrm{AF}$ & Rodentia & Muridae & Gerbillus & mackillingini & Mackillingin's Gerbil & 1.45 & 28 & extant & 0 & NA & LC & frug/browse \\
\hline AF & Rodentia & Muridae & Gerbillus & mauritaniae & Sudan Gerbil & 1.45 & 28 & extant & 0 & NA & DD & frug/browse \\
\hline AF & Rodentia & Muridae & Gerbillus & muriculus & Darfur Gerbil & 1.45 & 28 & extant & 0 & NA & DD & frug/browse \\
\hline AF & Rodentia & Muridae & Gerbillus & nancillus & Sudan Gerbil & 1.45 & 28 & extant & 0 & NA & DD & frug/browse \\
\hline $\mathrm{AF}$ & Rodentia & Muridae & Gerbillus & nanus & $\begin{array}{l}\text { Dwarf Gerbil, } \\
\text { Baluchistan Gerbil }\end{array}$ & 1.41 & 26 & extant & 0 & NA & LC & frug/browse \\
\hline AF & Rodentia & Muridae & Gerbillus & nigeriae & Nigerian Gerbil & 1.47 & 30 & extant & 0 & NA & LC & frug/browse \\
\hline AF & Rodentia & Muridae & Gerbillus & occiduus & Occidental Gerbil & 1.45 & 28 & extant & 0 & NA & DD & frug/browse \\
\hline AF & Rodentia & Muridae & Gerbillus & perpallidus & Pale Gerbil & 1.56 & 36 & extant & 0 & NA & LC & frug/browse \\
\hline AF & Rodentia & Muridae & Gerbillus & principulus & Principal Gerbil & 1.45 & 28 & extant & 0 & NA & DD & frug/browse \\
\hline AF & Rodentia & Muridae & Gerbillus & pulvinatus & Cushioned Gerbil & 1.45 & 28 & extant & 0 & NA & LC & frug/browse \\
\hline AF & Rodentia & Muridae & Gerbillus & pulvinatus & Cushioned Gerbil & 1.45 & 28 & extant & 0 & NA & LC & frug/browse \\
\hline AF & Rodentia & Muridae & Gerbillus & pusillus & Least Gerbil & 1.10 & 13 & extant & 0 & NA & LC & frug/browse \\
\hline AF & Rodentia & Muridae & Gerbillus & pusillus & Least Gerbil & 1.45 & 28 & extant & 0 & NA & LC & frug/browse \\
\hline $\mathrm{AF}$ & Rodentia & Muridae & Gerbillus & pusillus & Least Gerbil & 1.45 & 28 & extant & 0 & NA & LC & frug/browse \\
\hline AF & Rodentia & Muridae & Gerbillus & pyramidum & Greater Egyptian Gerbil & 1.67 & 46 & extant & 0 & NA & LC & frug/browse \\
\hline AF & Rodentia & Muridae & Gerbillus & rosalinda & Rosalinda Gerbil & 1.45 & 28 & extant & 0 & NA & LC & frug/browse \\
\hline AF & Rodentia & Muridae & Gerbillus & syrticus & Pygmy Gerbil & 1.45 & 28 & extant & 0 & NA & LC & frug/browse \\
\hline AF & Rodentia & Muridae & Gerbillus & tarabuli & Tarabul's Gerbil & 1.45 & 28 & extant & 0 & NA & LC & frug/browse \\
\hline AF & Rodentia & Muridae & Gerbillus & tarabuli & Tarabul's Gerbil & 1.45 & 28 & extant & 0 & NA & LC & frug/browse \\
\hline AF & Rodentia & Muridae & Gerbillus & vivax & Pleasant Gerbil & 1.45 & 28 & extant & 0 & NA & LC & frug/browse \\
\hline $\mathrm{AF}$ & Rodentia & Muridae & Gerbillus & watersi & Waters's Gerbil & 1.02 & 10 & extant & 0 & NA & LC & frug/browse \\
\hline AF & Rodentia & Muridae & Gerbillus & watersi & Waters's Gerbil & 1.45 & 28 & extant & 0 & NA & LC & frug/browse \\
\hline AF & Rodentia & Muridae & Girbilliscus & nigricauda & Black-tailed Gerbil & 2.09 & 123 & extant & 0 & NA & LC & frug/graze \\
\hline $\mathrm{AF}$ & Rodentia & Muridae & Grammomys & aridulus & $\begin{array}{l}\text { Arid Thicket Rat, Arid } \\
\text { Woodland Grammomys }\end{array}$ & 1.63 & 42 & extant & 0 & NA & DD & frug/browse \\
\hline AF & Rodentia & Muridae & Grammomys & buntingi & $\begin{array}{l}\text { Bunting's Grammomys, } \\
\text { Bunting's Thicket Rat } \\
\text { Gray-headed Thicket }\end{array}$ & 1.63 & 42 & extant & 0 & NA & DD & frug/browse \\
\hline AF & Rodentia & Muridae & Grammomys & caniceps & $\begin{array}{l}\text { Rat, Grey-headed } \\
\text { Thicket Rat }\end{array}$ & 1.63 & 42 & extant & 0 & NA & DD & frug/browse \\
\hline AF & Rodentia & Muridae & Grammomys & cometes & $\begin{array}{l}\text { Mozambique Thicket } \\
\text { Rat }\end{array}$ & 1.69 & 49 & extant & 0 & NA & LC & frug/browse \\
\hline $\mathrm{AF}$ & Rodentia & Muridae & Grammomys & dolichurus & Woodland Thicket Rat & 1.61 & 41 & extant & 0 & NA & LC & frug/browse \\
\hline AF & Rodentia & Muridae & Grammomys & dryas & Forest Thicket Rat & 1.63 & 42 & extant & Future & NA & NT & frug/browse \\
\hline AF & Rodentia & Muridae & Grammomys & gigas & Giant Thicket Rat & 1.63 & 42 & extant & Future & NA & EN & frug/browse \\
\hline AF & Rodentia & Muridae & Grammomys & ibeanus & Ruwenzori Thicket Rat & 1.61 & 41 & extant & 0 & NA & $\mathrm{LC}$ & frug/browse \\
\hline
\end{tabular}




\begin{tabular}{|c|c|c|c|c|c|c|c|c|c|c|c|c|}
\hline$A F$ & Rodentia & Muridae & Grammomys & macmillani & Macmillan's Thicket Rat & 1.56 & 36 & extant & 0 & NA & $\mathrm{LC}$ & frug/browse \\
\hline $\mathrm{AF}$ & Rodentia & Muridae & Grammomys & minnae & Ethiopian Thicket Rat & 1.56 & 36 & extant & Future & NA & Vu & frug/browse \\
\hline$A F$ & Rodentia & Muridae & Grammomys & rutilans & Shining Thicket Rat & 1.72 & 52 & extant & 0 & NA & LC & frug/browse \\
\hline$A F$ & Rodentia & Muridae & Heimyscus & fumosus & $\begin{array}{l}\text { African Smoky Mouse, } \\
\text { Smokey Heimyscus }\end{array}$ & 1.15 & 14 & extant & 0 & NA & $\mathrm{LC}$ & ;ect/frug/brol \\
\hline AF & Rodentia & Muridae & Hybomys & eisentrauti & $\begin{array}{l}\text { Cameroon Highland } \\
\text { Hybomys }\end{array}$ & 1.71 & 51 & extant & Future & NA & EN & frug \\
\hline AF & Rodentia & Muridae & Hybomys & lunaris & $\begin{array}{l}\text { Moon Striped Mouse, } \\
\text { Ruwenzori Hybomys } \\
\text { Liberian Forest }\end{array}$ & 1.71 & 51 & extant & Future & NA & VU & frug \\
\hline AF & Rodentia & Muridae & Hybomys & planifrons & $\begin{array}{l}\text { Hybomys, Miller's } \\
\text { Striped Mouse } \\
\text { Temminck's Striped }\end{array}$ & 1.70 & 50 & extant & 0 & NA & $\mathrm{LC}$ & frug \\
\hline AF & Rodentia & Muridae & Hybomys & trivirgatus & $\begin{array}{l}\text { Mouse, West African } \\
\text { Hybomys }\end{array}$ & 1.76 & 57 & extant & 0 & NA & LC & frug \\
\hline AF & Rodentia & Muridae & Hybomys & univittatus & $\begin{array}{l}\text { Peters' Hybomys, } \\
\text { Peters' Striped Mouse }\end{array}$ & 1.68 & 48 & extant & 0 & NA & $\mathrm{LC}$ & frug \\
\hline AF & Rodentia & Muridae & Hylomyscus & aeta & $\begin{array}{l}\text { Beaded Hylomyscus, } \\
\text { Beaded Wood Mouse }\end{array}$ & 1.30 & 20 & extant & 0 & NA & LC & frug/ginsect \\
\hline AF & Rodentia & Muridae & Hylomyscus & alleni & $\begin{array}{l}\text { Allen's Hylomyscus, } \\
\text { Allen's Wood Mouse }\end{array}$ & 1.30 & 20 & extant & 0 & NA & LC & frug/ginsect \\
\hline$A F$ & Rodentia & Muridae & Hylomyscus & baeri & $\begin{array}{l}\text { Baer's Hylomyscus, } \\
\text { Baer's Wood Mouse }\end{array}$ & 1.34 & 22 & extant & Future & NA & EN & frug/ginsect \\
\hline AF & Rodentia & Muridae & Hylomyscus & carillus & $\begin{array}{l}\text { Angolan Hylomyscus, } \\
\text { Angolan Wood Mouse }\end{array}$ & 1.34 & 22 & extant & 0 & NA & LC & frug/ginsect \\
\hline AF & Rodentia & Muridae & Hylomyscus & denniae & $\begin{array}{l}\text { Montane Wood Mouse, } \\
\text { Montane Hylomyscus }\end{array}$ & 1.56 & 36 & extant & 0 & NA & LC & frug/ginsect \\
\hline AF & Rodentia & Muridae & Hylomyscus & parvus & $\begin{array}{l}\text { Lesser Hylomyscus, } \\
\text { Little Wood Mouse }\end{array}$ & 1.16 & 14 & extant & 0 & NA & LC & frug/ginsect \\
\hline$A F$ & Rodentia & Muridae & Hylomyscus & stella & $\begin{array}{l}\text { Stella Wood Mouse, } \\
\text { Stella Hylomyscus }\end{array}$ & 1.30 & 20 & extant & 0 & NA & $\mathrm{LC}$ & frug/ginsect \\
\hline $\mathrm{AF}$ & Rodentia & Muridae & Lamottemys & okuensis & $\begin{array}{l}\text { Mount Oku Rat, Mt. Oku } \\
\text { Rat }\end{array}$ & & & extant & Future & NA & EN & \\
\hline AF & Rodentia & Muridae & Leimacomys & buettneri & $\begin{array}{l}\text { Groove-toothed Forest } \\
\text { Mouse }\end{array}$ & 1.74 & 55 & extinct & Holocene & NA & EX & Ginsect \\
\hline AF & Rodentia & Muridae & Lemniscomys & barbarus & $\begin{array}{l}\text { Barbary Lemniscomys, } \\
\text { Barbary Striped Grass } \\
\text { Mouse } \\
\text { Bellier's Lemniscomys, }\end{array}$ & 1.52 & 33 & extant & 0 & NA & LC & graze/frug \\
\hline AF & Rodentia & Muridae & Lemniscomys & bellieri & $\begin{array}{l}\text { Bellier's Lemniscomys, } \\
\text { Bellier's Striped Grass } \\
\text { Mouse }\end{array}$ & 1.63 & 43 & extant & 0 & NA & $\mathrm{LC}$ & graze/frug \\
\hline$A F$ & Rodentia & Muridae & Lemniscomys & griselda & $\begin{array}{l}\text { Griselda's } \\
\text { Lemniscomys, } \\
\text { Griselda's } \\
\text { Lemniscomys, } \\
\text { Griselda's Striped Grass } \\
\text { Mouse }\end{array}$ & 1.76 & 58 & extant & 0 & NA & LC & graze/frug \\
\hline
\end{tabular}


Hoogstral's Striped

Grass Mouse

\begin{tabular}{|c|c|c|c|c|}
\hline $\mathrm{AF}$ & Rodentia & Muridae & Lemniscomys & linulus \\
\hline AF & Rodentia & Muridae & Lemniscomys & macculus \\
\hline $\mathrm{AF}$ & Rodentia & Muridae & Lemniscomys & mittendorfi \\
\hline $\mathrm{AF}$ & Rodentia & Muridae & Lemniscomys & rosalia \\
\hline $\mathrm{AF}$ & Rodentia & Muridae & Lemniscomys & roseveari \\
\hline $\mathrm{AF}$ & Rodentia & Muridae & Lemniscomys & striatus \\
\hline $\mathrm{AF}$ & Rodentia & Muridae & Lophuromys & aquilus \\
\hline $\mathrm{AF}$ & Rodentia & Muridae & Lophuromys & flavopunctatus \\
\hline $\mathrm{AF}$ & Rodentia & Muridae & Lophuromys & luteogaster \\
\hline $\mathrm{AF}$ & Rodentia & Muridae & Lophuromys & medicaudatus \\
\hline $\mathrm{AF}$ & Rodentia & Muridae & Lophuromys & melanonyx \\
\hline $\mathrm{AF}$ & Rodentia & Muridae & Lophuromys & nudicaudus \\
\hline $\mathrm{AF}$ & Rodentia & Muridae & Lophuromys & rahmi \\
\hline AF & Rodentia & Muridae & Lophuromys & sikapusi \\
\hline $\mathrm{AF}$ & Rodentia & Muridae & Lophuromys & woosnami \\
\hline AF & Rodentia & Muridae & Malacomys & cansdalei \\
\hline AF & Rodentia & Muridae & Malacomys & edwardsi \\
\hline
\end{tabular}

Senegal Lemniscomys,

Senegal One-striped

Grass Mouse

Buffoon Lemniscomys,

Buffoon Striped Grass

Mouse

Mittendorf's

Lemniscomys,

Mittendorf's

Lemniscomys,

Mittendorf's Striped

Grass Mouse

Single-striped Grass

Mouse, Single-striped

Lemniscomys

Rosevear's

Lemniscomys,

Rosevear's

Romniscomys,

Grass Mouse

Typical Lemniscomys,

Typical Striped Grass

Mouse

Yellow-spotted Brush-

furred Rat

Yellow-spotted Brush-

furred Rat

Yellow-bellied Brush-

furred Rat

Medium-tailed Brush-

furred Rat

Black-clawed Brush-

furred Rat

Fire-bellied Brush-

furred Rat

Rahm's Brush-furred

Rat

Rusty-bellied Brush-

furred Rat

Woosnam's Brush-

furred Rat

1.63

extant

0

NA

DD

graze/frug

Cansdale's Swamp Rat

Edward's Swamp Rat

\section{4}

42

4

43

exta

Future

1.77

59

extant

0

NA

LC

graze/frug

1.63

43

extant

NA

DD

graze/frug

1.58

38

exta

1.65

1.7

1.65

1.65

1.01

1.65

1.58

1.87

1.62

1.90

1.81

45

45
60
45
45
10
45
38
74
42
80
65

exta

extant

extant

extant

extant

Future

extant

extant

Future

extant

extant

extant

extant
extant

0

0
0

0
0
0

0
0

$\begin{array}{llr}\text { NA } & \text { LC } & \text { graze/frug } \\ \text { NA } & \text { LC } & \text { ginsect } \\ \text { NA } & \text { LC } & \text { ginsect } \\ \text { NA } & \text { LC } & \text { ginsect } \\ \text { NA } & \text { VU } & \text { ginsect } \\ \text { NA } & \text { VU } & \text { ginsect } \\ \text { NA } & \text { LC } & \text { ginsect } \\ \text { NA } & \text { EN } & \text { ginsect } \\ \text { NA } & \text { LC } & \text { ginsect } \\ \text { NA } & \text { LC } & \text { ginsect } \\ \text { NA } & \text { LC } & \begin{array}{r}\text { ginsect/frug } \\ \text { ginsect/frug }\end{array}\end{array}$




\begin{tabular}{|c|c|c|c|c|c|c|c|c|c|c|c|c|}
\hline $\mathrm{AF}$ & Rodentia & Muridae & Malacomys & longipes & $\begin{array}{l}\text { Big-eared Swamp Rat } \\
\text { Southern African }\end{array}$ & 1.98 & 96 & extant & 0 & NA & LC & ginsect/frug \\
\hline AF & Rodentia & Muridae & Mastomys & coucha & $\begin{array}{l}\text { Mastomys, Southern } \\
\text { Multimammate Mouse } \\
\text { Guinea Multimammate }\end{array}$ & 1.64 & 44 & extant & 0 & NA & LC & frug/ginsect \\
\hline $\mathrm{AF}$ & Rodentia & Muridae & Mastomys & erythroleucus & $\begin{array}{l}\text { Mouse, Reddish-white } \\
\text { Mastomys }\end{array}$ & 1.75 & 56 & extant & 0 & NA & LC & frug/ginsect \\
\hline $\mathrm{AF}$ & Rodentia & Muridae & Mastomys & kollmannspergeri & $\begin{array}{l}\text { Kollmannsperger's } \\
\text { Mastomys }\end{array}$ & 1.72 & 53 & extant & 0 & NA & LC & frug/ginsect \\
\hline $\mathrm{AF}$ & Rodentia & Muridae & Mastomys & natalensis & $\begin{array}{l}\text { Natal Mastomys, Natal } \\
\text { Multimammate Mouse }\end{array}$ & 1.74 & 55 & extant & 0 & NA & $\mathrm{LC}$ & frug/ginsect \\
\hline $\mathrm{AF}$ & Rodentia & Muridae & Mastomys & natalensis & $\begin{array}{l}\text { Natal Mastomys, Natal } \\
\text { Multimammate Mouse } \\
\text { Dwarf Mastomys, }\end{array}$ & 1.79 & 62 & extant & 0 & NA & $\mathrm{LC}$ & frug/ginsect \\
\hline $\mathrm{AF}$ & Rodentia & Muridae & Mastomys & pernanus & $\begin{array}{l}\text { Dwarf Multimammate } \\
\text { Mouse } \\
\text { Shortridge's Mastomys, }\end{array}$ & 1.72 & 53 & extant & 0 & NA & DD & frug/ginsect \\
\hline $\mathrm{AF}$ & Rodentia & Muridae & Mastomys & shortridgei & $\begin{array}{l}\text { Shortridge's } \\
\text { Multimammate Mouse }\end{array}$ & 1.67 & 47 & extant & 0 & NA & LC & frug/ginsect \\
\hline $\mathrm{AF}$ & Rodentia & Muridae & Meriones & crassus & Sundevall's Jird & 1.90 & 80 & extant & 0 & NA & LC & frug/browse \\
\hline $\mathrm{AF}$ & Rodentia & Muridae & Meriones & libycus & Libyan Jird & 1.96 & 91 & extant & 0 & NA & LC & frug/browse \\
\hline $\mathrm{AF}$ & Rodentia & Muridae & Meriones & shawi & Shaw's Jird & 1.96 & 91 & extant & 0 & NA & $\mathrm{LC}$ & frug/browse \\
\hline $\mathrm{AF}$ & Rodentia & Muridae & Micaelamys & namaquensis & Namaqua Rock Ra & 1.70 & 51 & extant & 0 & NA & LC & \\
\hline $\mathrm{AF}$ & Rodentia & Muridae & Microdillus & peeli & $\begin{array}{l}\text { Somali Pygmy Gerbil } \\
\text { Ethiopian Striped }\end{array}$ & & & extant & 0 & NA & $\mathrm{LC}$ & \\
\hline $\mathrm{AF}$ & Rodentia & Muridae & Muriculus & imberbis & $\begin{array}{l}\text { Mouse, Striped-back } \\
\text { Mouse }\end{array}$ & 1.27 & 19 & extant & 0 & NA & $\mathrm{LC}$ & ginsect \\
\hline $\mathrm{AF}$ & Rodentia & Muridae & Mus & baoulei & Baoule's Mouse & 1.17 & 15 & extant & 0 & NA & LC & \\
\hline $\mathrm{AF}$ & Rodentia & Muridae & Mus & bufo & Toad Mouse & 0.96 & 9 & extant & 0 & NA & LC & frug/browse \\
\hline $\mathrm{AF}$ & Rodentia & Muridae & Mus & callewaerti & Callewaert's Mouse & 1.17 & 15 & extant & 0 & NA & $\mathrm{LC}$ & \\
\hline $\mathrm{AF}$ & Rodentia & Muridae & Mus & goundae & Gounda Mouse & 1.17 & 15 & extant & 0 & NA & $\mathrm{DD}$ & \\
\hline $\mathrm{AF}$ & Rodentia & Muridae & Mus & haussa & Hausa Mouse & 1.17 & 15 & extant & 0 & NA & LC & \\
\hline $\mathrm{AF}$ & Rodentia & Muridae & Mus & indutus & Desert Pygmy Mouse & 0.79 & 6 & extant & 0 & NA & LC & frug/browse \\
\hline $\mathrm{AF}$ & Rodentia & Muridae & Mus & mahomet & Mahomet Mouse & 1.00 & 10 & extant & 0 & NA & LC & frug/browse \\
\hline $\mathrm{AF}$ & Rodentia & Muridae & Mus & mattheyi & Matthey's Mouse & 1.17 & 15 & extant & 0 & NA & $\mathrm{LC}$ & \\
\hline $\mathrm{AF}$ & Rodentia & Muridae & Mus & minutoides & Pygmy Mouse & 0.85 & 7 & extant & 0 & NA & LC & frug/browse \\
\hline $\mathrm{AF}$ & Rodentia & Muridae & Mus & musculoides & NA & 0.78 & 6 & extant & 0 & NA & LC & frug/browse \\
\hline $\mathrm{AF}$ & Rodentia & Muridae & Mus & musculoides & & 1.17 & 15 & extant & 0 & NA & LC & \\
\hline $\mathrm{AF}$ & Rodentia & Muridae & Mus & musculus & House Mouse & 1.24 & 17 & extant & 0 & NA & LC & frug/browse \\
\hline $\mathrm{AF}$ & Rodentia & Muridae & Mus & neavei & Neave's Mouse & 1.17 & 15 & extant & 0 & NA & $\mathrm{DD}$ & \\
\hline $\mathrm{AF}$ & Rodentia & Muridae & Mus & orangiae & $\begin{array}{l}\text { Free State Pygmy } \\
\text { Mouse, Orange Mouse }\end{array}$ & 1.17 & 15 & extant & 0 & NA & LC & \\
\hline $\mathrm{AF}$ & Rodentia & Muridae & Mus & oubanguii & Oubangui Mouse & 1.17 & 15 & extant & 0 & NA & DD & \\
\hline $\mathrm{AF}$ & Rodentia & Muridae & Mus & setulosus & Peter's Mouse & 0.97 & 9 & extant & 0 & NA & LC & frug/browse \\
\hline $\mathrm{AF}$ & Rodentia & Muridae & Mus & setzeri & $\begin{array}{l}\text { Setzer's Mouse, } \\
\text { Setzer's Pygmy Mouse }\end{array}$ & 0.82 & 7 & extant & 0 & NA & LC & frug/browse \\
\hline $\mathrm{AF}$ & Rodentia & Muridae & Mus & sorella & $\begin{array}{l}\text { Thomas's Mouse, } \\
\text { Thomas's Pygmy Mouse }\end{array}$ & 1.17 & 15 & extant & 0 & NA & LC & \\
\hline
\end{tabular}


Algerian Mouse,

Western Mediterranean

Mouse, WESTERN

MEDITERRANEAN

1.11

MOUSE

AF Rodenti

Muridae

Mus

tenellus

Delicate Mouse

Gray-bellied Mouse,

Gray-bellied Pygmy

Mouse

African Groove-toothed

Mylomys

ans

Rat, Mill Rat

fumatus

Muridae

Myomys

Angolan

Multimammate Mouse,

Myomyscus angolensis

Angolan Myomyscus

Verreaux's Mouse,

Verreaux's White-

footed Rat

Sloggett's Vlei Rat

Myotomys

sloggetti

Nesokia

unisulcatus

$\begin{array}{lll}\text { AF } & \text { Rodentia } & \text { Muridae } \\ \text { AF } & \text { Rodentia } & \text { Muridae }\end{array}$

indica

AF Rodentia

Muridae

Oenomys

Oenomys

Otomys

Muridae

Rodentia

Otomys

Otomys

Otomys

Muridae

Rodentia

Muridae

Rodentia

Muridae

Rodentia

Rodentia

Rodentia

Muridae

Muridae

Muridae

Rodentia

Muridae

Otomys

Otomys

Otomys

Otomys

Otomys

Otomys

Pachyuromys

hypoxanthus

ornatus

anchietae

angoniensis

denti

irroratus

laminatus

maximus

occidentalis

saundersiae

tropicalis

typus

duprasi

AF Rodentia Muridae

Parotomys

brantsii

AF Rodentia

Muridae

Parotomys

littledalei

AF Rodentia

Muridae

Pelomys

campanae

Pelomys

fallax

Pelomys

hopkinsi

Short-tailed Bandicoot

Rat

Rufous-nosed Rats

Ghana Rufous-nosed

Rat

Angolan Vlei Rat

Angoni Vlei Rat

Dent's Vlei Rat

Southern African Vlei

Rat, Vlei Rat

Laminate Vlei Rat

Western Vlei Rat

Saunder's Vlei Rat
Tropical Vlei Rat

Typical Vlei Rat

Fat-tailed Gerbil

Brants's Whistling Rat,

Brants' Whistling Rat

Littledale's Whistling

Rat

Bell Groove-toothed

Swamp Rat

Creek Groove-toothed

Swamp Rat

Hopkins's Groove-

toothed Swamp Rat

Swamp Rat

isseli

$\begin{array}{cccc}1.61 & 41 & \text { extant } & 0 \\ 2.03 & 106 & \text { extant } & 0 \\ 2.10 & 125 & \text { extant } & 0 \\ 2.25 & 179 & \text { extant } & 0 \\ 1.95 & 90 & \text { extant } & 0 \\ 1.93 & 86 & \text { extant } & 0 \\ 2.11 & 130 & \text { extant } & 0 \\ 2.09 & 122 & \text { extant } & 0 \\ 2.08 & 120 & \text { extant } & 0 \\ 2.07 & 118 & \text { extant } & 0 \\ 2.16 & 145 & \text { extant } & 0 \\ 2.28 & 192 & \text { extant } & 0 \\ 2.11 & 130 & \text { extant } & \text { Future } \\ 2.01 & 103 & \text { extant } & 0 \\ 2.04 & 109 & \text { extant } & 0 \\ 2.11 & 130 & \text { extant } & 0 \\ 1.68 & 48 & \text { extant } & 0 \\ 2.11 & 130 & \text { extant } & 0 \\ 2.10 & 127 & \text { extant } & 0 \\ 1.90 & 80 & \text { extant } & 0 \\ 2.11 & 130 & \text { extant } & 0 \\ 1.90 & 80 & \text { extant } & 0 \\ 1.78 & 60 & \text { extant } & 0\end{array}$

graze
graze
graze
graze




\begin{tabular}{|c|c|c|c|c|c|c|c|c|c|c|c|c|}
\hline AF & Rodentia & Muridae & Pelomys & minor & $\begin{array}{l}\text { Least Groove-toothed } \\
\text { Swamp Rat }\end{array}$ & 1.70 & 50 & extant & 0 & NA & LC & graze \\
\hline $\mathrm{AF}$ & Rodentia & Muridae & Praomys & daltoni & $\begin{array}{l}\text { Dalton's Mouse, } \\
\text { Dalton's Praomys } \\
\text { East African Praomys, }\end{array}$ & 1.59 & 39 & extant & 0 & NA & LC & frug/ginsect \\
\hline AF & Rodentia & Muridae & Praomys & delectorum & $\begin{array}{l}\text { Delectable Soft-furred } \\
\text { Mouse }\end{array}$ & 1.56 & 36 & extant & 0 & NA & LC & ginsect/frug \\
\hline $\mathrm{AF}$ & Rodentia & Muridae & Praomys & derooi & $\begin{array}{l}\text { Deroo's Mouse, Deroo's } \\
\text { Praomys }\end{array}$ & 1.51 & 32 & extant & 0 & NA & $\mathrm{LC}$ & frug/ginsect \\
\hline $\mathrm{AF}$ & Rodentia & Muridae & Praomys & hartwigi & $\begin{array}{l}\text { Hartwig's Praomys, } \\
\text { Hartwig's Soft-furred } \\
\text { Mouse }\end{array}$ & 1.58 & 38 & extant & Future & NA & EN & ginsect/frug \\
\hline AF & Rodentia & Muridae & Praomys & jacksoni & $\begin{array}{l}\text { Jackson's Praomys, } \\
\text { Jackson's Soft-furred } \\
\text { Mouse }\end{array}$ & 1.62 & 42 & extant & 0 & NA & $\mathrm{LC}$ & ginsect/frug \\
\hline $\mathrm{AF}$ & Rodentia & Muridae & Praomys & lukolelae & Lukolela Swamp Rat & 1.58 & 38 & extant & 0 & NA & $\mathrm{LC}$ & ginsect/frug \\
\hline $\mathrm{AF}$ & Rodentia & Muridae & Praomys & minor & $\begin{array}{l}\text { Least Praomys, Least } \\
\text { Soft-furred Mouse } \\
\text { Misonne's Praomys, }\end{array}$ & 1.58 & 38 & extant & 0 & NA & DD & ginsect/frug \\
\hline $\mathrm{AF}$ & Rodentia & Muridae & Praomys & misonnei & $\begin{array}{l}\text { Misonne's Soft-furred } \\
\text { Mouse }\end{array}$ & 1.58 & 38 & extant & 0 & NA & LC & ginsect/frug \\
\hline $\mathrm{AF}$ & Rodentia & Muridae & Praomys & morio & $\begin{array}{l}\text { Cameroon Praomys, } \\
\text { Cameroon Soft-furred } \\
\text { Mouse } \\
\text { Muton's Soft-furred }\end{array}$ & 1.48 & 30 & extant & Future & NA & EN & ginsect/frug \\
\hline $\mathrm{AF}$ & Rodentia & Muridae & Praomys & mutoni & $\begin{array}{l}\text { Mouse, Riverine } \\
\text { Praomys } \\
\text { Forest Soft-furred }\end{array}$ & 1.58 & 38 & extant & 0 & NA & DD & ginsect/frug \\
\hline $\mathrm{AF}$ & Rodentia & Muridae & Praomys & rostratus & $\begin{array}{l}\text { Mouse, West African } \\
\text { Praomys } \\
\text { Tullberg's Soft-furred }\end{array}$ & 1.58 & 38 & extant & 0 & NA & LC & ginsect/frug \\
\hline $\mathrm{AF}$ & Rodentia & Muridae & Praomys & tullbergi & $\begin{array}{l}\text { Mouse, Tullberg's } \\
\text { Praomys }\end{array}$ & 1.55 & 36 & extant & 0 & NA & LC & ginsect/frug \\
\hline $\mathrm{AF}$ & Rodentia & Muridae & Praomys & verschureni & $\begin{array}{l}\text { Verschuren's Swamp } \\
\text { Rat }\end{array}$ & 1.69 & 49 & extant & 0 & NA & DD & ginsect/frug \\
\hline $\mathrm{AF}$ & Rodentia & Muridae & Psammomys & obesus & Fat Sand Rat & 2.17 & 150 & extant & 0 & NA & $\mathrm{LC}$ & Browse \\
\hline $\mathrm{AF}$ & Rodentia & Muridae & Psammomys & vexillaris & $\begin{array}{l}\text { Thin Sand Rat } \\
\text { Four-striped Grass }\end{array}$ & 2.17 & 150 & extant & 0 & NA & DD & Browse \\
\hline $\mathrm{AF}$ & Rodentia & Muridae & Rhabdomys & pumilio & $\begin{array}{l}\text { Mouse, Four-striped } \\
\text { Grass Rat }\end{array}$ & 1.61 & 41 & extant & 0 & NA & LC & graze/frug \\
\hline $\mathrm{AF}$ & Rodentia & Muridae & Sekeetamys & calurus & $\begin{array}{l}\text { Bushy-tailed Jird } \\
\text { Palestine Mole Rat, }\end{array}$ & 1.74 & 55 & extant & 0 & NA & LC & frug \\
\hline $\mathrm{AF}$ & Rodentia & Muridae & Spalax & ehrenbergi & $\begin{array}{l}\text { Middle East Blind Mole } \\
\text { Rat } \\
\text { Ethiopian White-footed }\end{array}$ & 2.25 & 176 & extant & 0 & NA & DD & Browse \\
\hline $\mathrm{AF}$ & Rodentia & Muridae & Stenocephalemys & albipes & $\begin{array}{l}\text { Mouse, White-footed } \\
\text { Stenocephalemys }\end{array}$ & 1.97 & 93 & extant & 0 & NA & LC & frug/ginsect \\
\hline $\mathrm{AF}$ & Rodentia & Muridae & Stenocephalemys & albocaudata & $\begin{array}{l}\text { Ethiopian Narrow- } \\
\text { headed Rat }\end{array}$ & 2.16 & 144 & extant & 0 & NA & LC & frug/ginsect \\
\hline $\mathrm{AF}$ & Rodentia & Muridae & Stenocephalemys & griseicauda & $\begin{array}{l}\text { Gray-tailed Narrow- } \\
\text { headed Rat }\end{array}$ & 1.93 & 86 & extant & 0 & NA & LC & frug/ginsect \\
\hline
\end{tabular}




\begin{tabular}{|c|c|c|c|c|c|c|c|c|c|c|c|c|}
\hline AF & Rodentia & Muridae & Stenocephalemys & ruppi & $\begin{array}{l}\text { Stenocephalemys, } \\
\text { Rupp's } \\
\text { Stenocephalemys }\end{array}$ & 1.69 & 49 & extant & 0 & NA & DD & frug/ginsect \\
\hline AF & Rodentia & Muridae & Stochomys & longicaudatus & Target Rat & 1.85 & 71 & extant & 0 & NA & LC & frug/ginsect \\
\hline AF & Rodentia & Muridae & Tatera & inclusus & Gorongoza Gerbil & 2.07 & 119 & extant & 0 & NA & LC & frug/graze \\
\hline AF & Rodentia & Muridae & Tatera & validus & Savanna Gerbil & 2.03 & 108 & extant & 0 & NA & LC & frug/graze \\
\hline AF & Rodentia & Muridae & Taterillus & arenarius & $\begin{array}{l}\text { Robbins's Tateril, Sahel } \\
\text { Gerbil }\end{array}$ & 1.67 & 47 & extant & 0 & NA & LC & frug/browse \\
\hline AF & Rodentia & Muridae & Taterillus & congicus & $\begin{array}{l}\text { Congo Gerbil, Congo } \\
\text { Tateril }\end{array}$ & 1.71 & 52 & extant & 0 & NA & LC & frug/browse \\
\hline AF & Rodentia & Muridae & Taterillus & emini & $\begin{array}{l}\text { Emin's Gerbil, Emin's } \\
\text { Tateril }\end{array}$ & 1.72 & 52 & extant & 0 & NA & LC & frug/browse \\
\hline AF & Rodentia & Muridae & Taterillus & gracilis & $\begin{array}{l}\text { Gracile Tateril, Slender } \\
\text { Gerbil }\end{array}$ & 1.68 & 48 & extant & 0 & NA & LC & frug/browse \\
\hline AF & Rodentia & Muridae & Taterillus & harringtoni & Emin's Gerbil & 1.71 & 52 & extant & 0 & NA & $\mathrm{LC}$ & frug/browse \\
\hline AF & Rodentia & Muridae & Taterillus & lacustris & $\begin{array}{l}\text { Lake Chad Gerbil, Lake } \\
\text { Chad Tateril }\end{array}$ & 1.71 & 52 & extant & 0 & NA & LC & frug/browse \\
\hline AF & Rodentia & Muridae & Taterillus & petteri & $\begin{array}{l}\text { Petter's Gerbil, Petter's } \\
\text { Tateril }\end{array}$ & 1.71 & 51 & extant & 0 & NA & LC & frug/browse \\
\hline AF & Rodentia & Muridae & Taterillus & pygargus & $\begin{array}{l}\text { Senegal Gerbil, Senegal } \\
\text { Tateril }\end{array}$ & 1.78 & 60 & extant & 0 & NA & $\mathrm{LC}$ & frug/browse \\
\hline AF & Rodentia & Muridae & Thallomys & loringi & Loring's Rat & 2.01 & 103 & extant & 0 & NA & LC & browse/frug \\
\hline AF & Rodentia & Muridae & Thallomys & nigricauda & Black-tailed Tree Rat & 2.10 & 125 & extant & 0 & NA & LC & browse/frug \\
\hline AF & Rodentia & Muridae & Thallomys & paedulcus & Acacia Rat & 1.91 & 81 & extant & 0 & NA & LC & browse/frug \\
\hline AF & Rodentia & Muridae & Thallomys & shortridgei & $\begin{array}{l}\text { Shortridge's Rat, } \\
\text { Shortrige's Rat }\end{array}$ & 2.01 & 103 & extant & 0 & NA & DD & browse/frug \\
\hline AF & Rodentia & Muridae & Thamnomys & kempi & Kemp's Thicket Rat & 1.88 & 75 & extant & Future & NA & Vu & browse/frug \\
\hline AF & Rodentia & Muridae & Thamnomys & venustus & Charming Thicket Rat & 1.79 & 62 & extant & Future & NA & vu & browse/frug \\
\hline AF & Rodentia & Muridae & Uranomys & ruddi & $\begin{array}{l}\text { Rudd's Bristle-furred } \\
\text { Rat, Rudd's Mouse }\end{array}$ & 1.61 & 40 & extant & 0 & NA & LC & ginsect/frug \\
\hline AF & Rodentia & Muridae & Zelotomys & hildegardeae & $\begin{array}{l}\text { Hildegarde's Broad- } \\
\text { headed Mouse, } \\
\text { Hildegarde's Zelotomys, } \\
\text { Hildegarde's Zelotomys }\end{array}$ & 1.78 & 60 & extant & 0 & NA & LC & ginsect \\
\hline AF & Rodentia & Muridae & Zelotomys & woosnami & $\begin{array}{l}\text { Woosnam's Broad- } \\
\text { headed Mouse, } \\
\text { Woosnam's Zelotomys, } \\
\text { Woosnam's Zelotomys } \\
\text { Kellen's Dormouse, }\end{array}$ & 1.73 & 54 & extant & 0 & NA & LC & frug/ginsect \\
\hline AF & Rodentia & Myoxidae & Graphiurus & kelleni & $\begin{array}{l}\text { Kellen's African } \\
\text { Dormouse } \\
\text { Kellen's Dormouse, }\end{array}$ & 1.02 & 11 & extant & 0 & NA & LC & ginsect/carn \\
\hline AF & Rodentia & Myoxidae & Graphiurus & kelleni & $\begin{array}{l}\text { Kellen's African } \\
\text { Dormouse }\end{array}$ & 1.44 & 28 & extant & 0 & NA & LC & ginsect/carn \\
\hline AF & Rodentia & Myoxidae & Graphiurus & nagtglasii & $\begin{array}{l}\text { Long-tailed Pouched } \\
\text { Rat, Lesser Pouched }\end{array}$ & 1.65 & 45 & extant & 0 & NA & $\mathrm{LC}$ & ginsect/carn \\
\hline AF & Rodentia & Nesomyidae & Beamys & hindei & $\begin{array}{l}\text { Rat, Lesser Hamster- } \\
\text { rat, Hinde's Pouched } \\
\text { Rat }\end{array}$ & 1.88 & 76 & extant & 0 & NA & LC & frug \\
\hline
\end{tabular}




\begin{tabular}{|c|c|c|c|c|c|c|c|c|c|c|c|c|}
\hline $\mathrm{AF}$ & Rodentia & Nesomyidae & Beamys & major & $\begin{array}{l}\text { Long-tailed Pouched } \\
\text { Rat }\end{array}$ & 1.88 & 76 & extant & 0 & NA & LC & \\
\hline AF & Rodentia & Nesomyidae & Cricetomys & emini & $\begin{array}{l}\text { Forest Giant Pouched } \\
\text { Rat, Giant Rat }\end{array}$ & 3.11 & 1300 & extant & 0 & NA & LC & frug/browse \\
\hline AF & Rodentia & Nesomyidae & Cricetomys & gambianus & $\begin{array}{l}\text { Gambian Rat, Northern } \\
\text { Giant Pouched Rat }\end{array}$ & 3.10 & 1271 & extant & 0 & NA & LC & frug/browse \\
\hline $\mathrm{AF}$ & Rodentia & Nesomyidae & Delanymys & brooksi & $\begin{array}{l}\text { Delany's Mouse, } \\
\text { Delany's Swamp Mouse }\end{array}$ & 0.70 & 5 & extant & Future & NA & VU & frug \\
\hline $\mathrm{AF}$ & Rodentia & Nesomyidae & Dendromus & insignis & $\begin{array}{l}\text { Montane African } \\
\text { Climbing Mouse, } \\
\text { Remarkable Climbing } \\
\text { Mouse }\end{array}$ & 1.04 & 11 & extant & 0 & NA & LC & frug \\
\hline AF & Rodentia & Nesomyidae & Dendromus & kahuziensis & $\begin{array}{l}\text { Mount Kahuzi African } \\
\text { Climbing Mouse, Mt. } \\
\text { Kahuzi Climbing Mouse } \\
\text { Lovat's African Climbing }\end{array}$ & 1.04 & 11 & extant & Future & NA & $C R$ & frug \\
\hline AF & Rodentia & Nesomyidae & Dendromus & lovati & $\begin{array}{l}\text { Mouse, Lovat's Climbing } \\
\text { Mouse } \\
\text { Gray African Climbing }\end{array}$ & 1.08 & 12 & extant & 0 & NA & LC & frug \\
\hline AF & Rodentia & Nesomyidae & Dendromus & melanotis & $\begin{array}{l}\text { Mouse, Gray Glimbing } \\
\text { Mouse }\end{array}$ & 0.96 & 9 & extant & 0 & NA & LC & frug \\
\hline AF & Rodentia & Nesomyidae & Dendromus & mesomelas & Brant's Climbing Mouse & 1.05 & 11 & extant & 0 & NA & LC & frug \\
\hline $\mathrm{AF}$ & Rodentia & Nesomyidae & Dendromus & messorius & Banana Climbing Mouse & 1.04 & 11 & extant & 0 & NA & LC & frug \\
\hline $\mathrm{AF}$ & Rodentia & Nesomyidae & Dendromus & mystacalis & $\begin{array}{l}\text { Chestnut Climbing } \\
\text { Mouse }\end{array}$ & 0.89 & 8 & extant & 0 & NA & LC & frug \\
\hline $\mathrm{AF}$ & Rodentia & Nesomyidae & Dendromus & nyikae & Nyika Climbing Mouse & 1.18 & 15 & extant & 0 & NA & LC & frug \\
\hline $\mathrm{AF}$ & Rodentia & Nesomyidae & Dendromus & oreas & $\begin{array}{l}\text { Cameroon Climbing } \\
\text { Mouse }\end{array}$ & 1.04 & 11 & extant & Future & NA & vU & frug \\
\hline $\mathrm{AF}$ & Rodentia & Nesomyidae & Dendromus & vernayi & $\begin{array}{l}\text { Vernay's Climbing } \\
\text { Mouse } \\
\text { Velvet African Climbing }\end{array}$ & 1.04 & 11 & extant & 0 & NA & DD & frug \\
\hline $\mathrm{AF}$ & Rodentia & Nesomyidae & Dendroprionomys & rousseloti & $\begin{array}{l}\text { Mouse, Velvet Climbing } \\
\text { Mouse }\end{array}$ & & & extant & 0 & NA & DD & frug \\
\hline AF & Rodentia & Nesomyidae & Malacothrix & typica & Gerbil Mouse & 1.13 & 13 & extant & 0 & NA & LC & frug/browse \\
\hline $\mathrm{AF}$ & Rodentia & Nesomyidae & Megadendromus & nikolausi & Nikolaus's Mouse & 1.72 & 52 & extant & 0 & NA & DD & frug \\
\hline $\mathrm{AF}$ & Rodentia & Nesomyidae & Mystromys & albicaudatus & $\begin{array}{l}\text { White-tailed Mouse, } \\
\text { White-tailed Rat }\end{array}$ & 1.94 & 87 & extant & Future & NA & EN & frug/browse \\
\hline $\mathrm{AF}$ & Rodentia & Nesomyidae & Petromyscus & barbouri & Barbour's Rock Mouse & 1.46 & 29 & extant & 0 & NA & LC & frug \\
\hline $\mathrm{AF}$ & Rodentia & Nesomyidae & Petromyscus & collinus & Pygmy Rock Mouse & 1.30 & 20 & extant & 0 & NA & LC & frug \\
\hline $\mathrm{AF}$ & Rodentia & Nesomyidae & Petromyscus & monticularis & $\begin{array}{l}\text { Brukkaros Pygmy Rock } \\
\text { Mouse }\end{array}$ & 1.32 & 21 & extant & 0 & NA & LC & frug \\
\hline $\mathrm{AF}$ & Rodentia & Nesomyidae & Petromyscus & shortridgei & $\begin{array}{l}\text { Shortridge's Rock } \\
\text { Mouse }\end{array}$ & 1.67 & 47 & extant & 0 & NA & LC & frug \\
\hline $\mathrm{AF}$ & Rodentia & Nesomyidae & Prionomys & batesi & $\begin{array}{l}\text { Dollman's Tree Mouse } \\
\text { Pouched Mouse, }\end{array}$ & 1.10 & 13 & extant & 0 & NA & DD & ginsect \\
\hline $\mathrm{AF}$ & Rodentia & Nesomyidae & Saccostomus & campestris & $\begin{array}{l}\text { Southern African } \\
\text { Pouched Mouse }\end{array}$ & 1.71 & 51 & extant & 0 & NA & LC & frug \\
\hline
\end{tabular}




\begin{tabular}{|c|c|c|c|c|c|c|c|c|c|c|c|c|}
\hline AF & Rodentia & Nesomyidae & Saccostomus & mearnsi & $\begin{array}{l}\text { East African Pouched } \\
\text { Mouse, Mearns's } \\
\text { Pouched Mouse }\end{array}$ & 1.66 & 46 & extant & 0 & NA & LC & frug \\
\hline $\mathrm{AF}$ & Rodentia & Nesomyidae & Steatomys & caurinus & $\begin{array}{l}\text { Northwestern Fat } \\
\text { Mouse }\end{array}$ & 1.65 & 45 & extant & 0 & NA & LC & frug/browse \\
\hline AF & Rodentia & Nesomyidae & Steatomys & cuppedius & Dainty Fat Mouse & 1.49 & 31 & extant & 0 & NA & LC & frug/browse \\
\hline AF & Rodentia & Nesomyidae & Steatomys & jacksoni & Jackson's Fat Mouse & 1.49 & 31 & extant & 0 & NA & DD & frug/browse \\
\hline AF & Rodentia & Nesomyidae & Steatomys & krebsii & Kreb's Fat Mouse & 1.32 & 21 & extant & 0 & NA & LC & frug/browse \\
\hline AF & Rodentia & Nesomyidae & Steatomys & parvus & Tiny Fat Mouse & 0.95 & 9 & extant & 0 & NA & LC & frug/browse \\
\hline AF & Rodentia & Nesomyidae & Steatomys & pratensis & Fat Mouse & 1.68 & 48 & extant & 0 & NA & LC & frug/browse \\
\hline AF & Rodentia & Pedetidae & Pedetes & capensis & $\begin{array}{l}\text { Spring Hare, Springhare, } \\
\text { Springhaas }\end{array}$ & 3.50 & 3155 & extant & 0 & NA & LC & graze \\
\hline AF & Rodentia & Petromuridae & Petromus & typicus & $\begin{array}{l}\text { Dassie Rat } \\
\text { Barbary Ground }\end{array}$ & 2.35 & 224 & extant & 0 & NA & LC & эraze/browse \\
\hline $\mathrm{AF}$ & Rodentia & Sciuridae & Atlantoxerus & getulus & $\begin{array}{l}\text { Squirrel, North African } \\
\text { Ground Squirrel } \\
\text { Western Palm Squirrel, }\end{array}$ & 2.40 & 251 & extant & 0 & NA & LC & frug \\
\hline $\mathrm{AF}$ & Rodentia & Sciuridae & Epixerus & ebii & $\begin{array}{l}\text { Ebian's Palm Squirrel, } \\
\text { Temminck's Giant } \\
\text { Squirrel }\end{array}$ & 2.77 & 592 & extant & 0 & NA & LC & frug \\
\hline $\mathrm{AF}$ & Rodentia & Sciuridae & Epixerus & wilsoni & Western Palm Squirrel & 2.59 & 388 & extant & 0 & NA & LC & frug \\
\hline $\mathrm{AF}$ & Rodentia & Sciuridae & Funisciurus & anerythrus & $\begin{array}{l}\text { Redness Tree Squirrel, } \\
\text { Thomas's Rope Squirrel }\end{array}$ & 2.34 & 218 & extant & 0 & NA & LC & frug/ginsect \\
\hline AF & Rodentia & Sciuridae & Funisciurus & bayonii & Lunda Rope Squirrel & 2.13 & 135 & extant & 0 & NA & DD & frug \\
\hline $\mathrm{AF}$ & Rodentia & Sciuridae & Funisciurus & carruthersi & $\begin{array}{l}\text { Carruther's Mountain } \\
\text { Squirrel }\end{array}$ & 2.46 & 286 & extant & 0 & NA & LC & frug \\
\hline AF & Rodentia & Sciuridae & Funisciurus & congicus & Congo Rope Squirrel & 2.05 & 112 & extant & 0 & NA & LC & frug/browse \\
\hline $\mathrm{AF}$ & Rodentia & Sciuridae & Funisciurus & isabella & $\begin{array}{l}\text { Lady Burton's Rope } \\
\text { Squirrel }\end{array}$ & 2.04 & 109 & extant & 0 & NA & LC & frug/browse \\
\hline $\mathrm{AF}$ & Rodentia & Sciuridae & Funisciurus & lemniscatus & $\begin{array}{l}\text { Ribboned Rope Squirrel } \\
\text { Orange Headed Tree }\end{array}$ & 2.15 & 141 & extant & 0 & NA & LC & frug/ginsect \\
\hline $\mathrm{AF}$ & Rodentia & Sciuridae & Funisciurus & leucogenys & $\begin{array}{l}\text { Squirrel, Red-cheeked } \\
\text { Rope Squirrel }\end{array}$ & 2.40 & 250 & extant & 0 & NA & LC & frug \\
\hline $\mathrm{AF}$ & Rodentia & Sciuridae & Funisciurus & pyrropus & $\begin{array}{l}\text { Fire-footed Rope } \\
\text { Squirrel }\end{array}$ & 2.47 & 297 & extant & 0 & NA & LC & frug/ginsect \\
\hline $\mathrm{AF}$ & Rodentia & Sciuridae & Funisciurus & substriatus & $\begin{array}{l}\text { Kintampo Rope Squirrel, } \\
\text { Winston's Tree Squirrel }\end{array}$ & 2.27 & 186 & extant & 0 & NA & DD & frug \\
\hline AF & Rodentia & Sciuridae & Heliosciurus & gambianus & Gambian Sun Squirrel & 2.47 & 295 & extant & 0 & NA & LC & frug \\
\hline AF & Rodentia & Sciuridae & Heliosciurus & mutabilis & Mutable Sun Squirrel & 2.59 & 390 & extant & 0 & NA & LC & frug/browse \\
\hline $\mathrm{AF}$ & Rodentia & Sciuridae & Heliosciurus & punctatus & $\begin{array}{l}\text { Small Sun Squirrel } \\
\text { Crab-eating Mongoose, }\end{array}$ & 2.24 & 174 & extant & 0 & NA & DD & frug \\
\hline $\mathrm{AF}$ & Rodentia & Sciuridae & Heliosciurus & rufobrachium & $\begin{array}{l}\text { Isabelline Red-legged } \\
\text { Sun Squirrel, Red- } \\
\text { legged Sun Squirrel }\end{array}$ & 2.57 & 372 & extant & 0 & NA & LC & frug \\
\hline AF & Rodentia & Sciuridae & Heliosciurus & ruwenzorii & Ruwenzori Sun Squirrel & 2.46 & 291 & extant & 0 & NA & LC & frug \\
\hline AF & Rodentia & Sciuridae & Heliosciurus & undulatus & Zanj Sun Squirrel & 2.54 & 347 & extant & 0 & NA & DD & frug \\
\hline $\mathrm{AF}$ & Rodentia & Sciuridae & Myosciurus & pumilio & African Pygmy Squirrel & 2.22 & 165 & extant & 0 & NA & LC & ginsect \\
\hline $\mathrm{AF}$ & Rodentia & Sciuridae & Paraxerus & alexandri & $\begin{array}{l}\text { Alexander's Bush } \\
\text { Squirrel }\end{array}$ & 1.72 & 52 & extant & 0 & NA & LC & ginsect/frug \\
\hline AF & Rodentia & Sciuridae & Paraxerus & boehmi & Boehm's Bush Squirrel & 1.85 & 70 & extant & 0 & NA & LC & ginsect/frug \\
\hline $\mathrm{AF}$ & Rodentia & Sciuridae & Paraxerus & серарі & Smith's Bush Squirrel & 2.26 & 180 & extant & 0 & NA & LC & frug/browse \\
\hline
\end{tabular}




\begin{tabular}{|c|c|c|c|c|c|c|c|c|c|c|c|c|}
\hline \multirow[b]{2}{*}{ AF } & \multirow[b]{2}{*}{ Rodentia } & \multirow[b]{2}{*}{ Sciuridae } & \multirow[b]{2}{*}{ Paraxerus } & \multirow[b]{2}{*}{ cooperi } & Cooper's Mountain & & & & & & & \\
\hline & & & & & $\begin{array}{l}\text { Squirrel, Cusorial } \\
\text { Akodont }\end{array}$ & 2.40 & 250 & extant & 0 & NA & DD & frug \\
\hline$A F$ & Rodentia & Sciuridae & Paraxerus & flavovittis & Striped Bush Squirrel & 2.20 & 160 & extant & 0 & NA & LC & frug/browse \\
\hline $\mathrm{AF}$ & Rodentia & Sciuridae & Paraxerus & lucifer & $\begin{array}{l}\text { Black And Red Bush } \\
\text { Squirrel }\end{array}$ & 2.85 & 700 & extant & 0 & NA & DD & frug/ginsect \\
\hline AF & Rodentia & Sciuridae & Paraxerus & ochraceus & Ochre Bush Squirrel & 2.02 & 104 & extant & 0 & NA & LC & frug/browse \\
\hline AF & Rodentia & Sciuridae & Paraxerus & palliatus & Red Bush Squirrel & 2.49 & 309 & extant & 0 & NA & LC & frug \\
\hline AF & Rodentia & Sciuridae & Paraxerus & poensis & Green Bush Squirrel & 2.10 & 125 & extant & 0 & NA & LC & frug \\
\hline AF & Rodentia & Sciuridae & Paraxerus & vexillarius & $\begin{array}{l}\text { Svynnerton's Bush } \\
\text { Squirrel }\end{array}$ & 2.83 & 675 & extant & Future & NA & NT & frug \\
\hline $\mathrm{AF}$ & Rodentia & Sciuridae & Paraxerus & vincenti & Vincent's Bush Squirrel & 2.42 & 263 & extant & Future & NA & EN & \\
\hline $\mathrm{AF}$ & Rodentia & Sciuridae & Protoxerus & aubinnii & Slender-tailed Squirrel & 2.54 & 350 & extant & 0 & NA & DD & frug \\
\hline $\mathrm{AF}$ & Rodentia & Sciuridae & Protoxerus & stangeri & $\begin{array}{l}\text { African Giant Squirrel, } \\
\text { Forest Giant Squirrel }\end{array}$ & 2.79 & 621 & extant & 0 & NA & LC & frug \\
\hline AF & Rodentia & Sciuridae & Xerus & erythropus & $\begin{array}{l}\text { Striped Ground Squirrel, } \\
\text { Geoffroy's Ground } \\
\text { Squirrel }\end{array}$ & 2.70 & 502 & extant & 0 & NA & LC & frug/browse \\
\hline AF & Rodentia & Sciuridae & Xerus & inauris & $\begin{array}{l}\text { South African Ground } \\
\text { Squirrel }\end{array}$ & 2.72 & 529 & extant & 0 & NA & LC & browse/frug \\
\hline AF & Rodentia & Sciuridae & Xerus & princeps & Damara Ground Squirrel & 2.80 & 636 & extant & 0 & NA & LC & browse/frug \\
\hline $\mathrm{AF}$ & Rodentia & Sciuridae & Xerus & rutilus & $\begin{array}{l}\text { Unstriped Ground } \\
\text { Squirrel }\end{array}$ & 2.50 & 317 & extant & 0 & NA & LC & browse/frug \\
\hline AF & Rodentia & Spalacidae & Tachyoryctes & ankoliae & & 2.54 & 348 & extant & 0 & NA & LC & \\
\hline AF & Rodentia & Spalacidae & Tachyoryctes & annectens & & 2.54 & 348 & extant & 0 & NA & LC & \\
\hline AF & Rodentia & Spalacidae & Tachyoryctes & audax & & 2.37 & 235 & extant & 0 & NA & LC & Browse \\
\hline AF & Rodentia & Spalacidae & Tachyoryctes & daemon & & 2.54 & 348 & extant & 0 & NA & LC & \\
\hline AF & Rodentia & Spalacidae & Tachyoryctes & macrocephalus & Giant Mole Rat & 2.78 & 597 & extant & Future & NA & EN & Browse \\
\hline AF & Rodentia & Spalacidae & Tachyoryctes & naivashae & & 2.54 & 348 & extant & 0 & NA & LC & \\
\hline AF & Rodentia & Spalacidae & Tachyoryctes & rex & & 2.54 & 348 & extant & 0 & NA & LC & \\
\hline $\mathrm{AF}$ & Rodentia & Spalacidae & Tachyoryctes & ruandae & & 2.54 & 348 & extant & 0 & NA & LC & \\
\hline AF & Rodentia & Spalacidae & Tachyoryctes & ruddi & & 2.54 & 348 & extant & 0 & NA & LC & \\
\hline AF & Rodentia & Spalacidae & Tachyoryctes & spalacinus & & 2.54 & 348 & extant & 0 & NA & LC & \\
\hline AF & Rodentia & Spalacidae & Tachyoryctes & splendens & East African Mole Rat & 2.33 & 212 & extant & 0 & NA & LC & Browse \\
\hline AF & Rodentia & Thryonomyidae & Thryonomys & gregorianus & Lesser Cane Rat & 3.43 & 2707 & extant & 0 & NA & LC & graze \\
\hline $\mathrm{AF}$ & Rodentia & Thryonomyidae & Thryonomys & swinderianus & $\begin{array}{l}\text { Greater Cane Rat } \\
\text { African Manatee, West }\end{array}$ & 3.50 & 3180 & extant & 0 & NA & LC & graze \\
\hline $\mathrm{AF}$ & Sirenia & Trichechidae & Trichechus & senegalensis & $\begin{array}{l}\text { African Manatee, } \\
\text { Seacow }\end{array}$ & 5.66 & 454000 & extant & Future & NA & $\mathrm{Vu}$ & Graze \\
\hline $\mathrm{AF}$ & Soricomorpha & Soricidae & Congosorex & polli & $\begin{array}{l}\text { Greater Congo Shrew, } \\
\text { Poll's Shrew }\end{array}$ & & & extant & 0 & NA & DD & ginsect \\
\hline $\mathrm{AF}$ & Soricomorpha & Soricidae & Crocidura & aleksandrisi & $\begin{array}{l}\text { Alexandrian Shrew, } \\
\text { Cyrenaica Shrew }\end{array}$ & 1.11 & 13 & extant & 0 & NA & LC & ginsect \\
\hline $\mathrm{AF}$ & Soricomorpha & Soricidae & Crocidura & allex & $\begin{array}{l}\text { East African Highland } \\
\text { Shrew, Highlands Shrew }\end{array}$ & 1.11 & 13 & extant & Future & NA & vu & ginsect \\
\hline AF & Soricomorpha & Soricidae & Crocidura & ansellorum & Ansell's Shrew & 1.11 & 13 & extant & Future & NA & EN & ginsect \\
\hline AF & Soricomorpha & Soricidae & Crocidura & attila & Hun Shrew & 1.11 & 13 & extant & 0 & NA & LC & ginsect \\
\hline $\mathrm{AF}$ & Soricomorpha & Soricidae & Crocidura & baileyi & Bailey's Shrew & 1.11 & 13 & extant & Future & NA & EN & ginsect \\
\hline AF & Soricomorpha & Soricidae & Crocidura & batesi & Bate's Shrew & 1.19 & 16 & extant & 0 & NA & LC & ginsect \\
\hline AF & Soricomorpha & Soricidae & Crocidura & bottegi & Bottego's Shrew & 0.54 & 4 & extant & 0 & NA & DD & ginsect \\
\hline
\end{tabular}




\begin{tabular}{|c|c|c|c|c|c|c|c|c|c|c|c|c|}
\hline$A F$ & Soricomorpha & Soricidae & Crocidura & bottegoides & Bale Shrew & 1.11 & 13 & extant & Future & NA & EN & ginsect \\
\hline$A F$ & Soricomorpha & Soricidae & Crocidura & buettikoferi & Buettikofer's Shrew & 1.11 & 13 & extant & Future & NA & NT & ginsect \\
\hline $\mathrm{AF}$ & Soricomorpha & Soricidae & Crocidura & caliginea & $\begin{array}{l}\text { African Dusky Shrew, } \\
\text { African Foggy Shrew }\end{array}$ & 1.11 & 13 & extant & 0 & NA & LC & ginsect \\
\hline AF & Soricomorpha & Soricidae & Crocidura & cinderella & Cinderella Shrew & 1.11 & 13 & extant & 0 & NA & LC & ginsect \\
\hline AF & Soricomorpha & Soricidae & Crocidura & congobelgica & $\begin{array}{l}\text { Congo Shrew, Congo } \\
\text { White-toothed Shrew }\end{array}$ & 1.11 & 13 & extant & 0 & NA & LC & ginsect \\
\hline AF & Soricomorpha & Soricidae & Crocidura & crenata & Long-footed Shrew & 0.81 & 7 & extant & 0 & NA & LC & ginsect \\
\hline AF & Soricomorpha & Soricidae & Crocidura & crossei & Crosse's Shrew & 0.84 & 7 & extant & 0 & NA & LC & ginsect \\
\hline $\mathrm{AF}$ & Soricomorpha & Soricidae & Crocidura & cyanea & $\begin{array}{l}\text { Reddish-gray Musk } \\
\text { Shrew }\end{array}$ & 0.97 & 9 & extant & 0 & NA & LC & ginsect \\
\hline AF & Soricomorpha & Soricidae & Crocidura & denti & Dent's Shrew & 0.85 & 7 & extant & 0 & NA & LC & ginsect \\
\hline AF & Soricomorpha & Soricidae & Crocidura & desperata & Desperate Shrew & 1.11 & 13 & extant & Future & NA & EN & ginsect \\
\hline $\mathrm{AF}$ & Soricomorpha & Soricidae & Crocidura & dolichura & Long-tailed Musk Shrew & 0.76 & 6 & extant & 0 & NA & LC & ginsect \\
\hline AF & Soricomorpha & Soricidae & Crocidura & douceti & Doucet's Musk Shrew & 0.63 & 4 & extant & 0 & NA & DD & ginsect \\
\hline AF & Soricomorpha & Soricidae & Crocidura & eisentrauti & Eisentraut's Shrew & 1.11 & 13 & extant & Future & NA & Vu & ginsect \\
\hline AF & Soricomorpha & Soricidae & Crocidura & elgonius & Elgon Shrew & 0.81 & 7 & extant & 0 & NA & LC & ginsect \\
\hline AF & Soricomorpha & Soricidae & Crocidura & erica & Heather Shrew & 1.11 & 13 & extant & 0 & NA & DD & ginsect \\
\hline AF & Soricomorpha & Soricidae & Crocidura & fischeri & Fischer's Shrew & 1.11 & 13 & extant & 0 & NA & DD & ginsect \\
\hline $\mathrm{AF}$ & Soricomorpha & Soricidae & Crocidura & flavescens & $\begin{array}{l}\text { Greater Red Musk } \\
\text { Shrew }\end{array}$ & 1.45 & 28 & extant & 0 & NA & LC & ginsect \\
\hline AF & Soricomorpha & Soricidae & Crocidura & floweri & Flower's Shrew & 1.11 & 13 & extant & 0 & NA & DD & ginsect \\
\hline AF & Soricomorpha & Soricidae & Crocidura & foxi & Fox's Shrew & 1.28 & 19 & extant & 0 & NA & LC & ginsect \\
\hline AF & Soricomorpha & Soricidae & Crocidura & fulvastra & Savanna Shrew & 1.10 & 13 & extant & 0 & NA & LC & ginsect \\
\hline AF & Soricomorpha & Soricidae & Crocidura & fumosa & $\begin{array}{l}\text { Smoky White-toothed } \\
\text { Shrew }\end{array}$ & 1.16 & 15 & extant & Future & NA & Vu & ginsect \\
\hline AF & Soricomorpha & Soricidae & Crocidura & fuscomurina & $\begin{array}{l}\text { Bicolored Musk Shrew, } \\
\text { Tiny Musk Shrew }\end{array}$ & 0.59 & 4 & extant & 0 & NA & LC & ginsect \\
\hline $\mathrm{AF}$ & Soricomorpha & Soricidae & Crocidura & glassi & Glass's Shrew & 1.11 & 13 & extant & Future & NA & Vu & ginsect \\
\hline AF & Soricomorpha & Soricidae & Crocidura & goliath & Goliath Shrew & 1.74 & 55 & extant & 0 & NA & LC & ginsect \\
\hline AF & Soricomorpha & Soricidae & Crocidura & gracilipes & $\begin{array}{l}\text { Peters' Musk Shrew } \\
\text { Large-headed Forest }\end{array}$ & 0.83 & 7 & extant & 0 & NA & DD & ginsect \\
\hline $\mathrm{AF}$ & Soricomorpha & Soricidae & Crocidura & grandiceps & $\begin{array}{l}\text { Shrew, Large-headed } \\
\text { Shrew }\end{array}$ & 1.36 & 23 & extant & Future & NA & NT & ginsect \\
\hline AF & Soricomorpha & Soricidae & Crocidura & grassei & Grasse's Shrew & 1.13 & 14 & extant & 0 & NA & LC & ginsect \\
\hline AF & Soricomorpha & Soricidae & Crocidura & greenwoodi & Greenwood's Shrew & 1.11 & 13 & extant & 0 & NA & LC & ginsect \\
\hline $\mathrm{AF}$ & Soricomorpha & Soricidae & Crocidura & harenna & Harenna Shrew & 1.11 & 13 & extant & Future & NA & CR & ginsect \\
\hline AF & Soricomorpha & Soricidae & Crocidura & hildegardeae & Hildegarde's Shrew & 0.98 & 10 & extant & 0 & NA & LC & ginsect \\
\hline AF & Soricomorpha & Soricidae & Crocidura & hirta & Lesser Red Musk Shrew & 1.20 & 16 & extant & 0 & NA & LC & ginsect \\
\hline$A F$ & Soricomorpha & Soricidae & Crocidura & jacksoni & Jackson's Shrew & 0.85 & 7 & extant & 0 & NA & LC & ginsect \\
\hline AF & Soricomorpha & Soricidae & Crocidura & kivuana & Kivu Shrew & 1.11 & 13 & extant & Future & NA & Vu & ginsect \\
\hline AF & Soricomorpha & Soricidae & Crocidura & lamottei & Lamotte's Shrew & 1.31 & 21 & extant & 0 & NA & LC & ginsect \\
\hline $\mathrm{AF}$ & Soricomorpha & Soricidae & Crocidura & Ianosa & $\begin{array}{l}\text { Kivu Long-haired Shrew, } \\
\text { Lemara Shrew }\end{array}$ & 1.11 & 13 & extant & Future & NA & EN & ginsect \\
\hline $\mathrm{AF}$ & Soricomorpha & Soricidae & Crocidura & latona & $\begin{array}{l}\text { Latona Shrew, Latona's } \\
\text { Shrew } \\
\text { Butiaba Naked-tailed }\end{array}$ & 1.11 & 13 & extant & 0 & NA & LC & ginsect \\
\hline AF & Soricomorpha & Soricidae & Crocidura & littoralis & $\begin{array}{l}\text { Shrew, Naked-tail } \\
\text { Shrew }\end{array}$ & 1.20 & 16 & extant & 0 & NA & LC & ginsect \\
\hline $\mathrm{AF}$ & Soricomorpha & Soricidae & Crocidura & longipes & Savanna Swamp Shrew & 1.29 & 19 & extant & 0 & NA & DD & ginsect \\
\hline
\end{tabular}




\begin{tabular}{|c|c|c|c|c|c|c|c|c|c|c|c|c|}
\hline $\mathrm{AF}$ & Soricomorpha & Soricidae & Crocidura & lucina & $\begin{array}{l}\text { Lucina's Shrew, } \\
\text { Moorland Shrew, } \\
\text { Morrland Shrew }\end{array}$ & 1.11 & 13 & extant & Future & NA & VU & ginsect \\
\hline $\mathrm{AF}$ & Soricomorpha & Soricidae & Crocidura & ludia & $\begin{array}{l}\text { Dramatic Shrew, Ludia's } \\
\text { Shrew }\end{array}$ & 1.11 & 13 & extant & 0 & NA & LC & ginsect \\
\hline $\mathrm{AF}$ & Soricomorpha & Soricidae & Crocidura & luna & $\begin{array}{l}\text { Greater Gray-brown } \\
\text { Musk Shrew }\end{array}$ & 1.16 & 15 & extant & 0 & NA & LC & ginsect \\
\hline $\mathrm{AF}$ & Soricomorpha & Soricidae & Crocidura & Iusitania & Mauritanian Shrew & 0.30 & 2 & extant & 0 & NA & LC & ginsect \\
\hline $\mathrm{AF}$ & Soricomorpha & Soricidae & Crocidura & macarthuri & Macarthur's Shrew & 1.11 & 13 & extant & 0 & NA & LC & ginsect \\
\hline $\mathrm{AF}$ & Soricomorpha & Soricidae & Crocidura & macmillani & Macmillan's Shrew & 1.11 & 13 & extant & Future & NA & Vu & ginsect \\
\hline $\mathrm{AF}$ & Soricomorpha & Soricidae & Crocidura & macowi & $\begin{array}{l}\text { Macow's Shrew, Nyiro } \\
\text { Shrew }\end{array}$ & 1.11 & 13 & extant & 0 & NA & DD & ginsect \\
\hline $\mathrm{AF}$ & Soricomorpha & Soricidae & Crocidura & manengubae & $\begin{array}{l}\text { Manenguba Shrew } \\
\text { Makwassie Musk }\end{array}$ & 1.11 & 13 & extant & Future & NA & $\mathrm{Vu}$ & ginsect \\
\hline $\mathrm{AF}$ & Soricomorpha & Soricidae & Crocidura & maquassiensis & $\begin{array}{l}\text { Shrew, Maquassie Musk } \\
\text { Shrew }\end{array}$ & 0.78 & 6 & extant & 0 & NA & LC & ginsect \\
\hline $\mathrm{AF}$ & Soricomorpha & Soricidae & Crocidura & mariquensis & Swamp Musk Shrew & 1.05 & 11 & extant & 0 & NA & LC & ginsect \\
\hline $\mathrm{AF}$ & Soricomorpha & Soricidae & Crocidura & maurisca & $\begin{array}{l}\text { Dark Shrew, Gracile } \\
\text { Naked-tailed Shrew }\end{array}$ & 1.15 & 14 & extant & 0 & NA & LC & ginsect \\
\hline $\mathrm{AF}$ & Soricomorpha & Soricidae & Crocidura & $\operatorname{monax}$ & $\begin{array}{l}\text { Kilimanjaro Shrew, } \\
\text { Rombo Shrew }\end{array}$ & 1.20 & 16 & extant & 0 & NA & LC & ginsect \\
\hline $\mathrm{AF}$ & Soricomorpha & Soricidae & Crocidura & montis & $\begin{array}{l}\text { Montane White-toothed } \\
\text { Shrew }\end{array}$ & 1.16 & 15 & extant & 0 & NA & LC & ginsect \\
\hline $\mathrm{AF}$ & Soricomorpha & Soricidae & Crocidura & muricauda & $\begin{array}{l}\text { Mouse-tailed Shrew, } \\
\text { West African Long- } \\
\text { tailed Shrew } \\
\text { Uganda Large-toothed }\end{array}$ & 1.11 & 13 & extant & 0 & NA & LC & ginsect \\
\hline $\mathrm{AF}$ & Soricomorpha & Soricidae & Crocidura & mutesae & $\begin{array}{l}\text { Shrew, Ugandan Musk } \\
\text { Shrew } \\
\text { Dwarf White-toothed }\end{array}$ & 1.11 & 13 & extant & 0 & NA & DD & ginsect \\
\hline $\mathrm{AF}$ & Soricomorpha & Soricidae & Crocidura & nana & $\begin{array}{l}\text { Shrew, Somali Dwarf } \\
\text { Shrew } \\
\text { Savanna Dwarf Shrew, }\end{array}$ & 1.11 & 13 & extant & 0 & NA & LC & ginsect \\
\hline $\mathrm{AF}$ & Soricomorpha & Soricidae & Crocidura & nanilla & $\begin{array}{l}\text { Tiny White-toothed } \\
\text { Shrew }\end{array}$ & 0.81 & 7 & extant & 0 & NA & LC & ginsect \\
\hline $\mathrm{AF}$ & Soricomorpha & Soricidae & Crocidura & nigeriae & $\begin{array}{l}\text { Nigerian Shrew } \\
\text { Blackish White-toothed }\end{array}$ & 1.36 & 23 & extant & 0 & NA & LC & ginsect \\
\hline $\mathrm{AF}$ & Soricomorpha & Soricidae & Crocidura & nigricans & $\begin{array}{l}\text { Shrew, Black White- } \\
\text { toothed Shrew }\end{array}$ & 1.30 & 20 & extant & 0 & NA & $\mathrm{LC}$ & ginsect \\
\hline $\mathrm{AF}$ & Soricomorpha & Soricidae & Crocidura & nigripes & Black-footed Shrew & 1.12 & 13 & extant & 0 & NA & LC & ginsect \\
\hline $\mathrm{AF}$ & Soricomorpha & Soricidae & Crocidura & nigrofusca & $\begin{array}{l}\text { African Black Shrew, } \\
\text { Tenebrous Shrew }\end{array}$ & 1.11 & 13 & extant & 0 & NA & LC & ginsect \\
\hline $\mathrm{AF}$ & Soricomorpha & Soricidae & Crocidura & nimbae & Nimba Shrew & 1.19 & 16 & extant & Future & NA & NT & ginsect \\
\hline $\mathrm{AF}$ & Soricomorpha & Soricidae & Crocidura & niobe & $\begin{array}{l}\text { Niobe's Shrew, Stony } \\
\text { Shrew } \\
\text { Obscure White-toothed }\end{array}$ & 1.20 & 16 & extant & Future & NA & NT & ginsect \\
\hline $\mathrm{AF}$ & Soricomorpha & Soricidae & Crocidura & obscurior & $\begin{array}{l}\text { Shrew, West African } \\
\text { Pygmy Shrew }\end{array}$ & 1.11 & 13 & extant & 0 & NA & LC & ginsect \\
\hline $\mathrm{AF}$ & Soricomorpha & Soricidae & Crocidura & olivieri & $\begin{array}{l}\text { Olivier's Shrew, African } \\
\text { Giant Shrew }\end{array}$ & 1.76 & 58 & extant & 0 & NA & LC & ginsect \\
\hline $\mathrm{AF}$ & Soricomorpha & Soricidae & Crocidura & parvipes & Small-footed Shrew & 1.11 & 13 & extant & 0 & NA & LC & ginsect \\
\hline
\end{tabular}




\begin{tabular}{|c|c|c|c|c|c|c|c|c|c|c|c|c|}
\hline AF & Soricomorpha & Soricidae & Crocidura & pasha & $\begin{array}{l}\text { Pasha Shrew, Sahelian } \\
\text { Tiny Shrew }\end{array}$ & 0.81 & 7 & extant & 0 & NA & LC & ginsect \\
\hline AF & Soricomorpha & Soricidae & Crocidura & phaeura & Guramba Shrew & 1.11 & 13 & extant & Future & NA & EN & ginsect \\
\hline AF & Soricomorpha & Soricidae & Crocidura & picea & $\begin{array}{l}\text { Cameroonian Shrew, } \\
\text { Pitch Shrew }\end{array}$ & 1.11 & 13 & extant & Future & NA & EN & ginsect \\
\hline AF & Soricomorpha & Soricidae & Crocidura & pitmani & Pitman's Shrew & 1.11 & 13 & extant & 0 & NA & DD & ginsect \\
\hline $\mathrm{AF}$ & Soricomorpha & Soricidae & Crocidura & planiceps & Flat-headed Shrew & 0.40 & 3 & extant & 0 & NA & DD & ginsect \\
\hline $\mathrm{AF}$ & Soricomorpha & Soricidae & Crocidura & poensis & Fraser's Musk Shrew & 1.22 & 17 & extant & 0 & NA & LC & ginsect \\
\hline $\mathrm{AF}$ & Soricomorpha & Soricidae & Crocidura & polia & $\begin{array}{l}\text { Fuscous Shrew, Polia's } \\
\text { Shrew }\end{array}$ & 1.11 & 13 & extant & 0 & NA & DD & ginsect \\
\hline AF & Soricomorpha & Soricidae & Crocidura & raineyi & $\begin{array}{l}\text { Rainey Shrew, Rainey's } \\
\text { Shrew }\end{array}$ & 1.16 & 15 & extant & 0 & NA & DD & ginsect \\
\hline $\mathrm{AF}$ & Soricomorpha & Soricidae & Crocidura & religiosa & Egyptian Pygmy Shrew & 0.85 & 7 & extant & 0 & NA & DD & ginsect \\
\hline $\mathrm{AF}$ & Soricomorpha & Soricidae & Crocidura & roosevelti & Roosvelt's Shrew & 1.11 & 13 & extant & 0 & NA & LC & ginsect \\
\hline $\mathrm{AF}$ & Soricomorpha & Soricidae & Crocidura & russula & $\begin{array}{l}\text { White-toothed Shrew, } \\
\text { Greater White-toothed } \\
\text { Shrew }\end{array}$ & 0.81 & 6 & extant & 0 & NA & LC & ginsect \\
\hline $\mathrm{AF}$ & Soricomorpha & Soricidae & Crocidura & selina & $\begin{array}{l}\text { Moon Shrew, Ugandan } \\
\text { Lowland Shrew }\end{array}$ & 1.11 & 13 & extant & 0 & NA & DD & ginsect \\
\hline AF & Soricomorpha & Soricidae & Crocidura & silacea & $\begin{array}{l}\text { Lesser Gray-brown } \\
\text { Musk Shrew }\end{array}$ & 0.90 & 8 & extant & 0 & NA & LC & ginsect \\
\hline $\mathrm{AF}$ & Soricomorpha & Soricidae & Crocidura & smithii & Desert Musk Shrew & 1.11 & 13 & extant & 0 & NA & LC & ginsect \\
\hline $\mathrm{AF}$ & Soricomorpha & Soricidae & Crocidura & somalica & Somali Shrew & 1.06 & 12 & extant & 0 & NA & LC & ginsect \\
\hline $\mathrm{AF}$ & Soricomorpha & Soricidae & Crocidura & stenocephala & $\begin{array}{l}\text { Kahuzi Swamp Shrew, } \\
\text { Narrow-headed Shrew }\end{array}$ & 1.11 & 13 & extant & Future & NA & EN & ginsect \\
\hline $\mathrm{AF}$ & Soricomorpha & Soricidae & Crocidura & suaveolens & $\begin{array}{l}\text { Lesser Shrew, Lesser } \\
\text { White-toothed Shrew }\end{array}$ & 0.70 & 5 & extant & 0 & NA & LC & ginsect \\
\hline $\mathrm{AF}$ & Soricomorpha & Soricidae & Crocidura & tansaniana & Tanzanian Shrew & 1.11 & 13 & extant & Future & NA & EN & ginsect \\
\hline $\mathrm{AF}$ & Soricomorpha & Soricidae & Crocidura & tarella & $\begin{array}{l}\text { Tarella Shrew, Ugandan } \\
\text { Shrew }\end{array}$ & 1.11 & 13 & extant & Future & NA & EN & ginsect \\
\hline $\mathrm{AF}$ & Soricomorpha & Soricidae & Crocidura & tarfayensis & $\begin{array}{l}\text { Tarfaya Shrew, Saharan } \\
\text { Shrew }\end{array}$ & 0.81 & 7 & extant & 0 & NA & DD & ginsect \\
\hline AF & Soricomorpha & Soricidae & Crocidura & telfordi & Telford's Shrew & 1.11 & 13 & extant & Future & NA & EN & ginsect \\
\hline $\mathrm{AF}$ & Soricomorpha & Soricidae & Crocidura & thalia & $\begin{array}{l}\text { Thalia Shrew, Thalia's } \\
\text { Shrew }\end{array}$ & 1.11 & 13 & extant & 0 & NA & LC & ginsect \\
\hline $\mathrm{AF}$ & Soricomorpha & Soricidae & Crocidura & theresae & Therese's Shrew & 1.25 & 18 & extant & 0 & NA & LC & ginsect \\
\hline $\mathrm{AF}$ & Soricomorpha & Soricidae & Crocidura & thomensis & $\begin{array}{l}\text { Sao Tom_Shrew, Sto } \\
\text { Tom_Shrew }\end{array}$ & 1.11 & 13 & extant & Future & NA & EN & ginsect \\
\hline $\mathrm{AF}$ & Soricomorpha & Soricidae & Crocidura & turba & $\begin{array}{l}\text { Tumultuous Shrew, } \\
\text { Turbo Shrew }\end{array}$ & 1.16 & 15 & extant & 0 & NA & LC & ginsect \\
\hline $\mathrm{AF}$ & Soricomorpha & Soricidae & Crocidura & ultima & Ultimate Shrew & 1.20 & 16 & extant & 0 & NA & DD & ginsect \\
\hline $\mathrm{AF}$ & Soricomorpha & Soricidae & Crocidura & usambarae & Usambara Shrew & 0.99 & 10 & extant & Future & NA & EN & ginsect \\
\hline $\mathrm{AF}$ & Soricomorpha & Soricidae & Crocidura & viaria & Savanna Path Shrew & 1.23 & 17 & extant & 0 & NA & LC & ginsect \\
\hline $\mathrm{AF}$ & Soricomorpha & Soricidae & Crocidura & voi & Voi Shrew & 1.11 & 13 & extant & 0 & NA & LC & ginsect \\
\hline AF & Soricomorpha & Soricidae & Crocidura & whitakeri & Whitaker's Shrew & 0.74 & 6 & extant & 0 & NA & LC & ginsect \\
\hline AF & Soricomorpha & Soricidae & Crocidura & wimmeri & Wimmer's Shrew & 1.37 & 24 & extant & Future & NA & CR & ginsect \\
\hline AF & Soricomorpha & Soricidae & Crocidura & xantippe & $\begin{array}{l}\text { Vermiculate Shrew, } \\
\text { Xanthippe's Shrew }\end{array}$ & 1.11 & 13 & extant & 0 & NA & LC & ginsect \\
\hline $\mathrm{AF}$ & Soricomorpha & Soricidae & Crocidura & yankariensis & Yankari Shrew & 0.70 & 5 & extant & 0 & NA & LC & ginsect \\
\hline AF & Soricomorpha & Soricidae & Crocidura & zaphiri & Zaphir's Shrew & 1.11 & 13 & extant & 0 & NA & DD & ginsect \\
\hline $\mathrm{AF}$ & Soricomorpha & Soricidae & Crocidura & zimmeri & $\begin{array}{l}\text { Upemba Shrew, } \\
\text { Zimmer's Shrew }\end{array}$ & 1.16 & 15 & extant & 0 & NA & DD & ginsect \\
\hline
\end{tabular}




\begin{tabular}{|c|c|c|c|c|c|c|c|c|c|c|c|c|}
\hline AF & Soricomorpha & Soricidae & Myosorex & babaulti & Babault's Mouse Shrew & 1.09 & 12 & extant & Future & NA & NT & ginsect \\
\hline AF & Soricomorpha & Soricidae & Myosorex & blarina & $\begin{array}{l}\text { Montane Mouse Shrew } \\
\text { Dark-footed Forest }\end{array}$ & 1.09 & 12 & extant & Future & NA & EN & ginsect \\
\hline $\mathrm{AF}$ & Soricomorpha & Soricidae & Myosorex & cafer & $\begin{array}{l}\text { Shrew, Dark-footed } \\
\text { Mouse Shrew }\end{array}$ & 1.13 & 13 & extant & 0 & NA & LC & ginsect \\
\hline $\mathrm{AF}$ & Soricomorpha & Soricidae & Myosorex & eisentrauti & $\begin{array}{l}\text { Eisentraut's Mouse } \\
\text { Shrew }\end{array}$ & 1.09 & 12 & extant & Future & NA & $C R$ & ginsect \\
\hline $\mathrm{AF}$ & Soricomorpha & Soricidae & Myosorex & geata & Geata Mouse Shrew & 0.97 & 9 & extant & Future & NA & EN & ginsect \\
\hline $\mathrm{AF}$ & Soricomorpha & Soricidae & Myosorex & longicaudatus & $\begin{array}{l}\text { Long-tailed Forest } \\
\text { Shrew }\end{array}$ & 1.11 & 13 & extant & Future & NA & Vu & ginsect \\
\hline $\mathrm{AF}$ & Soricomorpha & Soricidae & Myosorex & okuensis & Oku Mouse Shrew & 1.09 & 12 & extant & Future & NA & EN & ginsect \\
\hline $\mathrm{AF}$ & Soricomorpha & Soricidae & Myosorex & rumpii & Rumpi Mouse Shrew & 1.09 & 12 & extant & Future & NA & EN & ginsect \\
\hline $\mathrm{AF}$ & Soricomorpha & Soricidae & Myosorex & schalleri & Schaller's Mouse Shrew & 1.09 & 12 & extant & 0 & NA & DD & ginsect \\
\hline $\mathrm{AF}$ & Soricomorpha & Soricidae & Myosorex & sclateri & $\begin{array}{l}\text { Sclater's Mouse Shrew, } \\
\text { Sclater's Tiny Mouse } \\
\text { Shrew }\end{array}$ & 1.09 & 12 & extant & Future & NA & NT & ginsect \\
\hline $\mathrm{AF}$ & Soricomorpha & Soricidae & Myosorex & tenuis & Thin Mouse Shrew & 1.09 & 12 & extant & 0 & NA & DD & ginsect \\
\hline $\mathrm{AF}$ & Soricomorpha & Soricidae & Myosorex & varius & Forest Shrew & 1.12 & 13 & extant & 0 & NA & LC & ginsect \\
\hline $\mathrm{AF}$ & Soricomorpha & Soricidae & Paracrocidura & graueri & $\begin{array}{l}\text { Grauer's Large-headed } \\
\text { Shrew, Grauer's Shrew }\end{array}$ & 1.11 & 13 & extant & 0 & NA & DD & ginsect \\
\hline $\mathrm{AF}$ & Soricomorpha & Soricidae & Paracrocidura & maxima & $\begin{array}{l}\text { Greater Large-headed } \\
\text { Shrew, Greater Shrew } \\
\text { Lesser Large-headed }\end{array}$ & 1.11 & 13 & extant & Future & NA & NT & ginsect \\
\hline $\mathrm{AF}$ & Soricomorpha & Soricidae & Paracrocidura & schoutedeni & $\begin{array}{l}\text { Shrew, Schouteden's } \\
\text { Shrew }\end{array}$ & 1.11 & 13 & extant & 0 & NA & $\mathrm{LC}$ & ginsect \\
\hline $\mathrm{AF}$ & Soricomorpha & Soricidae & Ruwenzorisorex & suncoides & Ruwenzori Shrew & 1.26 & 18 & extant & Future & NA & VU & ginsect \\
\hline $\mathrm{AF}$ & Soricomorpha & Soricidae & Scutisorex & somereni & $\begin{array}{l}\text { Armored Shrew } \\
\text { Pygmy White-toothed } \\
\text { Shrew, White-toothed }\end{array}$ & 1.96 & 92 & extant & 0 & NA & LC & ginsect \\
\hline AF & Soricomorpha & Soricidae & Suncus & etruscus & $\begin{array}{l}\text { Pygmy Shrew, Savi's } \\
\text { Pygmy Shrew, Etruscan } \\
\text { Shrew }\end{array}$ & 0.28 & 2 & extant & 0 & NA & LC & ginsect \\
\hline AF & Soricomorpha & Soricidae & Suncus & infinitesimus & Least Dwarf Shrew & 0.48 & 3 & extant & 0 & NA & LC & ginsect \\
\hline $\mathrm{AF}$ & Soricomorpha & Soricidae & Suncus & lixus & Greater Dwarf Shrew & 0.69 & 5 & extant & 0 & NA & LC & ginsect \\
\hline AF & Soricomorpha & Soricidae & Suncus & megalura & Climbing Shrew & 0.67 & 5 & extant & 0 & NA & LC & ginsect \\
\hline AF & Soricomorpha & Soricidae & Suncus & murinus & $\begin{array}{l}\text { House Shrew, Asian } \\
\text { House Shrew } \\
\text { Gabon Dwarf Shrew, }\end{array}$ & 1.94 & 87 & extant & 0 & NA & LC & ginsect \\
\hline $\mathrm{AF}$ & Soricomorpha & Soricidae & Suncus & remyi & $\begin{array}{l}\text { Remy's Pygmy Shrew, } \\
\text { Remy's Shrew }\end{array}$ & 0.24 & 2 & extant & 0 & NA & LC & ginsect \\
\hline $\mathrm{AF}$ & Soricomorpha & Soricidae & Suncus & varilla & Lesser Dwarf Shrew & 0.70 & 5 & extant & 0 & NA & LC & ginsect \\
\hline $\mathrm{AF}$ & Soricomorpha & Soricidae & Surdisorex & norae & $\begin{array}{l}\text { Aberdare Mole Shrew, } \\
\text { Aberdare Shrew }\end{array}$ & 0.95 & 9 & extant & Future & NA & vU & ginsect \\
\hline $\mathrm{AF}$ & Soricomorpha & Soricidae & Surdisorex & polulus & $\begin{array}{l}\text { Mt. Kenya Mole Shrew, } \\
\text { Mt. Kenya Shrew }\end{array}$ & 0.95 & 9 & extant & Future & NA & VU & ginsect \\
\hline $\mathrm{AF}$ & Soricomorpha & Soricidae & Sylvisorex & granti & $\begin{array}{l}\text { Grant's Forest Shrew, } \\
\text { Grant's Shrew }\end{array}$ & 0.93 & 9 & extant & 0 & NA & LC & ginsect \\
\hline $\mathrm{AF}$ & Soricomorpha & Soricidae & Sylvisorex & howelli & $\begin{array}{l}\text { Howell's Forest Shrew, } \\
\text { Howell's Shrew }\end{array}$ & 0.64 & 4 & extant & Future & NA & EN & ginsect \\
\hline $\mathrm{AF}$ & Soricomorpha & Soricidae & Sylvisorex & isabellae & $\begin{array}{l}\text { Bioko Forest Shrew, } \\
\text { Isabella Shrew }\end{array}$ & 0.93 & 9 & extant & Future & NA & EN & ginsect \\
\hline
\end{tabular}




\begin{tabular}{|c|c|c|c|c|c|c|c|c|c|c|c|c|}
\hline $\mathrm{AF}$ & Soricomorpha & Soricidae & Sylvisorex & johnstoni & $\begin{array}{l}\text { Johnston's Forest } \\
\text { Shrew, Johnston's } \\
\text { Shrew }\end{array}$ & 0.50 & 3 & extant & 0 & NA & $\mathrm{LC}$ & ginsect \\
\hline AF & Soricomorpha & Soricidae & Sylvisorex & lunaris & $\begin{array}{l}\text { Moon Forest Shrew, } \\
\text { Crescent Shrew }\end{array}$ & 1.27 & 19 & extant & Future & NA & VU & ginsect \\
\hline AF & Soricomorpha & Soricidae & Sylvisorex & morio & $\begin{array}{l}\text { Arrogant Shrew, Mt. } \\
\text { Cameroon Forest Shrew }\end{array}$ & 0.93 & 9 & extant & Future & NA & EN & ginsect \\
\hline AF & Soricomorpha & Soricidae & Sylvisorex & ollula & $\begin{array}{l}\text { Forest Musk Shrew, } \\
\text { Greater Forest Shrew }\end{array}$ & 1.12 & 13 & extant & 0 & NA & $\mathrm{LC}$ & ginsect \\
\hline $\mathrm{AF}$ & Soricomorpha & Soricidae & Sylvisorex & oriundus & $\begin{array}{l}\text { Lesser Forest Shrew, } \\
\text { Mountain Shrew }\end{array}$ & 0.93 & 9 & extant & 0 & NA & DD & ginsect \\
\hline $\mathrm{AF}$ & Soricomorpha & Soricidae & Sylvisorex & vulcanorum & Volcano Shrew & 0.54 & 4 & extant & Future & NA & NT & ginsect \\
\hline AF & Tubulidentata & Orycteropodidae & Orycteropus & afer & Aardvark, Antbear & 4.72 & 52350 & extant & 0 & NA & $\mathrm{LC}$ & ginsect \\
\hline AUS & Carnivora & Canidae & Canis & lupus & dingo & 4.23 & 16800 & extant & 0 & NA & LC & Carn \\
\hline AUS & Dasyuromorphia & Dasyuridae & Antechinomys & laniger & Kultarr & 1.40 & 25 & extant & 0 & NA & LC & Ginsect \\
\hline AUS & Dasyuromorphia & Dasyuridae & Antechinus & bellus & $\begin{array}{l}\text { Fawn Antechinus, Fawn } \\
\text { Marsupial Mouse } \\
\text { Yellow-footed }\end{array}$ & 1.65 & 45 & extant & 0 & NA & LC & Ginsect \\
\hline AUS & Dasyuromorphia & Dasyuridae & Antechinus & flavipes & $\begin{array}{l}\text { Antechinus, Yellow- } \\
\text { footed Marsupial } \\
\text { Mouse } \\
\text { Atherton Antechinus, }\end{array}$ & 1.65 & 45 & extant & 0 & NA & LC & Ginsect/carn \\
\hline AUS & Dasyuromorphia & Dasyuridae & Antechinus & godmani & $\begin{array}{l}\text { Godman's Antechinus, } \\
\text { Atherton Marsupial } \\
\text { Mouse }\end{array}$ & 1.88 & 77 & extant & Future & NA & NT & Ginsect/carn \\
\hline AUS & Dasyuromorphia & Dasyuridae & Antechinus & leo & $\begin{array}{l}\text { Cinnamon Antechinus, } \\
\text { Iron Range Antechinus, } \\
\text { Cinnamon Marsupial } \\
\text { Mouse } \\
\text { Swamp Antechinus, } \\
\text { Swamp Marsupial }\end{array}$ & 1.87 & 74 & extant & 0 & NA & LC & Ginsect \\
\hline AUS & Dasyuromorphia & Dasyuridae & Antechinus & minimus & $\begin{array}{l}\text { Mouse, Little } \\
\text { Tasmanian Marsupial- } \\
\text { mouse }\end{array}$ & 1.73 & 54 & extant & 0 & NA & LC & Ginsect \\
\hline AUS & Dasyuromorphia & Dasyuridae & Antechinus & stuartii & $\begin{array}{l}\text { Brown Antechinus, } \\
\text { Stuart's Marsupial- } \\
\text { mouse, Brown }\end{array}$ & 1.44 & 28 & extant & 0 & NA & $\mathrm{LC}$ & Ginsect \\
\hline & & & & & $\begin{array}{l}\text { Marsupial Mouse } \\
\text { Dusky Antechinus, Dusky }\end{array}$ & & & & & & & \\
\hline AUS & Dasyuromorphia & Dasyuridae & Antechinus & swainsonii & $\begin{array}{l}\text { Marsupial Mouse, } \\
\text { Swainson's Marsupial- } \\
\text { mouse }\end{array}$ & 1.72 & 53 & extant & 0 & NA & LC & Ginsect \\
\hline AUS & Dasyuromorphia & Dasyuridae & Dasycercus & cristicauda & $\begin{array}{l}\text { Crest-tailed Mulgara } \\
\text { Kaluta, Little Red }\end{array}$ & 2.00 & 100 & extant & Future & NA & NT & Ginsect/carn \\
\hline AUS & Dasyuromorphia & Dasyuridae & Dasykaluta & rosamondae & $\begin{array}{l}\text { Kaluta, Little Red } \\
\text { Antechinus }\end{array}$ & 1.46 & 29 & extant & 0 & NA & $\mathrm{LC}$ & Ginsect/carn \\
\hline AUS & Dasyuromorphia & Dasyuridae & Dasyuroides & byrnei & Kowari & 2.02 & 104 & extant & Future & NA & VU & Ginsect/carn \\
\hline AUS & Dasyuromorphia & Dasyuridae & Dasyurus & geoffroii & Western Quoll, Chuditch & 3.04 & 1100 & extant & Future & NA & NT & carn/ginsect \\
\hline AUS & Dasyuromorphia & Dasyuridae & Dasyurus & hallucatus & Northern Quoll & 2.72 & 525 & extant & Future & NA & EN & Ginsect/carn \\
\hline AUS & Dasyuromorphia & Dasyuridae & Dasyurus & maculatus & Spotted-tailed Quoll & 3.74 & 5500 & extant & Future & NA & NT & carn/ginsect \\
\hline
\end{tabular}




\begin{tabular}{|c|c|c|c|c|c|c|c|c|c|c|c|c|}
\hline AUS & Dasyuromorphia & Dasyuridae & Dasyurus & viverrinus & Eastern Quoll & 3.04 & 1090 & extant & Future & NA & EN & Ginsect/carn \\
\hline AUS & Dasyuromorphia & Dasyuridae & Ningaui & ridei & Wongai Ningaui & 0.93 & 9 & extant & 0 & NA & LC & Ginsect \\
\hline AUS & Dasyuromorphia & Dasyuridae & Ningaui & timealeyi & Pilbara Ningaui & 0.76 & 6 & extant & 0 & NA & $\mathrm{LC}$ & Ginsect \\
\hline AUS & Dasyuromorphia & Dasyuridae & Ningaui & yvonneae & & 0.85 & 7 & extant & 0 & NA & LC & Ginsect \\
\hline AUS & Dasyuromorphia & Dasyuridae & Parantechinus & apicalis & $\begin{array}{l}\text { Dibbler, Southern } \\
\text { Dibbler }\end{array}$ & 1.84 & 69 & extant & Future & NA & EN & Ginsect/carn \\
\hline AUS & Dasyuromorphia & Dasyuridae & Phascogale & calura & $\begin{array}{l}\text { Red-tailed Phascogale, } \\
\text { Red-tailed Wambenger }\end{array}$ & 1.71 & 52 & extant & Future & NA & NT & Ginsect \\
\hline AUS & Dasyuromorphia & Dasyuridae & Phascogale & tapoatafa & Brush-tailed Phascogale & 2.29 & 194 & extant & Future & NA & NT & Ginsect/carn \\
\hline AUS & Dasyuromorphia & Dasyuridae & Planigale & gilesi & $\begin{array}{l}\text { Giles' Planigale, } \\
\text { Paucident Planigale }\end{array}$ & 0.96 & 9 & extant & 0 & NA & $\mathrm{LC}$ & Ginsect \\
\hline AUS & Dasyuromorphia & Dasyuridae & Planigale & ingrami & Long-tailed Planigale & 0.63 & 4 & extant & 0 & NA & $\mathrm{LC}$ & Ginsect \\
\hline AUS & Dasyuromorphia & Dasyuridae & Planigale & maculata & $\begin{array}{l}\text { Common Planigale, } \\
\text { Pygmy Planigale }\end{array}$ & 1.04 & 11 & extant & 0 & NA & LC & Ginsect \\
\hline AUS & Dasyuromorphia & Dasyuridae & Planigale & tenuirostris & Narrow-nosed Planigale & 0.78 & 6 & extant & 0 & NA & $\mathrm{LC}$ & Ginsect \\
\hline AUS & Dasyuromorphia & Dasyuridae & Pseudantechinus & bilarni & $\begin{array}{l}\text { Sandstone Antechinus, } \\
\text { Sandstone } \\
\text { Pseudantechinus } \\
\text { Fat-tailed }\end{array}$ & 1.44 & 28 & extant & Future & NA & NT & Ginsect \\
\hline AUS & Dasyuromorphia & Dasyuridae & Pseudantechinus & macdonnellensis & $\begin{array}{l}\text { Pseudantechinus, Fat- } \\
\text { tailed Antechinus } \\
\text { Ningbing }\end{array}$ & 1.51 & 33 & extant & 0 & NA & LC & Ginsect \\
\hline AUS & Dasyuromorphia & Dasyuridae & Pseudantechinus & ningbing & $\begin{array}{l}\text { Pseudantechinus, } \\
\text { Ningbing Antechinus } \\
\text { Woolley's }\end{array}$ & 1.30 & 20 & extant & 0 & NA & LC & Ginsect \\
\hline AUS & Dasyuromorphia & Dasyuridae & Pseudantechinus & woolleyae & $\begin{array}{l}\text { Pseudantechinus, } \\
\text { Woolley's Antechinus }\end{array}$ & 1.60 & 40 & extant & 0 & NA & $\mathrm{LC}$ & Ginsect \\
\hline AUS & Dasyuromorphia & Dasyuridae & Sarcophilus & harrisii & Tasmanian Devil & 4.00 & 10000 & extant & Future & NA & EN & Carn \\
\hline AUS & Dasyuromorphia & Dasyuridae & Sarcophilus & laniarius & large Tasmanian devil & 4.08 & 12000 & extinct & EP & 50 & EX & Carn \\
\hline AUS & Dasyuromorphia & Dasyuridae & Sminthopsis & aitkeni & $\begin{array}{l}\text { Kangaroo Island } \\
\text { Dunnart }\end{array}$ & 1.35 & 23 & extant & Future & NA & CR & Ginsect \\
\hline AUS & Dasyuromorphia & Dasyuridae & Sminthopsis & archeri & Chestnut Dunnart & 1.20 & 16 & extant & 0 & NA & DD & Ginsect \\
\hline AUS & Dasyuromorphia & Dasyuridae & Sminthopsis & butleri & Butler's Dunnart & 1.40 & 25 & extant & Future & NA & Vu & Ginsect \\
\hline AUS & Dasyuromorphia & Dasyuridae & Sminthopsis & crassicaudata & Fat-tailed Dunnart & 1.18 & 15 & extant & 0 & NA & LC & Ginsect \\
\hline AUS & Dasyuromorphia & Dasyuridae & Sminthopsis & dolichura & $\begin{array}{l}\text { Little Long-tailed } \\
\text { Dunnart }\end{array}$ & 1.13 & 14 & extant & 0 & NA & LC & Ginsect \\
\hline AUS & Dasyuromorphia & Dasyuridae & Sminthopsis & douglasi & Julia Creek Dunnart & 1.74 & 55 & extant & Future & NA & NT & Ginsect \\
\hline AUS & Dasyuromorphia & Dasyuridae & Sminthopsis & fuliginosus & Sooty Dunnart & 1.34 & 22 & extant & 0 & NA & DD & \\
\hline AUS & Dasyuromorphia & Dasyuridae & Sminthopsis & gilberti & Gilbert's Dunnart & 1.29 & 20 & extant & 0 & NA & LC & Ginsect \\
\hline AUS & Dasyuromorphia & Dasyuridae & Sminthopsis & granulipes & $\begin{array}{l}\text { White-tailed Dunnart } \\
\text { Grey-bellied Dunnart, }\end{array}$ & 1.40 & 25 & extant & 0 & NA & LC & Ginsect \\
\hline AUS & Dasyuromorphia & Dasyuridae & Sminthopsis & griseoventer & $\begin{array}{l}\text { Boullanger Island } \\
\text { Dunnart, Gray-bellied } \\
\text { Dunnart }\end{array}$ & 1.24 & 18 & extant & 0 & NA & $\mathrm{LC}$ & Ginsect \\
\hline AUS & Dasyuromorphia & Dasyuridae & Sminthopsis & hirtipes & Hairy-footed Dunnart & 1.18 & 15 & extant & 0 & NA & LC & Ginsect \\
\hline AUS & Dasyuromorphia & Dasyuridae & Sminthopsis & leucopus & White-footed Dunnart & 1.41 & 26 & extant & 0 & NA & LC & Ginsect \\
\hline AUS & Dasyuromorphia & Dasyuridae & Sminthopsis & longicaudata & Long-tailed Dunnart & 1.26 & 18 & extant & 0 & NA & LC & Ginsect \\
\hline AUS & Dasyuromorphia & Dasyuridae & Sminthopsis & macroura & Stripe-faced Dunnart & 1.30 & 20 & extant & 0 & NA & LC & Ginsect \\
\hline AUS & Dasyuromorphia & Dasyuridae & Sminthopsis & murina & $\begin{array}{l}\text { Common Dunnart, } \\
\text { Slender-tailed Dunnart }\end{array}$ & 1.23 & 17 & extant & 0 & NA & LC & Ginsect \\
\hline
\end{tabular}




\begin{tabular}{|c|c|c|c|c|c|c|c|c|c|c|c|c|}
\hline AUS & Dasyuromorphia & Dasyuridae & Sminthopsis & psammophila & $\begin{array}{l}\text { Ooldea Dunnart } \\
\text { Sandhill Dunnart, } \\
\text { Sandhill Sminthopsis, } \\
\text { Large Desert Marsupial- } \\
\text { mouse }\end{array}$ & 1.53 & 11 & extant & Future & NA & Vu & Ginsect \\
\hline AUS & Dasyuromorphia & Dasyuridae & Sminthopsis & virginiae & Red-cheeked Dunnart & 1.55 & 35 & extant & 0 & NA & $\mathrm{LC}$ & Ginsect \\
\hline AUS & Dasyuromorphia & Dasyuridae & Sminthopsis & youngsoni & $\begin{array}{l}\text { Lesser Hairy-footed } \\
\text { Dunnart }\end{array}$ & 1.00 & 10 & extant & 0 & NA & LC & Ginsect \\
\hline AUS & Dasyuromorphia & Myrmecobiidae & Myrmecobius & fasciatus & $\begin{array}{l}\text { Numbat, Banded } \\
\text { Anteater }\end{array}$ & 2.67 & 472 & extant & Future & NA & EN & Ginsect \\
\hline AUS & Dasyuromorphia & Phascolarctidae & Phascolarctos & stirtoni & giant koala & 4.15 & 14000 & extinct & EP & 50 & EX & Browse \\
\hline AUS & Dasyuromorphia & Thylacinidae & Thylacinus & cynocephalus & $\begin{array}{l}\text { Thylacine, Tasmanian } \\
\text { Tiger, Tasmanian Wolf } \\
\text { Feathertail Glider, }\end{array}$ & 4.48 & 30000 & extinct & Holocene & NA & EX & Carn \\
\hline AUS & Diprotodontia & Acrobatidae & Acrobates & pygmaeus & $\begin{array}{l}\text { Feather-tailed Glider, } \\
\text { Pygmy Gliding Possum } \\
\text { Mountain Pygmy }\end{array}$ & 1.08 & 12 & extant & 0 & NA & LC & Frug/ginsect \\
\hline AUS & Diprotodontia & Burramyidae & Burramys & parvus & $\begin{array}{l}\text { Possum, Broom's } \\
\text { Pygmy-possum, } \\
\text { Burramys } \\
\text { Long-tailed Pygmy } \\
\text { Possum, Long-tailed }\end{array}$ & 1.62 & 42 & extant & Future & NA & CR & Ginsect \\
\hline AUS & Diprotodontia & Burramyidae & Cercartetus & caudatus & $\begin{array}{l}\text { Pygmy-possum, Long- } \\
\text { tailed Dormouse- } \\
\text { phalanger } \\
\text { Western Pygmy } \\
\text { Possum, Western }\end{array}$ & 1.48 & 30 & extant & 0 & NA & $\mathrm{LC}$ & Frug \\
\hline AUS & Diprotodontia & Burramyidae & Cercartetus & concinnus & $\begin{array}{l}\text { Pygmy-possum, } \\
\text { Southwestern Pygmy } \\
\text { Possum, Mundarda } \\
\text { Little Pygmy Possum, } \\
\text { Tasmanian Pygmy- }\end{array}$ & 1.11 & 13 & extant & 0 & NA & $\mathrm{LC}$ & Frug/ginsect \\
\hline AUS & Diprotodontia & Burramyidae & Cercartetus & lepidus & $\begin{array}{l}\text { possum, Little Pygmy- } \\
\text { possum, Tasmanian } \\
\text { Pygmy Possum }\end{array}$ & 0.85 & 7 & extant & 0 & NA & $\mathrm{LC}$ & ginsect/frug \\
\hline AUS & Diprotodontia & Burramyidae & Cercartetus & nanus & $\begin{array}{l}\text { Eastern Pygmy Possum, } \\
\text { Eastern Pygmy-possum, } \\
\text { Common Dormouse- } \\
\text { phalanger }\end{array}$ & 1.38 & 24 & extant & 0 & NA & LC & Frug \\
\hline AUS & Diprotodontia & Diprotodonidae & Diprotodon & minor & & 5.95 & 900000 & extinct & EP & 46 & EX & Browse \\
\hline AUS & Diprotodontia & Diprotodonidae & Diprotodon & optatum & & 6.18 & 1500000 & extinct & EP & 46 & EX & Browse \\
\hline AUS & Diprotodontia & Diprotodonidae & Euowenia & grata & age? & 5.88 & 750000 & extinct & EP & 50 & EX & Browse \\
\hline AUS & Diprotodontia & Diprotodonidae & Nototherium & mitchelli & & 5.70 & 500000 & extinct & EP & 50 & EX & Browse \\
\hline AUS & Diprotodontia & Diprotodonidae & Palorchestes & azael & & 5.70 & 500000 & extinct & EP & 50 & EX & Browse \\
\hline AUS & Diprotodontia & Diprotodonidae & Palorchestes & parvus & & 5.00 & 100000 & extinct & LP & 122 & EX & Browse \\
\hline AUS & Diprotodontia & Diprotodonidae & Zygomaturus & trilobus & & 5.88 & 750000 & extinct & EP & 50 & EX & Browse \\
\hline AUS & Diprotodontia & Macropodidae & Dendrolagus & bennettianus & $\begin{array}{l}\text { Bennett's Tree } \\
\text { Kangaroo, } \\
\text { Tcharibbeena, Dusty } \\
\text { Tree-kangaroo }\end{array}$ & 4.04 & 10950 & extant & Future & NA & NT & Browse/frug \\
\hline
\end{tabular}




\begin{tabular}{|c|c|c|c|c|c|c|c|c|c|c|c|c|}
\hline AUS & Diprotodontia & Macropodidae & Dendrolagus & lumholtzi & $\begin{array}{l}\text { Lumholtz's Tree } \\
\text { Kangaroo, Lumholtz' } \\
\text { Tree-kangaroo, } \\
\text { Boongary }\end{array}$ & 3.83 & 6750 & extant & Future & NA & NT & Browse \\
\hline AUS & Diprotodontia & Macropodidae & Lagorchestes & asomatus & $\begin{array}{l}\text { Kuluwarri, Central Hare } \\
\text { Wallaby } \\
\text { Spectacled Hare }\end{array}$ & 3.18 & 1500 & extinct & Holocene & NA & EX & Graze/frug \\
\hline AUS & Diprotodontia & Macropodidae & Lagorchestes & conspicillatus & $\begin{array}{l}\text { Wallaby, Spectacled } \\
\text { Hare-wallaby }\end{array}$ & 3.48 & 3050 & extant & 0 & NA & LC & 3rowse/graze \\
\hline AUS & Diprotodontia & Macropodidae & Lagorchestes & hirsutus & $\begin{array}{l}\text { Rufous Hare Wallaby, } \\
\text { Wurrup, Mala }\end{array}$ & 3.10 & 1265 & extant & Future & NA & VU & Graze \\
\hline AUS & Diprotodontia & Macropodidae & Lagorchestes & leporides & $\begin{array}{l}\text { Eastern Hare Wallaby } \\
\text { Banded Hare Wallaby, }\end{array}$ & 3.48 & 3000 & extinct & Holocene & NA & EX & Graze \\
\hline AUS & Diprotodontia & Macropodidae & Lagostrophus & fasciatus & $\begin{array}{l}\text { Maning, Banded Hare- } \\
\text { wallaby }\end{array}$ & 3.23 & 1700 & extant & Future & NA & VU & 3rowse/graze \\
\hline AUS & Diprotodontia & Macropodidae & Macropus & agilis & $\begin{array}{l}\text { Agile Wallaby } \\
\text { Antilopine Wallaroo, }\end{array}$ & 4.18 & 15000 & extant & 0 & NA & LC & Graze \\
\hline AUS & Diprotodontia & Macropodidae & Macropus & antilopinus & $\begin{array}{l}\text { Antilopine Wallaby, } \\
\text { Antilopine Kangaroo }\end{array}$ & 4.44 & 27250 & extant & 0 & NA & LC & Graze \\
\hline AUS & Diprotodontia & Macropodidae & Macropus & bernardus & $\begin{array}{l}\text { Black Wallaroo, } \\
\text { Bernard's Wallaroo }\end{array}$ & 4.23 & 17000 & extant & Future & NA & NT & Graze \\
\hline AUS & Diprotodontia & Macropodidae & Macropus & dorsalis & Black-striped Wallaby & 4.05 & 11250 & extant & 0 & NA & LC & Graze \\
\hline AUS & Diprotodontia & Macropodidae & Macropus & eugenii & $\begin{array}{l}\text { Tammar Wallaby, } \\
\text { Tamar Wallaby }\end{array}$ & 3.81 & 6500 & extant & 0 & NA & LC & Graze \\
\hline AUS & Diprotodontia & Macropodidae & Macropus & ferragus & $\begin{array}{l}\text { Western Gray } \\
\text { Kangaroo, Kangaroo }\end{array}$ & 5.18 & 150000 & extinct & EP & 54 & EX & Graze \\
\hline AUS & Diprotodontia & Macropodidae & Macropus & fuliginosus & $\begin{array}{l}\text { Island Western Grey } \\
\text { Kangaroo, Western } \\
\text { Grey Kangaroo }\end{array}$ & 4.34 & 22000 & extant & 0 & NA & LC & Graze \\
\hline AUS & Diprotodontia & Macropodidae & Macropus & giganteus & Eastern Grey Kangaroo & 4.41 & 25875 & extant & 0 & NA & LC & Graze \\
\hline AUS & Diprotodontia & Macropodidae & Macropus & greyi & $\begin{array}{l}\text { Toolache Wallaby } \\
\text { Western Brush Wallaby, }\end{array}$ & 4.00 & 10000 & extinct & Holocene & NA & EX & Graze \\
\hline AUS & Diprotodontia & Macropodidae & Macropus & irma & $\begin{array}{l}\text { Kwoora, Western Bush } \\
\text { Wallaby }\end{array}$ & 3.90 & 8000 & extant & 0 & NA & LC & Graze \\
\hline AUS & Diprotodontia & Macropodidae & Macropus & parma & Parma Wallaby & 3.65 & 4500 & extant & Future & NA & NT & Graze \\
\hline AUS & Diprotodontia & Macropodidae & Macropus & parryi & Whiptail Wallaby & 4.13 & 13500 & extant & 0 & NA & LC & Graze \\
\hline AUS & Diprotodontia & Macropodidae & Macropus & pearsoni & & 5.18 & 150000 & extinct & EP & 50 & EX & Graze \\
\hline AUS & Diprotodontia & Macropodidae & Macropus & piltonesis & Common Wallaroo, & 4.48 & 30000 & extinct & EP & 50 & EX & Graze \\
\hline AUS & Diprotodontia & Macropodidae & Macropus & robustus & $\begin{array}{l}\text { Euro, Barrow Island } \\
\text { Euro, Hill Wallaroo }\end{array}$ & 4.33 & 21263 & extant & 0 & NA & LC & Graze \\
\hline AUS & Diprotodontia & Macropodidae & Macropus & rufogriseus & $\begin{array}{l}\text { Red-necked Wallaby, } \\
\text { Bennett's Wallaby }\end{array}$ & 4.23 & 16850 & extant & 0 & NA & LC & Graze \\
\hline AUS & Diprotodontia & Macropodidae & Macropus & rufus & Red Kangaroo & 4.67 & 46250 & extant & 0 & NA & LC & Graze \\
\hline AUS & Diprotodontia & Macropodidae & Macropus & thor & & 4.48 & 30000 & extinct & EP & 50 & EX & Graze \\
\hline AUS & Diprotodontia & Macropodidae & Onychogalea & fraenata & $\begin{array}{l}\text { Bridled Nailtail } \\
\text { Wallaby, Merrin, } \\
\text { Bridled Wallaby, Bridled } \\
\text { Nail-tailed Wallaby }\end{array}$ & 3.74 & 5500 & extant & Future & NA & VU & J̈raze/brows€ \\
\hline
\end{tabular}




\begin{tabular}{|c|c|c|c|c|c|c|c|c|c|c|c|c|}
\hline AUS & Diprotodontia & Macropodidae & Onychogalea & lunata & $\begin{array}{l}\text { Crescent Nailtail } \\
\text { Wallaby } \\
\text { Northern Nailtail }\end{array}$ & 3.54 & 3500 & extinct & Holocene & NA & EX & Graze \\
\hline AUS & Diprotodontia & Macropodidae & Onychogalea & unguifera & $\begin{array}{l}\text { Wallaby, Northern Nail- } \\
\text { tailed Wallaby }\end{array}$ & 3.82 & 6650 & extant & 0 & NA & LC & Browse \\
\hline AUS & Diprotodontia & Macropodidae & Petrogale & assimilis & Allied Rock Wallaby & 3.65 & 4500 & extant & 0 & NA & $\mathrm{LC}$ & Graze \\
\hline AUS & Diprotodontia & Macropodidae & Petrogale & brachyotis & $\begin{array}{l}\text { Short-eared Rock } \\
\text { Wallaby }\end{array}$ & 3.61 & 4050 & extant & 0 & NA & LC & Graze \\
\hline AUS & Diprotodontia & Macropodidae & Petrogale & burbidgei & Monjon & 3.10 & 1258 & extant & Future & NA & NT & Graze \\
\hline AUS & Diprotodontia & Macropodidae & Petrogale & concinna & Nabarlek & 3.15 & 1400 & extant & Future & NA & EN & כ̇raze/brows€ \\
\hline AUS & Diprotodontia & Macropodidae & Petrogale & godmani & Godman's Rock Wallaby & 3.68 & 4750 & extant & Future & NA & NT & Graze \\
\hline AUS & Diprotodontia & Macropodidae & Petrogale & inornata & $\begin{array}{l}\text { Unadorned Rock } \\
\text { Wallaby } \\
\text { Black-footed Rock }\end{array}$ & 3.66 & 4600 & extant & 0 & NA & LC & Graze \\
\hline AUS & Diprotodontia & Macropodidae & Petrogale & lateralis & $\begin{array}{l}\text { Wallaby, Black-flanked } \\
\text { Wallaby, Warru } \\
\text { Brush-tailed Rock }\end{array}$ & 3.67 & 4625 & extant & Future & NA & Vu & J̈raze/brows€ \\
\hline AUS & Diprotodontia & Macropodidae & Petrogale & penicillata & $\begin{array}{l}\text { Wallaby, Brush-tailed } \\
\text { Rock-wallaby }\end{array}$ & 3.85 & 7100 & extant & Future & NA & VU & Graze \\
\hline AUS & Diprotodontia & Macropodidae & Petrogale & persephone & $\begin{array}{l}\text { Proserpine Rock } \\
\text { Wallaby }\end{array}$ & 3.79 & 6200 & extant & Future & NA & EN & Graze \\
\hline AUS & Diprotodontia & Macropodidae & Petrogale & rothschildi & $\begin{array}{l}\text { Rothschild's Rock } \\
\text { Wallaby } \\
\text { Yellow-footed Rock }\end{array}$ & 3.53 & 3400 & extant & 0 & NA & LC & Graze \\
\hline AUS & Diprotodontia & Macropodidae & Petrogale & xanthopus & $\begin{array}{l}\text { Wallaby, Ring-tailed } \\
\text { Rock Wallaby }\end{array}$ & 3.93 & 8500 & extant & Future & NA & NT & Graze \\
\hline AUS & Diprotodontia & Macropodidae & Procoptodon & goliah & & 5.37 & 232000 & extinct & EP & 50 & EX & Browse \\
\hline AUS & Diprotodontia & Macropodidae & Procoptodon & pusio & & 4.88 & 75000 & extinct & EP & 50 & EX & Browse \\
\hline AUS & Diprotodontia & Macropodidae & Procoptodon & rapha & & 5.18 & 150000 & extinct & EP & 50 & EX & Browse \\
\hline AUS & Diprotodontia & Macropodidae & Procoptodon & texasensis & & 5.18 & 150000 & extinct & EP & 50 & EX & Browse \\
\hline AUS & Diprotodontia & Macropodidae & Protemnodon & anak & & 5.12 & 131000 & extinct & EP & 50 & EX & Browse \\
\hline AUS & Diprotodontia & Macropodidae & Protemnodon & brehus & & 5.00 & 100000 & extinct & EP & 50 & EX & Browse \\
\hline AUS & Diprotodontia & Macropodidae & Protemnodon & brehus & giant wallaby & 5.04 & 110000 & extinct & EP & 36 & EX & browse \\
\hline AUS & Diprotodontia & Macropodidae & Protemnodon & gilli & & 4.73 & 54000 & extinct & EP & NA & EX & browse \\
\hline AUS & Diprotodontia & Macropodidae & Protemnodon & roechus & & 5.22 & 166000 & extinct & EP & 46 & EX & Browse \\
\hline AUS & Diprotodontia & Macropodidae & Setonix & brachyurus & Quokka & 3.51 & 3250 & extant & Future & NA & Vu & Browse \\
\hline AUS & Diprotodontia & Macropodidae & Simosthenurus & brownei & & 4.70 & 50000 & extinct & EP & 50 & EX & Browse \\
\hline AUS & Diprotodontia & Macropodidae & Simosthenurus & gilli & & 4.48 & 30000 & extinct & EP & 50 & EX & Browse \\
\hline AUS & Diprotodontia & Macropodidae & Simosthenurus & maddocki & & 4.89 & 78000 & extinct & LP & 70 & EX & Browse \\
\hline AUS & Diprotodontia & Macropodidae & Simosthenurus & occidentalis & & 5.07 & 118000 & extinct & EP & 50 & EX & Browse \\
\hline AUS & Diprotodontia & Macropodidae & Simosthenurus & orientalis & & 4.88 & 75000 & extinct & EP & 50 & EX & Browse \\
\hline AUS & Diprotodontia & Macropodidae & Simosthenurus & pales & & 5.18 & 150000 & extinct & EP & 50 & EX & Browse \\
\hline AUS & Diprotodontia & Macropodidae & Sthenurus & andersoni & & 4.86 & 72000 & extinct & EP & 36 & EX & Browse \\
\hline AUS & Diprotodontia & Macropodidae & Sthenurus & atlas & & 5.18 & 150000 & extinct & EP & 54 & EX & Browse \\
\hline AUS & Diprotodontia & Macropodidae & Sthenurus & murrayi & $\begin{array}{l}\text { short-faced kangaroo; } \\
>50 \mathrm{~kg}\end{array}$ & 5.09 & 124400 & extinct & LP & NA & EX & browse \\
\hline AUS & Diprotodontia & Macropodidae & Sthenurus & oreas & & 5.00 & 100000 & extinct & EP & 50 & EX & Browse \\
\hline AUS & Diprotodontia & Macropodidae & Sthenurus & stirlingi & & 5.24 & 173000 & extinct & LP & 75 & EX & Browse \\
\hline AUS & Diprotodontia & Macropodidae & Sthenurus & tindalei & & 5.10 & 127000 & extinct & LP & 75 & EX & Browse \\
\hline AUS & Diprotodontia & Macropodidae & Thylogale & billardierii & $\begin{array}{l}\text { Tasmanian Pademelon, } \\
\text { Red-bellied Pademelon }\end{array}$ & 3.74 & 5450 & extant & 0 & NA & $\mathrm{LC}$ & Graze \\
\hline
\end{tabular}




\begin{tabular}{|c|c|c|c|c|c|c|c|c|c|c|c|c|}
\hline AUS & Diprotodontia & Macropodidae & Thylogale & stigmatica & Red-legged Pademelon & 3.66 & 4600 & extant & 0 & NA & LC & 3rowse/graze \\
\hline AUS & Diprotodontia & Macropodidae & Thylogale & thetis & Red-necked Pademelon & 3.73 & 5400 & extant & 0 & NA & LC & J̈raze/browse \\
\hline AUS & Diprotodontia & Macropodidae & Troposodon & minor & & 4.60 & 40000 & extinct & EP & 60 & EX & Browse \\
\hline AUS & Diprotodontia & Macropodidae & Wallabia & bicolor & Swamp Wallaby & 4.18 & 15000 & extant & 0 & NA & LC & Browse \\
\hline AUS & Diprotodontia & Petauridae & Dactylopsila & trivirgata & Striped Possum & 2.63 & 423 & extant & 0 & NA & LC & Ginsect \\
\hline AUS & Diprotodontia & Petauridae & Gymnobelideus & leadbeateri & Leadbeater's Possum & 2.10 & 127 & extant & Future & NA & $C R$ & Ginsect/frug \\
\hline AUS & Diprotodontia & Petauridae & Petaurus & australis & Yellow-bellied Glider & 2.76 & 575 & extant & Future & NA & NT & Frug \\
\hline AUS & Diprotodontia & Petauridae & Petaurus & breviceps & Sugar Glider & 2.11 & 128 & extant & 0 & NA & LC & Frug/ginsect \\
\hline AUS & Diprotodontia & Petauridae & Petaurus & gracilis & Mahogany Glider & 2.55 & 353 & extant & Future & NA & EN & Frug/ginsect \\
\hline AUS & Diprotodontia & Petauridae & Petaurus & norfolcensis & $\begin{array}{l}\text { Squirrel Glider } \\
\text { Northern Common }\end{array}$ & 2.36 & 230 & extant & 0 & NA & LC & Frug/ginsect \\
\hline AUS & Diprotodontia & Phalangeridae & Phalanger & orientalis & $\begin{array}{l}\text { Cuscus, Common } \\
\text { Phalanger, Grey Cuscus } \\
\text { Common Spotted }\end{array}$ & 3.27 & 1850 & extant & 0 & NA & $\mathrm{LC}$ & Frug/browse \\
\hline AUS & Diprotodontia & Phalangeridae & Spilocuscus & maculatus & $\begin{array}{l}\text { Cuscus, Spotted } \\
\text { Phalanger, Short-tailed } \\
\text { Spotted Cuscus }\end{array}$ & 3.81 & 6400 & extant & 0 & NA & LC & Browse \\
\hline AUS & Diprotodontia & Phalangeridae & Trichosurus & arnhemensis & $\begin{array}{l}\text { Northern brushtail } \\
\text { possum } \\
\text { Short-eared Brushtail }\end{array}$ & 3.11 & 1300 & extant & 0 & NA & DD & Browse/frug \\
\hline AUS & Diprotodontia & Phalangeridae & Trichosurus & caninus & $\begin{array}{l}\text { Possum, Mountain } \\
\text { Brushtail Possum } \\
\text { Common Brushtail }\end{array}$ & 3.54 & 3500 & extant & 0 & NA & $\mathrm{LC}$ & Browse/frug \\
\hline AUS & Diprotodontia & Phalangeridae & Trichosurus & vulpecula & $\begin{array}{l}\text { Possum, Silver-gray } \\
\text { Brushtail Possum }\end{array}$ & 3.42 & 2650 & extant & 0 & NA & $\mathrm{LC}$ & Browse/frug \\
\hline AUS & Diprotodontia & Phalangeridae & Wyulda & squamicaudata & $\begin{array}{l}\text { Scaly-tailed Possum, } \\
\text { Yilangal, llangurra }\end{array}$ & 3.22 & 1675 & extant & Future & NA & NT & Frug/browse \\
\hline AUS & Diprotodontia & Phascolarctidae & Phascolarctos & cinereus & Koala & 4.01 & 10250 & extant & Future & NA & Vu & Browse \\
\hline AUS & Diprotodontia & Potoroidae & Aepyprymnus & rufescens & $\begin{array}{l}\text { Rufous Bettong, Rufous } \\
\text { Rat-kangaroo }\end{array}$ & 3.51 & 3250 & extant & 0 & NA & $\mathrm{LC}$ & Browse \\
\hline AUS & Diprotodontia & Potoroidae & Bettongia & gaimardi & $\begin{array}{l}\text { Tasmanian Bettong, } \\
\text { Tasmanian Rat } \\
\text { Kangaroo, Southern } \\
\text { Bettong, Eastern } \\
\text { Bettong, Gaimard's } \\
\text { Bettong }\end{array}$ & 3.22 & 1660 & extant & Future & NA & NT & Browse/frug \\
\hline AUS & Diprotodontia & Potoroidae & Bettongia & lesueur & $\begin{array}{l}\text { Burrowing Bettong, } \\
\text { Lesueur's Rat Kangaroo, } \\
\text { Burrowing Rat- } \\
\text { kangaroo, Lesueur's } \\
\text { Bettong, Boodie }\end{array}$ & 3.18 & 1500 & extant & Future & NA & NT & Browse/frug \\
\hline AUS & Diprotodontia & Potoroidae & Bettongia & penicillata & $\begin{array}{l}\text { Woylie, Brush-tailed } \\
\text { Bettong, Brush-tailed } \\
\text { Rat-kangaroo } \\
\text { Desert Rat Kangaroo, } \\
\text { Plains Rat-kangaroo, }\end{array}$ & 3.11 & 1300 & extant & Future & NA & CR & Browse \\
\hline AUS & Diprotodontia & Potoroidae & Caloprymnus & campestris & $\begin{array}{l}\text { Buff-nosed Rat- } \\
\text { kangaroo, Desert Rat- } \\
\text { kangaroo }\end{array}$ & 2.95 & 900 & extinct & Holocene & NA & EX & Frug \\
\hline AUS & Diprotodontia & Potoroidae & Hypsiprymnodon & moschatus & Musky Rat Kangaroo & 2.72 & 520 & extant & 0 & NA & LC & Browse/frug \\
\hline AUS & Diprotodontia & Potoroidae & N.gen & spp & & 3.70 & 5000 & extinct & EP & NA & EX & Browse \\
\hline
\end{tabular}




\begin{tabular}{|c|c|c|c|c|c|c|c|c|c|c|c|c|}
\hline AUS & Diprotodontia & Potoroidae & Potorous & longipes & Long-footed Potoroo & 3.28 & 1900 & extant & Future & NA & Vu & Browse \\
\hline AUS & Diprotodontia & Potoroidae & Potorous & platyops & Broad-faced Potoroo & 2.70 & 500 & extinct & Holocene & NA & EX & Browse \\
\hline AUS & Diprotodontia & Potoroidae & Potorous & tridactylus & Long-nosed Potoroo & 3.04 & 1100 & extant & Future & NA & NT & Browse \\
\hline AUS & Diprotodontia & Potoroidae & Propleopus & oscillans & & 4.60 & 40000 & extinct & EP & 55 & EX & Browse \\
\hline AUS & Diprotodontia & Psuedocheiridae & Hemibelideus & lemuroides & $\begin{array}{l}\text { Lemuroid Ringtail } \\
\text { Possum }\end{array}$ & 2.98 & 953 & extant & Future & NA & NT & Browse \\
\hline AUS & Diprotodontia & Psuedocheiridae & Petauroides & volans & $\begin{array}{l}\text { Greater Glider, Greater } \\
\text { Gliding Possum }\end{array}$ & 3.11 & 1300 & extant & Future & NA & VU & Browse \\
\hline AUS & Diprotodontia & Psuedocheiridae & Petropseudes & dahli & $\begin{array}{l}\text { Rock Ringtail Possum, } \\
\text { Rock Possum } \\
\text { Common Ringtail }\end{array}$ & 3.21 & 1640 & extant & 0 & NA & LC & Frug/browse \\
\hline AUS & Diprotodontia & Psuedocheiridae & Pseudocheirus & peregrinus & $\begin{array}{l}\text { Possum, Common Ring- } \\
\text { tailed Possum, } \\
\text { Queensland Ringtail }\end{array}$ & 2.95 & 900 & extant & 0 & NA & LC & Browse \\
\hline AUS & Diprotodontia & Psuedocheiridae & Pseudochirops & archeri & $\begin{array}{l}\text { Green Ringtail Possum } \\
\text { Herbert River Ringtail }\end{array}$ & 3.04 & 1092 & extant & 0 & NA & LC & Browse \\
\hline AUS & Diprotodontia & Psuedocheiridae & Pseudochirulus & herbertensis & $\begin{array}{l}\text { Possum, Herbert River } \\
\text { Ringtail }\end{array}$ & 3.05 & 1115 & extant & 0 & NA & LC & Browse \\
\hline AUS & Diprotodontia & Tarsipedidae & Tarsipes & rostratus & Honey Possum & 0.95 & 9 & extant & 0 & NA & LC & Frug \\
\hline AUS & Diprotodontia & Vombatidae & Lasiorhinus & angustidens & & 4.70 & 50000 & extinct & EP & 50 & EX & Graze \\
\hline AUS & Diprotodontia & Vombatidae & Lasiorhinus & krefftii & $\begin{array}{l}\text { Northern Hairy-nosed } \\
\text { Wombat, Queensland } \\
\text { Hairy-nosed Wombat }\end{array}$ & 3.46 & 2900 & extant & Future & NA & $\mathrm{CR}$ & Graze \\
\hline AUS & Diprotodontia & Vombatidae & Lasiorhinus & latifrons & $\begin{array}{l}\text { Southern Hairy-nosed } \\
\text { Wombat }\end{array}$ & 4.41 & 25500 & extant & Future & NA & NT & Graze \\
\hline AUS & Diprotodontia & Vombatidae & Phascolomys & medius & & 4.70 & 50000 & extinct & EP & 46 & EX & Graze \\
\hline AUS & Diprotodontia & Vombatidae & Phascolonus & gigas & & 5.30 & 200000 & extinct & EP & 46 & EX & Graze \\
\hline AUS & Diprotodontia & Vombatidae & Ramsaya & magna & & 5.00 & 100000 & extinct & EP & NA & EX & Graze \\
\hline AUS & Diprotodontia & Vombatidae & Vombatus & hacketti & & 4.48 & 30000 & extinct & EP & 48 & EX & Graze \\
\hline AUS & Diprotodontia & Vombatidae & Vombatus & ursinus & $\begin{array}{l}\text { Common Wombat, } \\
\text { Coarse-haired Wombat }\end{array}$ & 4.41 & 26000 & extant & 0 & NA & LC & Graze \\
\hline AUS & Diprotodontia & Vombatidae & Warenja & wakefieldi & & 4.00 & 10000 & extinct & EP & NA & EX & Graze \\
\hline AUS & Lagomorpha & Leporidae & Lepus & capensis & $\begin{array}{l}\text { Cape Hare, Desert Hare, } \\
\text { Arabian Hare, Brown } \\
\text { Hare }\end{array}$ & 3.60 & 4000 & extant & 0 & NA & LC & J̄raze/browse \\
\hline AUS & Monotremata & Ornithorhynchidae & Ornithorhynchus & anatinus & $\begin{array}{l}\text { Platypus, Duck-billed } \\
\text { Platypus }\end{array}$ & 3.17 & 1484 & extant & Future & NA & NT & Ginsect \\
\hline AUS & Monotremata & Tachyglossidae & Megalibgwilia & ramsayi & & 4.00 & 10000 & extinct & LP & 104 & EX & Ginsect \\
\hline AUS & Monotremata & Tachyglossidae & Tachyglossus & aculeatus & $\begin{array}{l}\text { Short-beaked Echidna, } \\
\text { Kangaroo Island Echidna }\end{array}$ & 3.65 & 4500 & extant & 0 & NA & $\mathrm{LC}$ & Ginsect \\
\hline AUS & Monotremata & Tachyglossidae & Zaglossus & hacketti & $\begin{array}{l}\text { Gigantic Long Beaked } \\
\text { Echidna } \\
\text { Kakarratul, } \\
\text { Northwestern }\end{array}$ & 4.48 & 30000 & extinct & LP & 122 & EX & Ginsect \\
\hline AUS & Notoryctemorphia & Notoryctidae & Notoryctes & caurinus & $\begin{array}{l}\text { Marsupial Mole, } \\
\text { Northern Marsupial } \\
\text { Mole } \\
\text { Itjaritjari, Southern }\end{array}$ & 1.53 & 34 & extant & 0 & NA & LC & Ginsect \\
\hline AUS & Notoryctemorphia & Notoryctidae & Notoryctes & typhlops & $\begin{array}{l}\text { Marsupial Mole, } \\
\text { Marsupial Mole }\end{array}$ & 1.74 & 55 & extant & 0 & NA & LC & Ginsect \\
\hline AUS & Peramelemorphia & Chaeropodidae & Chaeropus & ecaudatus & Pig-footed Bandicoot & 2.30 & 200 & extinct & Holocene & NA & EX & rowse/ginsec \\
\hline
\end{tabular}




\begin{tabular}{|c|c|c|c|c|c|c|c|c|c|c|c|c|}
\hline AUS & Peramelemorphia & Peramelidae & Echymipera & rufescens & Long-nosed Echymipera & 3.04 & 1100 & extant & 0 & NA & LC & Ginsect/frug \\
\hline AUS & Peramelemorphia & Peramelidae & Isoodon & auratus & Golden Bandicoot & 2.63 & 425 & extant & Future & NA & Vu & insect/brows \\
\hline AUS & Peramelemorphia & Peramelidae & Isoodon & macrourus & $\begin{array}{l}\text { Northern Brown } \\
\text { Bandicoot } \\
\text { Southern Brown }\end{array}$ & 3.20 & 1600 & extant & 0 & NA & $\mathrm{LC}$ & insect/brows \\
\hline AUS & Peramelemorphia & Peramelidae & Isoodon & obesulus & $\begin{array}{l}\text { Bandicoot, Nuyts } \\
\text { Southern Brown } \\
\text { Bandicoot, Quenda }\end{array}$ & 2.89 & 775 & extant & 0 & NA & LC & Ginsect \\
\hline AUS & Peramelemorphia & Peramelidae & Perameles & bougainville & $\begin{array}{l}\text { Western Barred } \\
\text { Bandicoot, Barred } \\
\text { Bandicoot, Mal, Nymal, } \\
\text { Long-nosed Bandicoot }\end{array}$ & 2.35 & 226 & extant & Future & NA & Vu & Ginsect \\
\hline AUS & Peramelemorphia & Peramelidae & Perameles & eremiana & Desert Bandicoot & 2.70 & 500 & extinct & Holocene & NA & EX & Ginsect \\
\hline AUS & Peramelemorphia & Peramelidae & Perameles & gunnii & $\begin{array}{l}\text { Eastern Barred } \\
\text { Bandicoot }\end{array}$ & 2.94 & 870 & extant & Future & NA & NT & insect/brows \\
\hline AUS & Peramelemorphia & Peramelidae & Perameles & nasuta & Long-nosed Bandicoot & 2.99 & 975 & extant & 0 & NA & LC & insect/brows! \\
\hline AUS & Peramelemorphia & Thylacoleonidae & Thylacoleo & carnifex & & 5.06 & 115000 & extinct & EP & 48 & EX & Carn \\
\hline AUS & Peramelemorphia & Thylacomyidae & Macrotis & lagotis & $\begin{array}{l}\text { Bilby, Greater Rabbit- } \\
\text { eared Bandicoot, } \\
\text { Greater Bilby, Dalgyte }\end{array}$ & 3.13 & 1350 & extant & Future & NA & Vu & Ginsect/frug \\
\hline AUS & Peramelemorphia & Thylacomyidae & Macrotis & leucura & $\begin{array}{l}\text { Yallara, Lesser Bilby } \\
\text { White-footed Rabbit- } \\
\text { rat, White-footed }\end{array}$ & 2.55 & 354 & extinct & Holocene & NA & EX & frug/carn \\
\hline AUS & Rodentia & Muridae & Conilurus & albipes & $\begin{array}{l}\text { Rabbit Rat, Rabbit- } \\
\text { eared Tree-rat, White- } \\
\text { footed Tree-rat }\end{array}$ & 2.30 & 200 & extinct & Holocene & NA & EX & Browse/frug \\
\hline AUS & Rodentia & Muridae & Conilurus & penicillatus & $\begin{array}{l}\text { Brush-tailed Rabbit-rat, } \\
\text { Brush-tailed Tree-rat }\end{array}$ & 2.19 & 154 & extant & Future & NA & Vu & Browse/frug \\
\hline AUS & Rodentia & Muridae & Hydromys & chrysogaster & $\begin{array}{l}\text { Common Water Rat, } \\
\text { Golden-bellied Water } \\
\text { Rat }\end{array}$ & 2.78 & 606 & extant & 0 & NA & LC & Ginsect/carn \\
\hline AUS & Rodentia & Muridae & Leggadina & forresti & $\begin{array}{l}\text { Central Short-tailed } \\
\text { Mouse, Forrest's Mouse }\end{array}$ & 1.30 & 20 & extant & 0 & NA & $\mathrm{LC}$ & :/ginsect/brol \\
\hline AUS & Rodentia & Muridae & Leggadina & lakedownensis & $\begin{array}{l}\text { Northern Short-tailed } \\
\text { Mouse, Lakeland Downs } \\
\text { Mouse }\end{array}$ & 1.24 & 18 & extant & 0 & NA & LC & Frug \\
\hline AUS & Rodentia & Muridae & Leporillus & apicalis & $\begin{array}{l}\text { Lesser Stick-nest Rat, } \\
\text { White-tipped Stick-nest } \\
\text { Rat }\end{array}$ & 2.18 & 150 & extinct & Holocene & NA & EX & Browse \\
\hline AUS & Rodentia & Muridae & Leporillus & conditor & $\begin{array}{l}\text { Greater Stick-nest Rat, } \\
\text { House-building Rat }\end{array}$ & 2.54 & 350 & extant & Future & NA & Vu & Browse \\
\hline AUS & Rodentia & Muridae & Mastacomys & fuscus & $\begin{array}{l}\text { Broad-toothed Rat, } \\
\text { Broad-toothed Mouse }\end{array}$ & 2.09 & 122 & extant & Future & NA & NT & Frug \\
\hline AUS & Rodentia & Muridae & Melomys & burtoni & $\begin{array}{l}\text { Grassland Melomys, } \\
\text { Grassland Mosaic-tailed } \\
\text { Rat }\end{array}$ & 1.84 & 69 & extant & 0 & NA & LC & Browse/frug \\
\hline AUS & Rodentia & Muridae & Melomys & capensis & $\begin{array}{l}\text { Cape York Melomys, } \\
\text { Cape York Mosaic-tailed } \\
\text { Rat }\end{array}$ & 1.85 & 70 & extant & 0 & NA & LC & Browse/frug \\
\hline
\end{tabular}




\begin{tabular}{|c|c|c|c|c|c|c|c|c|c|c|c|c|}
\hline AUS & Rodentia & Muridae & Melomys & cervinipes & $\begin{array}{l}\text { Fawn-footed Melomys, } \\
\text { Fawn-footed Mosaic- } \\
\text { tailed Rat }\end{array}$ & 1.89 & 78 & extant & 0 & NA & LC & Browse/frug \\
\hline AUS & Rodentia & Muridae & Melomys & rubicola & $\begin{array}{l}\text { Bramble Cay Melomys, } \\
\text { Bramble Cay Mosaic- } \\
\text { tailed Rat }\end{array}$ & 2.00 & 100 & extant & Future & NA & $C R$ & Browse/frug \\
\hline AUS & Rodentia & Muridae & Mesembriomys & gouldii & Black-footed Tree-rat & 2.86 & 717 & extant & Future & NA & NT & Frug \\
\hline AUS & Rodentia & Muridae & Mesembriomys & macrurus & $\begin{array}{l}\text { Golden-backed Tree-rat } \\
\text { Spinifex Hopping }\end{array}$ & 2.43 & 267 & extant & 0 & NA & LC & Frug/ginsect \\
\hline AUS & Rodentia & Muridae & Notomys & alexis & $\begin{array}{l}\text { Mouse, Spinefex } \\
\text { Hopping Mouse }\end{array}$ & 1.54 & 35 & extant & 0 & NA & LC & Frug/browse \\
\hline AUS & Rodentia & Muridae & Notomys & amplus & $\begin{array}{l}\text { Short-tailed Hopping- } \\
\text { mouse }\end{array}$ & 1.95 & 90 & extinct & Holocene & NA & EX & Frug/browse \\
\hline AUS & Rodentia & Muridae & Notomys & aquilo & $\begin{array}{l}\text { Northern Hopping- } \\
\text { mouse }\end{array}$ & 1.59 & 39 & extant & Future & NA & EN & Frug/browse \\
\hline AUS & Rodentia & Muridae & Notomys & cervinus & Fawn Hopping-mouse & 1.54 & 35 & extant & Future & NA & Vu & Frug/browse \\
\hline AUS & Rodentia & Muridae & Notomys & fuscus & Dusky Hopping-mouse & 1.54 & 35 & extant & Future & NA & Vu & Frug/browse \\
\hline AUS & Rodentia & Muridae & Notomys & longicaudatus & $\begin{array}{l}\text { Long-tailed Hopping- } \\
\text { mouse }\end{array}$ & 2.00 & 100 & extinct & Holocene & NA & EX & Frug/browse \\
\hline AUS & Rodentia & Muridae & Notomys & macrotis & $\begin{array}{l}\text { Big-eared Hopping- } \\
\text { mouse }\end{array}$ & 1.95 & 90 & extinct & Holocene & NA & EX & Frug/browse \\
\hline AUS & Rodentia & Muridae & Notomys & mitchellii & $\begin{array}{l}\text { Mitchell's Hopping } \\
\text { Mouse }\end{array}$ & 1.72 & 52 & extant & 0 & NA & LC & Frug/browse \\
\hline AUS & Rodentia & Muridae & Notomys & mordax & $\begin{array}{l}\text { Darling Downs Hopping- } \\
\text { mouse }\end{array}$ & 1.95 & 90 & extinct & Holocene & NA & EX & Frug/browse \\
\hline AUS & Rodentia & Muridae & Notomys & sp1 & & 2.70 & 500 & extinct & Holocene & NA & EX & Frug/browse \\
\hline AUS & Rodentia & Muridae & Pogonomys & macrourus & Chestnut Tree Mouse & 1.80 & 63 & extant & 0 & NA & LC & Browse/frug \\
\hline AUS & Rodentia & Muridae & Pseudomys & albocinereus & $\begin{array}{l}\text { Ash-grey Mouse, Ash- } \\
\text { gray Mouse }\end{array}$ & 1.48 & 31 & extant & 0 & NA & LC & rowse/ginsec \\
\hline AUS & Rodentia & Muridae & Pseudomys & apodemoides & Silky Mouse & 1.30 & 20 & extant & 0 & NA & LC & Frug \\
\hline AUS & Rodentia & Muridae & Pseudomys & australis & Plains Mouse & 1.81 & 65 & extant & Future & NA & vu & Frug \\
\hline AUS & Rodentia & Muridae & Pseudomys & bolami & $\begin{array}{l}\text { Bolam's Mouse } \\
\text { Western Pebble Mouse, }\end{array}$ & 1.19 & 16 & extant & 0 & NA & LC & Frug \\
\hline AUS & Rodentia & Muridae & Pseudomys & chapmani & $\begin{array}{l}\text { Western Pebble-mound } \\
\text { Mouse, Pilbara Pebble } \\
\text { Mouse }\end{array}$ & 1.08 & 12 & extant & 0 & NA & LC & Frug \\
\hline AUS & Rodentia & Muridae & Pseudomys & delicatulus & $\begin{array}{l}\text { Delicate Mouse, Little } \\
\text { Native Mouse }\end{array}$ & 1.04 & 11 & extant & 0 & NA & LC & Frug \\
\hline AUS & Rodentia & Muridae & Pseudomys & desertor & $\begin{array}{l}\text { Desert Mouse, Brown } \\
\text { Desert Mouse } \\
\text { Djoongari, Shark Bay }\end{array}$ & 1.40 & 25 & extant & 0 & NA & LC & Browse \\
\hline AUS & Rodentia & Muridae & Pseudomys & fieldi & $\begin{array}{l}\text { Mouse, Alice Springs } \\
\text { Mouse } \\
\text { Djoongari, Shark Bay }\end{array}$ & 1.65 & 45 & extant & Future & NA & Vu & Frug \\
\hline AUS & Rodentia & Muridae & Pseudomys & fieldi & $\begin{array}{l}\text { Mouse, Alice Springs } \\
\text { Mouse }\end{array}$ & 1.65 & 45 & extant & Future & NA & $\mathrm{Vu}$ & Frug \\
\hline AUS & Rodentia & Muridae & Pseudomys & fumeus & Smoky Mouse & 1.85 & 70 & extant & Future & NA & EN & Frug/browse \\
\hline AUS & Rodentia & Muridae & Pseudomys & glaucus & $\begin{array}{l}\text { Blue-grey Mouse, Blue- } \\
\text { gray Mouse }\end{array}$ & 1.58 & 38 & extinct & Holocene & NA & EX & \\
\hline AUS & Rodentia & Muridae & Pseudomys & gouldii & Gould's Mouse & 1.70 & 50 & extinct & Holocene & NA & EX & Frug \\
\hline AUS & Rodentia & Muridae & Pseudomys & gracilicaudatus & Eastern Chestnut Mouse & 1.87 & 75 & extant & 0 & NA & LC & Frug/browse \\
\hline
\end{tabular}




\begin{tabular}{|c|c|c|c|c|c|c|c|c|c|c|c|c|}
\hline AUS & Rodentia & Muridae & Pseudomys & hermannsburgensis & Sandy Inland Mouse & 1.08 & 12 & extant & 0 & NA & LC & :/browse/gins \\
\hline AUS & Rodentia & Muridae & Pseudomys & higginsi & $\begin{array}{l}\text { Long-tailed Mouse } \\
\text { Central Pebble Mouse, }\end{array}$ & 1.83 & 67 & extant & 0 & NA & LC & wse/frug/gins \\
\hline AUS & Rodentia & Muridae & Pseudomys & johnsoni & $\begin{array}{l}\text { Central Pebble-mound } \\
\text { Mouse }\end{array}$ & 1.08 & 12 & extant & 0 & NA & LC & Frug/browse \\
\hline AUS & Rodentia & Muridae & Pseudomys & laborifex & Central Pebble Mouse & 1.08 & 12 & extant & 0 & NA & LC & Frug/browse \\
\hline AUS & Rodentia & Muridae & Pseudomys & nanus & $\begin{array}{l}\text { Western Chestnut } \\
\text { Mouse }\end{array}$ & 1.53 & 34 & extant & 0 & NA & LC & J̈raze/browse \\
\hline AUS & Rodentia & Muridae & Pseudomys & novaehollandiae & $\begin{array}{l}\text { New Holland Mouse, } \\
\text { Pookila }\end{array}$ & 1.30 & 20 & extant & Future & NA & Vu & Frug/ginsect \\
\hline AUS & Rodentia & Muridae & Pseudomys & occidentalis & Western Mouse & 1.53 & 34 & extant & 0 & NA & LC & Browse \\
\hline AUS & Rodentia & Muridae & Pseudomys & patrius & $\begin{array}{l}\text { Country Mouse, } \\
\text { Queensland Pebble- } \\
\text { mound Mouse }\end{array}$ & 1.58 & 38 & extant & 0 & NA & LC & \\
\hline AUS & Rodentia & Muridae & Pseudomys & pilligaensis & $\begin{array}{l}\text { Pilliga Mouse } \\
\text { Heath Mouse, }\end{array}$ & 1.04 & 11 & extant & 0 & NA & DD & Frug \\
\hline AUS & Rodentia & Muridae & Pseudomys & shortridgei & $\begin{array}{l}\text { Shortridge's Native } \\
\text { Mouse }\end{array}$ & 1.85 & 70 & extant & Future & NA & NT & Frug \\
\hline AUS & Rodentia & Muridae & Pseudomys & $s p 1$ & & 1.70 & 50 & extinct & Holocene & NA & EX & Frug \\
\hline AUS & Rodentia & Muridae & Rattus & colletti & Dusky Rat & 1.79 & 61 & extant & 0 & NA & LC & Browse \\
\hline AUS & Rodentia & Muridae & Rattus & exulans & Polynesian Rat & 1.60 & 40 & extant & 0 & NA & LC & Frug/browse \\
\hline AUS & Rodentia & Muridae & Rattus & fuscipes & Bush Rat & 2.12 & 133 & extant & 0 & NA & LC & insect/brows \\
\hline AUS & Rodentia & Muridae & Rattus & leucopus & Cape York Rat & 2.12 & 132 & extant & 0 & NA & LC & Ginsect/frug \\
\hline AUS & Rodentia & Muridae & Rattus & lutreolus & Australian Swamp Rat & 2.03 & 106 & extant & 0 & NA & LC & Browse/frug \\
\hline AUS & Rodentia & Muridae & Rattus & sordidus & $\begin{array}{l}\text { Canefield Rat, Dusky } \\
\text { Field Rat }\end{array}$ & 2.20 & 158 & extant & 0 & NA & LC & wse/frug/gins \\
\hline AUS & Rodentia & Muridae & Rattus & tunneyi & Pale Field Rat & 2.09 & 124 & extant & 0 & NA & LC & Browse \\
\hline AUS & Rodentia & Muridae & Rattus & villosissimus & $\begin{array}{l}\text { Long-haired Rat } \\
\text { White-tailed Giant Rat, }\end{array}$ & 2.13 & 134 & extant & 0 & NA & LC & Frug/browse \\
\hline AUS & Rodentia & Muridae & Uromys & caudimaculatus & $\begin{array}{l}\text { Mottled-tailed Giant } \\
\text { Rat, Giant White-tailed } \\
\text { Rat }\end{array}$ & 2.81 & 646 & extant & 0 & NA & LC & Frug \\
\hline AUS & Rodentia & Muridae & Uromys & hadrourus & $\begin{array}{l}\text { Pygmy White-tailed } \\
\text { Rat, Masked White- } \\
\text { tailed Rat, Thornton } \\
\text { Peak Melomys }\end{array}$ & 2.29 & 194 & extant & Future & NA & NT & Frug/ginsect \\
\hline AUS & Rodentia & Muridae & Xeromys & myoides & $\begin{array}{l}\text { Water Mouse, False } \\
\text { Water-rat }\end{array}$ & 1.61 & 41 & extant & Future & NA & $\mathrm{Vu}$ & Ginsect \\
\hline AUS & Rodentia & Muridae & Zyzomys & argurus & $\begin{array}{l}\text { Common Rock Rat, } \\
\text { Silver-tailed Rock Rat }\end{array}$ & 1.56 & 36 & extant & 0 & NA & LC & Browse/frug \\
\hline AUS & Rodentia & Muridae & Zyzomys & maini & $\begin{array}{l}\text { Arnhem Rock-rat, } \\
\text { Arnhem Land Rock Rat }\end{array}$ & 1.97 & 94 & extant & Future & NA & NT & Frug \\
\hline AUS & Rodentia & Muridae & Zyzomys & palatilis & $\begin{array}{l}\text { Carpentarian Rock-rat } \\
\text { Central Rock-rat, }\end{array}$ & 2.09 & 123 & extant & Future & NA & $C R$ & Frug \\
\hline AUS & Rodentia & Muridae & Zyzomys & pedunculatus & $\begin{array}{l}\text { Macdonnell Range Rock- } \\
\text { rat }\end{array}$ & 2.00 & 100 & extant & Future & NA & $C R$ & Frug \\
\hline AUS & Rodentia & Muridae & Zyzomys & woodwardi & $\begin{array}{l}\text { Kimberley Rock Rat, } \\
\text { Kimberly Rock Rat }\end{array}$ & 2.13 & 136 & extant & 0 & NA & LC & Frug \\
\hline AUS & Sirenia & Dugongidae & Dugong & dugon & Dugong, Sea Cow & 5.62 & 420000 & extant & Future & NA & Vu & Graze \\
\hline
\end{tabular}




\begin{tabular}{|c|c|c|c|c|c|c|c|c|c|c|c|c|}
\hline EA & Artiodactyla & Bovidae & Antilope & cervicapra & Blackbuck & 4.57 & 37500 & extant & Future & NA & NT & Graze \\
\hline EA & Artiodactyla & Bovidae & Bison & bonasus & European Bison, Wisent & 5.70 & 500000 & extant & Future & NA & vu & graze \\
\hline EA & Artiodactyla & Bovidae & Bison & priscus & steppe bison & 5.72 & 523600 & extinct & TP & 9.8 & EX & graze \\
\hline EA & Artiodactyla & Bovidae & Bos & frontalis & gayal & 5.90 & 800000 & extant & 0 & NA & LC & Graze \\
\hline EA & Artiodactyla & Bovidae & Bos & grunniens & & 5.52 & 333000 & extant & 0 & NA & LC & graze \\
\hline EA & Artiodactyla & Bovidae & Bos & javanicus & Banteng, Tembadau & 5.85 & 700000 & extant & Future & NA & EN & Graze \\
\hline EA & Artiodactyla & Bovidae & Bos & sauveli & Kouprey, Grey Ox & 5.90 & 800000 & extant & Future & NA & CR & Graze \\
\hline EA & Artiodactyla & Bovidae & Bos & taurus & cattle & 5.95 & 900000 & extant & 0 & NA & LC & Graze \\
\hline EA & Artiodactyla & Bovidae & Boselaphus & tragocamelus & Nilgai, Bluebuck & 5.23 & 169000 & extant & 0 & NA & LC & J̈raze/brows \\
\hline EA & Artiodactyla & Bovidae & Bubalus & bubalis & water buffalo & 4.57 & 37500 & extant & 0 & NA & LC & Graze \\
\hline EA & Artiodactyla & Bovidae & Bubalus & mephistopheles & $\begin{array}{l}\text { Short-horned water } \\
\text { buffalo }\end{array}$ & 5.63 & 431250 & extinct & Holocene & 3 & EX & graze \\
\hline EA & Artiodactyla & Bovidae & Budorcas & taxicolor & Takin & 5.48 & 302000 & extant & Future & NA & vu & J̈raze/browse \\
\hline EA & Artiodactyla & Bovidae & Capra & caucasica & $\begin{array}{l}\text { Western Tur, West } \\
\text { Caucasian Tur, Tur }\end{array}$ & 4.74 & 55000 & extant & Future & NA & EN & orowse/graze \\
\hline EA & Artiodactyla & Bovidae & Capra & cylindricornis & $\begin{array}{l}\text { Eastern Tur, East } \\
\text { Caucasian Tur }\end{array}$ & 4.70 & 50000 & extant & Future & NA & NT & orowse/graze \\
\hline EA & Artiodactyla & Bovidae & Capra & falconeri & Markhor & 4.61 & 41000 & extant & Future & NA & NT & orowse/graze \\
\hline EA & Artiodactyla & Bovidae & Capra & hircus & domestic goats & 4.88 & 75000 & extant & 0 & NA & LC & orowse/graze \\
\hline EA & Artiodactyla & Bovidae & Capra & ibex & Alpine Ibex, Ibex & 4.84 & 69333 & extant & 0 & NA & LC & orowse/graze \\
\hline EA & Artiodactyla & Bovidae & Capra & nubiana & $\begin{array}{l}\text { Nubian Ibex } \\
\text { Iberian Wild Goat, }\end{array}$ & 4.67 & 46250 & extant & Future & NA & vU & orowse/graze \\
\hline EA & Artiodactyla & Bovidae & Capra & pyrenaica & $\begin{array}{l}\text { Spanish Ibex, Pyrenean } \\
\text { Ibex }\end{array}$ & 4.70 & 50000 & extant & 0 & NA & LC & orowse/graze \\
\hline EA & Artiodactyla & Bovidae & Capra & sibirica & $\begin{array}{l}\text { Siberian Ibex, } \\
\text { Himalayan Ibex, Asiatic } \\
\text { Ibex }\end{array}$ & 5.11 & 130000 & extant & 0 & NA & LC & Jrowse/graze \\
\hline EA & Artiodactyla & Bovidae & Capricornis & sumatraensis & Sumatran Serow, Serow & 4.94 & 87500 & extant & Future & NA & vu & J̈aze/brows $\epsilon$ \\
\hline EA & Artiodactyla & Bovidae & Gazella & arabica & Arabian Gazelle & 4.08 & 12000 & extinct & Holocene & NA & EX & Browse \\
\hline EA & Artiodactyla & Bovidae & Gazella & bennettii & Chinkara, Indian Gazelle & 4.28 & 18917 & extant & 0 & NA & LC & Browse \\
\hline EA & Artiodactyla & Bovidae & Gazella & bilkis & $\begin{array}{l}\text { Queen of Sheba's } \\
\text { Gazelle, Yemen Gazelle }\end{array}$ & 4.69 & 48500 & extinct & Holocene & NA & EX & Browse \\
\hline EA & Artiodactyla & Bovidae & Gazella & dorcas & Dorcas Gazelle & 4.36 & 23000 & extant & Future & NA & vu & Browse \\
\hline EA & Artiodactyla & Bovidae & Gazella & gazella & Mountain Gazelle, Idmi & 4.36 & 23000 & extant & Future & NA & vu & Browse \\
\hline EA & Artiodactyla & Bovidae & Gazella & saudiya & Saudi Gazelle & 4.20 & 16000 & extinct & Holocene & NA & EX & Browse \\
\hline EA & Artiodactyla & Bovidae & Gazella & subgutturosa & Goitered Gazelle & 4.45 & 28500 & extant & Future & NA & vu & Browse \\
\hline EA & Artiodactyla & Bovidae & Hemitragus & hylocrius & $\begin{array}{l}\text { nilgiri tahr; Nilyiri wild } \\
\text { Goat }\end{array}$ & 4.88 & 75000 & extant & Future & NA & EN & J̄aze/brows $\epsilon$ \\
\hline EA & Artiodactyla & Bovidae & Hemitragus & jayakari & arabian tahr & 4.36 & 23000 & extant & Future & NA & EN & J̄raze/brows€ \\
\hline EA & Artiodactyla & Bovidae & Hemitragus & jemlahicus & Himalayan Tahr & 4.55 & 35200 & extant & Future & NA & NT & J̈raze/brows \\
\hline EA & Artiodactyla & Bovidae & Naemorhedus & baileyi & Red Goral & 4.44 & 27488 & extant & Future & NA & vu & Jraze/browse \\
\hline EA & Artiodactyla & Bovidae & Naemorhedus & caudatus & $\begin{array}{l}\text { Long-tailed Goral, } \\
\text { Chinese Goral }\end{array}$ & 4.43 & 27000 & extant & Future & NA & vu & J̈aze/brows $\epsilon$ \\
\hline EA & Artiodactyla & Bovidae & Naemorhedus & goral & Himalayan Goral, Goral & 4.45 & 28500 & extant & Future & NA & NT & Jraze/brows \\
\hline EA & Artiodactyla & Bovidae & Oryx & dammah & Scimitar-horned Oryx & 5.30 & 200000 & extinct & Holocene & NA & EW & zraze/browse \\
\hline EA & Artiodactyla & Bovidae & Oryx & leucoryx & $\begin{array}{l}\text { Arabian Oryx, White } \\
\text { Oryx }\end{array}$ & 4.94 & 87700 & extant & Future & NA & $\mathrm{vu}$ & zraze/browse \\
\hline EA & Artiodactyla & Bovidae & Ovibos & moschatus & Muskox, Musk Ox & 5.49 & 312500 & extinct & Holocene & 2.8 & EX & Graze \\
\hline
\end{tabular}




\begin{tabular}{|c|c|c|c|c|c|c|c|c|c|c|c|c|}
\hline EA & Artiodactyla & Bovidae & Ovis & ammon & Argali, Wild Sheep & 5.26 & 180000 & extant & Future & NA & NT & Graze \\
\hline EA & Artiodactyla & Bovidae & Ovis & aries & domestic sheep & 4.70 & 50000 & extant & 0 & NA & LC & Graze \\
\hline EA & Artiodactyla & Bovidae & Ovis & nivicola & Snow Sheep & 4.95 & 90000 & extant & 0 & NA & LC & Graze \\
\hline EA & Artiodactyla & Bovidae & Ovis & vignei & & 4.78 & 60000 & extant & Future & NA & Vu & Graze \\
\hline EA & Artiodactyla & Bovidae & Pantholops & hodgsonii & Chiru, Tibetan Antelope & 4.44 & 27500 & extant & Future & NA & EN & 3rowse/graze \\
\hline EA & Artiodactyla & Bovidae & Procapra & gutturosa & $\begin{array}{l}\text { Mongolian Gazelle, } \\
\text { Dzeren }\end{array}$ & 4.44 & 27750 & extant & 0 & NA & LC & J̈raze/brows \\
\hline EA & Artiodactyla & Bovidae & Procapra & picticaudata & Tibetan Gazelle, Goa & 4.30 & 20000 & extant & Future & NA & NT & J̄raze/brows \\
\hline EA & Artiodactyla & Bovidae & Procapra & przewalskii & Przewalski's Gazelle & 4.44 & 27500 & extant & Future & NA & EN & J̄raze/brows€ \\
\hline EA & Artiodactyla & Bovidae & Pseudois & nayaur & Blue Sheep, Bharal & 4.65 & 45000 & extant & 0 & NA & LC & Graze \\
\hline EA & Artiodactyla & Bovidae & Pseudois & schaeferi & $\begin{array}{l}\text { Dwarf Blue Sheep, } \\
\text { Dwarf Bharal } \\
\text { Pyrenean Chamois, }\end{array}$ & 4.47 & 29250 & extant & Future & NA & EN & Graze \\
\hline EA & Artiodactyla & Bovidae & Rupicapra & pyrenaica & $\begin{array}{l}\text { Abruzzo Chamois, } \\
\text { Southern Chamois, } \\
\text { Apennine Chamois }\end{array}$ & 4.48 & 30000 & extant & 0 & NA & LC & Browse \\
\hline EA & Artiodactyla & Bovidae & Rupicapra & rupicapra & $\begin{array}{l}\text { Northern Chamois, } \\
\text { Alpine Chamois, } \\
\text { Chamois, Balkan } \\
\text { Chamois }\end{array}$ & 4.42 & 26100 & extant & 0 & NA & LC & Browse \\
\hline EA & Artiodactyla & Bovidae & Saiga & tatarica & $\begin{array}{l}\text { Mongolian Saiga, Saiga } \\
\text { Antelope, Saiga }\end{array}$ & 4.46 & 29000 & extant & Future & NA & $\mathrm{CR}$ & graze \\
\hline EA & Artiodactyla & Bovidae & Spirocerus & kiakhtensis & Spiral-horned antelope & 5.90 & 790679 & extinct & EP & 37 & EX & Graze \\
\hline EA & Artiodactyla & Bovidae & Tetracerus & quadricornis & $\begin{array}{l}\text { Four-horned Antelope, } \\
\text { Chousingha } \\
\text { Bactrian Camel, Wild }\end{array}$ & 4.28 & 19000 & extant & Future & NA & vu & Graze \\
\hline EA & Artiodactyla & Camelidae & Camelus & bactrianus & $\begin{array}{l}\text { Bactrian Camel, Two- } \\
\text { humped Camel }\end{array}$ & 5.84 & 690000 & extant & Future & NA & CR & J̄raze/browse \\
\hline EA & Artiodactyla & Camelidae & Camelus & dromedarius & Arabian camel & 5.60 & 402000 & extant & 0 & NA & LC & J̈raze/brows€ \\
\hline EA & Artiodactyla & Camelidae & Camelus & knoblochi & Moose, Elk, Eurasian & 5.74 & 549541 & extinct & LP & 100 & EX & Jraze/browse \\
\hline EA & Artiodactyla & Cervidae & Alces & alces & $\begin{array}{l}\text { Moose, European Elk, } \\
\text { Eurasian Elk, Siberian } \\
\text { Elk } \\
\text { Chital, Indian Spotted }\end{array}$ & 5.55 & 355000 & extant & 0 & NA & LC & Browse \\
\hline EA & Artiodactyla & Cervidae & Axis & axis & $\begin{array}{l}\text { Deer, Axis Deer, Spotted } \\
\text { Deer }\end{array}$ & 4.85 & 70000 & extant & 0 & NA & LC & Browse \\
\hline EA & Artiodactyla & Cervidae & Axis & porcinus & $\begin{array}{l}\text { Hog Deer, Thai Hog } \\
\text { Deer, Indochina Hog } \\
\text { Deer, Indochinese Hog } \\
\text { Deer, Indian Hog Deer } \\
\text { European Roe Deer, }\end{array}$ & 4.54 & 35000 & extant & Future & NA & EN & Browse \\
\hline EA & Artiodactyla & Cervidae & Capreolus & capreolus & $\begin{array}{l}\text { Western Roe Deer, Roe } \\
\text { Deer, European Roe } \\
\text { Siberian Roe Deer, }\end{array}$ & 4.35 & 22500 & extant & 0 & NA & LC & Browse \\
\hline EA & Artiodactyla & Cervidae & Capreolus & pygargus & $\begin{array}{l}\text { Eastern Roe Deer, } \\
\text { Siberian Roe }\end{array}$ & 4.64 & 43752 & extant & 0 & NA & LC & Browse \\
\hline EA & Artiodactyla & Cervidae & Cervalces & latifrons & $\begin{array}{l}\text { broad-fronted moose; } \\
\text { stag moose }\end{array}$ & 5.93 & 850000 & extinct & TP & 12.6 & EX & browse \\
\hline
\end{tabular}




\begin{tabular}{|c|c|c|c|c|c|c|c|c|c|c|c|c|}
\hline EA & Artiodactyla & Cervidae & Cervus & elaphus & $\begin{array}{l}\text { Wapitl, Boknaran Deer, } \\
\text { Bukhara Red Deer, Elk, } \\
\text { Bactrian Deer, Wapiti, } \\
\text { Bactrian Red Deer } \\
\text { Sika Deer, Sika, Shansi }\end{array}$ & 5.22 & 166563 & extant & 0 & NA & LC & Browse \\
\hline EA & Artiodactyla & Cervidae & Cervus & nippon & $\begin{array}{l}\text { Sika, Japanese Sika } \\
\text { Deer } \\
\text { Fallow Deer, }\end{array}$ & 4.72 & 53000 & extant & 0 & NA & LC & Browse \\
\hline EA & Artiodactyla & Cervidae & Dama & dama & $\begin{array}{l}\text { Mesopotamian Fallow } \\
\text { Deer, Persian Fallow } \\
\text { Deer, FALLOW DEER } \\
\text { Persian Fallow Deer, }\end{array}$ & 4.75 & 56250 & extant & 0 & NA & $\mathrm{LC}$ & Browse \\
\hline EA & Artiodactyla & Cervidae & Dama & mesopotamica & $\begin{array}{l}\text { Mesopotamian Fallow } \\
\text { Deer }\end{array}$ & 4.86 & 72500 & extant & Future & NA & EN & Browse \\
\hline EA & Artiodactyla & Cervidae & Elaphodus & cephalophus & Tufted Deer & 4.53 & 33500 & extant & Future & NA & NT & Browse \\
\hline EA & Artiodactyla & Cervidae & Elaphurus & davidianus & $\begin{array}{l}\text { P re David's Deer, Pere } \\
\text { David's Deer }\end{array}$ & 5.17 & 149000 & extinct & Holocene & NA & EW & Browse \\
\hline EA & Artiodactyla & Cervidae & Hydropotes & inermis & Water Deer & 4.15 & 14000 & extant & Future & NA & Vu & Browse \\
\hline EA & Artiodactyla & Cervidae & Megaloceros & giganteus & Irish elk & 5.85 & 699842 & extinct & TP & 11 & EX & Browse \\
\hline EA & Artiodactyla & Cervidae & Muntiacus & crinifrons & $\begin{array}{l}\text { Black Muntjac, Hairy- } \\
\text { fronted Muntjac }\end{array}$ & 4.56 & 36141 & extant & Future & NA & Vu & Browse \\
\hline EA & Artiodactyla & Cervidae & Muntiacus & feae & Fea's Muntjac & 4.34 & 22000 & extant & 0 & NA & DD & Browse \\
\hline EA & Artiodactyla & Cervidae & Muntiacus & gongshanensis & $\begin{array}{l}\text { Gongshan Muntjac } \\
\text { Southern Red Muntjac, } \\
\text { Red Muntjac, Indian }\end{array}$ & 4.30 & 19907 & extant & 0 & NA & DD & Browse \\
\hline EA & Artiodactyla & Cervidae & Muntiacus & muntjak & $\begin{array}{l}\text { Muntjac, Bornean Red } \\
\text { Muntjac, Barking Deer, } \\
\text { Sundaland Red Muntjac } \\
\text { Reeves' Muntjac, } \\
\text { Reeve's Muntjac, }\end{array}$ & 4.15 & 14000 & extant & 0 & NA & $\mathrm{LC}$ & Browse \\
\hline EA & Artiodactyla & Cervidae & Muntiacus & reevesi & $\begin{array}{l}\text { Formosan Reeves' } \\
\text { Muntjac, Chinese } \\
\text { Muntjak, Reeves's } \\
\text { Muntjac }\end{array}$ & 4.13 & 13500 & extant & 0 & NA & LC & Browse \\
\hline EA & Artiodactyla & Cervidae & Przewalskium & albirostris & $\begin{array}{l}\text { White-lipped Deer, } \\
\text { Thorold's Deer }\end{array}$ & 5.10 & 125000 & extant & Future & NA & VU & Browse \\
\hline EA & Artiodactyla & Cervidae & Rucervus & duvaucelii & $\begin{array}{l}\text { Barasingha, Swamp } \\
\text { Deer }\end{array}$ & 5.16 & 143000 & extant & Future & NA & VU & Browse \\
\hline EA & Artiodactyla & Cervidae & Rucervus & eldii & $\begin{array}{l}\text { Eld's Deer, Thamin, } \\
\text { Brow-antlered Deer }\end{array}$ & 4.86 & 73000 & extant & Future & NA & EN & Browse \\
\hline EA & Artiodactyla & Cervidae & Rucervus & schomburgki & Schomburgk's Deer & 5.04 & 110000 & extinct & Holocene & NA & EX & Browse \\
\hline EA & Artiodactyla & Cervidae & Rusa & unicolor & $\begin{array}{l}\text { Sambar, Indian Sambar, } \\
\text { Sambar Deer }\end{array}$ & 5.33 & 211620 & extant & Future & NA & VU & \\
\hline EA & Artiodactyla & Cervidae & Sinomegaceros & yabei & giant deer & 5.70 & 500000 & extinct & EP & 45 & EX & browse \\
\hline EA & Artiodactyla & Hippopotamidae & Hippopotamus & amphibius & $\begin{array}{l}\text { Forest Musk Deer, } \\
\text { Chinese Forest Musk }\end{array}$ & 6.15 & 1417490 & extinct & $\mathrm{LP}$ & 117 & EX & graze \\
\hline EA & Artiodactyla & Moschidae & Moschus & berezovskii & $\begin{array}{l}\text { Deer, Dwarf Musk Deer, } \\
\text { South China Forest } \\
\text { Musk Deer }\end{array}$ & 3.95 & 9000 & extant & Future & NA & EN & Browse \\
\hline
\end{tabular}




\begin{tabular}{|c|c|c|c|c|c|c|c|c|c|c|c|c|}
\hline EA & Artiodactyla & Moschidae & Moschus & chrysogaster & $\begin{array}{l}\text { Alpine Musk Deer, } \\
\text { Himalayan Musk Deer }\end{array}$ & 4.16 & 14500 & extant & Future & NA & EN & Browse \\
\hline EA & Artiodactyla & Moschidae & Moschus & fuscus & $\begin{array}{l}\text { Black Musk Deer, Dusky } \\
\text { Musk Deer }\end{array}$ & 1.10 & 13 & extant & Future & NA & EN & \\
\hline EA & Artiodactyla & Moschidae & Moschus & moschiferus & Siberian Musk Deer & 4.11 & 13000 & extant & Future & NA & Vu & Browse \\
\hline EA & Artiodactyla & Suidae & Sus & barbatus & $\begin{array}{l}\text { Bearded Pig, Western } \\
\text { Bearded Pig } \\
\text { Indo-chinese Warty Pig, }\end{array}$ & 4.99 & 96667 & extant & Future & NA & VU & Browse/frug \\
\hline EA & Artiodactyla & Suidae & Sus & bucculentus & $\begin{array}{l}\text { Heude's Pig, Vietnam } \\
\text { Warty Pig }\end{array}$ & 4.89 & 76980 & extant & 0 & NA & DD & \\
\hline EA & Artiodactyla & Suidae & Sus & salvanius & $\begin{array}{l}\text { Pygmy Hog } \\
\text { Wild Boar, Eurasian }\end{array}$ & 3.89 & 7718 & extant & Future & NA & CR & Browse/frug \\
\hline EA & Artiodactyla & Suidae & Sus & scrofa & $\begin{array}{l}\text { Wild Pig, Ryukyu Islands } \\
\text { Wild Pig } \\
\text { White-spotted } \\
\text { Chevrotain, Chevrotain, }\end{array}$ & 5.07 & 117286 & extant & 0 & NA & LC & Browse/frug \\
\hline EA & Artiodactyla & Tragulidae & Moschiola & meminna & $\begin{array}{l}\text { Indian Spotted } \\
\text { Chevrotain, Indian } \\
\text { Mouse Deer, White- } \\
\text { spotted Mousedeer } \\
\text { Javan Chevrotain, } \\
\text { Kanchil, Javan }\end{array}$ & 3.39 & 2450 & extant & 0 & NA & LC & Browse/frug \\
\hline EA & Artiodactyla & Tragulidae & Tragulus & javanicus & $\begin{array}{l}\text { Mousedeer, Java } \\
\text { Mousedeer, Lesser } \\
\text { Mouse Deer } \\
\text { Greater Oriental } \\
\text { Chevrotain, Larger } \\
\text { Malay Chevrotain, }\end{array}$ & 3.52 & 3300 & extant & 0 & NA & DD & Browse/frug \\
\hline EA & Artiodactyla & Tragulidae & Tragulus & nари & $\begin{array}{l}\text { Greater Mousedeer, } \\
\text { Balabac Chevrotain, } \\
\text { Greater Indo-Malayan } \\
\text { Chevrotain, Larger } \\
\text { Mousedeer, Napu }\end{array}$ & 3.77 & 5900 & extant & 0 & NA & LC & Browse/frug \\
\hline EA & Carnivora & Ailuridae & Ailurus & fulgens & $\begin{array}{l}\text { Red Panda, Lesser } \\
\text { Panda, Red Cat-bear }\end{array}$ & 3.69 & 4900 & extant & Future & NA & EN & owse (bambo \\
\hline EA & Carnivora & Canidae & Canis & aureus & $\begin{array}{l}\text { Golden Jackal, Asiatic } \\
\text { Jackal, Common Jackal }\end{array}$ & 4.08 & 11958 & extant & 0 & NA & LC & carn/ginsect \\
\hline EA & Carnivora & Canidae & Canis & lupus & $\begin{array}{l}\text { Wolf } \\
\text { Dhole, Red Dog, Indian }\end{array}$ & 4.57 & 37000 & extant & 0 & NA & LC & carn \\
\hline EA & Carnivora & Canidae & Cuon & alpinus & $\begin{array}{l}\text { Wild Dog, Asiatic Wild } \\
\text { Dog }\end{array}$ & 4.11 & 12760 & extant & Future & NA & EN & carn \\
\hline EA & Carnivora & Canidae & Nyctereutes & procyonoides & Raccoon Dog & 3.61 & 4040 & extant & 0 & NA & LC & carn/invert \\
\hline EA & Carnivora & Canidae & Vulpes & bengalensis & Bengal Fox, Indian Fox & 3.44 & 2726 & extant & 0 & NA & LC & Carn \\
\hline EA & Carnivora & Canidae & Vulpes & cana & $\begin{array}{l}\text { Blanford's Fox, Steppe } \\
\text { Fox, Afghan Fox }\end{array}$ & 2.97 & 937 & extant & 0 & NA & LC & ginsect/carn \\
\hline EA & Carnivora & Canidae & Vulpes & corsac & Corsac Fox & 3.38 & 2400 & extant & 0 & NA & LC & Carn \\
\hline EA & Carnivora & Canidae & Vulpes & ferrilata & Tibetan Fox & 3.70 & 5000 & extant & 0 & NA & LC & Carn \\
\hline EA & Carnivora & Canidae & Vulpes & lagopus & $\begin{array}{l}\text { Arctic Fox, Polar Fox } \\
\text { Rôppell's Fox, Sand Fox, }\end{array}$ & 3.80 & 6285 & extant & 0 & NA & LC & Carn \\
\hline EA & Carnivora & Canidae & Vulpes & rueppellii & $\begin{array}{l}\text { Rôppell's Sand Fox, } \\
\text { Rôeppell's Fox }\end{array}$ & 3.39 & 2480 & extant & 0 & NA & LC & Carn \\
\hline
\end{tabular}




\begin{tabular}{|c|c|c|c|c|c|c|c|c|c|c|c|c|}
\hline EA & Carnivora & Canidae & Vulpes & vulpes & $\begin{array}{l}\text { Red Fox, Silver Fox, } \\
\text { Cross Fox }\end{array}$ & 3.77 & 5823 & extant & 0 & NA & LC & carn/ginsect \\
\hline EA & Carnivora & Canidae & Vulpes & $z e r d a$ & Fennec Fox & 3.04 & 1100 & extant & 0 & NA & LC & ginsect/carn \\
\hline EA & Carnivora & Felidae & Acinonyx & jubatus & $\begin{array}{l}\text { Cheetah, Hunting } \\
\text { Leopard }\end{array}$ & 4.70 & 50000 & extant & Future & NA & $\mathrm{VU}$ & Carn \\
\hline EA & Carnivora & Felidae & Caracal & caracal & $\begin{array}{l}\text { Caracal, Desert Lynx, } \\
\text { African Caracal, Asian } \\
\text { Caracal }\end{array}$ & 4.14 & 13750 & extant & 0 & NA & LC & Carn \\
\hline EA & Carnivora & Felidae & Catopuma & temminckii & $\begin{array}{l}\text { Asiatic Golden Cat, } \\
\text { Temminck's Cat, Golden } \\
\text { Cat } \\
\text { Chinese Mountain Cat, } \\
\text { Grass Cat, Chinese }\end{array}$ & 4.06 & 11500 & extant & Future & NA & NT & Carn \\
\hline EA & Carnivora & Felidae & Felis & bieti & $\begin{array}{l}\text { Alpine Steppe Cat, } \\
\text { Chinese Steppe Cat, } \\
\text { Chinese Desert Cat }\end{array}$ & 3.74 & 5500 & extant & Future & NA & vu & Carn \\
\hline EA & Carnivora & Felidae & Felis & chaus & $\begin{array}{l}\text { Jungle Cat, Swamp Cat, } \\
\text { Reed Cat }\end{array}$ & 3.86 & 7257 & extant & 0 & NA & LC & Carn \\
\hline EA & Carnivora & Felidae & Felis & manul & Pallas's cat & 3.54 & 3500 & extant & Future & NA & NT & Carn \\
\hline EA & Carnivora & Felidae & Felis & margarita & $\begin{array}{l}\text { Sand Cat, Sand Dune } \\
\text { Cat }\end{array}$ & 3.40 & 2525 & extant & Future & NA & NT & Carn \\
\hline EA & Carnivora & Felidae & Felis & silvestris & Wild Cat, Wildcat & 3.81 & 6400 & extant & 0 & NA & LC & Carn \\
\hline EA & Carnivora & Felidae & Homotherium & latidens & & 5.28 & 189000 & extinct & EP & 32 & EX & carn \\
\hline EA & Carnivora & Felidae & Lynx & $\operatorname{lynx}$ & Eurasian Lynx & 4.25 & 17950 & extant & 0 & NA & LC & Carn \\
\hline EA & Carnivora & Felidae & Lynx & pardinus & $\begin{array}{l}\text { Iberian Lynx, Spanish } \\
\text { Lynx, Pardel Lynx }\end{array}$ & 3.97 & 9400 & extant & Future & NA & EN & Carn \\
\hline EA & Carnivora & Felidae & Neofelis & nebulosa & Clouded Leopard & 4.29 & 19500 & extant & Future & NA & vu & Carn \\
\hline EA & Carnivora & Felidae & Panthera & leo & Lion, African Lion & 5.58 & 380189 & extinct & TP & 14 & EX & Carn \\
\hline EA & Carnivora & Felidae & Panthera & leo & Lion, African Lion & 5.21 & 161499 & extant & Future & NA & Vu & carn \\
\hline EA & Carnivora & Felidae & Panthera & pardus & Leopard & 4.66 & 45500 & extant & Future & NA & vu & Carn \\
\hline EA & Carnivora & Felidae & Panthera & tigris & Tiger & 5.21 & 162564 & extant & Future & NA & EN & Carn \\
\hline EA & Carnivora & Felidae & Pardofelis & marmorata & Marbled Cat & 3.51 & 3250 & extant & Future & NA & NT & Carn \\
\hline EA & Carnivora & Felidae & Prionailurus & bengalensis & Leopard Cat & 3.52 & 3300 & extant & 0 & NA & LC & Carn \\
\hline EA & Carnivora & Felidae & Prionailurus & rubiginosus & Rusty-spotted Cat & 3.13 & 1350 & extant & Future & NA & NT & Carn \\
\hline EA & Carnivora & Felidae & Prionailurus & viverrinus & Fishing Cat & 4.04 & 10850 & extant & Future & NA & EN & Carn \\
\hline EA & Carnivora & Felidae & Uncia & uncia & Snow leopard & 4.65 & 44167 & extant & Future & NA & EN & Carn \\
\hline EA & Carnivora & Herpestidae & Herpestes & brachyurus & Short-tailed Mongoose & 3.30 & 2000 & extant & Future & NA & NT & Carn \\
\hline EA & Carnivora & Herpestidae & Herpestes & edwardsii & common mongoose & 3.12 & 1324 & extant & 0 & NA & LC & Carn \\
\hline EA & Carnivora & Herpestidae & Herpestes & ichneumon & $\begin{array}{l}\text { Egyptian Mongoose, } \\
\text { Large Grey Mongoose }\end{array}$ & 3.88 & 7500 & extant & 0 & NA & LC & Carn \\
\hline EA & Carnivora & Herpestidae & Herpestes & javanicus & Javan Mongoose & 2.88 & 750 & extant & 0 & NA & LC & Carn \\
\hline EA & Carnivora & Herpestidae & Herpestes & palustris & bengal mongoose & 3.43 & 2683 & extant & 0 & NA & LC & \\
\hline EA & Carnivora & Herpestidae & Herpestes & smithii & Ruddy Mongoose & 3.27 & 1861 & extant & 0 & NA & LC & Carn \\
\hline EA & Carnivora & Herpestidae & Herpestes & urva & $\begin{array}{l}\text { Crab-eating Mongoose } \\
\text { Stripe-necked }\end{array}$ & 3.27 & 1863 & extant & 0 & NA & LC & Carn \\
\hline EA & Carnivora & Herpestidae & Herpestes & vitticollis & $\begin{array}{l}\text { Mongoose, Striped- } \\
\text { necked Mongoose }\end{array}$ & 3.48 & 2995 & extant & 0 & NA & LC & Carn \\
\hline EA & Carnivora & Herpestidae & Ichneumia & albicauda & White-tailed Mongoose & 3.54 & 3500 & extant & 0 & NA & LC & ginsect/carn \\
\hline EA & Carnivora & Hyaenidae & Crocuta & crocuta & Spotted Hyaena & 4.85 & 69984 & extinct & EP & 31 & EX & Carn \\
\hline EA & Carnivora & Hyaenidae & Hyaena & hyaena & Striped Hyaena & 4.57 & 37410 & extant & Future & NA & NT & Carn \\
\hline
\end{tabular}




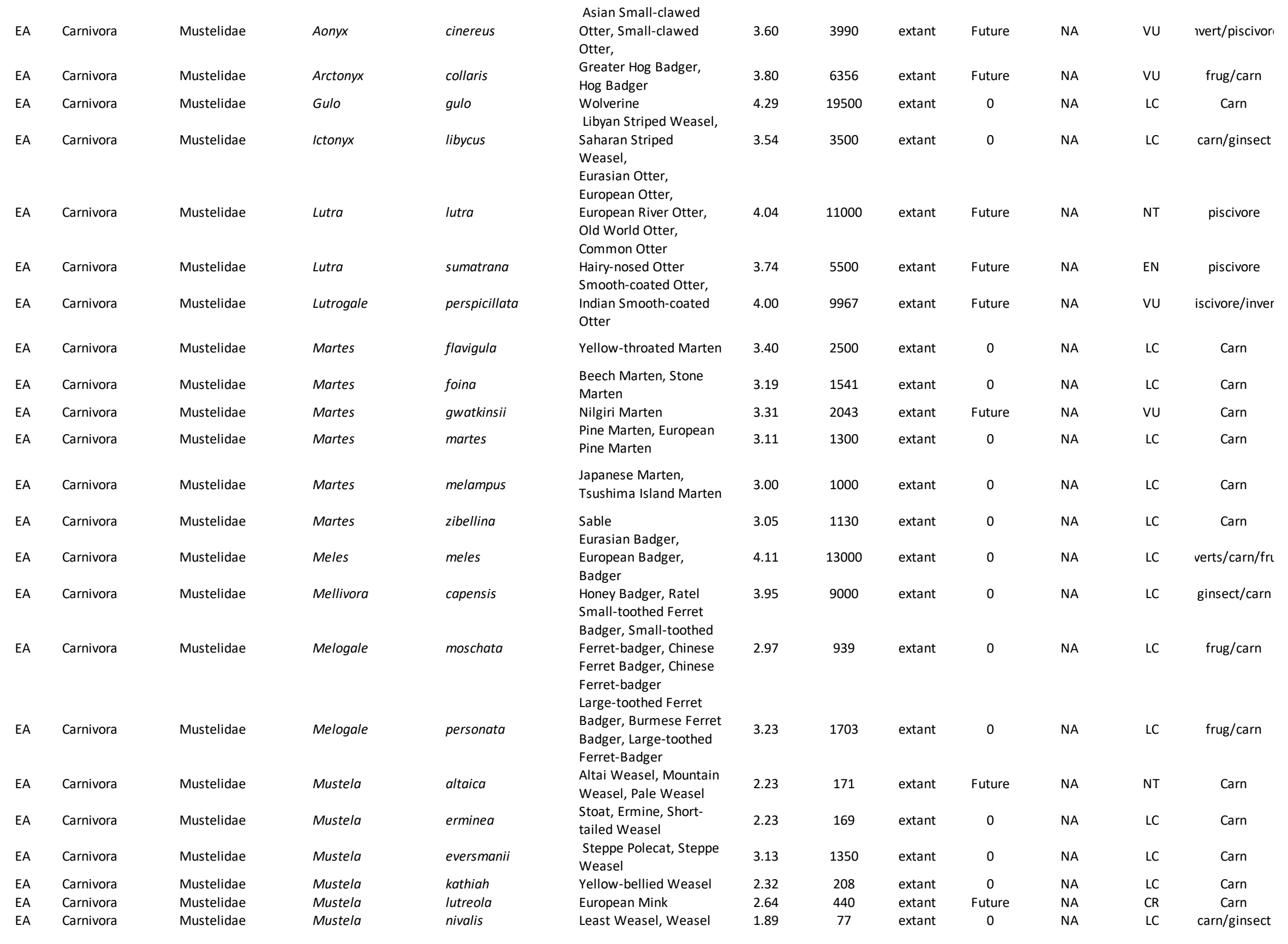




\begin{tabular}{|c|c|c|c|c|c|c|c|c|c|c|c|c|}
\hline EA & Carnivora & Mustelidae & Mustela & nudipes & $\begin{array}{l}\text { Malay Weasel, Malayan } \\
\text { Weasel }\end{array}$ & 2.70 & 500 & extant & 0 & NA & LC & Carn \\
\hline EA & Carnivora & Mustelidae & Mustela & putorius & $\begin{array}{l}\text { Western Polecat, } \\
\text { European Polecat }\end{array}$ & 2.86 & 731 & extant & 0 & NA & LC & carn/ginsect \\
\hline EA & Carnivora & Mustelidae & Mustela & sibirica & Siberian Weasel & 2.61 & 405 & extant & 0 & NA & LC & Carn \\
\hline EA & Carnivora & Mustelidae & Mustela & strigidorsa & $\begin{array}{l}\text { Stripe-backed Weasel, } \\
\text { Back-striped Weasel } \\
\text { Marbled Polecat, }\end{array}$ & 3.18 & 1500 & extant & 0 & NA & LC & Carn \\
\hline EA & Carnivora & Mustelidae & Vormela & peregusna & $\begin{array}{l}\text { European Marbled } \\
\text { Polecat }\end{array}$ & 2.65 & 450 & extant & Future & NA & Vu & Carn \\
\hline EA & Carnivora & Ursidae & Ailuropoda & melanoleuca & Giant Panda & 5.04 & 108400 & extant & Future & NA & EN & owse (bambo \\
\hline EA & Carnivora & Ursidae & Helarctos & malayanus & $\begin{array}{l}\text { Sun Bear, Malayan Sun } \\
\text { Bear }\end{array}$ & 4.66 & 46000 & extant & Future & NA & vu & Ginsect/frug \\
\hline EA & Carnivora & Ursidae & Melursus & ursinus & Sloth Bear & 5.00 & 100000 & extant & Future & NA & Vu & Ginsect/frug \\
\hline EA & Carnivora & Ursidae & Ursus & $\operatorname{arctos}$ & $\begin{array}{l}\text { Brown Bear, Grizzly } \\
\text { Bear }\end{array}$ & 5.35 & 221600 & extant & 0 & NA & LC & frug/carn \\
\hline EA & Carnivora & Ursidae & Ursus & maritimus & Polar Bear & 5.63 & 425000 & extant & Future & NA & Vu & Carn \\
\hline EA & Carnivora & Ursidae & Ursus & spelaeus & European cave bear & 5.48 & 300000 & extinct & EP & 31 & EX & carn \\
\hline EA & Carnivora & Ursidae & Ursus & thibetanus & $\begin{array}{l}\text { Asiatic Black Bear, } \\
\text { Himalayan Black Bear }\end{array}$ & 4.89 & 77500 & extant & Future & NA & vU & Frug \\
\hline EA & Carnivora & Viverridae & Arctictis & binturong & $\begin{array}{l}\text { Binturong, Bearcat } \\
\text { Small-toothed Palm }\end{array}$ & 4.11 & 13000 & extant & Future & NA & Vu & frug/carn \\
\hline EA & Carnivora & Viverridae & Arctogalidia & trivirgata & $\begin{array}{l}\text { Civet, Three-striped } \\
\text { Palm Civet } \\
\text { Owston's Civet, } \\
\text { Owston's Banded Civet, }\end{array}$ & 3.35 & 2250 & extant & 0 & NA & LC & רsect/carn/frı \\
\hline EA & Carnivora & Viverridae & Chrotogale & owstoni & $\begin{array}{l}\text { Owston's Banded Palm } \\
\text { Civet, Owston's Palm } \\
\text { Civet }\end{array}$ & 3.51 & 3250 & extant & Future & NA & EN & ect (earthwor \\
\hline EA & Carnivora & Viverridae & Cynogale & bennettii & $\begin{array}{l}\text { Otter Civet, Sunda Otter } \\
\text { Civet, Otter-civet }\end{array}$ & 3.65 & 4500 & extant & Future & NA & EN & ivore/invert/c \\
\hline EA & Carnivora & Viverridae & Diplogale & hosei & $\begin{array}{l}\text { Hose's Civet, Hose's } \\
\text { Palm Civet }\end{array}$ & & & extant & Future & NA & vu & \\
\hline EA & Carnivora & Viverridae & Genetta & genetta & Common Genet & 3.20 & 1600 & extant & 0 & NA & LC & carn/ginsect \\
\hline EA & Carnivora & Viverridae & Hemigalus & derbyanus & $\begin{array}{l}\text { Banded Civet, Banded } \\
\text { Palm Civet } \\
\text { Masked Palm Civet, }\end{array}$ & 3.40 & 2500 & extant & Future & NA & NT & invert/carn \\
\hline EA & Carnivora & Viverridae & Paguma & larvata & $\begin{array}{l}\text { Himalayan Palm Civet, } \\
\text { Gem-faced Civet }\end{array}$ & 3.63 & 4300 & extant & 0 & NA & LC & frug/carn \\
\hline EA & Carnivora & Viverridae & Paradoxurus & hermaphroditus & $\begin{array}{l}\text { Common Palm Civet, } \\
\text { Mentawai Palm Civet }\end{array}$ & 3.51 & 3200 & extant & 0 & NA & LC & frug/carn \\
\hline EA & Carnivora & Viverridae & Paradoxurus & jerdoni & $\begin{array}{l}\text { Brown Palm Civet, } \\
\text { Jerdon's Palm Civet }\end{array}$ & 3.44 & 2781 & extant & 0 & NA & LC & frug/carn \\
\hline EA & Carnivora & Viverridae & Prionodon & linsang & Banded Linsang & 2.85 & 700 & extant & 0 & NA & LC & carn/frug \\
\hline EA & Carnivora & Viverridae & Prionodon & pardicolor & Spotted Linsang & 2.71 & 512 & extant & 0 & NA & LC & carn/frug \\
\hline EA & Carnivora & Viverridae & Viverra & civettina & $\begin{array}{l}\text { Malabar Civet, Malabar } \\
\text { Large-spotted Civet }\end{array}$ & 4.11 & 12794 & extant & Future & NA & CR & carn/frug \\
\hline EA & Carnivora & Viverridae & Viverra & megaspila & Large-spotted Civet & 3.97 & 9250 & extant & Future & NA & EN & carn/frug \\
\hline EA & Carnivora & Viverridae & Viverra & tangalunga & $\begin{array}{l}\text { Malay Civet, Malayan } \\
\text { Civet, Oriental Civet }\end{array}$ & 4.00 & 10000 & extant & 0 & NA & LC & carn/frug \\
\hline EA & Carnivora & Viverridae & Viverra & zibetha & Large Indian Civet & 3.95 & 9000 & extant & 0 & NA & LC & carn/frug \\
\hline
\end{tabular}




\begin{tabular}{|c|c|c|c|c|c|c|c|c|c|c|c|c|}
\hline EA & Carnivora & Viverridae & Viverricula & indica & $\begin{array}{l}\text { Small Indian Civet, } \\
\text { Oriental Civet }\end{array}$ & 3.47 & 2980 & extant & 0 & NA & LC & carn/frug \\
\hline EA & Dermoptera & Cynocephalidae & Cynocephalus & volans & $\begin{array}{l}\text { Philippine Flying Lemur, } \\
\text { Flying Lemur }\end{array}$ & 3.10 & 1250 & extant & 0 & NA & LC & Browse \\
\hline EA & Dermoptera & Cynocephalidae & Galeopterus & variegatus & $\begin{array}{l}\text { Sunda Flying Lemur, } \\
\text { Malayan Flying Lemur }\end{array}$ & 3.00 & 1000 & extant & 0 & NA & LC & Browse \\
\hline EA & Erinaceomorpha & Erinaceidae & Echinosorex & gymnura & Moonrat, Gymnure & 2.98 & 957 & extant & 0 & NA & LC & ginsect \\
\hline EA & Erinaceomorpha & Erinaceidae & Erinaceus & amurensis & $\begin{array}{l}\text { Amur Hedgehog, } \\
\text { Manchurian Hedgehog }\end{array}$ & 2.87 & 745 & extant & 0 & NA & LC & \\
\hline EA & Erinaceomorpha & Erinaceidae & Erinaceus & concolor & $\begin{array}{l}\text { Southern White- } \\
\text { breasted Hedgehog, } \\
\text { Eastern European } \\
\text { Hedgehog } \\
\text { Western European }\end{array}$ & 2.86 & 719 & extant & 0 & NA & LC & ginsect \\
\hline EA & Erinaceomorpha & Erinaceidae & Erinaceus & europaeus & $\begin{array}{l}\text { Hedgehog, Western } \\
\text { Hedgehog, Northern } \\
\text { Hedgehog }\end{array}$ & 2.89 & 771 & extant & 0 & NA & LC & ginsect \\
\hline EA & Erinaceomorpha & Erinaceidae & Hemiechinus & auritus & $\begin{array}{l}\text { Long-eared Hedgehog } \\
\text { Collared Hedgehog, }\end{array}$ & 2.53 & 342 & extant & 0 & NA & LC & ginsect/carn \\
\hline EA & Erinaceomorpha & Erinaceidae & Hemiechinus & collaris & $\begin{array}{l}\text { Indian Long-eared } \\
\text { Hedgehog }\end{array}$ & 2.15 & 141 & extant & 0 & NA & LC & ginsect/carn \\
\hline EA & Erinaceomorpha & Erinaceidae & Hylomys & suillus & $\begin{array}{l}\text { Short-tailed Gymnure, } \\
\text { Lesser Gymnure }\end{array}$ & 1.81 & 64 & extant & 0 & NA & LC & Ginsect \\
\hline EA & Erinaceomorpha & Erinaceidae & Mesechinus & dauuricus & Daurian Hedgehog & 2.53 & 342 & extant & 0 & NA & LC & Ginsect \\
\hline EA & Erinaceomorpha & Erinaceidae & Mesechinus & hughi & $\begin{array}{l}\text { Hugh's Hedgehog, } \\
\text { Shaanxi Hedgehog }\end{array}$ & 2.53 & 342 & extant & 0 & NA & LC & \\
\hline EA & Erinaceomorpha & Erinaceidae & Paraechinus & aethiopicus & Desert Hedgehog & 2.27 & 188 & extant & 0 & NA & LC & ginsect/carn \\
\hline EA & Erinaceomorpha & Erinaceidae & Paraechinus & hypomelas & Brandt's Hedgehog & 2.33 & 213 & extant & 0 & NA & LC & ginsect/carn \\
\hline EA & Erinaceomorpha & Erinaceidae & Paraechinus & nudiventris & $\begin{array}{l}\text { Madras Hedgehog, Bare- } \\
\text { bellied Hedgehog }\end{array}$ & 2.28 & 190 & extant & 0 & NA & LC & \\
\hline EA & Hyracoidea & Procaviidae & Procavia & capensis & Rock Hyrax, Rock Dassie & 3.48 & 3030 & extant & 0 & NA & LC & కraze/browse \\
\hline EA & Insectivora & Erinaceidae & Neotetracus & sinensis & Shrew Gymnure & 1.94 & 87 & extant & 0 & NA & $\mathrm{LC}$ & Ginsect \\
\hline EA & Insectivora & Erinaceidae & Paraechinus & micropus & Indian Hedgehog & 2.23 & 171 & extant & 0 & NA & LC & ginsect/carn \\
\hline EA & Insectivora & Soricidae & Chodsigoa & lamula & Lamulate Shrew & 0.89 & 8 & extant & 0 & NA & LC & Ginsect \\
\hline EA & Insectivora & Soricidae & Chodsigoa & parca & Lowe's Shrew & 1.05 & 11 & extant & 0 & NA & LC & Ginsect \\
\hline EA & Insectivora & Soricidae & Chodsigoa & salenskii & Salenski's Shrew & 0.74 & 6 & extant & 0 & NA & DD & Ginsect \\
\hline EA & Insectivora & Soricidae & Crocidura & suaveolens & $\begin{array}{l}\text { Lesser Shrew, Lesser } \\
\text { White-toothed Shrew }\end{array}$ & 0.94 & 9 & extant & 0 & NA & LC & Ginsect \\
\hline EA & Insectivora & Soricidae & Eospalax & smithii & Smith's Zokor & 1.16 & 14 & extant & 0 & NA & LC & Ginsect \\
\hline EA & Insectivora & Soricidae & Episoriculus & leucops & $\begin{array}{l}\text { Long-tailed Brown- } \\
\text { toothed Shrew }\end{array}$ & 0.92 & 8 & extant & 0 & NA & LC & Ginsect \\
\hline EA & Insectivora & Soricidae & Neomys & teres & $\begin{array}{l}\text { Transcaucasian Water } \\
\text { Shrew }\end{array}$ & 1.11 & 13 & extant & 0 & NA & LC & \\
\hline EA & Insectivora & Soricidae & Soriculus & hypsibius & De Winton's Shrew & 1.18 & 15 & extant & 0 & NA & LC & Ginsect \\
\hline EA & Insectivora & Talpidae & Talpa & davidiana & P re David's Mole & 1.92 & 83 & extant & 0 & NA & DD & \\
\hline EA & Lagomorpha & Leporidae & Caprolagus & hispidus & $\begin{array}{l}\text { Hispid Hare, Assam } \\
\text { Rabbit, Bristly Rabbit } \\
\text { Cape Hare, Desert Hare, }\end{array}$ & 3.40 & 2497 & extant & Future & NA & EN & Browse \\
\hline EA & Lagomorpha & Leporidae & Lepus & capensis & $\begin{array}{l}\text { Arabian Hare, Brown } \\
\text { Hare }\end{array}$ & 3.60 & 4010 & extant & 0 & NA & LC & క̧raze/browse \\
\hline EA & Lagomorpha & Leporidae & Lepus & castroviejoi & Broom Hare & 3.44 & 2764 & extant & Future & NA & VU & graze \\
\hline
\end{tabular}




\begin{tabular}{|c|c|c|c|c|c|c|c|c|c|c|c|c|}
\hline EA & Lagomorpha & Leporidae & Lepus & comus & Yunnan Hare & 3.16 & 1460 & extant & 0 & NA & LC & graze/browse \\
\hline EA & Lagomorpha & Leporidae & Lepus & coreanus & Korean Hare & 3.44 & 2764 & extant & 0 & NA & $\mathrm{LC}$ & \\
\hline EA & Lagomorpha & Leporidae & Lepus & corsicanus & $\begin{array}{l}\text { Corsican Hare, } \\
\text { CORSICAN HARE, } \\
\text { Apennine Hare, } \\
\text { APPENINE HARE, Italian } \\
\text { Hare } \\
\text { European Hare, Brown }\end{array}$ & 3.48 & 3000 & extant & Future & NA & Vu & jraze/browse \\
\hline EA & Lagomorpha & Leporidae & Lepus & europaeus & $\begin{array}{l}\text { Hare, European Brown } \\
\text { Hare }\end{array}$ & 3.57 & 3740 & extant & 0 & NA & LC & zraze/browse \\
\hline EA & Lagomorpha & Leporidae & Lepus & granatensis & $\begin{array}{l}\text { Granada Hare, Iberian } \\
\text { Hare }\end{array}$ & 3.60 & 4010 & extant & 0 & NA & LC & эraze/browse \\
\hline EA & Lagomorpha & Leporidae & Lepus & mandshuricus & Manchurian Hare & 3.26 & 1800 & extant & 0 & NA & LC & sraze/browse \\
\hline EA & Lagomorpha & Leporidae & Lepus & nigricollis & $\begin{array}{l}\text { Indian Hare, Black- } \\
\text { naped Hare }\end{array}$ & 3.32 & 2100 & extant & 0 & NA & $\mathrm{LC}$ & sraze/browse \\
\hline EA & Lagomorpha & Leporidae & Lepus & oiostolus & Woolly Hare & 3.48 & 3000 & extant & 0 & NA & LC & эraze/browse \\
\hline EA & Lagomorpha & Leporidae & Lepus & peguensis & Burmese Hare & 3.32 & 2105 & extant & 0 & NA & $\mathrm{LC}$ & эraze/browse \\
\hline EA & Lagomorpha & Leporidae & Lepus & sinensis & Chinese Hare & 3.18 & 1510 & extant & 0 & NA & LC & эraze/browse \\
\hline EA & Lagomorpha & Leporidae & Lepus & timidus & $\begin{array}{l}\text { Mountain Hare, Arctic } \\
\text { Hare }\end{array}$ & 3.48 & 3048 & extant & 0 & NA & LC & jraze/browse \\
\hline EA & Lagomorpha & Leporidae & Lepus & tolai & Tolai Hare & 3.20 & 1589 & extant & 0 & NA & LC & Jraze/browse \\
\hline EA & Lagomorpha & Leporidae & Lepus & yarkandensis & Yarkand Hare & 3.44 & 2764 & extant & Future & NA & NT & కraze/browse \\
\hline EA & Lagomorpha & Leporidae & Oryctolagus & cuniculus & European Rabbit & 3.25 & 1767 & extant & Future & NA & NT & Jraze/browse \\
\hline EA & Lagomorpha & Ochotonidae & Ochotona & alpina & Alpine Pika, Altai Pika & 2.18 & 150 & extant & 0 & NA & $\mathrm{LC}$ & Graze \\
\hline EA & Lagomorpha & Ochotonidae & Ochotona & cansus & Gansu Pika, Gray Pika & 1.84 & 69 & extant & 0 & NA & LC & browse \\
\hline EA & Lagomorpha & Ochotonidae & Ochotona & curzoniae & $\begin{array}{l}\text { Plateau Pika, Black- } \\
\text { lipped Pika }\end{array}$ & 2.12 & 131 & extant & 0 & NA & LC & browse/frug \\
\hline EA & Lagomorpha & Ochotonidae & Ochotona & dauurica & Daurian Pika & 2.04 & 109 & extant & 0 & NA & LC & Graze \\
\hline EA & Lagomorpha & Ochotonidae & Ochotona & erythrotis & Chinese Red Pika & 2.26 & 181 & extant & 0 & NA & LC & Graze \\
\hline EA & Lagomorpha & Ochotonidae & Ochotona & forresti & Forrest's Pika & 2.20 & 157 & extant & 0 & NA & LC & browse/frug \\
\hline EA & Lagomorpha & Ochotonidae & Ochotona & gloveri & Glover's Pika & 2.20 & 157 & extant & 0 & NA & $\mathrm{LC}$ & browse/frug \\
\hline EA & Lagomorpha & Ochotonidae & Ochotona & hyperborea & Northern Pika & 2.08 & 120 & extant & 0 & NA & LC & Graze \\
\hline EA & Lagomorpha & Ochotonidae & Ochotona & iliensis & Ili Pika & 2.20 & 157 & extant & Future & NA & EN & \\
\hline EA & Lagomorpha & Ochotonidae & Ochotona & koslowi & $\begin{array}{l}\text { Kozlov's Pika, Koslov's } \\
\text { Pika }\end{array}$ & 2.20 & 157 & extant & Future & NA & EN & \\
\hline EA & Lagomorpha & Ochotonidae & Ochotona & ladacensis & Ladak Pika & 2.20 & 157 & extant & 0 & NA & LC & browse/frug \\
\hline EA & Lagomorpha & Ochotonidae & Ochotona & macrotis & Large-eared Pika & 2.41 & 260 & extant & 0 & NA & LC & Graze \\
\hline EA & Lagomorpha & Ochotonidae & Ochotona & nubrica & Nubra Pika & 2.20 & 157 & extant & 0 & NA & LC & browse/frug \\
\hline EA & Lagomorpha & Ochotonidae & Ochotona & pusilla & Steppe Pika, Little Pika & 2.15 & 143 & extant & 0 & NA & LC & Graze \\
\hline EA & Lagomorpha & Ochotonidae & Ochotona & roylei & Royle's Pika & 2.41 & 260 & extant & 0 & NA & LC & Graze \\
\hline EA & Lagomorpha & Ochotonidae & Ochotona & rufescens & Afghan Pika & 2.40 & 250 & extant & 0 & NA & LC & Graze \\
\hline EA & Lagomorpha & Ochotonidae & Ochotona & rutila & Turkestan Red Pika & 2.20 & 157 & extant & 0 & NA & LC & browse/frug \\
\hline EA & Lagomorpha & Ochotonidae & Ochotona & thibetana & Moupin Pika & 1.92 & 83 & extant & 0 & NA & LC & Graze \\
\hline EA & Lagomorpha & Ochotonidae & Ochotona & thomasi & Thomas's Pika & 2.20 & 157 & extant & 0 & NA & $\mathrm{LC}$ & browse/frug \\
\hline EA & Lagomorpha & Ochotonidae & Ochotona & gaoligongensis & Gaoligong pika & 2.19 & 154 & extant & 0 & NA & $\mathrm{DD}$ & browse/frug \\
\hline EA & Lagomorpha & Ochotonidae & Ochotona & himalaya & Himalayan Mouse Hare & 2.19 & 154 & extant & 0 & NA & LC & browse/frug \\
\hline EA & Lagomorpha & Ochotonidae & Ochotona & muliensis & Mull pika & 2.19 & 154 & extant & $q$ & NA & DD & browse/frug \\
\hline EA & Lagomorpha & Ochotonidae & Ochotona & pallasi & Mongolian Pika & 2.19 & 154 & extant & 0 & NA & LC & browse/frug \\
\hline EA & Monotremata & Tachyglossidae & Zaglossus & bruijnii & & 4.01 & 10140 & extant & Future & NA & $C R$ & Ginsect \\
\hline
\end{tabular}




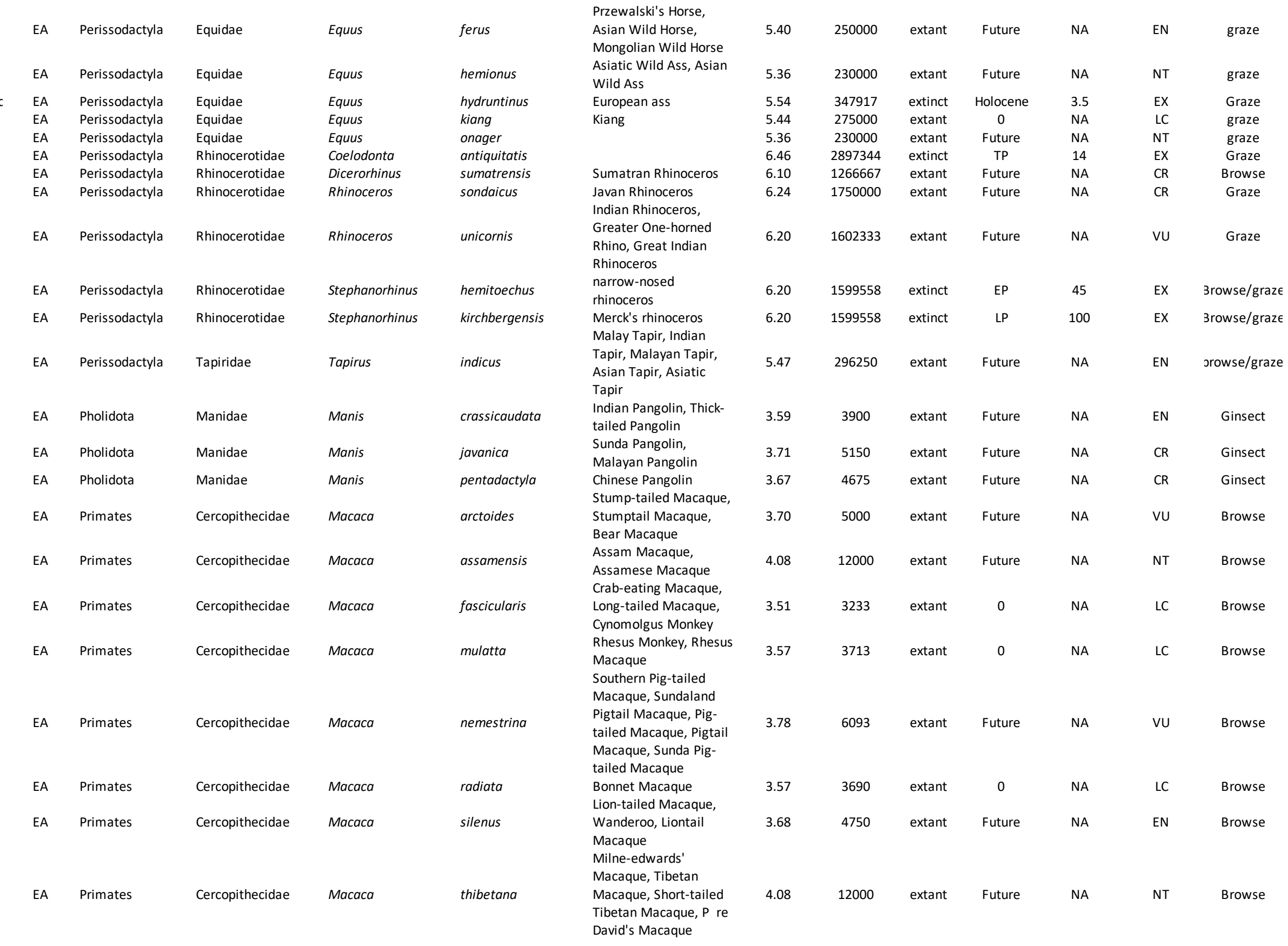




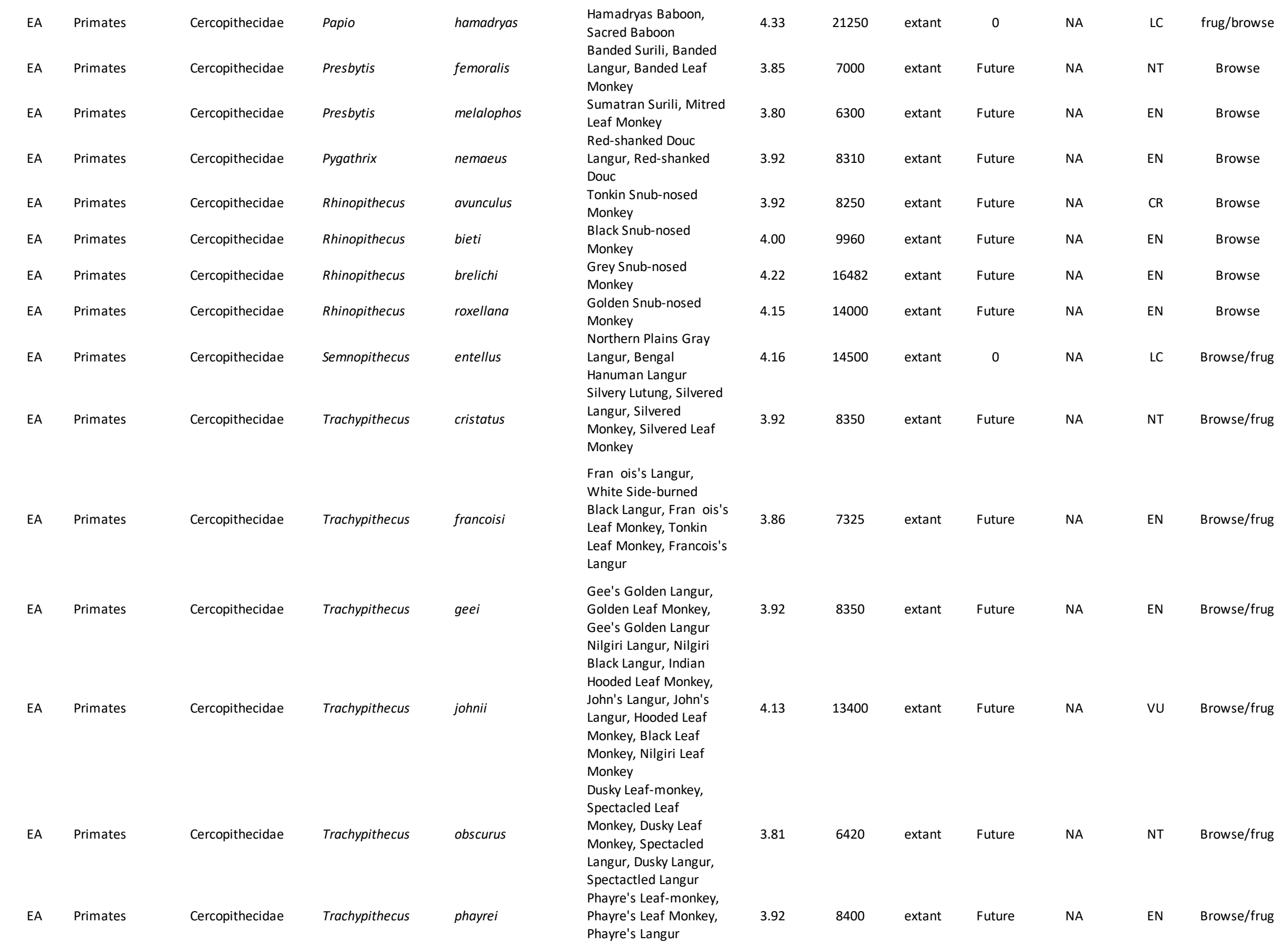




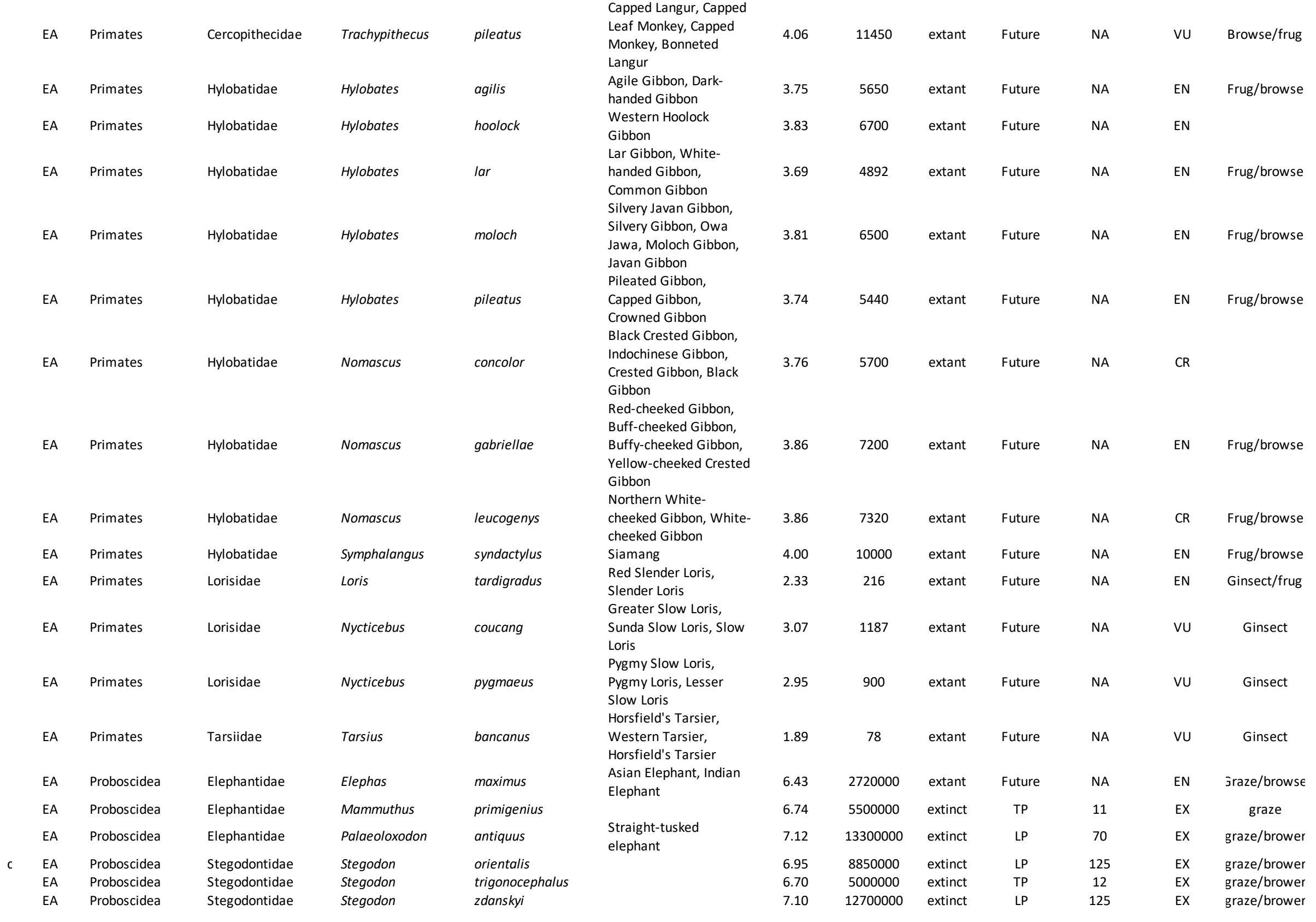




\begin{tabular}{|c|c|c|c|c|c|c|c|c|c|c|c|c|}
\hline & & & & & Zagros Mountains & & & & & & & \\
\hline EA & Rodentia & Calomyscidae & Calomyscus & bailwardi & $\begin{array}{l}\text { Calomyscus, Zagros } \\
\text { Mountains Mouse-like } \\
\text { Hamster }\end{array}$ & 1.31 & 20 & extant & 0 & NA & LC & Frug/browse \\
\hline EA & Rodentia & Calomyscidae & Calomyscus & baluchi & $\begin{array}{l}\text { Baluchi Brush-tailed } \\
\text { Mouse, Baluchi Mouse- } \\
\text { like Hamster, Pakistan } \\
\text { Calomyscus } \\
\text { Hotson's Brush-tailed }\end{array}$ & 1.27 & 19 & extant & 0 & NA & LC & \\
\hline EA & Rodentia & Calomyscidae & Calomyscus & hotsoni & $\begin{array}{l}\text { Mouse, Hotson's } \\
\text { Calomyscus, Hotson's } \\
\text { Mouse-like Hamster } \\
\text { Afghan Mouse-like }\end{array}$ & 1.27 & 19 & extant & 0 & NA & LC & \\
\hline EA & Rodentia & Calomyscidae & Calomyscus & mystax & $\begin{array}{l}\text { Hamster, Great Balkhan } \\
\text { Calomyscus } \\
\text { Tsolov's Mouse-like }\end{array}$ & 1.23 & 17 & extant & 0 & NA & LC & Frug/browse \\
\hline EA & Rodentia & Calomyscidae & Calomyscus & tsolovi & $\begin{array}{l}\text { Hamster, Syrian } \\
\text { Calomyscus }\end{array}$ & 1.27 & 19 & extant & 0 & NA & DD & \\
\hline EA & Rodentia & Calomyscidae & Calomyscus & urartensis & $\begin{array}{l}\text { Urar Calomyscus, } \\
\text { Urartsk Mouse-like } \\
\text { Hamster }\end{array}$ & 1.27 & 19 & extant & 0 & NA & LC & \\
\hline EA & Rodentia & Castoridae & Castor & fiber & Eurasian Beaver & 4.28 & 19000 & extant & 0 & NA & LC & Browse \\
\hline EA & Rodentia & Castoridae & Trogontherium & cuvieri & & 4.60 & 39994 & extinct & TP & 12 & EX & Browse \\
\hline EA & Rodentia & Cricetidae & Allocricetulus & curtatus & Mongolian Hamster & 1.68 & 48 & extant & 0 & NA & LC & Frug/browse \\
\hline EA & Rodentia & Cricetidae & Allocricetulus & eversmanni & Eversmann's Hamster & 2.06 & 116 & extant & 0 & NA & LC & Frug/browse \\
\hline EA & Rodentia & Cricetidae & Alticola & argentatus & Silver Mountain Vole & 1.58 & 38 & extant & 0 & NA & $\mathrm{LC}$ & J̈raze/brows€ \\
\hline EA & Rodentia & Cricetidae & Alticola & barakshin & $\begin{array}{l}\text { Gobi Altai Mountain } \\
\text { Vole }\end{array}$ & 1.58 & 38 & extant & 0 & NA & LC & \\
\hline EA & Rodentia & Cricetidae & Alticola & lemminus & $\begin{array}{l}\text { Lemming Vole, } \\
\text { Lemming Mountain } \\
\text { Vole }\end{array}$ & 1.58 & 38 & extant & 0 & NA & LC & \\
\hline EA & Rodentia & Cricetidae & Alticola & macrotis & $\begin{array}{l}\text { Large-eared Vole, Large- } \\
\text { eared Mountain Vole }\end{array}$ & 1.56 & 36 & extant & 0 & NA & LC & J̄raze/brows \\
\hline EA & Rodentia & Cricetidae & Alticola & montosa & $\begin{array}{l}\text { Central Kashmir Vole, } \\
\text { Kashmir Mountain Vole, } \\
\text { True's Vole }\end{array}$ & 1.58 & 38 & extant & Future & NA & Vu & \\
\hline EA & Rodentia & Cricetidae & Alticola & roylei & $\begin{array}{l}\text { Royle's Mountain Vole } \\
\text { Mongolian Silver Vole, }\end{array}$ & 1.57 & 37 & extant & Future & NA & NT & J̄aze/brows \\
\hline EA & Rodentia & Cricetidae & Alticola & semicanus & $\begin{array}{l}\text { Mongolian Mountain } \\
\text { Vole }\end{array}$ & 1.58 & 38 & extant & 0 & NA & LC & \\
\hline EA & Rodentia & Cricetidae & Alticola & stoliczkanus & $\begin{array}{l}\text { Stoliczka's Mountain } \\
\text { Vole }\end{array}$ & 1.44 & 27 & extant & 0 & NA & LC & J̄aze/brows \\
\hline EA & Rodentia & Cricetidae & Alticola & strelzowi & $\begin{array}{l}\text { Flat-headed Vole, } \\
\text { Strelzow's Mountain } \\
\text { Vole }\end{array}$ & 1.70 & 50 & extant & 0 & NA & LC & J̄raze/brows \\
\hline EA & Rodentia & Cricetidae & Alticola & tuvinicus & $\begin{array}{l}\text { Tuva Silver Vole, Tuva } \\
\text { Mountain Vole }\end{array}$ & 1.58 & 38 & extant & 0 & NA & LC & \\
\hline EA & Rodentia & Cricetidae & Arvicola & sapidus & $\begin{array}{l}\text { Southern Water Vole, } \\
\text { Southwestern Water } \\
\text { Vole }\end{array}$ & 2.34 & 220 & extant & Future & NA & Vu & J̄raze/brows \\
\hline
\end{tabular}




\begin{tabular}{|c|c|c|c|c|c|c|c|c|c|c|c|c|}
\hline EA & Rodentia & Cricetidae & Blanfordimys & afghanus & Afghan Vole & & & extant & 0 & NA & LC & \\
\hline EA & Rodentia & Cricetidae & Cansumys & canus & Gansu Hamster & & & extant & 0 & NA & LC & \\
\hline EA & Rodentia & Cricetidae & Chionomys & gud & $\begin{array}{l}\text { Caucasian Snow Vole, } \\
\text { Gudaur Snow Vole }\end{array}$ & 1.97 & 94 & extant & 0 & NA & LC & Jraze/brows \\
\hline EA & Rodentia & Cricetidae & Chionomys & nivalis & $\begin{array}{l}\text { European Snow Vole, } \\
\text { Snow Vole }\end{array}$ & 1.73 & 54 & extant & 0 & NA & LC & Jraze/brows \\
\hline EA & Rodentia & Cricetidae & Chionomys & roberti & Robert's Snow Vole & 1.97 & 94 & extant & 0 & NA & LC & J̄raze/brows \\
\hline EA & Rodentia & Cricetidae & Cricetulus & alticola & $\begin{array}{l}\text { Ladakh Hamster, } \\
\text { Tibetan Dwarf Hamster }\end{array}$ & 1.44 & 28 & extant & 0 & NA & LC & \\
\hline EA & Rodentia & Cricetidae & Cricetulus & barabensis & Striped Dwarf Hamster & 1.36 & 23 & extant & 0 & NA & LC & :/browse/gins \\
\hline EA & Rodentia & Cricetidae & Cricetulus & kamensis & Kam Dwarf Hamster & 1.44 & 28 & extant & 0 & NA & LC & \\
\hline EA & Rodentia & Cricetidae & Cricetulus & longicaudatus & $\begin{array}{l}\text { Long-tailed Dwarf } \\
\text { Hamster }\end{array}$ & 1.34 & 22 & extant & 0 & NA & LC & :/browse/gins \\
\hline EA & Rodentia & Cricetidae & Cricetulus & migratorius & $\begin{array}{l}\text { Gray Dwarf Hamster, } \\
\text { Grey Hamster } \\
\text { Sokolov's Dwarf }\end{array}$ & 1.59 & 39 & extant & 0 & NA & LC & :/browse/gins \\
\hline EA & Rodentia & Cricetidae & Cricetulus & sokolovi & $\begin{array}{l}\text { Hamster, Sokolov's } \\
\text { Dwarf Hamster }\end{array}$ & 1.44 & 28 & extant & 0 & NA & LC & \\
\hline EA & Rodentia & Cricetidae & Cricetus & cricetus & $\begin{array}{l}\text { Black-bellied Hamster, } \\
\text { Common Hamster } \\
\text { Arctic Lemming }\end{array}$ & 2.71 & 510 & extant & 0 & NA & LC & :/browse/gins \\
\hline EA & Rodentia & Cricetidae & Dicrostonyx & torquatus & $\begin{array}{l}\text { Palearctic Collared } \\
\text { Lemming }\end{array}$ & 1.93 & 85 & extant & 0 & NA & LC & 3rowse/graze \\
\hline EA & Rodentia & Cricetidae & Dinaromys & bogdanovi & $\begin{array}{l}\text { Balkan Snow Vole, } \\
\text { Martino's Snow Vole }\end{array}$ & 1.75 & 56 & extant & Future & NA & VU & 3rowse/graze \\
\hline EA & Rodentia & Cricetidae & Ellobius & alaicus & $\begin{array}{l}\text { Alai Mole Vole } \\
\text { Southern Mole Vole, }\end{array}$ & 1.90 & 79 & extant & 0 & NA & $\mathrm{DD}$ & browse/frug \\
\hline EA & Rodentia & Cricetidae & Ellobius & fuscocapillus & $\begin{array}{l}\text { Quetta Mole-vole, } \\
\text { Afghan Mole-vole } \\
\text { Transcaucasian Mole }\end{array}$ & 1.90 & 80 & extant & 0 & NA & LC & se (roots \& tu \\
\hline EA & Rodentia & Cricetidae & Ellobius & lutescens & $\begin{array}{l}\text { Vole, Mountain Mole } \\
\text { Vole }\end{array}$ & 2.07 & 117 & extant & 0 & NA & LC & se (roots \& tu \\
\hline EA & Rodentia & Cricetidae & Ellobius & talpinus & Northern Mole Vole & 1.60 & 40 & extant & 0 & NA & LC & se (roots \& tu \\
\hline EA & Rodentia & Cricetidae & Ellobius & tancrei & Zaisan Mole Vole & 1.90 & 79 & extant & 0 & NA & LC & browse/frug \\
\hline EA & Rodentia & Cricetidae & Eolagurus & luteus & Yellow Steppe Lemming & 1.41 & 26 & extant & 0 & NA & LC & J̈raze/browse \\
\hline EA & Rodentia & Cricetidae & Eolagurus & przewalskii & $\begin{array}{l}\text { Przewalski's Steppe } \\
\text { Lemming } \\
\text { Sichuan Red-backed }\end{array}$ & 1.41 & 26 & extant & 0 & NA & LC & \\
\hline EA & Rodentia & Cricetidae & Eothenomys & chinensis & $\begin{array}{l}\text { Vole, Sichuan Chinese } \\
\text { Vole, Pratt's Vole, } \\
\text { Pratt's Oriental Vole }\end{array}$ & 1.66 & 45 & extant & 0 & NA & LC & Jraze/brows \\
\hline EA & Rodentia & Cricetidae & Eothenomys & custos & $\begin{array}{l}\text { Southwest China Red- } \\
\text { backed Vole, Southwest } \\
\text { China Vole }\end{array}$ & 1.49 & 31 & extant & 0 & NA & LC & J̈raze/brows \\
\hline EA & Rodentia & Cricetidae & Eothenomys & melanogaster & $\begin{array}{l}\text { P_re David's Vole, P re } \\
\text { David's Vole }\end{array}$ & 1.43 & 27 & extant & 0 & NA & LC & Jraze/brows \\
\hline EA & Rodentia & Cricetidae & Eothenomys & olitor & $\begin{array}{l}\text { Black-eared Red-backed } \\
\text { Vole, Black-eared } \\
\text { Chinese Vole, Chaotung } \\
\text { Vole }\end{array}$ & 1.34 & 22 & extant & 0 & NA & LC & J̈raze/brows \\
\hline
\end{tabular}




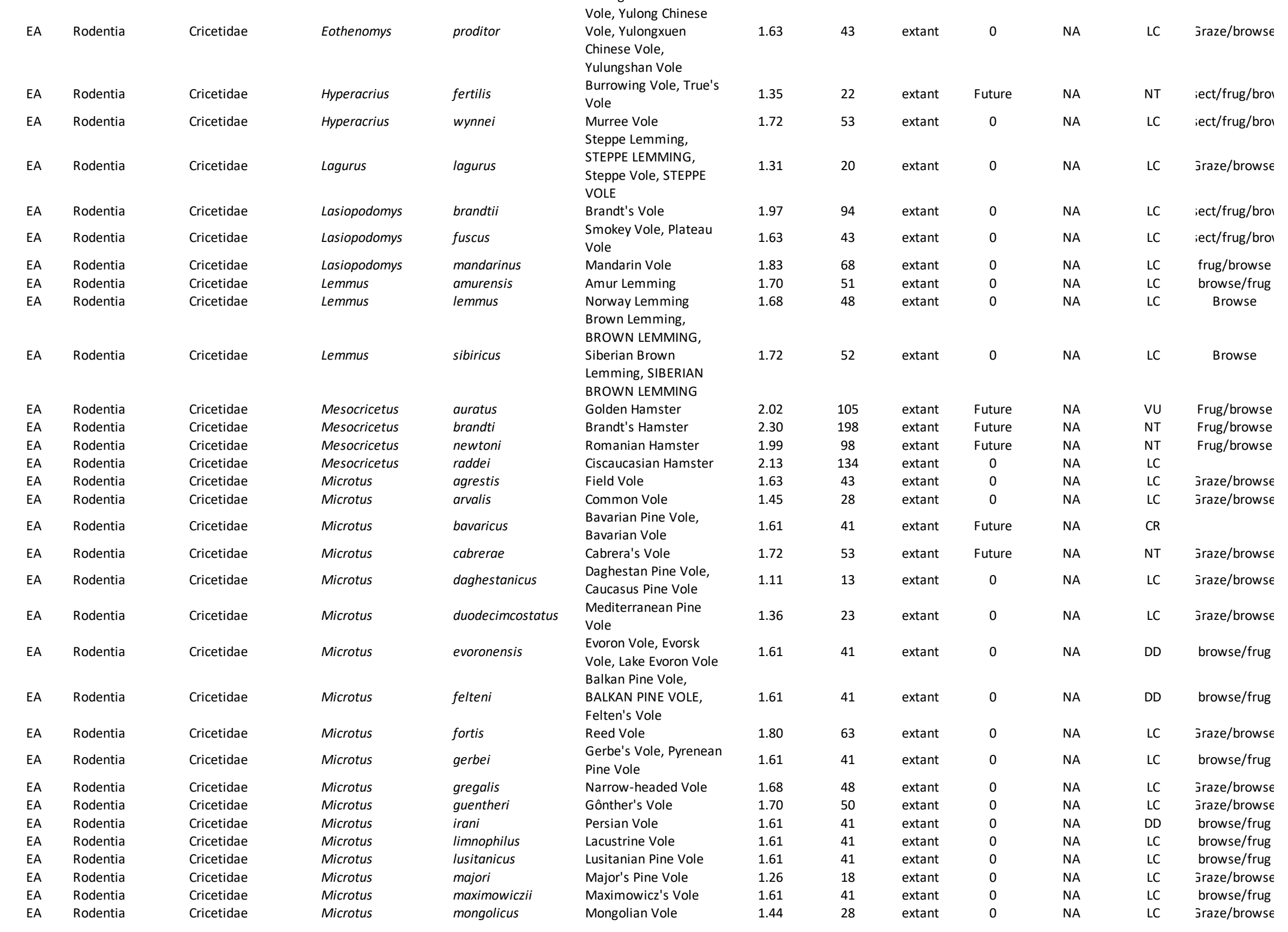




\begin{tabular}{|c|c|c|c|c|c|c|c|c|c|c|c|c|}
\hline EA & Rodentia & Cricetidae & Microtus & mujanensis & Muisk Vole & 1.61 & 41 & extant & 0 & NA & DD & browse/frug \\
\hline EA & Rodentia & Cricetidae & Microtus & multiplex & Alpine Pine Vole & 1.36 & 23 & extant & 0 & NA & LC & J̈raze/browse \\
\hline EA & Rodentia & Cricetidae & Microtus & oeconomus & Root Vole, Tundra Vole & 1.56 & 37 & extant & 0 & NA & $\mathrm{LC}$ & J̈raze/browse \\
\hline EA & Rodentia & Cricetidae & Microtus & savii & $\begin{array}{l}\text { Savi's Pine Vole } \\
\text { Schelkovnikov's Pine }\end{array}$ & 1.30 & 20 & extant & 0 & NA & LC & Jraze/brows \\
\hline EA & Rodentia & Cricetidae & Microtus & schelkovnikovi & $\begin{array}{l}\text { Vole, Schelkovnikov's } \\
\text { Pine Vole }\end{array}$ & 1.61 & 41 & extant & Future & NA & NT & \\
\hline EA & Rodentia & Cricetidae & Microtus & socialis & Social Vole & 1.68 & 48 & extant & 0 & NA & $\mathrm{LC}$ & Jraze/browse \\
\hline EA & Rodentia & Cricetidae & Microtus & subterraneus & $\begin{array}{l}\text { European Pine Vole, } \\
\text { Common Pine Vole }\end{array}$ & 1.29 & 20 & extant & 0 & NA & LC & J̈raze/brows€ \\
\hline EA & Rodentia & Cricetidae & Microtus & tatricus & Tatra Vole & 1.60 & 40 & extant & 0 & NA & LC & J̈raze/browse \\
\hline EA & Rodentia & Cricetidae & Microtus & thomasi & $\begin{array}{l}\text { Thomas's Pine Vole } \\
\text { Baluchistan Vole, }\end{array}$ & 1.61 & 41 & extant & 0 & NA & LC & browse/frug \\
\hline EA & Rodentia & Cricetidae & Microtus & transcaspicus & $\begin{array}{l}\text { Middle East Vole, } \\
\text { Transcaspian Vole }\end{array}$ & 1.61 & 41 & extant & 0 & NA & LC & browse/frug \\
\hline EA & Rodentia & Cricetidae & Myodes & centralis & $\begin{array}{l}\text { Tien Shan Red-backed } \\
\text { Vole }\end{array}$ & 1.51 & 32 & extant & 0 & NA & $\mathrm{LC}$ & Frug/browse \\
\hline EA & Rodentia & Cricetidae & Myopus & schisticolor & Wood Lemming & 1.40 & 25 & extant & 0 & NA & LC & ;ect/frug/brol \\
\hline EA & Rodentia & Cricetidae & Ondatra & zibethicus & Muskrat & 3.06 & 1150 & extant & 0 & NA & LC & Graze/carn \\
\hline EA & Rodentia & Cricetidae & Phodopus & campbelli & Campbell's Hamster & 1.37 & 23 & extant & 0 & NA & LC & ;ect/frug/brol \\
\hline EA & Rodentia & Cricetidae & Phodopus & roborovskii & $\begin{array}{l}\text { Desert Hamster, } \\
\text { Roborowski's Hamster }\end{array}$ & 1.27 & 19 & extant & 0 & NA & $\mathrm{LC}$ & ;ect/frug/brol \\
\hline EA & Rodentia & Cricetidae & Phodopus & sungorus & Dzhungarian Hamster & 1.53 & 34 & extant & 0 & NA & LC & ;ect/frug/brol \\
\hline EA & Rodentia & Cricetidae & Proedromys & bedfordi & Duke Of Bedford's Vole & & & extant & Future & NA & vu & \\
\hline EA & Rodentia & Cricetidae & Prometheomys & schaposchnikowi & Long-clawed Mole Vole & 1.88 & 75 & extant & Future & NA & NT & ;ect/frug/brol \\
\hline EA & Rodentia & Cricetidae & Tscherskia & triton & $\begin{array}{l}\text { Greater Long-tailed } \\
\text { Hamster }\end{array}$ & 2.58 & 380 & extant & 0 & NA & LC & Browse \\
\hline EA & Rodentia & Cricetidae & Volemys & millicens & $\begin{array}{l}\text { Sichuan Vole, Szechuan } \\
\text { Vole }\end{array}$ & 1.63 & 43 & extant & 0 & NA & DD & \\
\hline EA & Rodentia & Cricetidae & Volemys & musseri & Marie's Vole & 1.63 & 43 & extant & 0 & NA & DD & graze/ginsect \\
\hline EA & Rodentia & Dipodidae & Allactaga & balikunica & Balikun Jerboa & 2.17 & 147 & extant & 0 & NA & LC & \\
\hline EA & Rodentia & Dipodidae & Allactaga & bullata & Gobi Jerboa & 2.17 & 147 & extant & 0 & NA & LC & \\
\hline EA & Rodentia & Dipodidae & Allactaga & elater & Small Five-toed Jerboa & 1.77 & 59 & extant & 0 & NA & LC & Graze \\
\hline EA & Rodentia & Dipodidae & Allactaga & euphratica & Euphrates Jerboa & 2.37 & 232 & extant & Future & NA & NT & Graze \\
\hline EA & Rodentia & Dipodidae & Allactaga & firouzi & Iranian Jerboa & 2.17 & 147 & extant & 0 & NA & DD & \\
\hline EA & Rodentia & Dipodidae & Allactaga & hotsoni & $\begin{array}{l}\text { Hotson's Five-toed } \\
\text { Jerboa, Hotson's Jerboa }\end{array}$ & 1.88 & 77 & extant & 0 & NA & LC & Graze \\
\hline EA & Rodentia & Dipodidae & Allactaga & major & Great Jerboa & 2.54 & 350 & extant & 0 & NA & LC & Graze \\
\hline EA & Rodentia & Dipodidae & Allactaga & severtzovi & $\begin{array}{l}\text { Svertzov's Jerboa } \\
\text { Siberian Jerboa, }\end{array}$ & 2.20 & 159 & extant & 0 & NA & LC & Graze \\
\hline EA & Rodentia & Dipodidae & Allactaga & sibirica & $\begin{array}{l}\text { Mongolian Five-toed } \\
\text { Jerboa }\end{array}$ & 1.99 & 98 & extant & 0 & NA & LC & Graze \\
\hline EA & Rodentia & Dipodidae & Allactaga & vinogradovi & Vinogradov's Jerboa & 2.17 & 147 & extant & Future & NA & NT & \\
\hline EA & Rodentia & Dipodidae & Allactodipus & bobrinskii & Bobrinski's Jerboa & & & extant & 0 & NA & LC & \\
\hline EA & Rodentia & Dipodidae & Cardiocranius & paradoxus & $\begin{array}{l}\text { Five-toed Pygmy Jerboa } \\
\text { Hairy-footed Jerboa, }\end{array}$ & 0.93 & 9 & extant & 0 & NA & DD & Frug/browse \\
\hline EA & Rodentia & Dipodidae & Dipus & sagitta & $\begin{array}{l}\text { Northern Three-toed } \\
\text { Jerboa, NORTHERN } \\
\text { THREE-TOED JERBOA }\end{array}$ & 1.93 & 86 & extant & 0 & NA & LC & Frug/browse \\
\hline EA & Rodentia & Dipodidae & Eozapus & setchuanus & Chinese Jumping Mouse & 1.51 & 33 & extant & 0 & NA & LC & Frug/browse \\
\hline EA & Rodentia & Dipodidae & Eremodipus & lichtensteini & Lichtenstein's Jerboa & 1.71 & 51 & extant & 0 & NA & LC & Frug/browse \\
\hline
\end{tabular}




\begin{tabular}{|c|c|c|c|c|c|c|c|c|c|c|c|c|}
\hline EA & Rodentia & Dipodidae & Euchoreutes & naso & $\begin{array}{l}\text { Long-eared Jerboa } \\
\text { Blanford's Jerboa, }\end{array}$ & 1.38 & 24 & extant & 0 & NA & LC & Frug/browse \\
\hline EA & Rodentia & Dipodidae & Jaculus & blanfordi & $\begin{array}{l}\text { Blanford's Jerboa, } \\
\text { Greater Threetoed } \\
\text { Jerboa }\end{array}$ & 1.74 & 55 & extant & 0 & NA & $\mathrm{LC}$ & frug/graze \\
\hline EA & Rodentia & Dipodidae & Jaculus & blanfordi & $\begin{array}{l}\text { Blanford's Jerboa, } \\
\text { Blanford's Jerboa, } \\
\text { Greater Threetoed } \\
\text { Jerboa }\end{array}$ & 1.95 & 89 & extant & 0 & NA & LC & \\
\hline EA & Rodentia & Dipodidae & Jaculus & jaculus & Lesser Egyptian Jerboa & 1.75 & 57 & extant & 0 & NA & LC & frug/graze \\
\hline EA & Rodentia & Dipodidae & Jaculus & orientalis & Greater Egyptian Jerboa & 2.14 & 138 & extant & 0 & NA & LC & frug/graze \\
\hline EA & Rodentia & Dipodidae & Paradipus & ctenodactylus & Comb-toed Jerboa & 2.04 & 109 & extant & 0 & NA & LC & Frug/browse \\
\hline EA & Rodentia & Dipodidae & Pygeretmus & platyurus & Lesser Fat-tailed Jerboa & 1.72 & 52 & extant & 0 & NA & LC & \\
\hline EA & Rodentia & Dipodidae & Pygeretmus & pumilio & $\begin{array}{l}\text { Dwarf Fat-tailed Jerboa, } \\
\text { DWARF FAT-TAILED } \\
\text { JERBOA }\end{array}$ & 1.72 & 52 & extant & 0 & NA & LC & Frug/browse \\
\hline EA & Rodentia & Dipodidae & Pygeretmus & shitkovi & $\begin{array}{l}\text { Greater Fat-tailed } \\
\text { Jerboa } \\
\text { Baluchistan Pygmy }\end{array}$ & 1.72 & 52 & extant & Future & NA & NT & \\
\hline EA & Rodentia & Dipodidae & Salpingotulus & michaelis & $\begin{array}{l}\text { Jerboa, Dwarf Three- } \\
\text { toed Jerboa }\end{array}$ & 0.59 & 4 & extant & 0 & NA & DD & Frug/browse \\
\hline EA & Rodentia & Dipodidae & Salpingotus & crassicauda & $\begin{array}{l}\text { Thick-tailed Pygmy } \\
\text { Jerboa }\end{array}$ & & & extant & 0 & NA & DD & \\
\hline EA & Rodentia & Dipodidae & Salpingotus & heptneri & Heptner's Pygmy Jerboa & & & extant & 0 & NA & DD & \\
\hline EA & Rodentia & Dipodidae & Salpingotus & kozlovi & Kozlov's Pygmy Jerboa & & & extant & 0 & NA & LC & \\
\hline EA & Rodentia & Dipodidae & Salpingotus & pallidus & $\begin{array}{l}\text { Pale Pygmy Jerboa, } \\
\text { Pallid Pygmy Jerboa }\end{array}$ & & & extant & 0 & NA & DD & \\
\hline EA & Rodentia & Dipodidae & Salpingotus & thomasi & Thomas's pygmy jerboa & & & extant & 0 & NA & DD & $\mathrm{DD}$ \\
\hline EA & Rodentia & Dipodidae & Sicista & armenica & Armenian Birch Mouse & 1.00 & 10 & extant & Future & NA & EN & \\
\hline EA & Rodentia & Dipodidae & Sicista & betulina & Northern Birch Mouse & 0.90 & 8 & extant & 0 & NA & LC & Frug/browse \\
\hline EA & Rodentia & Dipodidae & Sicista & caucasica & Caucasian Birch Mouse & 1.00 & 10 & extant & Future & NA & VU & Frug/browse \\
\hline EA & Rodentia & Dipodidae & Sicista & caudata & Long-tailed Birch Mouse & 1.00 & 10 & extant & 0 & NA & DD & frug/browse \\
\hline EA & Rodentia & Dipodidae & Sicista & concolor & Chinese Birch Mouse & 1.00 & 10 & extant & 0 & NA & LC & frug/browse \\
\hline EA & Rodentia & Dipodidae & Sicista & kazbegica & Kazbeg Birch Mouse & 1.00 & 10 & extant & Future & NA & EN & \\
\hline EA & Rodentia & Dipodidae & Sicista & kluchorica & Kluchor Birch Mouse & 1.00 & 10 & extant & Future & NA & NT & \\
\hline EA & Rodentia & Dipodidae & Sicista & napaea & Altai Birch Mouse & 1.00 & 10 & extant & 0 & NA & LC & Frug/browse \\
\hline EA & Rodentia & Dipodidae & Sicista & pseudonapaea & $\begin{array}{l}\text { Grey Birch Mouse, Gray } \\
\text { Birch Mouse }\end{array}$ & 1.00 & 10 & extant & 0 & NA & DD & frug/browse \\
\hline EA & Rodentia & Dipodidae & Sicista & severtzovi & Severtzov's Birch Mouse & 1.00 & 10 & extant & 0 & NA & LC & frug/browse \\
\hline EA & Rodentia & Dipodidae & Sicista & strandi & Strand's Birch Mouse & 1.01 & 10 & extant & 0 & NA & LC & Frug/browse \\
\hline EA & Rodentia & Dipodidae & Sicista & subtilis & Southern Birch Mouse & 1.07 & 12 & extant & 0 & NA & LC & Frug/browse \\
\hline EA & Rodentia & Dipodidae & Sicista & tianshanica & $\begin{array}{l}\text { Tien Shan Birch Mouse } \\
\text { Andrews's Three-toed }\end{array}$ & 1.00 & 10 & extant & 0 & NA & LC & frug/browse \\
\hline EA & Rodentia & Dipodidae & Stylodipus & andrewsi & $\begin{array}{l}\text { Jerboa, Mongolian } \\
\text { Jerboa }\end{array}$ & 1.74 & 55 & extant & 0 & NA & LC & \\
\hline
\end{tabular}




\begin{tabular}{|c|c|c|c|c|c|c|c|c|c|c|c|c|}
\hline EA & Rodentia & Dipodidae & Stylodipus & sungorus & $\begin{array}{l}\text { Mongolian Three-toed } \\
\text { Jerboa }\end{array}$ & 1.74 & 55 & extant & 0 & NA & LC & \\
\hline EA & Rodentia & Dipodidae & Stylodipus & telum & $\begin{array}{l}\text { Thick-tailed Three-toed } \\
\text { Jerboa, THICK-TAILED } \\
\text { THREE-TOED JERBOA }\end{array}$ & 1.74 & 55 & extant & 0 & NA & $\mathrm{LC}$ & Frug/browse \\
\hline EA & Rodentia & Gliridae & Dryomys & laniger & Woolly Dormouse & 1.30 & 20 & extant & 0 & NA & DD & Frug/browse \\
\hline EA & Rodentia & Gliridae & Dryomys & nitedula & Forest Dormouse & 1.53 & 34 & extant & 0 & NA & LC & Frug/browse \\
\hline EA & Rodentia & Gliridae & Eliomys & melanurus & Asian Garden Dormouse & 1.87 & 75 & extant & 0 & NA & LC & frug/ginsect \\
\hline EA & Rodentia & Gliridae & Eliomys & quercinus & Garden Dormouse & 2.06 & 115 & extant & Future & NA & NT & frug/ginsect \\
\hline EA & Rodentia & Gliridae & Muscardinus & avellanarius & $\begin{array}{l}\text { Hazel Dormouse, } \\
\text { Common Dormouse }\end{array}$ & 1.44 & 28 & extant & 0 & NA & LC & \\
\hline EA & Rodentia & Gliridae & Myomimus & personatus & $\begin{array}{l}\text { Masked Mouse-tailed } \\
\text { Dormouse }\end{array}$ & & & extant & 0 & NA & DD & \\
\hline EA & Rodentia & Gliridae & Myomimus & roachi & $\begin{array}{l}\text { Mouse-tailed } \\
\text { Dormouse, Roach's } \\
\text { Mouse-tailed Dormouse }\end{array}$ & & & extant & Future & NA & Vu & \\
\hline EA & Rodentia & Gliridae & Myomimus & setzeri & $\begin{array}{l}\text { Setzer's Mouse-tailed } \\
\text { Dormouse }\end{array}$ & & & extant & 0 & NA & DD & \\
\hline EA & Rodentia & Gliridae & Selevinia & betpakdalaensis & Desert Dormouse & 1.45 & 28 & extant & 0 & NA & DD & Frug/browse \\
\hline EA & Rodentia & Heteromyidae & Dipodomys & californicus & California Kangaroo Rat & 1.93 & 85 & extant & 0 & NA & LC & Frug \\
\hline EA & Rodentia & Hystricidae & Atherurus & macrourus & $\begin{array}{l}\text { Asiatic Brush-tailed } \\
\text { Porcupine } \\
\text { Malayan Porcupine }\end{array}$ & 3.30 & 2000 & extant & 0 & NA & $\mathrm{LC}$ & frug/browse \\
\hline EA & Rodentia & Hystricidae & Hystrix & brachyura & $\begin{array}{l}\text { Vlalayan Porcupıne, } \\
\text { Himalayan Crestless } \\
\text { Porcupine }\end{array}$ & 3.90 & 8000 & extant & 0 & NA & $\mathrm{LC}$ & Browse \\
\hline EA & Rodentia & Hystricidae & Hystrix & cristata & $\begin{array}{l}\text { Crested Porcupine, } \\
\text { North African Crested } \\
\text { Porcupine }\end{array}$ & 4.10 & 12500 & extant & 0 & NA & $\mathrm{LC}$ & browse/frug \\
\hline EA & Rodentia & Hystricidae & Hystrix & indica & $\begin{array}{l}\text { Indian Crested } \\
\text { Porcupine }\end{array}$ & 4.17 & 14650 & extant & 0 & NA & $\mathrm{LC}$ & Browse/frug \\
\hline EA & Rodentia & Hystricidae & Trichys & fasciculata & $\begin{array}{l}\text { Long-tailed Porcupine } \\
\text { Cairo Spiny Mouse, }\end{array}$ & 3.19 & 1560 & extant & 0 & NA & $\mathrm{LC}$ & Browse/frug \\
\hline EA & Rodentia & Muridae & Acomys & cahirinus & $\begin{array}{l}\text { Greater Wilfred's } \\
\text { Mouse, Northeast } \\
\text { African Spiny Mouse } \\
\text { Asia Minor Spiny }\end{array}$ & 1.82 & 66 & extant & 0 & NA & $\mathrm{LC}$ & frug/browse \\
\hline EA & Rodentia & Muridae & Acomys & cilicicus & $\begin{array}{l}\text { Mouse, Turkish Spiny } \\
\text { Mouse }\end{array}$ & 1.51 & 33 & extant & 0 & NA & $\mathrm{DD}$ & \\
\hline EA & Rodentia & Muridae & Acomys & russatus & Golden Spiny Mouse & 1.70 & 50 & extant & 0 & NA & $\mathrm{LC}$ & frug/browse \\
\hline EA & Rodentia & Muridae & Alticola & albicaudus & $\begin{array}{l}\text { White-tailed Mountain } \\
\text { Vole }\end{array}$ & & & extant & 0 & NA & DD & \\
\hline EA & Rodentia & Muridae & Alticola & stracheyi & $\begin{array}{l}\text { Stoliczka's Mountain } \\
\text { Vole }\end{array}$ & & & extant & 0 & NA & LC & \\
\hline EA & Rodentia & Muridae & Apodemus & agrarius & Striped Field Mouse & 1.37 & 23 & extant & 0 & NA & LC & frug/ginsect \\
\hline EA & Rodentia & Muridae & Apodemus & alpicola & Alpine Field Mouse & 1.45 & 28 & extant & 0 & NA & LC & frug/ginsect \\
\hline EA & Rodentia & Muridae & Apodemus & arianus & $\begin{array}{l}\text { Yellow-necked Field } \\
\text { Mouse }\end{array}$ & 1.47 & 30 & extant & 0 & NA & $\mathrm{LC}$ & \\
\hline EA & Rodentia & Muridae & Apodemus & chevrieri & Chevrier's Field Mouse & 1.52 & 33 & extant & 0 & NA & LC & frug/ginsect \\
\hline EA & Rodentia & Muridae & Apodemus & draco & $\begin{array}{l}\text { South China Field } \\
\text { Mouse }\end{array}$ & 1.35 & 22 & extant & 0 & NA & LC & frug/ginsect \\
\hline
\end{tabular}




\begin{tabular}{|c|c|c|c|c|c|c|c|c|c|c|c|c|}
\hline EA & Rodentia & Muridae & Apodemus & flavicollis & $\begin{array}{l}\text { Yellow-necked Field } \\
\text { Mouse }\end{array}$ & 1.43 & 27 & extant & 0 & NA & LC & frug/ginsect \\
\hline EA & Rodentia & Muridae & Apodemus & fulvipectus & $\begin{array}{l}\text { Steppe Field Mouse } \\
\text { Nepalese Field Mouse, }\end{array}$ & 1.48 & 30 & extant & 0 & NA & LC & frug/ginsect \\
\hline EA & Rodentia & Muridae & Apodemus & gurkha & $\begin{array}{l}\text { Himalayan Wood } \\
\text { Mouse, Himalayan Field } \\
\text { Mouse }\end{array}$ & 1.60 & 40 & extant & Future & NA & EN & frug/ginsect \\
\hline EA & Rodentia & Muridae & Apodemus & hermonensis & $\begin{array}{l}\text { Steppe Field Mouse } \\
\text { Hyrcanian Field Mouse, }\end{array}$ & 1.32 & 21 & extant & 0 & NA & LC & frug/ginsect \\
\hline EA & Rodentia & Muridae & Apodemus & hyrcanicus & $\begin{array}{l}\text { Caucasian Field Mouse, } \\
\text { Caucasus Field Mouse, } \\
\text { Talysh Field Mouse } \\
\text { Large-eared Field }\end{array}$ & 1.47 & 30 & extant & Future & NA & NT & \\
\hline EA & Rodentia & Muridae & Apodemus & latronum & $\begin{array}{l}\text { Mouse, Sichuan Field } \\
\text { Mouse } \\
\text { Eastern Broad-toothed }\end{array}$ & 1.55 & 35 & extant & 0 & NA & LC & frug/ginsect \\
\hline EA & Rodentia & Muridae & Apodemus & mystacinus & $\begin{array}{l}\text { Field Mouse, Broad- } \\
\text { toothed Field Mouse }\end{array}$ & 1.66 & 46 & extant & 0 & NA & LC & frug/ginsect \\
\hline EA & Rodentia & Muridae & Apodemus & pallipes & $\begin{array}{l}\text { Himalayan Field Mouse, } \\
\text { Ward's Field Mouse }\end{array}$ & 1.47 & 30 & extant & 0 & NA & $\mathrm{LC}$ & \\
\hline EA & Rodentia & Muridae & Apodemus & peninsulae & Korean Field Mouse & 1.52 & 33 & extant & 0 & NA & LC & frug/ginsect \\
\hline EA & Rodentia & Muridae & Apodemus & ponticus & Black Sea Field Mouse & 1.47 & 30 & extant & 0 & NA & $\mathrm{LC}$ & \\
\hline EA & Rodentia & Muridae & Apodemus & rusiges & $\begin{array}{l}\text { Kashmir Field Mouse } \\
\text { Long-tailed Field }\end{array}$ & 1.47 & 30 & extant & 0 & NA & LC & \\
\hline EA & Rodentia & Muridae & Apodemus & sylvaticus & $\begin{array}{l}\text { Mouse, Wood Mouse, } \\
\text { Small Wood Mouse } \\
\text { Herb Field Mouse, Ural }\end{array}$ & 1.60 & 39 & extant & 0 & NA & $\mathrm{LC}$ & frug/ginsect \\
\hline EA & Rodentia & Muridae & Apodemus & uralensis & $\begin{array}{l}\text { Field Mouse, Pygmy } \\
\text { Field Mouse }\end{array}$ & 1.23 & 17 & extant & 0 & NA & LC & frug/ginsect \\
\hline EA & Rodentia & Muridae & Arvicanthis & niloticus & $\begin{array}{l}\text { African Grass Rat, } \\
\text { African Arvicanthis }\end{array}$ & 2.03 & 107 & extant & 0 & NA & LC & frug/graze \\
\hline EA & Rodentia & Muridae & Arvicola & amphibius & $\begin{array}{l}\text { European Water Vole, } \\
\text { Water Vole, Eurasian } \\
\text { Water Vole }\end{array}$ & 2.08 & 120 & extant & 0 & NA & LC & Jraze/brows€ \\
\hline EA & Rodentia & Muridae & Bandicota & bengalensis & $\begin{array}{l}\text { Lesser Bandicoot Rat, } \\
\text { Indian Mole-rat, Sind } \\
\text { Rice Rat }\end{array}$ & 2.18 & 150 & extant & 0 & NA & LC & 3rowse/graze \\
\hline EA & Rodentia & Muridae & Bandicota & indica & Greater Bandicoot Rat & 2.70 & 500 & extant & 0 & NA & $\mathrm{LC}$ & 3rowse/graze \\
\hline EA & Rodentia & Muridae & Bandicota & savilei & $\begin{array}{l}\text { Savile's Bandicoot Rat } \\
\text { Berdmore's Berylmys, }\end{array}$ & 2.30 & 199 & extant & 0 & NA & LC & 3rowse/graze \\
\hline EA & Rodentia & Muridae & Berylmys & berdmorei & $\begin{array}{l}\text { Small White-toothed } \\
\text { Rat } \\
\text { Bower's White-toothed }\end{array}$ & 2.37 & 235 & extant & 0 & NA & $\mathrm{LC}$ & :/browse/gins \\
\hline EA & Rodentia & Muridae & Berylmys & bowersi & $\begin{array}{l}\text { Rat, Bower's Rat, } \\
\text { Bower's Berylmys }\end{array}$ & 2.48 & 300 & extant & 0 & NA & $\mathrm{LC}$ & :/browse/gins \\
\hline EA & Rodentia & Muridae & Berylmys & mackenziei & $\begin{array}{l}\text { Kenneth's White- } \\
\text { toothed Rat, } \\
\text { Mackenzie's Berylmys, } \\
\text { Mackenzie's Rat }\end{array}$ & 2.41 & 256 & extant & 0 & NA & DD & :/browse/gins \\
\hline EA & Rodentia & Muridae & Berylmys & manipulus & $\begin{array}{l}\text { Manipur White-toothed } \\
\text { Rat, Manipur Berylmys }\end{array}$ & 2.06 & 116 & extant & 0 & NA & DD & :/browse/gins \\
\hline
\end{tabular}




\begin{tabular}{|c|c|c|c|c|c|c|c|c|c|c|c|c|}
\hline EA & Rodentia & Muridae & Blanfordimys & bucharicus & & & & extant & 0 & NA & LC & \\
\hline EA & Rodentia & Muridae & Brachiones & przewalskii & $\begin{array}{l}\text { Przewalski's Jird, } \\
\text { Przewalski's Gerbil }\end{array}$ & & & extant & 0 & NA & $\mathrm{LC}$ & \\
\hline EA & Rodentia & Muridae & Caryomys & eva & $\begin{array}{l}\text { Eva's Red-backed Vole, } \\
\text { Gansu Vole, Eva's Vole, } \\
\text { Ganzu Vole, Taozhou } \\
\text { Vole }\end{array}$ & 1.43 & 27 & extant & 0 & NA & LC & Jraze/browse \\
\hline EA & Rodentia & Muridae & Caryomys & inez & $\begin{array}{l}\text { Inez's Red-backed Vole, } \\
\text { Inez's Vole, Kolan Vole } \\
\text { Indochinese }\end{array}$ & 1.43 & 27 & extant & 0 & NA & LC & J̄raze/browse \\
\hline EA & Rodentia & Muridae & Chiromyscus & chiropus & $\begin{array}{l}\text { Chiromyscus, Fea's Tree } \\
\text { Rat }\end{array}$ & 1.89 & 78 & extant & 0 & NA & LC & rowse/ginsec \\
\hline EA & Rodentia & Muridae & Chiropodomys & gliroides & $\begin{array}{l}\text { Indomalayan Pencil- } \\
\text { tailed Tree Mouse, } \\
\text { Pencil-tailed Tree } \\
\text { Mouse }\end{array}$ & 1.40 & 25 & extant & 0 & NA & LC & Frug/browse \\
\hline EA & Rodentia & Muridae & Chodsigoa & smithii & Smith's Shrew & 2.58 & 379 & extant & Future & NA & NT & iect/frug/brol \\
\hline EA & Rodentia & Muridae & Cremnomys & cutchicus & $\begin{array}{l}\text { Cutch Rock-rat, Cutch } \\
\text { Rat }\end{array}$ & 1.78 & 60 & extant & 0 & NA & LC & iect/frug/brol \\
\hline EA & Rodentia & Muridae & Cremnomys & elvira & $\begin{array}{l}\text { Large Rock-rat, Elvira } \\
\text { Rat }\end{array}$ & 1.89 & 77 & extant & Future & NA & $C R$ & iect/frug/brol \\
\hline EA & Rodentia & Muridae & Dacnomys & millardi & $\begin{array}{l}\text { Millard's Rat } \\
\text { Neartic Collared }\end{array}$ & & & extant & 0 & NA & DD & \\
\hline EA & Rodentia & Muridae & Dicrostonyx & groenlandicus & $\begin{array}{l}\text { Lemming, Northern } \\
\text { Collared Lemming }\end{array}$ & 1.67 & 47 & extant & 0 & NA & LC & 3rowse/graze \\
\hline EA & Rodentia & Muridae & Diomys & crumpi & Crump's Mouse & & & extant & 0 & NA & DD & \\
\hline EA & Rodentia & Muridae & Dipodillus & dasyurus & Wagner's Gerbil & 1.37 & 24 & extant & 0 & NA & LC & frug/browse \\
\hline EA & Rodentia & Muridae & Eospalax & fontanierii & $\begin{array}{l}\text { Chinese Zokor, Plateau } \\
\text { Zokor }\end{array}$ & 2.39 & 245 & extant & 0 & NA & LC & iect/frug/brol \\
\hline EA & Rodentia & Muridae & Eospalax & rothschildi & Rothschild's Zokor & 2.38 & 240 & extant & 0 & NA & LC & ;ect/frug/brol \\
\hline EA & Rodentia & Muridae & Gerbillus & andersoni & Anderson's Gerbil & 1.39 & 25 & extant & 0 & NA & LC & frug/browse \\
\hline EA & Rodentia & Muridae & Gerbillus & aquilus & Swarthy Gerbil & 1.45 & 28 & extant & 0 & NA & LC & \\
\hline EA & Rodentia & Muridae & Gerbillus & cheesmani & Cheesman's Gerbil & 1.45 & 28 & extant & 0 & NA & LC & frug/browse \\
\hline EA & Rodentia & Muridae & Gerbillus & famulus & $\begin{array}{l}\text { Black-tufted Gerbil } \\
\text { Little Hairy-footed }\end{array}$ & 1.57 & 37 & extant & 0 & NA & LC & frug/browse \\
\hline EA & Rodentia & Muridae & Gerbillus & gleadowi & $\begin{array}{l}\text { Gerbil, Indian Hairy- } \\
\text { footed Gerbil }\end{array}$ & 1.38 & 24 & extant & 0 & NA & LC & frug/browse \\
\hline EA & Rodentia & Muridae & Gerbillus & henleyi & Pygmy Gerbil & 0.90 & 8 & extant & 0 & NA & LC & frug/browse \\
\hline EA & Rodentia & Muridae & Gerbillus & mesopotamiae & Mesopotamian Gerbil & 1.30 & 20 & extant & 0 & NA & LC & frug/browse \\
\hline EA & Rodentia & Muridae & Gerbillus & nanus & $\begin{array}{l}\text { Dwarf Gerbil, } \\
\text { Baluchistan Gerbil }\end{array}$ & 1.41 & 26 & extant & 0 & NA & LC & frug/browse \\
\hline EA & Rodentia & Muridae & Gerbillus & poecilops & Large Aden Gerbil & 1.50 & 32 & extant & 0 & NA & LC & frug/browse \\
\hline EA & Rodentia & Muridae & Golunda & ellioti & $\begin{array}{l}\text { Indian Bush-rat, Indian } \\
\text { Bush Rat }\end{array}$ & 1.75 & 57 & extant & 0 & NA & LC & ;ect/frug/brol \\
\hline EA & Rodentia & Muridae & Hadromys & humei & $\begin{array}{l}\text { Hume's Rat, Manipur } \\
\text { Bush Rat } \\
\text { Lesser Marmoset Rat }\end{array}$ & 1.77 & 59 & extant & Future & NA & EN & Frug/ginsect \\
\hline EA & Rodentia & Muridae & Hapalomys & delacouri & $\begin{array}{l}\text { Delacour's Marmoset } \\
\text { Rat }\end{array}$ & 1.81 & 64 & extant & Future & NA & vu & owse (bambo \\
\hline EA & Rodentia & Muridae & Hapalomys & longicaudatus & $\begin{array}{l}\text { Greater Marmoset Rat, } \\
\text { Marmoset Rat }\end{array}$ & 1.85 & 70 & extant & Future & NA & EN & owse (bambo \\
\hline
\end{tabular}




\begin{tabular}{|c|c|c|c|c|c|c|c|c|c|c|c|c|}
\hline EA & Rodentia & Muridae & Lenothrix & canus & $\begin{array}{l}\text { Sundaic Lenothrix, Gray } \\
\text { Tree Rat }\end{array}$ & 2.08 & 120 & extant & 0 & NA & LC & ;ect/browse/1 \\
\hline EA & Rodentia & Muridae & Leopoldamys & edwardsi & $\begin{array}{l}\text { Edward's Rat, } \\
\text { Edwards's Long-tailed } \\
\text { Giant Rat }\end{array}$ & 2.48 & 300 & extant & 0 & NA & LC & iect/browse/1 \\
\hline EA & Rodentia & Muridae & Leopoldamys & neilli & $\begin{array}{l}\text { Neill's Leopoldamys, } \\
\text { Neill's Long-tailed Giant } \\
\text { Rat }\end{array}$ & 2.34 & 219 & extant & 0 & NA & LC & ;ect/browse/1 \\
\hline EA & Rodentia & Muridae & Leopoldamys & sabanus & $\begin{array}{l}\text { Long-tailed Giant Rat, } \\
\text { Noisy Rat }\end{array}$ & 2.54 & 344 & extant & 0 & NA & LC & ;ect/browse/1 \\
\hline EA & Rodentia & Muridae & Madromys & blanfordi & $\begin{array}{l}\text { White-tailed Wood Rat, } \\
\text { Blanford's Rat, } \\
\text { Blanford's Rat } \\
\text { Malayan Mountain }\end{array}$ & 2.15 & 141 & extant & 0 & NA & LC & iect/frug/brol \\
\hline EA & Rodentia & Muridae & Maxomys & inas & $\begin{array}{l}\text { Maxomys, Malayan } \\
\text { Mountain Spiny Rat } \\
\text { Indochinese Mountain }\end{array}$ & 1.95 & 90 & extant & 0 & NA & $\mathrm{LC}$ & ;ect/browse/1 \\
\hline EA & Rodentia & Muridae & Maxomys & moi & $\begin{array}{l}\text { Maxomys, Mo's Spiny } \\
\text { Rat } \\
\text { Rajah Sundaic }\end{array}$ & 2.17 & 147 & extant & 0 & NA & LC & ;ect/browse/1 \\
\hline EA & Rodentia & Muridae & Maxomys & rajah & $\begin{array}{l}\text { Maxomys, Rajah Spiny } \\
\text { Rat }\end{array}$ & 2.18 & 150 & extant & Future & NA & Vu & ;ect/browse/1 \\
\hline EA & Rodentia & Muridae & Maxomys & surifer & $\begin{array}{l}\text { Indomalayan Maxomys, } \\
\text { Red Spiny Rat } \\
\text { Whitehead's Sundaic }\end{array}$ & 2.14 & 139 & extant & 0 & NA & LC & ;ect/browse/1 \\
\hline EA & Rodentia & Muridae & Maxomys & whiteheadi & $\begin{array}{l}\text { Maxomys, Whitehead's } \\
\text { Spiny Rat }\end{array}$ & 1.74 & 54 & extant & Future & NA & vU & ;ect/browse/1 \\
\hline EA & Rodentia & Muridae & Meriones & arimalius & Arabian Jird & 2.00 & 100 & extant & 0 & NA & LC & \\
\hline EA & Rodentia & Muridae & Meriones & chengi & $\begin{array}{l}\text { Cheng's Jird, Cheng's } \\
\text { Gerbil }\end{array}$ & 2.00 & 100 & extant & 0 & NA & LC & \\
\hline EA & Rodentia & Muridae & Meriones & crassus & Sundevall's Jird & 1.90 & 80 & extant & 0 & NA & LC & frug/browse \\
\hline EA & Rodentia & Muridae & Meriones & dahli & Dahl's Jird & 2.00 & 100 & extant & Future & NA & EN & \\
\hline EA & Rodentia & Muridae & Meriones & hurrianae & $\begin{array}{l}\text { Indian Desert Gerbil, } \\
\text { Indian Desert Jird }\end{array}$ & 1.85 & 70 & extant & 0 & NA & LC & frug/browse \\
\hline EA & Rodentia & Muridae & Meriones & libycus & Libyan Jird & 1.96 & 91 & extant & 0 & NA & LC & frug/browse \\
\hline EA & Rodentia & Muridae & Meriones & meridianus & $\begin{array}{l}\text { Mid-day Gerbil, Mid-day } \\
\text { Jird }\end{array}$ & 1.79 & 62 & extant & 0 & NA & LC & frug/browse \\
\hline EA & Rodentia & Muridae & Meriones & persicus & Persian Jird & 2.00 & 100 & extant & 0 & NA & LC & frug/browse \\
\hline EA & Rodentia & Muridae & Meriones & rex & King Jird & 2.10 & 125 & extant & 0 & NA & LC & frug/browse \\
\hline EA & Rodentia & Muridae & Meriones & sacramenti & $\begin{array}{l}\text { Buxton's Jird } \\
\text { Tamarisk Gerbil, }\end{array}$ & 2.30 & 200 & extant & Future & NA & VU & frug/browse \\
\hline EA & Rodentia & Muridae & Meriones & tamariscinus & $\begin{array}{l}\text { Tamarisk Jird, } \\
\text { TAMARISK JIRD }\end{array}$ & 2.08 & 120 & extant & 0 & NA & LC & frug/browse \\
\hline EA & Rodentia & Muridae & Meriones & tristrami & Tristram's Jird & 2.02 & 105 & extant & 0 & NA & LC & frug/browse \\
\hline EA & Rodentia & Muridae & Meriones & unguiculatus & $\begin{array}{l}\text { Mongolian Gerbil, } \\
\text { Mongolian Jird }\end{array}$ & 1.73 & 53 & extant & 0 & NA & LC & frug/browse \\
\hline EA & Rodentia & Muridae & Meriones & vinogradovi & Vinogradov's Jird & 2.18 & 150 & extant & 0 & NA & LC & frug/browse \\
\hline EA & Rodentia & Muridae & Meriones & zarudnyi & Zarundny's Jird & 1.92 & 82 & extant & 0 & NA & DD & frug/browse \\
\hline EA & Rodentia & Muridae & Micromys & minutus & $\begin{array}{l}\text { Eurasian Harvest } \\
\text { Mouse, Harvest Mouse }\end{array}$ & 0.78 & 6 & extant & 0 & NA & LC & iect/frug/brol \\
\hline EA & Rodentia & Muridae & Microtus & arvalis & Common Vole & 2.07 & 119 & extant & 0 & NA & LC & browse/frug \\
\hline EA & Rodentia & Muridae & Microtus & clarkei & Clarke's Vole & 2.07 & 119 & extant & 0 & NA & LC & browse/frug \\
\hline
\end{tabular}




\begin{tabular}{|c|c|c|c|c|c|c|c|c|c|c|c|c|}
\hline EA & Rodentia & Muridae & Microtus & daghestanicus & $\begin{array}{l}\text { Daghestan Pine Vole, } \\
\text { Caucasus Pine Vole }\end{array}$ & 2.07 & 119 & extant & 0 & NA & LC & browse/frug \\
\hline EA & Rodentia & Muridae & Microtus & hyperborus & & 2.07 & 119 & extant & 0 & NA & & browse/frug \\
\hline EA & Rodentia & Muridae & Microtus & ilaeus & $\begin{array}{l}\text { Kazakhstan Vole, Tien } \\
\text { Shan Vole }\end{array}$ & 2.07 & 119 & extant & 0 & NA & LC & browse/frug \\
\hline EA & Rodentia & Muridae & Microtus & levis & $\begin{array}{l}\text { East European Vole, } \\
\text { Sibling Vole }\end{array}$ & 1.57 & 37 & extant & 0 & NA & LC & Jraze/brows \\
\hline EA & Rodentia & Muridae & Microtus & middendorffi & $\begin{array}{l}\text { Middendorff's Vole } \\
\text { Baluchistan Vole, }\end{array}$ & 2.07 & 119 & extant & 0 & NA & LC & browse/frug \\
\hline EA & Rodentia & Muridae & Microtus & transcaspicus & $\begin{array}{l}\text { Middle East Vole, } \\
\text { Transcaspian Vole }\end{array}$ & 2.07 & 119 & extant & 0 & NA & LC & browse/frug \\
\hline EA & Rodentia & Muridae & Millardia & gleadowi & $\begin{array}{l}\text { Sand-coloured Metad, } \\
\text { Sand-colored Soft- } \\
\text { furred Rat }\end{array}$ & 1.49 & 31 & extant & 0 & NA & LC & ect/frug/brol \\
\hline EA & Rodentia & Muridae & Millardia & kathleenae & $\begin{array}{l}\text { Burmese Metad, Miss } \\
\text { Ryley's Soft-furred Rat } \\
\text { Kondana Rat, Kondana }\end{array}$ & 1.97 & 93 & extant & 0 & NA & LC & ect/frug/bro \\
\hline EA & Rodentia & Muridae & Millardia & kondana & $\begin{array}{l}\text { Soft-furred Rat, Large } \\
\text { Metad }\end{array}$ & 2.17 & 147 & extant & Future & NA & CR & iect/frug/brol \\
\hline EA & Rodentia & Muridae & Millardia & meltada & $\begin{array}{l}\text { Soft-furred Metad, Soft- } \\
\text { furred Field Rat, Soft- } \\
\text { furred Rat } \\
\text { Common Indian Field }\end{array}$ & 1.85 & 70 & extant & 0 & NA & LC & ect/frug/bro \\
\hline EA & Rodentia & Muridae & Mus & booduga & $\begin{array}{l}\text { Mouse, Little Indian } \\
\text { Field Mouse }\end{array}$ & 1.10 & 13 & extant & 0 & NA & LC & frug/browse \\
\hline EA & Rodentia & Muridae & Mus & caroli & $\begin{array}{l}\text { Ryukyu Mouse, Ricefield } \\
\text { Mouse }\end{array}$ & 1.05 & 11 & extant & 0 & NA & LC & frug/browse \\
\hline EA & Rodentia & Muridae & Mus & cervicolor & Fawn-colored Mouse & 1.25 & 18 & extant & 0 & NA & LC & frug/browse \\
\hline EA & Rodentia & Muridae & Mus & cookii & $\begin{array}{l}\text { Cook's Mouse, Ryley's } \\
\text { Spiny Mouse }\end{array}$ & 1.31 & 20 & extant & 0 & NA & LC & frug/browse \\
\hline EA & Rodentia & Muridae & Mus & famulus & $\begin{array}{l}\text { Bonhote's Mouse, } \\
\text { Servant Mouse }\end{array}$ & 1.36 & 23 & extant & Future & NA & EN & frug/browse \\
\hline EA & Rodentia & Muridae & Mus & macedonicus & $\begin{array}{l}\text { Macedonian Mouse, } \\
\text { Balkan Short-tailed } \\
\text { Mouse }\end{array}$ & 1.18 & 15 & extant & 0 & NA & LC & frug/browse \\
\hline EA & Rodentia & Muridae & Mus & musculus & $\begin{array}{l}\text { House Mouse } \\
\text { Gairdner's }\end{array}$ & 1.18 & 15 & extant & 0 & NA & $\mathrm{LC}$ & frug/browse \\
\hline EA & Rodentia & Muridae & Mus & pahari & $\begin{array}{l}\text { Shrewmouse, Sikkim } \\
\text { Mouse } \\
\text { Wroughton's Small }\end{array}$ & 1.41 & 26 & extant & 0 & NA & LC & frug/browse \\
\hline EA & Rodentia & Muridae & Mus & phillipsi & $\begin{array}{l}\text { Spiny Mouse, Phillips's } \\
\text { Mouse }\end{array}$ & 1.09 & 12 & extant & 0 & NA & LC & frug/browse \\
\hline EA & Rodentia & Muridae & Mus & platythrix & $\begin{array}{l}\text { Brown Spiny Mouse, } \\
\text { Flat-haired Mouse }\end{array}$ & 1.40 & 25 & extant & 0 & NA & LC & frug/browse \\
\hline EA & Rodentia & Muridae & Mus & saxicola & $\begin{array}{l}\text { Brown Spiny Mouse, } \\
\text { Rock-loving Mouse }\end{array}$ & 1.33 & 21 & extant & 0 & NA & LC & frug/browse \\
\hline EA & Rodentia & Muridae & Mus & shortridgei & $\begin{array}{l}\text { Shortridge's Mouse, } \\
\text { Shortridge's Mouse }\end{array}$ & 1.54 & 35 & extant & 0 & NA & LC & frug/browse \\
\hline EA & Rodentia & Muridae & Mus & spicilegus & $\begin{array}{l}\text { Mound-building Mouse, } \\
\text { Steppe Mouse }\end{array}$ & 1.15 & 14 & extant & 0 & NA & $\mathrm{LC}$ & frug/browse \\
\hline
\end{tabular}




\begin{tabular}{|c|c|c|c|c|c|c|c|c|c|c|c|c|}
\hline EA & Rodentia & Muridae & Mus & spretus & $\begin{array}{l}\text { Western Mediterranean } \\
\text { Mouse, WESTERN } \\
\text { MEDITERRANEAN } \\
\text { MOUSE }\end{array}$ & 1.11 & 13 & extant & 0 & NA & LC & frug/browse \\
\hline EA & Rodentia & Muridae & Mus & terricolor & Earth-colored Mouse & 0.95 & 9 & extant & 0 & NA & $\mathrm{LC}$ & frug/browse \\
\hline EA & Rodentia & Muridae & Myodes & glareolus & Bank Vole & 1.33 & 21 & extant & 0 & NA & $\mathrm{LC}$ & Frug/browse \\
\hline EA & Rodentia & Muridae & Myodes & regulus & $\begin{array}{l}\text { Southern Red-backed } \\
\text { Vole, Royal Vole, } \\
\text { Korean Red-backed Vole }\end{array}$ & 1.40 & 25 & extant & 0 & NA & LC & \\
\hline EA & Rodentia & Muridae & Myodes & rufocanus & $\begin{array}{l}\text { Grey Red-backed Vole, } \\
\text { GREY-SIDED VOLE, Gray } \\
\text { Red-backed Vole }\end{array}$ & 1.57 & 37 & extant & 0 & NA & $\mathrm{LC}$ & Frug/browse \\
\hline EA & Rodentia & Muridae & Myodes & rutilus & $\begin{array}{l}\text { Northern Red-backed } \\
\text { Vole, RED VOLE }\end{array}$ & 1.44 & 28 & extant & 0 & NA & $\mathrm{LC}$ & Frug/browse \\
\hline EA & Rodentia & Muridae & Myodes & shanseius & $\begin{array}{l}\text { Shanxi Red-backed } \\
\text { Vole, Shansei Vole }\end{array}$ & 1.40 & 25 & extant & 0 & NA & $\mathrm{LC}$ & \\
\hline EA & Rodentia & Muridae & Myomyscus & yemeni & $\begin{array}{l}\text { Yemeni Mouse, Yemen } \\
\text { White-footed Rat }\end{array}$ & 1.61 & 41 & extant & 0 & NA & $\mathrm{LC}$ & \\
\hline EA & Rodentia & Muridae & Myospalax & psilurus & & & & extant & 0 & NA & $\mathrm{LC}$ & \\
\hline EA & Rodentia & Muridae & Neodon & irene & $\begin{array}{l}\text { Irene's Mountain Vole, } \\
\text { Chinese Scrub Vole }\end{array}$ & 1.65 & 45 & extant & 0 & NA & LC & \\
\hline EA & Rodentia & Muridae & Neodon & juldaschi & Juniper Vole & 1.65 & 45 & extant & 0 & NA & LC & frug/browse \\
\hline EA & Rodentia & Muridae & Neodon & leucurus & & 1.65 & 45 & extant & 0 & NA & & \\
\hline EA & Rodentia & Muridae & Neodon & sikimensis & Sikkim Vole & 1.65 & 45 & extant & 0 & NA & LC & browse/frug \\
\hline EA & Rodentia & Muridae & Nesokia & bunnii & $\begin{array}{l}\text { Bunn's Short-tailed } \\
\text { Bandicoot Rat }\end{array}$ & 2.25 & 178 & extant & Future & NA & EN & \\
\hline EA & Rodentia & Muridae & Nesokia & indica & $\begin{array}{l}\text { Short-tailed Bandicoot } \\
\text { Rat } \\
\text { Anderson's Niviventer, }\end{array}$ & 2.25 & 178 & extant & 0 & NA & $\mathrm{LC}$ & graze/frug \\
\hline EA & Rodentia & Muridae & Niviventer & andersoni & $\begin{array}{l}\text { Anderson's White- } \\
\text { bellied Rat } \\
\text { Brahma White-bellied }\end{array}$ & 2.17 & 147 & extant & 0 & NA & LC & iect/frug/brol \\
\hline EA & Rodentia & Muridae & Niviventer & brahma & $\begin{array}{l}\text { Rat, Thomas' Chestnut } \\
\text { Rat }\end{array}$ & 1.99 & 97 & extant & 0 & NA & $\mathrm{LC}$ & iect/frug/brol \\
\hline EA & Rodentia & Muridae & Niviventer & confucianus & $\begin{array}{l}\text { Confucian Niviventer, } \\
\text { Chinese White-bellied } \\
\text { Rat } \\
\text { Sundaic Arboreal }\end{array}$ & 1.81 & 65 & extant & 0 & NA & LC & iect/frug/brol \\
\hline EA & Rodentia & Muridae & Niviventer & cremoriventer & $\begin{array}{l}\text { Niviventer, Dark-tailed } \\
\text { Tree Rat }\end{array}$ & 1.82 & 66 & extant & Future & NA & Vu & iect/frug/brol \\
\hline EA & Rodentia & Muridae & Niviventer & eha & $\begin{array}{l}\text { Little Himalayan Rat, } \\
\text { Smoke-bellied Rat }\end{array}$ & 1.73 & 54 & extant & 0 & NA & $\mathrm{LC}$ & eect/frug/brol \\
\hline EA & Rodentia & Muridae & Niviventer & excelsior & $\begin{array}{l}\text { Sichuan Niviventer, } \\
\text { Large White-bellied Rat }\end{array}$ & 2.01 & 102 & extant & 0 & NA & LC & iect/frug/brol \\
\hline EA & Rodentia & Muridae & Niviventer & fulvescens & $\begin{array}{l}\text { Chestnut White-bellied } \\
\text { Rat }\end{array}$ & 1.90 & 80 & extant & 0 & NA & LC & iect/frug/brol \\
\hline EA & Rodentia & Muridae & Niviventer & hinpoon & $\begin{array}{l}\text { Limestone Niviventer, } \\
\text { Limestone Rat }\end{array}$ & 1.79 & 61 & extant & 0 & NA & $\mathrm{DD}$ & iect/frug/brol \\
\hline
\end{tabular}




\begin{tabular}{|c|c|c|c|c|c|c|c|c|c|c|c|c|}
\hline EA & Rodentia & Muridae & Niviventer & langbianis & $\begin{array}{l}\text { Lang Bian White-bellied } \\
\text { Rat, Dark-tailed Rat }\end{array}$ & 1.84 & 70 & extant & 0 & NA & LC & iect/frug/brol \\
\hline EA & Rodentia & Muridae & Niviventer & niviventer & $\begin{array}{l}\text { Himalayan White- } \\
\text { bellied Rat, White- } \\
\text { bellied Rat } \\
\text { Montane Bornean }\end{array}$ & 2.00 & 100 & extant & 0 & NA & LC & iect/frug/brol \\
\hline EA & Rodentia & Muridae & Niviventer & rapit & $\begin{array}{l}\text { Niviventer, Long-tailed } \\
\text { Mountain Rat } \\
\text { Indochinese Mountain }\end{array}$ & 1.94 & 87 & extant & 0 & NA & LC & ;ect/frug/brol \\
\hline EA & Rodentia & Muridae & Niviventer & tenaster & $\begin{array}{l}\text { Niviventer, Tenasserim } \\
\text { White-bellied Rat } \\
\text { Malay Peninsula }\end{array}$ & 2.01 & 103 & extant & 0 & NA & LC & ;ect/frug/brol \\
\hline EA & Rodentia & Muridae & Pithecheir & parvus & $\begin{array}{l}\text { Pithecheir, Malayan } \\
\text { Tree Rat }\end{array}$ & 2.03 & 106 & extant & 0 & NA & DD & ;ect/frug/brol \\
\hline EA & Rodentia & Muridae & Psammomys & obesus & Fat Sand Rat & 2.17 & 150 & extant & 0 & NA & LC & Browse \\
\hline EA & Rodentia & Muridae & Rattus & andamanensis & $\begin{array}{l}\text { Indochinese Forest Rat, } \\
\text { Sikkim Rat }\end{array}$ & 2.11 & 129 & extant & 0 & NA & LC & ;ect/frug/brol \\
\hline EA & Rodentia & Muridae & Rattus & annandalei & $\begin{array}{l}\text { Annandale's Sundaic } \\
\text { Rat, Annandale's Rat }\end{array}$ & 2.30 & 198 & extant & 0 & NA & LC & iect/frug/brol \\
\hline EA & Rodentia & Muridae & Rattus & argentiventer & $\begin{array}{l}\text { Ricefield Rat, Rice-field } \\
\text { Rat }\end{array}$ & 2.12 & 133 & extant & 0 & NA & LC & ;ect/frug/brol \\
\hline EA & Rodentia & Muridae & Rattus & exulans & Polynesian Rat & 1.60 & 40 & extant & 0 & NA & $\mathrm{LC}$ & ;ect/frug/brol \\
\hline EA & Rodentia & Muridae & Rattus & losea & $\begin{array}{l}\text { Losea Rat, Lesser Rice- } \\
\text { field Rat }\end{array}$ & 1.89 & 77 & extant & 0 & NA & LC & ;ect/frug/brol \\
\hline EA & Rodentia & Muridae & Rattus & nitidus & Himalayan Field Rat & 2.13 & 134 & extant & 0 & NA & LC & ;ect/frug/brol \\
\hline EA & Rodentia & Muridae & Rattus & norvegicus & Brown Rat & 2.55 & 357 & extant & 0 & NA & LC & ;ect/frug/brol \\
\hline EA & Rodentia & Muridae & Rattus & osgoodi & $\begin{array}{l}\text { Osgood's Vietnamese } \\
\text { Rat, Osgood's Rat }\end{array}$ & 1.97 & 93 & extant & 0 & NA & $\mathrm{LC}$ & ;ect/frug/brol \\
\hline EA & Rodentia & Muridae & Rattus & ranjiniae & $\begin{array}{l}\text { Ranjini's Field Rat, } \\
\text { Kerala Rat }\end{array}$ & 2.08 & 120 & extant & Future & NA & EN & ;ect/frug/brol \\
\hline EA & Rodentia & Muridae & Rattus & rattus & $\begin{array}{l}\text { House Rat, Ship Rat, } \\
\text { Roof Rat, Black Rat }\end{array}$ & 2.25 & 178 & extant & 0 & NA & LC & ;ect/frug/brol \\
\hline EA & Rodentia & Muridae & Rattus & tanezumi & $\begin{array}{l}\text { Oriental House Rat, } \\
\text { Tanezumi Rat }\end{array}$ & 1.94 & 87 & extant & 0 & NA & LC & iect/frug/brol \\
\hline EA & Rodentia & Muridae & Rattus & tiomanicus & $\begin{array}{l}\text { Malaysian Field Rat, } \\
\text { Malayan Field Rat }\end{array}$ & 2.11 & 128 & extant & 0 & NA & LC & ;ect/frug/bror \\
\hline EA & Rodentia & Muridae & Rattus & turkestanicus & Himalayan Ra & 2.13 & 136 & extant & 0 & NA & LC & ;ect/frug/brol \\
\hline EA & Rodentia & Muridae & Rhombomys & opimus & Great Gerbil & 2.16 & 144 & extant & 0 & NA & LC & 3rowse/graze \\
\hline EA & Rodentia & Muridae & Sekeetamys & calurus & Bushy-tailed Jird & 1.74 & 55 & extant & 0 & NA & LC & frug \\
\hline EA & Rodentia & Muridae & Spalax & leucodon & $\begin{array}{l}\text { Lesser Mole Rat, Lesser } \\
\text { Blind Mole Rat }\end{array}$ & 2.25 & 178 & extant & 0 & NA & DD & Browse \\
\hline EA & Rodentia & Muridae & Spalax & nehringi & $\begin{array}{l}\text { Nehring's Blind Mole } \\
\text { Rat }\end{array}$ & 2.25 & 176 & extant & 0 & NA & DD & Browse \\
\hline EA & Rodentia & Muridae & Sundamys & muelleri & $\begin{array}{l}\text { Môller's Sundamys, } \\
\text { Môller's Giant Sunda } \\
\text { Rat }\end{array}$ & 2.52 & 334 & extant & 0 & NA & LC & ;ect/frug/brol \\
\hline EA & Rodentia & Muridae & Tatera & indica & $\begin{array}{l}\text { Indian Gerbil } \\
\text { Sort-furred Tree Mouse, }\end{array}$ & 2.22 & 165 & extant & 0 & NA & LC & frug/graze \\
\hline EA & Rodentia & Muridae & Typhlomys & cinereus & $\begin{array}{l}\text { Chinese Pygmy } \\
\text { Dormouse, Chapa } \\
\text { Pygmy Dormouse }\end{array}$ & & & extant & 0 & NA & LC & \\
\hline
\end{tabular}




\begin{tabular}{|c|c|c|c|c|c|c|c|c|c|c|c|c|}
\hline EA & Rodentia & Muridae & Vandeleuria & oleracea & $\begin{array}{l}\text { Climbing Mouse, } \\
\text { Indomalayan } \\
\text { Vandeleuria } \\
\text { Vernay's Climbing }\end{array}$ & 1.00 & 10 & extant & 0 & NA & LC & ;ect/frug/brol \\
\hline EA & Rodentia & Muridae & Vernaya & fulva & $\begin{array}{l}\text { Mouse, Red Climbing } \\
\text { Mouse }\end{array}$ & 1.40 & 25 & extant & 0 & NA & LC & ;ect/frug/brol \\
\hline EA & Rodentia & Myoxidae & Dryomys & sichuanensis & Sichuan Dormouse & 1.48 & 30 & extant & 0 & NA & DD & \\
\hline EA & Rodentia & Myoxidae & Myохиs & glis & $\begin{array}{l}\text { Edible Dormouse } \\
\text { Malabar Spiny }\end{array}$ & 2.10 & 125 & extant & 0 & NA & LC & \\
\hline EA & Rodentia & Platacanthomyidae & Platacanthomys & lasiurus & $\begin{array}{l}\text { Dormouse, Malabar } \\
\text { Spiny Tree Mouse, Spiny } \\
\text { Tree Mouse } \\
\text { Sort-furred Tree Mouse }\end{array}$ & 1.88 & 75 & extant & Future & NA & VU & ;ect/frug/brol \\
\hline EA & Rodentia & Platacanthomyidae & Typhlomys & cinereus & $\begin{array}{l}\text { Chinese Pygmy } \\
\text { Dormouse, Chapa } \\
\text { Pygmy Dormouse }\end{array}$ & 1.49 & 31 & extant & 0 & NA & LC & ;ect/frug/brol \\
\hline EA & Rodentia & Sciuridae & Aeretes & melanopterus & $\begin{array}{l}\text { Northern Chinese Flying } \\
\text { Squirrel, North Chinese } \\
\text { Flying Squirrel, Groove- } \\
\text { toothed Flying Squirrel }\end{array}$ & & & extant & Future & NA & NT & \\
\hline EA & Rodentia & Sciuridae & Aeromys & tephromelas & $\begin{array}{l}\text { Black Flying Squirrel, } \\
\text { Large Black Flying } \\
\text { Squirrel }\end{array}$ & 3.10 & 1250 & extant & 0 & NA & DD & Frug \\
\hline EA & Rodentia & Sciuridae & Belomys & pearsonii & $\begin{array}{l}\text { Hairy-footed Flying } \\
\text { Squirrel } \\
\text { Namdapha Flying }\end{array}$ & 2.34 & 217 & extant & 0 & NA & DD & Frug \\
\hline EA & Rodentia & Sciuridae & Biswamoyopterus & biswasi & $\begin{array}{l}\text { Squirrel, Namdapha } \\
\text { Giant Flying Squirrel }\end{array}$ & 3.21 & 1613 & extant & Future & NA & CR & Frug \\
\hline EA & Rodentia & Sciuridae & Callosciurus & caniceps & Grey-bellied Squirrel & 2.41 & 257 & extant & 0 & NA & LC & Frug \\
\hline EA & Rodentia & Sciuridae & Callosciurus & erythraeus & Pallas's Squirrel & 2.45 & 280 & extant & 0 & NA & LC & Frug \\
\hline EA & Rodentia & Sciuridae & Callosciurus & finlaysonii & Finlayson's Squirrel & 2.44 & 278 & extant & 0 & NA & LC & Frug \\
\hline EA & Rodentia & Sciuridae & Callosciurus & inornatus & Inornate Squirrel & 2.51 & 325 & extant & 0 & NA & LC & Frug \\
\hline EA & Rodentia & Sciuridae & Callosciurus & nigrovittatus & Black-striped Squirrel & 2.31 & 202 & extant & Future & NA & NT & Frug \\
\hline EA & Rodentia & Sciuridae & Callosciurus & notatus & Plantain Squirrel & 2.28 & 190 & extant & 0 & NA & LC & Frug \\
\hline EA & Rodentia & Sciuridae & Callosciurus & phayrei & Phayre's Squirrel & 2.43 & 269 & extant & 0 & NA & LC & Frug \\
\hline EA & Rodentia & Sciuridae & Callosciurus & prevostii & Prevost's Squirrel & 2.60 & 400 & extant & 0 & NA & LC & Frug \\
\hline EA & Rodentia & Sciuridae & Callosciurus & pygerythrus & $\begin{array}{l}\text { Hoary-bellied Squirrel, } \\
\text { Irrawaddy Squirrel }\end{array}$ & 2.40 & 252 & extant & 0 & NA & LC & Frug \\
\hline EA & Rodentia & Sciuridae & Callosciurus & quinquestriatus & Anderson's Squirrel & 2.44 & 273 & extant & Future & NA & NT & \\
\hline EA & Rodentia & Sciuridae & Dremomys & lokriah & $\begin{array}{l}\text { Orange-bellied } \\
\text { Himalayan Squirrel } \\
\text { Perny's Long-nosed }\end{array}$ & 2.36 & 227 & extant & 0 & NA & LC & Frug \\
\hline EA & Rodentia & Sciuridae & Dremomys & pernyi & $\begin{array}{l}\text { Squirrel, Servaline } \\
\text { Genet }\end{array}$ & 2.30 & 199 & extant & 0 & NA & LC & Frug \\
\hline EA & Rodentia & Sciuridae & Dremomys & pyrrhomerus & Red-hipped Squirrel & 2.32 & 209 & extant & 0 & NA & LC & \\
\hline EA & Rodentia & Sciuridae & Dremomys & rufigenis & $\begin{array}{l}\text { Asian Red-cheeked } \\
\text { Squirrel, Haussa Genet }\end{array}$ & 2.30 & 200 & extant & 0 & NA & LC & Frug \\
\hline EA & Rodentia & Sciuridae & Eoglaucomys & fimbriatus & $\begin{array}{l}\text { Small Kashmir Flying } \\
\text { Squirrel }\end{array}$ & 2.71 & 510 & extant & 0 & NA & LC & Frug \\
\hline EA & Rodentia & Sciuridae & Eupetaurus & cinereus & Woolly Flying Squirrel & 3.40 & 2500 & extant & Future & NA & EN & Frug \\
\hline
\end{tabular}




\begin{tabular}{|c|c|c|c|c|c|c|c|c|c|c|c|c|}
\hline EA & Rodentia & Sciuridae & Funambulus & layardi & $\begin{array}{l}\text { Layard's Palm Squirrel } \\
\text { Common Palm Squirrel, }\end{array}$ & 2.14 & 137 & extant & Future & NA & vu & \\
\hline EA & Rodentia & Sciuridae & Funambulus & palmarum & $\begin{array}{l}\text { Indian Palm Squirrel, } \\
\text { Three-striped Palm } \\
\text { Squirrel } \\
\text { Five-striped Palm }\end{array}$ & 2.14 & 137 & extant & 0 & NA & LC & \\
\hline EA & Rodentia & Sciuridae & Funambulus & pennantii & $\begin{array}{l}\text { Squirrel, Northern Palm } \\
\text { Squirrel }\end{array}$ & 2.30 & 200 & extant & 0 & NA & LC & Frug \\
\hline EA & Rodentia & Sciuridae & Funambulus & sublineatus & $\begin{array}{l}\text { Dusky-striped Squirrel, } \\
\text { Dusky Palm Squirrel } \\
\text { Western Ghats Striped } \\
\text { Squirrel, Western Ghats }\end{array}$ & 1.85 & 71 & extant & Future & NA & vU & Frug \\
\hline EA & Rodentia & Sciuridae & Funambulus & tristriatus & $\begin{array}{l}\text { Squirrel, Jungle Striped } \\
\text { Squirrel, Jungle Palm } \\
\text { Squirrel }\end{array}$ & 2.14 & 139 & extant & 0 & NA & LC & Frug \\
\hline EA & Rodentia & Sciuridae & Hylopetes & alboniger & $\begin{array}{l}\text { African Linsang, } \\
\text { Particolored Flying } \\
\text { Squirrel }\end{array}$ & 2.41 & 255 & extant & 0 & NA & LC & Frug \\
\hline EA & Rodentia & Sciuridae & Hylopetes & baberi & $\begin{array}{l}\text { Small Kashmir Flying } \\
\text { Squirrel }\end{array}$ & 2.85 & 712 & extant & 0 & NA & LC & Frug \\
\hline EA & Rodentia & Sciuridae & Hylopetes & lepidus & $\begin{array}{l}\text { Gray-cheeked Flying } \\
\text { Squirrel } \\
\text { Indochinese Flying }\end{array}$ & 1.70 & 50 & extant & 0 & NA & DD & Frug \\
\hline EA & Rodentia & Sciuridae & Hylopetes & phayrei & $\begin{array}{l}\text { Squirrel, Phayre's Flying } \\
\text { Squirrel }\end{array}$ & 2.36 & 227 & extant & 0 & NA & LC & Frug \\
\hline EA & Rodentia & Sciuridae & Hylopetes & spadiceus & $\begin{array}{l}\text { Red-cheeked Flying } \\
\text { Squirrel }\end{array}$ & 1.70 & 50 & extant & 0 & NA & LC & Frug \\
\hline EA & Rodentia & Sciuridae & lomys & horsfieldii & Javanese Flying Squirrel & 2.08 & 120 & extant & 0 & NA & LC & browse/frug \\
\hline EA & Rodentia & Sciuridae & Lariscus & insignis & $\begin{array}{l}\text { Three-striped Ground } \\
\text { Squirrel }\end{array}$ & 2.30 & 200 & extant & 0 & NA & LC & Frug/browse \\
\hline EA & Rodentia & Sciuridae & Marmota & baibacina & $\begin{array}{l}\text { Altai Marmot, Gray } \\
\text { Marmot, Grey Marmot }\end{array}$ & 3.88 & 7500 & extant & 0 & NA & LC & Graze \\
\hline EA & Rodentia & Sciuridae & Marmota & bobak & Bobak Marmot & 3.74 & 5500 & extant & 0 & NA & LC & Graze \\
\hline EA & Rodentia & Sciuridae & Marmota & camtschatica & Black-capped Marmot & 3.54 & 3500 & extant & 0 & NA & LC & Graze \\
\hline EA & Rodentia & Sciuridae & Marmota & caudata & Long-tailed Marmot & 3.64 & 4350 & extant & 0 & NA & LC & Graze \\
\hline EA & Rodentia & Sciuridae & Marmota & himalayana & $\begin{array}{l}\text { Himalayan Marmot, } \\
\text { Karakoram Marmot }\end{array}$ & 3.70 & 5000 & extant & 0 & NA & LC & Graze \\
\hline EA & Rodentia & Sciuridae & Marmota & marmota & Alpine Marmot & 3.30 & 2010 & extant & 0 & NA & LC & Graze \\
\hline EA & Rodentia & Sciuridae & Marmota & menzbieri & $\begin{array}{l}\text { Menzbier's Marmot } \\
\text { Mongolian Marmot, }\end{array}$ & 3.51 & 3250 & extant & Future & NA & vU & Graze \\
\hline EA & Rodentia & Sciuridae & Marmota & sibirica & $\begin{array}{l}\text { Siberian Marmot, } \\
\text { Tarbagan Marmot }\end{array}$ & 3.90 & 8000 & extant & Future & NA & EN & Graze \\
\hline EA & Rodentia & Sciuridae & Menetes & berdmorei & $\begin{array}{l}\text { Indochinese Ground } \\
\text { Squirrel }\end{array}$ & 2.28 & 190 & extant & 0 & NA & LC & \\
\hline EA & Rodentia & Sciuridae & Petaurillus & kinlochii & $\begin{array}{l}\text { Selangor Pygmy Flying } \\
\text { Squirrel }\end{array}$ & 1.60 & 40 & extant & 0 & NA & DD & Ginsect \\
\hline EA & Rodentia & Sciuridae & Petaurista & alborufus & $\begin{array}{l}\text { Red And White Giant } \\
\text { Flying Squirrel } \\
\text { Spotted Giant Flying }\end{array}$ & 3.63 & 4290 & extant & 0 & NA & LC & Frug/browse \\
\hline EA & Rodentia & Sciuridae & Petaurista & elegans & $\begin{array}{l}\text { Squirrel, Grey-headed } \\
\text { Flying Squirrel }\end{array}$ & 2.98 & 954 & extant & 0 & NA & LC & Frug/browse \\
\hline
\end{tabular}




\begin{tabular}{|c|c|c|c|c|c|c|c|c|c|c|c|c|}
\hline EA & Rodentia & Sciuridae & Petaurista & leucogenys & $\begin{array}{l}\text { Japanese Giant Flying } \\
\text { Squirrel }\end{array}$ & 3.31 & 2052 & extant & 0 & NA & LC & \\
\hline EA & Rodentia & Sciuridae & Petaurista & magnificus & $\begin{array}{l}\text { Hodgson's Giant Flying } \\
\text { Squirrel } \\
\text { Bhutan Giant Flying }\end{array}$ & 3.17 & 1476 & extant & 0 & NA & LC & Frug/browse \\
\hline EA & Rodentia & Sciuridae & Petaurista & nobilis & $\begin{array}{l}\text { Squirrel, Gray's Giant } \\
\text { Flying Squirrel, Noble } \\
\text { Giant Flying Squirrel }\end{array}$ & 3.43 & 2710 & extant & Future & NA & NT & Frug/browse \\
\hline EA & Rodentia & Sciuridae & Petaurista & petaurista & $\begin{array}{l}\text { Common Giant Flying } \\
\text { Squirrel, Red Giant } \\
\text { Flying Squirrel } \\
\text { Large Brown Flying }\end{array}$ & 3.13 & 1335 & extant & 0 & NA & LC & Frug/browse \\
\hline EA & Rodentia & Sciuridae & Petaurista & philippensis & $\begin{array}{l}\text { Squirrel, Indian Giant } \\
\text { Flying Squirrel }\end{array}$ & 3.23 & 1700 & extant & 0 & NA & LC & Frug/browse \\
\hline EA & Rodentia & Sciuridae & Petaurista & xanthotis & $\begin{array}{l}\text { Chinese Giant Flying } \\
\text { Squirrel }\end{array}$ & 3.28 & 1900 & extant & 0 & NA & LC & Frug/browse \\
\hline EA & Rodentia & Sciuridae & Petinomys & fuscocapillus & $\begin{array}{l}\text { Travancore Flying } \\
\text { Squirrel }\end{array}$ & 2.90 & 795 & extant & Future & NA & NT & Frug/browse \\
\hline EA & Rodentia & Sciuridae & Petinomys & genibarbis & $\begin{array}{l}\text { Whiskered Flying } \\
\text { Squirrel }\end{array}$ & 2.04 & 110 & extant & Future & NA & VU & Frug/browse \\
\hline EA & Rodentia & Sciuridae & Petinomys & setosus & $\begin{array}{l}\text { Temminck's Flying } \\
\text { Squirrel }\end{array}$ & 1.58 & 38 & extant & Future & NA & VU & Frug/browse \\
\hline EA & Rodentia & Sciuridae & Petinomys & vordermanni & $\begin{array}{l}\text { Vordermann's Flying } \\
\text { Squirrel }\end{array}$ & 1.60 & 40 & extant & Future & NA & Vu & Frug/browse \\
\hline EA & Rodentia & Sciuridae & Pteromys & volans & $\begin{array}{l}\text { Russian Flying Squirrel, } \\
\text { Siberian Flying Squirrel }\end{array}$ & 2.20 & 158 & extant & 0 & NA & LC & Frug/browse \\
\hline EA & Rodentia & Sciuridae & Pteromyscus & pulverulentus & $\begin{array}{l}\text { Smoky Flying Squirrel } \\
\text { Pale Giant Squirrel, }\end{array}$ & 2.60 & 400 & extant & Future & NA & EN & Frug \\
\hline EA & Rodentia & Sciuridae & Ratufa & affinis & $\begin{array}{l}\text { Cream-coloured Giant } \\
\text { Squirrel }\end{array}$ & 3.05 & 1125 & extant & Future & NA & NT & Frug/browse \\
\hline EA & Rodentia & Sciuridae & Ratufa & bicolor & $\begin{array}{l}\text { Black Giant Squirrel, } \\
\text { Malayan Giant Squirrel }\end{array}$ & 3.31 & 2050 & extant & Future & NA & NT & Frug/browse \\
\hline EA & Rodentia & Sciuridae & Ratufa & indica & $\begin{array}{l}\text { Indian Giant Squirrel, } \\
\text { Malabar Giant Squirrel } \\
\text { Sri Lankan Giant }\end{array}$ & 3.03 & 1060 & extant & 0 & NA & LC & Frug/browse \\
\hline EA & Rodentia & Sciuridae & Ratufa & macroura & $\begin{array}{l}\text { Squirrel, Grizzled Giant } \\
\text { Squirrel }\end{array}$ & 3.11 & 1280 & extant & Future & NA & NT & Frug/browse \\
\hline EA & Rodentia & Sciuridae & Rhinosciurus & laticaudatus & Shrew-faced Squirrel & 2.34 & 221 & extant & Future & NA & NT & Ginsect/frug \\
\hline EA & Rodentia & Sciuridae & Sciurotamias & davidianus & $\begin{array}{l}\text { P_re David's Rock } \\
\text { Squirrel }\end{array}$ & 2.64 & 434 & extant & 0 & NA & LC & Browse \\
\hline EA & Rodentia & Sciuridae & Sciurotamias & forresti & Forrest's Rock Squirrel & 2.64 & 434 & extant & 0 & NA & LC & Browse \\
\hline EA & Rodentia & Sciuridae & Sciurus & anomalus & $\begin{array}{l}\text { Caucasian Squirrel, } \\
\text { Persian Squirrel }\end{array}$ & 2.41 & 256 & extant & 0 & NA & LC & Frug \\
\hline EA & Rodentia & Sciuridae & Sciurus & vulgaris & $\begin{array}{l}\text { Eurasian Red Squirrel, } \\
\text { Red Squirrel }\end{array}$ & 2.52 & 333 & extant & 0 & NA & LC & Frug \\
\hline EA & Rodentia & Sciuridae & Spermophilopsis & leptodactylus & $\begin{array}{l}\text { Long-clawed Ground } \\
\text { Squirrel }\end{array}$ & 2.78 & 600 & extant & 0 & NA & LC & \\
\hline EA & Rodentia & Sciuridae & Spermophilus & alashanicus & Alashan Ground Squirrel & 2.54 & 350 & extant & 0 & NA & LC & \\
\hline
\end{tabular}




\begin{tabular}{|c|c|c|c|c|c|c|c|c|c|c|c|c|}
\hline EA & Rodentia & Sciuridae & Spermophilus & citellus & $\begin{array}{l}\text { European Ground } \\
\text { Squirrel, European } \\
\text { Squirrel, European } \\
\text { Souslik }\end{array}$ & 2.46 & 290 & extant & Future & NA & Vu & Graze/frug \\
\hline EA & Rodentia & Sciuridae & Spermophilus & dauricus & Daurian Ground Squirrel & 2.21 & 161 & extant & 0 & NA & LC & Graze/frug \\
\hline EA & Rodentia & Sciuridae & Spermophilus & erythrogenys & $\begin{array}{l}\text { Red-cheeked Ground } \\
\text { Squirrel }\end{array}$ & 2.54 & 350 & extant & 0 & NA & LC & \\
\hline EA & Rodentia & Sciuridae & Spermophilus & fulvus & Yellow Ground Squirrel & 2.78 & 596 & extant & 0 & NA & LC & Graze/frug \\
\hline EA & Rodentia & Sciuridae & Spermophilus & major & Russet Ground Squirrel & 2.54 & 350 & extant & 0 & NA & LC & \\
\hline EA & Rodentia & Sciuridae & Spermophilus & musicus & $\begin{array}{l}\text { Caucasian Mountain } \\
\text { Ground Squirrel }\end{array}$ & 2.54 & 350 & extant & Future & NA & NT & \\
\hline EA & Rodentia & Sciuridae & Spermophilus & parryii & Arctic Ground Squirrel & 2.88 & 760 & extant & 0 & NA & LC & Frug/browse \\
\hline EA & Rodentia & Sciuridae & Spermophilus & pygmaeus & Little Ground Squirrel & 2.13 & 136 & extant & 0 & NA & LC & Graze/frug \\
\hline EA & Rodentia & Sciuridae & Spermophilus & relictus & $\begin{array}{l}\text { Tien Shan Ground } \\
\text { Squirrel }\end{array}$ & 2.78 & 600 & extant & 0 & NA & LC & Graze/frug \\
\hline EA & Rodentia & Sciuridae & Spermophilus & suslicus & $\begin{array}{l}\text { Speckled Ground } \\
\text { Squirrel, Spotted Souslik }\end{array}$ & 2.40 & 252 & extant & Future & NA & NT & Graze/frug \\
\hline EA & Rodentia & Sciuridae & Spermophilus & undulatus & $\begin{array}{l}\text { Long-tailed Ground } \\
\text { Squirrel }\end{array}$ & 2.92 & 840 & extant & 0 & NA & LC & Graze/frug \\
\hline EA & Rodentia & Sciuridae & Spermophilus & xanthoprymnus & $\begin{array}{l}\text { Asia Minor Ground } \\
\text { Squirrel }\end{array}$ & 2.46 & 290 & extant & Future & NA & NT & Graze/frug \\
\hline EA & Rodentia & Sciuridae & Sundasciurus & hippurus & Horse-tailed Squirrel & 2.70 & 500 & extant & Future & NA & NT & Browse/frug \\
\hline EA & Rodentia & Sciuridae & Sundasciurus & lowii & Low's Squirrel & 1.95 & 90 & extant & 0 & NA & LC & Browse/frug \\
\hline EA & Rodentia & Sciuridae & Sundasciurus & tenuis & Slender Squirrel & 1.88 & 75 & extant & 0 & NA & LC & Browse/frug \\
\hline EA & Rodentia & Sciuridae & Tamias & sibiricus & Siberian Chipmunk & 1.93 & 85 & extant & 0 & NA & LC & Frug \\
\hline EA & Rodentia & Sciuridae & Tamiops & macclellandi & $\begin{array}{l}\text { Himalayan Striped } \\
\text { Squirrel }\end{array}$ & 1.74 & 55 & extant & 0 & NA & LC & Frug/ginsect \\
\hline EA & Rodentia & Sciuridae & Tamiops & maritimus & $\begin{array}{l}\text { Maritime Striped } \\
\text { Squirrel }\end{array}$ & 1.85 & 70 & extant & 0 & NA & LC & Frug/ginsect \\
\hline EA & Rodentia & Sciuridae & Tamiops & rodolphei & $\begin{array}{l}\text { Cambodian Striped } \\
\text { Squirrel }\end{array}$ & 1.75 & 56 & extant & 0 & NA & LC & Frug/ginsect \\
\hline EA & Rodentia & Sciuridae & Tamiops & swinhoei & $\begin{array}{l}\text { Swinhoe's Striped } \\
\text { Squirrel }\end{array}$ & 1.93 & 85 & extant & 0 & NA & LC & Frug/ginsect \\
\hline EA & Rodentia & Sciuridae & Trogopterus & xanthipes & $\begin{array}{l}\text { Complex-toothed Flying } \\
\text { Squirrel }\end{array}$ & 2.87 & 745 & extant & Future & NA & NT & Frug/ginsect \\
\hline EA & Rodentia & Spalacidae & Cannomys & badius & $\begin{array}{l}\text { Lesser Bamboo Rat, Bay } \\
\text { Bamboo Rat }\end{array}$ & 2.81 & 650 & extant & 0 & NA & LC & owse (bambo \\
\hline EA & Rodentia & Spalacidae & Myospalax & aspalax & False Zokor & 2.35 & 225 & extant & 0 & NA & LC & \\
\hline EA & Rodentia & Spalacidae & Myospalax & myospalax & $\begin{array}{l}\text { Siberian Zokor, Altai } \\
\text { Zokor }\end{array}$ & 2.35 & 225 & extant & 0 & NA & LC & iect/frug/brol \\
\hline EA & Rodentia & Spalacidae & Myospalax & psilurus & $\begin{array}{l}\text { Transbaikal Zokor, } \\
\text { Manchurian Zokor }\end{array}$ & 2.35 & 225 & extant & 0 & NA & LC & \\
\hline EA & Rodentia & Spalacidae & Rhizomys & pruinosus & Hoary Bamboo Rat & 3.39 & 2450 & extant & 0 & NA & LC & owse (bambo \\
\hline EA & Rodentia & Spalacidae & Rhizomys & sinensis & Chinese Bamboo Rat & 2.94 & 865 & extant & 0 & NA & LC & owse (bambo \\
\hline EA & Rodentia & Spalacidae & Rhizomys & sumatrensis & $\begin{array}{l}\text { Indomalayan Bamboo } \\
\text { Rat, Large Bamboo Rat }\end{array}$ & 2.40 & 250 & extant & 0 & NA & LC & owse (bambo \\
\hline EA & Rodentia & Spalacidae & Spalax & arenarius & $\begin{array}{l}\text { Sandy Mole Rat, SANDY } \\
\text { MOLE RAT }\end{array}$ & 2.72 & 520 & extant & Future & NA & EN & Browse \\
\hline
\end{tabular}


Giant Blind Mole Rat,

GIANT BLIND MOLE

RAT, Giant Mole Rat,

GIANT MOLE RAT,

495

extant

Future

NA

VU

Russian Mole Rat,

RUSSIAN MOLE RAT

Balkan Blind Mole Rat,

Balkan Mole Rat,

2.69

Bukovin Mole Rat

Greater Blind Mole Rat,

GREATER BLIND MOLE

RAT, Greater Mole Rat,

GREATER MOLE RAT

Podolian Mole Rat,

Podolsk Mole Rat

Pen-tailed Treeshrew,

Pen-tailed Tree Shrew

Madras Treeshrew,

Indian Tree Shrew,

Madras Tree Shrew

Treeshrew, Northern

Smooth-tailed Tree

Shrew

EA Scandentia

Tupaiidae

Dendrogale

murina

EA Scandentia Tupaiidae

Tupaia

belangeri

A Scandentia

Tupaiidae

Tupaia

glis

EA Scandentia Tupaiidae

Tupaia

minor

EA Soricomorpha Soricidae

Anourosorex

Northern Treeshrew,

Northern Tree Shrew

Common Treeshrew,

Common Tree Shrew

Lesser Treeshrew,

Pygmy Tree Shrew

Mole-shrew, Chinese

Mole Shrew, Chinese

Short-tailed Shrew,

Mole Shrew, Sichuan

Burrowing Shrew

Asiatic Short-tailed

Shrew, Northern Short-

tailed Shrew, Sichuan

Short-tailed Shrew

Burmese Short-tailed

Shrew, Southern Short-

tailed Shrew, Ward's

Short-tailed Shrew

Malayan Water Shrew,

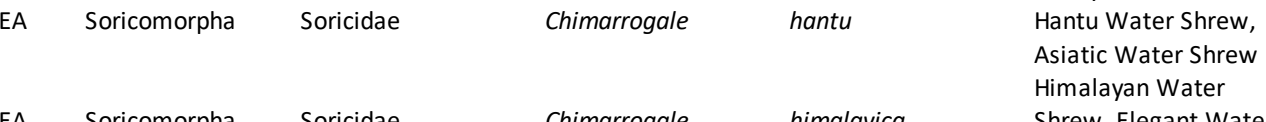

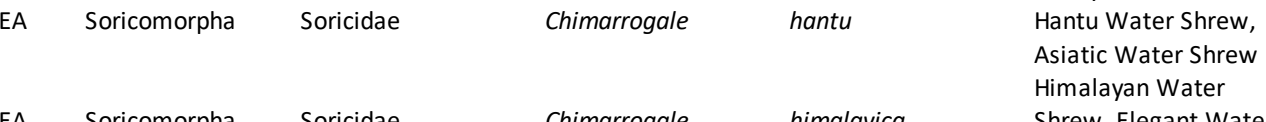

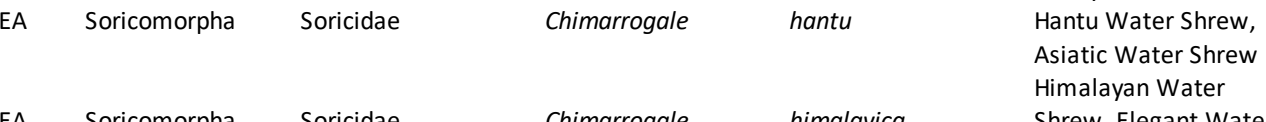

EA Soricomorpha Soricidae Chimarrogale himalayica $\begin{aligned} & \text { Shrew, Elegant Wate } \\ & \text { Shrew }\end{aligned}$

Chinese Water Shrew,

Styan's Water Shrew
Chimarrogale styani
EA Soricomorpha Soricidae

(2.6.

$\begin{array}{llllll}2.67 & 470 & \text { extant } & 0 & \text { NA } & \text { LC }\end{array}$

$2.69 \quad 495 \quad$ extant Future NA VU

$\begin{array}{llllll}1.63 & 43 & \text { extant } & 0 & \text { NA } & \text { LC }\end{array}$

\begin{tabular}{|c|c|c|c|c|}
\hline 1.70 & 50 & extant & 0 & NA \\
\hline
\end{tabular}

$\begin{array}{lllll}2.30 & 200 \quad \text { extant } & 0 & \text { NA } & \text { LC }\end{array}$

$\begin{array}{llllll}2.20 & 159 & \text { extant } & 0 & \text { NA } & \text { LC }\end{array}$

$\begin{array}{llllll}1.85 & 70 & \text { extant } & 0 & \text { NA } & \text { LC }\end{array}$

$\begin{array}{lllllll}1.30 & 20 & \text { extant } & 0 & \text { NA } & \text { LC } & \text { Ginsect }\end{array}$

$\begin{array}{llllll}2.20 & 160 \quad \text { extant } & 0 & \text { NA } & \text { LC } & \text { Ginsect/frug }\end{array}$

LC Ginsect




\begin{tabular}{|c|c|c|c|c|c|c|c|c|c|c|c|c|}
\hline EA & Soricomorpha & Soricidae & Crocidura & arabica & Arabian Shrew & 1.11 & 13 & extant & 0 & NA & LC & Ginsect \\
\hline EA & Soricomorpha & Soricidae & Crocidura & armenica & Armenian Shrew & 1.11 & 13 & extant & 0 & NA & $\mathrm{DD}$ & Ginsect \\
\hline EA & Soricomorpha & Soricidae & Crocidura & attenuata & $\begin{array}{l}\text { Grey Shrew, } \\
\text { Indochinese Shrew }\end{array}$ & 1.15 & 14 & extant & 0 & NA & LC & Ginsect \\
\hline EA & Soricomorpha & Soricidae & Crocidura & dhofarensis & Dhofarian Shrew & 1.11 & 13 & extant & 0 & NA & DD & Ginsect \\
\hline EA & Soricomorpha & Soricidae & Crocidura & fuliginosa & Southeast Asian Shrew & 1.08 & 12 & extant & 0 & NA & $\mathrm{LC}$ & Ginsect \\
\hline EA & Soricomorpha & Soricidae & Crocidura & horsfieldii & Horsefield's Shrew & 0.70 & 5 & extant & 0 & NA & $\mathrm{DD}$ & Ginsect \\
\hline EA & Soricomorpha & Soricidae & Crocidura & lasiura & $\begin{array}{l}\text { Ussuri Shrew } \\
\text { Bicolored Shrew, }\end{array}$ & 1.17 & 15 & extant & 0 & NA & LC & Ginsect \\
\hline EA & Soricomorpha & Soricidae & Crocidura & leucodon & $\begin{array}{l}\text { Bicoloured White- } \\
\text { toothed Shrew }\end{array}$ & 0.99 & 10 & extant & 0 & NA & $\mathrm{LC}$ & Ginsect \\
\hline EA & Soricomorpha & Soricidae & Crocidura & malayana & Malayan Shrew & 1.11 & 13 & extant & 0 & NA & LC & Ginsect \\
\hline EA & Soricomorpha & Soricidae & Crocidura & monticola & Sunda Shrew & 0.82 & 7 & extant & 0 & NA & LC & Ginsect \\
\hline EA & Soricomorpha & Soricidae & Crocidura & pergrisea & Pale Grey Shrew & 1.00 & 10 & extant & 0 & NA & DD & Ginsect \\
\hline EA & Soricomorpha & Soricidae & Crocidura & pullata & $\begin{array}{l}\text { Kashmir White-toothed } \\
\text { Shrew, Dusky Shrew } \\
\text { White-toothed Shrew, }\end{array}$ & 1.11 & 13 & extant & 0 & NA & DD & Ginsect \\
\hline EA & Soricomorpha & Soricidae & Crocidura & russula & $\begin{array}{l}\text { Greater White-toothed } \\
\text { Shrew }\end{array}$ & 1.00 & 10 & extant & 0 & NA & LC & ginsect \\
\hline EA & Soricomorpha & Soricidae & Crocidura & serezkyensis & Serezkaya Shrew & 1.11 & 13 & extant & 0 & NA & LC & Ginsect \\
\hline EA & Soricomorpha & Soricidae & Crocidura & sibirica & Siberian Shrew & 1.11 & 13 & extant & 0 & NA & LC & Ginsect \\
\hline EA & Soricomorpha & Soricidae & Crocidura & suaveolens & $\begin{array}{l}\text { Lesser Shrew, Lesser } \\
\text { White-toothed Shrew }\end{array}$ & 0.73 & 5 & extant & 0 & NA & $\mathrm{LC}$ & ginsect \\
\hline EA & Soricomorpha & Soricidae & Crocidura & susiana & Iranian Shrew & 1.11 & 13 & extant & 0 & NA & DD & Ginsect \\
\hline EA & Soricomorpha & Soricidae & Crocidura & zarudnyi & Zarudny's Shrew & 0.83 & 7 & extant & 0 & NA & LC & Ginsect \\
\hline EA & Soricomorpha & Soricidae & Diplomesodon & pulchellum & Piebald Shrew & 1.04 & 11 & extant & 0 & NA & LC & Ginsect \\
\hline EA & Soricomorpha & Soricidae & Episoriculus & caudatus & $\begin{array}{l}\text { Hodgson's Brown- } \\
\text { toothed Shrew } \\
\text { Arboreal Brown-toothed }\end{array}$ & 0.96 & 9 & extant & 0 & NA & LC & Ginsect \\
\hline EA & Soricomorpha & Soricidae & Episoriculus & macrurus & $\begin{array}{l}\text { Shrew, Long-tailed } \\
\text { Mountain Shrew } \\
\text { Elegant Water Shrew, }\end{array}$ & 1.13 & 14 & extant & 0 & NA & LC & Ginsect \\
\hline EA & Soricomorpha & Soricidae & Nectogale & elegans & $\begin{array}{l}\text { Web-footed Water } \\
\text { Shrew }\end{array}$ & 1.51 & 33 & extant & 0 & NA & LC & Ginsect \\
\hline EA & Soricomorpha & Soricidae & Neomys & anomalus & $\begin{array}{l}\text { Southern Water Shrew, } \\
\text { Mediterranean Water } \\
\text { Shrew, Miller's Water } \\
\text { Shrew }\end{array}$ & 1.20 & 16 & extant & 0 & NA & LC & Ginsect \\
\hline EA & Soricomorpha & Soricidae & Neomys & fodiens & $\begin{array}{l}\text { Eurasian Water Shrew, } \\
\text { Water Shrew, Northern } \\
\text { Water Shrew }\end{array}$ & 1.16 & 14 & extant & 0 & NA & LC & Ginsect \\
\hline EA & Soricomorpha & Soricidae & Sorex & alpinus & Alpine Shrew & 0.89 & 8 & extant & Future & NA & NT & Ginsect \\
\hline EA & Soricomorpha & Soricidae & Sorex & araneus & $\begin{array}{l}\text { Common Shrew, } \\
\text { Eurasian Shrew }\end{array}$ & 1.00 & 10 & extant & 0 & NA & LC & Ginsect \\
\hline EA & Soricomorpha & Soricidae & Sorex & asper & $\begin{array}{l}\text { Tien Shan Shrew } \\
\text { Lesser Stripe-backed }\end{array}$ & 0.90 & 8 & extant & 0 & NA & LC & Ginsect \\
\hline EA & Soricomorpha & Soricidae & Sorex & bedfordiae & $\begin{array}{l}\text { Shrew, Lesser Striped } \\
\text { Shrew }\end{array}$ & 0.82 & 7 & extant & 0 & NA & $\mathrm{LC}$ & Ginsect \\
\hline EA & Soricomorpha & Soricidae & Sorex & buchariensis & $\begin{array}{l}\text { Pamir Shrew } \\
\text { Laxmann's Shrew, }\end{array}$ & 0.83 & 7 & extant & 0 & NA & LC & Ginsect \\
\hline EA & Soricomorpha & Soricidae & Sorex & caecutiens & $\begin{array}{l}\text { MASKED SHREW, } \\
\text { Masked Shrew }\end{array}$ & 0.73 & 5 & extant & 0 & NA & LC & Ginsect \\
\hline
\end{tabular}




\begin{tabular}{|c|c|c|c|c|c|c|c|c|c|c|c|c|}
\hline EA & Soricomorpha & Soricidae & Sorex & camtschatica & Kamchatcka Shrew & 0.83 & 7 & extant & 0 & NA & LC & Ginsect \\
\hline EA & Soricomorpha & Soricidae & Sorex & cansulus & Gansu Shrew & 0.83 & 7 & extant & 0 & NA & DD & Ginsect \\
\hline EA & Soricomorpha & Soricidae & Sorex & coronatus & $\begin{array}{l}\text { Crowned Shrew, } \\
\text { Millet's Shrew } \\
\text { Stripe-backed Shrew, }\end{array}$ & 0.98 & 10 & extant & 0 & NA & LC & Ginsect \\
\hline EA & Soricomorpha & Soricidae & Sorex & cylindricauda & $\begin{array}{l}\text { Greater Stripe-backed } \\
\text { Shrew }\end{array}$ & 0.83 & 7 & extant & 0 & NA & LC & Ginsect \\
\hline EA & Soricomorpha & Soricidae & Sorex & daphaenodon & $\begin{array}{l}\text { Large-toothed Siberian } \\
\text { Shrew }\end{array}$ & 0.83 & 7 & extant & 0 & NA & LC & Ginsect \\
\hline EA & Soricomorpha & Soricidae & Sorex & excelsus & $\begin{array}{l}\text { Highland Shrew, Lofty } \\
\text { Shrew }\end{array}$ & 0.83 & 7 & extant & 0 & NA & LC & Ginsect \\
\hline EA & Soricomorpha & Soricidae & Sorex & gracillimus & Slender Shrew & 0.70 & 5 & extant & 0 & NA & LC & Ginsect \\
\hline EA & Soricomorpha & Soricidae & Sorex & granarius & $\begin{array}{l}\text { Iberian Shrew, Lagranja } \\
\text { Shrew }\end{array}$ & 0.80 & 6 & extant & 0 & NA & LC & Ginsect \\
\hline EA & Soricomorpha & Soricidae & Sorex & isodon & $\begin{array}{l}\text { Even-toothed Shrew, } \\
\text { TAIGA SHREW, Taiga } \\
\text { Shrew }\end{array}$ & 1.02 & 11 & extant & 0 & NA & LC & Ginsect \\
\hline EA & Soricomorpha & Soricidae & Sorex & kozlovi & $\begin{array}{l}\text { Kozlov's Shrew } \\
\text { Eurasian Least Shrew, }\end{array}$ & 0.83 & 7 & extant & 0 & NA & DD & Ginsect \\
\hline EA & Soricomorpha & Soricidae & Sorex & minutissimus & $\begin{array}{l}\text { Least Siberian Shrew, } \\
\text { Miniscule Shrew, Least } \\
\text { Shrew }\end{array}$ & 0.43 & 3 & extant & 0 & NA & LC & Ginsect \\
\hline EA & Soricomorpha & Soricidae & Sorex & minutus & $\begin{array}{l}\text { Eurasian Pygmy Shrew, } \\
\text { Pygmy Shrew }\end{array}$ & 0.65 & 5 & extant & 0 & NA & LC & Ginsect \\
\hline EA & Soricomorpha & Soricidae & Sorex & mirabilis & Ussuri Shrew & 0.83 & 7 & extant & 0 & NA & DD & Ginsect \\
\hline EA & Soricomorpha & Soricidae & Sorex & planiceps & Kashmir Shrew & 0.83 & 7 & extant & 0 & NA & LC & Ginsect \\
\hline EA & Soricomorpha & Soricidae & Sorex & portenkoi & Portenko's Shrew & 0.83 & 7 & extant & 0 & NA & DD & Ginsect \\
\hline EA & Soricomorpha & Soricidae & Sorex & raddei & Radde's Shrew & 1.00 & 10 & extant & 0 & NA & LC & Ginsect \\
\hline EA & Soricomorpha & Soricidae & Sorex & roboratus & Flat-skulled Shrew & 0.96 & 9 & extant & 0 & NA & $\mathrm{LC}$ & Ginsect \\
\hline EA & Soricomorpha & Soricidae & Sorex & samniticus & Appenine Shrew & 0.92 & 8 & extant & 0 & NA & LC & Ginsect \\
\hline EA & Soricomorpha & Soricidae & Sorex & satunini & Caucasian Shrew & 0.83 & 7 & extant & 0 & NA & LC & Ginsect \\
\hline EA & Soricomorpha & Soricidae & Sorex & sinalis & $\begin{array}{l}\text { Chinese Shrew, Dusky } \\
\text { Shrew }\end{array}$ & 0.97 & 9 & extant & 0 & NA & DD & Ginsect \\
\hline EA & Soricomorpha & Soricidae & Sorex & thibetanus & Tibetan Shrew & 0.83 & 7 & extant & 0 & NA & DD & Ginsect \\
\hline EA & Soricomorpha & Soricidae & Sorex & tundrensis & $\begin{array}{l}\text { Tundra Shrew, TUNDRA } \\
\text { SHREW }\end{array}$ & 0.91 & 8 & extant & 0 & NA & LC & Ginsect \\
\hline EA & Soricomorpha & Soricidae & Sorex & unguiculatus & Long-clawed Shrew & 1.00 & 10 & extant & 0 & NA & LC & Ginsect \\
\hline EA & Soricomorpha & Soricidae & Sorex & volnuchini & Caucasian Pygmy Shrew & 0.83 & 7 & extant & 0 & NA & LC & Ginsect \\
\hline EA & Soricomorpha & Soricidae & Soriculus & nigrescens & $\begin{array}{l}\text { Himalayan Shrew, } \\
\text { Sikkim Large-clawed } \\
\text { Shrew }\end{array}$ & 1.15 & 14 & extant & 0 & NA & LC & Ginsect \\
\hline EA & Soricomorpha & Soricidae & Suncus & dayi & $\begin{array}{l}\text { Day's Shrew } \\
\text { Pygmy White-toothed } \\
\text { Shrew, White-toothed }\end{array}$ & 0.97 & 9 & extant & Future & NA & EN & Ginsect \\
\hline EA & Soricomorpha & Soricidae & Suncus & etruscus & $\begin{array}{l}\text { Pygmy Shrew, Savi's } \\
\text { Pygmy Shrew, Etruscan } \\
\text { Shrew }\end{array}$ & 0.37 & 2 & extant & 0 & NA & LC & ginsect \\
\hline EA & Soricomorpha & Soricidae & Suncus & malayanus & $\begin{array}{l}\text { Malayan Pygmy Shrew } \\
\text { Hill Shrew, Montane }\end{array}$ & 0.70 & 5 & extant & 0 & NA & LC & Ginsect \\
\hline EA & Soricomorpha & Soricidae & Suncus & montanus & $\begin{array}{l}\text { Shrew, Sri Lanka } \\
\text { Highland Shrew }\end{array}$ & 1.26 & 18 & extant & Future & NA & $\mathrm{Vu}$ & Ginsect \\
\hline
\end{tabular}




\begin{tabular}{|c|c|c|c|c|c|c|c|c|c|c|c|c|}
\hline EA & Soricomorpha & Soricidae & Suncus & murinus & $\begin{array}{l}\text { House Shrew, Asian } \\
\text { House Shrew }\end{array}$ & 1.83 & 68 & extant & 0 & NA & LC & ginsect \\
\hline EA & Soricomorpha & Soricidae & Suncus & stoliczkanus & Anderson's Shrew & 0.97 & 9 & extant & 0 & NA & LC & Ginsect \\
\hline EA & Soricomorpha & Talpidae & Desmana & moschata & $\begin{array}{l}\text { Russian Desman, } \\
\text { RUSSIAN DESMAN }\end{array}$ & 2.58 & 383 & extant & Future & NA & Vu & Ginsect \\
\hline EA & Soricomorpha & Talpidae & Euroscaptor & grandis & Greater Chinese Mole & 1.78 & 60 & extant & 0 & NA & LC & \\
\hline EA & Soricomorpha & Talpidae & Euroscaptor & klossi & $\begin{array}{l}\text { Kloss's Mole, Kloss's } \\
\text { Mole }\end{array}$ & 1.78 & 60 & extant & 0 & NA & LC & \\
\hline EA & Soricomorpha & Talpidae & Euroscaptor & longirostris & Long-nosed Mole & 1.78 & 60 & extant & 0 & NA & LC & \\
\hline EA & Soricomorpha & Talpidae & Euroscaptor & micrura & Himalayan Mole & 1.78 & 60 & extant & 0 & NA & LC & Ginsect \\
\hline EA & Soricomorpha & Talpidae & Euroscaptor & parvidens & Small-toothed Mole & 1.78 & 60 & extant & 0 & NA & DD & \\
\hline EA & Soricomorpha & Talpidae & Galemys & pyrenaicus & Pyrenean Desman & 1.78 & 60 & extant & Future & NA & Vu & Ginsect \\
\hline EA & Soricomorpha & Talpidae & Mogera & wogura & $\begin{array}{l}\text { Japanese Mole, Large } \\
\text { Japanese Mole } \\
\text { White-tailed Mole, }\end{array}$ & 2.52 & 329 & extant & 0 & NA & LC & Ginsect \\
\hline EA & Soricomorpha & Talpidae & Parascaptor & leucura & $\begin{array}{l}\text { Indian Mole, Assamese } \\
\text { Mole }\end{array}$ & 1.66 & 46 & extant & 0 & NA & LC & Ginsect \\
\hline EA & Soricomorpha & Talpidae & Scapanulus & oweni & Gansu Mole & 1.56 & 37 & extant & 0 & NA & LC & Ginsect \\
\hline EA & Soricomorpha & Talpidae & Scaptochirus & moschatus & Short-faced Mole & 1.96 & 91 & extant & 0 & NA & $\mathrm{LC}$ & Ginsect \\
\hline EA & Soricomorpha & Talpidae & Scaptonyx & fusicaudus & Long-tailed Mole & 1.29 & 19 & extant & 0 & NA & LC & Ginsect \\
\hline EA & Soricomorpha & Talpidae & Talpa & altaica & Siberian Mole & 1.93 & 85 & extant & 0 & NA & $\mathrm{LC}$ & Ginsect \\
\hline EA & Soricomorpha & Talpidae & Talpa & caeca & $\begin{array}{l}\text { Blind Mole, } \\
\text { Mediterranean Mole }\end{array}$ & 1.97 & 93 & extant & 0 & NA & LC & Ginsect \\
\hline EA & Soricomorpha & Talpidae & Talpa & caucasica & Caucasian Mole & 1.95 & 90 & extant & 0 & NA & LC & Ginsect \\
\hline EA & Soricomorpha & Talpidae & Talpa & europaea & $\begin{array}{l}\text { European Mole, } \\
\text { Common Mole }\end{array}$ & 1.89 & 77 & extant & 0 & NA & LC & Ginsect \\
\hline EA & Soricomorpha & Talpidae & Talpa & levantis & $\begin{array}{l}\text { Levantine Mole, Levant } \\
\text { Mole }\end{array}$ & 1.95 & 90 & extant & 0 & NA & LC & Ginsect \\
\hline EA & Soricomorpha & Talpidae & Talpa & occidentalis & Iberian Mole & 1.73 & 54 & extant & 0 & NA & LC & Ginsect \\
\hline EA & Soricomorpha & Talpidae & Talpa & romana & Roman Mole & 1.97 & 93 & extant & 0 & NA & LC & Ginsect \\
\hline EA & Soricomorpha & Talpidae & Talpa & stankovici & $\begin{array}{l}\text { Balkan Mole, BALKAN } \\
\text { MOLE, Stankovic's Mole }\end{array}$ & 1.92 & 83 & extant & 0 & NA & LC & \\
\hline EA & Soricomorpha & Talpidae & Uropsilus & andersoni & Anderson's Shrew Mole & 1.20 & 16 & extant & 0 & NA & DD & \\
\hline EA & Soricomorpha & Talpidae & Uropsilus & gracilis & Gracile Shrew Mole & 1.20 & 16 & extant & 0 & NA & LC & \\
\hline EA & Soricomorpha & Talpidae & Uropsilus & investigator & Inquisitive Shrew Mole & 1.20 & 16 & extant & 0 & NA & DD & \\
\hline EA & Soricomorpha & Talpidae & Uropsilus & soricipes & $\begin{array}{l}\text { Chinese Shrew Mole } \\
\text { Pronghorn, American }\end{array}$ & 1.20 & 16 & extant & 0 & NA & LC & Ginsect \\
\hline NA & Artiodactyla & Antilocapridae & Antilocapra & americana & $\begin{array}{l}\text { Pronghorn, Mexican } \\
\text { Pronghorn }\end{array}$ & 4.66 & 46083 & extant & 0 & NA & LC & zraze/browse \\
\hline NA & Artiodactyla & Antilocapridae & Capromeryx & mexicana & might be syn of C.minor & 4.18 & 15000 & extinct & TP & 12 & EX & żaze/browse \\
\hline NA & Artiodactyla & Antilocapridae & Capromeryx & minor & & 4.00 & 10000 & extinct & TP & 12 & EX & zraze/browse \\
\hline NA & Artiodactyla & Antilocapridae & Stockoceros & conklingi & & 4.71 & 51000 & extinct & TP & 12 & EX & э̧raze/browse \\
\hline NA & Artiodactyla & Antilocapridae & Stockoceros & onusrosagris & & 4.74 & 55000 & extinct & TP & 12 & EX & зraze/browse \\
\hline NA & Artiodactyla & Antilocapridae & Tetrameryx & shuleri & & 4.78 & 60000 & extinct & TP & 12 & EX & э̧raze/browse \\
\hline NA & Artiodactyla & Bovidae & Bison & bison & American Bison & 5.76 & 579255 & extant & Future & NA & NT & Graze \\
\hline NA & Artiodactyla & Bovidae & Bison & latifrons & steppe bison; LP? & 5.95 & 900000 & extinct & TP & 20 & EX & graze \\
\hline NA & Artiodactyla & Bovidae & Bison & priscus & $\begin{array}{l}\text { Replaced by B. } \\
\text { latifrons? }\end{array}$ & 5.95 & 900000 & extinct & TP & 12 & EX & graze \\
\hline
\end{tabular}




\begin{tabular}{|c|c|c|c|c|c|c|c|c|c|c|c|c|}
\hline NA & Artiodactyla & Bovidae & Bootherium & bombifrons & $\begin{array}{l}\text { Harlan's muskox, } \\
\text { woodland muskox }\end{array}$ & 5.63 & 423000 & extinct & TP & 11 & EX & graze \\
\hline NA & Artiodactyla & Bovidae & Euceratherium & collinum & shrub-ox & 5.65 & 450000 & extinct & TP & 11.5 & EX & graze \\
\hline NA & Artiodactyla & Bovidae & Oreamnos & americanus & $\begin{array}{l}\text { Mountain Goat, Rocky } \\
\text { Mountain Goat }\end{array}$ & 4.86 & 72500 & extant & 0 & NA & LC & graze/browse \\
\hline NA & Artiodactyla & Bovidae & Oreamnos & harringtoni & $\begin{array}{l}\text { Harrington's mountain } \\
\text { goat }\end{array}$ & 4.88 & 75000 & extinct & TP & 11 & EX & graze/browse \\
\hline NA & Artiodactyla & Bovidae & Ovibos & moschatus & Muskox, Musk Ox & 5.57 & 368502 & extant & 0 & NA & LC & Graze \\
\hline NA & Artiodactyla & Bovidae & Ovis & canadensis & $\begin{array}{l}\text { Bighorn Sheep, } \\
\text { Mountain Sheep }\end{array}$ & 4.87 & 74645 & extant & 0 & NA & $\mathrm{LC}$ & Graze \\
\hline NA & Artiodactyla & Bovidae & Ovis & dalli & $\begin{array}{l}\text { Thinhorn Sheep, Dall's } \\
\text { Sheep }\end{array}$ & 4.75 & 55651 & extant & 0 & NA & $\mathrm{LC}$ & Graze \\
\hline NA & Artiodactyla & Bovidae & Saiga & spp. & & 4.70 & 50000 & extinct & TP & NA & EX & graze \\
\hline NA & Artiodactyla & Camelidae & Camelops & hesternus & & 6.04 & 1100000 & extinct & TP & 11 & EX & J̄aze/brows \\
\hline NA & Artiodactyla & Camelidae & Camelops & huerfanensis & & 5.85 & 700000 & extinct & TP & 12 & EX & J̈raze/brows \\
\hline NA & Artiodactyla & Camelidae & Hemiauchenia & macrocephala & large-headed Ilama & 5.04 & 110000 & extinct & TP & 11 & EX & graze \\
\hline NA & Artiodactyla & Camelidae & Palaeolama & mirifica & $\begin{array}{l}\text { stout-legged Ilama } \\
\text { Moose, Elk, Eurasian }\end{array}$ & 5.35 & 223000 & extinct & TP & 11 & EX & browse \\
\hline NA & Artiodactyla & Cervidae & Alces & alces & $\begin{array}{l}\text { Moose, European Elk, } \\
\text { Eurasian Elk, Siberian } \\
\text { Elk }\end{array}$ & 5.56 & 358996 & extant & 0 & NA & $\mathrm{LC}$ & Browse \\
\hline NA & Artiodactyla & Cervidae & Cervalces & scotti & $\begin{array}{l}\text { stag moose } \\
\text { Red Deer, Bactrian }\end{array}$ & 5.80 & 632000 & extinct & TP & 11 & EX & browse \\
\hline NA & Artiodactyla & Cervidae & Cervus & elaphus & $\begin{array}{l}\text { Wapiti, Bokharan Deer, } \\
\text { Bukhara Red Deer, Elk, } \\
\text { Bactrian Deer, Wapiti, } \\
\text { Bactrian Red Deer }\end{array}$ & 5.34 & 217751 & extant & 0 & NA & $\mathrm{LC}$ & Browse \\
\hline NA & Artiodactyla & Cervidae & Cervus & whitneyi & & 5.20 & 157500 & extinct & TP & 12 & EX & browse \\
\hline NA & Artiodactyla & Cervidae & Mazama & americana & Red Brocket & 4.36 & 22800 & extant & 0 & NA & $\mathrm{DD}$ & Browse \\
\hline NA & Artiodactyla & Cervidae & Navahoceros & fricki & $\begin{array}{l}\text { American mountain } \\
\text { deer } \\
\text { Mule Deer, Black-tailed }\end{array}$ & 5.40 & 250000 & extinct & TP & 11 & EX & browse \\
\hline NA & Artiodactyla & Cervidae & Odocoileus & hemionus & $\begin{array}{l}\text { Deer, Cedros Island } \\
\text { Mule Deer, Cedros } \\
\text { Island Black-tailed Deer }\end{array}$ & 4.73 & 54213 & extant & 0 & NA & LC & Browse \\
\hline NA & Artiodactyla & Cervidae & Odocoileus & virginianus & $\begin{array}{l}\text { White-tailed Deer, Key } \\
\text { Deer, Key Deer Toy Deer }\end{array}$ & 4.74 & 55509 & extant & 0 & NA & LC & Browse \\
\hline NA & Artiodactyla & Cervidae & Rangifer & tarandus & $\begin{array}{l}\text { Reindeer, Caribou, } \\
\text { Peary Caribou }\end{array}$ & 4.93 & 86034 & extant & Future & NA & Vu & эraze/browse \\
\hline NA & Artiodactyla & Tayassuidae & Mylohyus & nasutus & long-nosed peccary & 4.88 & 75000 & extinct & TP & 10 & EX & browse \\
\hline NA & Artiodactyla & Tayassuidae & Pecari & tajacu & Collared Peccary & 4.33 & 21267 & extant & 0 & NA & LC & Frug/browse \\
\hline NA & Artiodactyla & Tayassuidae & Platygonus & compressus & $\begin{array}{l}\text { flat headed peccary } \\
\text { m1 14.4 x 12.1; Lg Body }\end{array}$ & 5.13 & 135000 & extinct & TP & 11 & EX & browse \\
\hline NA & Artiodactyla & Tayassuidae & Platygonus & vetus & $\begin{array}{l}\text { Mass }(\mathrm{g})=3.2 *(\mathrm{lg} \\
14.4)+2.694=11.229\end{array}$ & 4.88 & 75282 & extinct & TP & 12 & EX & browse \\
\hline NA & Artiodactyla & Tayassuidae & Tayassu & pecari & White-lipped Peccary & 4.51 & 32234 & extant & Future & NA & Vu & Frug/browse \\
\hline NA & Carnivora & Canidae & Canis & dirus & & 4.81 & 65000 & extinct & TP & 12 & EX & carn \\
\hline NA & Carnivora & Canidae & Canis & latrans & Coyote & 4.13 & 13406 & extant & 0 & NA & LC & Carn \\
\hline NA & Carnivora & Canidae & Canis & lupus & Wolf & 4.63 & 42750 & extant & 0 & NA & LC & Carn \\
\hline
\end{tabular}




\begin{tabular}{|c|c|c|c|c|c|c|c|c|c|c|c|c|}
\hline NA & Carnivora & Canidae & Cuon & alpinus & $\begin{array}{l}\text { Dhole, Red Dog, Indian } \\
\text { Wild Dog, Asiatic Wild } \\
\text { Dog }\end{array}$ & 4.23 & 17000 & extinct & TP & 12 & EX & carn \\
\hline NA & Carnivora & Canidae & Urocyon & cinereoargenteus & $\begin{array}{l}\text { Tree Fox, Gray Fox } \\
\text { Island Fox, California }\end{array}$ & 3.58 & 3834 & extant & 0 & NA & $\mathrm{LC}$ & Carn \\
\hline NA & Carnivora & Canidae & Urocyon & littoralis & $\begin{array}{l}\text { Channel Island Fox, } \\
\text { Channel Islands Fox, } \\
\text { Island Gray Fox, Island } \\
\text { Grey Fox }\end{array}$ & 3.28 & 1896 & extant & Future & NA & NT & carn/frug \\
\hline NA & Carnivora & Canidae & Vulpes & lagopus & Arctic Fox, Polar Fox & 3.54 & 3450 & extant & 0 & NA & LC & Carn \\
\hline NA & Carnivora & Canidae & Vulpes & velox & Swift Fox & 3.34 & 2198 & extant & 0 & NA & LC & Carn \\
\hline NA & Carnivora & Canidae & Vulpes & vulpes & $\begin{array}{l}\text { Red Fox, Silver Fox, } \\
\text { Cross Fox }\end{array}$ & 3.62 & 4132 & extant & 0 & NA & $\mathrm{LC}$ & Carn \\
\hline NA & Carnivora & Felidae & Felis & amnicola & & 3.70 & 5000 & extinct & TP & 11 & EX & carn \\
\hline NA & Carnivora & Felidae & Herpailurus & yaguarondi & Jaguarundi, Eyra Cat & 3.95 & 9000 & extant & 0 & NA & $\mathrm{LC}$ & Carn \\
\hline NA & Carnivora & Felidae & Homotherium & serum & & 5.28 & 189000 & extinct & TP & 11 & EX & carn \\
\hline NA & Carnivora & Felidae & Leopardus & pardalis & $\begin{array}{l}\text { Ocelot } \\
\text { Oncilla, Little Spotted }\end{array}$ & 4.08 & 11900 & extant & 0 & NA & $\mathrm{LC}$ & Carn \\
\hline NA & Carnivora & Felidae & Leopardus & tigrinus & $\begin{array}{l}\text { Cat, Tiger Cat, Little } \\
\text { Tiger Cat }\end{array}$ & 3.35 & 2250 & extant & Future & NA & VU & Carn \\
\hline NA & Carnivora & Felidae & Leopardus & wiedii & Margay, Tree Ocelot & 3.51 & 3250 & extant & Future & NA & NT & Carn \\
\hline NA & Carnivora & Felidae & Lynx & canadensis & $\begin{array}{l}\text { Canada Lynx, American } \\
\text { Lynx }\end{array}$ & 3.97 & 9373 & extant & 0 & NA & $\mathrm{LC}$ & Carn \\
\hline NA & Carnivora & Felidae & $\operatorname{Lyn} x$ & rufus & Bobcat, Bay Lynx & 3.95 & 8904 & extant & 0 & NA & $\mathrm{LC}$ & Carn \\
\hline NA & Carnivora & Felidae & Miracinonyx & trumani & & 4.94 & 88000 & extinct & TP & 11 & EX & carn \\
\hline NA & Carnivora & Felidae & Panthera & leo & Lion, African Lion & 5.64 & 433200 & extinct & TP & 11 & EX & carn \\
\hline NA & Carnivora & Felidae & Panthera & onca & $\begin{array}{l}\text { Jaguar } \\
\text { Puma, Mountain Lion, }\end{array}$ & 5.00 & 100000 & extant & Future & NA & NT & Carn \\
\hline NA & Carnivora & Felidae & Puma & concolor & $\begin{array}{l}\text { Cougar, Red Tiger, Deer } \\
\text { Tiger }\end{array}$ & 4.71 & 51600 & extant & 0 & NA & LC & Carn \\
\hline NA & Carnivora & Felidae & Smilodon & fatalis & $\begin{array}{l}\text { saber-toothed cat } \\
\text { American Hog-nosed } \\
\text { Skunk, White-backed }\end{array}$ & 5.60 & 400000 & extinct & TP & 11 & EX & carn \\
\hline NA & Carnivora & Mephitidae & Conepatus & leuconotus & $\begin{array}{l}\text { Hog-nosed Skunk, } \\
\text { Eastern Hog-nosed } \\
\text { Skunk } \\
\text { Striped Hog-nosed }\end{array}$ & 3.54 & 3500 & extant & 0 & NA & $\mathrm{LC}$ & Ginsect/carr \\
\hline NA & Carnivora & Mephitidae & Conepatus & semistriatus & $\begin{array}{l}\text { Skunk, Amazonian Hog- } \\
\text { nosed Skunk }\end{array}$ & 3.08 & 1200 & extant & 0 & NA & LC & Ginsect/carr \\
\hline NA & Carnivora & Mephitidae & Mephitis & macroura & Hooded Skunk & 2.90 & 801 & extant & 0 & NA & LC & Ginsect/carr \\
\hline NA & Carnivora & Mephitidae & Mephitis & mephitis & Striped Skunk & 3.32 & 2085 & extant & 0 & NA & LC & Ginsect/carr \\
\hline NA & Carnivora & Mephitidae & Spilogale & putorius & $\begin{array}{l}\text { Eastern Spotted Skunk, } \\
\text { Plains Spotted Skunk, } \\
\text { Allegheny Spotted } \\
\text { Skunk, Appalachian } \\
\text { Spotted Skunk, Spotted } \\
\text { Skunk }\end{array}$ & 2.53 & 341 & extant & Future & NA & Vu & carn/frug \\
\hline NA & Carnivora & Mephitidae & Spilogale & pygmaea & Pygmy Spotted Skunk & 2.37 & 235 & extant & Future & NA & Vu & carn/frug \\
\hline NA & Carnivora & Mustelidae & Brachyprotoma & obtusata & short-faced skunk & 3.65 & 4500 & extinct & TP & 12 & EX & ginsect/frug \\
\hline
\end{tabular}




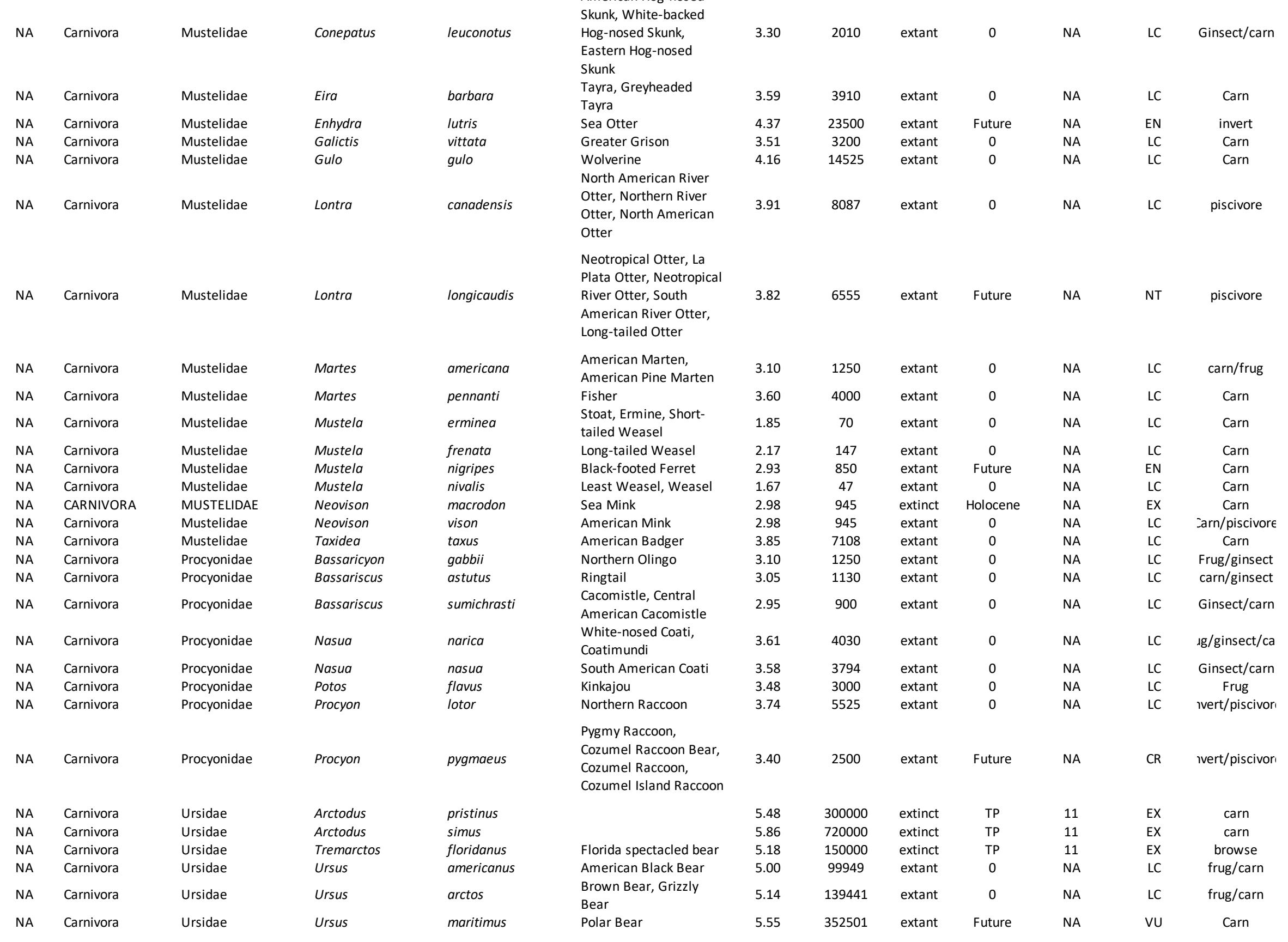




\begin{tabular}{|c|c|c|c|c|c|c|c|c|c|c|c|c|}
\hline NA & Cingulata & Dasypodidae & Cabassous & centralis & $\begin{array}{l}\text { Northern Naked-tailed } \\
\text { Armadillo }\end{array}$ & 3.58 & 3810 & extant & 0 & NA & DD & Ginsect \\
\hline NA & Cingulata & Dasypodidae & Dasypus & bellus & $\begin{array}{l}\text { beautiful armadillo } \\
\text { Nine-banded Armadillo, }\end{array}$ & 4.65 & 45000 & extinct & TP & 11 & EX & \\
\hline NA & Cingulata & Dasypodidae & Dasypus & novemcinctus & $\begin{array}{l}\text { Common Long-nosed } \\
\text { Armadillo }\end{array}$ & 3.62 & 4204 & extant & 0 & NA & LC & Ginsect/carn \\
\hline NA & Cingulata & Dasypodidae & Holmesi & septentriolis & & 5.40 & 250000 & extinct & TP & 11 & EX & browse \\
\hline NA & Cingulata & Glyptodontidae & Glyptotherium & floridanum & & 6.04 & 1100000 & extinct & TP & 12 & EX & browse \\
\hline NA & Cingulata & Glyptodontidae & Glyptotherium & mexicanum & & 6.04 & 1100000 & extinct & TP & NA & EX & browse \\
\hline NA & Didelphimorphia & Didelphidae & Caluromys & derbianus & $\begin{array}{l}\text { Central American } \\
\text { Woolly Opossum, } \\
\text { Derby's Woolly } \\
\text { Opossum }\end{array}$ & 2.47 & 297 & extant & 0 & NA & LC & :/browse/gins \\
\hline NA & Didelphimorphia & Didelphidae & Chironectes & minimus & $\begin{array}{l}\text { Water Opossum, Yapok } \\
\text { Common Opossum, }\end{array}$ & 2.98 & 946 & extant & 0 & NA & LC & ivert/piscivor \\
\hline NA & Didelphimorphia & Didelphidae & Didelphis & marsupialis & $\begin{array}{l}\text { Southern Opossum, } \\
\text { Black-eared Opossum }\end{array}$ & 3.04 & 1091 & extant & 0 & NA & LC & carn/frug \\
\hline NA & Didelphimorphia & Didelphidae & Didelphis & virginiana & Virginia Opossum & 3.34 & 2195 & extant & 0 & NA & LC & carn/frug \\
\hline NA & Didelphimorphia & Didelphidae & Marmosa & mexicana & $\begin{array}{l}\text { Mexican Mouse } \\
\text { Opossum }\end{array}$ & 1.69 & 49 & extant & 0 & NA & LC & ginsect/frug \\
\hline NA & Didelphimorphia & Didelphidae & Micoureus & alstoni & $\begin{array}{l}\text { Alston's Woolly Mouse } \\
\text { Opossum }\end{array}$ & 2.12 & 132 & extant & 0 & NA & LC & ginsect/frug \\
\hline NA & Didelphimorphia & Didelphidae & Philander & opossum & $\begin{array}{l}\text { Gray Four-eyed } \\
\text { Opossum, Guaiki }\end{array}$ & 2.88 & 750 & extant & 0 & NA & LC & Carn \\
\hline NA & Didelphimorphia & Didelphidae & Tlacuatzin & canescens & $\begin{array}{l}\text { Grayish Mouse } \\
\text { Opossum }\end{array}$ & 1.78 & 60 & extant & 0 & NA & LC & ginsect/frug \\
\hline NA & Lagomorpha & Leporidae & Aztlanolagus & agilis & & 3.30 & 2000 & extinct & TP & 10 & EX & browse \\
\hline NA & Lagomorpha & Leporidae & Brachylagus & idahoensis & Pygmy Rabbit & 2.54 & 350 & extant & 0 & NA & LC & browse \\
\hline NA & Lagomorpha & Leporidae & Lepus & alleni & $\begin{array}{l}\text { Antelope Jackrabbit } \\
\text { Snowshoe Hare, }\end{array}$ & 3.57 & 3685 & extant & 0 & NA & LC & 3rowse/graze \\
\hline NA & Lagomorpha & Leporidae & Lepus & americanus & $\begin{array}{l}\text { Snowshoe Rabbit, } \\
\text { Varying Hare }\end{array}$ & 3.23 & 1710 & extant & 0 & NA & LC & Graze \\
\hline NA & Lagomorpha & Leporidae & Lepus & arcticus & Arctic Hare & 3.64 & 4405 & extant & 0 & NA & LC & Graze \\
\hline NA & Lagomorpha & Leporidae & Lepus & californicus & Black-tailed Jackrabbit & 3.38 & 2422 & extant & 0 & NA & LC & Graze \\
\hline NA & Lagomorpha & Leporidae & Lepus & callotis & $\begin{array}{l}\text { White-sided Jackrabbit } \\
\text { Tehuantepec Jackrabbit, } \\
\text { Tropical Hare, }\end{array}$ & 3.40 & 2500 & extant & Future & NA & NT & Graze \\
\hline NA & Lagomorpha & Leporidae & Lepus & flavigularis & $\begin{array}{l}\text { Tehuantepec Jack } \\
\text { Rabbit, Tehuantepec } \\
\text { Hare }\end{array}$ & 3.48 & 3000 & extant & Future & NA & EN & Browse \\
\hline NA & Lagomorpha & Leporidae & Lepus & othus & Alaskan Hare & 3.68 & 4806 & extant & 0 & NA & LC & Graze \\
\hline NA & Lagomorpha & Leporidae & Lepus & townsendii & White-tailed Jackrabbit & 3.19 & 1555 & extant & 0 & NA & LC & Graze \\
\hline NA & Lagomorpha & Leporidae & Romerolagus & diazi & Volcano Rabbit & 2.68 & 477 & extant & Future & NA & EN & Browse \\
\hline NA & Lagomorpha & Leporidae & Sylvilagus & aquaticus & Swamp Rabbit & 3.33 & 2136 & extant & 0 & NA & LC & Graze \\
\hline NA & Lagomorpha & Leporidae & Sylvilagus & audubonii & $\begin{array}{l}\text { Desert Cottontail, } \\
\text { Audubon's Cottontail }\end{array}$ & 2.95 & 890 & extant & 0 & NA & LC & Graze \\
\hline NA & Lagomorpha & Leporidae & Sylvilagus & bachmani & $\begin{array}{l}\text { Brush Rabbit, Riparian } \\
\text { Brush Rabbit }\end{array}$ & 2.81 & 643 & extant & 0 & NA & LC & Graze \\
\hline NA & Lagomorpha & Leporidae & Sylvilagus & brasiliensis & Tapeti, Forest Rabbit & 2.98 & 950 & extant & 0 & NA & LC & Browse \\
\hline NA & Lagomorpha & Leporidae & Sylvilagus & cunicularius & Mexican Cottontail & 3.48 & 3000 & extant & 0 & NA & LC & Browse \\
\hline NA & Lagomorpha & Leporidae & Sylvilagus & dicei & Dice's Cottontail & 3.14 & 1378 & extant & 0 & NA & DD & Browse \\
\hline NA & Lagomorpha & Leporidae & Sylvilagus & floridanus & Eastern Cottontail & 3.07 & 1173 & extant & 0 & NA & LC & Graze \\
\hline
\end{tabular}




\begin{tabular}{|c|c|c|c|c|c|c|c|c|c|c|c|c|}
\hline NA & Lagomorpha & Leporidae & Sylvilagus & graysoni & $\begin{array}{l}\text { Tres Marias Cottontail, } \\
\text { Tres Marias Rabbit }\end{array}$ & 2.98 & 964 & extant & Future & NA & EN & Browse \\
\hline NA & Lagomorpha & Leporidae & Sylvilagus & insonus & $\begin{array}{l}\text { Omilteme Cottontail, } \\
\text { Omiltemi Rabbit }\end{array}$ & 3.48 & 3000 & extant & Future & NA & EN & Browse \\
\hline NA & Lagomorpha & Leporidae & Sylvilagus & leonensis & & 3.18 & 1500 & extinct & TP & NA & EX & browse \\
\hline NA & Lagomorpha & Leporidae & Sylvilagus & nuttallii & $\begin{array}{l}\text { Mountain Cottontail, } \\
\text { Nuttall's Cottontail } \\
\text { Marsh Rabbit, Lower }\end{array}$ & 2.88 & 755 & extant & 0 & NA & LC & Graze \\
\hline NA & Lagomorpha & Leporidae & Sylvilagus & palustris & $\begin{array}{l}\text { Keys Rabbit, Lower Keys } \\
\text { Marsh Rabbit, Key } \\
\text { Rabbit }\end{array}$ & 3.18 & 1500 & extant & 0 & NA & LC & Browse \\
\hline NA & Lagomorpha & Leporidae & Sylvilagus & transitionalis & $\begin{array}{l}\text { New England Cottontail, } \\
\text { Wood Rabbit }\end{array}$ & 2.82 & 657 & extant & Future & NA & VU & Graze \\
\hline NA & Lagomorpha & Ochotonidae & Ochotona & collaris & $\begin{array}{l}\text { Collared Pika } \\
\text { American Pika, Little }\end{array}$ & 2.11 & 129 & extant & 0 & NA & LC & Graze \\
\hline NA & Lagomorpha & Ochotonidae & Ochotona & princeps & $\begin{array}{l}\text { Chief Hare, Cony, Rock } \\
\text { Rabbit, Whistling Hare }\end{array}$ & 2.20 & 158 & extant & 0 & NA & LC & Graze \\
\hline NA & Perissodactyla & Equidae & Equus & alaskae & & 5.57 & 372000 & extinct & TP & 11 & EX & graze \\
\hline NA & Perissodactyla & Equidae & Equus & caballas & & 5.81 & 648000 & extinct & TP & NA & EX & graze \\
\hline NA & Perissodactyla & Equidae & Equus & complicatus & & 5.60 & 400000 & extinct & TP & 11 & EX & graze \\
\hline NA & Perissodactyla & Equidae & Equus & conversidens & & 5.49 & 306000 & extinct & TP & 11 & EX & graze \\
\hline NA & Perissodactyla & Equidae & Equus & giganteus & & 5.60 & 400000 & extinct & TP & 11 & EX & graze \\
\hline NA & Perissodactyla & Equidae & Equus & mexicanus & & 5.76 & 574000 & extinct & TP & NA & EX & graze \\
\hline NA & Perissodactyla & Equidae & Equus & scotti & & 5.74 & 555000 & extinct & TP & 11 & EX & graze \\
\hline NA & Perissodactyla & Tapiridae & Tapirus & bairdii & $\begin{array}{l}\text { Baird's Tapir, Central } \\
\text { American Tapir }\end{array}$ & 5.48 & 299999 & extant & Future & NA & EN & 3rowse/graze \\
\hline NA & Perissodactyla & Tapiridae & Tapirus & californicus & & 5.60 & 400000 & extinct & TP & 11 & EX & graze \\
\hline NA & Perissodactyla & Tapiridae & Tapirus & copei & Cope's tapir & 5.50 & 317000 & extinct & TP & NA & EX & graze \\
\hline NA & Perissodactyla & Tapiridae & Tapirus & veroensis & & 5.44 & 275000 & extinct & TP & 11 & EX & graze \\
\hline NA & Pilosa & Bradypodidae & Bradypus & variegatus & $\begin{array}{l}\text { Brown-throated Sloth, } \\
\text { Brown-throated Three- } \\
\text { toed Sloth, Bolivian } \\
\text { Three-toed Sloth }\end{array}$ & 3.64 & 4335 & extant & 0 & NA & LC & Browse \\
\hline NA & Pilosa & Cyclopedidae & Cyclopes & didactylus & $\begin{array}{l}\text { Silky Anteater, Pygmy } \\
\text { Anteater }\end{array}$ & 2.52 & 330 & extant & 0 & NA & LC & Ginsect \\
\hline NA & Pilosa & Megalonychidae & Choloepus & hoffmanni & $\begin{array}{l}\text { Hoffmann's Two-toed } \\
\text { Sloth }\end{array}$ & 3.78 & 6000 & extant & 0 & NA & LC & Browse \\
\hline NA & Pilosa & Megalonychidae & Megalonyx & jeffersonii & & 5.78 & 600000 & extinct & TP & 11 & EX & browse \\
\hline NA & Pilosa & Megalonychidae & Nothrotherium & shastense & & & & extinct & TP & 12 & EX & Browse \\
\hline NA & Pilosa & Megatheriidae & Eremotherium & laurillardi & & 6.54 & 3500000 & extinct & TP & 11 & EX & browse \\
\hline NA & Pilosa & Megatheriidae & Nothrotheriops & shastensis & Shasta ground sloth & 5.48 & 300000 & extinct & TP & 11 & EX & browse \\
\hline NA & Pilosa & Mylodontidae & Paramylodon & harlani & Harlan's ground sloth & 6.20 & 1587000 & extinct & TP & 11 & EX & browse \\
\hline NA & Pilosa & Myrmecophagidae & Myrmecophaga & tridactyla & Giant Anteater & 4.35 & 22333 & extant & Future & NA & VU & Ginsect \\
\hline NA & Pilosa & Myrmecophagidae & Tamandua & mexicana & $\begin{array}{l}\text { Northern Tamandua } \\
\text { Colombian Night }\end{array}$ & 3.62 & 4210 & extant & 0 & NA & LC & Ginsect \\
\hline NA & Primates & Aotidae & Aotus & lemurinus & $\begin{array}{l}\text { Monkey, Lemurine } \\
\text { Night Monkey, Gray- } \\
\text { bellied Night Monkey }\end{array}$ & 2.94 & 873 & extant & Future & NA & VU & Frug/browse \\
\hline
\end{tabular}


Monkey, Ecuadoria

Mantled Howling

Howler

Yucat`n Black Howler

Monkey, Mexican Black

Howler Monkey,

Guatemalan Black

Howler Monkey,

Guatemalan Howler,

Guatemalan Howling

Monkey, Lawrence's

Howler Monkey,

Guatemalan Black

Howler, Black Howling

Monkey

Geoffroy's Spide

Monkey, Black-handed

Spider Monkey, Central

American Spider

Atelidae

Ateles

geoffroyi

Monkey, Centra

American Spider

Monkey, Black-headed

Spider Monkey, Mono

Colorado

Cebidae

Cebus

capucinus

white-headed capuchin Black-crowned Central

merican Squirrel

Monkey, Central

NA Primates

Cebidae

Saimiri

oerstedi

merican Squirrel

Monkey, Red-backed

Squirrel Monkey

\begin{tabular}{|c|c|c|c|c|}
\hline NA & Proboscidea & Elephantidae & Mammuthus & columbi \\
\hline NA & Proboscidea & Elephantidae & Mammuthus & primigenius \\
\hline NA & Proboscidea & Gomphotheriidae & Cuvieronius & $s p$ \\
\hline NA & Proboscidea & Mammutidae & Mammut & americanum \\
\hline NA & Rodentia & Agoutidae & Cuniculus & paca \\
\hline NA & Rodentia & Aplodontiidae & Aplodontia & rufa \\
\hline NA & Rodentia & Castoridae & Castor & canadensis \\
\hline NA & Rodentia & Castoridae & Castoroides & ohioensis \\
\hline NA & Rodentia & Cricetidae & Arborimus & albipes \\
\hline NA & Rodentia & Cricetidae & Arborimus & longicaudus \\
\hline NA & Rodentia & Cricetidae & Arborimus & pomo \\
\hline NA & Rodentia & Cricetidae & Baiomys & musculus \\
\hline NA & Rodentia & Cricetidae & Baiomys & taylori \\
\hline
\end{tabular}

85

extant

Spotted Paca

extinct

8200000 extinct

5000000 extinct

Spotted Paca

Arena Mountain Beaver

Arena Mount

American Beaver

White-footed Vole

Red Tree Vole

Sonoma Tree Vole,

California Red Tree

Mouse

Southern Pygmy Mouse

Northern Pygmy Mouse

3.00

extinct

8000

extant

1004

extant

21820 extant

150000 extant

22 extant

extant

Future

extan

extant

TP
TP
TP
TP
0

0
0
TP
0
Future
Future
0
0
0

$\begin{array}{lc}\text { EX } & \text { graze } \\ \text { EX } & \text { graze } \\ \text { EX } & \text { browse } \\ \text { EX } & \text { browse } \\ \text { LC } & \text { Frug/browse } \\ \text { LC } & \text { Browse } \\ \text { LC } & \text { Browse } \\ \text { EX } & \text { browse } \\ \text { LC } & \text { Browse } \\ \text { NT } & \text { Browse } \\ & \\ \text { NT } & \text { Browse } \\ & \\ \text { LC } & \text { Browse } \\ \text { LC } & \text { Nse/frug/gins }\end{array}$




\begin{tabular}{|c|c|c|c|c|c|c|c|c|c|c|c|c|}
\hline NA & Rodentia & Cricetidae & Dicrostonyx & groenlandicus & $\begin{array}{l}\text { Neartic Collared } \\
\text { Lemming, Northern } \\
\text { Collared Lemming } \\
\text { Ungava Collared }\end{array}$ & 1.74 & 54 & extant & 0 & NA & LC & 3rowse/graze \\
\hline NA & Rodentia & Cricetidae & Dicrostonyx & hudsonius & $\begin{array}{l}\text { Lemming, Labrador } \\
\text { Collared Lemming } \\
\text { Nelson's Collared } \\
\text { Lemming, Nelson's }\end{array}$ & 1.76 & 57 & extant & 0 & NA & LC & 3rowse/graze \\
\hline NA & Rodentia & Cricetidae & Dicrostonyx & nelsoni & $\begin{array}{l}\text { Collred Lemming, } \\
\text { Ungava Collared } \\
\text { Lemming }\end{array}$ & 1.80 & 63 & extant & 0 & NA & LC & \\
\hline NA & Rodentia & Cricetidae & Dicrostonyx & nunatakensis & $\begin{array}{l}\text { Ogilvie Mountains } \\
\text { Collared Lemming }\end{array}$ & 1.80 & 63 & extant & 0 & NA & LC & \\
\hline NA & Rodentia & Cricetidae & Dicrostonyx & richardsoni & $\begin{array}{l}\text { Richardson's Collared } \\
\text { Lemming }\end{array}$ & 1.74 & 55 & extant & 0 & NA & LC & 3rowse/graze \\
\hline NA & Rodentia & Cricetidae & Habromys & chinanteco & $\begin{array}{l}\text { Chinanteco Deer Mouse } \\
\text { Slender-tailed Deer }\end{array}$ & 1.60 & 40 & extant & Future & NA & CR & Frug/ginsect \\
\hline NA & Rodentia & Cricetidae & Habromys & lepturus & $\begin{array}{l}\text { Mouse, Slender Tailed } \\
\text { Deer Mouse } \\
\text { Crested-tailed Deer }\end{array}$ & 1.93 & 85 & extant & Future & NA & $C R$ & Frug/ginsect \\
\hline NA & Rodentia & Cricetidae & Habromys & lophurus & $\begin{array}{l}\text { Mouse, Crested Tailed } \\
\text { Deer Mouse }\end{array}$ & 1.60 & 40 & extant & Future & NA & NT & Frug/ginsect \\
\hline NA & Rodentia & Cricetidae & Habromys & simulatus & Jico Deer Mouse & 1.60 & 40 & extant & Future & NA & EN & Frug/ginsect \\
\hline NA & Rodentia & Cricetidae & Hodomys & alleni & $\begin{array}{l}\text { Allen's Woodrat, Allen's } \\
\text { Wood Rat }\end{array}$ & 2.57 & 368 & extant & 0 & NA & LC & Browse \\
\hline NA & Rodentia & Cricetidae & Isthmomys & flavidus & Yellow Isthmus Rat & 2.37 & 234 & extant & Future & NA & NT & Frug/ginsect \\
\hline NA & Rodentia & Cricetidae & Isthmomys & pirrensis & Mt. Pirri Isthmus Rat & 2.14 & 138 & extant & 0 & NA & LC & Frug/ginsect \\
\hline NA & Rodentia & Cricetidae & Lemmiscus & curtatus & $\begin{array}{l}\text { Sagebrush Vole } \\
\text { Brown Lemming, } \\
\text { BROWN LEMMING, }\end{array}$ & 1.45 & 28 & extant & 0 & NA & LC & 3rowse/graze \\
\hline NA & Rodentia & Cricetidae & Lemmus & sibiricus & $\begin{array}{l}\text { Siberian Brown } \\
\text { Lemming, SIBERIAN } \\
\text { BROWN LEMMING } \\
\text { Oaxaca Giant Deer }\end{array}$ & 1.72 & 52 & extant & 0 & NA & LC & Browse \\
\hline NA & Rodentia & Cricetidae & Megadontomys & cryophilus & $\begin{array}{l}\text { Mouse, Oaxacan Giant } \\
\text { Deer Mouse }\end{array}$ & 2.05 & 111 & extant & Future & NA & EN & Frug/ginsect \\
\hline NA & Rodentia & Cricetidae & Megadontomys & nelsoni & $\begin{array}{l}\text { Nelson's Giant Deer } \\
\text { Mouse }\end{array}$ & 2.05 & 111 & extant & Future & NA & EN & Frug/ginsect \\
\hline NA & Rodentia & Cricetidae & Megadontomys & thomasi & $\begin{array}{l}\text { Thomas's Giant Deer } \\
\text { Mouse }\end{array}$ & 2.05 & 111 & extant & Future & NA & EN & Frug/ginsect \\
\hline NA & Rodentia & Cricetidae & Microtus & californicus & $\begin{array}{l}\text { Amargosa Vole, } \\
\text { California Vole }\end{array}$ & 1.76 & 57 & extant & 0 & NA & LC & J̈raze/brows \\
\hline NA & Rodentia & Cricetidae & Microtus & canicaudus & Gray-tailed Vole & 1.45 & 28 & extant & 0 & NA & LC & כ̇raze/brows€ \\
\hline NA & Rodentia & Cricetidae & Microtus & chrotorrhinus & $\begin{array}{l}\text { Rock Vole, Southern } \\
\text { Rock Vole }\end{array}$ & 1.59 & 39 & extant & 0 & NA & LC & Jraze/browse \\
\hline NA & Rodentia & Cricetidae & Microtus & guatemalensis & $\begin{array}{l}\text { Guatemalan Vole } \\
\text { Long-tailed Vole, San }\end{array}$ & 1.62 & 42 & extant & Future & NA & NT & Jraze/brows \\
\hline NA & Rodentia & Cricetidae & Microtus & longicaudus & $\begin{array}{l}\text { Bernardino Long-tailed } \\
\text { Vole }\end{array}$ & 1.67 & 47 & extant & 0 & NA & LC & $\mathrm{g} /$ browse/gra \\
\hline NA & Rodentia & Cricetidae & Microtus & mexicanus & Mexican Vole & 1.54 & 35 & extant & 0 & NA & LC & Graze \\
\hline NA & Rodentia & Cricetidae & Microtus & miurus & Singing Vole & 1.61 & 41 & extant & 0 & NA & LC & Browse \\
\hline
\end{tabular}




\begin{tabular}{|c|c|c|c|c|c|c|c|c|c|c|c|c|}
\hline NA & Rodentia & Cricetidae & Microtus & montanus & $\begin{array}{l}\text { Montane Vole, } \\
\text { Pahranagat Valley } \\
\text { Montane Vole }\end{array}$ & 1.56 & 36 & extant & 0 & NA & LC & Graze \\
\hline NA & Rodentia & Cricetidae & Microtus & oaxacensis & Tarabundi Vole & 1.59 & 39 & extant & Future & NA & EN & Jraze/browse \\
\hline NA & Rodentia & Cricetidae & Microtus & ochrogaster & Prairie Vole & 1.58 & 38 & extant & 0 & NA & LC & 3rowse/graze \\
\hline NA & Rodentia & Cricetidae & Microtus & oeconomus & Root Vole, Tundra Vole & 1.51 & 32 & extant & 0 & NA & LC & J̈raze/browse \\
\hline NA & Rodentia & Cricetidae & Microtus & oregoni & Creeping Vole & 1.31 & 20 & extant & 0 & NA & LC & 3rowse/graze \\
\hline NA & Rodentia & Cricetidae & Microtus & pennsylvanicus & $\begin{array}{l}\text { Chihuahua Vole, } \\
\text { Meadow Vole }\end{array}$ & 1.57 & 37 & extant & 0 & NA & LC & J̈raze/brows \\
\hline NA & Rodentia & Cricetidae & Microtus & pinetorum & Woodland Vole & 1.42 & 26 & extant & 0 & NA & LC & Browse/frug \\
\hline NA & Rodentia & Cricetidae & Microtus & quasiater & Jalapan Pine Vole & 1.60 & 40 & extant & Future & NA & NT & Jraze/browse \\
\hline NA & Rodentia & Cricetidae & Microtus & richardsoni & Water Vole & 1.93 & 85 & extant & 0 & NA & LC & Browse \\
\hline NA & Rodentia & Cricetidae & Microtus & townsendii & $\begin{array}{l}\text { Townsend's Vole, } \\
\text { Triangle Island Vole }\end{array}$ & 1.81 & 65 & extant & 0 & NA & LC & 3rowse/graze \\
\hline NA & Rodentia & Cricetidae & Microtus & umbrosus & Zempoaltepec Vole & 1.62 & 42 & extant & Future & NA & EN & Jraze/browse \\
\hline NA & Rodentia & Cricetidae & Microtus & xanthognathus & Taiga Vole & 2.10 & 126 & extant & 0 & NA & LC & Graze/frug \\
\hline NA & Rodentia & Cricetidae & Neacomys & pictus & $\begin{array}{l}\text { Painted Bristly Mouse } \\
\text { Nelson And Coldman's }\end{array}$ & 1.24 & 17 & extant & 0 & NA & DD & \\
\hline NA & Rodentia & Cricetidae & Nelsonia & goldmani & $\begin{array}{l}\text { Woodrat, Nelson And } \\
\text { Goodman's Wood Rat }\end{array}$ & 1.90 & 80 & extant & Future & NA & EN & Browse \\
\hline NA & Rodentia & Cricetidae & Nelsonia & neotomodon & $\begin{array}{l}\text { Diminutive Woodrat, } \\
\text { Diminutive Wood Rat }\end{array}$ & 1.90 & 80 & extant & Future & NA & NT & Browse \\
\hline NA & Rodentia & Cricetidae & Neofiber & alleni & Round-tailed Muskrat & 2.42 & 266 & extant & 0 & NA & LC & Jraze/browse \\
\hline NA & Rodentia & Cricetidae & Neotoma & albigula & $\begin{array}{l}\text { White-throated } \\
\text { Woodrat }\end{array}$ & 2.31 & 206 & extant & 0 & NA & LC & Browse \\
\hline NA & Rodentia & Cricetidae & Neotoma & angustapalata & Tamaulipan Woodrat & 2.30 & 198 & extant & Future & NA & EN & Browse \\
\hline NA & Rodentia & Cricetidae & Neotoma & chrysomelas & $\begin{array}{l}\text { Nicaraguan Woodrat } \\
\text { Bushy-tailed Woodrat, }\end{array}$ & 2.32 & 211 & extant & 0 & NA & LC & Browse \\
\hline NA & Rodentia & Cricetidae & Neotoma & cinerea & $\begin{array}{l}\text { Packrat, Bushy-talied } \\
\text { Woodrat, Woodrat }\end{array}$ & 2.48 & 299 & extant & 0 & NA & LC & Browse \\
\hline NA & Rodentia & Cricetidae & Neotoma & devia & Arizona Woodrat & 2.30 & 200 & extant & 0 & NA & LC & Browse \\
\hline NA & Rodentia & Cricetidae & Neotoma & floridana & $\begin{array}{l}\text { Eastern Woodrat, Key } \\
\text { Largo Woodrat }\end{array}$ & 2.39 & 245 & extant & 0 & NA & LC & Browse \\
\hline NA & Rodentia & Cricetidae & Neotoma & fuscipes & $\begin{array}{l}\text { Dusky-footed Woodrat, } \\
\text { San Joaquin Valley } \\
\text { Woodrat }\end{array}$ & 2.36 & 230 & extant & 0 & NA & LC & Browse \\
\hline NA & Rodentia & Cricetidae & Neotoma & goldmani & Goldman's Woodrat & 2.30 & 198 & extant & 0 & NA & LC & Browse \\
\hline NA & Rodentia & Cricetidae & Neotoma & lepida & Desert Woodrat & 2.21 & 164 & extant & 0 & NA & LC & Browse \\
\hline NA & Rodentia & Cricetidae & Neotoma & mexicana & Mexican Woodrat & 2.31 & 203 & extant & 0 & NA & LC & browse/frug \\
\hline NA & Rodentia & Cricetidae & Neotoma & micropus & $\begin{array}{l}\text { Southern Plains } \\
\text { Woodrat }\end{array}$ & 2.38 & 237 & extant & 0 & NA & LC & Browse/frug \\
\hline NA & Rodentia & Cricetidae & Neotoma & nelsoni & Nelson's Woodrat & 2.30 & 198 & extant & Future & NA & $C R$ & Browse \\
\hline NA & Rodentia & Cricetidae & Neotoma & palatina & $\begin{array}{l}\text { Bolano's Woodrat, } \\
\text { Bola_os Woodrat }\end{array}$ & 2.30 & 198 & extant & Future & NA & vU & Browse \\
\hline NA & Rodentia & Cricetidae & Neotoma & phenax & Sonoran Woodrat & 2.36 & 227 & extant & Future & NA & NT & Browse \\
\hline NA & Rodentia & Cricetidae & Neotoma & stephensi & Stephen's Woodrat & 2.18 & 153 & extant & 0 & NA & LC & Browse \\
\hline NA & Rodentia & Cricetidae & Neotomodon & alstoni & Mexican Volcano Mouse & 1.60 & 40 & extant & 0 & NA & LC & Frug/browse \\
\hline NA & Rodentia & Cricetidae & Nyctomys & sumichrasti & Vesper Rat & 1.69 & 49 & extant & 0 & NA & LC & Frug/browse \\
\hline NA & Rodentia & Cricetidae & Ochrotomys & nuttalli & Golden Mouse & 1.35 & 22 & extant & 0 & NA & LC & Frug/ginsect \\
\hline NA & Rodentia & Cricetidae & Oligoryzomys & fulvescens & Fulvous Pygmy Rice Rat & 1.40 & 25 & extant & 0 & NA & LC & Frug/ginsect \\
\hline
\end{tabular}




\begin{tabular}{|c|c|c|c|c|c|c|c|c|c|c|c|c|}
\hline NA & Rodentia & Cricetidae & Oligoryzomys & vegetus & $\begin{array}{l}\text { Sprightly Pygmy Rice } \\
\text { Rat }\end{array}$ & 1.40 & 25 & extant & 0 & NA & LC & \\
\hline NA & Rodentia & Cricetidae & Ondatra & zibethicus & Muskrat & 2.99 & 981 & extant & 0 & NA & $\mathrm{LC}$ & Graze/carn \\
\hline NA & Rodentia & Cricetidae & Onychomys & arenicola & $\begin{array}{l}\text { Mearns's Grasshopper } \\
\text { Mouse }\end{array}$ & 1.48 & 30 & extant & 0 & NA & $\mathrm{LC}$ & Ginsect/carn \\
\hline NA & Rodentia & Cricetidae & Onychomys & leucogaster & $\begin{array}{l}\text { Northern Grasshopper } \\
\text { Mouse }\end{array}$ & 1.45 & 28 & extant & 0 & NA & LC & Ginsect/carn \\
\hline NA & Rodentia & Cricetidae & Onychomys & torridus & $\begin{array}{l}\text { Southern Grasshopper } \\
\text { Mouse }\end{array}$ & 1.40 & 25 & extant & 0 & NA & LC & Ginsect/carn \\
\hline NA & Rodentia & Cricetidae & Oryzomys & alfaroi & Alfaro's Rice Rat & 1.52 & 33 & extant & 0 & NA & $\mathrm{LC}$ & frug/carn \\
\hline NA & Rodentia & Cricetidae & Oryzomys & chapmani & & 1.70 & 50 & extant & 0 & NA & $\mathrm{LC}$ & frug/carn \\
\hline NA & Rodentia & Cricetidae & Oryzomys & couesi & Coues's Rice Rat & 1.84 & 69 & extant & 0 & NA & LC & invert \\
\hline NA & Rodentia & Cricetidae & Oryzomys & devius & Boquete rice rat & 1.75 & 57 & extant & 0 & NA & $\mathrm{LC}$ & frug/carn \\
\hline NA & Rodentia & Cricetidae & Oryzomys & dimidiatus & Thomas's Rice Rat & 1.75 & 57 & extant & 0 & NA & $\mathrm{LC}$ & frug/carn \\
\hline NA & Rodentia & Cricetidae & Oryzomys & melanotis & Black-eared Rice Rat & 1.70 & 50 & extant & 0 & NA & $\mathrm{LC}$ & frug/carn \\
\hline NA & Rodentia & Cricetidae & Oryzomys & palustris & Marsh Rice Rat & 1.73 & 54 & extant & 0 & NA & $\mathrm{LC}$ & invert \\
\hline NA & Rodentia & Cricetidae & Oryzomys & rhabdops & Striped Rice Rat & 1.75 & 57 & extant & Future & NA & Vu & frug/carn \\
\hline NA & Rodentia & Cricetidae & Oryzomys & rostratus & & 1.75 & 57 & extant & 0 & NA & LC & frug/carn \\
\hline NA & Rodentia & Cricetidae & Oryzomys & saturatior & & 1.75 & 57 & extant & Future & NA & NT & frug/carn \\
\hline NA & Rodentia & Cricetidae & Osgoodomys & banderanus & Michoacan Deer Mouse & 1.70 & 50 & extant & 0 & NA & LC & ginsect/frug \\
\hline NA & Rodentia & Cricetidae & Otonyctomys & hatti & $\begin{array}{l}\text { Hatt's Vesper Mouse, } \\
\text { Hatt's Vesper Rat }\end{array}$ & 1.56 & 36 & extant & 0 & NA & LC & Frug \\
\hline NA & Rodentia & Cricetidae & Ototylomys & phyllotis & Big-eared Climbing Rat & 2.08 & 120 & extant & 0 & NA & LC & Frug/browse \\
\hline NA & Rodentia & Cricetidae & Peromyscus & attwateri & $\begin{array}{l}\text { Texas Deermouse, } \\
\text { Texas Mouse }\end{array}$ & 1.45 & 28 & extant & 0 & NA & LC & :/browse/gins \\
\hline NA & Rodentia & Cricetidae & Peromyscus & aztecus & Aztec Mouse & 1.60 & 40 & extant & 0 & NA & LC & ginsect/frug \\
\hline NA & Rodentia & Cricetidae & Peromyscus & boylii & Brush Mouse & 1.33 & 21 & extant & 0 & NA & LC & Frug \\
\hline NA & Rodentia & Cricetidae & Peromyscus & bullatus & $\begin{array}{l}\text { Perote Deer Mouse, } \\
\text { Perote Mouse }\end{array}$ & 1.60 & 40 & extant & Future & NA & $C R$ & ginsect/frug \\
\hline NA & Rodentia & Cricetidae & Peromyscus & californicus & California Mouse & 1.64 & 44 & extant & 0 & NA & $\mathrm{LC}$ & Frug/browse \\
\hline NA & Rodentia & Cricetidae & Peromyscus & crinitus & Canyon Mouse & 1.25 & 18 & extant & 0 & NA & LC & iect/frug/brol \\
\hline NA & Rodentia & Cricetidae & Peromyscus & difficilis & $\begin{array}{l}\text { Rock Mouse, Zacatecan } \\
\text { Deer Mouse }\end{array}$ & 1.45 & 28 & extant & 0 & NA & $\mathrm{LC}$ & ginsect/frug \\
\hline NA & Rodentia & Cricetidae & Peromyscus & eremicus & Cactus Mouse & 1.37 & 24 & extant & 0 & NA & LC & :/ginsect/brol \\
\hline NA & Rodentia & Cricetidae & Peromyscus & eva & Eva's Desert Mouse & 1.34 & 22 & extant & 0 & NA & $\mathrm{LC}$ & ginsect/frug \\
\hline NA & Rodentia & Cricetidae & Peromyscus & furvus & $\begin{array}{l}\text { Blackish Deer Mouse } \\
\text { Cotton Deermouse, }\end{array}$ & 1.52 & 33 & extant & 0 & NA & DD & ginsect/frug \\
\hline NA & Rodentia & Cricetidae & Peromyscus & gossypinus & $\begin{array}{l}\text { Cotton Mouse, Key } \\
\text { Largo Cotton Mouse }\end{array}$ & 1.47 & 29 & extant & 0 & NA & LC & ginsect/frug \\
\hline NA & Rodentia & Cricetidae & Peromyscus & grandis & $\begin{array}{l}\text { Big Deer Mouse, Large } \\
\text { Deer Mouse }\end{array}$ & 1.85 & 71 & extant & Future & NA & NT & ginsect/frug \\
\hline NA & Rodentia & Cricetidae & Peromyscus & gratus & $\begin{array}{l}\text { Osgood's Mouse } \\
\text { Guatemalan Deer }\end{array}$ & 1.44 & 27 & extant & 0 & NA & LC & ginsect/frug \\
\hline NA & Rodentia & Cricetidae & Peromyscus & guatemalensis & $\begin{array}{l}\text { Mouse, Guatemalan } \\
\text { Deermouse }\end{array}$ & 1.60 & 40 & extant & 0 & NA & $\mathrm{LC}$ & ginsect/frug \\
\hline NA & Rodentia & Cricetidae & Peromyscus & gymnotis & $\begin{array}{l}\text { Naked-eared Deer } \\
\text { Mouse }\end{array}$ & 1.60 & 40 & extant & 0 & NA & $\mathrm{LC}$ & ginsect/frug \\
\hline NA & Rodentia & Cricetidae & Peromyscus & hooperi & $\begin{array}{l}\text { Hooper's Deer Mouse, } \\
\text { Hooper's Mouse }\end{array}$ & 1.56 & 36 & extant & 0 & NA & LC & ginsect/frug \\
\hline NA & Rodentia & Cricetidae & Peromyscus & leucopus & White-footed Mouse & 1.33 & 21 & extant & 0 & NA & LC & iect/frug/brol \\
\hline
\end{tabular}




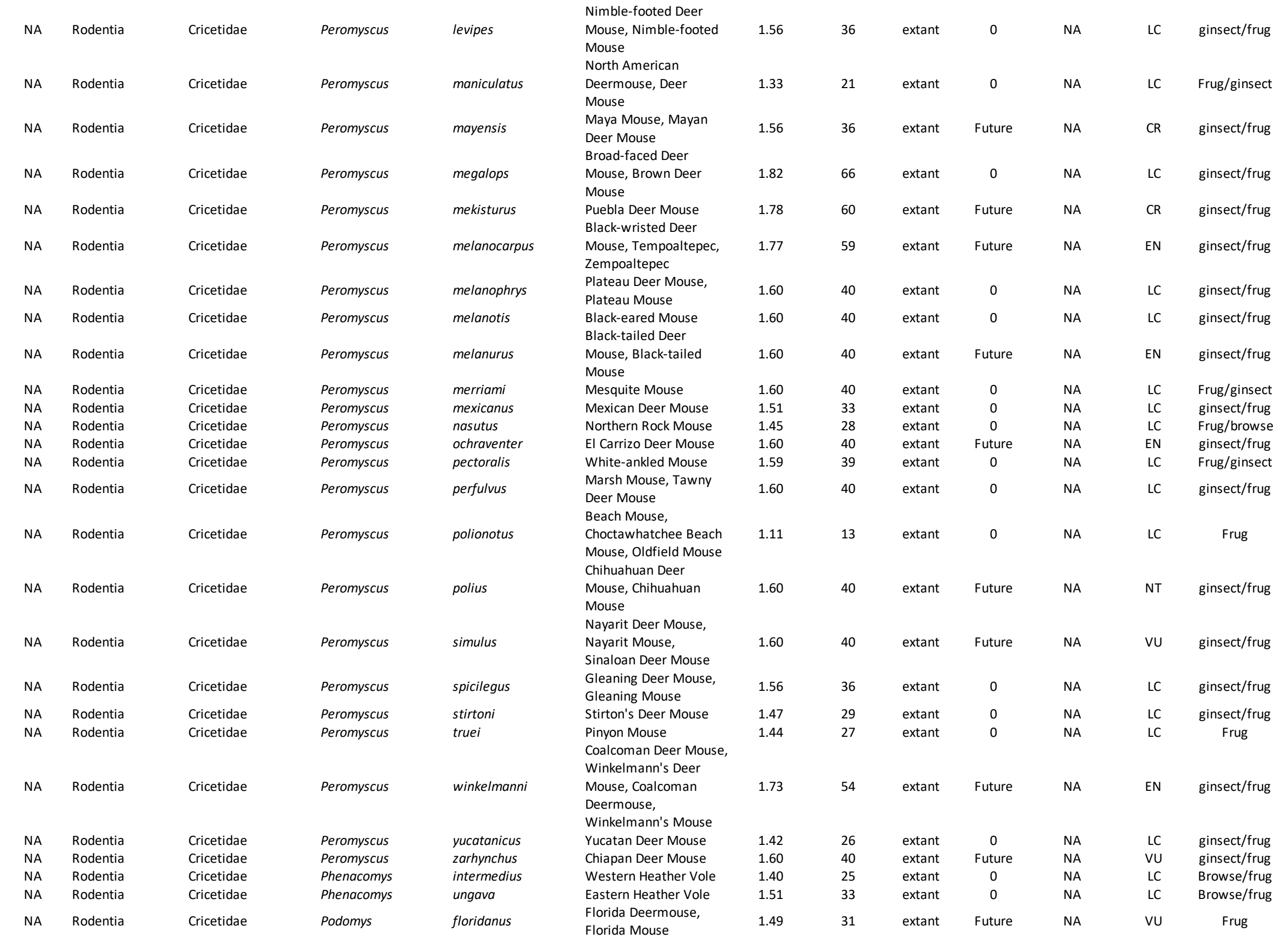




\begin{tabular}{|c|c|c|c|c|c|c|c|c|c|c|c|c|}
\hline NA & Rodentia & Cricetidae & Reithrodontomys & brevirostris & $\begin{array}{l}\text { Short-nosed Harvest } \\
\text { Mouse }\end{array}$ & 1.11 & 13 & extant & 0 & NA & $\mathrm{LC}$ & Frug \\
\hline NA & Rodentia & Cricetidae & Reithrodontomys & burti & Sonoran Harvest Mouse & 1.30 & 20 & extant & 0 & NA & DD & Frug \\
\hline NA & Rodentia & Cricetidae & Reithrodontomys & chrysopsis & Volcano Harvest Mouse & 1.28 & 19 & extant & 0 & NA & LC & Frug \\
\hline NA & Rodentia & Cricetidae & Reithrodontomys & creper & Chiriqui Harvest Mouse & 1.36 & 23 & extant & 0 & NA & LC & Frug \\
\hline NA & Rodentia & Cricetidae & Reithrodontomys & fulvescens & Fulvous Harvest Mouse & 1.06 & 11 & extant & 0 & NA & $\mathrm{LC}$ & ginsect/frug \\
\hline NA & Rodentia & Cricetidae & Reithrodontomys & gracilis & Slender Harvest Mouse & 1.09 & 12 & extant & 0 & NA & LC & Frug \\
\hline NA & Rodentia & Cricetidae & Reithrodontomys & hirsutus & Hairy Harvest Mouse & 1.30 & 20 & extant & Future & NA & Vu & Frug \\
\hline NA & Rodentia & Cricetidae & Reithrodontomys & humulis & Eastern Harvest Mouse & 0.92 & 8 & extant & 0 & NA & $\mathrm{LC}$ & Frug/ginsect \\
\hline NA & Rodentia & Cricetidae & Reithrodontomys & megalotis & $\begin{array}{l}\text { Southern Marsh Harvest } \\
\text { Mouse, Western } \\
\text { Harvest Mouse }\end{array}$ & 0.97 & 9 & extant & 0 & NA & $\mathrm{LC}$ & Frug/ginsect \\
\hline NA & Rodentia & Cricetidae & Reithrodontomys & mexicanus & Mexican Harvest Mouse & 1.28 & 19 & extant & 0 & NA & LC & Frug \\
\hline NA & Rodentia & Cricetidae & Reithrodontomys & microdon & $\begin{array}{l}\text { Small-toothed Harvest } \\
\text { Mouse }\end{array}$ & 1.30 & 20 & extant & 0 & NA & LC & Frug \\
\hline NA & Rodentia & Cricetidae & Reithrodontomys & montanus & Plains Harvest Mouse & 1.04 & 11 & extant & 0 & NA & LC & Frug/ginsect \\
\hline NA & Rodentia & Cricetidae & Reithrodontomys & paradoxus & $\begin{array}{l}\text { Nicaraguan Harvest } \\
\text { Mouse }\end{array}$ & 1.19 & 15 & extant & 0 & NA & DD & Frug \\
\hline NA & Rodentia & Cricetidae & Reithrodontomys & raviventris & $\begin{array}{l}\text { Salt-marsh Harvest } \\
\text { Mouse }\end{array}$ & 1.04 & 11 & extant & Future & NA & EN & Frug \\
\hline NA & Rodentia & Cricetidae & Reithrodontomys & rodriguezi & $\begin{array}{l}\text { Rodriguez's Harvest } \\
\text { Mouse }\end{array}$ & 1.19 & 15 & extant & 0 & NA & LC & Frug \\
\hline NA & Rodentia & Cricetidae & Reithrodontomys & sumichrasti & $\begin{array}{l}\text { Sumichrast's Harvest } \\
\text { Mouse }\end{array}$ & 1.28 & 19 & extant & 0 & NA & LC & Frug \\
\hline NA & Rodentia & Cricetidae & Reithrodontomys & tenuirostris & $\begin{array}{l}\text { Narrow-nosed Harvest } \\
\text { Mouse }\end{array}$ & 1.30 & 20 & extant & Future & NA & Vu & Frug \\
\hline NA & Rodentia & Cricetidae & Reithrodontomys & zacatecae & $\begin{array}{l}\text { Zacatecas Harvest } \\
\text { Mouse }\end{array}$ & 1.19 & 15 & extant & 0 & NA & $\mathrm{LC}$ & Frug \\
\hline NA & Rodentia & Cricetidae & Rheomys & mexicanus & Mexican Water Mouse & 1.60 & 40 & extant & Future & NA & EN & invert \\
\hline NA & Rodentia & Cricetidae & Rheomys & thomasi & Thomas's Water Mouse & 1.60 & 40 & extant & Future & NA & NT & invert \\
\hline NA & Rodentia & Cricetidae & Rheomys & underwoodi & $\begin{array}{l}\text { Underwood's Water } \\
\text { Mouse }\end{array}$ & 1.59 & 39 & extant & 0 & NA & LC & invert \\
\hline NA & Rodentia & Cricetidae & Scotinomys & teguina & $\begin{array}{l}\text { Alston's Brown Mouse, } \\
\text { Short-tailed Singing } \\
\text { Mouse }\end{array}$ & 1.05 & 11 & extant & 0 & NA & LC & Ginsect \\
\hline NA & Rodentia & Cricetidae & Scotinomys & xerampelinus & $\begin{array}{l}\text { Chiriqui Brown Mouse, } \\
\text { Long-tailed Singing } \\
\text { Mouse }\end{array}$ & 1.18 & 15 & extant & 0 & NA & LC & Ginsect \\
\hline NA & Rodentia & Cricetidae & Sigmodon & alleni & $\begin{array}{l}\text { Allen's Cotton Rat } \\
\text { Arizona Cotton Rat, }\end{array}$ & 2.25 & 179 & extant & Future & NA & vu & frug/carn \\
\hline NA & Rodentia & Cricetidae & Sigmodon & arizonae & $\begin{array}{l}\text { Colorado River Cotton } \\
\text { Rat }\end{array}$ & 2.30 & 198 & extant & 0 & NA & LC & J̈raze/brows $\epsilon$ \\
\hline NA & Rodentia & Cricetidae & Sigmodon & fulviventer & $\begin{array}{l}\text { Tawny-bellied Cotton } \\
\text { Rat }\end{array}$ & 2.33 & 214 & extant & 0 & NA & LC & J̈raze/brows $€$ \\
\hline NA & Rodentia & Cricetidae & Sigmodon & hispidus & Hispid Cotton Rat & 1.97 & 92 & extant & 0 & NA & LC & J̈raze/brows \\
\hline NA & Rodentia & Cricetidae & Sigmodon & leucotis & White-eared Cotton Rat & 2.13 & 136 & extant & 0 & NA & LC & frug/carn \\
\hline
\end{tabular}




\begin{tabular}{|c|c|c|c|c|c|c|c|c|c|c|c|c|}
\hline NA & Rodentia & Cricetidae & Sigmodon & mascotensis & $\begin{array}{l}\text { Jaliscan Cotton Rat, } \\
\text { West Mexican Cotton } \\
\text { Rat }\end{array}$ & 2.08 & 120 & extant & 0 & NA & LC & frug/carn \\
\hline NA & Rodentia & Cricetidae & Sigmodon & ochrognathus & $\begin{array}{l}\text { Yellow-nosed Cotton } \\
\text { Rat }\end{array}$ & 2.09 & 122 & extant & 0 & NA & $\mathrm{LC}$ & Jraze/brows \\
\hline NA & Rodentia & Cricetidae & Sigmodontomys & alfari & $\begin{array}{l}\text { Alfaro's Rice Water Rat, } \\
\text { Short-tailed } \\
\text { Sigmodontomys }\end{array}$ & 1.70 & 50 & extant & 0 & NA & $\mathrm{LC}$ & Ginsect/frug \\
\hline NA & Rodentia & Cricetidae & Sigmodontomys & aphrastus & $\begin{array}{l}\text { Harris's Rice Water Rat, } \\
\text { Long-tailed } \\
\text { Sigmodontomys }\end{array}$ & 1.85 & 71 & extant & 0 & NA & DD & Ginsect/frug \\
\hline NA & Rodentia & Cricetidae & Synaptomys & borealis & $\begin{array}{l}\text { Northern Bog Lemming, } \\
\text { Okanagan Bog } \\
\text { Lemming }\end{array}$ & 1.33 & 21 & extant & 0 & NA & $\mathrm{LC}$ & Jraze/browse \\
\hline NA & Rodentia & Cricetidae & Synaptomys & cooperi & $\begin{array}{l}\text { Kansas Bog Lemming, } \\
\text { Southern Bog Lemming }\end{array}$ & 1.50 & 32 & extant & 0 & NA & LC & Jraze/browse \\
\hline NA & Rodentia & Cricetidae & Tylomys & bullaris & Chiapan Climbing Rat & 2.45 & 280 & extant & Future & NA & CR & Frug/browse \\
\hline NA & Rodentia & Cricetidae & Tylomys & fulviventer & $\begin{array}{l}\text { Fulvous-bellied } \\
\text { Climbing Rat }\end{array}$ & 2.34 & 217 & extant & 0 & NA & DD & Frug/browse \\
\hline NA & Rodentia & Cricetidae & Tylomys & nudicaudus & $\begin{array}{l}\text { Peter's Climbing Rat, } \\
\text { Peters's Climbing Rat } \\
\text { Panam^ Climbing Rat, }\end{array}$ & 2.00 & 100 & extant & 0 & NA & $\mathrm{LC}$ & Frug/browse \\
\hline NA & Rodentia & Cricetidae & Tylomys & panamensis & $\begin{array}{l}\text { Panamanian Climbing } \\
\text { Rat }\end{array}$ & 2.34 & 217 & extant & 0 & NA & $\mathrm{DD}$ & Frug/browse \\
\hline NA & Rodentia & Cricetidae & Tylomys & tumbalensis & $\begin{array}{l}\text { Tumbal` Climbing Rat, } \\
\text { Tumbala Climbing Rat }\end{array}$ & 2.45 & 280 & extant & Future & NA & $C R$ & Frug/browse \\
\hline NA & Rodentia & Cricetidae & Tylomys & watsoni & Watson's Climbing Rat & 2.38 & 240 & extant & 0 & NA & LC & Frug/browse \\
\hline NA & Rodentia & Cricetidae & Xenomys & nelsoni & $\begin{array}{l}\text { Magdalena Rat, } \\
\text { Magdelena Wood Rat }\end{array}$ & 2.11 & 130 & extant & Future & NA & EN & Ginsect \\
\hline NA & Rodentia & Dasyproctidae & Dasyprocta & mexicana & Mexican Agouti & 3.70 & 5000 & extant & Future & NA & $C R$ & Frug/browse \\
\hline NA & Rodentia & Dasyproctidae & Dasyprocta & punctata & $\begin{array}{l}\text { Central American } \\
\text { Agouti }\end{array}$ & 3.43 & 2675 & extant & 0 & NA & LC & Frug/browse \\
\hline NA & Rodentia & Dipodidae & Napaeozapus & insignis & $\begin{array}{l}\text { Woodland Jumping } \\
\text { Mouse }\end{array}$ & 1.35 & 22 & extant & 0 & NA & $\mathrm{LC}$ & Nse/frug/gins \\
\hline NA & Rodentia & Dipodidae & Zapus & hudsonius & $\begin{array}{l}\text { Meadow Jumping } \\
\text { Mouse }\end{array}$ & 1.23 & 17 & extant & 0 & NA & $\mathrm{LC}$ & Frug/ginsect \\
\hline NA & Rodentia & Dipodidae & Zapus & princeps & $\begin{array}{l}\text { Pacific Jumping Mouse } \\
\text { Pacific Jumping Mouse, }\end{array}$ & 1.46 & 29 & extant & 0 & NA & $\mathrm{LC}$ & Frug/ginsect \\
\hline NA & Rodentia & Dipodidae & Zapus & trinotatus & $\begin{array}{l}\text { Point Reyes Jumping } \\
\text { Mouse }\end{array}$ & 1.44 & 28 & extant & 0 & NA & LC & :/browse/gins \\
\hline NA & Rodentia & Echimyidae & Hoplomys & gymnurus & Armored Rat & 2.38 & 240 & extant & 0 & NA & LC & Frug \\
\hline NA & Rodentia & Erethizontidae & Erethizon & dorsatum & & 3.85 & 7085 & extant & 0 & NA & $\mathrm{LC}$ & 3rowse/graze \\
\hline NA & Rodentia & Erethizontidae & Sphiggurus & mexicanus & $\begin{array}{l}\text { Mexican Hairy Dwarf } \\
\text { Porcupine }\end{array}$ & 3.30 & 2000 & extant & 0 & NA & $\mathrm{LC}$ & Browse/frug \\
\hline NA & Rodentia & Geomyidae & Cratogeomys & castanops & $\begin{array}{l}\text { Yellow-faced Pocket } \\
\text { Gopher }\end{array}$ & 2.40 & 252 & extant & 0 & NA & $\mathrm{LC}$ & 3rowse/graze \\
\hline NA & Rodentia & Geomyidae & Cratogeomys & fumosus & Smoky Pocket Gopher & 2.18 & 150 & extant & 0 & NA & LC & Browse/frug \\
\hline NA & Rodentia & Geomyidae & Cratogeomys & fumosus & Smoky Pocket Gopher & 2.18 & 150 & extant & 0 & NA & LC & Browse/frug \\
\hline NA & Rodentia & Geomyidae & Cratogeomys & gymnurus & $\begin{array}{l}\text { Smoky Pocket Gopher } \\
\text { Merriam's Pocket }\end{array}$ & 2.78 & 600 & extant & 0 & NA & $\mathrm{LC}$ & Browse/frug \\
\hline NA & Rodentia & Geomyidae & Cratogeomys & merriami & $\begin{array}{l}\text { Gopher, Merriam's } \\
\text { Pocket Gopher }\end{array}$ & 2.62 & 420 & extant & 0 & NA & $\mathrm{LC}$ & Browse/frug \\
\hline
\end{tabular}




\begin{tabular}{|c|c|c|c|c|c|c|c|c|c|c|c|c|}
\hline NA & Rodentia & Geomyidae & Cratogeomys & tylorhinus & & 2.40 & 249 & extant & Future & NA & & Browse/frug \\
\hline NA & Rodentia & Geomyidae & Cratogeomys & zinseri & & 2.18 & 150 & extant & 0 & NA & & Browse/frug \\
\hline NA & Rodentia & Geomyidae & Geomys & arenarius & Desert Pocket Gopher & 2.31 & 206 & extant & Future & NA & NT & 3rowse/graze \\
\hline NA & Rodentia & Geomyidae & Geomys & bursarius & Plains Pocket Gopher & 2.31 & 204 & extant & 0 & NA & LC & 3rowse/graze \\
\hline NA & Rodentia & Geomyidae & Geomys & personatus & $\begin{array}{l}\text { Texas Pocket Gopher } \\
\text { Cumberland Island }\end{array}$ & 2.60 & 397 & extant & 0 & NA & LC & 3rowse/graze \\
\hline NA & Rodentia & Geomyidae & Geomys & pinetis & $\begin{array}{l}\text { Pocket Gopher, } \\
\text { Southeastern Pocket } \\
\text { Gopher }\end{array}$ & 1.93 & 85 & extant & 0 & NA & LC & 3rowse/graze \\
\hline NA & Rodentia & Geomyidae & Geomys & tropicalis & Tropical Pocket Gopher & 2.54 & 350 & extant & Future & NA & $C R$ & Frug \\
\hline NA & Rodentia & Geomyidae & Orthogeomys & cavator & Chiriqui Pocket Gopher & 2.81 & 650 & extant & 0 & NA & LC & Browse/frug \\
\hline NA & Rodentia & Geomyidae & Orthogeomys & cherriei & $\begin{array}{l}\text { Cherrie's Pocket } \\
\text { Gopher }\end{array}$ & 2.35 & 225 & extant & 0 & NA & LC & Browse/frug \\
\hline NA & Rodentia & Geomyidae & Orthogeomys & cuniculus & Oaxacan Pocket Gopher & 2.70 & 500 & extant & 0 & NA & DD & Browse/frug \\
\hline NA & Rodentia & Geomyidae & Orthogeomys & grandis & Giant Pocket Gopher & 2.70 & 500 & extant & 0 & NA & LC & Browse/frug \\
\hline NA & Rodentia & Geomyidae & Orthogeomys & heterodus & Variable Pocket Gopher & 2.79 & 615 & extant & 0 & NA & LC & Browse/frug \\
\hline NA & Rodentia & Geomyidae & Orthogeomys & hispidus & Hispid Pocket Gopher & 2.70 & 500 & extant & 0 & NA & LC & Browse/frug \\
\hline NA & Rodentia & Geomyidae & Orthogeomys & lanius & Big Pocket Gopher & 2.70 & 500 & extant & Future & NA & $C R$ & Browse/frug \\
\hline NA & Rodentia & Geomyidae & Orthogeomys & matagalpae & & 2.67 & 464 & extant & 0 & NA & & Browse/frug \\
\hline NA & Rodentia & Geomyidae & Orthogeomys & thaeleri & & 2.67 & 464 & extant & 0 & NA & & Browse/frug \\
\hline NA & Rodentia & Geomyidae & Orthogeomys & underwoodi & $\begin{array}{l}\text { Underwood's Pocket } \\
\text { Gopher }\end{array}$ & 2.40 & 250 & extant & 0 & NA & LC & Browse/frug \\
\hline NA & Rodentia & Geomyidae & Pappogeomys & alcorni & Alcorn's Pocket Gopher & 2.18 & 150 & extant & Future & NA & $C R$ & Browse/frug \\
\hline NA & Rodentia & Geomyidae & Pappogeomys & bulleri & Buller's Pocket Gopher & 2.18 & 150 & extant & 0 & NA & LC & Browse/frug \\
\hline NA & Rodentia & Geomyidae & Thomomys & bottae & Botta's Pocket Gopher & 2.06 & 115 & extant & 0 & NA & LC & 3rowse/graze \\
\hline NA & Rodentia & Geomyidae & Thomomys & bulbivorus & Camas Pocket Gopher & 2.56 & 360 & extant & 0 & NA & LC & 3rowse/graze \\
\hline NA & Rodentia & Geomyidae & Thomomys & clusius & $\begin{array}{l}\text { Wyoming Pocket } \\
\text { Gopher }\end{array}$ & 1.76 & 58 & extant & 0 & NA & LC & 3rowse/graze \\
\hline NA & Rodentia & Geomyidae & Thomomys & idahoensis & Idaho Pocket Gopher & 1.83 & 67 & extant & 0 & NA & LC & 3rowse/graze \\
\hline NA & Rodentia & Geomyidae & Thomomys & mazama & $\begin{array}{l}\text { Western Pocket Gopher, } \\
\text { Puget Sound Pocket } \\
\text { Gopher, Mazama } \\
\text { Pocket Gopher }\end{array}$ & 1.88 & 75 & extant & 0 & NA & LC & 3rowse/graze \\
\hline NA & Rodentia & Geomyidae & Thomomys & microdon & & 1.88 & 75 & extinct & TP & NA & EX & browse \\
\hline NA & Rodentia & Geomyidae & Thomomys & monticola & $\begin{array}{l}\text { Mountain Pocket } \\
\text { Gopher }\end{array}$ & 1.90 & 80 & extant & 0 & NA & LC & 3rowse/graze \\
\hline NA & Rodentia & Geomyidae & Thomomys & orientalis & Northern Pocket & 1.98 & 95 & extinct & TP & NA & EX & browse \\
\hline NA & Rodentia & Geomyidae & Thomomys & talpoides & $\begin{array}{l}\text { Gopher, Vancouver } \\
\text { Pocket Gopher }\end{array}$ & 2.11 & 130 & extant & 0 & NA & LC & 3rowse/graze \\
\hline NA & Rodentia & Geomyidae & Thomomys & townsendii & $\begin{array}{l}\text { Townsend's Pocket } \\
\text { Gopher }\end{array}$ & 2.42 & 263 & extant & 0 & NA & LC & 3rowse/graze \\
\hline NA & Rodentia & Geomyidae & Thomomys & umbrinus & $\begin{array}{l}\text { Animas Mountains } \\
\text { Pocket Gopher, } \\
\text { Southern Pocket Gopher }\end{array}$ & 2.22 & 166 & extant & 0 & NA & LC & 3rowse/graze \\
\hline NA & Rodentia & Geomyidae & Zygogeomys & trichopus & $\begin{array}{l}\text { Michoacan Pocket } \\
\text { Gopher } \\
\text { Little Desert Pocket }\end{array}$ & 2.70 & 500 & extant & Future & NA & EN & Browse/frug \\
\hline NA & Rodentia & Heteromyidae & Chaetodipus & arenarius & $\begin{array}{l}\text { Mouse, Sand Pocket } \\
\text { Mouse }\end{array}$ & 1.36 & 23 & extant & 0 & NA & LC & Frug/browse \\
\hline
\end{tabular}




\begin{tabular}{|c|c|c|c|c|c|c|c|c|c|c|c|c|}
\hline NA & Rodentia & Heteromyidae & Chaetodipus & artus & $\begin{array}{l}\text { Narrow-skulled Pocket } \\
\text { Mouse }\end{array}$ & 1.32 & 21 & extant & 0 & NA & LC & Frug/browse \\
\hline NA & Rodentia & Heteromyidae & Chaetodipus & baileyi & Bailey's Pocket Mouse & 1.42 & 26 & extant & 0 & NA & LC & Frug/browse \\
\hline NA & Rodentia & Heteromyidae & Chaetodipus & californicus & California Pocket Mouse & 1.34 & 22 & extant & 0 & NA & LC & Frug \\
\hline NA & Rodentia & Heteromyidae & Chaetodipus & fallax & $\begin{array}{l}\text { San Diego Pocket } \\
\text { Mouse }\end{array}$ & 1.27 & 19 & extant & 0 & NA & LC & Frug \\
\hline NA & Rodentia & Heteromyidae & Chaetodipus & formosus & $\begin{array}{l}\text { Long-tailed Pocket } \\
\text { Mouse }\end{array}$ & 1.29 & 19 & extant & 0 & NA & LC & Frug/browse \\
\hline NA & Rodentia & Heteromyidae & Chaetodipus & goldmani & $\begin{array}{l}\text { Goldman's Pocket } \\
\text { Mouse }\end{array}$ & 1.36 & 23 & extant & Future & NA & NT & Frug/browse \\
\hline NA & Rodentia & Heteromyidae & Chaetodipus & hispidus & Hispid Pocket Mouse & 1.51 & 32 & extant & 0 & NA & LC & Frug/browse \\
\hline NA & Rodentia & Heteromyidae & Chaetodipus & intermedius & Rock Pocket Mouse & 1.22 & 17 & extant & 0 & NA & LC & Frug \\
\hline NA & Rodentia & Heteromyidae & Chaetodipus & lineatus & $\begin{array}{l}\text { Lined Pocket Gopher, } \\
\text { Lined Pocket Mouse }\end{array}$ & 1.36 & 23 & extant & 0 & NA & DD & Frug \\
\hline NA & Rodentia & Heteromyidae & Chaetodipus & nelsoni & Nelson's Pocket Mouse & 1.20 & 16 & extant & 0 & NA & LC & Frug \\
\hline NA & Rodentia & Heteromyidae & Chaetodipus & penicillatus & Desert Pocket Mouse & 1.23 & 17 & extant & 0 & NA & LC & Frug \\
\hline NA & Rodentia & Heteromyidae & Chaetodipus & pernix & $\begin{array}{l}\text { Sinaloan Pocket Mouse, } \\
\text { Sinaloan Pocket Gopher }\end{array}$ & 1.23 & 17 & extant & 0 & NA & LC & Frug/browse \\
\hline NA & Rodentia & Heteromyidae & Chaetodipus & spinatus & Spiny Pocket Mouse & 1.21 & 16 & extant & 0 & NA & LC & Frug \\
\hline NA & Rodentia & Heteromyidae & Dipodomys & agilis & Agile Kangaroo Rat & 1.76 & 58 & extant & 0 & NA & LC & Frug \\
\hline NA & Rodentia & Heteromyidae & Dipodomys & deserti & Desert Kangaroo Rat & 2.02 & 105 & extant & 0 & NA & LC & Frug \\
\hline NA & Rodentia & Heteromyidae & Dipodomys & elator & Texas Kangaroo Rat & 1.89 & 78 & extant & Future & NA & Vu & Frug \\
\hline NA & Rodentia & Heteromyidae & Dipodomys & elephantinus & & 1.86 & 72 & extant & 0 & NA & & Frug \\
\hline NA & Rodentia & Heteromyidae & Dipodomys & gravipes & $\begin{array}{l}\text { San Quintin Kangaroo } \\
\text { Rat } \\
\text { Heermann's Kangaroo }\end{array}$ & 1.92 & 84 & extant & Future & NA & CR & Frug \\
\hline NA & Rodentia & Heteromyidae & Dipodomys & heermanni & $\begin{array}{l}\text { Rat, Morro Bay } \\
\text { Kangaroo-rat, Morro } \\
\text { Bay Kangaroo Rat }\end{array}$ & 1.86 & 72 & extant & 0 & NA & LC & Frug/browse \\
\hline NA & Rodentia & Heteromyidae & Dipodomys & ingens & Giant Kangaroo Rat & 2.13 & 134 & extant & Future & NA & EN & Frug \\
\hline NA & Rodentia & Heteromyidae & Dipodomys & merriami & $\begin{array}{l}\text { Merriam's Kangaroo } \\
\text { Rat }\end{array}$ & 1.62 & 42 & extant & 0 & NA & LC & Frug \\
\hline NA & Rodentia & Heteromyidae & Dipodomys & microps & $\begin{array}{l}\text { Chisel-toothed } \\
\text { Kangaroo Rat, } \\
\text { Houserock Chisel- } \\
\text { toothed Kangaroo Rat, } \\
\text { House Rock Valley } \\
\text { Kangaroo Rat }\end{array}$ & 1.74 & 55 & extant & 0 & NA & LC & browse/frug \\
\hline NA & Rodentia & Heteromyidae & Dipodomys & nelsoni & $\begin{array}{l}\text { Nelson's Kangaroo Rat } \\
\text { Fresno Kangaroo Rat, }\end{array}$ & 1.95 & 89 & extant & 0 & NA & LC & Frug \\
\hline NA & Rodentia & Heteromyidae & Dipodomys & nitratoides & $\begin{array}{l}\text { San Joaquin Kangaroo } \\
\text { Rat }\end{array}$ & 1.64 & 44 & extant & Future & NA & VU & Frug \\
\hline NA & Rodentia & Heteromyidae & Dipodomys & ordii & Ord's Kangaroo Rat & 1.78 & 60 & extant & 0 & NA & LC & Frug/browse \\
\hline NA & Rodentia & Heteromyidae & Dipodomys & panamintinus & Panamint Kangaroo Rat & 1.87 & 75 & extant & 0 & NA & LC & Frug \\
\hline NA & Rodentia & Heteromyidae & Dipodomys & phillipsii & $\begin{array}{l}\text { Phillip's Kangaroo Rat } \\
\text { Banner-tailed Kangaroo }\end{array}$ & 1.61 & 41 & extant & 0 & NA & LC & Frug \\
\hline NA & Rodentia & Heteromyidae & Dipodomys & spectabilis & $\begin{array}{l}\text { Rat, Phillips's Kangaroo } \\
\text { Rat }\end{array}$ & 2.13 & 136 & extant & Future & NA & NT & Frug \\
\hline NA & Rodentia & Heteromyidae & Dipodomys & stephensi & Stephens' Kangaroo Rat & 1.84 & 70 & extant & Future & NA & EN & Frug \\
\hline
\end{tabular}




\begin{tabular}{|c|c|c|c|c|c|c|c|c|c|c|c|c|}
\hline NA & Rodentia & Heteromyidae & Dipodomys & venustus & $\begin{array}{l}\text { Narrow-faced Kangaroo } \\
\text { Rat }\end{array}$ & 1.86 & 72 & extant & 0 & NA & $\mathrm{LC}$ & Frug \\
\hline NA & Rodentia & Heteromyidae & Heteromys & desmarestianus & $\begin{array}{l}\text { Desmarest's Spiny } \\
\text { Pocket Mouse }\end{array}$ & 1.86 & 73 & extant & 0 & NA & $\mathrm{LC}$ & Frug/browse \\
\hline NA & Rodentia & Heteromyidae & Heteromys & gaumeri & $\begin{array}{l}\text { Gaumer's Spiny Pocket } \\
\text { Mouse }\end{array}$ & 1.80 & 64 & extant & 0 & NA & LC & Frug/browse \\
\hline NA & Rodentia & Heteromyidae & Heteromys & goldmani & & 1.93 & 85 & extant & 0 & NA & LC & Frug/browse \\
\hline NA & Rodentia & Heteromyidae & Heteromys & nelsoni & $\begin{array}{l}\text { Nelson's Spiny Pocket } \\
\text { Mouse }\end{array}$ & 1.73 & 54 & extant & Future & NA & EN & Frug/browse \\
\hline NA & Rodentia & Heteromyidae & Heteromys & oresterus & $\begin{array}{l}\text { Mountain Spiny Pocket } \\
\text { Mouse }\end{array}$ & 1.87 & 75 & extant & 0 & NA & $\mathrm{LC}$ & Frug/browse \\
\hline NA & Rodentia & Heteromyidae & Liomys & adspersus & $\begin{array}{l}\text { Panamanian Spiny } \\
\text { Pocket Mouse }\end{array}$ & 1.81 & 65 & extant & 0 & NA & $\mathrm{LC}$ & Frug/browse \\
\hline NA & Rodentia & Heteromyidae & Liomys & irroratus & $\begin{array}{l}\text { Mexican Spiny Pocket } \\
\text { Mouse }\end{array}$ & 1.70 & 50 & extant & 0 & NA & $\mathrm{LC}$ & Frug \\
\hline NA & Rodentia & Heteromyidae & Liomys & pictus & $\begin{array}{l}\text { Painted Spiny Pocket } \\
\text { Mouse }\end{array}$ & 1.60 & 40 & extant & 0 & NA & LC & Frug/browse \\
\hline NA & Rodentia & Heteromyidae & Liomys & salvini & $\begin{array}{l}\text { Salvin's Spiny Pocket } \\
\text { Mouse }\end{array}$ & 1.62 & 42 & extant & 0 & NA & $\mathrm{LC}$ & Frug/browse \\
\hline NA & Rodentia & Heteromyidae & Liomys & spectabilis & $\begin{array}{l}\text { Jaliscan Spiny Pocket } \\
\text { Mouse }\end{array}$ & 1.81 & 65 & extant & Future & NA & EN & Frug/browse \\
\hline NA & Rodentia & Heteromyidae & Microdipodops & megacephalus & $\begin{array}{l}\text { Dark Kangaroo Mouse, } \\
\text { Owyhee River Kangaroo } \\
\text { Mouse }\end{array}$ & 1.02 & 10 & extant & 0 & NA & LC & Frug/ginsect \\
\hline NA & Rodentia & Heteromyidae & Microdipodops & pallidus & $\begin{array}{l}\text { Pale Kangaroo Mouse, } \\
\text { Soda Spring Valley } \\
\text { Kangaroo Mouse }\end{array}$ & 1.10 & 13 & extant & 0 & NA & LC & Frug \\
\hline NA & Rodentia & Heteromyidae & Perognathus & alticola & $\begin{array}{l}\text { White-eared Pocket } \\
\text { Mouse }\end{array}$ & 1.38 & 24 & extant & Future & NA & vu & Frug/browse \\
\hline NA & Rodentia & Heteromyidae & Perognathus & amplus & Arizona Pocket Mouse & 1.07 & 12 & extant & 0 & NA & LC & Frug \\
\hline NA & Rodentia & Heteromyidae & Perognathus & fasciatus & $\begin{array}{l}\text { Olive-backed Pocket } \\
\text { Mouse }\end{array}$ & 1.06 & 12 & extant & 0 & NA & $\mathrm{LC}$ & Frug \\
\hline NA & Rodentia & Heteromyidae & Perognathus & flavescens & Plains Pocket Mouse & 0.94 & 9 & extant & 0 & NA & LC & Frug/browse \\
\hline NA & Rodentia & Heteromyidae & Perognathus & flavus & $\begin{array}{l}\text { Silky Pocket Mouse } \\
\text { Salinas Pocket Mouse, }\end{array}$ & 0.89 & 8 & extant & 0 & NA & LC & Frug/browse \\
\hline NA & Rodentia & Heteromyidae & Perognathus & inornatus & $\begin{array}{l}\text { San Joaquin Pocket } \\
\text { Mouse }\end{array}$ & 1.04 & 11 & extant & 0 & NA & LC & Frug \\
\hline NA & Rodentia & Heteromyidae & Perognathus & longimembris & $\begin{array}{l}\text { Little Pocket Mouse, Los } \\
\text { Angeles Little Pocket } \\
\text { Mouse, Los Angeles } \\
\text { Pocket Mouse }\end{array}$ & 0.88 & 8 & extant & 0 & NA & LC & Frug \\
\hline & & & & & Great Basin Pocket & & & & & & & \\
\hline NA & Rodentia & Heteromyidae & Perognathus & merriami & $\begin{array}{l}\text { Mouse, Merriam's } \\
\text { Pocket Mouse }\end{array}$ & 0.84 & 7 & extant & 0 & NA & LC & Frug \\
\hline NA & Rodentia & Heteromyidae & Perognathus & parvus & $\begin{array}{l}\text { Great Basin Pocket } \\
\text { Mouse }\end{array}$ & 1.34 & 22 & extant & 0 & NA & LC & Frug \\
\hline NA & Rodentia & Heteromyidae & Perognathus & xanthanotus & $\begin{array}{l}\text { Great Basin Pocket } \\
\text { Mouse }\end{array}$ & 1.38 & 24 & extant & 0 & NA & $\mathrm{LC}$ & Frug \\
\hline NA & Rodentia & Hydrochoeridae & Neochoerus & oesopi & & 4.78 & 60000 & extinct & TP & 12 & EX & graze \\
\hline NA & Rodentia & Hydrochoeridae & Neochoerus & pinckneyi & & 4.85 & 70000 & extinct & TP & 11 & EX & graze \\
\hline NA & Rodentia & Muridae & Microtus & ochrogaster & Prairie Vole & 2.30 & 200 & extant & 0 & NA & LC & graze \\
\hline
\end{tabular}




\begin{tabular}{|c|c|c|c|c|c|c|c|c|c|c|c|c|}
\hline NA & Rodentia & Muridae & Myodes & californicus & $\begin{array}{l}\text { Western Red-backed } \\
\text { Vole }\end{array}$ & 1.26 & 18 & extant & 0 & NA & $\mathrm{LC}$ & Browse \\
\hline NA & Rodentia & Muridae & Myodes & gapperi & $\begin{array}{l}\text { Revillagigedo Island } \\
\text { Red-backed Vole, } \\
\text { Southern Red-backed } \\
\text { Vole }\end{array}$ & 1.28 & 19 & extant & 0 & NA & $\mathrm{LC}$ & :/browse/gins \\
\hline NA & Rodentia & Muridae & Myodes & rutilus & $\begin{array}{l}\text { Northern Red-backed } \\
\text { Vole, RED VOLE }\end{array}$ & 1.46 & 29 & extant & 0 & NA & $\mathrm{LC}$ & Frug/browse \\
\hline NA & Rodentia & Muridae & Peromyscus & cochrani & & 1.32 & 21 & extinct & TP & NA & EX & ginsect/frug \\
\hline NA & Rodentia & Muridae & Peromyscus & imperfectus & & 1.54 & 35 & extinct & TP & NA & EX & ginsect/frug \\
\hline NA & Rodentia & Muridae & Peromyscus & maniculatus & $\begin{array}{l}\text { North American } \\
\text { Deermouse, Deer } \\
\text { Mouse }\end{array}$ & 1.39 & 25 & extant & 0 & NA & $\mathrm{LC}$ & ginsect/frug \\
\hline NA & Rodentia & Muridae & Rhipidomys & scandens & $\begin{array}{l}\text { Broad-footed Climbing } \\
\text { Mouse }\end{array}$ & & & extant & 0 & NA & LC & DD \\
\hline NA & Rodentia & Muridae & Synaptomys & australis & & 1.72 & 53 & extinct & TP & NA & EX & ginsect/frug \\
\hline NA & Rodentia & Muridae & Synaptomys & bunkeri & & 1.30 & 20 & extinct & TP & NA & EX & ginsect/frug \\
\hline NA & Rodentia & Sciuridae & Ammospermophilus & harrisii & $\begin{array}{l}\text { Harris's Antelope } \\
\text { Squirrel }\end{array}$ & 2.09 & 122 & extant & 0 & NA & LC & Frug \\
\hline NA & Rodentia & Sciuridae & Ammospermophilus & interpres & Texas Antelope Squirrel & 2.04 & 110 & extant & 0 & NA & LC & Frug \\
\hline NA & Rodentia & Sciuridae & Ammospermophilus & leucurus & $\begin{array}{l}\text { White-tailed Antelope } \\
\text { Squirrel } \\
\text { Nelson's Antelope } \\
\text { Squirrel, San Joaquin }\end{array}$ & 2.02 & 104 & extant & 0 & NA & $\mathrm{LC}$ & Frug/ginsect \\
\hline NA & Rodentia & Sciuridae & Ammospermophilus & nelsoni & $\begin{array}{l}\text { Antelope Ground } \\
\text { Squirrel, San Joaquin } \\
\text { Antelope Squirrel }\end{array}$ & 2.21 & 160 & extant & Future & NA & EN & Frug/ginsect \\
\hline NA & Rodentia & Sciuridae & Cynomys & gunnisoni & $\begin{array}{l}\text { Gunnison's Prairie Dog, } \\
\text { Ruddy Mongoose }\end{array}$ & 2.97 & 925 & extant & 0 & NA & LC & J̈raze/brows $\epsilon$ \\
\hline NA & Rodentia & Sciuridae & Cynomys & leucurus & White-tailed Prairie Dog & 2.96 & 908 & extant & 0 & NA & LC & J̈raze/brows \\
\hline NA & Rodentia & Sciuridae & Cynomys & ludovicianus & $\begin{array}{l}\text { Arizona Black-tailed } \\
\text { Prairie Dog, Black-tailed } \\
\text { Prairie Dog }\end{array}$ & 3.13 & 1364 & extant & 0 & NA & LC & J̈raze/brows $\epsilon$ \\
\hline NA & Rodentia & Sciuridae & Cynomys & mexicanus & $\begin{array}{l}\text { Mexican Prairie Dog, } \\
\text { Mexican Prairie Marmot }\end{array}$ & 2.95 & 900 & extant & Future & NA & EN & Jraze/brows \\
\hline NA & Rodentia & Sciuridae & Cynomys & parvidens & Utah Prairie Dog & 2.95 & 900 & extant & Future & NA & EN & Jraze/brows $\epsilon$ \\
\hline NA & Rodentia & Sciuridae & Glaucomys & sabrinus & $\begin{array}{l}\text { Carolina Flying Squirrel, } \\
\text { Northern Flying Squirrel }\end{array}$ & 2.22 & 166 & extant & 0 & NA & LC & Frug/ginsect \\
\hline NA & Rodentia & Sciuridae & Glaucomys & volans & $\begin{array}{l}\text { Mexican Flying Squirrel, } \\
\text { Southern Flying Squirrel }\end{array}$ & 1.81 & 64 & extant & 0 & NA & LC & Frug/ginsect \\
\hline NA & Rodentia & Sciuridae & Marmota & broweri & $\begin{array}{l}\text { Alaska Marmot } \\
\text { Hoary Marmot, }\end{array}$ & 3.53 & 3405 & extant & 0 & NA & $\mathrm{LC}$ & Graze \\
\hline NA & Rodentia & Sciuridae & Marmota & caligata & $\begin{array}{l}\text { Montague Island Hoary } \\
\text { Marmot }\end{array}$ & 3.86 & 7230 & extant & 0 & NA & LC & Graze \\
\hline NA & Rodentia & Sciuridae & Marmota & flaviventris & Yellow-bellied Marmot & 3.53 & 3350 & extant & 0 & NA & LC & Graze \\
\hline NA & Rodentia & Sciuridae & Marmota & $\operatorname{monax}$ & Woodchuck & 3.58 & 3802 & extant & 0 & NA & LC & J̄raze/brows $\epsilon$ \\
\hline NA & Rodentia & Sciuridae & Marmota & olympus & Olympic Marmot & 3.80 & 6300 & extant & 0 & NA & LC & Graze \\
\hline
\end{tabular}




\begin{tabular}{|c|c|c|c|c|c|c|c|c|c|c|c|c|}
\hline NA & Rodentia & Sciuridae & Marmota & vancouverensis & $\begin{array}{l}\text { Marmot, Chinese Ferret } \\
\text { Badgers, Vancouver } \\
\text { Marmot }\end{array}$ & 3.80 & 6300 & extant & Future & NA & $C R$ & Graze \\
\hline NA & Rodentia & Sciuridae & Sciurus & aberti & Abert's Squirrel & 2.80 & 624 & extant & 0 & NA & $\mathrm{LC}$ & browse/frug \\
\hline NA & Rodentia & Sciuridae & Sciurus & alleni & Allen's Squirrel & 2.64 & 435 & extant & 0 & NA & LC & Frug \\
\hline NA & Rodentia & Sciuridae & Sciurus & arizonensis & $\begin{array}{l}\text { Arizona Gray Squirrel } \\
\text { Mexican Gray Squirrel, }\end{array}$ & 2.81 & 647 & extant & 0 & NA & DD & Frug \\
\hline NA & Rodentia & Sciuridae & Sciurus & aureogaster & $\begin{array}{l}\text { Mexican Grey Squirrel, } \\
\text { Red-bellied Squirrel } \\
\text { Eastern Gray Squirrel, }\end{array}$ & 2.77 & 595 & extant & 0 & NA & LC & Frug \\
\hline NA & Rodentia & Sciuridae & Sciurus & carolinensis & $\begin{array}{l}\text { Gray Squirrel, Grey } \\
\text { Squirrel }\end{array}$ & 2.70 & 507 & extant & 0 & NA & LC & Frug \\
\hline NA & Rodentia & Sciuridae & Sciurus & colliaei & Collie's Squirrel & 2.70 & 498 & extant & 0 & NA & LC & Frug \\
\hline NA & Rodentia & Sciuridae & Sciurus & deppei & Deppe's Squirrel & 2.28 & 190 & extant & 0 & NA & LC & Frug \\
\hline NA & Rodentia & Sciuridae & Sciurus & granatensis & Red-tailed Squirrel & 2.40 & 250 & extant & 0 & NA & LC & Frug \\
\hline NA & Rodentia & Sciuridae & Sciurus & griseus & Western Gray Squirrel & 2.86 & 731 & extant & 0 & NA & LC & Frug/browse \\
\hline NA & Rodentia & Sciuridae & Sciurus & nayaritensis & $\begin{array}{l}\text { Mexican Fox Squirrel } \\
\text { Bryant's Fox Squirrel, }\end{array}$ & 2.84 & 697 & extant & 0 & NA & LC & Frug \\
\hline NA & Rodentia & Sciuridae & Sciurus & niger & $\begin{array}{l}\text { Delmarva Fox Squirrel, } \\
\text { Eastern Fox Squirrel, } \\
\text { Fox Squirrel }\end{array}$ & 2.88 & 762 & extant & 0 & NA & LC & Frug \\
\hline NA & Rodentia & Sciuridae & Sciurus & oculatus & Peters's Squirrel & 2.81 & 650 & extant & 0 & NA & $\mathrm{LC}$ & Frug \\
\hline NA & Rodentia & Sciuridae & Sciurus & richmondi & Richmond's Squirrel & 2.31 & 205 & extant & Future & NA & NT & Frug \\
\hline NA & Rodentia & Sciuridae & Sciurus & variegatoides & Variegated Squirrel & 2.69 & 485 & extant & 0 & NA & LC & Frug \\
\hline NA & Rodentia & Sciuridae & Sciurus & yucatanensis & Yucatan Squirrel & 2.35 & 225 & extant & 0 & NA & LC & Frug \\
\hline NA & Rodentia & Sciuridae & Spermophilus & adocetus & $\begin{array}{l}\text { Lesser Tropical Ground } \\
\text { Squirrel }\end{array}$ & 2.10 & 125 & extant & 0 & NA & LC & J̈raze/brows€ \\
\hline NA & Rodentia & Sciuridae & Spermophilus & annulatus & $\begin{array}{l}\text { Ring-tailed Ground } \\
\text { Squirrel }\end{array}$ & 2.70 & 500 & extant & 0 & NA & LC & Jraze/brows€ \\
\hline NA & Rodentia & Sciuridae & Spermophilus & armatus & Uinta Ground Squirrel & 2.50 & 313 & extant & 0 & NA & LC & Graze/frug \\
\hline NA & Rodentia & Sciuridae & Spermophilus & atricapillus & $\begin{array}{l}\text { Baja California Rock } \\
\text { Squirrel }\end{array}$ & 2.74 & 551 & extant & Future & NA & EN & J̈raze/brows \\
\hline NA & Rodentia & Sciuridae & Spermophilus & beecheyi & $\begin{array}{l}\text { California Ground } \\
\text { Squirrel }\end{array}$ & 2.76 & 578 & extant & 0 & NA & LC & Frug/browse \\
\hline NA & Rodentia & Sciuridae & Spermophilus & beldingi & $\begin{array}{l}\text { Belding's Ground } \\
\text { Squirrel }\end{array}$ & 2.45 & 280 & extant & 0 & NA & LC & Graze \\
\hline NA & Rodentia & Sciuridae & Spermophilus & brunneus & $\begin{array}{l}\text { Norhern Idaho Ground } \\
\text { Squirrel }\end{array}$ & 2.48 & 300 & extant & Future & NA & $C R$ & Jraze/brows $\epsilon$ \\
\hline NA & Rodentia & Sciuridae & Spermophilus & canus & $\begin{array}{l}\text { Merriam's Ground } \\
\text { Squirrel }\end{array}$ & 2.54 & 350 & extant & 0 & NA & $\mathrm{LC}$ & Jraze/brows $\epsilon$ \\
\hline NA & Rodentia & Sciuridae & Spermophilus & columbianus & $\begin{array}{l}\text { Columbian Ground } \\
\text { Squirrel }\end{array}$ & 2.69 & 493 & extant & 0 & NA & LC & Frug/browse \\
\hline NA & Rodentia & Sciuridae & Spermophilus & elegans & $\begin{array}{l}\text { Wyoming Ground } \\
\text { Squirrel }\end{array}$ & 2.66 & 454 & extant & 0 & NA & LC & Frug/browse \\
\hline NA & Rodentia & Sciuridae & Spermophilus & franklinii & $\begin{array}{l}\text { Franklin's Ground } \\
\text { Squirrel }\end{array}$ & 2.56 & 363 & extant & 0 & NA & LC & rowse/ginsec \\
\hline NA & Rodentia & Sciuridae & Spermophilus & lateralis & $\begin{array}{l}\text { Golden Mantled } \\
\text { Ground Squirrel }\end{array}$ & 2.28 & 191 & extant & 0 & NA & LC & wse/frug/gins \\
\hline NA & Rodentia & Sciuridae & Spermophilus & madrensis & $\begin{array}{l}\text { Sierra Madre Ground } \\
\text { Squirrel }\end{array}$ & 2.44 & 275 & extant & Future & NA & NT & J̈raze/brows€ \\
\hline
\end{tabular}




\begin{tabular}{|c|c|c|c|c|c|c|c|c|c|c|c|c|}
\hline NA & Rodentia & Sciuridae & Spermophilus & mexicanus & $\begin{array}{l}\text { Mexican Ground } \\
\text { Squirrel }\end{array}$ & 2.28 & 190 & extant & 0 & NA & LC & :/browse/gins \\
\hline NA & Rodentia & Sciuridae & Spermophilus & mohavensis & $\begin{array}{l}\text { Mohave Ground } \\
\text { Squirrel }\end{array}$ & 2.28 & 190 & extant & Future & NA & NT & Frug/browse \\
\hline NA & Rodentia & Sciuridae & Spermophilus & mollis & Piute Ground Squirrel & 2.22 & 165 & extant & 0 & NA & $\mathrm{LC}$ & browse/frug \\
\hline NA & Rodentia & Sciuridae & Spermophilus & parryii & Arctic Ground Squirrel & 2.88 & 760 & extant & 0 & NA & LC & Frug/browse \\
\hline NA & Rodentia & Sciuridae & Spermophilus & perotensis & Perote Ground Squirrel & 2.15 & 140 & extant & Future & NA & EN & J̈raze/browse \\
\hline NA & Rodentia & Sciuridae & Spermophilus & richardsonii & $\begin{array}{l}\text { Richardson's Ground } \\
\text { Squirrel }\end{array}$ & 2.61 & 406 & extant & 0 & NA & $\mathrm{LC}$ & Graze \\
\hline NA & Rodentia & Sciuridae & Spermophilus & saturatus & $\begin{array}{l}\text { Cascade Golden- } \\
\text { mantled Ground } \\
\text { Squirrel }\end{array}$ & 2.34 & 220 & extant & 0 & NA & LC & browse/frug \\
\hline NA & Rodentia & Sciuridae & Spermophilus & spilosoma & Spotted Ground Squirrel & 1.95 & 89 & extant & 0 & NA & LC & Graze/frug \\
\hline NA & Rodentia & Sciuridae & Spermophilus & tereticaudus & $\begin{array}{l}\text { Round-tailed Ground } \\
\text { Squirrel }\end{array}$ & 2.19 & 156 & extant & 0 & NA & LC & Frug/browse \\
\hline NA & Rodentia & Sciuridae & Spermophilus & townsendii & $\begin{array}{l}\text { Townsend's Ground } \\
\text { Squirrel }\end{array}$ & 2.33 & 213 & extant & Future & NA & VU & Frug/browse \\
\hline NA & Rodentia & Sciuridae & Spermophilus & tridecemlineatus & $\begin{array}{l}\text { Thirteen-lined Ground } \\
\text { Squirrel }\end{array}$ & 2.12 & 132 & extant & 0 & NA & LC & Frug/ginsect \\
\hline NA & Rodentia & Sciuridae & Spermophilus & variegatus & Rock Squirrel & 2.84 & 692 & extant & 0 & NA & $\mathrm{LC}$ & Frug/ginsect \\
\hline NA & Rodentia & Sciuridae & Spermophilus & washingtoni & $\begin{array}{l}\text { Washington Ground } \\
\text { Squirrel } \\
\text { Bangs's Mountain }\end{array}$ & 2.32 & 208 & extant & Future & NA & NT & J̄raze/brows $\epsilon$ \\
\hline NA & Rodentia & Sciuridae & Syntheosciurus & brochus & $\begin{array}{l}\text { Squirrel, Mountain } \\
\text { Squirrel, Neotropical } \\
\text { Montane Squirrel }\end{array}$ & 2.12 & 133 & extant & Future & NA & NT & \\
\hline NA & Rodentia & Sciuridae & Tamias & alpinus & Alpine Chipmunk & 1.56 & 37 & extant & 0 & NA & $\mathrm{LC}$ & Frug/browse \\
\hline NA & Rodentia & Sciuridae & Tamias & amoenus & Yellow-pine Chipmunk & 1.70 & 51 & extant & 0 & NA & $\mathrm{LC}$ & Frug/browse \\
\hline NA & Rodentia & Sciuridae & Tamias & aristus & & 2.16 & 143 & extinct & TP & NA & EX & frug \\
\hline NA & Rodentia & Sciuridae & Tamias & bulleri & Buller's Chipmunk & 2.00 & 100 & extant & Future & NA & Vu & Frug \\
\hline NA & Rodentia & Sciuridae & Tamias & canipes & Gray-footed Chipmunk & 1.85 & 70 & extant & 0 & NA & LC & Frug \\
\hline NA & Rodentia & Sciuridae & Tamias & cinereicollis & Gray-collared Chipmunk & 1.79 & 62 & extant & 0 & NA & LC & Frug \\
\hline NA & Rodentia & Sciuridae & Tamias & dorsalis & Cliff Chipmunk & 1.85 & 71 & extant & 0 & NA & $\mathrm{LC}$ & Frug/ginsect \\
\hline NA & Rodentia & Sciuridae & Tamias & durangae & Durango Chipmunk & 1.93 & 85 & extant & 0 & NA & $\mathrm{LC}$ & Frug \\
\hline NA & Rodentia & Sciuridae & Tamias & merriami & $\begin{array}{l}\text { Merriam's Chipmunk } \\
\text { Least Chipmunk, New }\end{array}$ & 1.87 & 75 & extant & 0 & NA & $\mathrm{LC}$ & Frug \\
\hline NA & Rodentia & Sciuridae & Tamias & minimus & $\begin{array}{l}\text { Mexico Least Chipmunk, } \\
\text { Pe_asco Least } \\
\text { Chipmunk, Selkirk Least } \\
\text { Chipmunk }\end{array}$ & 2.13 & 135 & extant & 0 & NA & LC & Frug \\
\hline NA & Rodentia & Sciuridae & Tamias & obscurus & $\begin{array}{l}\text { California Chipmunk, } \\
\text { Chaparral Chipmunk }\end{array}$ & 1.86 & 73 & extant & 0 & NA & LC & Frug \\
\hline NA & Rodentia & Sciuridae & Tamias & ochrogenys & $\begin{array}{l}\text { Yellow-cheeked } \\
\text { Chipmunk }\end{array}$ & 1.96 & 92 & extant & 0 & NA & $\mathrm{LC}$ & Frug/browse \\
\hline NA & Rodentia & Sciuridae & Tamias & palmeri & Palmer's Chipmunk & 1.84 & 69 & extant & Future & NA & EN & Frug \\
\hline NA & Rodentia & Sciuridae & Tamias & panamintinus & Panamint Chipmunk & 1.72 & 52 & extant & 0 & NA & LC & Frug/browse \\
\hline NA & Rodentia & Sciuridae & Tamias & quadrimaculatus & $\begin{array}{l}\text { Long-eared Chipmunk } \\
\text { Colorado Chipmunk, }\end{array}$ & 1.93 & 85 & extant & 0 & NA & LC & Frug/ginsect \\
\hline NA & Rodentia & Sciuridae & Tamias & quadrivittatus & $\begin{array}{l}\text { Organ Mountains } \\
\text { Chipmunk }\end{array}$ & 1.79 & 62 & extant & 0 & NA & LC & Frug \\
\hline
\end{tabular}




\begin{tabular}{|c|c|c|c|c|c|c|c|c|c|c|c|c|}
\hline NA & Rodentia & Sciuridae & Tamias & ruficaudus & Red-tailed Chipmunk & 1.83 & 68 & extant & 0 & NA & LC & Frug \\
\hline NA & Rodentia & Sciuridae & Tamias & rufus & Hopi Chipmunk & 1.73 & 54 & extant & 0 & NA & LC & Frug \\
\hline NA & Rodentia & Sciuridae & Tamias & senex & $\begin{array}{l}\text { Allen's Chipmunk, } \\
\text { Shadow Chipmunk }\end{array}$ & 1.95 & 89 & extant & 0 & NA & LC & Frug \\
\hline NA & Rodentia & Sciuridae & Tamias & siskiyou & Siskiyou Chipmunk & 1.88 & 75 & extant & 0 & NA & LC & Frug/ginsect \\
\hline NA & Rodentia & Sciuridae & Tamias & sonomae & Sonoma Chipmunk & 1.88 & 75 & extant & 0 & NA & LC & Frug \\
\hline NA & Rodentia & Sciuridae & Tamias & speciosus & Lodgepole Chipmunk & 1.79 & 62 & extant & 0 & NA & LC & Frug \\
\hline NA & Rodentia & Sciuridae & Tamias & striatus & Eastern Chipmunk & 2.05 & 112 & extant & 0 & NA & LC & Frug \\
\hline NA & Rodentia & Sciuridae & Tamias & townsendii & $\begin{array}{l}\text { Townsend's Chipmunk } \\
\text { Hidden Forest }\end{array}$ & 1.87 & 75 & extant & 0 & NA & LC & Frug \\
\hline NA & Rodentia & Sciuridae & Tamias & umbrinus & $\begin{array}{l}\text { Chipmunk, Uinta } \\
\text { Chipmunk }\end{array}$ & 1.80 & 63 & extant & 0 & NA & LC & Frug \\
\hline NA & Rodentia & Sciuridae & Tamiasciurus & douglasii & $\begin{array}{l}\text { Douglas's Squirrel } \\
\text { Red Squirrel, Mount }\end{array}$ & 2.35 & 225 & extant & 0 & NA & LC & Frug \\
\hline NA & Rodentia & Sciuridae & Tamiasciurus & hudsonicus & $\begin{array}{l}\text { Graham Red Squirrel, } \\
\text { Mountain Weasel }\end{array}$ & 2.30 & 201 & extant & 0 & NA & LC & Frug \\
\hline NA & Rodentia & Sciuridae & Tamiasciurus & mearnsi & Mearns's Squirrel & 2.33 & 213 & extant & Future & NA & EN & frug/carn \\
\hline NA & Sirenia & Trichechidae & Trichechus & manatus & $\begin{array}{l}\text { American Manatee, } \\
\text { West Indian Manatee }\end{array}$ & 5.64 & 433333 & extant & Future & NA & VU & Graze \\
\hline NA & Soricomorpha & Soricidae & Blarina & brevicauda & $\begin{array}{l}\text { Northern Short-tailed } \\
\text { Shrew }\end{array}$ & 1.45 & 28 & extant & 0 & NA & LC & Ginsect \\
\hline NA & Soricomorpha & Soricidae & Blarina & carolinensis & $\begin{array}{l}\text { Southern Short-tailed } \\
\text { Shrew }\end{array}$ & 1.13 & 14 & extant & 0 & NA & LC & Ginsect \\
\hline NA & Soricomorpha & Soricidae & Blarina & hylophaga & $\begin{array}{l}\text { Elliot's Short-tailed } \\
\text { Shrew } \\
\text { Ender's Small-eared }\end{array}$ & 1.16 & 15 & extant & 0 & NA & LC & Ginsect \\
\hline NA & Soricomorpha & Soricidae & Cryptotis & endersi & $\begin{array}{l}\text { Shrew, Enders' S Small- } \\
\text { eared Shrew } \\
\text { Goldman's Broad- } \\
\text { clawed Shrew, }\end{array}$ & 0.90 & 8 & extant & Future & NA & EN & Ginsect \\
\hline NA & Soricomorpha & Soricidae & Cryptotis & goldmani & $\begin{array}{l}\text { Goldman's Small-eared } \\
\text { Shrew, Goldman's } \\
\text { Small Eared Shrew } \\
\text { Goodwin's Broad- } \\
\text { clawed Shrew, }\end{array}$ & 0.90 & 8 & extant & 0 & NA & LC & Ginsect \\
\hline NA & Soricomorpha & Soricidae & Cryptotis & goodwini & $\begin{array}{l}\text { Goodwin's Small-eared } \\
\text { Shrew, Goodwin's } \\
\text { Small Eared Shrew } \\
\text { Talamancan Small- }\end{array}$ & 0.85 & 7 & extant & 0 & NA & LC & Ginsect \\
\hline NA & Soricomorpha & Soricidae & Cryptotis & gracilis & $\begin{array}{l}\text { eared Shrew, } \\
\text { Talamancan Small } \\
\text { Eared Shrew } \\
\text { Honduran Small-eared }\end{array}$ & 0.85 & 7 & extant & Future & NA & VU & Ginsect \\
\hline NA & Soricomorpha & Soricidae & Cryptotis & hondurensis & $\begin{array}{l}\text { Shrew, Honduran Small } \\
\text { Eared Shrew }\end{array}$ & 0.70 & 5 & extant & 0 & NA & DD & Ginsect \\
\hline NA & Soricomorpha & Soricidae & Cryptotis & magna & $\begin{array}{l}\text { Big Mexican Small- } \\
\text { eared Shrew }\end{array}$ & 0.85 & 7 & extant & Future & NA & VU & Ginsect \\
\hline NA & Soricomorpha & Soricidae & Cryptotis & mexicana & $\begin{array}{l}\text { Mexican Small-eared } \\
\text { Shrew }\end{array}$ & 0.85 & 7 & extant & 0 & NA & LC & Ginsect \\
\hline NA & Soricomorpha & Soricidae & Cryptotis & nigrescens & $\begin{array}{l}\text { Blackish Small-eared } \\
\text { Shrew }\end{array}$ & 0.73 & 5 & extant & 0 & NA & LC & Ginsect \\
\hline
\end{tabular}




\begin{tabular}{|c|c|c|c|c|c|c|c|c|c|c|c|c|}
\hline NA & Soricomorpha & Soricidae & Cryptotis & parva & $\begin{array}{l}\text { Least Shrew, North } \\
\text { American Least Shrew }\end{array}$ & 0.70 & 5 & extant & 0 & NA & LC & Ginsect \\
\hline NA & Soricomorpha & Soricidae & Megasorex & gigas & Mexican Shrew & 1.30 & 20 & extant & 0 & NA & LC & Ginsect \\
\hline NA & Soricomorpha & Soricidae & Notiosorex & crawfordi & Desert Shrew & 0.64 & 4 & extant & 0 & NA & LC & Ginsect \\
\hline NA & Soricomorpha & Soricidae & Sorex & alaskanus & $\begin{array}{l}\text { Glacier Bay Water } \\
\text { Shrew }\end{array}$ & 1.15 & 14 & extant & 0 & NA & $\mathrm{DD}$ & Ginsect \\
\hline NA & Soricomorpha & Soricidae & Sorex & arcticus & Arctic Shrew & 0.91 & 8 & extant & 0 & NA & LC & Ginsect \\
\hline NA & Soricomorpha & Soricidae & Sorex & arizonae & Arizona Shrew & 0.38 & 2 & extant & 0 & NA & LC & Ginsect \\
\hline NA & Soricomorpha & Soricidae & Sorex & bairdi & Baird's Shrew & 0.92 & 8 & extant & 0 & NA & LC & Ginsect \\
\hline NA & Soricomorpha & Soricidae & Sorex & bendirii & $\begin{array}{l}\text { Marsh Shrew, Pacific } \\
\text { Water Shrew }\end{array}$ & 1.21 & 16 & extant & 0 & NA & $\mathrm{LC}$ & Ginsect \\
\hline NA & Soricomorpha & Soricidae & Sorex & cinereus & $\begin{array}{l}\text { Cinereus Shrew, } \\
\text { Masked Shrew }\end{array}$ & 0.67 & 5 & extant & 0 & NA & LC & Ginsect \\
\hline NA & Soricomorpha & Soricidae & Sorex & dispar & Long-tailed Shrew & 0.70 & 5 & extant & 0 & NA & LC & Ginsect \\
\hline NA & Soricomorpha & Soricidae & Sorex & emarginatus & Zacatecas Shrew & 0.85 & 7 & extant & 0 & NA & $\mathrm{LC}$ & Ginsect \\
\hline NA & Soricomorpha & Soricidae & Sorex & fumeus & Smoky Shrew & 0.89 & 8 & extant & 0 & NA & LC & Ginsect \\
\hline NA & Soricomorpha & Soricidae & Sorex & gaspensis & Long-tailed Shrew & 0.46 & 3 & extant & 0 & NA & $\mathrm{LC}$ & Ginsect \\
\hline NA & Soricomorpha & Soricidae & Sorex & haydeni & $\begin{array}{l}\text { Prairie Shrew, Hayden's } \\
\text { Shrew }\end{array}$ & 0.54 & 4 & extant & 0 & NA & LC & Ginsect \\
\hline NA & Soricomorpha & Soricidae & Sorex & hoyi & $\begin{array}{l}\text { American Pygmy Shrew, } \\
\text { Pygmy Shrew }\end{array}$ & 0.41 & 3 & extant & 0 & NA & LC & Ginsect \\
\hline NA & Soricomorpha & Soricidae & Sorex & longirostris & Southeastern Shrew & 0.55 & 4 & extant & 0 & NA & LC & Ginsect \\
\hline NA & Soricomorpha & Soricidae & Sorex & lyelli & Mount Lyell Shrew & 0.70 & 5 & extant & 0 & NA & LC & Ginsect \\
\hline NA & Soricomorpha & Soricidae & Sorex & macrodon & Large-toothed Shrew & 0.85 & 7 & extant & Future & NA & VU & Ginsect \\
\hline NA & Soricomorpha & Soricidae & Sorex & merriami & Merriam's Shrew & 0.77 & 6 & extant & 0 & NA & LC & Ginsect \\
\hline NA & Soricomorpha & Soricidae & Sorex & milleri & $\begin{array}{l}\text { Carmen Mountain } \\
\text { Shrew }\end{array}$ & 0.85 & 7 & extant & Future & NA & VU & Ginsect \\
\hline NA & Soricomorpha & Soricidae & Sorex & monticolus & $\begin{array}{l}\text { Dusky Shrew, Montane } \\
\text { Shrew }\end{array}$ & 0.72 & 5 & extant & 0 & NA & LC & Ginsect \\
\hline NA & Soricomorpha & Soricidae & Sorex & nanus & Dwarf Shrew & 0.38 & 2 & extant & 0 & NA & LC & Ginsect \\
\hline NA & Soricomorpha & Soricidae & Sorex & oreopolus & $\begin{array}{l}\text { Mexican Long-tailed } \\
\text { Shrew }\end{array}$ & 0.85 & 7 & extant & 0 & NA & $\mathrm{LC}$ & Ginsect \\
\hline NA & Soricomorpha & Soricidae & Sorex & ornatus & Ornate Shrew & 0.70 & 5 & extant & 0 & NA & $\mathrm{LC}$ & Ginsect \\
\hline NA & Soricomorpha & Soricidae & Sorex & pacificus & Pacific Shrew & 0.83 & 7 & extant & 0 & NA & $\mathrm{LC}$ & Ginsect \\
\hline NA & Soricomorpha & Soricidae & Sorex & palustris & $\begin{array}{l}\text { American Water Shrew, } \\
\text { Water Shrew }\end{array}$ & 1.13 & 13 & extant & 0 & NA & LC & Ginsect \\
\hline NA & Soricomorpha & Soricidae & Sorex & preblei & Preble's Shrew & 0.49 & 3 & extant & 0 & NA & LC & Ginsect \\
\hline NA & Soricomorpha & Soricidae & Sorex & saussurei & Saussure's Shrew & 0.67 & 5 & extant & 0 & NA & LC & Ginsect \\
\hline NA & Soricomorpha & Soricidae & Sorex & sclateri & Sclater's Shrew & 0.85 & 7 & extant & Future & NA & CR & Ginsect \\
\hline NA & Soricomorpha & Soricidae & Sorex & sonomae & Fog Shrew & 0.92 & 8 & extant & 0 & NA & $\mathrm{LC}$ & Ginsect \\
\hline NA & Soricomorpha & Soricidae & Sorex & stizodon & San Cristobal Shrew & 0.85 & 7 & extant & Future & NA & CR & Ginsect \\
\hline NA & Soricomorpha & Soricidae & Sorex & tenellus & Inyo Shrew & 0.57 & 4 & extant & 0 & NA & $\mathrm{LC}$ & Ginsect \\
\hline NA & Soricomorpha & Soricidae & Sorex & trowbridgii & Trowbridge's Shrew & 0.58 & 4 & extant & 0 & NA & LC & Ginsect \\
\hline NA & Soricomorpha & Soricidae & Sorex & tundrensis & $\begin{array}{l}\text { Tundra Shrew, TUNDRA } \\
\text { SHREW }\end{array}$ & 0.88 & 8 & extant & 0 & NA & LC & Ginsect \\
\hline NA & Soricomorpha & Soricidae & Sorex & ugyunak & Barren Ground Shrew & 0.56 & 4 & extant & 0 & NA & $\mathrm{LC}$ & Ginsect \\
\hline NA & Soricomorpha & Soricidae & Sorex & vagrans & Vagrant Shrew & 0.77 & 6 & extant & 0 & NA & $\mathrm{LC}$ & Ginse \\
\hline NA & Soricomorpha & Soricidae & Sorex & ventralis & Chestnut-bellied Shrew & 0.85 & 7 & extant & 0 & NA & LC & Ginsect \\
\hline NA & Soricomorpha & Soricidae & Sorex & veraepacis & Verapaz Shrew & 0.88 & 8 & extant & 0 & NA & $\mathrm{LC}$ & Ginsect \\
\hline NA & Soricomorpha & Talpidae & Condylura & cristata & Star-nosed Mole & 1.92 & 84 & extant & 0 & NA & LC & Ginsect \\
\hline
\end{tabular}




\begin{tabular}{|c|c|c|c|c|c|c|c|c|c|c|c|c|}
\hline NA & Soricomorpha & Talpidae & Neurotrichus & gibbsii & $\begin{array}{l}\text { Shrew-mole, American } \\
\text { Shrew Mole, American } \\
\text { Shrew-mole }\end{array}$ & 0.95 & 9 & extant & 0 & NA & LC & Ginsect \\
\hline NA & Soricomorpha & Talpidae & Parascalops & breweri & Hairy-tailed Mole & 1.71 & 51 & extant & 0 & NA & LC & Ginsect \\
\hline NA & Soricomorpha & Talpidae & Scalopus & aquaticus & Eastern Mole & 1.96 & 91 & extant & 0 & NA & LC & Ginsect \\
\hline NA & Soricomorpha & Talpidae & Scapanus & latimanus & Broad-footed Mole & 1.74 & 55 & extant & 0 & NA & LC & Ginsect \\
\hline NA & Soricomorpha & Talpidae & Scapanus & orarius & Coast Mole & 1.79 & 61 & extant & 0 & NA & LC & Ginsect \\
\hline NA & Soricomorpha & Talpidae & Scapanus & townsendii & Townsend's Mole & 2.15 & 142 & extant & 0 & NA & LC & Ginsect \\
\hline SA & Artiodactyla & Camelidae & Eulamaops & parallelus & btwn 10-20 14C yrs & 5.18 & 150000 & extinct & TP & 15 & EX & Graze \\
\hline SA & Artiodactyla & Camelidae & Hemiauchenia & paradoxa & & 5.48 & 300000 & extinct & TP & 10 & EX & graze \\
\hline SA & Artiodactyla & Camelidae & Lama & glama & Ilama & 5.15 & 142500 & extant & 0 & NA & LC & эraze/browse \\
\hline SA & Artiodactyla & Camelidae & Lama & gracilis & extinct small Ilama & 4.70 & 50000 & extinct & TP & 15 & EX & graze \\
\hline SA & Artiodactyla & Camelidae & Lama & guanicoe & Guanaco & 5.08 & 120000 & extant & 0 & NA & LC & కraze/browse \\
\hline SA & Artiodactyla & Camelidae & Palaeolama & reissi & & 6.00 & 1000000 & extinct & TP & 11.9 & EX & Browse \\
\hline SA & Artiodactyla & Camelidae & Vicugna & vicugna & Vicuna, Vicu_a, Vicugna & 4.68 & 47500 & extant & 0 & NA & LC & graze \\
\hline SA & Artiodactyla & Cervidae & Agalmaceros & blicki & & 4.78 & 60000 & extinct & TP & 12 & EX & Browse \\
\hline SA & Artiodactyla & Cervidae & Antifer & niemeyeri & LP to Holocene & & & extinct & TP & 11.2 & EX & Browse \\
\hline SA & Artiodactyla & Cervidae & Blastocerus & dichotomus & $\begin{array}{l}\text { Marsh Deer } \\
\text { Taruca, Peruvian } \\
\text { Guemal, Peruvian }\end{array}$ & 4.94 & 86666 & extant & Future & NA & vU & క̧raze/browse \\
\hline SA & Artiodactyla & Cervidae & Hippocamelus & antisensis & $\begin{array}{l}\text { Huemul, North Andean } \\
\text { Deer, North Andean } \\
\text { Huemul } \\
\text { Patagonian Huemul, } \\
\text { Chilean Guemal, South }\end{array}$ & 4.84 & 68599 & extant & Future & NA & VU & эraze/browse \\
\hline SA & Artiodactyla & Cervidae & Hippocamelus & bisulcus & $\begin{array}{l}\text { Andean Deer, South } \\
\text { Andean Huemul, } \\
\text { Chilean Huemul }\end{array}$ & 4.85 & 70000 & extant & Future & NA & EN & эraze/browse \\
\hline SA & Artiodactyla & Cervidae & Mazama & americana & Red Brocket & 4.36 & 22800 & extant & 0 & NA & DD & Browse \\
\hline SA & Artiodactyla & Cervidae & Mazama & bricenii & $\begin{array}{l}\text { M_rida Brocket, Rufous } \\
\text { Brocket, Meroia Brocket }\end{array}$ & 4.22 & 16500 & extant & Future & NA & VU & Browse \\
\hline SA & Artiodactyla & Cervidae & Mazama & chunyi & $\begin{array}{l}\text { Peruvian Dwarf Brocket, } \\
\text { Chunyi, Dwarf Brocket }\end{array}$ & 4.22 & 16500 & extant & Future & NA & VU & Browse \\
\hline SA & Artiodactyla & Cervidae & Mazama & gouazoupira & $\begin{array}{l}\text { Gray Brocket, Brown } \\
\text { Brocket }\end{array}$ & 4.21 & 16300 & extant & 0 & NA & LC & Browse \\
\hline SA & Artiodactyla & Cervidae & Mazama & nana & $\begin{array}{l}\text { Brazilian Dwarf Brocket, } \\
\text { Pygmy Brocket }\end{array}$ & 4.22 & 16500 & extant & Future & NA & VU & Browse \\
\hline SA & Artiodactyla & Cervidae & Mazama & rufina & $\begin{array}{l}\text { Dwarf Red Brocket, } \\
\text { Little Red Brocket Deer, } \\
\text { Little Red Brocket }\end{array}$ & 4.41 & 26000 & extant & Future & NA & VU & Browse \\
\hline SA & Artiodactyla & Cervidae & Morenelaphus & brachyceros & & 4.70 & 50000 & extinct & TP & 12 & EX & Graze \\
\hline SA & Artiodactyla & Cervidae & Odocoileus & virginianus & $\begin{array}{l}\text { White-tailed Deer, Key } \\
\text { Deer, Key Deer Toy Deer }\end{array}$ & 4.74 & 55509 & extant & 0 & NA & LC & Browse \\
\hline SA & Artiodactyla & Cervidae & Ozotoceros & bezoarticus & $\begin{array}{l}\text { Pampas Deer } \\
\text { too old? But see }\end{array}$ & 4.60 & 40000 & extant & Future & NA & NT & క̧raze/browse \\
\hline SA & Artiodactyla & Cervidae & Paraceros & fragilis & $\begin{array}{l}\text { Encyclopedia of } \\
\text { Quaternary Science }\end{array}$ & 5.48 & 300000 & extinct & TP & NA & EX & Jraze/brows€ \\
\hline SA & Artiodactyla & Cervidae & Pudu & mephistophiles & Northern Pudu & 3.98 & 9600 & extant & Future & NA & VU & Browse \\
\hline
\end{tabular}




\begin{tabular}{|c|c|c|c|c|c|c|c|c|c|c|c|c|}
\hline SA & Artiodactyla & Cervidae & Pudu & puda & $\begin{array}{l}\text { Southern Pudu, Chilean } \\
\text { Pudu }\end{array}$ & 3.99 & 9750 & extant & Future & NA & NT & Browse \\
\hline SA & Artiodactyla & Tayassuidae & Catagonus & wagneri & Chacoan Peccary, Tagua & 4.55 & 35566 & extant & Future & NA & EN & Frug/browse \\
\hline SA & Artiodactyla & Tayassuidae & Pecari & tajacu & Collared Peccary & 4.33 & 21267 & extant & 0 & NA & $\mathrm{LC}$ & Frug/browse \\
\hline SA & Artiodactyla & Tayassuidae & Tayassu & pecari & $\begin{array}{l}\text { White-lipped Peccary } \\
\text { Short-eared Dog, Small- }\end{array}$ & 4.51 & 32234 & extant & Future & NA & Vu & Frug/browse \\
\hline SA & Carnivora & Canidae & Atelocynus & microtis & $\begin{array}{l}\text { eared Dog, Small-eared } \\
\text { Zorro, Short-eared Fox }\end{array}$ & 3.89 & 7750 & extant & Future & NA & NT & Carn \\
\hline SA & Carnivora & Canidae & Canis & dirus & & 4.70 & 50000 & extinct & TP & 12 & EX & carn \\
\hline SA & Carnivora & Canidae & Cerdocyon & thous & $\begin{array}{l}\text { Crab-eating Fox, Crab- } \\
\text { eating Zorro, Savannah } \\
\text { Fox, Common Zorro }\end{array}$ & 3.72 & 5240 & extant & 0 & NA & LC & carn/ginsect \\
\hline SA & Carnivora & Canidae & Chrysocyon & brachyurus & Maned Wolf & 4.37 & 23250 & extant & Future & NA & NT & carn/ginsect \\
\hline SA & Carnivora & Canidae & Dusicyon & avus & & 4.11 & 13000 & extinct & TP & 11.2 & EX & Carn \\
\hline SA & Carnivora & Canidae & Lycalopex & culpaeus & $\begin{array}{l}\text { Culpeo, Andean Fox } \\
\text { South American Grey }\end{array}$ & 3.99 & 9832 & extant & 0 & NA & LC & carn/ginsect \\
\hline SA & Carnivora & Canidae & Lycalopex & griseus & $\begin{array}{l}\text { Fox, Argentine Gray } \\
\text { Fox, Grey Zorro } \\
\text { Pampas Fox, Azara's }\end{array}$ & 3.92 & 8280 & extant & 0 & NA & LC & $\mathrm{rn} /$ ginsect/frı \\
\hline SA & Carnivora & Canidae & Lycalopex & gymnocercus & $\begin{array}{l}\text { Zorro, Azara's Fox, } \\
\text { Azara's Fox } \\
\text { Sechuran Fox, Peruvian }\end{array}$ & 3.67 & 4690 & extant & 0 & NA & $\mathrm{LC}$ & $\mathrm{rn} / \mathrm{ginsect} / \mathrm{fr}$ \\
\hline SA & Carnivora & Canidae & Lycalopex & sechurae & $\begin{array}{l}\text { Desert Fox, Sechuran } \\
\text { Desert Fox }\end{array}$ & 3.60 & 4000 & extant & Future & NA & NT & $\mathrm{rn} / \mathrm{ginsect} / \mathrm{fr} \mathrm{I}$ \\
\hline SA & Carnivora & Canidae & Lycalopex & vetulus & $\begin{array}{l}\text { Hoary Fox, Small- } \\
\text { toothed Dog, Hoary } \\
\text { Zorro }\end{array}$ & 3.73 & 5350 & extant & 0 & NA & LC & $\mathrm{rn} / \mathrm{ginsect} / \mathrm{fr}$ \\
\hline SA & Carnivora & Canidae & Protocyon & orcesi & & 4.40 & 25000 & extinct & TP & 12 & EX & Carn \\
\hline SA & Carnivora & Canidae & Protocyon & troglodytes & & 4.20 & 16000 & extinct & TP & 12 & EX & Carn \\
\hline SA & Carnivora & Canidae & Pseudalopex & peruanus & & 3.81 & 6430 & extinct & TP & NA & EX & $\mathrm{rn} /$ ginsect/frı \\
\hline SA & Carnivora & Canidae & Speothos & pacivorus & & 3.95 & 9000 & extinct & TP & 12 & EX & Carn \\
\hline SA & Carnivora & Canidae & Speothos & venaticus & $\begin{array}{l}\text { Bush Dog, Vinegar Dog, } \\
\text { Savannah Dog }\end{array}$ & 3.78 & 6000 & extant & Future & NA & NT & Carn \\
\hline SA & Carnivora & Canidae & Theriodictis & platensis & & 4.43 & 27000 & extinct & TP & 11 & EX & :arn/omnivore \\
\hline SA & Carnivora & Canidae & Theriodictis & tarijensis & & 4.43 & 27000 & extinct & TP & 11 & EX & :arn/omnivore \\
\hline SA & Carnivora & Canidae & Urocyon & cinereoargenteus & Tree Fox, Gray Fox & 3.58 & 3834 & extant & 0 & NA & LC & carn/frug \\
\hline SA & Carnivora & Felidae & Herpailurus & yaguarondi & Jaguarundi, Eyra Cat & 3.83 & 6750 & extant & 0 & NA & LC & Carn \\
\hline SA & Carnivora & Felidae & Leopardus & colocolo & $\begin{array}{l}\text { Pampas Cat, Chilean } \\
\text { Pampa Cat }\end{array}$ & 3.59 & 3935 & extant & Future & NA & NT & Carn \\
\hline SA & Carnivora & Felidae & Leopardus & geoffroyi & Geoffroy's Cat & 3.56 & 3590 & extant & 0 & NA & $\mathrm{LC}$ & Carn \\
\hline SA & Carnivora & Felidae & Leopardus & guigna & $\begin{array}{l}\text { Gui_a, Kodkod, Chilean } \\
\text { Cat }\end{array}$ & 3.35 & 2230 & extant & Future & NA & VU & Carn \\
\hline SA & Carnivora & Felidae & Leopardus & pardalis & $\begin{array}{l}\text { Ocelot } \\
\text { Oncilla, Little Spotted }\end{array}$ & 4.08 & 11900 & extant & 0 & NA & LC & Carn \\
\hline SA & Carnivora & Felidae & Leopardus & tigrinus & $\begin{array}{l}\text { Cat, Tiger Cat, Little } \\
\text { Tiger Cat }\end{array}$ & 3.35 & 2250 & extant & Future & NA & VU & Carn \\
\hline SA & Carnivora & Felidae & Leopardus & wiedii & Margay, Tree Ocelot & 3.51 & 3250 & extant & Future & NA & NT & Carn \\
\hline SA & Carnivora & Felidae & Oreailurus & jacobita & $\begin{array}{l}\text { Andean Cat, Mountain } \\
\text { Cat }\end{array}$ & 3.96 & 9170 & extant & Future & NA & EN & Carn \\
\hline SA & Carnivora & Felidae & Panthera & leo & Lion, African Lion & 5.64 & 433200 & extinct & TP & 11 & EX & Carn \\
\hline SA & Carnivora & Felidae & Panthera & onca & Jaguar & 5.00 & 100000 & extant & Future & NA & NT & Carn \\
\hline
\end{tabular}




\begin{tabular}{|c|c|c|c|c|c|c|c|c|c|c|c|c|}
\hline SA & Carnivora & Felidae & Puma & concolor & $\begin{array}{l}\text { Puma, Mountain Lion, } \\
\text { Cougar, Red Tiger, Deer } \\
\text { Tiger }\end{array}$ & 4.71 & 51600 & extant & 0 & NA & LC & Carn \\
\hline SA & Carnivora & Felidae & Smilodon & fatalis & saber-toothed cat & 5.64 & 433200 & extinct & TP & 11 & EX & carn \\
\hline SA & Carnivora & Felidae & Smilodon & populator & & 5.65 & 442000 & extinct & TP & 11.2 & EX & Carn \\
\hline SA & Carnivora & Mephitidae & Conepatus & chinga & $\begin{array}{l}\text { Molina's Hog-nosed } \\
\text { Skunk } \\
\text { Humboldt's Hog-nosed }\end{array}$ & 3.28 & 1918 & extant & 0 & NA & $\mathrm{LC}$ & Ginsect \\
\hline SA & Carnivora & Mephitidae & Conepatus & humboldtii & $\begin{array}{l}\text { Skunk, Patagonian Hog- } \\
\text { nosed Skunk } \\
\text { Striped Hog-nosed }\end{array}$ & 2.52 & 328 & extant & 0 & NA & $\mathrm{LC}$ & Ginsect \\
\hline SA & Carnivora & Mephitidae & Conepatus & semistriatus & $\begin{array}{l}\text { Skunk, Amazonian Hog- } \\
\text { nosed Skunk }\end{array}$ & 3.08 & 1200 & extant & 0 & NA & LC & ginsect/carn \\
\hline SA & Carnivora & Mustelidae & Eira & barbara & $\begin{array}{l}\text { Tayra, Greyheaded } \\
\text { Tayra }\end{array}$ & 3.59 & 3910 & extant & 0 & NA & $\mathrm{LC}$ & Carn \\
\hline SA & Carnivora & Mustelidae & Galictis & cuja & Lesser Grison & 3.00 & 1000 & extant & 0 & NA & LC & carn/ginsect \\
\hline SA & Carnivora & Mustelidae & Galictis & vittata & Greater Grison & 3.51 & 3200 & extant & 0 & NA & LC & Carn \\
\hline SA & Carnivora & Mustelidae & Lontra & felina & Marine Otter, Sea Cat & 4.49 & 30600 & extant & Future & NA & EN & iscivore/inver \\
\hline SA & Carnivora & Mustelidae & Lontra & longicaudis & $\begin{array}{l}\text { Neotropical Otter, La } \\
\text { Plata Otter, Neotropical } \\
\text { River Otter, South } \\
\text { American River Otter, } \\
\text { Long-tailed Otter }\end{array}$ & 3.82 & 6555 & extant & Future & NA & NT & iscivore/inver \\
\hline SA & Carnivora & Mustelidae & Lontra & provocax & $\begin{array}{l}\text { Southern River Otter, } \\
\text { Huillin }\end{array}$ & 3.88 & 7500 & extant & Future & NA & EN & iscivore/inver \\
\hline SA & Carnivora & Mustelidae & Lyncodon & patagonicus & Patagonian Weasel & 2.35 & 225 & extant & 0 & NA & $\mathrm{LC}$ & Carn \\
\hline SA & Carnivora & Mustelidae & Mustela & africana & $\begin{array}{l}\text { Amazon Weasel, } \\
\text { Tropical Weasel }\end{array}$ & 2.73 & 537 & extant & 0 & NA & LC & Jarn/piscivore \\
\hline SA & Carnivora & Mustelidae & Mustela & felipei & Colombian Weasel & 2.32 & 211 & extant & Future & NA & Vu & Zarn/piscivore \\
\hline SA & Carnivora & Mustelidae & Mustela & frenata & Long-tailed Weasel & 2.17 & 147 & extant & 0 & NA & LC & Carn \\
\hline SA & Carnivora & Mustelidae & Pteronura & brasiliensis & $\begin{array}{l}\text { Giant Otter, Giant } \\
\text { Brazilian Otter }\end{array}$ & 4.38 & 24000 & extant & Future & NA & EN & iscivore/inver \\
\hline SA & Carnivora & Procyonidae & Bassaricyon & alleni & Eastern Lowland Olingo & 3.09 & 1235 & extant & 0 & NA & $\mathrm{LC}$ & Frug/ginsect \\
\hline SA & Carnivora & Procyonidae & Bassaricyon & beddardi & eastern lowland olingo & 3.09 & 1235 & extant & 0 & NA & LC & Frug/ginsect \\
\hline SA & Carnivora & Procyonidae & Bassaricyon & gabbii & Northern Olingo & 3.10 & 1250 & extant & 0 & NA & LC & Frug/ginsect \\
\hline SA & Carnivora & Procyonidae & Nasua & narica & $\begin{array}{l}\text { White-nosed Coati, } \\
\text { Coatimundi }\end{array}$ & 3.61 & 4030 & extant & 0 & NA & LC & $\mathrm{dg} / \mathrm{ginsect} / \mathrm{ca}$ \\
\hline SA & Carnivora & Procyonidae & Nasua & nasua & South American Coati & 3.58 & 3794 & extant & 0 & NA & $\mathrm{LC}$ & $\mathrm{lg} / \mathrm{ginsect} / \mathrm{ca}$ \\
\hline SA & Carnivora & Procyonidae & Nasuella & olivacea & $\begin{array}{l}\text { Western Mountain } \\
\text { Coati }\end{array}$ & 3.13 & 1340 & extant & Future & NA & NT & Ginsect \\
\hline SA & Carnivora & Procyonidae & Potos & flavus & Kinkajou & 3.48 & 3000 & extant & 0 & NA & LC & Frug \\
\hline SA & Carnivora & Procyonidae & Procyon & cancrivorus & Crab-eating Raccoon & 3.84 & 6950 & extant & 0 & NA & LC & invert \\
\hline SA & Carnivora & Ursidae & Arctotherium & bonariensis & short-faced bears & 5.78 & 600000 & extinct & TP & 11 & EX & Carn \\
\hline SA & Carnivora & Ursidae & Arctotherium & tarijense & short-faced bears & 5.60 & 400000 & extinct & TP & 11.2 & EX & carn \\
\hline SA & Carnivora & Ursidae & Arctotherium & wingei & short-faced bears & 2.18 & 150 & extinct & TP & 10 & EX & carn \\
\hline SA & Carnivora & Ursidae & Tremarctos & ornatus & $\begin{array}{l}\text { Spectacled Bear, } \\
\text { Andean Bear }\end{array}$ & 5.15 & 140001 & extant & Future & NA & VU & Frug \\
\hline SA & Cingulata & Dasypodidae & Cabassous & centralis & $\begin{array}{l}\text { Northern Naked-tailed } \\
\text { Armadillo }\end{array}$ & 3.58 & 3810 & extant & 0 & NA & DD & Ginsect \\
\hline SA & Cingulata & Dasypodidae & Cabassous & chacoensis & $\begin{array}{l}\text { Chacoan Naked-tailed } \\
\text { Armadillo }\end{array}$ & 3.17 & 1490 & extant & Future & NA & NT & Ginsect \\
\hline
\end{tabular}




\begin{tabular}{|c|c|c|c|c|c|c|c|c|c|c|c|c|}
\hline SA & Cingulata & Dasypodidae & Cabassous & tatouay & $\begin{array}{l}\text { Greater Naked-tailed } \\
\text { Armadillo }\end{array}$ & 3.73 & 5350 & extant & 0 & NA & $\mathrm{LC}$ & Ginsect \\
\hline SA & Cingulata & Dasypodidae & Cabassous & unicinctus & $\begin{array}{l}\text { Southern Naked-Tailed } \\
\text { Armadillo }\end{array}$ & 3.68 & 4800 & extant & 0 & NA & LC & Ginsect \\
\hline SA & Cingulata & Dasypodidae & Calyptophractus & retusus & $\begin{array}{l}\text { Greater Fairy Armadillo, } \\
\text { Burmeister's Armadillo, } \\
\text { Chacoan Fairy } \\
\text { Armadillo, Greater Pichi } \\
\text { Ciego }\end{array}$ & 2.11 & 130 & extant & 0 & NA & DD & insect/brows! \\
\hline SA & Cingulata & Dasypodidae & Chaetophractus & nationi & $\begin{array}{l}\text { Screaming Hairy } \\
\text { Armadillo }\end{array}$ & 3.33 & 2150 & extant & 0 & NA & $\mathrm{LC}$ & ginsect/carn \\
\hline SA & Cingulata & Dasypodidae & Chaetophractus & vellerosus & $\begin{array}{l}\text { Screaming Hairy } \\
\text { Armadillo }\end{array}$ & 3.01 & 1030 & extant & 0 & NA & LC & ginsect/carn \\
\hline SA & Cingulata & Dasypodidae & Chaetophractus & villosus & $\begin{array}{l}\text { Large Hairy Armadillo, } \\
\text { Big Hairy Armadillo } \\
\text { Pink Fairy Armadillo, }\end{array}$ & 3.66 & 4540 & extant & 0 & NA & LC & ginsect/carn \\
\hline SA & Cingulata & Dasypodidae & Chlamyphorus & truncatus & $\begin{array}{l}\text { Pichiciego, Lesser Pichi } \\
\text { Ciego, Lesser Fairy } \\
\text { Armadillo }\end{array}$ & 1.65 & 44 & extant & 0 & NA & DD & insect/brows! \\
\hline SA & Cingulata & Dasypodidae & Dasypus & hybridus & $\begin{array}{l}\text { Southern Long-Nosed } \\
\text { Armadillo }\end{array}$ & 3.18 & 1500 & extant & Future & NA & NT & ginsect/carn \\
\hline SA & Cingulata & Dasypodidae & Dasypus & kappleri & $\begin{array}{l}\text { Greater Long-nosed } \\
\text { Armadillo }\end{array}$ & 3.98 & 9500 & extant & 0 & NA & LC & ginsect/carn \\
\hline SA & Cingulata & Dasypodidae & Dasypus & novemcinctus & $\begin{array}{l}\text { Nine-banded Armadillo, } \\
\text { Common Long-nosed } \\
\text { Armadillo }\end{array}$ & 3.62 & 4204 & extant & 0 & NA & LC & ginsect/carn \\
\hline SA & Cingulata & Dasypodidae & Dasypus & pilosus & $\begin{array}{l}\text { Hairy Long-nosed } \\
\text { Armadillo } \\
\text { Northern Long-nosed }\end{array}$ & 3.65 & 4445 & extant & 0 & NA & DD & ginsect/carn \\
\hline SA & Cingulata & Dasypodidae & Dasypus & sabanicola & $\begin{array}{l}\text { Armadillo, Llanos Long- } \\
\text { nosed Armadillo }\end{array}$ & 3.06 & 1150 & extant & Future & NA & NT & ginsect/carn \\
\hline SA & Cingulata & Dasypodidae & Dasypus & septemcinctus & $\begin{array}{l}\text { Brazilian Lesser Long- } \\
\text { nosed Armadillo, Seven- } \\
\text { banded Armadillo }\end{array}$ & 3.18 & 1527 & extant & 0 & NA & $\mathrm{LC}$ & Ginsect \\
\hline SA & Cingulata & Dasypodidae & Euphractus & sexcinctus & $\begin{array}{l}\text { Yellow Armadillo, Six- } \\
\text { Banded Armadillo }\end{array}$ & 3.68 & 4783 & extant & 0 & NA & $\mathrm{LC}$ & Nse/ginsect/c \\
\hline SA & Cingulata & Dasypodidae & Eutatus & seguini & & 5.16 & 144400 & extinct & TP & 11 & EX & Browse \\
\hline SA & Cingulata & Dasypodidae & Holmesi & occidentalis & & 5.30 & 200000 & extinct & TP & 12 & EX & browse \\
\hline SA & Cingulata & Dasypodidae & Holmesi & paulacoutoi & & 5.10 & 125000 & extinct & TP & 12 & EX & browse \\
\hline SA & Cingulata & Dasypodidae & Pampatherium & humboldtii & & 5.18 & 150000 & extinct & TP & 12 & EX & Ginsect \\
\hline SA & Cingulata & Dasypodidae & Pampatherium & typum & & 5.30 & 200000 & extinct & TP & 11 & EX & Ginsect \\
\hline SA & Cingulata & Dasypodidae & Priodontes & maximus & Giant Armadillo & 4.66 & 45360 & extant & Future & NA & Vu & Ginsect \\
\hline SA & Cingulata & Dasypodidae & Propraopus & grandis & & 4.67 & 47000 & extinct & TP & NA & EX & Ginsect \\
\hline SA & Cingulata & Dasypodidae & Tolypeutes & matacus & $\begin{array}{l}\text { Southern Three-banded } \\
\text { Armadillo }\end{array}$ & 3.08 & 1200 & extant & Future & NA & NT & Ginsect \\
\hline SA & Cingulata & Dasypodidae & Tolypeutes & tricinctus & $\begin{array}{l}\text { Brazilian Three-banded } \\
\text { Armadillo }\end{array}$ & 3.17 & 1487 & extant & Future & NA & Vu & Ginsect \\
\hline SA & Cingulata & Dasypodidae & Zaedyus & pichiy & Pichi & 3.14 & 1380 & extant & Future & NA & NT & ginsect/carn \\
\hline SA & Cingulata & Glyptodontidae & Chlamydotherium & humboldtii & & 5.24 & 175000 & extinct & TP & 12 & EX & Browse \\
\hline SA & Cingulata & Glyptodontidae & Doedicurus & clavicaudatus & & 6.17 & 1468000 & extinct & TP & 10 & EX & Browse \\
\hline SA & Cingulata & Glyptodontidae & Glyptodon & clavipes & & 6.30 & 2000000 & extinct & TP & 10 & EX & Browse \\
\hline
\end{tabular}




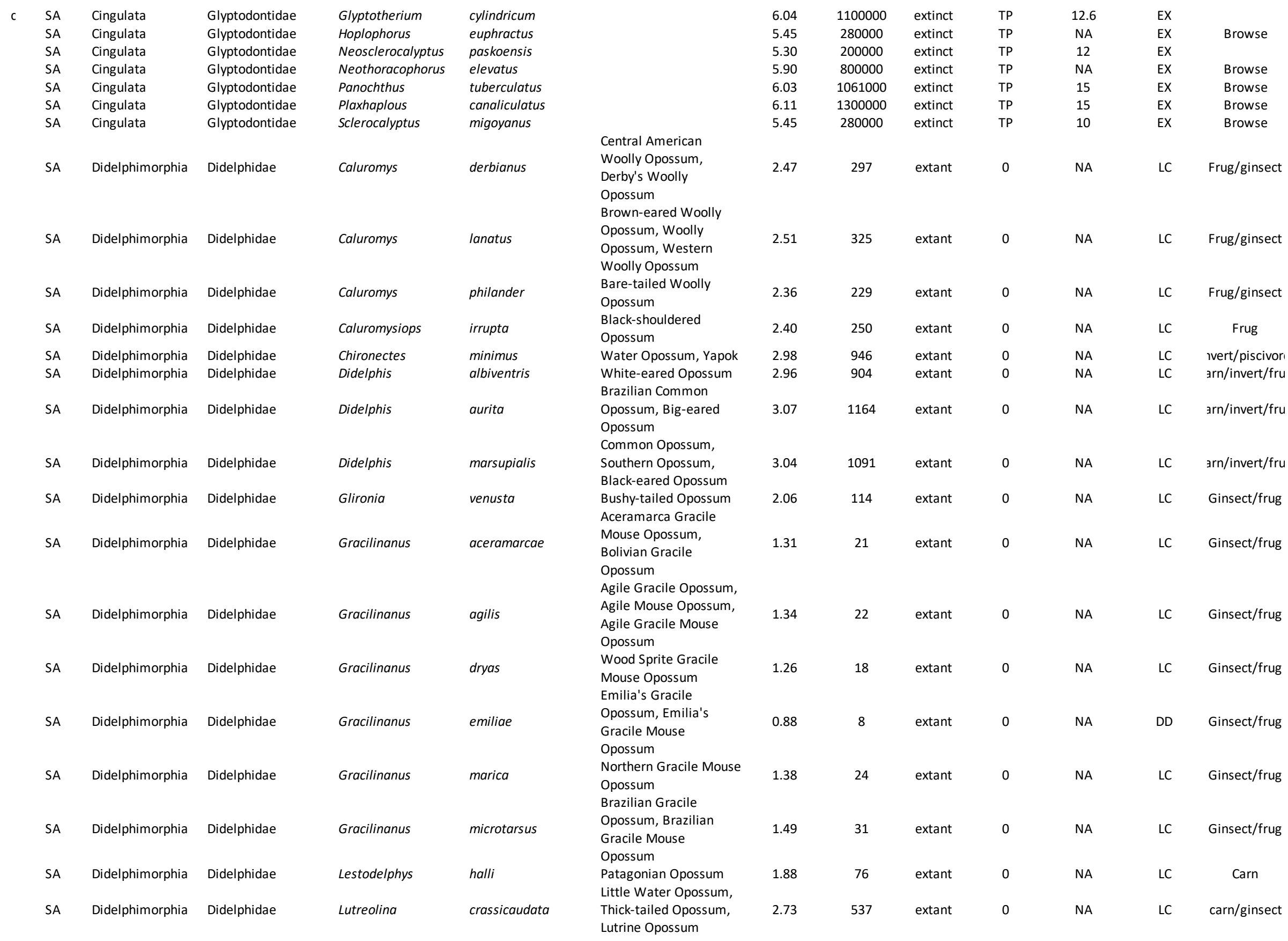




\begin{tabular}{|c|c|c|c|c|c|c|c|c|c|c|c|c|}
\hline SA & Didelphimorphia & Didelphidae & Makalata & macrura & Long-tailed Tree Rat & 1.32 & 21 & extant & 0 & NA & LC & ginsect/carn \\
\hline SA & Didelphimorphia & Didelphidae & Marmosa & andersoni & $\begin{array}{l}\text { Anderson's Mouse } \\
\text { Opossum }\end{array}$ & 1.68 & 47 & extant & 0 & NA & DD & Ginsect/frug \\
\hline SA & Didelphimorphia & Didelphidae & Marmosa & lepida & $\begin{array}{l}\text { Little Rufous Mouse } \\
\text { Opossum }\end{array}$ & 1.15 & 14 & extant & 0 & NA & LC & Ginsect/frug \\
\hline SA & Didelphimorphia & Didelphidae & Marmosa & mexicana & $\begin{array}{l}\text { Mexican Mouse } \\
\text { Opossum } \\
\text { Linnaeus's Mouse }\end{array}$ & 1.69 & 49 & extant & 0 & NA & LC & Ginsect/frug \\
\hline SA & Didelphimorphia & Didelphidae & Marmosa & murina & $\begin{array}{l}\text { Opossum, Murine } \\
\text { Mouse Opossum }\end{array}$ & 1.41 & 26 & extant & 0 & NA & LC & Ginsect/frug \\
\hline SA & Didelphimorphia & Didelphidae & Marmosa & robinsoni & $\begin{array}{l}\text { Robinson's Mouse } \\
\text { Opossum }\end{array}$ & 1.75 & 57 & extant & 0 & NA & LC & Ginsect/frug \\
\hline SA & Didelphimorphia & Didelphidae & Marmosa & rubra & Red Mouse Opossum & 1.80 & 63 & extant & 0 & NA & DD & Ginsect/frug \\
\hline SA & Didelphimorphia & Didelphidae & Marmosa & tyleriana & Tyler's Mouse Opossum & 1.51 & 32 & extant & 0 & NA & DD & Ginsect/frug \\
\hline SA & Didelphimorphia & Didelphidae & Marmosa & xerophila & $\begin{array}{l}\text { Dryland Mouse } \\
\text { Opossum }\end{array}$ & 1.66 & 46 & extant & Future & NA & VU & Ginsect/frug \\
\hline SA & Didelphimorphia & Didelphidae & Marmosops & cracens & $\begin{array}{l}\text { Slim-faced Slender } \\
\text { Mouse Opossum }\end{array}$ & 1.41 & 26 & extant & 0 & NA & $\mathrm{DD}$ & Ginsect/frug \\
\hline SA & Didelphimorphia & Didelphidae & Marmosops & fuscatus & $\begin{array}{l}\text { Gray-bellied Slender } \\
\text { Mouse Opossum }\end{array}$ & 1.78 & 60 & extant & 0 & NA & $\mathrm{DD}$ & Ginsect/frug \\
\hline SA & Didelphimorphia & Didelphidae & Marmosops & handleyi & $\begin{array}{l}\text { Handley's Slender } \\
\text { Mouse Opossum }\end{array}$ & 1.49 & 31 & extant & Future & NA & CR & Ginsect/frug \\
\hline SA & Didelphimorphia & Didelphidae & Marmosops & impavidus & $\begin{array}{l}\text { Andean Slender Mouse } \\
\text { Opossum } \\
\text { Grey Slender Mouse }\end{array}$ & 1.61 & 41 & extant & 0 & NA & LC & Ginsect/frug \\
\hline SA & Didelphimorphia & Didelphidae & Marmosops & incanus & $\begin{array}{l}\text { Opossum, Gray Slender } \\
\text { Mouse Opossum, Gray } \\
\text { Slender Opossum }\end{array}$ & 1.79 & 62 & extant & 0 & NA & LC & Ginsect/frug \\
\hline SA & Didelphimorphia & Didelphidae & Marmosops & invictus & $\begin{array}{l}\text { Slaty Slender Mouse } \\
\text { Opossum }\end{array}$ & 1.47 & 29 & extant & 0 & NA & LC & Ginsect/frug \\
\hline SA & Didelphimorphia & Didelphidae & Marmosops & noctivagus & $\begin{array}{l}\text { White-bellied Slender } \\
\text { Mouse Opossum } \\
\text { Delicate Slender Mouse }\end{array}$ & 1.32 & 21 & extant & 0 & NA & LC & Ginsect/frug \\
\hline SA & Didelphimorphia & Didelphidae & Marmosops & parvidens & $\begin{array}{l}\text { Opossum, Delicate } \\
\text { Slender Opossum, } \\
\text { Opossum-souris D_licat }\end{array}$ & 1.18 & 15 & extant & 0 & NA & LC & Ginsect/frug \\
\hline SA & Didelphimorphia & Didelphidae & Metachirus & nudicaudatus & $\begin{array}{l}\text { Brown Four-eyed } \\
\text { Opossum }\end{array}$ & 2.57 & 375 & extant & 0 & NA & LC & Ginsect/frug \\
\hline SA & Didelphimorphia & Didelphidae & Micoureus & alstoni & $\begin{array}{l}\text { Alston's Woolly Mouse } \\
\text { Opossum }\end{array}$ & 2.12 & 132 & extant & 0 & NA & LC & Ginsect/frug \\
\hline SA & Didelphimorphia & Didelphidae & Micoureus & constantiae & & 1.95 & 90 & extant & 0 & NA & LC & Ginsect/frug \\
\hline SA & Didelphimorphia & Didelphidae & Micoureus & demerarae & & 1.88 & 76 & extant & 0 & NA & LC & Ginsect/frug \\
\hline SA & Didelphimorphia & Didelphidae & Micoureus & regina & & 2.07 & 119 & extant & 0 & NA & LC & Ginsect/frug \\
\hline SA & Didelphimorphia & Didelphidae & Monodelphis & adusta & $\begin{array}{l}\text { Sepia Short-tailed } \\
\text { Opossum }\end{array}$ & 1.54 & 35 & extant & 0 & NA & LC & Ginsect \\
\hline SA & Didelphimorphia & Didelphidae & Monodelphis & americana & $\begin{array}{l}\text { Northern Three-striped } \\
\text { Opossum, Three-striped } \\
\text { Short-tailed Opossum }\end{array}$ & 1.29 & 20 & extant & 0 & NA & LC & ginsect/carn \\
\hline
\end{tabular}




\begin{tabular}{|c|c|c|c|c|}
\hline SA & Didelphimorphia & Didelphidae & Monodelphis & brevicaudat \\
\hline SA & Didelphimorphia & Didelphidae & Monodelphis & dimidiata \\
\hline SA & Didelphimorphia & Didelphidae & Monodelphis & domestica \\
\hline SA & Didelphimorphia & Didelphidae & Monodelphis & emiliae \\
\hline SA & Didelphimorphia & Didelphidae & Monodelphis & iheringi \\
\hline SA & Didelphimorphia & Didelphidae & Monodelphis & kunsi \\
\hline SA & Didelphimorphia & Didelphidae & Monodelphis & osgoodi \\
\hline SA & Didelphimorphia & Didelphidae & Monodelphis & rubida \\
\hline SA & Didelphimorphia & Didelphidae & Monodelphis & scalops \\
\hline SA & Didelphimorphia & Didelphidae & Monodelphis & sorex \\
\hline SA & Didelphimorphia & Didelphidae & Monodelphis & theresa \\
\hline SA & Didelphimorphia & Didelphidae & Monodelphis & unistriata \\
\hline SA & Didelphimorphia & Didelphidae & Philander & andersoni \\
\hline SA & Didelphimorphia & Didelphidae & Philander & opossum \\
\hline SA & Didelphimorphia & Didelphidae & Thylamys & elegans \\
\hline SA & Didelphimorphia & Didelphidae & Thylamys & pallidior \\
\hline SA & Didelphimorphia & Didelphidae & Thylamys & pusillus \\
\hline SA & Didelphimorphia & Didelphidae & Thylamys & velutinus \\
\hline SA & Insectivora & Soricidae & Cryptotis & avia \\
\hline SA & Lagomorpha & Leporidae & Sylvilagus & brasiliensis \\
\hline SA & Lagomorpha & Leporidae & Sylvilagus & floridanus \\
\hline SA & Litopterna & Macraucheniidae & Macrauchenia & patachonica \\
\hline
\end{tabular}

Northern Red-sided

Opossum, Red-legged

Short-tailed Opossum

Southern Short-tailed

Opossum, Yellow-sided

Opossum, Eastern Short-

tailed Opossum

Grey Short-tailed

Opossum, Gray Short-

tailed Opossum

Emilia's Short-tailed

Opossum

Ihering's Short-tailed

Opossum, Ihering's

Three-striped Opossum

Pygmy Short-tailed

Opossum

Osgood's Short-tailed

Opossum

Three-striped Short-

tailed Opossum

Long-nosed Short-tailed

Opossum

Long-nosed Short-tailed

Opossum

Single-striped Opossum

Anderson's Four-eyed

Opossum, Black Four-

eyed Opossum

Gray Four-eyed

Opossum, Guaiki

Elegant Fat-tailed

Mouse Opossum,

Elegant Fat-tailed

Opossum

Pallid Fat-tailed

Opossum, White-bellied

Fat-tailed Mouse

Opossum

Small Fat-tailed

Opossum

Dwarf Fat-tailed Mouse Opossum, Velvety Fattailed Opossum

Thomas' Small-eared Shrew

Tapeti, Forest Rabbit

Eastern Cottontail
1.84

.

1.76

1.85

1.60

2.05

1.05

2.05

2.05

1.66

2.87

extant

7

55

421

NA

LC

Ginsect

政

DA

ginsect/carn

$\begin{array}{ll}71 & \text { extant } \\ 40 & \text { extant } \\ 112 & \text { extant } \\ 11 & \text { extant } \\ 112 & \text { extant } \\ 46 & \text { extant } \\ 741 & \text { extant } \\ 48 & \text { extant } \\ 112 & \text { extant } \\ & \end{array}$

extant

o

$\begin{array}{ccc}\text { NA } & \text { LC } & \text { ginsect/carn } \\ \text { NA } & \text { LC } & \text { Ginsect } \\ \text { NA } & \text { DD } & \text { ginsect/carn } \\ \text { NA } & \text { LC } & \text { Ginsect } \\ \text { NA } & \text { LC } & \text { ginsect/carn } \\ \text { NA } & \text { LC } & \text { Ginsect } \\ \text { NA } & \text { LC } & \text { Ginsect } \\ \text { NA } & \text { LC } & \text { Ginsect } \\ \text { NA } & \text { LC } & \text { ginsect/carn } \\ \text { NA } & \text { CR } & \text { Ginsect } \\ \text { NA } & \text { LC } & \text { carn/ginsect } \\ \text { NA } & \text { LC } & \text { carn/ginsect } \\ \text { NA } & \text { LC } & \text { ginsect/carn } \\ \text { NA } & \text { LC } & \text { ginsect/carn } \\ & & \\ \text { NA } & & \end{array}$

NA

LC ginsect/carn

NA

LC ginsect/carn

$\begin{array}{ccc}11 & \text { extant } & 0 \\ 950 & \text { extant } & 0 \\ 1173 & \text { extant } & 0 \\ 988000 & \text { extinct } & \text { TP }\end{array}$

$\begin{array}{ll}\text { LC } & \text { Ginsect } \\ \text { LC } & \text { Browse } \\ \text { LC } & \text { Browse }\end{array}$




\begin{tabular}{|c|c|c|c|c|c|c|c|c|c|c|c|c|}
\hline SA & Litopterna & Macraucheniidae & Windhausenia & $s p$. & & 5.85 & 700000 & extinct & TP & 12 & EX & Graze \\
\hline SA & Microbiotheria & Microbiotheriidae & Dromiciops & gliroides & Monito del Monte & 1.35 & 22 & extant & Future & NA & NT & Ginsect \\
\hline SA & Notoungulata & Toxodontidae & Mixotoxodon & larensis & & 6.00 & 1000000 & extinct & TP & 12 & EX & 3rowse/graz€ \\
\hline SA & Notoungulata & Toxodontidae & Toxodon & bilobidens & & 6.04 & 1100000 & extinct & TP & 12 & EX & 3rowse/graz€ \\
\hline SA & Notoungulata & Toxodontidae & Toxodon & paradoxus & & 6.00 & 1000000 & extinct & TP & 10 & EX & 3rowse/graze \\
\hline SA & Notoungulata & Toxodontidae & Toxodon & platensis & & 6.22 & 1642000 & extinct & TP & 12 & EX & 3rowse/graze \\
\hline \multirow[t]{2}{*}{ SA } & Notoungulata & Toxodontidae & Toxodon & protoburmeisteri & & 6.04 & 1100000 & extinct & TP & NA & EX & 3rowse/graze \\
\hline & & & & & Gray-bellied Shrew & & & & & & & \\
\hline SA & Paucituberculata & Caenolestidae & Caenolestes & caniventer & $\begin{array}{l}\text { Opossum, Pale-bellied } \\
\text { Shrew Opossum, Gray- } \\
\text { bellied Caenolestid } \\
\text { Blackish Shrew }\end{array}$ & 1.60 & 40 & extant & Future & NA & NT & ginsect/carn \\
\hline SA & Paucituberculata & Caenolestidae & Caenolestes & convelatus & $\begin{array}{l}\text { Opossum, Northern } \\
\text { Shrew Opossum, } \\
\text { Northern Caenolestid }\end{array}$ & 1.60 & 40 & extant & Future & NA & Vu & ginsect/carn \\
\hline SA & Paucituberculata & Caenolestidae & Caenolestes & fuliginosus & $\begin{array}{l}\text { Silky Shrew Opossum, } \\
\text { Ecuadorean Shrew- } \\
\text { opossum, Dusky } \\
\text { Caenolestid, Common } \\
\text { Grey Shrew Opossum }\end{array}$ & 1.44 & 28 & extant & 0 & NA & $\mathrm{LC}$ & ginsect/carn \\
\hline SA & Paucituberculata & Caenolestidae & Lestoros & inca & $\begin{array}{l}\text { Incan Shrew Opossum } \\
\text { Chilean Caenolestid, }\end{array}$ & 1.32 & 21 & extant & 0 & NA & LC & ginsect/carn \\
\hline SA & Paucituberculata & Caenolestidae & Rhyncholestes & raphanurus & $\begin{array}{l}\text { Chilean Shrew } \\
\text { Opossum, Long-nosed } \\
\text { Caenolestid }\end{array}$ & 1.32 & 21 & extant & Future & NA & NT & ginsect/carn \\
\hline SA & Perissodactyla & Equidae & Equus & andium & & 5.34 & 220000 & extinct & TP & 11.9 & EX & graze \\
\hline SA & Perissodactyla & Equidae & Equus & insulatus & & 5.55 & 351000 & extinct & TP & 11 & EX & graze \\
\hline SA & Perissodactyla & Equidae & Equus & lasallei & & 5.54 & 350000 & extinct & TP & 11 & EX & graze \\
\hline SA & Perissodactyla & Equidae & Equus & neogeus & allochthonous sp & 5.58 & 378000 & extinct & TP & 11.9 & EX & graze \\
\hline SA & Perissodactyla & Equidae & Equus & santaeelenae & & 5.54 & 350000 & extinct & TP & 11 & EX & graze \\
\hline SA & Perissodactyla & Equidae & Hippidion & principale & allochthonous sp & 5.71 & 511000 & extinct & TP & 12 & EX & Graze \\
\hline SA & Perissodactyla & Equidae & Hippidion & $s p$ & & 5.42 & 265000 & extinct & TP & 11.9 & EX & Graze \\
\hline SA & Perissodactyla & Equidae & Onohippidium & saldiasi & & 5.49 & 310700 & extinct & TP & 13.4 & EX & Graze \\
\hline SA & Perissodactyla & Tapiridae & Tapirus & bairdii & $\begin{array}{l}\text { Baird's Tapir, Central } \\
\text { American Tapir }\end{array}$ & 5.48 & 299999 & extant & Future & NA & EN & orowse/graze \\
\hline SA & Perissodactyla & Tapiridae & Tapirus & pinchaque & $\begin{array}{l}\text { Mountain Tapir, Woolly } \\
\text { Tapir, Andean Tapir } \\
\text { Lowland Tapir, South }\end{array}$ & 5.17 & 148950 & extant & Future & NA & EN & orowse/graze \\
\hline SA & Perissodactyla & Tapiridae & Tapirus & terrestris & $\begin{array}{l}\text { American Tapir, } \\
\text { Brazilian Tapir } \\
\text { Maned Three-toed }\end{array}$ & 5.32 & 207501 & extant & Future & NA & vu & orowse/graze \\
\hline SA & Pilosa & Bradypodidae & Bradypus & torquatus & $\begin{array}{l}\text { Sloth, Maned Sloth, } \\
\text { Brazilian Three-toed } \\
\text { Sloth }\end{array}$ & 3.59 & 3900 & extant & Future & NA & Vu & Browse \\
\hline SA & Pilosa & Bradypodidae & Bradypus & tridactylus & $\begin{array}{l}\text { Pale-throated Three- } \\
\text { toed Sloth, Pale- } \\
\text { throated Sloth }\end{array}$ & 3.59 & 3850 & extant & 0 & NA & $\mathrm{LC}$ & Browse \\
\hline SA & Pilosa & Bradypodidae & Bradypus & variegatus & $\begin{array}{l}\text { Brown-throated Sloth, } \\
\text { Brown-throated Three- } \\
\text { toed Sloth, Bolivian } \\
\text { Three-toed Sloth }\end{array}$ & 3.64 & 4335 & extant & 0 & NA & LC & Browse \\
\hline
\end{tabular}




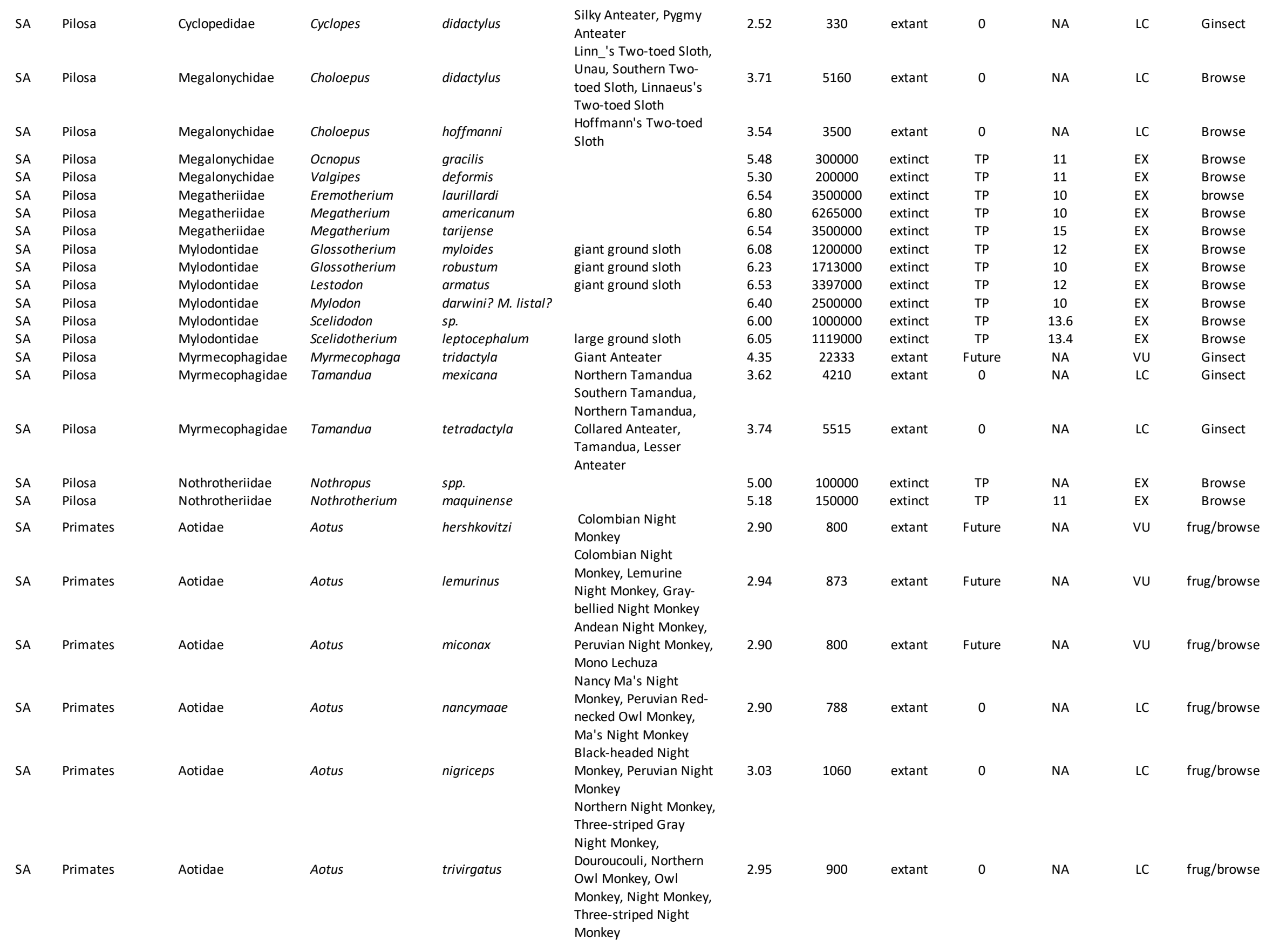


Spix's Night Monkey,

Spix's Owl Monkey,

Noisy Night Monkey,

Colombian Gray Night

Monkey, Night Monkey

Red-handed Howler

Monkey, Black and Red

Howler, Red-handed

Howler, Red-handed

Howling Monkey

Black-and-gold Howler

Monkey, Black Howling

Monkey, Black Howler

Monkey, Black Howler

Mantled Howler

Monkey, South Pacific

Blackish Howling

Monkey, Ecuadorian

Mantled Howling

Monkey, Mantled

Howler

Bolivian Red Howler

Monkey, Bolivian Red

Howling Monkey,

Bolivian Red Howler

Juruá Red Howler

Monkey

White-bellied Spider

Monkey, White-fronted

Spider Monkey, Long-

haired Spider Monkey

Black-faced Black

Spider Monkey,

Peruvian Black Spider

Monkey, Peruvian

Spider Monkey, Chamek

Spider Monkey

Brown-headed Spider

Monkey, Black-headed

Spider Monkey

White-cheeked Spider

Monkey, White-

whiskered Spider

Monkey

Guiana Spider Monkey, Red-faced Black Spider Monkey, Red-faced

Spider Monkey, Black

2.94

3.77

3.

3.79

3.

3.78

3.78

3.96

3.78
873

extant

(

3.81

6400

extant

Future

VU Browse/frug

3.77

5862

extant

0

NA

LC Browse/frug

3.86

7275

extant

0

NA

LC Browse/frug

9100

extan

Future

NA

CR

frug/browse

6000

extant

Future

NA

EN frug/browse

Spider Monkey 


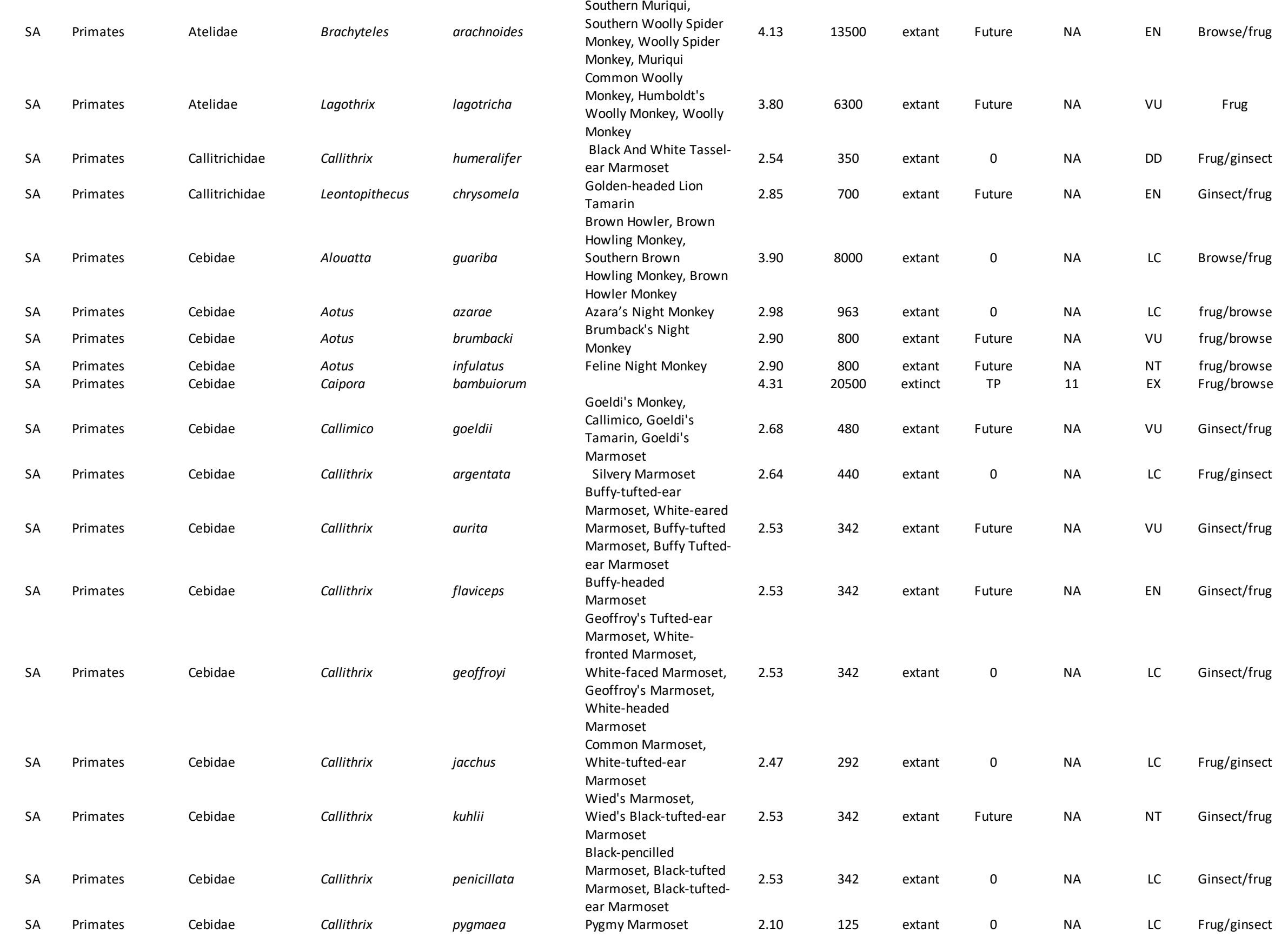




\begin{tabular}{|c|c|c|c|c|c|c|c|c|c|c|c|c|}
\hline SA & Primates & Cebidae & Cebus & albifrons & $\begin{array}{l}\text { Humboldt's White- } \\
\text { fronted Capuchin, White- } \\
\text { fronted Capuchin }\end{array}$ & 3.42 & 2629 & extant & 0 & NA & $\mathrm{LC}$ & Frug/ginsect \\
\hline SA & Primates & Cebidae & Cebus & apella & & 3.40 & 2500 & extant & 0 & NA & $\mathrm{LC}$ & Frug/ginsect \\
\hline SA & Primates & Cebidae & Cebus & capucinus & white-headed capuchin & 3.44 & 2733 & extant & 0 & NA & LC & Frug/ginsect \\
\hline SA & Primates & Cebidae & Cebus & olivaceus & wedge-capped capuchin & 3.41 & 2600 & extant & 0 & NA & $\mathrm{LC}$ & Frug/ginsect \\
\hline SA & Primates & Cebidae & Leontopithecus & chrysopygus & $\begin{array}{l}\text { Black Lion Tamarin, } \\
\text { Golden-rumped Lion } \\
\text { Tamarin }\end{array}$ & 2.85 & 700 & extant & Future & NA & EN & Ginsect/frug \\
\hline SA & Primates & Cebidae & Leontopithecus & rosalia & $\begin{array}{l}\text { Golden Lion Tamarin } \\
\text { Peruvian Yellow-tailed }\end{array}$ & 2.73 & 536 & extant & Future & NA & EN & Frug/ginsect \\
\hline SA & Primates & Cebidae & Oreonax & flavicauda & $\begin{array}{l}\text { Woolly Monkey, Yellow- } \\
\text { tailed Woolly Monkey } \\
\text { Brazilian Bare-faced } \\
\text { Tamarin, Pied Bare- }\end{array}$ & 3.83 & 6800 & extant & Future & NA & $C R$ & Frug \\
\hline SA & Primates & Cebidae & Saguinus & bicolor & $\begin{array}{l}\text { faced Tamarin, Pied } \\
\text { Bare-face Tamarin, Pied } \\
\text { Tamarin } \\
\text { Saddleback Tamarin, }\end{array}$ & 2.67 & 465 & extant & Future & NA & EN & Frug/ginsect \\
\hline SA & Primates & Cebidae & Saguinus & fuscicollis & $\begin{array}{l}\text { Andean Saddle-back } \\
\text { Tamarin } \\
\text { Geoffroy's Tamarin, }\end{array}$ & 2.59 & 387 & extant & 0 & NA & $\mathrm{LC}$ & Frug/ginsect \\
\hline SA & Primates & Cebidae & Saguinus & geoffroyi & $\begin{array}{l}\text { Red Crested Bare-face } \\
\text { Tamarin, Geoffroy's } \\
\text { Tamarin }\end{array}$ & 2.69 & 486 & extant & 0 & NA & $\mathrm{LC}$ & Frug/ginsect \\
\hline SA & Primates & Cebidae & Saguinus & imperator & $\begin{array}{l}\text { Emperor Tamarin, Black- } \\
\text { chinned Emperor } \\
\text { Tamarin }\end{array}$ & 2.60 & 400 & extant & 0 & NA & $\mathrm{LC}$ & Frug/ginsect \\
\hline SA & Primates & Cebidae & Saguinus & inustus & $\begin{array}{l}\text { Mottled-face Tamarin, } \\
\text { Mottle-face Tamarin } \\
\text { Red-chested Mustached }\end{array}$ & 2.61 & 410 & extant & 0 & NA & LC & Frug/ginsect \\
\hline SA & Primates & Cebidae & Saguinus & labiatus & $\begin{array}{l}\text { Tamarin, Red-bellied } \\
\text { Tamarin }\end{array}$ & 2.76 & 575 & extant & 0 & NA & $\mathrm{LC}$ & Frug/ginsect \\
\hline SA & Primates & Cebidae & Saguinus & leucopus & $\begin{array}{l}\text { Silvery-brown Tamarin, } \\
\text { White-footed Tamarin, } \\
\text { Silvery-brown Bare-face } \\
\text { Tamarin } \\
\text { Golden-handed } \\
\text { Tamarin, Midas }\end{array}$ & 2.64 & 440 & extant & Future & NA & EN & Frug/ginsect \\
\hline SA & Primates & Cebidae & Saguinus & midas & $\begin{array}{l}\text { Tamarin, Red-handed } \\
\text { Tamarin, Yellow- } \\
\text { handed Tamarin }\end{array}$ & 2.73 & 540 & extant & 0 & NA & LC & Frug/ginsect \\
\hline SA & Primates & Cebidae & Saguinus & mystax & $\begin{array}{l}\text { Moustached Tamarin, } \\
\text { Spix's Moustached } \\
\text { Tamarin, Black-chested } \\
\text { Mustached Tamarin } \\
\text { Black Mantle Tamarin }\end{array}$ & 2.79 & 618 & extant & 0 & NA & LC & Frug/ginsect \\
\hline SA & Primates & Cebidae & Saguinus & nigricollis & $\begin{array}{l}\text { Hern^ndez-camacho's } \\
\text { Black Mantle Tamarin }\end{array}$ & 2.66 & 462 & extant & 0 & NA & $\mathrm{LC}$ & Ginsect/frug \\
\hline
\end{tabular}




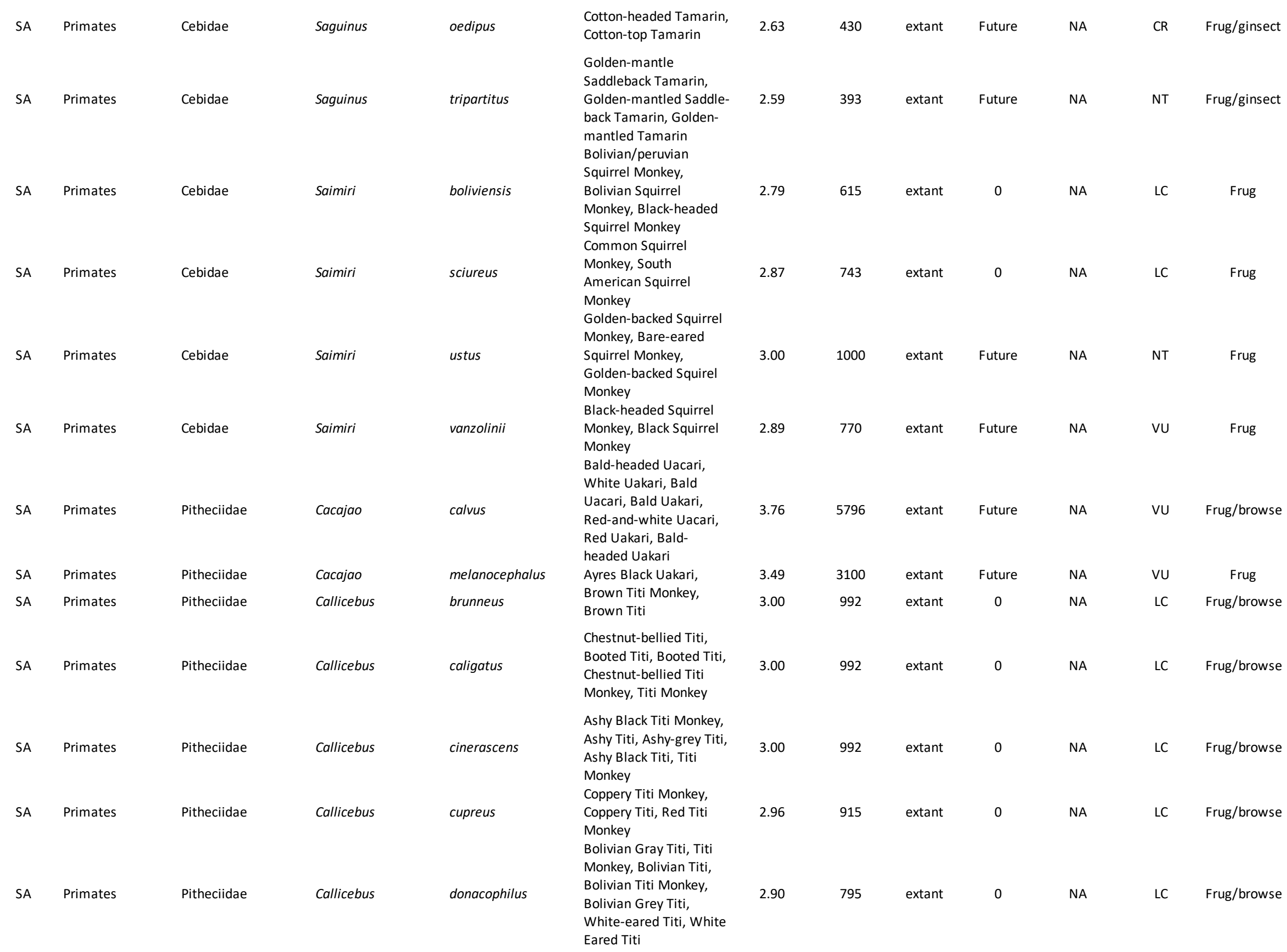




\begin{tabular}{|c|c|c|c|c|c|c|c|c|c|c|c|c|}
\hline SA & Primates & Pitheciidae & Callicebus & dubius & $\begin{array}{l}\text { Hershkovitz's Titi, } \\
\text { Dubius Titi, Doubtful Titi } \\
\text { Monkey } \\
\text { Hoffmann's Titi }\end{array}$ & 3.00 & 992 & extant & 0 & NA & LC & Frug/browse \\
\hline SA & Primates & Pitheciidae & Callicebus & hoffmannsi & $\begin{array}{l}\text { Monkey, Hoffmanns's } \\
\text { Titi }\end{array}$ & 3.00 & 992 & extant & 0 & NA & LC & Frug/browse \\
\hline SA & Primates & Pitheciidae & Callicebus & modestus & $\begin{array}{l}\text { Beni Titi Monkey, Rio } \\
\text { Beni Titi, Modest Titi, } \\
\text { Bolivian Titi, Titi } \\
\text { Monkey } \\
\text { Red-bellied Titi Monkey, }\end{array}$ & 3.00 & 992 & extant & Future & NA & EN & Frug/browse \\
\hline SA & Primates & Pitheciidae & Callicebus & moloch & $\begin{array}{l}\text { Dusky Titi, Red-bellied } \\
\text { Titi, Titi Monkey } \\
\text { San Martin Titi Monkey, }\end{array}$ & 2.93 & 855 & extant & 0 & NA & LC & Browse/frug \\
\hline SA & Primates & Pitheciidae & Callicebus & oenanthe & $\begin{array}{l}\text { Rio Mayo Titi Monkey, } \\
\text { Rio Mayo Titi, Andean } \\
\text { Titi Monkey } \\
\text { Olalla Brothers' Titi, } \\
\text { Olalla's Titi Monkey, }\end{array}$ & 3.00 & 992 & extant & Future & NA & $\mathrm{CR}$ & Frug/browse \\
\hline SA & Primates & Pitheciidae & Callicebus & olallae & $\begin{array}{l}\text { Olalla's Titi, Beni Titi } \\
\text { Monkey, Ollala } \\
\text { Brothers' Titi } \\
\text { Atlantic Titi, Northern }\end{array}$ & 3.00 & 992 & extant & Future & NA & EN & Frug/browse \\
\hline SA & Primates & Pitheciidae & Callicebus & personatus & $\begin{array}{l}\text { Masked Titi, Masked } \\
\text { Titi }\end{array}$ & 3.13 & 1350 & extant & Future & NA & VU & Frug/browse \\
\hline SA & Primates & Pitheciidae & Callicebus & torquatus & $\begin{array}{l}\text { Widow Monkey, } \\
\text { Collared Titi, Yellow } \\
\text { Handed Titi }\end{array}$ & 3.02 & 1050 & extant & 0 & NA & LC & Frug \\
\hline SA & Primates & Pitheciidae & Chiropotes & albinasus & $\begin{array}{l}\text { White-nosed Saki, Red- } \\
\text { nosed Bearded Saki, } \\
\text { White-nosed Bearded } \\
\text { Saki, Red-nosed Saki }\end{array}$ & 3.46 & 2900 & extant & Future & NA & EN & Frug \\
\hline SA & Primates & Pitheciidae & Chiropotes & satanas & $\begin{array}{l}\text { Black Bearded Saki, } \\
\text { Black Saki, Bearded } \\
\text { Saki, Brown-bearded } \\
\text { Saki }\end{array}$ & 3.48 & 3000 & extant & Future & NA & CR & Frug \\
\hline SA & Primates & Pitheciidae & Pithecia & aequatorialis & Equatorial Saki & 3.35 & 2250 & extant & 0 & NA & LC & Frug/browse \\
\hline SA & Primates & Pitheciidae & Pithecia & albicans & Buffy Saki, White Saki & 3.45 & 2800 & extant & Future & NA & VU & Frug/browse \\
\hline SA & Primates & Pitheciidae & Pithecia & irrorata & $\begin{array}{l}\text { Vanzolini's Bald-faced } \\
\text { Saki }\end{array}$ & 3.35 & 2241 & extant & 0 & NA & DD & Frug/browse \\
\hline SA & Primates & Pitheciidae & Pithecia & monachus & Miller's Saki & 3.19 & 1538 & extant & 0 & NA & DD & Frug/browse \\
\hline SA & Primates & Pitheciidae & Pithecia & pithecia & White-faced Saki & 3.14 & 1375 & extant & 0 & NA & LC & Frug/browse \\
\hline SA & Proboscidea & Gomphotheriidae & Cuvieronius & hyodon & & 6.70 & 5000000 & extinct & TP & 11.9 & EX & browse \\
\hline SA & Proboscidea & Gomphotheriidae & Stegomastodon & platensis & & 6.79 & 6193000 & extinct & TP & 11.9 & EX & Browse \\
\hline SA & Proboscidea & Gomphotheriidae & Stegomastodon & superbus & & 6.88 & 7580000 & extinct & TP & 11.9 & EX & Browse \\
\hline SA & Proboscidea & Gomphotheriidae & Stegomastodon & waringi & & 6.78 & 6000000 & extinct & TP & 10 & EX & Browse \\
\hline SA & Rodentia & Abrocomidae & Abrocoma & bennettii & Bennett's Chinchilla Rat & 2.40 & 251 & extant & 0 & NA & LC & Browse/frug \\
\hline SA & Rodentia & Abrocomidae & Abrocoma & boliviensis & Bolivian Chinchilla Rat & 2.20 & 158 & extant & Future & NA & CR & Browse/frug \\
\hline SA & Rodentia & Abrocomidae & Abrocoma & cinerea & Ashy Chinchilla Rat & 2.40 & 250 & extant & 0 & NA & LC & Browse/frug \\
\hline SA & Rodentia & Agoutidae & Cuniculus & paca & Spotted Paca & 3.90 & 8000 & extant & 0 & NA & LC & frug/browse \\
\hline
\end{tabular}




\begin{tabular}{|c|c|c|c|c|c|c|c|c|c|c|c|c|}
\hline SA & Rodentia & Agoutidae & Cuniculus & taczanowskii & Mountain Paca & 3.95 & 9000 & extant & Future & NA & NT & \\
\hline SA & Rodentia & Caviidae & Cavia & aperea & Brazilian Guinea Pig & 2.74 & 549 & extant & 0 & NA & LC & Browse/frug \\
\hline SA & Rodentia & Caviidae & Cavia & fulgida & Shiny Guinea Pig & 2.45 & 283 & extant & 0 & NA & LC & Browse/frug \\
\hline SA & Rodentia & Caviidae & Cavia & magna & Greater Guinea Pig & 2.66 & 460 & extant & 0 & NA & LC & Browse/frug \\
\hline SA & Rodentia & Caviidae & Cavia & porcellus & guinea pig & 2.86 & 728 & extant & 0 & NA & LC & Browse/frug \\
\hline SA & Rodentia & Caviidae & Cavia & tschudii & $\begin{array}{l}\text { Montane Guinea Pig } \\
\text { Patagonian Cavy, }\end{array}$ & 3.00 & 1000 & extant & 0 & NA & LC & Browse/frug \\
\hline SA & Rodentia & Caviidae & Dolichotis & patagonum & $\begin{array}{l}\text { Patagonian Hare, } \\
\text { Patagonian Mara }\end{array}$ & 3.90 & 8000 & extant & Future & NA & NT & Browse \\
\hline SA & Rodentia & Caviidae & Dolichotis & salinicola & $\begin{array}{l}\text { Dwarf Patagonian Cavy, } \\
\text { Dwarf Mara, Dwarf } \\
\text { Patagonian Hare, } \\
\text { Chacoan Mara }\end{array}$ & 3.20 & 1600 & extant & 0 & NA & LC & Browse \\
\hline SA & Rodentia & Caviidae & Galea & flavidens & Yellow-toothed Cavy & 2.65 & 450 & extant & 0 & NA & LC & z̧raze/browse \\
\hline SA & Rodentia & Caviidae & Galea & musteloides & $\begin{array}{l}\text { Common Yellow- } \\
\text { toothed Cavy }\end{array}$ & 2.68 & 480 & extant & 0 & NA & LC & graze/browse \\
\hline SA & Rodentia & Caviidae & Galea & spixii & $\begin{array}{l}\text { Spix's Yellow-toothed } \\
\text { Cavy }\end{array}$ & 2.51 & 326 & extant & 0 & NA & LC & graze/browse \\
\hline SA & Rodentia & Caviidae & Kerodon & rupestris & Rock Cavy & 2.90 & 800 & extant & 0 & NA & LC & Browse \\
\hline SA & Rodentia & Caviidae & Microcavia & australis & Southern Mountain Cavy & 2.46 & 286 & extant & 0 & NA & $\mathrm{LC}$ & Browse/frug \\
\hline SA & Rodentia & Caviidae & Microcavia & niata & Andean Mountain Cavy & 2.41 & 255 & extant & 0 & NA & LC & Browse/frug \\
\hline SA & Rodentia & Caviidae & Microcavia & shiptoni & $\begin{array}{l}\text { Shipton's Mountain } \\
\text { Cavy }\end{array}$ & 2.27 & 185 & extant & Future & NA & NT & Browse/frug \\
\hline SA & Rodentia & Chinchillidae & Chinchilla & chinchilla & Short-tailed Chinchilla & 2.70 & 500 & extant & Future & NA & $C R$ & Browse/frug \\
\hline SA & Rodentia & Chinchillidae & Chinchilla & Ianigera & $\begin{array}{l}\text { Long-tailed Chinchilla, } \\
\text { Chinchilla }\end{array}$ & 2.62 & 417 & extant & Future & NA & $C R$ & Browse/frug \\
\hline SA & Rodentia & Chinchillidae & Lagidium & peruanum & $\begin{array}{l}\text { Southern Mountain } \\
\text { Viscacha } \\
\text { Southern Mountain }\end{array}$ & 3.09 & 1220 & extant & 0 & NA & LC & Browse/frug \\
\hline SA & Rodentia & Chinchillidae & Lagidium & viscacia & $\begin{array}{l}\text { Viscacha, Southern } \\
\text { Viscacha }\end{array}$ & 3.19 & 1540 & extant & 0 & NA & LC & Browse/frug \\
\hline SA & Rodentia & Chinchillidae & Lagidium & wolffsohni & Wolffsohn's Viscacha & 3.43 & 2682 & extant & 0 & NA & DD & Browse/frug \\
\hline SA & Rodentia & Chinchillidae & Lagostomus & maximus & Plains Viscacha & 3.67 & 4648 & extant & 0 & NA & LC & graze/frug \\
\hline SA & Rodentia & Cricetidae & Abrawayaomys & ruschii & Ruschi's Rat & 1.80 & 63 & extant & 0 & NA & LC & frug/browse \\
\hline SA & Rodentia & Cricetidae & Aepeomys & lugens & $\begin{array}{l}\text { Olive Montane Mouse, } \\
\text { M_rida Aepeomys }\end{array}$ & 1.57 & 37 & extant & 0 & NA & LC & Ginsect \\
\hline SA & Rodentia & Cricetidae & Akodon & aerosus & $\begin{array}{l}\text { Highland Grass Mouse, } \\
\text { Yungas Akodont } \\
\text { Colombian Grass }\end{array}$ & 1.78 & 60 & extant & 0 & NA & LC & iect/frug/brol \\
\hline SA & Rodentia & Cricetidae & Akodon & affinis & $\begin{array}{l}\text { Mouse, Cordillera } \\
\text { Occidental Akodont } \\
\text { White-bellied Grass }\end{array}$ & 1.40 & 25 & extant & 0 & NA & LC & ;ect/frug/brol \\
\hline SA & Rodentia & Cricetidae & Akodon & albiventer & $\begin{array}{l}\text { Mouse, White-bellied } \\
\text { Akodont }\end{array}$ & 1.34 & 22 & extant & 0 & NA & LC & iect/frug/brol \\
\hline SA & Rodentia & Cricetidae & Akodon & azarae & $\begin{array}{l}\text { Azara's Grass Mouse, } \\
\text { Azara's Akodont }\end{array}$ & 1.40 & 25 & extant & 0 & NA & LC & ;ect/frug/brol \\
\hline SA & Rodentia & Cricetidae & Akodon & bogotensis & Bogota Grass Mouse & 1.11 & 13 & extant & 0 & NA & LC & ;ect/frug/brol \\
\hline SA & Rodentia & Cricetidae & Akodon & boliviensis & $\begin{array}{l}\text { Bolivian Grass Mouse, } \\
\text { Bolivian Akodont }\end{array}$ & 1.44 & 28 & extant & 0 & NA & LC & ;ect/frug/brol \\
\hline SA & Rodentia & Cricetidae & Akodon & budini & $\begin{array}{l}\text { Budin's Grass Mouse, } \\
\text { Budin's Akodont }\end{array}$ & 1.43 & 27 & extant & 0 & NA & LC & ;ect/frug/brol \\
\hline
\end{tabular}




\begin{tabular}{|c|c|c|c|c|c|c|c|c|c|c|c|c|}
\hline SA & Rodentia & Cricetidae & Akodon & cursor & $\begin{array}{l}\text { Cursor Grass Mouse, } \\
\text { Cusorial Akodont }\end{array}$ & 1.60 & 40 & extant & 0 & NA & LC & ;ect/frug/brol \\
\hline SA & Rodentia & Cricetidae & Akodon & dayi & $\begin{array}{l}\text { Day's Grass Mouse, } \\
\text { Dusky Akodont }\end{array}$ & 1.51 & 33 & extant & 0 & NA & LC & ;ect/frug/brol \\
\hline SA & Rodentia & Cricetidae & Akodon & dolores & $\begin{array}{l}\text { C, rdoba Akodont, } \\
\text { Dolorous Grass Mouse }\end{array}$ & 1.70 & 51 & extant & 0 & NA & LC & ;ect/frug/brol \\
\hline SA & Rodentia & Cricetidae & Akodon & fumeus & $\begin{array}{l}\text { Smoky Grass Mouse, } \\
\text { Smoky Akodont } \\
\text { Intelligent Grass }\end{array}$ & 1.36 & 23 & extant & 0 & NA & LC & ;ect/frug/brol \\
\hline SA & Rodentia & Cricetidae & Akodon & iniscatus & $\begin{array}{l}\text { Mouse, Patagonian } \\
\text { Akodont }\end{array}$ & 1.46 & 29 & extant & 0 & NA & LC & ;ect/frug/brol \\
\hline SA & Rodentia & Cricetidae & Akodon & juninensis & $\begin{array}{l}\text { Junin Grass Mouse, } \\
\text { Junín Akodont }\end{array}$ & 1.59 & 39 & extant & 0 & NA & LC & ;ect/frug/brol \\
\hline SA & Rodentia & Cricetidae & Akodon & kofordi & $\begin{array}{l}\text { Koford's Grass Mouse, } \\
\text { Koford's Akodont }\end{array}$ & 1.47 & 30 & extant & 0 & NA & LC & ;ect/frug/brol \\
\hline SA & Rodentia & Cricetidae & Akodon & latebricola & $\begin{array}{l}\text { Ecuadorean Grass } \\
\text { Mouse } \\
\text { Lindbergh's Grass }\end{array}$ & 1.59 & 39 & extant & Future & NA & VU & ;ect/frug/brol \\
\hline SA & Rodentia & Cricetidae & Akodon & lindberghi & $\begin{array}{l}\text { Mouse, Lindbergh's } \\
\text { Akodont }\end{array}$ & 1.42 & 26 & extant & 0 & NA & DD & ;ect/frug/brol \\
\hline SA & Rodentia & Cricetidae & Akodon & mimus & $\begin{array}{l}\text { Thespian Grass Mouse, } \\
\text { Hocicudo-like Akodont }\end{array}$ & 1.38 & 24 & extant & 0 & NA & LC & ;ect/frug/brol \\
\hline SA & Rodentia & Cricetidae & Akodon & molinae & $\begin{array}{l}\text { Molina's Grass Mouse, } \\
\text { Molina's Akodont }\end{array}$ & 1.52 & 33 & extant & 0 & NA & LC & ;ect/frug/brol \\
\hline SA & Rodentia & Cricetidae & Akodon & mollis & $\begin{array}{l}\text { Soft Grass Mouse, Soft- } \\
\text { furred Akodont }\end{array}$ & 1.48 & 30 & extant & 0 & NA & LC & ;ect/frug/brol \\
\hline SA & Rodentia & Cricetidae & Akodon & neocenus & $\begin{array}{l}\text { Neuqu_n Akodont, } \\
\text { Neuquen Grass Mouse }\end{array}$ & 1.63 & 42 & extant & 0 & NA & DD & ;ect/frug/brol \\
\hline SA & Rodentia & Cricetidae & Akodon & orophilus & $\begin{array}{l}\text { El Dorado Grass Mouse, } \\
\text { Utcubamba Akodont }\end{array}$ & 1.59 & 39 & extant & 0 & NA & LC & ;ect/frug/brol \\
\hline SA & Rodentia & Cricetidae & Akodon & sanctipaulensis & $\begin{array}{l}\text { Sao Paulo Grass Mouse, } \\
\text { Sto Paulo Akodont }\end{array}$ & 1.43 & 27 & extant & 0 & NA & DD & ;ect/frug/brol \\
\hline SA & Rodentia & Cricetidae & Akodon & serrensis & $\begin{array}{l}\text { Serrado Do Mar Grass } \\
\text { Mouse, Serrado Mar } \\
\text { Akodont, Serrado Mar } \\
\text { Grass Mouse } \\
\text { Cochabamba Grass }\end{array}$ & 1.45 & 28 & extant & 0 & NA & LC & ;ect/frug/brol \\
\hline SA & Rodentia & Cricetidae & Akodon & siberiae & $\begin{array}{l}\text { Mouse, Cochabamba } \\
\text { Akodont } \\
\text { Gray-bellied Grass }\end{array}$ & 1.54 & 35 & extant & Future & NA & NT & ;ect/frug/brol \\
\hline SA & Rodentia & Cricetidae & Akodon & simulator & $\begin{array}{l}\text { Mouse, White-throated } \\
\text { Akodont } \\
\text { Spegazzini's Grass }\end{array}$ & 1.63 & 43 & extant & 0 & NA & LC & ;ect/frug/brol \\
\hline SA & Rodentia & Cricetidae & Akodon & spegazzinii & $\begin{array}{l}\text { Mouse, Spegazzini's } \\
\text { Akodont }\end{array}$ & 1.46 & 29 & extant & 0 & NA & LC & ;ect/frug/brol \\
\hline SA & Rodentia & Cricetidae & Akodon & subfuscus & $\begin{array}{l}\text { Puno Grass Mouse, } \\
\text { Puno Akodont }\end{array}$ & 1.48 & 30 & extant & 0 & NA & LC & ;ect/frug/brol \\
\hline SA & Rodentia & Cricetidae & Akodon & surdus & $\begin{array}{l}\text { Silent Grass Mouse, } \\
\text { Slate-bellied Akodont }\end{array}$ & 1.59 & 39 & extant & Future & NA & VU & ;ect/frug/brol \\
\hline
\end{tabular}




\begin{tabular}{|c|c|c|c|c|c|c|c|c|c|c|c|c|}
\hline SA & Rodentia & Cricetidae & Akodon & sylvanus & $\begin{array}{l}\text { Forest Grass Mouse, } \\
\text { Woodland Akodont }\end{array}$ & 1.59 & 39 & extant & 0 & NA & $\mathrm{LC}$ & ect/frug/brol \\
\hline SA & Rodentia & Cricetidae & Akodon & toba & $\begin{array}{l}\text { Chaco Grass Mouse, } \\
\text { Toba Akodont } \\
\text { Cloud Forest Grass }\end{array}$ & 1.71 & 51 & extant & 0 & NA & $\mathrm{LC}$ & ;ect/frug/brol \\
\hline SA & Rodentia & Cricetidae & Akodon & torques & $\begin{array}{l}\text { Mouse, Cloud Forest } \\
\text { Akodont }\end{array}$ & 1.59 & 39 & extant & 0 & NA & $\mathrm{LC}$ & ect/frug/brol \\
\hline SA & Rodentia & Cricetidae & Akodon & varius & $\begin{array}{l}\text { Variable Grass Mouse, } \\
\text { Variable Akodont }\end{array}$ & 1.60 & 40 & extant & 0 & NA & DD & ect/frug/brol \\
\hline SA & Rodentia & Cricetidae & Andalgalomys & olrogi & $\begin{array}{l}\text { Olrog's Pericote, Olrog's } \\
\text { Chaco Mouse }\end{array}$ & 1.52 & 33 & extant & 0 & NA & $\mathrm{LC}$ & Frug \\
\hline SA & Rodentia & Cricetidae & Andalgalomys & pearsoni & $\begin{array}{l}\text { Pearson's Chaco Mouse, } \\
\text { Pearson's Pericote }\end{array}$ & 1.40 & 25 & extant & 0 & NA & LC & Frug \\
\hline SA & Rodentia & Cricetidae & Andinomys & edax & Andean Mouse & 1.84 & 70 & extant & 0 & NA & $\mathrm{LC}$ & Browse \\
\hline SA & Rodentia & Cricetidae & Anotomys & leander & $\begin{array}{l}\text { Ecuadoran } \\
\text { Ichthyomyine, Ecuador } \\
\text { Fish-eating Rat, Aquatic } \\
\text { Rat, Fish-eating Rat }\end{array}$ & 1.82 & 66 & extant & Future & NA & Vu & ivert/piscivor \\
\hline SA & Rodentia & Cricetidae & Auliscomys & boliviensis & $\begin{array}{l}\text { Bolivian Big-eared } \\
\text { Mouse, Bolivian } \\
\text { Pericote }\end{array}$ & 1.82 & 66 & extant & 0 & NA & LC & Browse \\
\hline SA & Rodentia & Cricetidae & Auliscomys & pictus & $\begin{array}{l}\text { Painted Big-eared } \\
\text { Mouse, Colorful } \\
\text { Pericote }\end{array}$ & 1.74 & 55 & extant & 0 & NA & $\mathrm{LC}$ & Browse \\
\hline SA & Rodentia & Cricetidae & Auliscomys & sublimis & $\begin{array}{l}\text { Andean Big-eared } \\
\text { Mouse, Lofty Pericote }\end{array}$ & 1.58 & 38 & extant & 0 & NA & $\mathrm{LC}$ & Browse \\
\hline SA & Rodentia & Cricetidae & Bibimys & chacoensis & $\begin{array}{l}\text { Chaco Crimson-nosed } \\
\text { Rat, Chacoan Akodont } \\
\text { Large-lipped Crimson- }\end{array}$ & 1.45 & 28 & extant & 0 & NA & $\mathrm{LC}$ & graze \\
\hline SA & Rodentia & Cricetidae & Bibimys & labiosus & $\begin{array}{l}\text { Large-lipped Crimson- } \\
\text { nosed Rat, Lagoa Santa } \\
\text { Akodont }\end{array}$ & 1.46 & 29 & extant & 0 & NA & $\mathrm{LC}$ & graze \\
\hline SA & Rodentia & Cricetidae & Bibimys & torresi & $\begin{array}{l}\text { Torres's Crimson-nosed } \\
\text { Rat, Torres's Akodont }\end{array}$ & 1.45 & 28 & extant & Future & NA & NT & graze \\
\hline SA & Rodentia & Cricetidae & Blarinomys & breviceps & $\begin{array}{l}\text { Brazilian Shrew Mouse, } \\
\text { Brazilian Shrew-mouse, } \\
\text { Blarinine Akodont }\end{array}$ & 1.57 & 37 & extant & 0 & NA & $\mathrm{LC}$ & Ginsect \\
\hline SA & Rodentia & Cricetidae & Calomys & boliviae & $\begin{array}{l}\text { Bolivian Vesper Mouse, } \\
\text { Bolivian Laucha }\end{array}$ & 1.43 & 27 & extant & 0 & NA & LC & Browse \\
\hline SA & Rodentia & Cricetidae & Calomys & callidus & $\begin{array}{l}\text { Crafty Vesper Mouse, } \\
\text { Reclusive Laucha }\end{array}$ & 1.43 & 27 & extant & 0 & NA & LC & Browse \\
\hline SA & Rodentia & Cricetidae & Calomys & callosus & $\begin{array}{l}\text { Large Vesper Mouse, } \\
\text { Big Laucha } \\
\text { Hummelinck's Vesper }\end{array}$ & 1.65 & 45 & extant & 0 & NA & $\mathrm{LC}$ & Browse \\
\hline SA & Rodentia & Cricetidae & Calomys & hummelincki & $\begin{array}{l}\text { Mouse, Hummelinck's } \\
\text { Laucha }\end{array}$ & 1.43 & 27 & extant & 0 & NA & LC & Browse \\
\hline SA & Rodentia & Cricetidae & Calomys & laucha & $\begin{array}{l}\text { Small Vesper Mouse, } \\
\text { Little Laucha }\end{array}$ & 1.15 & 14 & extant & 0 & NA & LC & Browse \\
\hline SA & Rodentia & Cricetidae & Calomys & lepidus & $\begin{array}{l}\text { Andean Vesper Mouse, } \\
\text { Graceful Laucha }\end{array}$ & 1.42 & 27 & extant & 0 & NA & $\mathrm{LC}$ & Browse \\
\hline
\end{tabular}




\begin{tabular}{|c|c|c|c|c|c|c|c|c|c|c|c|c|}
\hline SA & Rodentia & Cricetidae & Calomys & musculinus & $\begin{array}{l}\text { Drylands Vesper Mouse, } \\
\text { Drylands Laucha }\end{array}$ & 1.30 & 20 & extant & 0 & NA & LC & Browse \\
\hline SA & Rodentia & Cricetidae & Calomys & sorellus & $\begin{array}{l}\text { Peruvian Vesper Mouse, } \\
\text { Peruvian Laucha }\end{array}$ & 1.30 & 20 & extant & 0 & NA & LC & Browse \\
\hline SA & Rodentia & Cricetidae & Calomys & tener & $\begin{array}{l}\text { Delicate Vesper Mouse, } \\
\text { Delicate Laucha }\end{array}$ & 1.14 & 14 & extant & 0 & NA & $\mathrm{LC}$ & Browse \\
\hline SA & Rodentia & Cricetidae & Chelemys & macronyx & $\begin{array}{l}\text { Andean Long-clawed } \\
\text { Mouse, Andean Long- } \\
\text { clawed Akodont }\end{array}$ & 1.87 & 73 & extant & 0 & NA & $\mathrm{LC}$ & insect/brows! \\
\hline SA & Rodentia & Cricetidae & Chelemys & megalonyx & $\begin{array}{l}\text { Large Long-clawed } \\
\text { Mouse, Large Long- } \\
\text { clawed Akodont }\end{array}$ & 1.71 & 51 & extant & Future & NA & NT & insect/brows \\
\hline SA & Rodentia & Cricetidae & Chibchanomys & trichotis & $\begin{array}{l}\text { Chibchan Water Mouse, } \\
\text { Chibchan Ichthyomyine }\end{array}$ & 1.70 & 50 & extant & 0 & NA & DD & Ginsect \\
\hline SA & Rodentia & Cricetidae & Chilomys & instans & $\begin{array}{l}\text { Colombian Forest } \\
\text { Mouse, Andean } \\
\text { Chilomys }\end{array}$ & 1.28 & 19 & extant & 0 & NA & LC & ;ect/frug/brol \\
\hline SA & Rodentia & Cricetidae & Chinchillula & sahamae & $\begin{array}{l}\text { Altiplano Chincilla } \\
\text { Mouse, Achallo }\end{array}$ & 2.23 & 170 & extant & 0 & NA & LC & Browse \\
\hline SA & Rodentia & Cricetidae & Delomys & dorsalis & $\begin{array}{l}\text { Striped Atlantic Forest } \\
\text { Rat }\end{array}$ & 1.83 & 67 & extant & 0 & NA & LC & frug/browse \\
\hline SA & Rodentia & Cricetidae & Delomys & sublineatus & $\begin{array}{l}\text { Pallid Atlantic Forest } \\
\text { Rat }\end{array}$ & 1.95 & 90 & extant & 0 & NA & LC & frug/browse \\
\hline SA & Rodentia & Cricetidae & Eligmodontia & moreni & Monte Gerbil Mouse & 1.26 & 18 & extant & 0 & NA & LC & Frug \\
\hline SA & Rodentia & Cricetidae & Eligmodontia & morgani & Morgan's Gerbil Mouse & 1.22 & 17 & extant & 0 & NA & LC & Frug \\
\hline SA & Rodentia & Cricetidae & Eligmodontia & puerulus & $\begin{array}{l}\text { Andean Gerbil Mouse } \\
\text { Eastern Patagonian }\end{array}$ & 1.45 & 29 & extant & 0 & NA & LC & Frug \\
\hline SA & Rodentia & Cricetidae & Eligmodontia & typus & $\begin{array}{l}\text { Laucha, Highland Gerbil } \\
\text { Mouse }\end{array}$ & 1.24 & 17 & extant & 0 & NA & LC & Frug \\
\hline SA & Rodentia & Cricetidae & Euneomys & chinchilloides & $\begin{array}{l}\text { Patagonian Chincilla } \\
\text { Mouse }\end{array}$ & 1.94 & 88 & extant & 0 & NA & DD & Browse \\
\hline SA & Rodentia & Cricetidae & Euneomys & fossor & Burrowing Euneomys & 1.92 & 83 & extant & 0 & NA & DD & Browse \\
\hline SA & Rodentia & Cricetidae & Euneomys & mordax & Biting Chincilla Mouse & 1.91 & 82 & extant & 0 & NA & LC & Browse \\
\hline SA & Rodentia & Cricetidae & Euneomys & petersoni & $\begin{array}{l}\text { Peterson's Chincilla } \\
\text { Mouse }\end{array}$ & 1.92 & 83 & extant & 0 & NA & $\mathrm{LC}$ & Browse \\
\hline SA & Rodentia & Cricetidae & Galenomys & garleppi & Gerlepp's Mouse & 1.77 & 59 & extant & 0 & NA & DD & Browse/frug \\
\hline SA & Rodentia & Cricetidae & Geoxus & valdivianus & $\begin{array}{l}\text { Long-clawed Mole } \\
\text { Mouse }\end{array}$ & 1.50 & 32 & extant & 0 & NA & LC & Ginsect \\
\hline SA & Rodentia & Cricetidae & Graomys & domorum & Pale Leaf-eared Mouse & 2.01 & 102 & extant & 0 & NA & $\mathrm{LC}$ & graze/frug \\
\hline SA & Rodentia & Cricetidae & Graomys & edithae & $\begin{array}{l}\text { Edith's Leaf-eared } \\
\text { Mouse }\end{array}$ & 1.61 & 40 & extant & 0 & NA & DD & graze/frug \\
\hline SA & Rodentia & Cricetidae & Graomys & griseoflavus & Gray Leaf-eared Mouse & 1.83 & 67 & extant & 0 & NA & LC & graze/frug \\
\hline SA & Rodentia & Cricetidae & Holochilus & brasiliensis & Web-footed Marsh Rat & 2.19 & 155 & extant & 0 & NA & LC & zraze/browse \\
\hline SA & Rodentia & Cricetidae & Holochilus & chacarius & Chaco Marsh Rat & 2.31 & 204 & extant & 0 & NA & LC & graze/browse \\
\hline SA & Rodentia & Cricetidae & Holochilus & sciureus & Marsh Rat & 2.23 & 171 & extant & 0 & NA & LC & కraze/browse \\
\hline SA & Rodentia & Cricetidae & Ichthyomys & hydrobates & Crab-eating Rat & 1.82 & 66 & extant & Future & NA & NT & ivert/piscivor \\
\hline SA & Rodentia & Cricetidae & Ichthyomys & pittieri & Pittier's Crab-eating Rat & 1.84 & 69 & extant & Future & NA & Vu & ivert/piscivor \\
\hline
\end{tabular}




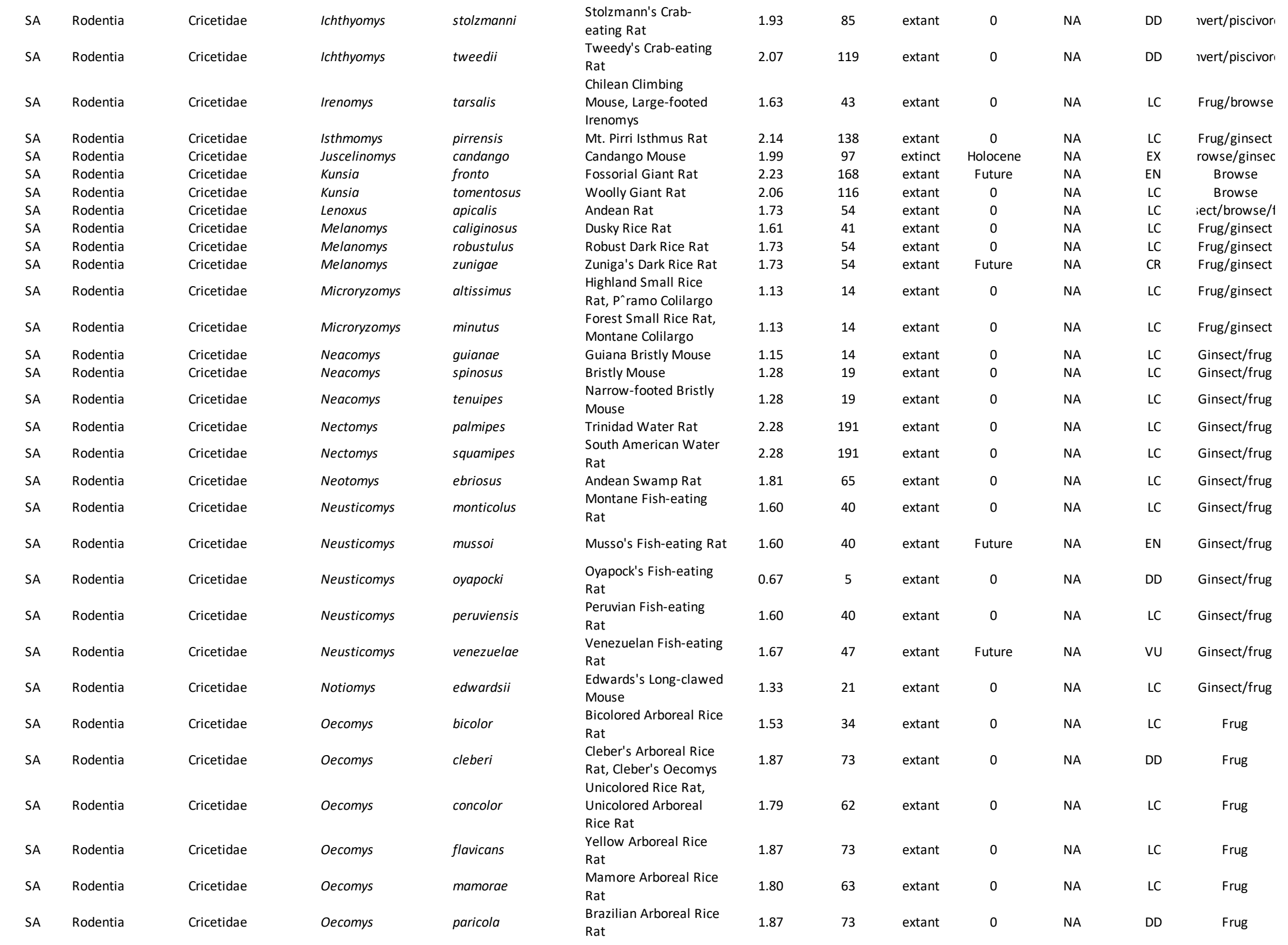




\begin{tabular}{|c|c|c|c|c|c|c|c|c|c|c|c|c|}
\hline SA & Rodentia & Cricetidae & Oecomys & phaeotis & Dusky Arboreal Rice Rat & 1.87 & 73 & extant & 0 & NA & LC & Frug \\
\hline SA & Rodentia & Cricetidae & Oecomys & rex & King Arboreal Rice Rat & 1.87 & 73 & extant & 0 & NA & $\mathrm{LC}$ & Frug \\
\hline SA & Rodentia & Cricetidae & Oecomys & roberti & $\begin{array}{l}\text { Robert's Arboreal Rice } \\
\text { Rat }\end{array}$ & 1.87 & 73 & extant & 0 & NA & LC & Frug \\
\hline SA & Rodentia & Cricetidae & Oecomys & rutilus & Red Arboreal Rice Rat & 1.87 & 73 & extant & 0 & NA & LC & Frug \\
\hline SA & Rodentia & Cricetidae & Oecomys & speciosus & Arboreal Rice Rat & 1.87 & 73 & extant & 0 & NA & LC & Frug \\
\hline SA & Rodentia & Cricetidae & Oecomys & superans & $\begin{array}{l}\text { Foothill Arboreal Rice } \\
\text { Rat }\end{array}$ & 1.87 & 73 & extant & 0 & NA & LC & Frug \\
\hline SA & Rodentia & Cricetidae & Oecomys & trinitatis & $\begin{array}{l}\text { Long-furred Rice Rat, } \\
\text { Trinidad Arboreal Rice } \\
\text { Rat }\end{array}$ & 1.87 & 73 & extant & 0 & NA & LC & Frug \\
\hline SA & Rodentia & Cricetidae & Oligoryzomys & andinus & Andean Pygmy Rice Rat & 1.40 & 25 & extant & 0 & NA & LC & Frug/ginsect \\
\hline SA & Rodentia & Cricetidae & Oligoryzomys & arenalis & Sandy Pygmy Rice Rat & 1.40 & 25 & extant & 0 & NA & LC & Frug/ginsect \\
\hline SA & Rodentia & Cricetidae & Oligoryzomys & chacoensis & $\begin{array}{l}\text { Chacoan Pygmy Rice } \\
\text { Rat }\end{array}$ & 1.36 & 23 & extant & 0 & NA & LC & Frug/ginsect \\
\hline SA & Rodentia & Cricetidae & Oligoryzomys & delticola & Delta Pygmy Rice Rat & 1.47 & 29 & extant & 0 & NA & LC & Frug/ginsect \\
\hline SA & Rodentia & Cricetidae & Oligoryzomys & destructor & $\begin{array}{l}\text { Destructive Pygmy Rice } \\
\text { Rat }\end{array}$ & 1.40 & 25 & extant & 0 & NA & LC & Frug/ginsect \\
\hline SA & Rodentia & Cricetidae & Oligoryzomys & eliurus & $\begin{array}{l}\text { Brazilian Pygmy Rice } \\
\text { Rat }\end{array}$ & 1.48 & 30 & extant & 0 & NA & LC & Frug/ginsect \\
\hline SA & Rodentia & Cricetidae & Oligoryzomys & flavescens & Yellow Pygmy Rice Rat & 1.33 & 21 & extant & 0 & NA & LC & Frug/ginsect \\
\hline SA & Rodentia & Cricetidae & Oligoryzomys & fulvescens & Fulvous Pygmy Rice Rat & 1.40 & 25 & extant & 0 & NA & $\mathrm{LC}$ & Frug/ginsect \\
\hline SA & Rodentia & Cricetidae & Oligoryzomys & griseolus & Grayish Pygmy Rice Rat & 1.40 & 25 & extant & 0 & NA & LC & Frug/ginsect \\
\hline SA & Rodentia & Cricetidae & Oligoryzomys & longicaudatus & $\begin{array}{l}\text { Long-tailed Pygmy Rice } \\
\text { Rat }\end{array}$ & 1.43 & 27 & extant & 0 & NA & LC & Frug/ginsect \\
\hline SA & Rodentia & Cricetidae & Oligoryzomys & magellanicus & $\begin{array}{l}\text { Magellanic Pygmy Rice } \\
\text { Rat }\end{array}$ & 1.40 & 25 & extant & 0 & NA & $\mathrm{LC}$ & Frug/ginsect \\
\hline SA & Rodentia & Cricetidae & Oligoryzomys & microtis & $\begin{array}{l}\text { Small-eared Pygmy Rice } \\
\text { Rat } \\
\text { Black-footed Pygmy }\end{array}$ & 1.35 & 23 & extant & 0 & NA & $\mathrm{LC}$ & Frug/ginsect \\
\hline SA & Rodentia & Cricetidae & Oligoryzomys & nigripes & $\begin{array}{l}\text { Rice Rat, Delta Pygmy } \\
\text { Rice Rat }\end{array}$ & 1.31 & 21 & extant & 0 & NA & LC & Frug/ginsect \\
\hline SA & Rodentia & Cricetidae & Oryzomys & albigularis & Tomes's Rice Rat & 1.78 & 61 & extant & 0 & NA & LC & Frug/ginsect \\
\hline SA & Rodentia & Cricetidae & Oryzomys & alfaroi & & 1.52 & 33 & extant & 0 & NA & LC & Frug/ginsect \\
\hline SA & Rodentia & Cricetidae & Oryzomys & auriventer & & 1.78 & 61 & extant & 0 & NA & LC & Frug/ginsect \\
\hline SA & Rodentia & Cricetidae & Oryzomys & balneator & Peruvian Rice Rat & 1.78 & 61 & extant & 0 & NA & DD & Frug/ginsect \\
\hline SA & Rodentia & Cricetidae & Oryzomys & bolivaris & Bolivar Rice Rat & 1.78 & 61 & extant & 0 & NA & LC & Frug/ginsect \\
\hline SA & Rodentia & Cricetidae & Oryzomys & couesi & Coues's Rice Rat & 1.84 & 69 & extant & 0 & NA & LC & Frug/ginsect \\
\hline SA & Rodentia & Cricetidae & Oryzomys & gorgasi & Gorgas's Rice Rat & 1.78 & 61 & extant & Future & NA & EN & Frug/ginsect \\
\hline SA & Rodentia & Cricetidae & Oryzomys & hammondi & Hammond's Rice Rat & 1.78 & 61 & extant & Future & NA & EN & Frug/ginsect \\
\hline SA & Rodentia & Cricetidae & Oryzomys & keaysi & Keays's Rice Rat & 1.77 & 58 & extant & 0 & NA & LC & Frug/ginsect \\
\hline SA & Rodentia & Cricetidae & Oryzomys & Iamia & Monster Rice Rat & 1.78 & 61 & extant & Future & NA & EN & Frug/ginsect \\
\hline SA & Rodentia & Cricetidae & Oryzomys & legatus & & 1.79 & 62 & extant & 0 & NA & LC & Frug/ginsect \\
\hline SA & Rodentia & Cricetidae & Oryzomys & levipes & & 1.78 & 61 & extant & 0 & NA & LC & Frug/ginsect \\
\hline SA & Rodentia & Cricetidae & Oryzomys & macconnelli & & 1.76 & 58 & extant & 0 & NA & LC & Frug/ginsect \\
\hline SA & Rodentia & Cricetidae & Oryzomys & nitidus & & 1.74 & 55 & extant & 0 & NA & LC & Frug/ginsect \\
\hline SA & Rodentia & Cricetidae & Oryzomys & polius & Gray Rice Rat & 1.78 & 61 & extant & 0 & NA & DD & Frug/ginsect \\
\hline SA & Rodentia & Cricetidae & Oryzomys & subflavus & Terraced Rice Rat & 1.70 & 50 & extant & 0 & NA & LC & Frug/ginsect \\
\hline SA & Rodentia & Cricetidae & Oryzomys & talamancae & & 1.74 & 55 & extant & 0 & NA & LC & Frug/ginsect \\
\hline SA & Rodentia & Cricetidae & Oryzomys & yunganus & & 1.78 & 61 & extant & 0 & NA & LC & Frug/ginsect \\
\hline SA & Rodentia & Cricetidae & Oxymycterus & akodontius & Argentine Hocicudo & 1.83 & 68 & extant & 0 & NA & $\mathrm{DD}$ & Ginsect \\
\hline
\end{tabular}




\begin{tabular}{|c|c|c|c|c|c|c|c|c|c|c|c|c|}
\hline SA & Rodentia & Cricetidae & Oxymycterus & angularis & Angular Hocicudo & 1.83 & 68 & extant & 0 & NA & LC & Ginsect \\
\hline SA & Rodentia & Cricetidae & Oxymycterus & delator & Spy Hocicudo & 1.91 & 82 & extant & 0 & NA & LC & Ginsect \\
\hline SA & Rodentia & Cricetidae & Oxymycterus & hiska & Small Hocicudo & 1.83 & 68 & extant & 0 & NA & LC & Ginsect \\
\hline SA & Rodentia & Cricetidae & Oxymycterus & hispidus & Hispid Hocicudo & 1.57 & 37 & extant & 0 & NA & LC & Ginsect \\
\hline SA & Rodentia & Cricetidae & Oxymycterus & hucucha & Quechuan Hocicudo & 1.83 & 68 & extant & Future & NA & EN & Ginsect \\
\hline SA & Rodentia & Cricetidae & Oxymycterus & inca & Incan Hocicudo & 1.54 & 35 & extant & 0 & NA & LC & Ginsect \\
\hline SA & Rodentia & Cricetidae & Oxymycterus & nasutus & Long-nosed Hocicudo & 1.83 & 68 & extant & 0 & NA & LC & Ginsect \\
\hline SA & Rodentia & Cricetidae & Oxymycterus & paramensis & Paramo Hocicudo & 1.62 & 42 & extant & 0 & NA & LC & Ginsect \\
\hline SA & Rodentia & Cricetidae & Oxymycterus & roberti & Robert's Hocicudo & 1.92 & 83 & extant & 0 & NA & LC & Ginsect \\
\hline SA & Rodentia & Cricetidae & Oxymycterus & rufus & Red Hocicudo & 1.88 & 75 & extant & 0 & NA & LC & Ginsect \\
\hline SA & Rodentia & Cricetidae & Phaenomys & ferrugineus & $\begin{array}{l}\text { Rio De Janeiro Arboreal } \\
\text { Rat }\end{array}$ & 1.97 & 94 & extant & Future & NA & Vu & iect/frug/brol \\
\hline SA & Rodentia & Cricetidae & Phyllotis & amicus & $\begin{array}{l}\text { Friendly Leaf-eared } \\
\text { Mouse }\end{array}$ & 1.31 & 20 & extant & 0 & NA & LC & Frug/browse \\
\hline SA & Rodentia & Cricetidae & Phyllotis & andium & $\begin{array}{l}\text { Andean Leaf-eared } \\
\text { Mouse }\end{array}$ & 1.72 & 53 & extant & 0 & NA & LC & Frug/browse \\
\hline SA & Rodentia & Cricetidae & Phyllotis & caprinus & $\begin{array}{l}\text { Capricorn Leaf-eared } \\
\text { Mouse }\end{array}$ & 1.71 & 51 & extant & 0 & NA & LC & Frug/browse \\
\hline SA & Rodentia & Cricetidae & Phyllotis & darwini & $\begin{array}{l}\text { Darwin's Leaf-eared } \\
\text { Mouse }\end{array}$ & 1.71 & 51 & extant & 0 & NA & LC & Frug/browse \\
\hline SA & Rodentia & Cricetidae & Phyllotis & definitus & $\begin{array}{l}\text { Definitive Leaf-eared } \\
\text { Mouse }\end{array}$ & 1.95 & 89 & extant & Future & NA & EN & Frug/browse \\
\hline SA & Rodentia & Cricetidae & Phyllotis & haggardi & $\begin{array}{l}\text { Haggard's Leaf-eared } \\
\text { Mouse }\end{array}$ & 1.63 & 43 & extant & 0 & NA & LC & Frug/browse \\
\hline SA & Rodentia & Cricetidae & Phyllotis & magister & $\begin{array}{l}\text { Master Leaf-eared } \\
\text { Mouse }\end{array}$ & 1.84 & 69 & extant & 0 & NA & LC & Frug/browse \\
\hline SA & Rodentia & Cricetidae & Phyllotis & osgoodi & $\begin{array}{l}\text { Osgood's Leaf-eared } \\
\text { Mouse }\end{array}$ & 1.65 & 45 & extant & 0 & NA & DD & Frug/browse \\
\hline SA & Rodentia & Cricetidae & Phyllotis & osilae & $\begin{array}{l}\text { Bunchgrass Leaf-eared } \\
\text { Mouse }\end{array}$ & 1.69 & 49 & extant & 0 & NA & LC & Frug/browse \\
\hline SA & Rodentia & Cricetidae & Phyllotis & wolffsohni & $\begin{array}{l}\text { Wolffsohn's Leaf-eared } \\
\text { Mouse }\end{array}$ & 1.63 & 43 & extant & 0 & NA & LC & Frug/browse \\
\hline SA & Rodentia & Cricetidae & Phyllotis & xanthopygus & $\begin{array}{l}\text { Yellow-rumped Leaf- } \\
\text { eared Mouse }\end{array}$ & 1.75 & 56 & extant & 0 & NA & LC & Frug/browse \\
\hline SA & Rodentia & Cricetidae & Podoxymys & roraimae & Roraima Mouse & 1.53 & 34 & extant & Future & NA & VU & iect/frug/brol \\
\hline SA & Rodentia & Cricetidae & Pseudoryzomys & simplex & Brazilian False Rice Rat & 1.71 & 51 & extant & 0 & NA & LC & Frug/ginsect \\
\hline SA & Rodentia & Cricetidae & Punomys & lemminus & Puna Mouse & 1.93 & 85 & extant & Future & NA & VU & Browse \\
\hline SA & Rodentia & Cricetidae & Reithrodon & auritus & Bunny Rat & 1.85 & 71 & extant & 0 & NA & LC & graze \\
\hline SA & Rodentia & Cricetidae & Reithrodontomys & darienensis & Darien Harvest Mouse & 1.11 & 13 & extant & 0 & NA & LC & Frug \\
\hline SA & Rodentia & Cricetidae & Reithrodontomys & mexicanus & Mexican Harvest Mouse & 1.11 & 13 & extant & 0 & NA & LC & Frug \\
\hline SA & Rodentia & Cricetidae & Rhagomys & rufescens & $\begin{array}{l}\text { Brazilian Arboreal } \\
\text { Mouse }\end{array}$ & 1.33 & 21 & extant & Future & NA & NT & iect/frug/brol \\
\hline SA & Rodentia & Cricetidae & Rheomys & raptor & $\begin{array}{l}\text { Goldman's Water } \\
\text { Mouse }\end{array}$ & 1.58 & 38 & extant & 0 & NA & LC & invert \\
\hline SA & Rodentia & Cricetidae & Rhipidomys & austrinus & $\begin{array}{l}\text { Southern Climbing } \\
\text { Mouse }\end{array}$ & 1.95 & 89 & extant & 0 & NA & LC & Frug/ginsect \\
\hline SA & Rodentia & Cricetidae & Rhipidomys & caucensis & Cauca Climbing Mouse & 1.95 & 89 & extant & 0 & NA & DD & Frug/ginsect \\
\hline SA & Rodentia & Cricetidae & Rhipidomys & conesi & Coues's Climbing Mouse & 1.95 & 89 & extant & 0 & NA & LC & Frug/ginsect \\
\hline SA & Rodentia & Cricetidae & Rhipidomys & fulviventer & $\begin{array}{l}\text { Buff-bellied Climbing } \\
\text { Mouse }\end{array}$ & 1.95 & 89 & extant & 0 & NA & $\mathrm{LC}$ & Frug/ginsect \\
\hline
\end{tabular}




\begin{tabular}{|c|c|c|c|c|c|c|c|c|c|c|c|c|}
\hline SA & Rodentia & Cricetidae & Rhipidomys & latimanus & $\begin{array}{l}\text { Broad-footed Climbing } \\
\text { Mouse }\end{array}$ & 1.76 & 58 & extant & 0 & NA & LC & Frug/ginsect \\
\hline SA & Rodentia & Cricetidae & Rhipidomys & leucodactylus & $\begin{array}{l}\text { White-footed Climbing } \\
\text { Mouse }\end{array}$ & 1.90 & 80 & extant & 0 & NA & LC & Frug/ginsect \\
\hline SA & Rodentia & Cricetidae & Rhipidomys & macconnelli & $\begin{array}{l}\text { Macconnell's Climbing } \\
\text { Mouse }\end{array}$ & 1.62 & 42 & extant & 0 & NA & LC & Frug/ginsect \\
\hline SA & Rodentia & Cricetidae & Rhipidomys & mastacalis & $\begin{array}{l}\text { Long-tailed Climbing } \\
\text { Mouse }\end{array}$ & 1.89 & 78 & extant & 0 & NA & LC & Frug/ginsect \\
\hline SA & Rodentia & Cricetidae & Rhipidomys & nitela & $\begin{array}{l}\text { Splendid Climbing } \\
\text { Mouse }\end{array}$ & 1.95 & 89 & extant & 0 & NA & $\mathrm{LC}$ & Frug/ginsect \\
\hline SA & Rodentia & Cricetidae & Rhipidomys & ochrogaster & $\begin{array}{l}\text { Yellow-bellied Climbing } \\
\text { Mouse }\end{array}$ & 1.95 & 89 & extant & 0 & NA & DD & Frug/ginsect \\
\hline SA & Rodentia & Cricetidae & Rhipidomys & venezuelae & $\begin{array}{l}\text { Venezuelan Climbing } \\
\text { Mouse }\end{array}$ & 1.95 & 90 & extant & 0 & NA & LC & Frug/ginsect \\
\hline SA & Rodentia & Cricetidae & Rhipidomys & venustus & $\begin{array}{l}\text { Charming Climbing } \\
\text { Mouse }\end{array}$ & 1.95 & 89 & extant & 0 & NA & LC & Frug/ginsect \\
\hline SA & Rodentia & Cricetidae & Rhipidomys & wetzeli & $\begin{array}{l}\text { Wetzel's Climbing } \\
\text { Mouse }\end{array}$ & 1.95 & 89 & extant & 0 & NA & LC & Frug/ginsect \\
\hline SA & Rodentia & Cricetidae & Scapteromys & tumidus & Swamp Rat & 2.16 & 146 & extant & 0 & NA & LC & Ginsect/frug \\
\hline SA & Rodentia & Cricetidae & Scolomys & melanops & $\begin{array}{l}\text { South American Spiny } \\
\text { Mouse }\end{array}$ & 1.42 & 27 & extant & 0 & NA & LC & Ginsect/frug \\
\hline SA & Rodentia & Cricetidae & Scolomys & ucayalensis & Ucayali Spiny Mouse & 1.42 & 27 & extant & 0 & NA & LC & Ginsect/frug \\
\hline SA & Rodentia & Cricetidae & Sigmodon & alstoni & Alston's Cotton Rat & 1.75 & 56 & extant & 0 & NA & LC & frug/carn \\
\hline SA & Rodentia & Cricetidae & Sigmodon & hispidus & Hispid Cotton Rat & 1.97 & 92 & extant & 0 & NA & LC & frug/carn \\
\hline SA & Rodentia & Cricetidae & Sigmodon & inopinatus & Unexpected Cotton Rat & 2.15 & 141 & extant & Future & NA & Vu & frug/carn \\
\hline SA & Rodentia & Cricetidae & Sigmodon & peruanus & $\begin{array}{l}\text { Peruvian Cotton Rat } \\
\text { Alfaro's Rice Water Rat, }\end{array}$ & 2.13 & 135 & extant & 0 & NA & LC & frug/carn \\
\hline SA & Rodentia & Cricetidae & Sigmodontomys & alfari & $\begin{array}{l}\text { Short-tailed } \\
\text { Sigmodontomys }\end{array}$ & 1.70 & 50 & extant & 0 & NA & LC & Ginsect/frug \\
\hline SA & Rodentia & Cricetidae & Thalpomys & cerradensis & Cerrado Mouse & 1.38 & 24 & extant & 0 & NA & LC & Ginsect \\
\hline SA & Rodentia & Cricetidae & Thalpomys & lasiotis & $\begin{array}{l}\text { Hairy-eared Cerrado } \\
\text { Mouse }\end{array}$ & 1.38 & 24 & extant & 0 & NA & LC & Ginsect \\
\hline SA & Rodentia & Cricetidae & Thomasomys & aureus & Golden Oldfield Mouse & 1.94 & 88 & extant & 0 & NA & LC & Browse \\
\hline SA & Rodentia & Cricetidae & Thomasomys & baeops & Beady-eyed Mouse & 1.89 & 77 & extant & 0 & NA & LC & Browse \\
\hline SA & Rodentia & Cricetidae & Thomasomys & bombycinus & Silky Oldfield Mouse & 2.06 & 115 & extant & 0 & NA & DD & Browse \\
\hline SA & Rodentia & Cricetidae & Thomasomys & cinereiventer & $\begin{array}{l}\text { Ashy-bellied Oldfield } \\
\text { Mouse }\end{array}$ & 1.89 & 77 & extant & 0 & NA & LC & Browse \\
\hline SA & Rodentia & Cricetidae & Thomasomys & cinereus & $\begin{array}{l}\text { Ash-colored Oldfield } \\
\text { Mouse }\end{array}$ & 1.89 & 77 & extant & 0 & NA & LC & Browse \\
\hline SA & Rodentia & Cricetidae & Thomasomys & daphne & $\begin{array}{l}\text { Daphne's Oldfield } \\
\text { Mouse }\end{array}$ & 1.89 & 77 & extant & 0 & NA & LC & Browse \\
\hline SA & Rodentia & Cricetidae & Thomasomys & eleusis & $\begin{array}{l}\text { Peruvian Oldfield } \\
\text { Mouse }\end{array}$ & 1.89 & 77 & extant & 0 & NA & LC & Browse \\
\hline SA & Rodentia & Cricetidae & Thomasomys & gracilis & Slender Oldfield Mouse & 1.89 & 77 & extant & Future & NA & NT & Browse \\
\hline SA & Rodentia & Cricetidae & Thomasomys & hylophilus & $\begin{array}{l}\text { Woodland Oldfield } \\
\text { Mouse }\end{array}$ & 1.89 & 77 & extant & Future & NA & EN & Browse \\
\hline SA & Rodentia & Cricetidae & Thomasomys & incanus & Inca Oldfield Mouse & 1.89 & 77 & extant & Future & NA & Vu & Browse \\
\hline SA & Rodentia & Cricetidae & Thomasomys & kalinowskii & $\begin{array}{l}\text { Kalinowski's Oldfield } \\
\text { Mouse }\end{array}$ & 1.89 & 77 & extant & Future & NA & vU & Browse \\
\hline SA & Rodentia & Cricetidae & Thomasomys & ladewi & Ladew's Oldfield Mouse & 1.89 & 77 & extant & 0 & NA & LC & Browse \\
\hline SA & Rodentia & Cricetidae & Thomasomys & laniger & Butcher Oldfield Mouse & 1.55 & 36 & extant & 0 & NA & LC & Browse \\
\hline
\end{tabular}




\begin{tabular}{|c|c|c|c|c|c|c|c|c|c|c|c|c|}
\hline SA & Rodentia & Cricetidae & Thomasomys & monochromos & $\begin{array}{l}\text { Unicolored Oldfield } \\
\text { Mouse }\end{array}$ & 1.89 & 77 & extant & Future & NA & EN & Browse \\
\hline SA & Rodentia & Cricetidae & Thomasomys & niveipes & $\begin{array}{l}\text { Snow-footed Oldfield } \\
\text { Mouse }\end{array}$ & 1.89 & 77 & extant & 0 & NA & LC & Browse \\
\hline SA & Rodentia & Cricetidae & Thomasomys & notatus & $\begin{array}{l}\text { Distinguished Oldfield } \\
\text { Mouse }\end{array}$ & 1.89 & 77 & extant & 0 & NA & $\mathrm{LC}$ & Browse \\
\hline SA & Rodentia & Cricetidae & Thomasomys & oreas & $\begin{array}{l}\text { Montane Oldfield } \\
\text { Mouse }\end{array}$ & 1.89 & 77 & extant & 0 & NA & $\mathrm{LC}$ & Browse \\
\hline SA & Rodentia & Cricetidae & Thomasomys & paramorum & Paramo Oldfield Mouse & 1.89 & 77 & extant & 0 & NA & $\mathrm{LC}$ & Browse \\
\hline SA & Rodentia & Cricetidae & Thomasomys & pyrrhonotus & $\begin{array}{l}\text { Thomas's Oldfield } \\
\text { Mouse }\end{array}$ & 1.89 & 77 & extant & Future & NA & Vu & Browse \\
\hline SA & Rodentia & Cricetidae & Thomasomys & rhoadsi & $\begin{array}{l}\text { Rhoads's Oldfield } \\
\text { mouse }\end{array}$ & 1.89 & 77 & extant & 0 & NA & $\mathrm{LC}$ & Browse \\
\hline SA & Rodentia & Cricetidae & Thomasomys & rosalinda & $\begin{array}{l}\text { Rosalinda's Oldfield } \\
\text { Mouse }\end{array}$ & 1.89 & 77 & extant & 0 & NA & DD & Browse \\
\hline SA & Rodentia & Cricetidae & Thomasomys & silvestris & Forest Oldfield Mouse & 1.89 & 77 & extant & 0 & NA & LC & Browse \\
\hline SA & Rodentia & Cricetidae & Thomasomys & taczanowskii & $\begin{array}{l}\text { Taczanowski's Oldfield } \\
\text { Mouse }\end{array}$ & 1.89 & 77 & extant & 0 & NA & $\mathrm{LC}$ & Browse \\
\hline SA & Rodentia & Cricetidae & Thomasomys & vestitus & Dressy Oldfield Mouse & 1.88 & 77 & extant & 0 & NA & $\mathrm{LC}$ & Browse \\
\hline SA & Rodentia & Cricetidae & Tylomys & mirae & Mira Climbing Rat & 2.26 & 184 & extant & 0 & NA & $\mathrm{LC}$ & Frug/browse \\
\hline SA & Rodentia & Cricetidae & Wiedomys & pyrrhorhinos & Red-nosed Mouse & 1.67 & 47 & extant & 0 & NA & $\mathrm{LC}$ & Frug/ginsect \\
\hline SA & Rodentia & Cricetidae & Wilfredomys & oenax & $\begin{array}{l}\text { Greater Wilfred's } \\
\text { Mouse }\end{array}$ & 1.67 & 47 & extant & Future & NA & EN & Browse \\
\hline SA & Rodentia & Cricetidae & Zygodontomys & brevicauda & $\begin{array}{l}\text { Short-tailed Cane } \\
\text { Mouse }\end{array}$ & 1.72 & 52 & extant & 0 & NA & $\mathrm{LC}$ & Frug \\
\hline SA & Rodentia & Cricetidae & Zygodontomys & brunneus & Brown Cane Mouse & 1.88 & 76 & extant & 0 & NA & $\mathrm{LC}$ & Frug \\
\hline SA & Rodentia & Ctenomyidae & Ctenomys & argentinus & Argentine Tuco-tuco & 2.34 & 221 & extant & Future & NA & NT & Browse/frug \\
\hline SA & Rodentia & Ctenomyidae & Ctenomys & australis & Southern Tuco-tuco & 2.56 & 362 & extant & Future & NA & EN & Browse/frug \\
\hline SA & Rodentia & Ctenomyidae & Ctenomys & azarae & Azara's Tuco-tuco & 2.60 & 400 & extant & Future & NA & Vu & Browse/frug \\
\hline SA & Rodentia & Ctenomyidae & Ctenomys & boliviensis & Bolivian Tuco-tuco & 2.60 & 400 & extant & 0 & NA & LC & Browse/frug \\
\hline SA & Rodentia & Ctenomyidae & Ctenomys & boliviensis & Bolivian Tuco-tuco & 2.73 & 535 & extant & 0 & NA & LC & Browse/frug \\
\hline SA & Rodentia & Ctenomyidae & Ctenomys & bonettoi & Bonetto's Tuco-tuco & 2.31 & 202 & extant & Future & NA & EN & Browse/frug \\
\hline SA & Rodentia & Ctenomyidae & Ctenomys & brasiliensis & Brazilian Tuco-tuco & 2.60 & 400 & extant & 0 & NA & DD & Browse/frug \\
\hline SA & Rodentia & Ctenomyidae & Ctenomys & colburni & Colburn's Tuco-tuco & 2.60 & 400 & extant & 0 & NA & DD & Browse/frug \\
\hline SA & Rodentia & Ctenomyidae & Ctenomys & conoveri & Conover's Tuco-tuco & 2.93 & 860 & extant & 0 & NA & $\mathrm{LC}$ & Browse/frug \\
\hline SA & Rodentia & Ctenomyidae & Ctenomys & dorsalis & Chacoan Tuco-tuco & 2.22 & 166 & extant & 0 & NA & DD & Browse/frug \\
\hline SA & Rodentia & Ctenomyidae & Ctenomys & emilianus & Emily's Tuco-tuco & 2.46 & 285 & extant & Future & NA & NT & Browse/frug \\
\hline SA & Rodentia & Ctenomyidae & Ctenomys & frater & Forest Tuco-tuco & 2.24 & 173 & extant & 0 & NA & LC & Browse/frug \\
\hline SA & Rodentia & Ctenomyidae & Ctenomys & fulvus & Tawny Tuco-tuco & 2.42 & 262 & extant & 0 & NA & $\mathrm{LC}$ & Browse/frug \\
\hline SA & Rodentia & Ctenomyidae & Ctenomys & haigi & Haig's Tuco-tuco & 2.21 & 164 & extant & 0 & NA & $\mathrm{LC}$ & Browse/frug \\
\hline SA & Rodentia & Ctenomyidae & Ctenomys & knighti & Catamarca Tuco-tuco & 2.50 & 316 & extant & 0 & NA & $\mathrm{DD}$ & Browse/frug \\
\hline SA & Rodentia & Ctenomyidae & Ctenomys & latro & Mottled Tuco-tuco & 2.28 & 192 & extant & Future & NA & Vu & Browse/frug \\
\hline SA & Rodentia & Ctenomyidae & Ctenomys & leucodon & $\begin{array}{l}\text { White-toothed Tuco- } \\
\text { tuco }\end{array}$ & 2.39 & 244 & extant & 0 & NA & LC & Browse/frug \\
\hline SA & Rodentia & Ctenomyidae & Ctenomys & lewisi & $\begin{array}{l}\text { Lewis's Tuco-tuco, } \\
\text { Lewis' Tuco-tuco }\end{array}$ & 2.07 & 117 & extant & 0 & NA & LC & Browse/frug \\
\hline SA & Rodentia & Ctenomyidae & Ctenomys & magellanicus & Magellanic Tuco-tuco & 2.43 & 272 & extant & Future & NA & Vu & Browse/frug \\
\hline SA & Rodentia & Ctenomyidae & Ctenomys & maulinus & Maule Tuco-tuco & 2.33 & 215 & extant & 0 & NA & $\mathrm{LC}$ & Browse/frug \\
\hline SA & Rodentia & Ctenomyidae & Ctenomys & mendocinus & Mendoza Tuco-tuco & 2.21 & 163 & extant & 0 & NA & LC & Browse/frug \\
\hline SA & Rodentia & Ctenomyidae & Ctenomys & minutus & Tiny Tuco-tuco & 1.96 & 92 & extant & 0 & NA & DD & Browse/frug \\
\hline SA & Rodentia & Ctenomyidae & Ctenomys & occultus & Furtive Tuco-tuco & 2.18 & 150 & extant & Future & NA & EN & Browse/frug \\
\hline SA & Rodentia & Ctenomyidae & Ctenomys & opimus & Highland Tuco-tuco & 2.56 & 362 & extant & 0 & NA & LC & Browse/frug \\
\hline
\end{tabular}




\begin{tabular}{|c|c|c|c|c|c|c|c|c|c|c|c|c|}
\hline SA & Rodentia & Ctenomyidae & Ctenomys & pearsoni & Pearson's Tuco-tuco & 2.33 & 212 & extant & Future & NA & NT & Browse/frug \\
\hline SA & Rodentia & Ctenomyidae & Ctenomys & perrensis & Goya Tuco-tuco & 2.60 & 400 & extant & 0 & NA & LC & Browse/frug \\
\hline SA & Rodentia & Ctenomyidae & Ctenomys & peruanus & Peruvian Tuco-tuco & 2.69 & 490 & extant & 0 & NA & LC & Browse/frug \\
\hline SA & Rodentia & Ctenomyidae & Ctenomys & pontifex & San Luis Tuco-tuco & 2.60 & 400 & extant & 0 & NA & DD & Browse/frug \\
\hline SA & Rodentia & Ctenomyidae & Ctenomys & porteousi & Porteous's Tuco-tuco & 2.28 & 192 & extant & Future & NA & NT & Browse/frug \\
\hline SA & Rodentia & Ctenomyidae & Ctenomys & saltarius & Salta Tuco-tuco & 2.36 & 230 & extant & 0 & NA & DD & Browse/frug \\
\hline SA & Rodentia & Ctenomyidae & Ctenomys & sericeus & Silky Tuco-tuco & 2.60 & 400 & extant & 0 & NA & $\mathrm{DD}$ & Browse/frug \\
\hline SA & Rodentia & Ctenomyidae & Ctenomys & sociabilis & Social Tuco-tuco & 2.60 & 400 & extant & Future & NA & CR & Browse/frug \\
\hline SA & Rodentia & Ctenomyidae & Ctenomys & steinbachi & Steinbach's Tuco-tuco & 2.59 & 385 & extant & 0 & NA & $\mathrm{LC}$ & Browse/frug \\
\hline SA & Rodentia & Ctenomyidae & Ctenomys & talarum & Talas Tuco-tuco & 2.12 & 132 & extant & 0 & NA & LC & Browse/frug \\
\hline SA & Rodentia & Ctenomyidae & Ctenomys & torquatus & Collared Tuco-tuco & 2.32 & 209 & extant & 0 & NA & LC & Browse/frug \\
\hline SA & Rodentia & Ctenomyidae & Ctenomys & tuconax & Robust Tuco-tuco & 2.40 & 249 & extant & 0 & NA & DD & Browse/frug \\
\hline SA & Rodentia & Ctenomyidae & Ctenomys & tucumanus & Tucuman Tuco-tuco & 2.34 & 217 & extant & 0 & NA & DD & Browse/frug \\
\hline SA & Rodentia & Ctenomyidae & Ctenomys & validus & Strong Tuco-tuco & 2.37 & 233 & extant & 0 & NA & DD & Browse/frug \\
\hline SA & Rodentia & Dasyproctidae & Dasyprocta & azarae & Azara's Agouti & 3.36 & 2310 & extant & 0 & NA & DD & frug/browse \\
\hline SA & Rodentia & Dasyproctidae & Dasyprocta & cristata & Red-rumped Agouti & 3.42 & 2650 & extant & 0 & NA & $\mathrm{LC}$ & frug/browse \\
\hline SA & Rodentia & Dasyproctidae & Dasyprocta & fuliginosa & Black Agouti & 3.54 & 3500 & extant & 0 & NA & LC & frug/browse \\
\hline SA & Rodentia & Dasyproctidae & Dasyprocta & guamara & Orinoco Agouti & 3.42 & 2650 & extant & Future & NA & NT & frug/browse \\
\hline SA & Rodentia & Dasyproctidae & Dasyprocta & kalinowskii & Kalinowski Agouti & 3.42 & 2650 & extant & 0 & NA & DD & frug/browse \\
\hline SA & Rodentia & Dasyproctidae & Dasyprocta & leporina & $\begin{array}{l}\text { Red-rumped Agouti, } \\
\text { Brazilian Agouti }\end{array}$ & 3.48 & 3020 & extant & 0 & NA & LC & frug/browse \\
\hline SA & Rodentia & Dasyproctidae & Dasyprocta & prymnolopha & Black-rumped Agouti & 3.46 & 2900 & extant & 0 & NA & $\mathrm{LC}$ & frug/browse \\
\hline SA & Rodentia & Dasyproctidae & Dasyprocta & punctata & $\begin{array}{l}\text { Central American } \\
\text { Agouti }\end{array}$ & 3.43 & 2675 & extant & 0 & NA & $\mathrm{LC}$ & frug/browse \\
\hline SA & Rodentia & Dasyproctidae & Myoprocta & acouchy & Red Acouchi & 2.78 & 600 & extant & 0 & NA & LC & frug/browse \\
\hline SA & Rodentia & Dasyproctidae & Myoprocta & acouchy & Red Acouchi & 3.11 & 1280 & extant & 0 & NA & LC & frug/browse \\
\hline SA & Rodentia & Dinomyidae & Dinomys & branickii & Pacarana & 4.10 & 12500 & extant & Future & NA & Vu & Frug \\
\hline SA & Rodentia & Echimyidae & Callistomys & pictus & $\begin{array}{l}\text { Painted Tree-rat, } \\
\text { Painted Tree Rat }\end{array}$ & 2.72 & 519 & extant & Future & NA & EN & Frug/browse \\
\hline SA & Rodentia & Echimyidae & Carterodon & sulcidens & Owl's Spiny Rat & 2.06 & 114 & extant & 0 & NA & DD & graze \\
\hline SA & Rodentia & Echimyidae & Clyomys & bishopi & $\begin{array}{l}\text { Broad-headed Spiny } \\
\text { Rat }\end{array}$ & 1.48 & 30 & extant & 0 & NA & $\mathrm{LC}$ & Browse \\
\hline SA & Rodentia & Echimyidae & Clyomys & laticeps & Broad-headed Spiny Rat & 2.30 & 201 & extant & 0 & NA & LC & Browse \\
\hline SA & Rodentia & Echimyidae & Dactylomys & boliviensis & Bolivian Bamboo Rat & 2.86 & 729 & extant & 0 & NA & $\mathrm{LC}$ & Browse \\
\hline SA & Rodentia & Echimyidae & Dactylomys & dactylinus & Amazon Bamboo Rat & 2.81 & 650 & extant & 0 & NA & LC & Browse \\
\hline SA & Rodentia & Echimyidae & Dactylomys & peruanus & Peruvian Bamboo Rat & 2.58 & 382 & extant & 0 & NA & DD & Browse \\
\hline SA & Rodentia & Echimyidae & Diplomys & caniceps & $\begin{array}{l}\text { Arboreal Soft-furred } \\
\text { Spiny Rat }\end{array}$ & 2.60 & 395 & extant & 0 & NA & $\mathrm{DD}$ & Frug/browse \\
\hline SA & Rodentia & Echimyidae & Diplomys & labilis & Rufous Tree Rat & 2.36 & 227 & extant & 0 & NA & $\mathrm{LC}$ & Frug/browse \\
\hline SA & Rodentia & Echimyidae & Diplomys & rufodorsalis & Red Crested Tree Rat & 2.16 & 145 & extant & Future & NA & CR & Frug/browse \\
\hline SA & Rodentia & Echimyidae & Echimys & chrysurus & White-faced Tree Rat & 2.81 & 652 & extant & 0 & NA & LC & Frug/browse \\
\hline SA & Rodentia & Echimyidae & Echimys & saturnus & $\begin{array}{l}\text { Dark Spiny Tree-rat, } \\
\text { Dark Tree Rat }\end{array}$ & 2.80 & 627 & extant & 0 & NA & DD & Frug/browse \\
\hline SA & Rodentia & Echimyidae & Echimys & semivillosus & & 2.30 & 200 & extant & 0 & NA & & Frug/browse \\
\hline SA & Rodentia & Echimyidae & Euryzygomatomys & spinosus & Guiara & 2.27 & 187 & extant & 0 & NA & LC & Browse/frug \\
\hline SA & Rodentia & Echimyidae & Hoplomys & gymnurus & Armored Rat & 2.38 & 240 & extant & 0 & NA & $\mathrm{LC}$ & Frug \\
\hline SA & Rodentia & Echimyidae & Isothrix & bistriata & $\begin{array}{l}\text { Yellow-crowned Brush- } \\
\text { tailed Rat }\end{array}$ & 2.65 & 445 & extant & 0 & NA & $\mathrm{LC}$ & Frug/browse \\
\hline SA & Rodentia & Echimyidae & Isothrix & pagurus & Plain Brush-tailed Rat & 2.32 & 210 & extant & 0 & NA & LC & Frug/browse \\
\hline SA & Rodentia & Echimyidae & Kannabateomys & amblyonyx & Atlantic Bamboo Rat & 2.78 & 600 & extant & 0 & NA & LC & Browse \\
\hline
\end{tabular}




\begin{tabular}{|c|c|c|c|c|c|c|c|c|c|c|c|c|}
\hline SA & Rodentia & Echimyidae & Lonchothrix & emiliae & $\begin{array}{l}\text { Tuft-tailed Spiny Tree } \\
\text { Rat }\end{array}$ & 2.14 & 138 & extant & 0 & NA & LC & Browse \\
\hline SA & Rodentia & Echimyidae & Makalata & didelphoides & Brazilian Spiny Tree Rat & 2.60 & 400 & extant & 0 & NA & LC & Frug \\
\hline SA & Rodentia & Echimyidae & Makalata & didelphoides & Brazilian Spiny Tree Rat & 2.03 & 108 & extant & 0 & NA & LC & \\
\hline SA & Rodentia & Echimyidae & Makalata & grandis & Giant Tree Rat & 2.77 & 584 & extant & 0 & NA & LC & Frug/browse \\
\hline SA & Rodentia & Echimyidae & Makalata & macrura & Long-tailed Tree Rat & 2.64 & 439 & extant & 0 & NA & LC & Frug/browse \\
\hline SA & Rodentia & Echimyidae & Makalata & obscurus & Dusky Spiny Tree Rat & 2.03 & 108 & extant & 0 & NA & DD & Frug/ginsect \\
\hline SA & Rodentia & Echimyidae & Makalata & rhipidurus & & 2.50 & 315 & extant & 0 & NA & DD & Frug/browse \\
\hline SA & Rodentia & Echimyidae & Mesomys & hispidus & Spiny Tree Rat & 2.24 & 175 & extant & 0 & NA & LC & Frug/ginsect \\
\hline SA & Rodentia & Echimyidae & Mesomys & leniceps & $\begin{array}{l}\text { Woolly-headed Spiny } \\
\text { Tree Rat }\end{array}$ & 2.03 & 108 & extant & 0 & NA & $\mathrm{DD}$ & Frug/ginsect \\
\hline SA & Rodentia & Echimyidae & Mesomys & stimulax & $\begin{array}{l}\text { Para Spiny Tree Rat, } \\
\text { Surinam Spiny Tree Rat }\end{array}$ & 2.03 & 108 & extant & 0 & NA & LC & Frug/ginsect \\
\hline SA & Rodentia & Echimyidae & Olallamys & albicauda & White-tailed Olalla Rat & 2.44 & 274 & extant & 0 & NA & DD & Browse \\
\hline SA & Rodentia & Echimyidae & Olallamys & edax & Greedy Olalla Rat & 2.31 & 206 & extant & 0 & NA & DD & Browse \\
\hline SA & Rodentia & Echimyidae & Phyllomys & blainvillii & $\begin{array}{l}\text { Golden Atlantic Tree } \\
\text { Rat }\end{array}$ & 2.39 & 243 & extant & 0 & NA & LC & Frug/browse \\
\hline SA & Rodentia & Echimyidae & Phyllomys & braziliensis & Red-nosed Tree Rat & 2.49 & 313 & extant & Future & NA & EN & Frug/browse \\
\hline SA & Rodentia & Echimyidae & Phyllomys & dasythrix & Drab Atlantic Tree Rat & 2.41 & 260 & extant & 0 & NA & LC & Frug/browse \\
\hline SA & Rodentia & Echimyidae & Phyllomys & lamarum & Pallid Atlantic Tree Rat & 2.33 & 215 & extant & 0 & NA & DD & Frug/browse \\
\hline SA & Rodentia & Echimyidae & Phyllomys & nigrispinus & $\begin{array}{l}\text { Black-spined Atlantic } \\
\text { Tree Rat }\end{array}$ & 2.35 & 224 & extant & 0 & NA & LC & Frug/browse \\
\hline SA & Rodentia & Echimyidae & Phyllomys & thomasi & Giant Atlantic Tree Rat & 2.75 & 560 & extant & Future & NA & EN & Frug/browse \\
\hline SA & Rodentia & Echimyidae & Phyllomys & unicolor & Unicolored Tree Rat & 2.64 & 439 & extant & Future & NA & CR & Frug/browse \\
\hline SA & Rodentia & Echimyidae & Proechimys & brevicauda & Huallaga Spiny Rat & 2.45 & 285 & extant & 0 & NA & LC & Frug \\
\hline SA & Rodentia & Echimyidae & Proechimys & brevicauda & Huallaga Spiny Rat & 2.45 & 285 & extant & 0 & NA & LC & Frug \\
\hline SA & Rodentia & Echimyidae & Proechimys & brevicauda & Huallaga Spiny Rat & 2.45 & 285 & extant & 0 & NA & LC & Frug \\
\hline SA & Rodentia & Echimyidae & Proechimys & canicollis & Colombian Spiny Rat & 2.45 & 285 & extant & 0 & NA & LC & Frug \\
\hline SA & Rodentia & Echimyidae & Proechimys & chrysaeolus & Boyaca Spiny Rat & 2.45 & 285 & extant & 0 & NA & DD & Frug \\
\hline SA & Rodentia & Echimyidae & Proechimys & cuvieri & Cuvier's Spiny Rat & 2.52 & 330 & extant & 0 & NA & LC & Frug \\
\hline SA & Rodentia & Echimyidae & Proechimys & decumanus & Pacific Spiny Rat & 2.45 & 285 & extant & Future & NA & VU & Frug \\
\hline SA & Rodentia & Echimyidae & Proechimys & goeldii & Goeldi's Spiny Rat & 2.45 & 285 & extant & 0 & NA & LC & Frug \\
\hline SA & Rodentia & Echimyidae & Proechimys & guairae & Guaira Spiny Rat & 2.60 & 400 & extant & 0 & NA & LC & Frug \\
\hline SA & Rodentia & Echimyidae & Proechimys & guyannensis & Cayenne Spiny Rat & 2.45 & 285 & extant & 0 & NA & LC & Frug \\
\hline SA & Rodentia & Echimyidae & Proechimys & guyannensis & Cayenne Spiny Rat & 2.50 & 316 & extant & 0 & NA & LC & Frug \\
\hline SA & Rodentia & Echimyidae & Proechimys & hoplomyoides & Guyanan Spiny Rat & 2.45 & 285 & extant & 0 & NA & DD & Frug \\
\hline SA & Rodentia & Echimyidae & Proechimys & longicaudatus & Long-tailed Spiny Rat & 2.31 & 205 & extant & 0 & NA & LC & Frug \\
\hline SA & Rodentia & Echimyidae & Proechimys & magdalenae & Boyaca Spiny Rat & 2.45 & 285 & extant & 0 & NA & DD & Frug \\
\hline SA & Rodentia & Echimyidae & Proechimys & mincae & Minca Spiny Rat & 2.45 & 285 & extant & 0 & NA & DD & Frug \\
\hline SA & Rodentia & Echimyidae & Proechimys & oconnelli & O'Connell's Spiny Rat & 2.45 & 285 & extant & 0 & NA & DD & Frug \\
\hline SA & Rodentia & Echimyidae & Proechimys & oris & Roberto's Spiny Rat & 2.45 & 285 & extant & 0 & NA & LC & Frug \\
\hline SA & Rodentia & Echimyidae & Proechimys & poliopus & Guaira Spiny Rat & 2.45 & 285 & extant & 0 & NA & LC & Frug \\
\hline SA & Rodentia & Echimyidae & Proechimys & quadruplicatus & Napo Spiny Rat & 2.45 & 285 & extant & 0 & NA & LC & Frug \\
\hline SA & Rodentia & Echimyidae & Proechimys & quadruplicatus & Napo Spiny Rat & 2.45 & 285 & extant & 0 & NA & LC & Frug \\
\hline SA & Rodentia & Echimyidae & Proechimys & semispinosus & Tome's Spiny Rat & 2.45 & 285 & extant & 0 & NA & LC & Frug \\
\hline SA & Rodentia & Echimyidae & Proechimys & semispinosus & Tome's Spiny Rat & 2.56 & 361 & extant & 0 & NA & LC & Frug \\
\hline SA & Rodentia & Echimyidae & Proechimys & simonsi & Simon's Spiny Rat & 2.45 & 285 & extant & 0 & NA & LC & Frug \\
\hline SA & Rodentia & Echimyidae & Proechimys & simonsi & Simon's Spiny Rat & 2.45 & 285 & extant & 0 & NA & LC & Frug \\
\hline SA & Rodentia & Echimyidae & Proechimys & steerei & Steere's Spiny Rat & 2.45 & 285 & extant & 0 & NA & LC & Frug \\
\hline SA & Rodentia & Echimyidae & Proechimys & urichi & & 2.45 & 285 & extant & 0 & NA & DD & Frug \\
\hline
\end{tabular}




\begin{tabular}{|c|c|c|c|c|c|c|c|c|c|c|c|c|}
\hline SA & Rodentia & Echimyidae & Thrichomys & apereoides & $\begin{array}{l}\text { Common Punare, } \\
\text { Punare, Rabudo }\end{array}$ & 2.53 & 339 & extant & 0 & NA & LC & Frug \\
\hline SA & Rodentia & Echimyidae & Trinomys & albispinus & White-spined Spiny Rat & 2.45 & 285 & extant & 0 & NA & LC & Frug \\
\hline SA & Rodentia & Echimyidae & Trinomys & dimidiatus & Atlantic Spiny Rat & 2.22 & 168 & extant & 0 & NA & LC & Frug \\
\hline SA & Rodentia & Echimyidae & Trinomys & iheringi & Ihering's Spiny Rat & 2.31 & 203 & extant & 0 & NA & $\mathrm{LC}$ & Frug \\
\hline SA & Rodentia & Echimyidae & Trinomys & myosuros & $\begin{array}{l}\text { mouse-tailed Atlantic } \\
\text { spiny-rat }\end{array}$ & 2.45 & 285 & extant & 0 & NA & LC & Frug \\
\hline SA & Rodentia & Echimyidae & Trinomys & setosus & $\begin{array}{l}\text { Hairy Spiny Rat } \\
\text { Bristle-spined Rat }\end{array}$ & 2.45 & 285 & extant & 0 & NA & LC & Frug \\
\hline SA & Rodentia & Erethizontidae & Chaetomys & subspinosus & $\begin{array}{l}\text { Bristle-spined } \\
\text { Porcupine, Thin-spined } \\
\text { Porcupine }\end{array}$ & 3.11 & 1300 & extant & Future & NA & vu & Frug \\
\hline SA & Rodentia & Erethizontidae & Coendou & bicolor & $\begin{array}{l}\text { Bicolor-spined } \\
\text { Porcupine }\end{array}$ & 3.61 & 4050 & extant & 0 & NA & LC & Browse/frug \\
\hline SA & Rodentia & Erethizontidae & Coendou & nycthemera & Koopman's Porcupine & 3.48 & 3000 & extant & 0 & NA & DD & Browse/frug \\
\hline SA & Rodentia & Erethizontidae & Coendou & prehensilis & Brazilian Porcupine & 3.64 & 4400 & extant & 0 & NA & LC & Browse/frug \\
\hline SA & Rodentia & Erethizontidae & Coendou & rothschildi & & 3.30 & 2000 & extant & 0 & NA & DD & Browse/frug \\
\hline SA & Rodentia & Erethizontidae & Echinoprocta & rufescens & Stump-tailed Porcupine & 2.92 & 832 & extant & 0 & NA & LC & Browse/frug \\
\hline SA & Rodentia & Erethizontidae & Sphiggurus & insidiosus & $\begin{array}{l}\text { Bahia Hairy Dwarf } \\
\text { Porcupine }\end{array}$ & 3.00 & 1000 & extant & 0 & NA & LC & Browse/frug \\
\hline SA & Rodentia & Erethizontidae & Sphiggurus & spinosus & & 2.88 & 751 & extant & 0 & NA & & Browse/frug \\
\hline SA & Rodentia & Erethizontidae & Sphiggurus & vestitus & $\begin{array}{l}\text { Brown Hairy Dwarf } \\
\text { Porcupine }\end{array}$ & 2.87 & 736 & extant & 0 & NA & DD & Browse/frug \\
\hline SA & Rodentia & Erethizontidae & Sphiggurus & villosus & $\begin{array}{l}\text { Paraguay Hairy Dwarf } \\
\text { Porcupine }\end{array}$ & 3.24 & 1750 & extant & 0 & NA & LC & Browse/frug \\
\hline SA & Rodentia & Geomyidae & Orthogeomys & dariensis & Caribbean Spiny Pocket & 2.64 & 438 & extant & 0 & NA & LC & Browse/frug \\
\hline SA & Rodentia & Heteromyidae & Heteromys & anomalus & $\begin{array}{l}\text { Mouse, Trinidad Spiny } \\
\text { Pocket Mouse }\end{array}$ & 1.85 & 70 & extant & 0 & NA & LC & Frug/browse \\
\hline SA & Rodentia & Heteromyidae & Heteromys & australis & $\begin{array}{l}\text { Southern Spiny Pocket } \\
\text { Mouse }\end{array}$ & 2.43 & 267 & extant & 0 & NA & LC & Frug/browse \\
\hline SA & Rodentia & Heteromyidae & Heteromys & desmarestianus & $\begin{array}{l}\text { Desmarest's Spiny } \\
\text { Pocket Mouse }\end{array}$ & 1.86 & 73 & extant & 0 & NA & LC & Frug/browse \\
\hline SA & Rodentia & Heteromyidae & Liomys & adspersus & $\begin{array}{l}\text { Panamanian Spiny } \\
\text { Pocket Mouse }\end{array}$ & 1.81 & 65 & extant & 0 & NA & LC & Frug/browse \\
\hline SA & Rodentia & Hydrochoeridae & Hydrochoerus & hydrochaeris & Capybara & 4.80 & 62450 & extant & 0 & NA & LC & \\
\hline SA & Rodentia & Hydrochoeridae & Neochoerus & sulcidens & & 5.18 & 150000 & extinct & TP & 11 & EX & Graze \\
\hline SA & Rodentia & Muridae & Abrothrix & andinus & $\begin{array}{l}\text { Andean Akodont, } \\
\text { Andean Altiplano } \\
\text { Mouse, Andean Grass } \\
\text { Mouse }\end{array}$ & 1.26 & 18 & extant & 0 & NA & LC & rowse/ginsec \\
\hline SA & Rodentia & Muridae & Abrothrix & illuteus & $\begin{array}{l}\text { Gray Akodont, Gray } \\
\text { Grass Mouse }\end{array}$ & 1.68 & 48 & extant & Future & NA & NT & iect/frug/brol \\
\hline SA & Rodentia & Muridae & Abrothrix & jelskii & $\begin{array}{l}\text { Jelski's Altiplano } \\
\text { Mouse, Ornate Akodont, } \\
\text { Ornate Grass Mouse }\end{array}$ & 1.54 & 35 & extant & 0 & NA & LC & rowse/ginsec \\
\hline SA & Rodentia & Muridae & Abrothrix & lanosus & $\begin{array}{l}\text { Woolly Grass Mouse, } \\
\text { Woolly Akodont }\end{array}$ & 1.51 & 33 & extant & 0 & NA & LC & ;ect/frug/brol \\
\hline
\end{tabular}




\begin{tabular}{|c|c|c|c|c|c|c|c|c|c|c|c|c|}
\hline SA & Rodentia & Muridae & Abrothrix & longipilis & $\begin{array}{l}\text { Long-haired Akodont, } \\
\text { Long-haired Grass } \\
\text { Mouse }\end{array}$ & 1.57 & 38 & extant & 0 & NA & LC & iect/frug/brol \\
\hline SA & Rodentia & Muridae & Abrothrix & olivaceus & $\begin{array}{l}\text { Olive Grass Mouse, } \\
\text { Olive-coloured Akodont }\end{array}$ & 1.59 & 39 & extant & 0 & NA & $\mathrm{LC}$ & iect/frug/brol \\
\hline SA & Rodentia & Muridae & Abrothrix & sanborni & $\begin{array}{l}\text { Sanborn's Grass Mouse, } \\
\text { Sanborn's Akodont }\end{array}$ & 1.39 & 25 & extant & Future & NA & NT & iect/frug/brol \\
\hline SA & Rodentia & Muridae & Akodon & lutescens & $\begin{array}{l}\text { Altiplano Grass Mouse, } \\
\text { Altiplano Akodont }\end{array}$ & 1.32 & 21 & extant & 0 & NA & LC & iect/frug/brol \\
\hline SA & Rodentia & Muridae & Akodon & nigrita & $\begin{array}{l}\text { Blackish Grass Mouse } \\
\text { Ihering's Hocicudo, }\end{array}$ & 1.30 & 20 & extant & 0 & NA & $\mathrm{LC}$ & iect/frug/brol \\
\hline SA & Rodentia & Muridae & Brucepattersonius & iheringi & $\begin{array}{l}\text { Ihering's Brucie, } \\
\text { Ihering's Akodont }\end{array}$ & 1.63 & 43 & extant & 0 & NA & $\mathrm{LC}$ & Ginsect \\
\hline SA & Rodentia & Muridae & Deltamys & kempi & Kemp's Grass Mouse & 1.42 & 26 & extant & 0 & NA & LC & iect/frug/brol \\
\hline SA & Rodentia & Muridae & Handleyomys & fuscatus & Dusky Montane Mouse & 1.69 & 50 & extant & 0 & NA & LC & Ginsect \\
\hline SA & Rodentia & Muridae & Handleyomys & intectus & Colombian Rice Rat & 1.78 & 61 & extant & 0 & NA & LC & Frug/ginsect \\
\hline SA & Rodentia & Muridae & Juliomys & pictipes & $\begin{array}{l}\text { Lesser Wilfred's Mouse, } \\
\text { Contreras' Juliomys }\end{array}$ & 1.36 & 23 & extant & 0 & NA & LC & Browse \\
\hline SA & Rodentia & Muridae & Juscelinomys & talpinus & & & & extinct & TP & 11 & EX & \\
\hline SA & Rodentia & Muridae & Loxodontomys & micropus & $\begin{array}{l}\text { Southern Pericote, } \\
\text { Southern Big-eared } \\
\text { Mouse } \\
\text { Lund's Amphibious Rat, }\end{array}$ & 1.86 & 73 & extant & 0 & NA & $\mathrm{LC}$ & Browse \\
\hline SA & Rodentia & Muridae & Lundomys & molitor & $\begin{array}{l}\text { Lund's Amphibious Rat, } \\
\text { Greater Marsh Rat }\end{array}$ & 2.38 & 239 & extant & 0 & NA & LC & zraze/browse \\
\hline SA & Rodentia & Muridae & Necromys & amoenus & Pleasant Bolo Mouse & 1.46 & 29 & extant & 0 & NA & LC & Ginsect \\
\hline SA & Rodentia & Muridae & Necromys & lactens & $\begin{array}{l}\text { Rufous-bellied Bolo } \\
\text { Mouse }\end{array}$ & 1.52 & 33 & extant & 0 & NA & $\mathrm{LC}$ & Ginsect \\
\hline SA & Rodentia & Muridae & Necromys & lasiurus & Hairy-tailed Bolo Mouse & 1.60 & 40 & extant & 0 & NA & LC & Ginsect \\
\hline SA & Rodentia & Muridae & Necromys & obscurus & Dark Bolo Mouse & 1.61 & 41 & extant & Future & NA & NT & Ginsect \\
\hline SA & Rodentia & Muridae & Necromys & punctulatus & Spotted Bolo Mouse & 1.57 & 37 & extant & 0 & NA & DD & Ginsect \\
\hline SA & Rodentia & Muridae & Necromys & temchuki & Temchuk's Bolo Mouse & 1.67 & 47 & extant & 0 & NA & $\mathrm{LC}$ & Ginsect \\
\hline SA & Rodentia & Muridae & Necromys & urichi & $\begin{array}{l}\text { Northern Grass Mouse } \\
\text { Amazonian Mouse, }\end{array}$ & 1.53 & 34 & extant & 0 & NA & LC & iect/frug/brol \\
\hline SA & Rodentia & Muridae & Nectomys & rattus & $\begin{array}{l}\text { Small-footed Bristly } \\
\text { Mouse }\end{array}$ & 2.40 & 249 & extant & 0 & NA & LC & Ginsect/frug \\
\hline SA & Rodentia & Muridae & Oryzomys & buccinatus & & 2.00 & 100 & extant & 0 & NA & LC & Frug/ginsect \\
\hline SA & Rodentia & Muridae & Oryzomys & capito & & 1.76 & 58 & extant & 0 & NA & $\mathrm{LC}$ & Frug/ginsect \\
\hline SA & Rodentia & Muridae & Oryzomys & intermedius & Russet Rice Rat & 1.78 & 61 & extant & 0 & NA & $\mathrm{LC}$ & Frug/ginsect \\
\hline SA & Rodentia & Muridae & Oryzomys & kelloggi & & 1.78 & 61 & extant & 0 & NA & LC & Frug/ginsect \\
\hline SA & Rodentia & Muridae & Oryzomys & oniscus & & 1.78 & 61 & extant & Future & NA & NT & Frug/ginsect \\
\hline SA & Rodentia & Muridae & Oryzomys & ratticeps & & 2.16 & 144 & extant & 0 & NA & LC & Frug/ginsect \\
\hline SA & Rodentia & Muridae & Oryzomys & xantheolus & Yellowish Rice Rat & 1.90 & 80 & extant & 0 & NA & $\mathrm{LC}$ & Frug/ginsect \\
\hline SA & Rodentia & Muridae & Paralomys & gerbillus & $\begin{array}{l}\text { Gerbil Leaf-eared } \\
\text { Mouse }\end{array}$ & 1.24 & 17 & extant & 0 & NA & LC & Frug/browse \\
\hline SA & Rodentia & Muridae & Phyllotis & bonariensis & $\begin{array}{l}\text { Buenos Aires Leaf- } \\
\text { eared Mouse }\end{array}$ & 1.63 & 43 & extant & Future & NA & NT & Frug/browse \\
\hline SA & Rodentia & Muridae & Thomasomys & ischyurus & $\begin{array}{l}\text { Strong-tailed Oldfield } \\
\text { Mouse }\end{array}$ & 1.89 & 77 & extant & 0 & NA & LC & Browse \\
\hline
\end{tabular}




\begin{tabular}{|c|c|c|c|c|c|c|c|c|c|c|c|c|}
\hline SA & Rodentia & Myocastoridae & Myocastor & coypus & Coypu, Nutria & 3.84 & 6937 & extant & 0 & NA & LC & Browse \\
\hline SA & Rodentia & Octodontidae & Aconaemys & fuscus & Chilean Rock Rat & 2.13 & 134 & extant & 0 & NA & LC & Browse/frug \\
\hline SA & Rodentia & Octodontidae & Aconaemys & sagei & Sage's Rock Rat & 1.98 & 97 & extant & 0 & NA & DD & Browse/frug \\
\hline SA & Rodentia & Octodontidae & Dicolpomys & fossor & & 2.16 & 144 & extinct & TP & 11 & EX & 3rowse/graz€ \\
\hline SA & Rodentia & Octodontidae & Octodon & bridgesi & Bridges's Degu & 2.18 & 150 & extant & Future & NA & Vu & ize/browse/fi \\
\hline SA & Rodentia & Octodontidae & Octodon & degus & Degu & 2.32 & 210 & extant & 0 & NA & LC & Ize/browse/fi \\
\hline SA & Rodentia & Octodontidae & Octodon & lunatus & Moon-toothed Degu & 2.37 & 233 & extant & Future & NA & NT & ize/browse/fi \\
\hline SA & Rodentia & Octodontidae & Octodontomys & gliroides & Mountain Degu & 2.18 & 150 & extant & 0 & NA & LC & Frug \\
\hline SA & Rodentia & Octodontidae & Octomys & $\operatorname{mimax}$ & Viscacha Rat & 2.12 & 131 & extant & 0 & NA & LC & Browse \\
\hline SA & Rodentia & Octodontidae & Spalacopus & cyanus & Coruro & 2.11 & 128 & extant & 0 & NA & LC & Browse \\
\hline SA & Rodentia & Octodontidae & Tympanoctomys & barrerae & $\begin{array}{l}\text { Plains Viscacha Rat, } \\
\text { Red Vizcacha Rat }\end{array}$ & 1.85 & 70 & extant & Future & NA & NT & Browse \\
\hline SA & Rodentia & Sciuridae & Microsciurus & alfari & $\begin{array}{l}\text { Central American Dwarf } \\
\text { Squirrel }\end{array}$ & 1.94 & 88 & extant & 0 & NA & LC & Ginsect/frug \\
\hline SA & Rodentia & Sciuridae & Microsciurus & flaviventer & Amazon Dwarf Squirrel & 1.96 & 92 & extant & 0 & NA & $\mathrm{DD}$ & Ginsect/frug \\
\hline SA & Rodentia & Sciuridae & Microsciurus & mimulus & Western Dwarf Squirrel & 2.08 & 120 & extant & 0 & NA & LC & Ginsect/frug \\
\hline SA & Rodentia & Sciuridae & Microsciurus & santanderensis & $\begin{array}{l}\text { Santander Dwarf } \\
\text { Squirrel }\end{array}$ & 2.00 & 100 & extant & 0 & NA & DD & Ginsect/frug \\
\hline SA & Rodentia & Sciuridae & Sciurillus & pusillus & $\begin{array}{l}\text { Neotropical Pygmy } \\
\text { Squirrel }\end{array}$ & 1.59 & 39 & extant & 0 & NA & DD & Browse \\
\hline SA & Rodentia & Sciuridae & Sciurus & aestuans & Guianan Squirrel & 2.27 & 185 & extant & 0 & NA & LC & Frug \\
\hline SA & Rodentia & Sciuridae & Sciurus & flammifer & Fiery Squirrel & 3.63 & 4293 & extant & 0 & NA & DD & Frug \\
\hline SA & Rodentia & Sciuridae & Sciurus & gilvigularis & $\begin{array}{l}\text { Yellow-throated } \\
\text { Squirrel }\end{array}$ & 2.90 & 803 & extant & 0 & NA & DD & Frug \\
\hline SA & Rodentia & Sciuridae & Sciurus & granatensis & Red-tailed Squirrel & 2.40 & 250 & extant & 0 & NA & LC & Frug \\
\hline SA & Rodentia & Sciuridae & Sciurus & ignitus & Bolivian Squirrel & 2.28 & 190 & extant & 0 & NA & DD & Frug \\
\hline SA & Rodentia & Sciuridae & Sciurus & igniventris & $\begin{array}{l}\text { Northern Amazon Red } \\
\text { Squirrel }\end{array}$ & 2.85 & 700 & extant & 0 & NA & LC & Frug \\
\hline SA & Rodentia & Sciuridae & Sciurus & pucheranii & Andean Squirrel & 2.90 & 803 & extant & 0 & NA & DD & Frug \\
\hline SA & Rodentia & Sciuridae & Sciurus & pyrrhinus & JunÍn Red Squirrel & 2.68 & 482 & extant & 0 & NA & DD & Frug \\
\hline SA & Rodentia & Sciuridae & Sciurus & sanborni & Sanborn's Squirrel & 2.13 & 136 & extant & 0 & NA & DD & Frug \\
\hline SA & Rodentia & Sciuridae & Sciurus & spadiceus & $\begin{array}{l}\text { Southern Amazon Red } \\
\text { Squirrel }\end{array}$ & 2.61 & 403 & extant & 0 & NA & LC & Frug \\
\hline SA & Rodentia & Sciuridae & Sciurus & stramineus & $\begin{array}{l}\text { Guayaquil Squirrel } \\
\text { Amazonian Manatee, }\end{array}$ & 2.64 & 433 & extant & 0 & NA & $\mathrm{LC}$ & Frug \\
\hline SA & Sirenia & Trichechidae & Trichechus & inunguis & $\begin{array}{l}\text { South American } \\
\text { Manatee }\end{array}$ & 5.68 & 480000 & extant & Future & NA & vU & Graze \\
\hline SA & Soricomorpha & Soricidae & Cryptotis & meridensis & $\begin{array}{l}\text { Merida Small-eared } \\
\text { Shrew }\end{array}$ & 1.06 & 12 & extant & 0 & NA & LC & Ginsect \\
\hline SA & Soricomorpha & Soricidae & Cryptotis & montivaga & $\begin{array}{l}\text { Ecuadorean Small- } \\
\text { eared Shrew, } \\
\text { Wandering Small-eared } \\
\text { Shrew }\end{array}$ & 1.05 & 11 & extant & 0 & NA & LC & Ginsect \\
\hline SA & Soricomorpha & Soricidae & Cryptotis & nigrescens & $\begin{array}{l}\text { Blackish Small-eared } \\
\text { Shrew }\end{array}$ & 1.00 & 10 & extant & 0 & NA & LC & Ginsect \\
\hline SA & Soricomorpha & Soricidae & Cryptotis & squamipes & $\begin{array}{l}\text { Scaly-footed Small- } \\
\text { eared Shrew, Western } \\
\text { Colombian Small-eared } \\
\text { Shrew } \\
\text { Thomas' Small-eared }\end{array}$ & 1.04 & 11 & extant & 0 & NA & LC & Ginsect \\
\hline SA & Soricomorpha & Soricidae & Cryptotis & thomasi & $\begin{array}{l}\text { Shrew, Thomas's Small- } \\
\text { eared Shrew }\end{array}$ & 1.10 & 13 & extant & 0 & NA & LC & Ginsect \\
\hline
\end{tabular}


Table S7. Cenozoic mammal data. Taxonomy, first and last appearance, PBDB life habit, PBDB diet, and taxon rank at which the diet assignment was made were taken from the the Paleobiology Database (175). Body sizes were compiled from the literature $(38,103-209)$ or estimated from regressions on teeth. Criteria for Recoded Diet and Recoded groups are described in the SI.

\begin{tabular}{|c|c|c|c|c|c|c|c|c|c|c|}
\hline Order & Family & Genus_species & FAD & LAD & In Mass (g) & PBDB life habit & PBDB diet & $\begin{array}{l}\text { taxon rank at which } \\
\text { diet assignment was } \\
\text { made }\end{array}$ & $\begin{array}{l}\text { Recoded Diet: } \\
\text { Carnivore/Omnivore/ } \\
\text { Herbivore/Insectivore }\end{array}$ & $\begin{array}{l}\text { Recoded } \\
\text { groups:Rodent/ } \\
\text { Herbiovore } \\
\text { guild/Carnivore } \\
\text { guild }\end{array}$ \\
\hline Carnivora & Ursidae & Ursus americanus & 2 & 0.00349 & 10.51 & 8 ground dwelling & herbivore, carnivore & species & omnivore & carnivore guild \\
\hline Artiodactyla & Hippopotamidae & Hippopotamus amphibius & 3.96 & 0.00385 & 14.5406407 & 8 amphibious & grazer, browser & species & herbivore & herbivore guild \\
\hline Carnivora & Procyonidae & Nasua nasua & 0.00491 & 0.00491 & 8.46404604 & 9 scansorial & carnivore, omnivore & species & carnivore & carnivore guild \\
\hline Rodentia & Hydrochoeridae & Hydrochoerus hydrochaeris & 0.4535 & 0.00491 & 11.4186147 & 9 amphibious & herbivore & species & herbivore & Rodentia \\
\hline Artiodactyla & Cervidae & Rangifer tarandus & 1.6845 & 0.0049225 & 11.84 & 9 ground dwelling & grazer, browser & species & herbivore & herbivore guild \\
\hline Proboscidea & Elephantidae & Mammuthus primigenius & 1.6845 & 0.0057 & 15.2707443 & 4 ground dwelling & grazer & species & herbivore & herbivore guild \\
\hline Artiodactyla & Bovidae & Gazella leptoceros & 0.006 & 0.006 & 10.0162451 & 5 ground dwelling & grazer, browser & species & herbivore & herbivore guild \\
\hline Carnivora & Odobenidae & Odobenus rosmarus & 0.006 & 0.006 & 14.3461388 & 1 amphibious & insectivore & species & insectivore & carnivore guild \\
\hline Rodentia & Heteromyidae & Dipodomys spectabilis & 0.069 & 0.006 & & 7 saltatorial & granivore & species & herbivore & Rodentia \\
\hline Rodentia & Cricetidae & Neotoma micropus & 0.3 & 0.006 & 5.43 & 7 ground dwelling & herbivore & species & herbivore & Rodentia \\
\hline Rodentia & Cricetidae & Peromyscus maniculatus & 0.3 & 0.006 & 2.748 & 5 ground dwelling & herbivore & species & herbivore & Rodentia \\
\hline Rodentia & Cricetidae & Reithrodontomys montanus & 0.3 & 0.006 & 2.5 & 8 ground dwelling & herbivore & species & herbivore & Rodentia \\
\hline Carnivora & Procyonidae & Bassariscus astutus & 0.3 & 0.006 & 7.547 & 5 arboreal & frugivore, carnivore & species & omnivore & carnivore guild \\
\hline Eulipotyphla & Soricidae & Notiosorex crawfordi & 0.5 & 0.006 & 2.06073920 & 8 ground dwelling & omnivore & species & omnivore & NA \\
\hline Rodentia & Heteromyidae & Chaetodipus hispidus & 1 & 0.006 & 2.80 & 2 ground dwelling & granivore, browser & species & herbivore & Rodentia \\
\hline Rodentia & Cricetidae & Sigmodon hispidus & 1.15 & 0.006 & 4.69 & 1 ground dwelling & herbivore & species & herbivore & Rodentia \\
\hline Rodentia & Sciuridae & Spermophilus variegatus & 1.294 & 0.006 & 5.810 & 5 semifossorial & granivore, frugivore & species & herbivore & Rodentia \\
\hline Rodentia & Cricetidae & Neotoma albigula & 1.294 & 0.006 & 5.20 & 1 ground dwelling & herbivore & species & herbivore & Rodentia \\
\hline Carnivora & Phocidae & Mirounga leonina & 1.3 & 0.006 & 15.1216788 & 7 amphibious & piscivore, carnivore & species & carnivore & carnivore guild \\
\hline Artiodactyla & Bovidae & Bison antiquus & 1.3 & 0.006 & 13.8293260 & 7 ground dwelling & grazer & species & herbivore & herbivore guild \\
\hline Artiodactyla & Capridae & Ovis canadensis & 1.3 & 0.006 & 11.041 & 5 ground dwelling & grazer, browser & species & herbivore & herbivore guild \\
\hline Artiodactyla & Bovidae & Pelorovis antiquus & 1.3 & 0.006 & 13.8155105 & 6 ground dwelling & grazer, browser & species & herbivore & herbivore guild \\
\hline Proboscidea & Elephantidae & Loxodonta africana & 1.3 & 0.006 & 16.1180956 & 5 ground dwelling & grazer, browser & species & herbivore & herbivore guild \\
\hline Rodentia & Sciuridae & Marmota monax & 1.3 & 0.006 & 7.945 & 5 semifossorial & granivore, frugivore & species & herbivore & Rodentia \\
\hline Rodentia & Sciuridae & Tamias striatus & 1.3 & 0.006 & 3.83 & 5 scansorial & granivore, frugivore & species & herbivore & Rodentia \\
\hline Rodentia & Sciuridae & Tamiasciurus hudsonicus & 1.3 & 0.006 & 4.808 & 5 arboreal & granivore, frugivore & species & herbivore & Rodentia \\
\hline Rodentia & Cricetidae & Clethrionomys gapperi & 1.3 & 0.006 & 4.098 & 5 ground dwelling & herbivore & species & herbivore & Rodentia \\
\hline Rodentia & Cricetidae & Microtus pennsylvanicus & 1.3 & 0.006 & 4.41 & 1 ground dwelling & herbivore & species & herbivore & Rodentia \\
\hline Rodentia & Cricetidae & Neotoma floridana & 1.3 & 0.006 & 5.622 & 5 ground dwelling & herbivore & species & herbivore & Rodentia \\
\hline Rodentia & Cricetidae & Ondatra zibethicus & 1.3 & 0.006 & 7.634 & 5 amphibious & herbivore & species & herbivore & Rodentia \\
\hline Rodentia & Cricetidae & Mictomys borealis & 1.3 & 0.006 & $4.37 \xi$ & & & & NA & Rodentia \\
\hline Rodentia & Sciuridae & Sciurus carolinensis & 1.6 & 0.006 & 5.608 & 5 arboreal & granivore, frugivore & species & herbivore & Rodentia \\
\hline Artiodactyla & Bovidae & Bos primigenius & 1.6845 & 0.006 & 13.8638648 & 4 ground dwelling & grazer, browser & genus & herbivore & herbivore guild \\
\hline Carnivora & Canidae & Urocyon cinereoargenteus & 1.75 & 0.006 & 8.71 & 4 scansorial & carnivore, omnivore & species & carnivore & carnivore guild \\
\hline Carnivora & Mustelidae & Lontra canadensis & 1.85 & 0.006 & 7.886 & 5 amphibious & carnivore, omnivore & species & carnivore & carnivore guild \\
\hline Carnivora & Mustelidae & Mustela frenata & 1.85 & 0.006 & 4.29 & 7 scansorial & carnivore, omnivore & species & carnivore & carnivore guild \\
\hline Carnivora & Mustelidae & Spilogale putorius & 2.1 & 0.006 & 5.368 & 5 scansorial & carnivore, omnivore & species & carnivore & carnivore guild \\
\hline Artiodactyla & Cervidae & Odocoileus hemionus & 2.3 & 0.006 & 11.6 & 3 ground dwelling & browser & species & herbivore & herbivore guild \\
\hline Carnivora & Felidae & Puma concolor & 2.35 & 0.006 & 10.351 & 5 scansorial & carnivore & species & carnivore & carnivore guild \\
\hline Carnivora & Canidae & Canis lupus & 3.094 & 0.006 & 10.795 & 5 ground dwelling & carnivore & species & carnivore & carnivore guild \\
\hline Carnivora & Canidae & Canis latrans & 3.1 & 0.006 & 10.06 & 4 ground dwelling & carnivore, omnivore & species & carnivore & carnivore guild \\
\hline Carnivora & Procyonidae & Procyon lotor & 3.35 & 0.006 & 8.63 & 7 scansorial & omnivore, carnivore & species & omnivore & carnivore guild \\
\hline Carnivora & Mustelidae & Mephitis mephitis & 3.5 & 0.006 & 7.87 & 9 ground dwelling & carnivore, omnivore & species & carnivore & carnivore guild \\
\hline Carnivora & Felidae & Lynx rufus & 3.6 & 0.006 & 8.82350469 & 7 scansorial & carnivore & species & carnivore & carnivore guild \\
\hline Artiodactyla & Cervidae & Odocoileus virginianus & 3.65 & 0.006 & 11.139 & 5 ground dwelling & browser & species & herbivore & herbivore guild \\
\hline
\end{tabular}




\begin{tabular}{|c|c|c|c|c|c|c|c|c|c|}
\hline Rodentia & Castoridae & Castor canadensis & 3.65 & 0.006 & 9.3425 amphibious & browser & species & herbivore & Rodentia \\
\hline Lipotyphla & Talpidae & Scalopus aquaticus & 3.65 & 0.006 & 3.034952987 fossorial & insectivore & species & insectivore & NA \\
\hline Carnivora & Felidae & Panthera leo & 3.96 & 0.006 & 12.39623091 scansorial & carnivore & species & carnivore & carnivore guild \\
\hline Artiodactyla & Cervidae & Cervus elaphus & 3.96 & 0.006 & 12.6765 ground dwelling & grazer & species & herbivore & herbivore guild \\
\hline Proboscidea & Mammutidae & Mammut americanum & 5.95 & 0.006 & 11.89477331 ground dwelling & browser & species & herbivore & herbivore guild \\
\hline Rodentia & Sciuridae & Ammospermophilus leucurus & 0.1 & 0.00941 & 4.14 semifossorial & granivore, frugivore & species & herbivore & Rodentia \\
\hline Rodentia & Sciuridae & Marmota flaviventris & 0.3 & 0.00941 & 7.6375 semifossorial & granivore, frugivore & species & herbivore & Rodentia \\
\hline Rodentia & Cricetidae & Neotoma lepida & 0.3 & 0.00941 & 5.0635 ground dwelling & herbivore & species & herbivore & Rodentia \\
\hline Rodentia & Cricetidae & Lemmiscus curtatus & 0.8 & 0.00941 & 3.7055 ground dwelling & herbivore & species & herbivore & Rodentia \\
\hline Carnivora & Mustelidae & Brachyprotoma obtusata & 0.85 & 0.00941 & 6.104597616 scansorial & carnivore, omnivore & species & carnivore & carnivore guild \\
\hline Carnivora & Mustelidae & Mustela vison & 0.85 & 0.00941 & 5.612 amphibious & carnivore, omnivore & species & carnivore & carnivore guild \\
\hline Artiodactyla & Antilocapridae & Antilocapra americana & 1.294 & 0.00941 & 10.538 ground dwelling & browser, grazer & species & herbivore & herbivore guild \\
\hline Carnivora & Felidae & Smilodon fatalis & 1.3 & 0.00941 & 12.04480319 scansorial & carnivore & species & carnivore & carnivore guild \\
\hline Artiodactyla & Bovidae & Bootherium bombifrons & 1.3 & 0.00941 & 13.53229259 ground dwelling & grazer, browser & species & herbivore & herbivore guild \\
\hline Perissodactyla & Equidae & Equus conversidens & 1.3 & 0.00941 & 12.54499091 ground dwelling & grazer & species & herbivore & herbivore guild \\
\hline Carnivora & Canidae & Vulpes vulpes & 1.6845 & 0.00941 & 9.211 ground dwelling & carnivore, omnivore & species & carnivore & carnivore guild \\
\hline Rodentia & Cricetidae & Peromyscus truei & 2.15 & 0.00941 & 3.3175 ground dwelling & herbivore & species & herbivore & Rodentia \\
\hline Artiodactyla & Camelidae & Camelops hesternus & 2.3 & 0.00941 & 13.52862689 ground dwelling & browser, browser & species & herbivore & herbivore guild \\
\hline Rodentia & Cricetidae & Neotoma cinerea & 3.1 & 0.00941 & 5.597 ground dwelling & herbivore & species & herbivore & Rodentia \\
\hline Carnivora & Canidae & Vulpes velox & 3.4 & 0.00941 & 8.5405 ground dwelling & carnivore, omnivore & species & carnivore & carnivore guild \\
\hline Perissodactyla & Equidae & Equus scotti & 4.125 & 0.00941 & 13.072 ground dwelling & grazer & species & herbivore & herbivore guild \\
\hline Artiodactyla & Camelidae & Hemiauchenia macrocephala & 4.2 & 0.00941 & 11.62100299 ground dwelling & browser, grazer & species & herbivore & herbivore guild \\
\hline Carnivora & Felidae & Panthera onca & 1.6845 & 0.01002 & 11.253 scansorial & carnivore & species & carnivore & carnivore guild \\
\hline Carnivora & Felidae & Smilodon populator & 1.6845 & 0.01002 & 11.91818044 scansorial & carnivore & species & carnivore & carnivore guild \\
\hline Xenarthra & Megatheriidae & Eremotherium laurillardi & 12.809 & 0.01002 & 15.08386843 ground dwelling & herbivore & species & herbivore & herbivore guild \\
\hline Artiodactyla & Cervidae & Cervalces scotti & 1.3 & 0.01023 & 13.35 ground dwelling & grazer, browser & species & herbivore & herbivore guild \\
\hline Perissodactyla & Equidae & Equus occidentalis & 1.3 & 0.010965 & 13.09348384 ground dwelling & grazer & species & herbivore & herbivore guild \\
\hline Proboscidea & Elephantidae & Mammuthus columbi & 12.809 & 0.0112 & 16.14170861 ground dwelling & grazer & species & herbivore & herbivore guild \\
\hline Carnivora & Ursidae & Arctodus simus & 1.95 & 0.01132 & 13.02501881 ground dwelling & herbivore, carnivore & species & omnivore & carnivore guild \\
\hline Carnivora & Mustelidae & Martes pennanti & 0.3 & 0.011385 & 8.048788284 & & & NA & carnivore guild \\
\hline Carnivora & Mustelidae & Mustela erminea & 0.55 & 0.011385 & 3.775 scansorial & carnivore, omnivore & species & carnivore & carnivore guild \\
\hline Rodentia & Cricetidae & Phenacomys intermedius & 0.8 & 0.011385 & 4.353 ground dwelling & herbivore & species & herbivore & Rodentia \\
\hline Rodentia & Castoridae & Castoroides ohioensis & 1.1 & 0.011385 & 11.92279428 amphibious & browser & species & herbivore & Rodentia \\
\hline Rodentia & Cricetidae & Microtus xanthognathus & 1.3 & 0.011385 & 4.8225 ground dwelling & herbivore & species & herbivore & Rodentia \\
\hline Artiodactyla & Tayassuidae & Platygonus compressus & 1.3 & 0.011385 & 10.77669864 ground dwelling & browser, grazer & species & herbivore & herbivore guild \\
\hline Rodentia & Erethizontidae & Erethizon dorsatum & 1.55 & 0.011385 & 8.751 arboreal & herbivore & species & herbivore & Rodentia \\
\hline Eulipotyphla & Soricidae & Sorex cinereus & 2.2 & 0.011385 & 1.4645 ground dwelling & omnivore & species & omnivore & NA \\
\hline Rodentia & Sciuridae & Spermophilus tridecemlineatus & 1.3 & 0.01141 & 4.3945 semifossorial & granivore, frugivore & species & herbivore & Rodentia \\
\hline Xenarthra & Megalonychidae & Megalonyx jeffersonii & 1.6845 & 0.011445 & 13.37300465 arboreal & folivore & species & herbivore & herbivore guild \\
\hline Perissodactyla & Tapiridae & Tapirus merriami & 2 & 0.01145 & 13.32 amphibious & browser & species & herbivore & herbivore guild \\
\hline Perissodactyla & Equidae & Equus fraternus & 1.55 & 0.01155 & 11.65508789 ground dwelling & grazer & species & herbivore & herbivore guild \\
\hline Rodentia & Sciuridae & Cynomys ludovicianus & 1 & 0.01163 & 6.652 semifossorial & granivore, frugivore & species & herbivore & Rodentia \\
\hline Rodentia & Geomyidae & Geomys bursarius & 1.3 & 0.01163 & 4.1775 fossorial & browser & species & herbivore & Rodentia \\
\hline Carnivora & Ursidae & Ursus arctos & 1.6845 & 0.0117 & 11.18 ground dwelling & herbivore, carnivore & species & omnivore & carnivore guild \\
\hline Carnivora & Mustelidae & Mustela nigripes & 0.3 & 0.01228 & 5.52 scansorial & carnivore, omnivore & species & carnivore & carnivore guild \\
\hline Carnivora & Mustelidae & Mustela nivalis & 1.3 & 0.01228 & 1.25 scansorial & carnivore, omnivore & species & carnivore & carnivore guild \\
\hline Carnivora & Mustelidae & Gulo gulo & 1.6845 & 0.01228 & 8.9 scansorial & carnivore, omnivore & species & carnivore & carnivore guild \\
\hline Carnivora & Mustelidae & Taxidea taxus & 3.5 & 0.01228 & 7.5485 semifossorial & carnivore, omnivore & species & carnivore & carnivore guild \\
\hline Rodentia & Cricetidae & Onychomys leucogaster & 0.4 & 0.014695 & 3.6065 ground dwelling & herbivore & species & herbivore & Rodentia \\
\hline Rodentia & Cricetidae & Synaptomys cooperi & 1.3 & 0.014695 & 4.588 ground dwelling & herbivore & species & herbivore & Rodentia \\
\hline Xenarthra & Mylodontidae & Paramylodon harlani & 1.85 & 0.014695 & 13.95843272 ground dwelling & herbivore & species & herbivore & herbivore guild \\
\hline Artiodactyla & Bovidae & Bison priscus & 1.6845 & 0.015275 & 13.81551056 ground dwelling & grazer & species & herbivore & herbivore guild \\
\hline
\end{tabular}




\begin{tabular}{|c|c|c|}
\hline Carnivora & Canidae & Canis dirus \\
\hline Carnivora & Felidae & Leopardus pardalis \\
\hline Carnivora & Canidae & Protocyon troglodytes \\
\hline Rodentia & Cricetidae & Microtus ochrogaster \\
\hline Rodentia & Zapodidae & Zapus hudsonius \\
\hline Eulipotyphla & Soricidae & Blarina brevicauda \\
\hline Rodentia & Sciuridae & Cynomys niobrarius \\
\hline Rodentia & Cricetidae & Neotoma pygmaea \\
\hline Rodentia & Cricetidae & Neotoma findleyi \\
\hline Rodentia & Cricetidae & Peromyscus boylii \\
\hline Xenarthra & Nothrotheriidae & Nothrotheriops shastensis \\
\hline Rodentia & Sciuridae & Glaucomys sabrinus \\
\hline Rodentia & Cricetidae & Neofiber leonardi \\
\hline Artiodactyla & Cervidae & Alces alces \\
\hline Perissodactyla & Equidae & Equus complicatus \\
\hline Artiodactyla & Tayassuidae & Mylohyus fossilis \\
\hline Rodentia & Sciuridae & Sciurus aberti \\
\hline Rodentia & Cricetidae & Neotoma mexicana \\
\hline Rodentia & Cricetidae & Microtus californicus \\
\hline Carnivora & Felidae & Leopardus wiedii \\
\hline Artiodactyla & Bovidae & Euceratherium collinum \\
\hline Xenarthra & Dasypodidae & Dasypus bellus \\
\hline Carnivora & Mustelidae & Conepatus robustus \\
\hline Artiodactyla & Cervidae & Elaphodus cephalophus \\
\hline Artiodactyla & Capridae & Ovibos pallantis \\
\hline Perissodactyla & Equidae & Hippidion saldiasi \\
\hline Rodentia & Sciuridae & Sciurus niger \\
\hline Rodentia & Sciuridae & Spermophilus armatus \\
\hline Rodentia & Cricetidae & Neotoma stephensi \\
\hline Artiodactyla & Antilocapridae & Capromeryx minor \\
\hline Eulipotyphla & Soricidae & Sorex cinerus \\
\hline Carnivora & Canidae & Alopex lagopus \\
\hline Rodentia & Sciuridae & Spermophilus parryii \\
\hline Rodentia & Aplodontidae & Aplodontia rufa \\
\hline Carnivora & Felidae & Lynx canadensis \\
\hline Carnivora & Mustelidae & Conepatus leuconotus \\
\hline Artiodactyla & Bovidae & Bison latifrons \\
\hline Rodentia & Sciuridae & Tamias aristus \\
\hline Rodentia & Cricetidae & Neotoma fuscipes \\
\hline Artiodactyla & Cervidae & Navahoceros fricki \\
\hline Rodentia & Sciuridae & Cynomys spenceri \\
\hline Rodentia & Cricetidae & Peromyscus progressus \\
\hline Rodentia & Cricetidae & Reithrodontomys fulvescens \\
\hline Artiodactyla & Antilocapridae & Capromeryx furcifer \\
\hline Artiodactyla & Antilocapridae & Stockoceros conklingi \\
\hline Artiodactyla & Antilocapridae & $\begin{array}{l}\text { Tetrameryx shuleri } \\
\text { Megatapirus augustus }\end{array}$ \\
\hline Perissodactyla & Equidae & Equus niobrarensis \\
\hline Rodentia & Cricetidae & Sigmodon bakeri \\
\hline Rodentia & Sciuridae & Spermophilus richardsonii \\
\hline Rodentia & Sciuridae & Cynomys gunnisoni \\
\hline Carnivora & Felidae & Homotherium serum \\
\hline
\end{tabular}

\begin{tabular}{|c|c|c|c|c|}
\hline 1.6845 & 0153375 & 11.24033787 ground dwelling & carnivore, omnivore & \\
\hline 0.156 & 0.02006 & 9.011142726 scansorial & carnivore & pecies \\
\hline 0.4535 & 0.02006 & 10.27643727 ground dwelling & carnivore, omnivore & pecies \\
\hline 0.85 & 0.025643 & 4.17 ground dwelling & herbivore & pecies \\
\hline 1.3 & 0.025643 & 2.701 saltatorial & herbivore & pecies \\
\hline 2.75 & 0.025643 & 2.9355 ground dwelling & omnivore & pecies \\
\hline 0.3 & 0.0283 & 7.024 semifossorial & granivore, frugivore & pecies \\
\hline 29375 & 0.029375 & 4.78 ground dwelling & herbivore & species \\
\hline 0.069 & 0.029375 & 5.32 ground dwelling & ivore & pecies \\
\hline 0.069 & 0.029375 & dwelling & & species \\
\hline 2 & 0.029375 & 12.35479821 & & \\
\hline 0.4535 & 0.0294 & 4.15 gliding & granivore, frugivore & species \\
\hline 1.3 & 0.02987 & 6.71 amphibious & herbivore & species \\
\hline 1.6845 & 0.02987 & 13.35 ground dwelling & grazer, browser & species \\
\hline 1.6845 & 0.02987 & 12.84772836 ground dwelling & grazer & species \\
\hline 1.8 & 0.02987 & 10.9153034 ground dwelling & browser, grazer & species \\
\hline 0.1 & 0.056 & 5.79 arboreal & granivore, frugivore & pecies \\
\hline 0.25 & 0.056 & 5.1515 ground dwelling & & species \\
\hline 1.05 & 0.056 & 4.765 grou & & species \\
\hline 1.1 & 0.063 & 8.038 arboreal & carnivore & pecies \\
\hline 1.294 & 0.063 & 13.10004329 ground dwelling & grazer, brow & species \\
\hline 3.65 & 0.063 & 9.56029277 semifossorial & insectivore, $\mathrm{f}$ & species \\
\hline 0.069 & 0.069 & 8.664627705 scansorial & carnivore, omnivore & species \\
\hline 0.069 & 0.069 & 10.43071047 gro & & becies \\
\hline 0.069 & 0.069 & $12.65500767 \mathrm{~g}$ & & genus \\
\hline 0.069 & 0.069 & 12.4800112 ground dwelling & gra & species \\
\hline 0.069 & 0.069 & $5.94 \mathrm{arb}$ & graniv & species \\
\hline 0.069 & 0.069 & $4.642 \mathrm{sem}$ & granivore, fru & species \\
\hline 0.069 & 0.069 & 4.93 ground dwelling & herbivore & species \\
\hline 0.069 & & 8.8655 gro & $\mathrm{br}$ & pecies \\
\hline 0.069 & & $1.098612289 \mathrm{grc}$ & & enus \\
\hline 0.1 & 069 & dwelling & ca & species \\
\hline 0.1 & 0.069 & 5.9275 semifossorial & grar & species \\
\hline 0.156 & 0.069 & $6.8 \mathrm{se}$ & & pecies \\
\hline 0.2 & 0.069 & 9.229445038 scansorial & carnivore & pecies \\
\hline 0.3 & .069 & $6.811 \mathrm{sca}$ & carnivore, omnivore & species \\
\hline 0.3 & 069 & 13.85004933 ground dwelling & grazer & becies \\
\hline 0.3 & 69 & & granivore, frugivore & species \\
\hline 0.3 & & $\mathrm{ng}$ & & becies \\
\hline 0.4 & & elling & & \\
\hline 0.4 & 069 & fossorial & grar & pecies \\
\hline 0.4 & .069 & nd dwelling & & pecies \\
\hline 0.4 & .069 & nd dwelling & & pecies \\
\hline 0.4 & .069 & 9.460603074 ground dwelling & ser, grazer & species \\
\hline 0.4 & 069 & 10.87741198 ground dwelling & & species \\
\hline 0.4 & 069 & $11.04730652 \mathrm{grc}$ & & species \\
\hline 0.4535 & & 13.26289014 & & \\
\hline 1.006 & & 12.94596828 grou & & \\
\hline 1.05 & & & & $\mathrm{ci}$ \\
\hline 1.2 & 069 & 5.007 semifossorial & granivore, frugivore & species \\
\hline 1.294 & 069 & 6.551 semifossorial & granivore, frugivore & species \\
\hline 1.3 & 0.069 & 11.98931494 scansorial & carnivore & species \\
\hline
\end{tabular}

$\begin{array}{ll}\text { carnivore } & \text { carnivore guild } \\ \text { carnivore } & \text { carnivore guild } \\ \text { carnivore } & \text { carnivore guild } \\ \text { herbivore } & \text { Rodentia } \\ \text { herbivore } & \text { Rodentia } \\ \text { omnivore } & \text { NA } \\ \text { herbivore } & \text { Rodentia } \\ \text { herbivore } & \text { Rodentia } \\ \text { herbivore } & \text { Rodentia } \\ \text { herbivore } & \text { Rodentia } \\ \text { NA } & \text { NA } \\ \text { herbivore } & \text { Rodentia } \\ \text { herbivore } & \text { Rodentia } \\ \text { herbivore } & \text { herbivore guild } \\ \text { herbivore } & \text { herbivore guild } \\ \text { herbivore } & \text { herbivore guild } \\ \text { herbivore } & \text { Rodentia } \\ \text { herbivore } & \text { Rodentia } \\ \text { herbivore } & \text { Rodentia } \\ \text { carnivore } & \text { carnivore guild } \\ \text { herbivore } & \text { herbivore guild } \\ \text { omnivore } & \text { NA } \\ \text { carnivore } & \text { carnivore guild } \\ \text { herbivore } & \text { herbivore guild } \\ \text { herbivore } & \text { herbivore guild } \\ \text { herbivore } & \text { herbivore guild } \\ \text { herbivore } & \text { Rodentia } \\ \text { herbivore } & \text { Rodentia } \\ \text { herbivore } & \text { Rodentia } \\ \text { herbivore } & \text { herbivore guild } \\ \text { omnivore } & \text { NA } \\ \text { carnivore } & \text { carnivore guild } \\ \text { herbivore } & \text { Rodentia } \\ \text { herbivore } & \text { Rodentia } \\ \text { carnivore } & \text { carnivore guild } \\ \text { carnivore } & \text { carnivore guild } \\ \text { herbivore } & \text { herbivore guild } \\ \text { herbivore } & \text { Rodentia } \\ \text { herbivore } & \text { Rodentia } \\ \text { herbivore } & \text { herbivore guild } \\ \text { herbivore } & \text { Rodentia } \\ \text { herbivore } & \text { Rodentia } \\ \text { herbivore } & \text { Rodentia } \\ \text { herbivore } & \text { herbivore guild } \\ \text { herbivore } & \text { herbivore guild } \\ \text { herbivore } & \text { herbivore guild } \\ \text { NA } & \text { NA } \\ \text { herbivore } & \text { herbivore guild } \\ \text { herbivore } & \\ \text { herbivore } & \text { Rerbivore } \\ \text { carnivore } & \end{array}$




\begin{tabular}{|c|c|c|}
\hline Xenarthra & Pampatheriidae & Holmesina septentrionalis \\
\hline Rodentia & Cricetidae & Peromyscus polionotus \\
\hline Rodentia & Cricetidae & Reithrodontomys humulis \\
\hline Artiodactyla & Camelidae & Palaeolama mirifica \\
\hline Perissodactyla & Equidae & Equus hydruntinus \\
\hline Perissodactyla & Rhinocerotidae & Coelodonta antiquitatis \\
\hline Perissodactyla & Rhinocerotidae & Stephanorhinus hemitoechus \\
\hline Perissodactyla & Equidae & Equus leidyi \\
\hline Xenarthra & Megalonychidae & Megalonyx wheatleyi \\
\hline Proboscidea & Elephantidae & Mammuthus imperator \\
\hline Rodentia & Cricetidae & Ondatra annectens \\
\hline Eulipotyphla & Soricidae & Blarina carolinensis \\
\hline Rodentia & Cricetidae & Peromyscus cragini \\
\hline Artiodactyla & Tayassuidae & Platygonus vetus \\
\hline Rodentia & Cricetidae & Onychomys pedroensis \\
\hline Carnivora & Felidae & Miracinonyx inexpectatus \\
\hline Carnivora & Ursidae & Arctodus pristinus \\
\hline Perissodactyla & Equidae & Equus francisci \\
\hline Carnivora & Felidae & Smilodon gracilis \\
\hline Rodentia & Hydrochoeridae & Neochoerus aesopi \\
\hline Carnivora & Felidae & Miracinonyx studeri \\
\hline Proboscidea & Elephantidae & Stegodon orientalis \\
\hline Carnivora & Mustelidae & Mustela jacksoni \\
\hline Rodentia & Cricetidae & Predicrostonyx hopkinsi \\
\hline Rodentia & Cricetidae & Phenacomys deeringensis \\
\hline Rodentia & Cricetidae & Lasiopodomys deceitensis \\
\hline Rodentia & Cricetidae & Mictomys kansasensis \\
\hline Hicanodonta & Sclerocalyptidae & Glyptotherium floridanum \\
\hline Rodentia & Cricetidae & Mictomys meltoni \\
\hline Rodentia & Cricetidae & Microtus paroperarius \\
\hline Rodentia & Cricetidae & Microtus meadensis \\
\hline Rodentia & Cricetidae & Peromyscus berendsensis \\
\hline Carnivora & Mustelidae & Martes diluviana \\
\hline Carnivora & Canidae & Canis armbrusteri \\
\hline Carnivora & Canidae & Canis edwardii \\
\hline Rodentia & Cricetidae & Allophaiomys pliocaenicus \\
\hline & & Dama mesopotamica \\
\hline Perissodactyla & Equidae & Equus hemionus \\
\hline Proboscidea & Gomphotheriidae & Stegomastodon platensis \\
\hline Carnivora & Mustelidae & Martes foina \\
\hline Proboscidea & Elephantidae & Mammuthus trogontherii \\
\hline Carnivora & Ursidae & Ursus spelaeus \\
\hline Carnivora & Canidae & Canis adustus \\
\hline Perissodactyla & Rhinocerotidae & Ceratotherium simum \\
\hline Rodentia & Cricetidae & Microtus Ilanensis \\
\hline Artiodactyla & Camelidae & Hemiauchenia seymourensis \\
\hline Artiodactyla & Camelidae & Camelops minidokae \\
\hline Rodentia & Geomyidae & Thomomys potomacensis \\
\hline Xenarthra & Megatheriidae & Eremotherium eomigrans \\
\hline Rodentia & Cricetidae & Atopomys texensis \\
\hline Rodentia & Zapodidae & Zapus sandersi \\
\hline Rodentia & Cricetidae & Ondatra idahoensis \\
\hline
\end{tabular}

\begin{tabular}{|c|c|c|c|c|}
\hline 1.3 & 0.069 & 11.523 ground dwelling & omnivore & species \\
\hline 1.5 & 0.069 & 2.418 ground dwelling & herbivore & species \\
\hline 1.5 & 0.069 & 2.1525 ground dwelling & herbivore & species \\
\hline 1.55 & 0.069 & 12.00651158 ground dwelling & browser & species \\
\hline 1.6845 & 0.069 & 12.57671978 ground dwelling & grazer & genus \\
\hline 1.6845 & 0.069 & 14.87930487 ground dwelling & browser & species \\
\hline 1.6845 & 0.069 & 14.15168798 ground dwelling & browser & species \\
\hline 1.8 & 0.069 & 12.28077294 ground dwelling & grazer & species \\
\hline 1.8 & 0.069 & 12.85443924 arboreal & folivore & species \\
\hline 2.15 & 0.069 & 15.79803632 ground dwelling & grazer & species \\
\hline 2.15 & 0.069 & 6.822 amphibious & herbivore & species \\
\hline 2.15 & 0.069 & 2.562 ground dwelling & omnivore & species \\
\hline 2.2 & 0.069 & 2.7395 ground dwelling & herbivore & species \\
\hline 2.25 & 0.069 & 11.1691249 ground dwelling & browser, grazer & species \\
\hline 2.5 & 0.069 & 3.588 ground dwelling & herbivore & species \\
\hline 2.95 & 0.069 & 10.8342098 scansorial & carnivore & species \\
\hline 2.95 & 0.069 & 11.53894069 ground dwelling & herbivore, carnivore & species \\
\hline 3 & 0.069 & 12.28727832 ground dwelling & grazer & species \\
\hline 3.65 & 0.069 & 10.869 scansorial & carnivore & species \\
\hline 3.65 & 0.069 & 11.612 ground dwelling & herbivore & species \\
\hline 4.2 & 0.069 & 10.44648449 scansorial & carnivore & species \\
\hline 12.809 & 0.069 & 14.50858867 ground dwelling & grazer, browser & genus \\
\hline 0.1 & 0.1 & 6.675194185 scansorial & carnivore, omnivore & species \\
\hline 0.1 & 0.1 & 5.385 ground dwelling & herbivore & species \\
\hline 0.3 & 0.1 & 5.2 ground dwelling & herbivore & species \\
\hline 1.3 & 0.1 & 5.2 ground dwelling & herbivore & species \\
\hline 2.3 & 0.1 & 4.399 ground dwelling & herbivore & species \\
\hline 1.3 & 0.156 & 13.24041304 ground dwelling & omnivore & species \\
\hline 0.55 & 0.2 & 4.159 ground dwelling & herbivore & species \\
\hline 1.3 & 0.2 & 4.0905 ground dwelling & herbivore & species \\
\hline 2.15 & 0.2 & 4.24 ground dwelling & herbivore & species \\
\hline 0.85 & 0.3 & 2.58 ground dwelling & herbivore & species \\
\hline 0.85 & 0.3 & 8.942722519 & & \\
\hline 3.1 & 0.3 & 10.79059719 ground dwelling & carnivore, omnivore & species \\
\hline 3.15 & 0.3 & 10.54015577 ground dwelling & carnivore, omnivore & species \\
\hline 3.96 & 0.3 & 3.848 ground dwelling & herbivore & species \\
\hline 1.3 & 0.3905 & 11.19056355 ground dwelling & grazer, browser & species \\
\hline 1.3 & 0.3905 & 12.57671978 & & \\
\hline 0.3965 & 0.3965 & 8.374501983 ground dwelling & grazer & species \\
\hline 1.3 & 0.3965 & 7.958576904 scansorial & carnivore, omnivore & species \\
\hline 1.6845 & 0.3965 & 16.06879874 ground dwelling & grazer & species \\
\hline 3.094 & 0.3965 & 12.61125855 ground dwelling & herbivore, carnivore & species \\
\hline 3.96 & 0.3965 & 9.233366223 ground dwelling & carnivore, omnivore & species \\
\hline 11.521 & 0.3965 & 15.0964444 ground dwelling & grazer & species \\
\hline 0.6 & 0.4 & 4.3 ground dwelling & herbivore & species \\
\hline 0.7 & 0.4 & 12.237 & & \\
\hline 1.15 & 0.4 & 13.65893477 ground dwelling & browser, browser & species \\
\hline 1.3 & 0.4 & 4.14 fossorial & browser & species \\
\hline 1.6 & 0.4 & 14.76500393 ground dwelling & herbivore & species \\
\hline 1.6 & 0.4 & 3.0755 ground dwelling & herbivore & species \\
\hline 2.8 & 0.4 & 2.9395 saltatorial & herbivore & species \\
\hline 3 & 0.4 & 6.191 amphibious & herbivore & species \\
\hline
\end{tabular}

\begin{tabular}{|c|c|}
\hline & \\
\hline herbivore & Rodentia \\
\hline herbivore & Rodentia \\
\hline herbivore & herbivore guild \\
\hline herbivore & herbivore guild \\
\hline herbivore & herbivore guild \\
\hline herbivore & herbivore guild \\
\hline herbivore & herbivore guild \\
\hline herbivore & herbivore guild \\
\hline herbivore & herbivore guild \\
\hline herbivore & Rodentia \\
\hline omnivore & NA \\
\hline herbivore & Rodentia \\
\hline herbivore & herbivore guild \\
\hline herbivore & Rodentia \\
\hline carnivore & carnivore guild \\
\hline omnivore & carnivore guild \\
\hline herbivore & herbivore guild \\
\hline carnivore & carnivore guild \\
\hline herbivore & Rodentia \\
\hline carnivore & carnivore guild \\
\hline herbivore & herbivore guild \\
\hline carnivore & carnivore guild \\
\hline herbivore & Rodentia \\
\hline herbivore & Rodentia \\
\hline herbivore & Rodentia \\
\hline herbivore & Rodentia \\
\hline omnivore & NA \\
\hline herbivore & Rodentia \\
\hline herbivore & Rodentia \\
\hline herbivore & Rodentia \\
\hline herbivore & Rodentia \\
\hline NA & carnivore guild \\
\hline carnivore & carnivore guild \\
\hline carnivore & carnivore guild \\
\hline herbivore & Rodentia \\
\hline herbivore & herbivore guild \\
\hline NA & NA \\
\hline herbivore & herbivore guild \\
\hline carnivore & carnivore guild \\
\hline herbivore & herbivore guild \\
\hline omnivore & carnivore guild \\
\hline carnivore & carnivore guild \\
\hline herbivore & herbivore guild \\
\hline herbivore & Rodentia \\
\hline NA & NA \\
\hline herbivore & herbivore guild \\
\hline herbivore & Rodentia \\
\hline herbivore & herbivore guild \\
\hline herbivore & Rodentia \\
\hline herbivore & Rodentia \\
\hline herbivore & Rodentia \\
\hline
\end{tabular}




\begin{tabular}{|c|c|c|}
\hline Artiodactyla & Camelidae & Camelops traviswhitei \\
\hline Rodentia & Castoridae & Castor californicus \\
\hline Artiodactyla & Cervidae & Megaloceros savini \\
\hline Perissodactyla & Rhinocerotidae & Stephanorhinus kirchbergensis \\
\hline Artiodactyla & & Nemorhaedus goral \\
\hline \multirow[t]{2}{*}{ Proboscidea } & Elephantidae & Elephas antiquus \\
\hline & & Gigantopithecus blacki \\
\hline \multirow[t]{2}{*}{ Artiodactyla } & Camelidae & Camelus thomasi \\
\hline & & Dama clactoniana \\
\hline Artiodactyla & Cervidae & Praemegaceros verticornis \\
\hline Carnivora & Canidae & Canis mosbachensis \\
\hline Artiodactyla & Bovidae & Bison schoetensacki \\
\hline Perissodactyla & Equidae & Hippidion principale \\
\hline Perissodactyla & Rhinocerotidae & Stephanorhinus hundsheimensis \\
\hline Carnivora & Ursidae & Ursus deningeri \\
\hline \multirow[t]{2}{*}{ Carnivora } & Canidae & Canis arnensis \\
\hline & & Theropithecus oswaldi \\
\hline Carnivora & Hyaenidae & Pachycrocuta brevirostris \\
\hline Carnivora & Felidae & Panthera gombaszoegensis \\
\hline Carnivora & Canidae & Canis etruscus \\
\hline Proboscidea & Elephantidae & Mammuthus meridionalis \\
\hline Perissodactyla & Rhinocerotidae & Stephanorhinus etruscus \\
\hline Carnivora & Hyaenidae & Pliocrocuta perrieri \\
\hline Carnivora & Felidae & Homotherium crenatidens \\
\hline Rodentia & Heteromyidae & Dipodomys pattersoni \\
\hline Rodentia & Cricetidae & Proneofiber guildayi \\
\hline Rodentia & Cricetidae & Neotoma spelaea \\
\hline Rodentia & Cricetidae & Sigmodon curtisi \\
\hline Rodentia & Sciuridae & Spermophilus Iorisrusselli \\
\hline Eulipotyphla & Soricidae & Sorex cudahyensis \\
\hline Eulipotyphla & Soricidae & Sorex lacustris \\
\hline Eulipotyphla & Soricidae & Sorex megapalustris \\
\hline Eulipotyphla & Soricidae & Sorex pratensis \\
\hline Xenarthra & Nothrotheriidae & Nothrotheriops texanus \\
\hline Rodentia & Cricetidae & Reithrodontomys moorei \\
\hline Artiodactyla & Camelidae & Camelops sulcatus \\
\hline Carnivora & Canidae & Cuon texanus \\
\hline Hicanodonta & Sclerocalyptidae & Glyptotherium arizonae \\
\hline Proboscidea & Gomphotheriidae & Cuvieronius tropicus \\
\hline Rodentia & Cricetidae & Onychomys hollisteri \\
\hline Artiodactyla & Tayassuidae & Platygonus bicalcaratus \\
\hline Rodentia & Cricetidae & Mictomys vetus \\
\hline Rodentia & Castoridae & Dipoides stovalli \\
\hline Perissodactyla & Equidae & Equus idahoensis \\
\hline Carnivora & Hyaenidae & Chasmaporthetes ossifragus \\
\hline Rodentia & Geomyidae & Thomomys gidleyi \\
\hline Artiodactyla & Camelidae & Gigantocamelus spatulus \\
\hline Rodentia & Erethizontidae & Erethizon kleini \\
\hline Rodentia & Sciuridae & Spermophilus meadensis \\
\hline Rodentia & Sciuridae & Spermophilus bensoni \\
\hline Rodentia & Heteromyidae & Perognathus pearlettensis \\
\hline Artiodactyla & Hippopotamidae & Hippopotamus gorgops \\
\hline
\end{tabular}

$\begin{array}{r}3.5 \\ 10.65 \\ 0.4535 \\ 0.4535 \\ 0.4535 \\ 0.4535 \\ 0.4535 \\ 1.3 \\ 1.3 \\ 1.357 \\ 1.6845 \\ 1.6845 \\ 1.6845 \\ 1.6845 \\ 1.6845 \\ 2.894 \\ 3.094 \\ 3.96 \\ 3.96 \\ 3.96 \\ 3.96 \\ 3.96 \\ 4.266 \\ 6.289 \\ 0.5 \\ 0.5 \\ 1.3 \\ 2.6 \\ 0.55 \\ 0.55 \\ 0.55 \\ 0.55 \\ 0.55 \\ 1.8 \\ 2.15 \\ 0.6 \\ 0.672 \\ 0.6 \\ 4.2 \\ 10.25 \\ 1.75 \\ 4.125 \\ 3 \\ 0.85 \\ 2.9 \\ 3.4 \\ 5.25 \\ 4.95 \\ 1.5 \\ \hline\end{array}$

0.413 .86447297 ground dwelling browser, browser species

$0.4 \quad 9.47$ amphibious browser 0.453512 .39711814 ground dwelling grazer, browser genus 0.453514 .28523792 ground dwelling browser genus $\begin{array}{ll}0.4535 & 10.46294666 \mathrm{NA}\end{array}$ 0.453515 .68751224

$0.4535 \quad 12.85619415$ ground dwelling omnivore 0.453513 .12473503 ground dwelling browser, grazer $0.453511 .60733145 \mathrm{NA}$ NA

0.453512 .26817338 ground dwelling grazer, browser 0.45359 .797499571 ground dwelling carnivore, omnivore genus 0.453513 .34348061 ground dwelling grazer 0.453513 .14776088 ground dwelling grazer, browser spenus 0.453514 .15168798 ground dwelling browser species 0.453512 .52376032 ground dwelling herbivore, carnivore genus 0.453510 .30867346 ground dwelling carnivore, omnivore genus 0.453511 .47210347 arboreal frugivore, folivore species 0.453511 .69482969 ground dwelling carnivore genus 0.453511 .40700655 scansorial carnivore species 0.453510 .30867346 ground dwelling carnivore, omnivore genus 0.453515 .65067088 ground dwelling grazer species 0.453514 .15168798 ground dwelling browser species 0.453511 .28266696 ground dwelling carnivore species 0.453512 .35106644 scansorial carnivore genus $\begin{array}{llll}0.5 & 4.22 \text { saltatorial } & \text { granivore } & \text { species }\end{array}$

$0.5 \quad 6.844$ ground dwelling herbivore species

$0.5 \quad 5.451$ ground dwelling herbivore species

$0.5 \quad 4.548$ ground dwelling herbivore species

$0.55 \quad 5.115$ semifossorial granivore, frugivore species $\begin{array}{ll}0.55 & 1.895548891 \text { ground dwelling omnivore species }\end{array}$ $\begin{array}{ll}0.55 & 2.407438134 \text { ground dwelling omnivore species }\end{array}$ 0.552 .899640406 ground dwelling omnivore species $\begin{array}{ll}0.552 .017572923 \text { ground dwelling omnivore species } \\ 0.55 & 12.68512893\end{array}$ 0.5512 .68512893 ground dwelling herbivore species $0.55 \quad 2.406$ ground dwelling herbivore species

$0.6 \quad 13.811$ ground dwelling browser, browser species $0.6 \quad 11.413$

0.713 .57938308 ground dwelling omnivore species

$0.7 \quad 15.347$ ground dwelling browser species

$0.75 \quad 3.195$ ground dwelling herbivore species

0.7511 .25280939 ground dwelling browser, grazer species

$0.8 \quad 4.728$ ground dwelling herbivore species 0.858 .098201926 amphibious browser species

0.8513 .124 ground dwelling grazer species

0.8511 .82335784 ground dwelling carnivore species

0.93 .6215 fossorial browser species

$1 \quad 14.463888$ ground dwelling browser, browser species

$\begin{array}{rrll}1.1 & 8.258 \text { arboreal } & \text { herbivore } & \text { species } \\ 1.15 & 4.61 \text { semifossorial } & \text { granivore, frugivore } & \text { species }\end{array}$

$1.15 \quad 5.2175$

1.201 .9325 ground dwelling granivore, browser species

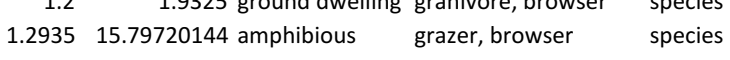

\begin{tabular}{|c|c|}
\hline herbivore & herbivore guild \\
\hline herbivore & Rodentia \\
\hline herbivore & herbivore guild \\
\hline herbivore & herbivore guild \\
\hline NA & NA \\
\hline NA & NA \\
\hline omnivore & NA \\
\hline herbivore & herbivore guild \\
\hline NA & NA \\
\hline herbivore & herbivore guild \\
\hline carnivore & carnivore guild \\
\hline herbivore & herbivore guild \\
\hline herbivore & herbivore guild \\
\hline herbivore & herbivore guild \\
\hline omnivore & carnivore guild \\
\hline carnivore & carnivore guild \\
\hline herbivore & herbivore guild \\
\hline carnivore & carnivore guild \\
\hline carnivore & carnivore guild \\
\hline carnivore & carnivore guild \\
\hline herbivore & herbivore guild \\
\hline herbivore & herbivore guild \\
\hline carnivore & carnivore guild \\
\hline carnivore & carnivore guild \\
\hline herbivore & Rodentia \\
\hline herbivore & Rodentia \\
\hline herbivore & Rodentia \\
\hline herbivore & Rodentia \\
\hline herbivore & Rodentia \\
\hline omnivore & NA \\
\hline omnivore & NA \\
\hline omnivore & NA \\
\hline omnivore & NA \\
\hline herbivore & herbivore guild \\
\hline herbivore & Rodentia \\
\hline herbivore & herbivore guild \\
\hline NA & carnivore guild \\
\hline omnivore & NA \\
\hline herbivore & herbivore guild \\
\hline herbivore & Rodentia \\
\hline herbivore & herbivore guild \\
\hline herbivore & Rodentia \\
\hline herbivore & Rodentia \\
\hline herbivore & herbivore guild \\
\hline carnivore & carnivore guild \\
\hline herbivore & Rodentia \\
\hline herbivore & herbivore guild \\
\hline herbivore & Rodentia \\
\hline herbivore & Rodentia \\
\hline NA & Rodentia \\
\hline herbivore & Rodentia \\
\hline herbivore & herbivore guild \\
\hline
\end{tabular}




\begin{tabular}{|c|c|c|c|}
\hline Artiodactyla & Bovidae & Antidorcas bondi & 3.0565 \\
\hline Artiodactyla & Bovidae & Antidorcas recki & 3.96 \\
\hline Artiodactyla & Bovidae & Kobus sigmoidalis & 3.96 \\
\hline Proboscidea & Elephantidae & Elephas recki & 3.96 \\
\hline Artiodactyla & Suidae & Metridiochoerus andrewsi & 3.96 \\
\hline Artiodactyla & Bovidae & Gazella janenschi & 11.521 \\
\hline Proboscidea & Deinotheriidae & Deinotherium bozasi & 11.521 \\
\hline Perissodactyla & Rhinocerotidae & Elasmotherium sibiricum & 1.294 \\
\hline Artiodactyla & Suidae & Hippopotamodon antiquus & 9.2 \\
\hline Perissodactyla & Rhinocerotidae & Stephanorhinus megarhinus & 9.427 \\
\hline Proboscidea & Mammutidae & Mammut borsoni & 9.427 \\
\hline Carnivora & Felidae & Megantereon nihowanensis & 1.3 \\
\hline Carnivora & Canidae & Vulpes chikushanensis & 1.3 \\
\hline Artiodactyla & Cervidae & Megaloceros giganteus & 1.3 \\
\hline Artiodactyla & Camelidae & Camelus knoblochi & 1.3 \\
\hline Rodentia & Castoridae & Castoroides leiseyorum & 1.3 \\
\hline \multirow{2}{*}{ Artiodactyla } & Cervidae & Alces latifrons & 1.3 \\
\hline & & Ourebia ourebi & 1.3 \\
\hline Carnivora & Canidae & Lycaon lycaonoides & 1.6845 \\
\hline Carnivora & Canidae & Xenocyon falconeri & 1.6845 \\
\hline Artiodactyla & Bovidae & Antidorcas australis & 1.6845 \\
\hline Artiodactyla & Hippopotamidae & Hippopotamus antiquus & 1.6845 \\
\hline Artiodactyla & Cervidae & Metacervulus capreolinus & 1.6845 \\
\hline Artiodactyla & Bovidae & Leptobos etruscus & 2.672 \\
\hline Hicanodonta & Sclerocalyptidae & Glyptotherium texanum & 3.3 \\
\hline Rodentia & Heteromyidae & Perognathus gidleyi & 3.5 \\
\hline Carnivora & Ursidae & Ursus etruscus & 3.96 \\
\hline Carnivora & Felidae & Acinonyx pardinensis & 4.266 \\
\hline Carnivora & Felidae & Lynx issiodorensis & 4.466 \\
\hline Carnivora & & Borophagus diversidens & 4.6 \\
\hline Artiodactyla & Camelidae & Paracamelus gigas & 7.098 \\
\hline Perissodactyla & Rhinocerotidae & Ceratotherium praecox & 12.809 \\
\hline \multirow[t]{3}{*}{ Artiodactyla } & Bovidae & Bison degiulii & 1.6845 \\
\hline & & Pseudodama farnetensis & 1.6845 \\
\hline & Cricetidae & Ophiomys parvus & 2.8 \\
\hline Rodentia & Cricetidae & Sigmodon minor & 5.25 \\
\hline Perissodactyla & Equidae & Equus simplicidens & 4.6 \\
\hline Artiodactyla & Camelidae & Hemiauchenia gracilis & 3.35 \\
\hline Xenarthra & Pampatheriidae & Holmesina floridanus & 3.65 \\
\hline Carnivora & Mustelidae & Trigonictis macrodon & 4.5 \\
\hline Xenarthra & Megalonychidae & Megalonyx leptostomus & 4.5 \\
\hline Rodentia & Cricetidae & Reithrodontomys wetmorei & 4.7 \\
\hline Rodentia & Erethizontidae & Erethizon poyeri & 1.55 \\
\hline \multirow[t]{2}{*}{ Rodentia } & Cricetidae & Sigmodon libitinus & 1.8 \\
\hline & & Pachyarmatherium leiseyi & 2.3 \\
\hline Carnivora & Mustelidae & Satherium piscinarium & 3.9 \\
\hline Carnivora & Mustelidae & Trigonictis cookii & 3.9 \\
\hline Rodentia & Cricetidae & Peromyscus hagermanensis & 4 \\
\hline \multirow[t]{2}{*}{ Rodentia } & Hydrochoeridae & Neochoerus dichroplax & 4.2 \\
\hline & & Metacervoceros rhenanus & 1.606 \\
\hline Artiodactyla & Cervidae & Eucladoceros ctenoides & 2.672 \\
\hline Artiodactyla & Cervidae & Croizetoceros ramosus & 3.96 \\
\hline
\end{tabular}

1.293510 .43071047 ground dwelling grazer, browser genus 1.293510 .23959591 ground dwelling grazer, browser 1.293511 .63496247 ground dwelling grazer, browser 1.293516 .30041721

1.293511 .91818044 ground dwelling omnivore 1.293510 .30867346 ground dwelling grazer, browser

1.293516 .67485021 ground dwelling grazer, browser

$1.294 \quad 15.42494847$ ground dwelling browser

1.29413 .14315571 ground dwelling omnivore

1.29414 .07800526 ground dwelling browser

1.29415 .78422081 ground dwelling browser

1.311 .08003947 scansorial carnivore

1.38 .765941449 ground dwelling carnivore, omnivore

1.3 13.45860987 ground dwelling grazer, browser

$\begin{array}{ll}1.3 & 13.21683843 \text { ground dwelling browser, grazer }\end{array}$

1.3

11.733 amphibious browser

1.312 .89908169

NA

1.310 .17742611 ground dwelling carnivore

species

1.310 .16591319 ground dwelling carnivore, omnivore genus

1.310 .59189143 ground dwelling grazer, browser genus

1.314 .90463331 amphibious grazer, browser species

1.310 .5504449 ground dwelling grazer, browser genus

1.312 .89908169 ground dwelling grazer, browser genus

1.312 .34904681 ground dwelling omnivore species

1.3 2.4765 ground dwelling granivore, browser species

1.311 .40700655 ground dwelling herbivore, carnivore genus

1.310 .81984735 scansorial carnivore genus

1.310 .30867346 scansorial carnivore genus

$\begin{array}{lll}1.3 & 11.20740799 \text { ground dwelling carnivore species }\end{array}$

1.314 .21385778

1.314 .55694296 ground dwelling grazer genus

$1.357 \quad 13.41025558$ ground dwelling grazer

$1.357 \quad 11.3839807 \mathrm{NA} \quad \mathrm{NA}$

NA

$1.4 \quad 3.918$ ground dwelling herbivore

1.4512 .94069272

1.510 .94687279 ground dwelling browser, grazer species

$1.5 \quad 11.137$ ground dwelling omnivore species

1.57 .6145 scansorial carnivore, omnivore species

1.512 .69075604 arboreal folivore species

$1.5 \quad 2.1635$ ground dwelling herbivore species

1.558 .969 arboreal herbivore species

1.554 .329 ground dwelling herbivore species

1.559 .643420647 ground dwelling omnivore species

1.558 .597 scansorial carnivore, omnivore species

1.558 .588 scansorial carnivore, omnivore species

$1.55 \quad 2.9935$ ground dwelling herbivore $\quad$ species

$1.55 \quad 12.2115$

$\begin{array}{ll}1.606 & 11.15602478 \mathrm{NA}\end{array}$

160612.44086726 ground dwelling grazer, browser

$\begin{array}{lll}1.606 & 11.28957471 \text { ground dwelling grazer, browser } & \begin{array}{l}\text { genus } \\ \text { species }\end{array}\end{array}$

\begin{tabular}{|c|c|}
\hline herbivore & herbivore guild \\
\hline herbivore & herbivore guild \\
\hline herbivore & herbivore guild \\
\hline NA & NA \\
\hline omnivore & NA \\
\hline herbivore & herbivore guild \\
\hline herbivore & herbivore guild \\
\hline herbivore & herbivore guild \\
\hline omnivore & NA \\
\hline herbivore & herbivore guild \\
\hline herbivore & herbivore guild \\
\hline carnivore & carnivore guild \\
\hline carnivore & carnivore guild \\
\hline herbivore & herbivore guild \\
\hline herbivore & herbivore guild \\
\hline herbivore & Rodentia \\
\hline NA & NA \\
\hline NA & NA \\
\hline carnivore & carnivore guild \\
\hline carnivore & carnivore guild \\
\hline herbivore & herbivore guild \\
\hline herbivore & herbivore guild \\
\hline herbivore & herbivore guild \\
\hline herbivore & herbivore guild \\
\hline omnivore & NA \\
\hline herbivore & Rodentia \\
\hline omnivore & carnivore guild \\
\hline carnivore & carnivore guild \\
\hline carnivore & carnivore guild \\
\hline carnivore & carnivore guild \\
\hline NA & NA \\
\hline herbivore & herbivore guild \\
\hline herbivore & herbivore guild \\
\hline NA & NA \\
\hline herbivore & herbivore guild \\
\hline herbivore & Rodentia \\
\hline NA & NA \\
\hline herbivore & herbivore guild \\
\hline omnivore & NA \\
\hline carnivore & carnivore guild \\
\hline herbivore & herbivore guild \\
\hline herbivore & Rodentia \\
\hline herbivore & Rodentia \\
\hline herbivore & Rodentia \\
\hline omnivore & NA \\
\hline carnivore & carnivore guild \\
\hline carnivore & carnivore guild \\
\hline herbivore & Rodentia \\
\hline NA & Rodentia \\
\hline NA & NA \\
\hline herbivore & herbivore guild \\
\hline herbivore & herbivore guild \\
\hline
\end{tabular}




\begin{tabular}{|c|c|c|c|c|c|c|c|c|c|}
\hline Carnivora & Felidae & Lynx pardinus & 1.6845 & 1.6845 & 9.141262819 scansorial & carnivore & species & carnivore & carnivore guild \\
\hline Carnivora & Hyaenidae & Pachycrocuta licenti & 1.6845 & 1.6845 & 11.78002534 ground dwelling & carnivore & genus & carnivore & carnivore guild \\
\hline Carnivora & Mustelidae & Meles iberica & 1.6845 & 1.6845 & 9.210340372 semifossorial & carnivore, omnivore & species & carnivore & carnivore guild \\
\hline Artiodactyla & Cervidae & Axis eurygonos & 1.6845 & 1.6845 & 11.3839807 ground dwelling & grazer & genus & herbivore & herbivore guild \\
\hline Artiodactyla & Cervidae & Eucladoceros tetraceros & 1.6845 & 1.6845 & 12.40632848 ground dwelling & grazer, browser & genus & herbivore & herbivore guild \\
\hline \multirow{2}{*}{ Artiodactyla } & Bovidae & Leptobos vallisarni & 1.6845 & 1.6845 & 12.84151706 ground dwelling & grazer, browser & genus & herbivore & herbivore guild \\
\hline & & Megaceroides solilhacus & 1.6845 & 1.6845 & $12.41784141 \mathrm{NA}$ & NA & genus & NA & NA \\
\hline Rodentia & Cricetidae & Microtus pliocaenicus & 1.6845 & 1.6845 & 3.85 & & & NA & Rodentia \\
\hline Carnivora & Felidae & Megantereon cultridens & 3.96 & 1.6845 & 11.05010586 scansorial & carnivore & species & carnivore & carnivore guild \\
\hline Carnivora & Canidae & Vulpes alopecoides & 3.96 & 1.6845 & 9.304746361 ground dwelling & carnivore, omnivore & genus & carnivore & carnivore guild \\
\hline Artiodactyla & Bovidae & Gazellospira torticornis & 3.96 & 1.6845 & 11.89745718 ground dwelling & grazer, browser & genus & herbivore & herbivore guild \\
\hline Artiodactyla & Cervidae & Libralces gallicus & 3.96 & 1.6845 & 12.9290153 ground dwelling & grazer, browser & genus & herbivore & herbivore guild \\
\hline Artiodactyla & Bovidae & Gazella borbonica & 4.266 & 1.6845 & 10.08532271 ground dwelling & grazer, browser & genus & herbivore & herbivore guild \\
\hline Lagomorpha & Leporidae & Hypolagus furlongi & 3.35 & 1.7 & 5.758 ground dwelling & grazer, browser & species & herbivore & herbivore guild \\
\hline Rodentia & Sciuridae & Spermophilus cragini & 1.75 & 1.75 & 6.365 semifossorial & granivore, frugivore & species & herbivore & Rodentia \\
\hline Rodentia & Zapodidae & Zapus burti & 1.75 & 1.75 & 3.0585 saltatorial & herbivore & species & herbivore & Rodentia \\
\hline Eulipotyphla & Soricidae & Sorex taylori & 3.5 & 1.75 & 2.01936836 ground dwelling & omnivore & species & omnivore & NA \\
\hline Rodentia & Heteromyidae & Dipodomys hibbardi & 3.6 & 1.75 & 2.8805 saltatorial & granivore & species & herbivore & Rodentia \\
\hline Rodentia & Cricetidae & Neotoma taylori & 5.25 & 1.75 & 5.492 ground dwelling & herbivore & species & herbivore & Rodentia \\
\hline Perissodactyla & Equidae & Hippotherium primigenium & 12.629 & 1.806 & 12.52145774 ground dwelling & grazer, browser & species & herbivore & herbivore guild \\
\hline Rodentia & Cricetidae & Sigmodon lindsayi & 1.85 & 1.85 & 4.7925 ground dwelling & herbivore & species & herbivore & Rodentia \\
\hline Carnivora & Felidae & Puma lacustris & 3.35 & 1.85 & 9.856323744 & & & NA & carnivore guild \\
\hline Eulipotyphla & Soricidae & Notiosorex jacksoni & 3.5 & 1.85 & 2.441699545 ground dwelling & omnivore & species & omnivore & NA \\
\hline Rodentia & Erethizontidae & Erethizon bathygnathum & 2.6 & 1.95 & 9.124 arboreal & herbivore & species & herbivore & Rodentia \\
\hline Carnivora & Procyonidae & Bassariscus casei & 3.5 & 1.95 & 7.403347911 arboreal & frugivore, carnivore & species & omnivore & carnivore guild \\
\hline Rodentia & Cricetidae & Ondatra minor & 3.9 & 2 & 5.867 amphibious & herbivore & species & herbivore & Rodentia \\
\hline Xenarthra & Mylodontidae & Glossotherium chapadmalense & 4.9 & 2 & 12.646068 ground dwelling & herbivore & species & herbivore & herbivore guild \\
\hline Proboscidea & Gomphotheriidae & Stegomastodon mirificus & 4.25 & 2.1 & 14.899 ground dwelling & grazer & species & herbivore & herbivore guild \\
\hline Rodentia & Cricetidae & Bensonomys arizonae & 5.25 & 2.1 & 2.954910279 & & & NA & Rodentia \\
\hline Rodentia & Cricetidae & Hibbardomys zakrzewskii & 2.15 & 2.15 & 4.4325 ground dwelling & herbivore & species & herbivore & Rodentia \\
\hline Rodentia & Zapodidae & Javazapus weeksi & 2.15 & 2.15 & 2.48 ground dwelling & herbivore & species & herbivore & Rodentia \\
\hline Rodentia & Cricetidae & Mimomys dakotaensis & 2.15 & 2.15 & 4.3015 ground dwelling & herbivore & species & herbivore & Rodentia \\
\hline Rodentia & Zapodidae & Zapus sykesae & 2.15 & 2.15 & 2.84 saltatorial & herbivore & species & herbivore & Rodentia \\
\hline Rodentia & Cricetidae & Guildayomys hibbardi & 2.4 & 2.15 & 4.4125 ground dwelling & herbivore & species & herbivore & Rodentia \\
\hline Rodentia & Cricetidae & Hibbardomys marthae & 2.4 & 2.15 & 4.439 ground dwelling & herbivore & species & herbivore & Rodentia \\
\hline Rodentia & Cricetidae & Pliophenacomys osborni & 2.8 & 2.15 & 4.458 ground dwelling & herbivore & species & herbivore & Rodentia \\
\hline \multirow[t]{2}{*}{ Rodentia } & Heteromyidae & Prodipodomys idahoensis & 5.75 & 2.15 & 3.1105 ground dwelling & granivore, browser & species & herbivore & Rodentia \\
\hline & & Pseudodama nestii & 2.197 & 2.197 & $11.64417282 \mathrm{NA}$ & NA & genus & NA & NA \\
\hline Carnivora & Mustelidae & Meles thorali & 2.672 & 2.197 & 9.680067731 semifossorial & carnivore, omnivore & genus & carnivore & carnivore guild \\
\hline Artiodactyla & Bovidae & Gallogoral meneghinii & 2.672 & 2.197 & 11.82147187 ground dwelling & grazer, browser & genus & herbivore & herbivore guild \\
\hline Artiodactyla & Cervidae & Eucladoceros dicranios & 3.96 & 2.197 & 12.44086726 ground dwelling & grazer, browser & genus & herbivore & herbivore guild \\
\hline Artiodactyla & Bovidae & Leptobos stenometopon & 3.96 & 2.197 & 11.89054942 ground dwelling & grazer, browser & genus & herbivore & herbivore guild \\
\hline Artiodactyla & Bovidae & Procamptoceras brivatense & 3.96 & 2.197 & 11.58891077 ground dwelling & grazer, browser & genus & herbivore & herbivore guild \\
\hline Carnivora & Canidae & Nyctereutes megamastoides & 3.96 & 2.197 & 9.903418485 ground dwelling & omnivore, carnivore & genus & omnivore & carnivore guild \\
\hline Carnivora & Hyaenidae & Chasmaporthetes lunensis & 4.266 & 2.197 & 11.23661525 ground dwelling & carnivore & genus & carnivore & carnivore guild \\
\hline Carnivora & Mustelidae & Baranogale antiqua & 4.55 & 2.197 & 8.517262259 scansorial & carnivore, omnivore & genus & carnivore & carnivore guild \\
\hline Rodentia & Cricetidae & Hibbardomys voorhiesi & 2.5 & 2.2 & 4.509 ground dwelling & herbivore & species & herbivore & Rodentia \\
\hline Rodentia & Sciuridae & Spermophilus boothi & 3 & 2.2 & 4.873505363 semifossorial & granivore, frugivore & species & herbivore & Rodentia \\
\hline Rodentia & Cricetidae & Pliolemmus antiquus & 3 & 2.2 & 5.30822753 ground dwelling & herbivore & species & herbivore & Rodentia \\
\hline Eulipotyphla & Soricidae & Sorex sandersi & 3 & 2.2 & 2.058549929 ground dwelling & omnivore & species & omnivore & NA \\
\hline Rodentia & Cricetidae & Bensonomys meadensis & 3.4 & 2.2 & 2.923161581 & & & NA & Rodentia \\
\hline Perissodactyla & Equidae & Nannippus peninsulatus & 5.5 & 2.2 & 11.152 ground dwelling & grazer & species & herbivore & herbivore guild \\
\hline
\end{tabular}




\begin{tabular}{|c|c|c|}
\hline Carnivora & Felidae & Homotherium idahoensis \\
\hline Lagomorpha & Leporidae & Hypolagus gidleyi \\
\hline Rodentia & Cricetidae & Nebraskomys mcgrewi \\
\hline Rodentia & Cricetidae & Reithrodontomys galushai \\
\hline Carnivora & Mustelidae & Mustela rexroadensis \\
\hline Eulipotyphla & Soricidae & Cryptotis kansasensis \\
\hline Rodentia & Heteromyidae & Prodipodomys centralis \\
\hline Perissodactyla & Equidae & Hipparion dietrichi \\
\hline Artiodactyla & Bovidae & Palaeoryx pallasi \\
\hline Artiodactyla & Giraffidae & Helladotherium duvernoyi \\
\hline Rodentia & Cricetidae & Sigmodon hudspethensis \\
\hline Perissodactyla & Equidae & Equus cumminsii \\
\hline Lagomorpha & Leporidae & Notolagus lepusculus \\
\hline Lagomorpha & Leporidae & Nekrolagus progressus \\
\hline Artiodactyla & Giraffidae & Giraffa gracilis \\
\hline Artiodactyla & Suidae & Propotamochoerus hysudricus \\
\hline Rodentia & Cricetidae & Neotoma quadriplicata \\
\hline Rodentia & Cricetidae & Hibbardomys fayae \\
\hline Rodentia & Cricetidae & Pliophenacomys dixonensis \\
\hline Eulipotyphla & Soricidae & Planisorex dixonensis \\
\hline Rodentia & Cricetidae & Ondatra meadensis \\
\hline Artiodactyla & Camelidae & Capricamelus gettyi \\
\hline Rodentia & Castoridae & Procastoroides idahoensis \\
\hline Rodentia & Cricetidae & Neotoma fossilis \\
\hline Lagomorpha & Leporidae & Hypolagus edensis \\
\hline Perissodactyla & Tapiridae & Tapirus arvernensis \\
\hline Carnivora & Ursidae & Ursus minimus \\
\hline Rodentia & Geomyidae & Thomomys carsonensis \\
\hline Rodentia & Cricetidae & Pliophenacomys primaevus \\
\hline Rodentia & Sciuridae & Spermophilus howelli \\
\hline Carnivora & Canidae & Canis lepophagus \\
\hline \multirow{2}{*}{ Carnivora } & Mustelidae & Buisnictis burrowsi \\
\hline & Cricetidae & Ophiomys fricki \\
\hline Rodentia & Sciuridae & Spermophilus johnstoni \\
\hline Rodentia & Sciuridae & Spermophilus meltoni \\
\hline Rodentia & Cricetidae & Hibbardomys skinneri \\
\hline Rodentia & Cricetidae & Repomys arizonensis \\
\hline Proboscidea & Mammutidae & Mammut raki \\
\hline Artiodactyla & Camelidae & Blancocamelus meadei \\
\hline Proboscidea & Gomphotheriidae & Stegomastodon primitivus \\
\hline Rodentia & Cricetidae & Ogmodontomys poaphagus \\
\hline Rodentia & Cricetidae & Peromyscus kansasensis \\
\hline Rodentia & Cricetidae & Reithrodontomys rexroadensis \\
\hline Rodentia & Castoridae & Procastoroides sweeti \\
\hline Artiodactyla & Antilocapridae & Capromeryx tauntonensis \\
\hline \multirow[t]{3}{*}{ Rodentia } & Castoridae & Dipoides rexroadensis \\
\hline & Cricetidae & Ophiomys magilli \\
\hline & & Proboscidipparion sinense \\
\hline Artiodactyla & Bovidae & Gazella sinensis \\
\hline Artiodactyla & Bovidae & Madoqua avifluminis \\
\hline Carnivora & Viverridae & Viverra leakeyi \\
\hline Lagomorpha & Leporidae & Alilepus wilsoni \\
\hline
\end{tabular}

$\begin{array}{r}2.6 \\ 8.1 \\ 3 \\ 2.5 \\ 7.25 \\ 2.55 \\ 3.5 \\ 8.225 \\ 9.427 \\ 9.427 \\ 2.6 \\ 4.125 \\ 4.7 \\ 7.6 \\ 3.96 \\ 9.427 \\ 5.25 \\ 2.75 \\ 2.75 \\ 3 \\ 4 \\ 2.8 \\ 3.35 \\ 3.4 \\ 11.8 \\ 4.266 \\ 4.266 \\ 2.9 \\ 3.4 \\ 8.3 \\ 7.4 \\ 3 \\ 3 \\ 3 \\ 3 \\ 3.994 \\ 3.96 \\ 3.96 \\ 3.4 \\ 3 \\ \hline 3 \\ 3.3 \\ 3.3 \\ 3.5 \\ 3.5 \\ 3.5 \\ 3.5 \\ 3.5 \\ 4.2 \\ 4.5 \\ 4.6 \\ \hline\end{array}$

\begin{tabular}{|c|c|c|c|}
\hline 2.35 & 12.138 scansorial & carnivore & species \\
\hline 2.35 & 6.679 ground dwelling & grazer, browser & species \\
\hline 2.4 & 3.831 ground dwelling & herbivore & pecies \\
\hline 2.5 & 2.386 ground dwelling & herbivore & oecies \\
\hline 2.5 & 4.856405501 scansorial & carnivore, omnivore & pecies \\
\hline 2.55 & 3.509 ground dwelling & omnivore & pecies \\
\hline 2.55 & 3.057 ground dwelling & granivore, browser & ecies \\
\hline 2.588 & 12.15534671 ground dwelling & browser & oecies \\
\hline 2.588 & 12.20600358 ground dwelling & grazer, browser & genus \\
\hline 2.588 & 13.81551056 ground dwelling & browser & genus \\
\hline 2.6 & 4.217 ground dwelling & herbivore & species \\
\hline 2.6 & 12.50564397 & & \\
\hline 2.6 & 5.1975 ground dwelling & grazer, & species \\
\hline 2.6 & 7.32 ground dwelling & grazer, browser & species \\
\hline 2.672 & 13.6543296 ground dwelling & browser & genus \\
\hline 2.672 & 12.0010735 ground $\mathrm{dw}$ & & species \\
\hline 2.7 & 6.527 gro & herb & pecies \\
\hline 2.75 & 4.474 ground dwelling & herbivore & secies \\
\hline 2.75 & 4.411 ground $d x$ & herbivore & pecies \\
\hline 2.75 & 2.443 ground dwelling & omnivore & pecies \\
\hline 2.75 & 6.287 amphibious & herbivore & pecies \\
\hline 2.8 & 13.79248471 grol & browser, browser & pecies \\
\hline 2.8 & $11.1095 \mathrm{amp}$ & browser & species \\
\hline 2.8 & $5.243 \mathrm{grc}$ & & pecies \\
\hline 2.8 & 5.694 gro & grazer, browser & oecies \\
\hline 2.894 & 12.24054235 amphibious & browser & pecies \\
\hline 2.894 & 10.5964966 ground dwelling & herbivore, carnivore & senus \\
\hline 2.9 & 3.372 fossorial & browser & species \\
\hline 2.9 & 4.1265 ground dwelling & herbivc & ecies \\
\hline 2.9 & 4.94 se & frugivore & species \\
\hline 2.95 & 9.862654776 ground dwelling & omnivore & species \\
\hline 3 & 6.68949201 scansorial & carnivore, omnivore & species \\
\hline 3 & 4.076314608 ground dwelling & herbivore & species \\
\hline 3 & 5.183698737 semifossorial & granivore, frugivore & oecies \\
\hline 3 & 3.513948796 semifossorial & granivore, frugivore & oecies \\
\hline 3 & 4.666 ground dwelling & herbiv & jecies \\
\hline 3 & 3.988 & & oecies \\
\hline 3 & $15.031 \mathrm{~g}$ & & secies \\
\hline 3 & $14.17876382 \mathrm{grc}$ & & ecies \\
\hline 3 & $14.85 \mathrm{grc}$ & & oecies \\
\hline 3 & 4.88 ground dwelling & herbivore & species \\
\hline 3 & 3.734132255 ground dwelling & herbis & species \\
\hline 3 & 2.298 ground dwelling & & species \\
\hline 3 & 10.46 amphibious & browser & species \\
\hline 3 & 9.867117364 ground dwelling & & species \\
\hline 3 & $8.454969602 \mathrm{am}$ & & ecies \\
\hline 3 & 4.710441653 gro & & ecies \\
\hline 094 & 12.23133201 grol & browser & genus \\
\hline 3.094 & 10.04387618 ground dwelling & grazer, browser & genus \\
\hline 3.094 & 8.517262259 ground dwelling & grazer, browser & genus \\
\hline 3.094 & 8.006088368 ground dwelling & carnivore, omnivore & species \\
\hline 3.1 & 4.724 ground dwelling & grazer, browser & species \\
\hline
\end{tabular}

$\begin{array}{ll}\text { carnivore } & \text { carnivore guild } \\ \text { herbivore } & \text { herbivore guild } \\ \text { herbivore } & \text { Rodentia } \\ \text { herbivore } & \text { Rodentia } \\ \text { carnivore } & \text { carnivore guild } \\ \text { omnivore } & \text { NA } \\ \text { herbivore } & \text { Rodentia } \\ \text { herbivore } & \text { herbivore guild } \\ \text { herbivore } & \text { herbivore guild } \\ \text { herbivore } & \text { herbivore guild } \\ \text { herbivore } & \text { Rodentia } \\ \text { NA } & \text { NA } \\ \text { herbivore } & \text { herbivore guild } \\ \text { herbivore } & \text { herbivore guild } \\ \text { herbivore } & \text { herbivore guild } \\ \text { omnivore } & \text { NA } \\ \text { herbivore } & \text { Rodentia } \\ \text { herbivore } & \text { Rodentia } \\ \text { herbivore } & \text { Rodentia } \\ \text { omnivore } & \text { NA } \\ \text { herbivore } & \text { Rodentia } \\ \text { herbivore } & \text { herbivore guild } \\ \text { herbivore } & \text { Rodentia } \\ \text { herbivore } & \text { Rodentia } \\ \text { herbivore } & \text { herbivore guild } \\ \text { herbivore } & \text { herbivore guild } \\ \text { omnivore } & \text { carnivore guild } \\ \text { herbivore } & \text { Rodentia } \\ \text { herbivore } & \text { Rodentia } \\ \text { herbivore } & \text { Rodentia } \\ \text { carnivore } & \text { carnivore guild } \\ \text { carnivore } & \text { carnivore guild } \\ \text { herbivore } & \text { herbivore guild } \\ \text { herbivore } & \text { Rodentia } \\ \text { herbivore } & \text { Rodentia } \\ \text { herbivore } & \text { Rodentia } \\ \text { herbivore } & \text { Rodentia } \\ \text { herbivore } & \text { herbivore guild } \\ \text { herbivore } & \text { herbivore guild } \\ \text { herbivore } & \text { herbivore guild } \\ \text { herbivore } & \text { Rodentia } \\ \text { herbivore } & \text { Rodentia } \\ \text { herbivore } & \text { Rodentia } \\ \text { herbivore } & \text { Rodentia } \\ \text { herbivore } & \text { herbivore guild } \\ \text { herbivore } & \text { Rodentia } \\ \text { herbivore } & \text { herbivore guild } \\ \text { herbivore } & \text { herbivore guild } \\ \text { herbivore } & \text { herbivore guild } \\ \text { herbivore } & \text { herbivore guild } \\ \text { carnivore } & \text { herbivore guild } \\ \text { herbivore } & \end{array}$




\begin{tabular}{|c|c|c|}
\hline Rodentia & Sciuridae & Paenemarmota barbouri \\
\hline Rodentia & Heteromyidae & Prodipodomys timoteoensis \\
\hline \multirow[t]{2}{*}{ Rodentia } & Cricetidae & Peromyscus baumgartneri \\
\hline & Cricetidae & Ophiomys taylori \\
\hline Carnivora & Felidae & Homotherium johnstoni \\
\hline Lipotyphla & Talpidae & Hesperoscalops blancoensis \\
\hline Carnivora & Felidae & Dinofelis palaeoonca \\
\hline Carnivora & Mustelidae & Spilogale rexroadi \\
\hline Rodentia & Heteromyidae & Perognathus rexroadensis \\
\hline Carnivora & Procyonidae & Procyon rexroadensis \\
\hline Lagomorpha & Leporidae & Aluralagus bensonensis \\
\hline Rodentia & Geomyidae & Nerterogeomys minor \\
\hline Lagomorpha & Leporidae & Hypolagus ringoldensis \\
\hline Carnivora & Mustelidae & Buisnictis breviramus \\
\hline Eulipotyphla & Soricidae & Sorex meltoni \\
\hline Eulipotyphla & Soricidae & Paracryptotis gidleyi \\
\hline Rodentia & Sciuridae & Spermophilus russelli \\
\hline Carnivora & Ursidae & Plionarctos harroldorum \\
\hline Lagomorpha & Leporidae & Alilepus vagus \\
\hline Carnivora & & Borophagus hilli \\
\hline Perissodactyla & Equidae & Hippotherium emsliei \\
\hline Carnivora & Ursidae & Agriotherium schneideri \\
\hline Lagomorpha & Leporidae & Hypolagus vetus \\
\hline Artiodactyla & Camelidae & Paracamelus alexejevi \\
\hline Perissodactyla & Equidae & Nannippus beckensis \\
\hline Eulipotyphla & Soricidae & Beckiasorex hibbardi \\
\hline Lagomorpha & Leporidae & Pratilepus kansasensis \\
\hline Lipotyphla & Talpidae & Hesperoscalops rexroadi \\
\hline Rodentia & Cricetidae & Bensonomys eliasi \\
\hline Proboscidea & Gomphotheriidae & Rhynchotherium praecursor \\
\hline Rodentia & Cricetidae & Baiomys rexroadi \\
\hline Rodentia & Cricetidae & Symmetrodontomys simplicidens \\
\hline Rodentia & Cricetidae & Onychomys gidleyi \\
\hline Carnivora & Felidae & Felis rexroadensis \\
\hline Carnivora & Mustelidae & Brachyopsigale dubius \\
\hline Lagomorpha & Leporidae & Hypolagus regalis \\
\hline Eulipotyphla & Soricidae & Paracryptotis rex \\
\hline Carnivora & Felidae & Homotherium crusafonti \\
\hline Carnivora & Mustelidae & Martes foxi \\
\hline Carnivora & Mustelidae & Mustela meltoni \\
\hline Carnivora & Canidae & Urocyon progressus \\
\hline Rodentia & Cricetidae & Nebraskomys rexroadensis \\
\hline Rodentia & Cricetidae & Pliophenacomys finneyi \\
\hline Rodentia & Zapodidae & Zapus rinkeri \\
\hline Rodentia & Geomyidae & Geomys jacobi \\
\hline Artiodactyla & Camelidae & Megatylopus cochrani \\
\hline Rodentia & Cricetidae & Baiomys kolbi \\
\hline Rodentia & Sciuridae & Spermophilus rexroadensis \\
\hline Carnivora & Mustelidae & Buisnictis schoffi \\
\hline Carnivora & Felidae & Megantereon hesperus \\
\hline Eulipotyphla & Soricidae & Cryptotis adamsi \\
\hline Carnivora & Canidae & Nyctereutes donnezani \\
\hline
\end{tabular}

\begin{tabular}{|c|c|c|c|c|}
\hline 3.95 & 3.15 & 9.24 scansorial & granivore, frugivore & species \\
\hline 3.2 & 3.2 & 3.068 ground dwelling & granivore, browser & species \\
\hline 3.5 & 3.2 & 2.899939792 ground dwelling & herbivore & species \\
\hline 3.85 & 3.2 & 4.08 ground dwelling & herbivore & species \\
\hline 3.3 & 3.3 & 11.816 scansorial & carnivore & species \\
\hline 3.3 & 3.3 & 4.306547943 fossorial & insectivore & species \\
\hline 3.35 & 3.3 & 10.977 scansorial & carnivore & species \\
\hline 3.5 & 3.3 & 6.282 scansorial & carnivore, omnivore & species \\
\hline 3.5 & 3.3 & 2.903 ground dwelling & granivore, browser & species \\
\hline 3.5 & 3.3 & 9.413 scansorial & omnivore, carnivore & species \\
\hline 4.2 & 3.3 & 4.925 ground dwelling & grazer, browser & species \\
\hline 4.7 & 3.3 & 3.586 & & \\
\hline 7.4 & 3.3 & 7.708068306 ground dwelling & grazer, browser & species \\
\hline 3.75 & 3.35 & 6.653045671 scansorial & carnivore, omnivore & species \\
\hline 3.75 & 3.35 & 1.24477594 ground dwelling & omnivore & species \\
\hline 3.9 & 3.35 & 3.3365 ground dwelling & omnivore & species \\
\hline 4.5 & 3.35 & 5.111987788 semifossorial & granivore, frugivore & species \\
\hline 4.5 & 3.35 & 10.78991375 ground dwelling & herbivore, carnivore & species \\
\hline 7.25 & 3.35 & 5.308 ground dwelling & grazer, browser & species \\
\hline 10 & 3.35 & 10.9023181 ground dwelling & carnivore & species \\
\hline 10 & 3.35 & 11.777 & & \\
\hline 10.1 & 3.35 & 12.53995674 ground dwelling & carnivore, omnivore & species \\
\hline 16.65 & 3.35 & 6.9625 ground dwelling & grazer, browser & species \\
\hline 3.394 & 3.394 & 14.10563628 ground dwelling & browser, browser & species \\
\hline 3.4 & 3.4 & 11.06906204 ground dwelling & grazer & species \\
\hline 3.4 & 3.4 & 1.851 ground dwelling & omnivore & species \\
\hline 3.5 & 3.4 & 6.88 ground dwelling & grazer, browser & species \\
\hline 3.5 & 3.4 & 3.801733077 fossorial & insectivore & species \\
\hline 3.5 & 3.4 & 2.749441612 & & \\
\hline 3.6 & 3.4 & 15.12 & & \\
\hline 4.95 & 3.4 & 2.092096392 ground dwelling & herbivore & species \\
\hline 4.95 & 3.4 & 3.3055 ground dwelling & herbivore & species \\
\hline 5.25 & 3.4 & 3.143730923 ground dwelling & herbivore & species \\
\hline 7.25 & 3.4 & 9.961 scansorial & carnivore & species \\
\hline 7.4 & 3.4 & 6.653045671 scansorial & carnivore, omnivore & species \\
\hline 7.4 & 3.4 & 7.729726186 ground dwelling & grazer, browser & species \\
\hline 11.4 & 3.4 & 3.7395 ground dwelling & omnivore & species \\
\hline 3.5 & 3.5 & 11.445 scansorial & carnivore & species \\
\hline 3.5 & 3.5 & 5.088672403 scansorial & carnivore, omnivore & species \\
\hline 3.5 & 3.5 & 6.122 scansorial & carnivore, omnivore & species \\
\hline 3.5 & 3.5 & 8.938278406 scansorial & carnivore, omnivore & species \\
\hline 3.5 & 3.5 & 3.955937706 ground dwelling & herbivore & species \\
\hline 3.5 & 3.5 & 4.146 ground dwelling & herbivore & species \\
\hline 3.5 & 3.5 & 3.325 saltatorial & herbivore & species \\
\hline 3.5 & 3.5 & 4.01319373 fossorial & browser & species \\
\hline 4.5 & 3.5 & 13.87974235 ground dwelling & browser & species \\
\hline 5.75 & 3.5 & 2.424653848 ground dwelling & herbivore & species \\
\hline 6.55 & 3.5 & 4.544062402 semifossorial & granivore, frugivore & species \\
\hline 7.4 & 3.5 & 4.687 scansorial & carnivore, omnivore & species \\
\hline 7.6 & 3.5 & 10.91739447 scansorial & carnivore & species \\
\hline 9.45 & 3.5 & 2.5925 ground dwelling & omnivore & species \\
\hline 7.016 & 3.7 & 8.986989618 ground dwelling & omnivore, carnivore & genus \\
\hline
\end{tabular}

\begin{tabular}{|c|c|}
\hline herbivore & Rodentia \\
\hline herbivore & Rodentia \\
\hline herbivore & Rodentia \\
\hline herbivore & herbivore guild \\
\hline carnivore & carnivore guild \\
\hline insectivore & NA \\
\hline carnivore & carnivore guild \\
\hline carnivore & carnivore guild \\
\hline herbivore & Rodentia \\
\hline omnivore & carnivore guild \\
\hline herbivore & herbivore guild \\
\hline NA & Rodentia \\
\hline herbivore & herbivore guild \\
\hline carnivore & carnivore guild \\
\hline omnivore & NA \\
\hline omnivore & NA \\
\hline herbivore & Rodentia \\
\hline omnivore & carnivore guild \\
\hline herbivore & herbivore guild \\
\hline carnivore & carnivore guild \\
\hline NA & NA \\
\hline omnivore & carnivore guild \\
\hline herbivore & herbivore guild \\
\hline herbivore & herbivore guild \\
\hline herbivore & herbivore guild \\
\hline omnivore & NA \\
\hline herbivore & herbivore guild \\
\hline insectivore & NA \\
\hline NA & Rodentia \\
\hline NA & Rodentia \\
\hline herbivore & Rodentia \\
\hline herbivore & Rodentia \\
\hline herbivore & Rodentia \\
\hline carnivore & carnivore guild \\
\hline carnivore & carnivore guild \\
\hline herbivore & herbivore guild \\
\hline omnivore & NA \\
\hline carnivore & carnivore guild \\
\hline carnivore & carnivore guild \\
\hline carnivore & carnivore guild \\
\hline carnivore & carnivore guild \\
\hline herbivore & Rodentia \\
\hline herbivore & Rodentia \\
\hline herbivore & Rodentia \\
\hline herbivore & Rodentia \\
\hline herbivore & herbivore guild \\
\hline herbivore & Rodentia \\
\hline herbivore & Rodentia \\
\hline carnivore & carnivore guild \\
\hline carnivore & carnivore guild \\
\hline omnivore & NA \\
\hline omnivore & carnivore guild \\
\hline
\end{tabular}




\begin{tabular}{|c|c|c|c|c|c|c|c|c|c|}
\hline Carnivora & Mustelidae & Sminthosinis bowleri & 3.75 & 3.75 & 4.61 scansorial & carnivore, omnivore & species & carnivore & carnivore guild \\
\hline Rodentia & Heteromyidae & Oregonomys magnus & 3.75 & 3.75 & 3.046 ground dwelling & granivore, browser & species & herbivore & Rodentia \\
\hline Rodentia & Heteromyidae & Perognathus maldei & 3.9 & 3.75 & 2.384 ground dwelling & granivore, browser & species & herbivore & Rodentia \\
\hline Rodentia & Geomyidae & Pliogeomys parvus & 3.9 & 3.75 & 2.3345 fossorial & browser & species & herbivore & Rodentia \\
\hline Eulipotyphla & Soricidae & Sorex powersi & 4 & 3.75 & 2.058344963 ground dwelling & omnivore & species & omnivore & NA \\
\hline Eulipotyphla & Soricidae & Sorex hagermanensis & 3.9 & 3.9 & 1.997959899 ground dwelling & omnivore & species & omnivore & NA \\
\hline Rodentia & Castoridae & Procastoroides intermedius & 4.5 & 3.9 & 9.469 amphibious & browser & species & herbivore & Rodentia \\
\hline Carnivora & Ursidae & Ursus abstrusus & 4.5 & 3.9 & 10.64168191 ground dwelling & herbivore, carnivore & species & omnivore & carnivore guild \\
\hline Carnivora & Mustelidae & Limnonyx sinerizi & 3.96 & 3.96 & 8.293911505 scansorial & carnivore, omnivore & species & carnivore & carnivore guild \\
\hline Carnivora & Mustelidae & Martes woodwardi & 3.96 & 3.96 & 8.16036157 scansorial & carnivore, omnivore & genus & carnivore & carnivore guild \\
\hline Carnivora & Mustelidae & Parataxidea crassa & 3.96 & 3.96 & 8.411343345 scansorial & carnivore, omnivore & genus & carnivore & carnivore guild \\
\hline Artiodactyla & Camelidae & Paracamelus alutensis & 3.96 & 3.96 & 13.407953 ground dwelling & browser, browser & genus & herbivore & herbivore guild \\
\hline Perissodactyla & Equidae & Sinohippus zitteli & 4.466 & 3.96 & 12.71948005 ground dwelling & browser & species & herbivore & herbivore guild \\
\hline Artiodactyla & Bovidae & Gazella gaudryi & 6.325 & 3.96 & 9.797499571 ground dwelling & grazer, browser & genus & herbivore & herbivore guild \\
\hline Artiodactyla & Suidae & Propotamochoerus provincialis & 7.016 & 3.96 & 11.98265282 ground dwelling & omnivore & genus & omnivore & NA \\
\hline Artiodactyla & Bovidae & Oioceros rothi & 7.498 & 3.96 & 10.71392844 ground dwelling & grazer, browser & genus & herbivore & herbivore guild \\
\hline Artiodactyla & & Pliocervus pentelici & 7.498 & 3.96 & 11.28957471 ground dwelling & grazer, browser & genus & herbivore & herbivore guild \\
\hline Perissodactyla & Equidae & Hippotherium brachypus & 7.498 & 3.96 & 12.35797419 ground dwelling & grazer, browser & genus & herbivore & herbivore guild \\
\hline Artiodactyla & Giraffidae & Bohlinia attica & 7.498 & 3.96 & 13.38492715 ground dwelling & browser & species & herbivore & herbivore guild \\
\hline Carnivora & Mustelidae & Plesiogulo monspessulanus & 7.604 & 3.96 & 11.51292546 scansorial & carnivore & genus & carnivore & carnivore guild \\
\hline Carnivora & Ursidae & Indarctos atticus & 8.225 & 3.96 & 12.76553176 ground dwelling & herbivore, carnivore & species & omnivore & carnivore guild \\
\hline Carnivora & Felidae & Amphimachairodus giganteus & 8.47 & 3.96 & 12.25435786 scansorial & carnivore & species & carnivore & carnivore guild \\
\hline Carnivora & Ailuridae & Simocyon primigenius & 8.47 & 3.96 & 11.10751239 ground dwelling & carnivore, omnivore & species & carnivore & carnivore guild \\
\hline Perissodactyla & Chalicotheriidae & Ancylotherium pentelicum & 8.47 & 3.96 & 14.83785834 ground dwelling & browser & species & herbivore & herbivore guild \\
\hline Carnivora & Felidae & Metailurus major & 8.725 & 3.96 & 11.28957471 scansorial & carnivore & genus & carnivore & carnivore guild \\
\hline Carnivora & Felidae & Metailurus parvulus & 9.427 & 3.96 & 10.54583973 scansorial & carnivore & genus & carnivore & carnivore guild \\
\hline Artiodactyla & Cervidae & Cervavitus variabilis & 9.427 & 3.96 & 11.08234205 ground dwelling & grazer, browser & genus & herbivore & herbivore guild \\
\hline Artiodactyla & Bovidae & Gazella capricornis & 9.427 & 3.96 & 9.997824474 ground dwelling & grazer, browser & genus & herbivore & herbivore guild \\
\hline Artiodactyla & Bovidae & Gazella deperdita & 9.427 & 3.96 & 10.04387618 ground dwelling & grazer, browser & genus & herbivore & herbivore guild \\
\hline Artiodactyla & Bovidae & Palaeoreas lindermayeri & 9.427 & 3.96 & 10.71392844 ground dwelling & grazer, browser & genus & herbivore & herbivore guild \\
\hline Artiodactyla & Bovidae & Protragelaphus skouzesi & 9.427 & 3.96 & 11.08234205 ground dwelling & grazer, browser & genus & herbivore & herbivore guild \\
\hline \multirow[t]{2}{*}{ Artiodactyla } & Bovidae & Tragoportax amalthea & 9.427 & 3.96 & 11.75239431 ground dwelling & grazer, browser & genus & herbivore & herbivore guild \\
\hline & & Cremohipparion mediterraneum & 9.427 & 3.96 & $12.13922861 \mathrm{NA}$ & NA & genus & NA & NA \\
\hline Artiodactyla & Suidae & Microstonyx major & 9.427 & 3.96 & 12.70796713 ground dwelling & omnivore & species & omnivore & NA \\
\hline Carnivora & Hyaenidae & Lycyaena chaeretis & 10.154 & 3.96 & 10.5964966 ground dwelling & carnivore & genus & carnivore & carnivore guild \\
\hline Carnivora & Hyaenidae & Ictitherium viverrinum & 10.4 & 3.96 & 9.797499571 ground dwelling & carnivore & species & carnivore & carnivore guild \\
\hline Perissodactyla & Rhinocerotidae & Dihoplus schleiermacheri & 12.154 & 3.96 & 13.99741478 ground dwelling & browser & species & herbivore & herbivore guild \\
\hline Carnivora & Hyaenidae & Adcrocuta eximia & 12.629 & 3.96 & 11.15602478 ground dwelling & carnivore & genus & carnivore & carnivore guild \\
\hline Artiodactyla & Bovidae & Simatherium demissum & 12.809 & 3.96 & 14.50168092 ground dwelling & grazer, browser & genus & herbivore & herbivore guild \\
\hline Carnivora & Hyaenidae & Hyaenictis graeca & 13.789 & 3.96 & 10.91425334 ground dwelling & carnivore & genus & carnivore & carnivore guild \\
\hline Artiodactyla & Bovidae & Prostrepsiceros rotundicornis & 14.81 & 3.96 & 10.46294666 ground dwelling & grazer, browser & genus & herbivore & herbivore guild \\
\hline Artiodactyla & Bovidae & Protoryx carolinae & 14.81 & 3.96 & 11.98265282 ground dwelling & grazer, browser & genus & herbivore & herbivore guild \\
\hline Proboscidea & Deinotheriidae & Deinotherium giganteum & 14.81 & 3.96 & 16.67485021 ground dwelling & grazer, browser & species & herbivore & herbivore guild \\
\hline Carnivora & Mustelidae & Martes filholi & 15.804 & 3.96 & 7.695239381 scansorial & carnivore, omnivore & genus & carnivore & carnivore guild \\
\hline Rodentia & Cricetidae & Repomys panacaensis & 4.6 & 4 & 3.652 ground dwelling & herbivore & species & herbivore & Rodentia \\
\hline Rodentia & Cricetidae & Bensonomys elachys & 5.75 & 4 & 2.140066163 & & & NA & Rodentia \\
\hline Proboscidea & Gomphotheriidae & Rhynchotherium falconeri & 6.7 & 4.1 & 15.311 ground dwelling & browser, grazer & species & herbivore & herbivore guild \\
\hline Lagomorpha & Leporidae & Pewelagus mexicanus & 4.2 & 4.2 & 5.224 ground dwelling & grazer, browser & species & herbivore & herbivore guild \\
\hline Rodentia & Sciuridae & Spermophilus matachicensis & 5.75 & 4.2 & 5.399 semifossorial & granivore, frugivore & species & herbivore & Rodentia \\
\hline Artiodactyla & Antilocapridae & Hexobelomeryx fricki & 5.8 & 4.2 & 10.23806 ground dwelling & browser, grazer & species & herbivore & herbivore guild \\
\hline Carnivora & Hyaenidae & Chasmaporthetes kani & 4.266 & 4.266 & 11.33562641 ground dwelling & carnivore & genus & carnivore & carnivore guild \\
\hline Artiodactyla & Bovidae & Gazella emilii & 4.266 & 4.266 & 10.20275455 ground dwelling & grazer, browser & species & herbivore & herbivore guild \\
\hline
\end{tabular}




\begin{tabular}{|c|c|}
\hline Artiodactyla & Bovidae \\
\hline Perissodactyla & Equidae \\
\hline Perissodactyla & Equidae \\
\hline Perissodactyla & Rhinocerotidae \\
\hline Perissodactyla & Rhinocerotidae \\
\hline Artiodactyla & Camelidae \\
\hline Artiodactyla & Tayassuidae \\
\hline Perissodactyla & Equidae \\
\hline Proboscidea & Deinotheriidae \\
\hline Artiodactyla & Giraffidae \\
\hline Carnivora & Felidae \\
\hline Proboscidea & Gomphotheriidae \\
\hline Rodentia & Cricetidae \\
\hline Rodentia & Sciuridae \\
\hline Carnivora & Canidae \\
\hline Rodentia & Cricetidae \\
\hline Lagomorpha & Leporidae \\
\hline Rodentia & Cricetidae \\
\hline Eulipotyphla & Soricidae \\
\hline Rodentia & Cricetidae \\
\hline Rodentia & Cricetidae \\
\hline Rodentia & Cricetidae \\
\hline Rodentia & Cricetidae \\
\hline Rodentia & Cricetidae \\
\hline Artiodactyla & Bovidae \\
\hline Carnivora & Hyaenidae \\
\hline Rodentia & Cricetidae \\
\hline Rodentia & Heteromyidae \\
\hline Rodentia & Cricetidae \\
\hline Rodentia & Castoridae \\
\hline Rodentia & Cricetidae \\
\hline Rodentia & Cricetidae \\
\hline Rodentia & Cricetidae \\
\hline Rodentia & Heteromyidae \\
\hline Rodentia & Cricetidae \\
\hline Rodentia & Heteromyidae \\
\hline Rodentia & Cricetidae \\
\hline Perissodactyla & Equidae \\
\hline Perissodactyla & Equidae \\
\hline Rodentia & Cricetidae \\
\hline Perissodactyla & Equidae \\
\hline Artiodactyla & Camelidae \\
\hline Carnivora & Hyaenidae \\
\hline Artiodactyla & Cervidae \\
\hline Rodentia & Sciuridae \\
\hline Perissodactyla & Rhinocerotidae \\
\hline Perissodactyla & Equidae \\
\hline Carnivora & Felidae \\
\hline Rodentia & Cricetidae \\
\hline Perissodactyla & Equidae \\
\hline Artiodactyla & Tayassuidae \\
\hline Artiodactyla & Antilocapridae \\
\hline
\end{tabular}

Tchaltacerus longicornis

Hipparion longipes

Chilotherium haberer

Aceratherium incisivum

Titanotylopus nebraskensis

Platygonus pearcei

Hipparion hippidiodus

Deinotherium gigantium

Palaeotragus germaini

Machairodus aphanistus

Gomphotherium angustidens

Peromyscus nosher

Paenemarmota sawrockensis

Eucyon davisi

Mimomys panacaensis

Lepoides lepoides

acobsomys verdensis

Notiosorex repenningi

Prosigmodon holocuspis

Neotoma vaughani

Prosigmodon chihuahuensis

Prosigmodon oroscoi

Bensonomys baskini

Tragoportax cyrenaicus

Hyaenictitherium parvum

Repomys maxumi

Perognathus mclaughlini

Paraneotoma sawrockensis

Dipoides wilsoni

Antecalomys vasquez

Repomys gustelyi

Paronychomys lemredfieldi

Oregonomys sargenti

Antecalomys valensis

Cupidinimus bidahochiensis

Bensonomys gidleyi

Dinohippus mexicanus

Neohipparion eurystyle

Bensonomys winklerorum

Astrohippus stockii

Hemiauchenia vera

Hyaenictitherium wongii

Cervavitus novorossiae

Paenemarmota mexicana

Teleoceras hicksi

Nannippus minor

Machairodus coloradensis

Bensonomys lindsayi

Pseudhipparion simpsoni

Catagonus brachydontus

Hexameryx simpsoni
4.26610 .20275455 ground dwelling grazer, browser

4.26612 .69184903 ground dwelling browser

$4.266 \quad 12.27738372$ ground dwelling browser

4.26613 .45860987 ground dwelling browser

4.26613 .90991655 ground dwelling browser

4.3514 .49016799 ground dwelling browser, browser

4.4511 .13237764 ground dwelling browser, grazer

$4.466 \quad 12.18067514$ ground dwelling browser

4.46616 .67485021 ground dwelling grazer, browser

4.46613 .30433667 ground dwelling browser, grazer

4.46612 .30040957 scansorial carnivore

4.46615 .04278841 ground dwelling browser

$4.5 \quad 2.767$ ground dwelling herbivore

8.6905 scansorial granivore, frugivore

4.5 9.394928968 ground dwelling carnivore, omnivore species

4.64 .4585 ground dwelling herbivore $\quad$ species

species

$\begin{array}{lrl}4.7 & 2.875842181 \text { ground dwelling omnivore } & \text { species } \\ 4.7 & 4.7325 \text { ground dwelling herbivore } & \text { species }\end{array}$

$\begin{array}{lll}4.7 & 5.301 \text { ground dwelling herbivore } & \text { species }\end{array}$

$4.7 \quad 4.302$ ground dwelling herbivore species

$4.7 \quad 3.351$ ground dwelling herbivore species

$4.7 \quad 3.449987546$

$\begin{array}{lll}4.917 & 12.10008466 \text { ground dwelling grazer, browser }\end{array}$

4.91710 .54583973 ground dwelling carnivore

$4.95 \quad 4.8095$ ground dwelling herbivore

species

$4.95 \quad 4.814919763$

$4.95 \quad 6.9685$ amphibious browser 4.05

$\begin{array}{lll}5.05 & 2.58 \text { ground dwelling herbivore } & \text { species }\end{array}$

$5.05 \quad 4.4$ ground dwelling herbivore species

$5.05 \quad 3.0005$ ground dwelling herbivore species

$5.05 \quad 3.2615$ ground dwelling granivore, browser species

$5.05 \quad 2.6115$ ground dwelling herbivore species

$5.25 \quad 3.307$ ground dwelling granivore, browser species

$5.25 \quad 2.541601993$

5.512 .74078478 ground dwelling browser species

5.511 .95449806 ground dwelling grazer species

$\begin{array}{ll}5.65 & 2.424802726\end{array}$

$\begin{array}{lll}5.65 & 11.73481397 \text { ground dwelling browser species }\end{array}$

5.6512 .41557561

5.72310 .38465877 ground dwelling carnivore genus

5.72311 .28957471 ground dwelling grazer, browser genus

5.759 .126 scansorial granivore, frugivore species

5.7514 .84200938 amphibious grazer species

$\begin{array}{ll}5.75 & 10.99903089 \text { ground dwelling grazer species }\end{array}$

$5.75 \quad 12.30528419$

5.82 .545502549 ground dwelling herbivore species

$\begin{array}{ll}5.8 & 10.74380399 \text { ground dwelling grazer }\end{array}$

species

5.811 .56485356

$\begin{array}{ll}5.8 & 10.55420041 \text { ground dwelling browser, grazer species }\end{array}$

$\begin{array}{ll}\text { herbivore } & \text { herbivore guild } \\ \text { herbivore } & \text { herbivore guild } \\ \text { herbivore } & \text { herbivore guild } \\ \text { herbivore } & \text { herbivore guild } \\ \text { herbivore } & \text { herbivore guild } \\ \text { herbivore } & \text { herbivore guild } \\ \text { herbivore } & \text { herbivore guild } \\ \text { herbivore } & \text { herbivore guild } \\ \text { herbivore } & \text { herbivore guild } \\ \text { herbivore } & \text { herbivore guild } \\ \text { carnivore } & \text { carnivore guild } \\ \text { herbivore } & \text { herbivore guild } \\ \text { herbivore } & \text { Rodentia } \\ \text { herbivore } & \text { Rodentia } \\ \text { carnivore } & \text { carnivore guild } \\ \text { herbivore } & \text { Rodentia } \\ \text { herbivore } & \text { herbivore guild } \\ \text { herbivore } & \text { Rodentia } \\ \text { omnivore } & \text { NA } \\ \text { herbivore } & \text { Rodentia } \\ \text { herbivore } & \text { Rodentia } \\ \text { herbivore } & \text { Rodentia } \\ \text { herbivore } & \text { Rodentia } \\ \text { NA } & \text { Rodentia } \\ \text { herbivore } & \text { herbivore guild } \\ \text { carnivore } & \text { carnivore guild } \\ \text { herbivore } & \text { Rodentia } \\ \text { herbivore } & \text { Rodentia } \\ \text { NA } & \text { Rodentia } \\ \text { herbivore } & \text { Rodentia } \\ \text { herbivore } & \text { Rodentia } \\ \text { herbivore } & \text { Rodentia } \\ \text { herbivore } & \text { Rodentia } \\ \text { herbivore } & \text { Rodentia } \\ \text { herbivore } & \text { Rodentia } \\ \text { herbivore } & \text { Rodentia } \\ \text { NA } & \text { Rodentia } \\ \text { herbivore } & \text { herbivore guild } \\ \text { herbivore } & \text { herbivore guild } \\ \text { NA } & \text { Rodentia } \\ \text { herbivore } & \text { herbivore guild } \\ \text { NA } & \text { NA } \\ \text { carnivore } & \text { carnivore guild } \\ \text { herbivore } & \text { herbivore guild } \\ \text { herbivore } & \text { Rodentia } \\ \text { herbivore } & \text { herbivore guild } \\ \text { herbivore } & \text { herborild } \\ \text { NA } & \text { herbivore } \\ \text { herbivore } & \\ \text { Nerbivore } & \end{array}$




\begin{tabular}{|c|c|c|c|}
\hline Carnivora & Canidae & Canis ferox & 10.1 \\
\hline Artiodactyla & Camelidae & Megatylopus matthewi & 10.1 \\
\hline Perissodactyla & Equidae & Dinohippus interpolatus & 10.25 \\
\hline Perissodactyla & Equidae & Nannippus aztecus & 11.2 \\
\hline Carnivora & Canidae & Vulpes stenognathus & 11.3 \\
\hline Artiodactyla & Antilocapridae & Osbornoceros osborni & 11.5 \\
\hline Carnivora & & Borophagus secundus & 11.6 \\
\hline Artiodactyla & Antilocapridae & Plioceros blicki & 13.1 \\
\hline Carnivora & Felidae & Pseudaelurus intrepidus & 17.35 \\
\hline Rodentia & Sciuridae & Spermophilus wellingtonensis & 9.45 \\
\hline Rodentia & Cricetidae & Paronychomys alticuspis & 6.05 \\
\hline Rodentia & Cricetidae & Bensonomys yazhi & 6.05 \\
\hline Carnivora & Mustelidae & Plesiogulo marshalli & 9.7 \\
\hline Rodentia & Castoridae & Dipoides williamsi & 11.1 \\
\hline Carnivora & Hyaenidae & Belbus beaumonti & 6.073 \\
\hline Carnivora & Hyaenidae & Ictitherium intuberculatum & 6.073 \\
\hline Artiodactyla & Bovidae & Nisidorcas planicornis & 8.225 \\
\hline Artiodactyla & Bovidae & Pachytragus laticeps & 8.47 \\
\hline Artiodactyla & Bovidae & Pseudotragus capricornis & 9.427 \\
\hline Perissodactyla & Rhinocerotidae & Chilotherium schlosseri & 9.427 \\
\hline Artiodactyla & Bovidae & Gazella dorcadoides & 6.289 \\
\hline \multirow[t]{2}{*}{ Artiodactyla } & Bovidae & Prostrepsiceros houtumschindleri & 6.289 \\
\hline & & Cremohipparion matthewi & 8.47 \\
\hline Artiodactyla & Giraffidae & Samotherium boissieri & 9.427 \\
\hline Artiodactyla & Bovidae & Protoryx crassicornis & 9.427 \\
\hline Artiodactyla & Bovidae & Tragoportax rugosifrons & 9.427 \\
\hline Carnivora & Hyaenidae & Hyaenictitherium hyaenoides & 12.629 \\
\hline Artiodactyla & Giraffidae & Giraffokeryx punjabiensis & 14.81 \\
\hline Perissodactyla & Equidae & Dinohippus leidyanus & 11.1 \\
\hline Perissodactyla & Rhinocerotidae & Acerorhinus palaeosinensis & 6.325 \\
\hline Perissodactyla & Equidae & Hipparion plocodus & 6.325 \\
\hline Artiodactyla & Giraffidae & Palaeotragus microdon & 10.154 \\
\hline Artiodactyla & Suidae & Chleuastochoerus stehlini & 10.154 \\
\hline Carnivora & Hyaenidae & Thalassictis robusta & 10.4 \\
\hline Carnivora & Mustelidae & Sivaonyx hessicus & 6.4 \\
\hline Artiodactyla & Tragulidae & Dorcatherium jourdani & 9.2 \\
\hline Artiodactyla & Protoceratidae & Kyptoceras amatorum & 6.45 \\
\hline Rodentia & Sciuridae & Spermophilus dotti & 6.55 \\
\hline Artiodactyla & Camelidae & Megacamelus merriami & 7.4 \\
\hline Carnivora & Ursidae & Plionarctos edensis & 8.3 \\
\hline Artiodactyla & Palaeomerycidae & Pediomeryx hemphillensis & 10.45 \\
\hline Rodentia & Eomyidae & Kansasimys wilsoni & 6.85 \\
\hline Rodentia & Cricetidae & Paronychomys tuttlei & 6.85 \\
\hline Rodentia & Heteromyidae & Prodipodomys kansensis & 7.15 \\
\hline Carnivora & & Borophagus parvus & 8.5 \\
\hline Carnivora & Mustelidae & Plesiogulo lindsayi & 9.15 \\
\hline Rodentia & Heteromyidae & Perognathus stevei & 7 \\
\hline Rodentia & Sciuridae & Spermophilus shotwelli & 7.4 \\
\hline Rodentia & Heteromyidae & Oregonomys pebblespringsensis & 7.95 \\
\hline Lagomorpha & Leporidae & Hypolagus oregonensis & 9.7 \\
\hline Lipotyphla & Talpidae & Scapanus proceridens & 10.35 \\
\hline Carnivora & Mustelidae & Pliotaxidea nevadensis & 10.45 \\
\hline
\end{tabular}

5.8 10.129 ground dwelling carnivore, omnivore species

$5.8 \quad 1439778788$ ground dwelling browser

5.812 .52724365 ground dwelling browser species

$\begin{array}{ll}5.8 & 10.96203089 \text { ground dwelling grazer species }\end{array}$

$5.8 \quad 8.961$ ground dwelling carnivore, omnivore species

$5.8 \quad 9.76$ ground dwelling browser, grazer species

5.810 .50807682 ground dwelling carnivore species

5.811 .59682884 ground dwelling browser, grazer species

5.810 .2825 scansorial carnivore species

$5.9 \quad 5.6885$ semifossorial granivore, frugivore species

6.052 .8405 ground dwelling herbivore species

$6.05 \quad 2.219203484$

6.059 .578200991 scansorial carnivore species

6.057 .2415 amphibious browser species

6.07310 .71392844 ground dwelling carnivore genus

6.07310 .30867346 ground dwelling carnivore

6.07310 .08532271 ground dwelling grazer, browser genus

6.07311 .65338316 ground dwelling grazer, browser

6.07310 .81984735 ground dwelling grazer, browser genus

$\begin{array}{lll}6.073 & 13.86616743 \text { ground dwelling browser genus }\end{array}$

6.28910 .04387618 ground dwelling grazer, browser genus

6.28910 .73695429 ground dwelling grazer, browser genus

$6.28911 .56127975 \mathrm{NA}$

6.28913 .30433667 ground dwelling grazer

6.28911 .34944192 ground dwelling grazer, browser genus

6.28911 .82147187 ground dwelling grazer, browser genus

6.28910 .89583266 ground dwelling carnivore genus

6.28912 .80007053 ground dwelling browser species

6.312 .31883025

6.32514 .12866213 ground dwelling browser

6.32511 .72015812 ground dwelling browser

6.32510 .71162585 ground dwelling omnivore species

6.3259 .735329773 ground dwelling carnivore species

6.49 .546517796 scansorial carnivore, omnivore species

6.411 .28957471 ground dwelling frugivore, grazer species

6.4512 .71635187 ground dwelling browser species

$6.55 \quad 5.603$ semifossorial granivore, frugivore species

6.714 .61918143 ground dwelling browser, browser species

6.7 $\quad 10.95$ ground dwelling herbivore, carnivore species

6.711 .83897026 ground dwelling browser, grazer species

$6.85 \quad 5.431$ ground dwelling herbivore species

$6.85 \quad 3.653$ ground dwelling herbivore species

6.852 .549 ground dwelling granivore, browser species

6.8510 .20575818 ground dwelling carnivore species

6.859 .932581129 scansorial carnivore species

2.3685 ground dwelling granivore, browser species

5.672 semifossorial granivore, frugivore species

3.279 ground dwelling granivore, browser species

6.8755 ground dwelling grazer, browser species

4.1895 fossorial insectivore species

6.5945 scansorial carnivore, omnivore species

$\begin{array}{ll}\text { carnivore } & \text { carnivore guild } \\ \text { herbivore } & \text { herbivore guild } \\ \text { herbivore } & \text { herbivore guild } \\ \text { herbivore } & \text { herbivore guild } \\ \text { carnivore } & \text { carnivore guild } \\ \text { herbivore } & \text { herbivore guild } \\ \text { carnivore } & \text { carnivore guild } \\ \text { herbivore } & \text { herbivore guild } \\ \text { carnivore } & \text { carnivore guild } \\ \text { herbivore } & \text { Rodentia } \\ \text { herbivore } & \text { Rodentia } \\ \text { NA } & \text { Rodentia } \\ \text { carnivore } & \text { carnivore guild } \\ \text { herbivore } & \text { Rodentia } \\ \text { carnivore } & \text { carnivore guild } \\ \text { carnivore } & \text { carnivore guild } \\ \text { herbivore } & \text { herbivore guild } \\ \text { herbivore } & \text { herbivore guild } \\ \text { herbivore } & \text { herbivore guild } \\ \text { herbivore } & \text { herbivore guild } \\ \text { herbivore } & \text { herbivore guild } \\ \text { herbivore } & \text { herbivore guild } \\ \text { NA } & \text { NA } \\ \text { herbivore } & \text { herbivore guild } \\ \text { herbivore } & \text { herbivore guild } \\ \text { herbivore } & \text { herbivore guild } \\ \text { carnivore } & \text { carnivore guild } \\ \text { herbivore } & \text { herbivore guild } \\ \text { herbivore } & \text { herbivore guild } \\ \text { herbivore } & \text { herbivore guild } \\ \text { herbivore } & \text { herbivore guild } \\ \text { herbivore } & \text { herbivore guild } \\ \text { omnivore } & \text { NA } \\ \text { carnivore } & \text { carnivore guild } \\ \text { carnivore } & \text { carnivore guild } \\ \text { herbivore } & \text { herbivore guild } \\ \text { herbivore } & \text { herbivore guild } \\ \text { herbivore } & \text { Rodentia } \\ \text { herbivore } & \text { herbivore guild } \\ \text { omnivore } & \text { carnivore guild } \\ \text { herbivore } & \text { herbivore guild } \\ \text { herbivore } & \text { Rodentia } \\ \text { herbivore } & \text { Rodentia } \\ \text { herbivore } & \text { Rodentia } \\ \text { carnivore } & \text { carnivore guild } \\ \text { carnivore } & \text { carnivore guild } \\ \text { herbivore } & \text { Rodentia } \\ \text { herbivore } & \text { Rodentia } \\ \text { herbivore } & \text { Rodentia } \\ \text { herbivore } & \text { herbivore guild } \\ \text { insectivore } & \text { Narnivore }\end{array}$




\begin{tabular}{|c|c|c|c|c|c|c|c|c|c|}
\hline Rodentia & Sciuridae & Spermophilus wilsoni & 10.85 & 7 & 5.5135 semifossorial & granivore, frugivore & species & herbivore & Rodentia \\
\hline Carnivora & Mustelidae & Martes andersoni & 7.016 & 7.016 & 7.313010255 scansorial & carnivore, omnivore & genus & carnivore & carnivore guild \\
\hline \multirow[t]{2}{*}{ Artiodactyla } & Bovidae & Maremmia hauptii & 7.016 & 7.016 & 10.81984735 ground dwelling & grazer, browser & genus & herbivore & herbivore guild \\
\hline & & Ouzocerus gracilis & 7.016 & 7.016 & $10.71392844 \mathrm{NA}$ & NA & genus & NA & NA \\
\hline Perissodactyla & Equidae & Hipparion matthewi & 8.47 & 7.016 & 11.98 ground dwelling & browser & species & herbivore & herbivore guild \\
\hline Artiodactyla & Bovidae & Maremmia lorenzi & 9.427 & 7.016 & 11.22510233 ground dwelling & grazer, browser & species & herbivore & herbivore guild \\
\hline Artiodactyla & Bovidae & Tyrrhenotragus gracillimus & 9.427 & 7.016 & 9.546517796 ground dwelling & grazer, browser & species & herbivore & herbivore guild \\
\hline Carnivora & Mustelidae & Eomellivora wimani & 11.8 & 7.016 & 10.71392844 scansorial & carnivore, omnivore & species & carnivore & carnivore guild \\
\hline Artiodactyla & Suidae & Propotamochoerus palaeochoerus & 11.925 & 7.016 & 11.69482969 ground dwelling & omnivore & genus & omnivore & NA \\
\hline Artiodactyla & Tragulidae & Dorcatherium minus & 13.789 & 7.016 & 8.800480225 ground dwelling & frugivore, grazer & species & herbivore & herbivore guild \\
\hline Rodentia & Heteromyidae & Perognathus dunklei & 7.15 & 7.15 & 2.071 ground dwelling & granivore, browser & species & herbivore & Rodentia \\
\hline Rodentia & Cricetidae & Onychomys martini & 7.15 & 7.15 & 2.911 ground dwelling & herbivore & species & herbivore & Rodentia \\
\hline Artiodactyla & Camelidae & Alforjas taylori & 8.4 & 7.15 & 13.19486134 ground dwelling & browser, browser & species & herbivore & herbivore guild \\
\hline Perissodactyla & Equidae & Astrohippus ansae & 8.4 & 7.15 & 12.10597826 ground dwelling & browser & species & herbivore & herbivore guild \\
\hline Carnivora & Felidae & Adelphailurus kansensis & 8.5 & 7.15 & 10.0535 scansorial & carnivore & species & carnivore & carnivore guild \\
\hline Carnivora & Mustelidae & Martinogale alveodens & 9.15 & 7.15 & 5.406960334 scansorial & carnivore, omnivore & species & carnivore & carnivore guild \\
\hline Rodentia & Eomyidae & Kansasimys dubius & 9.25 & 7.15 & 4.9095 ground dwelling & herbivore & species & herbivore & Rodentia \\
\hline Perissodactyla & Rhinocerotidae & Aphelops mutilus & 11.3 & 7.15 & 15.22898489 ground dwelling & browser & species & herbivore & herbivore guild \\
\hline Artiodactyla & Camelidae & Megatylopus gigas & 11.5 & 7.15 & 14.2115985 ground dwelling & browser & species & herbivore & herbivore guild \\
\hline Lipotyphla & Talpidae & Hesperoscalops mcgrewi & 7.4 & 7.25 & 5.225 fossorial & insectivore & species & insectivore & NA \\
\hline Rodentia & Sciuridae & Marmota vetus & 7.4 & 7.25 & 6.4905 & & & NA & Rodentia \\
\hline Carnivora & Mustelidae & Pliotaxidea garberi & 7.65 & 7.25 & 7.567287386 scansorial & carnivore, omnivore & species & carnivore & carnivore guild \\
\hline Rodentia & Sciuridae & Spermophilus matthewi & 10.55 & 7.25 & 5.991 semifossorial & granivore, frugivore & species & herbivore & Rodentia \\
\hline Xenarthra & Megalonychidae & Megalonyx curvidens & 10.65 & 7.25 & 12.12838134 arboreal & folivore & species & herbivore & herbivore guild \\
\hline Perissodactyla & Equidae & Nannippus lenticularis & 11.1 & 7.25 & 11.4922022 & & & NA & NA \\
\hline Rodentia & Mylagaulidae & Ceratogaulus hatcheri & 11.7 & 7.25 & 7.396316533 ground dwelling & herbivore & species & herbivore & Rodentia \\
\hline Rodentia & Sciuridae & Paratamias tarassus & 7.4 & 7.4 & 3.31 scansorial & granivore, frugivore & species & herbivore & Rodentia \\
\hline Rodentia & Sciuridae & Spermophilus gidleyi & 7.75 & 7.4 & 4.604 semifossorial & granivore, frugivore & species & herbivore & Rodentia \\
\hline Perissodactyla & Rhinocerotidae & Teleoceras fossiger & 11.5 & 7.4 & 14.56337895 amphibious & grazer & species & herbivore & herbivore guild \\
\hline Carnivora & & Epicyon haydeni & 13.1 & 7.4 & 11.17111265 ground dwelling & carnivore, omnivore & species & carnivore & carnivore guild \\
\hline Carnivora & Mustelidae & Promephitis hootoni & 7.498 & 7.498 & 8.130427963 scansorial & carnivore, omnivore & genus & carnivore & carnivore guild \\
\hline Artiodactyla & Bovidae & Pseudotragus parvidens & 7.498 & 7.498 & 10.84057062 ground dwelling & grazer, browser & genus & herbivore & herbivore guild \\
\hline Perissodactyla & Equidae & Plesiohipparion longipes & 7.498 & 7.498 & 12.27738372 ground dwelling & browser & genus & herbivore & herbivore guild \\
\hline Perissodactyla & Rhinocerotidae & Stephanorhinus pikermiensis & 7.498 & 7.498 & 13.90991655 ground dwelling & browser & species & herbivore & herbivore guild \\
\hline Artiodactyla & Suidae & Microstonyx erymanthius & 8.225 & 7.498 & 12.50533964 ground dwelling & omnivore & genus & omnivore & NA \\
\hline \multirow[t]{2}{*}{ Perissodactyla } & Rhinocerotidae & Ceratotherium neumayri & 8.47 & 7.498 & 13.99741478 ground dwelling & grazer & species & herbivore & herbivore guild \\
\hline & & Cremohipparion moldavicum & 9.427 & 7.498 & $11.91127269 \mathrm{NA}$ & NA & genus & NA & NA \\
\hline Carnivora & & Epicyon saevus & 13.25 & 7.55 & 10.862 ground dwelling & carnivore, omnivore & species & carnivore & carnivore guild \\
\hline Rodentia & Cricetidae & Basirepomys robertsi & 7.6 & 7.6 & 3.943017916 ground dwelling & herbivore & species & herbivore & Rodentia \\
\hline Artiodactyla & Tayassuidae & Mylohyus elmorei & 7.6 & 7.6 & 11.59635666 ground dwelling & browser, grazer & species & herbivore & herbivore guild \\
\hline Artiodactyla & Antilocapridae & Ottoceros peacevalleyensis & 7.6 & 7.6 & 9.644 ground dwelling & browser, grazer & species & herbivore & herbivore guild \\
\hline Carnivora & Felidae & Lynx rexroadensis & 7.6 & 7.6 & 9.883529257 & & & NA & carnivore guild \\
\hline Carnivora & Mustelidae & Enhydritherium terraenovae & 9.05 & 7.6 & 9.737565016 scansorial & carnivore, omnivore & species & carnivore & carnivore guild \\
\hline Rodentia & Heteromyidae & Diprionomys minimus & 9.6 & 7.6 & 2.5 ground dwelling & granivore, browser & species & herbivore & Rodentia \\
\hline Carnivora & Procyonidae & Arctonasua eurybates & 10.45 & 7.6 & 9.675005156 scansorial & frugivore, carnivore & species & omnivore & carnivore guild \\
\hline Artiodactyla & Gelocidae & Floridameryx floridanus & 10.95 & 7.6 & 9.01 ground dwelling & grazer, browser & species & herbivore & herbivore guild \\
\hline Carnivora & & Borophagus pugnator & 11.4 & 7.6 & 10.58152217 ground dwelling & carnivore & species & carnivore & carnivore guild \\
\hline Perissodactyla & Equidae & Hippotherium ingenuum & 11.7 & 7.6 & 11.72759757 & & & NA & NA \\
\hline Perissodactyla & Tapiridae & Tapirus polkensis & 13.1 & 7.6 & 11.8875 amphibious & browser & species & herbivore & herbivore guild \\
\hline Carnivora & Mustelidae & Mellivora benfieldi & 7.604 & 7.604 & 8.293911505 semifossorial & carnivore, omnivore & genus & carnivore & carnivore guild \\
\hline Perissodactyla & Equidae & Neohipparion leptode & 11.2 & 7.65 & 12.6825003 ground dwelling & grazer & species & herbivore & herbivore guild \\
\hline Rodentia & Castoridae & Dipoides vallicula & 11.5 & 7.65 & 6.748 amphibious & browser & species & herbivore & Rodentia \\
\hline
\end{tabular}




\begin{tabular}{|c|c|}
\hline Rodentia & Cricetidae \\
\hline Artiodactyla & Tayassuidae \\
\hline Carnivora & Ursidae \\
\hline Carnivora & Mustelidae \\
\hline Rodentia & Castoridae \\
\hline Proboscidea & Gomphotheriidae \\
\hline Carnivora & Felidae \\
\hline Carnivora & Felidae \\
\hline Perissodactyla & Rhinocerotidae \\
\hline Rodentia & Geomyidae \\
\hline Lipotyphla & Talpidae \\
\hline Carnivora & Felidae \\
\hline Artiodactyla & Antilocapridae \\
\hline Artiodactyla & Tayassuidae \\
\hline Lagomorpha & Ochotonidae \\
\hline Rodentia & Zapodidae \\
\hline Carnivora & Hyaenidae \\
\hline Carnivora & Mustelidae \\
\hline Carnivora & Mustelidae \\
\hline Artiodactyla & Cervidae \\
\hline Artiodactyla & Giraffidae \\
\hline Carnivora & Ursidae \\
\hline Artiodactyla & Bovidae \\
\hline Carnivora & Hyaenidae \\
\hline \multicolumn{2}{|l|}{ Artiodactyla } \\
\hline Artiodactyla & Bovidae \\
\hline Carnivora & Mustelidae \\
\hline Carnivora & Ursidae \\
\hline Artiodactyla & Tragulidae \\
\hline Rodentia & Cricetidae \\
\hline Rodentia & Cricetidae \\
\hline Rodentia & Cricetidae \\
\hline Rodentia & Cricetidae \\
\hline Rodentia & Sciuridae \\
\hline Rodentia & Cricetidae \\
\hline Lagomorpha & Leporidae \\
\hline Artiodactyla & Cervidae \\
\hline Artiodactyla & Bovidae \\
\hline Artiodactyla & Bovidae \\
\hline Artiodactyla & Bovidae \\
\hline Artiodactyla & Bovidae \\
\hline Artiodactyla & Bovidae \\
\hline Artiodactyla & Giraffidae \\
\hline Artiodactyla & Giraffidae \\
\hline Perissodactyla & Rhinocerotidae \\
\hline Perissodactyla & Rhinocerotidae \\
\hline Perissodactyla & Rhinocerotidae \\
\hline Carnivora & Ursidae \\
\hline Artiodactyla & Bovidae \\
\hline Artiodactyla & Suidae \\
\hline Carnivora & Amphicyonidae \\
\hline
\end{tabular}

Pliotomodon primitivus Platygonus oregonensis Indarctos oregonensis

Lutravus halli

Dipoides stirtoni

Amebelodon floridanus

Pratifelis martini

Nimravides hibbardi

Aphelops kimballensis

Progeomys sulcatus

Hesperoscalops ruficervus

ynx proterolyncis

Texoceros altidens

Prosthennops serus

Ochotona spanglei

Pliozapus solus

Allohyaena kadici

Plesiogulo brachygnathus

Baranogale adroveri

Cervavitulus mimus

Birgerbohlinia schaubi

Promegantereon ogygia

Indarctos arctoides

Gazella schlosseri

Thalassictis hipparionum

Amphiprox anocerus

Miotragocerus pannoniae

Martes sansaniensis

Ursavus primaevus

Dorcatherium naui

Bensonomys hershkovitzi

gmodontomys pipecreekensis

Pliophenacomys koenigswaldi

Symmetrodontomys daamsi

Paenemarmota nevadensis

Prosomys mimus

Hypolagus fontinalis

Cervavitus sarmaticus

Damalavus boroccoi

Mesembriacerus melentisi

Oioceros wegneri

Protoryx solignaci

Selenoportax vexillarius

Palaeotragus pavlowae

Palaeotragus robinsoni

Chilotherium anderssoni

Chilotherium persiae

Subchilotherium intermedium

Agriotherium inexpetans

Criotherium argalioides

Parachleuastochoerus huenermanni

Amphicyon palaeindicus

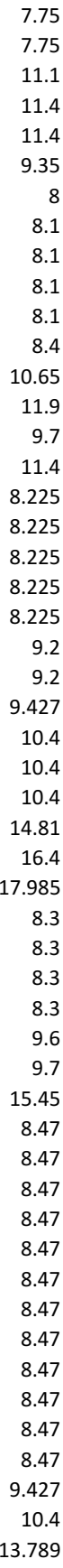

7.754 .678 ground dwelling herbivore species

7.7510 .54759143 ground dwelling browser, grazer species

$7.75 \quad 12.8195$ ground dwelling herbivore, carnivore species

7.758 .058326153 scansorial carnivore, omnivore species

7.757 .506221893 amphibious browser

7.9514 .938 ground dwelling browser species

811.6045 scansorial carnivore species

8.110 .946 scansorial carnivore species

8.114 .39806459 ground dwelling browser species

8.11 .722356187 fossorial browser species

8.14 .667 fossorial insectivore species

8.19 .238132309 scansorial carnivore species

8.19 .894248974 ground dwelling browser species

8.110 .99340916 ground dwelling herbivore, omnivore species

$8.2 \quad 4.44$ ground dwelling herbivore species

8.2 2.93 ground dwelling herbivore species

8.22511 .22510233 ground dwelling carnivore species

8.2259 .903418485 scansorial carnivore genus

8.2257 .600833392 scansorial carnivore, omnivore genus

8.2259 .903418485 ground dwelling grazer, browser genus

8.22513 .5921598 ground dwelling browser genus

$8.22510 .69090259 \mathrm{NA}$ NA

8.22511 .98265282 ground dwelling herbivore, carnivore species

8.22510 .04387618 ground dwelling grazer, browser genus

8.2259 .868879709 ground dwelling carnivore genus

8.22510 .81984735 ground dwelling grazer, browser genus

8.22511 .28957471 ground dwelling grazer, browser genus

8.2258 .699166481 scansorial carnivore, omnivore genus

$8.225 \quad 10.36$ ground dwelling herbivore, carnivore species

8.22510 .49057768 ground dwelling frugivore, grazer species

$\begin{array}{ll}8.3 & 2.921175333 \text { ground dwelling herbivore } \\ 8.3 & \text { species }\end{array}$

$8.3 \quad 4.32$ ground dwelling herbivore species

$8.3 \quad 4.65$ ground dwelling herbivore species

$8.3 \quad 3.02$ ground dwelling herbivore species

8.38 .942 scansorial granivore, frugivore species

8.3 3.4725 ground dwelling herbivore species

8.3 6.14 ground dwelling grazer, browser species

$8.47 \quad 11.28957471$ ground dwelling grazer, browser genus

8.4711 .08234205 ground dwelling grazer, browser genus

8.4711 .00175157 ground dwelling grazer, browser genus

8.4711 .00175157 ground dwelling grazer, browser genus

8.4711 .28957471 ground dwelling grazer, browser genus

8.4712 .19909582 ground dwelling grazer, browser genus

8.4712 .34646127 ground dwelling browser, grazer genus

8.4712 .89908169 ground dwelling browser, grazer genus

8.4713 .90991655 ground dwelling browser species

$8.47 \quad 13.22835136$ ground dwelling carnivore, omnivore species

$8.47 \quad 12.20600358$ ground dwelling grazer, browser

8.4710 .57116816 ground dwelling omnivore genus

$\begin{array}{lll}8.47 & 11.77542017 \text { ground dwelling carnivore } & \text { genus }\end{array}$

carnivore guild

Rodentia

herbivore guild

carnivore guild

carnivore guild

herbivore guild

Rodentia

NA

carnivore guild

herbivore guild

NA

herbivore guild

Rodentia

carnivore guild

carnivore guild

carnivore guild

herbivore guild

herbivore guild

NA

carnivore guild

herbivore guild

carnivore guild

herbivore guild

herbivore guild

carnivore guild

carnivore guild

herbivore guild

Rodentia

Rodentia

Rodentia

Rodentia

Rodentia

Rodentia

herbivore guild

herbivore guild

herbivore guild

herbivore guild

herbivore guild

herbivore guild

herbivore guild

herbivore guild

herbivore guild

herbivore guild

herbivore guild

herbivore guild

carnivore guild

herbivore guild

carnivore guild 


\begin{tabular}{|c|c|c|c|}
\hline Artiodactyla & Tragulidae & Dorcatherium majus & 13.789 \\
\hline Artiodactyla & Bovidae & Gazella lydekkeri & 13.789 \\
\hline Artiodactyla & Bovidae & Sivaceros gradiens & 13.789 \\
\hline Artiodactyla & Palaeomerycidae & Palaeomeryx eminens & 13.789 \\
\hline Artiodactyla & Suidae & Conohyus sindiensis & 14.81 \\
\hline Artiodactyla & Suidae & Listriodon pentapotamiae & 14.81 \\
\hline Perissodactyla & Rhinocerotidae & Hispanotherium matritense & 16.435 \\
\hline Perissodactyla & Rhinocerotidae & Brachypotherium perimense & 18.45 \\
\hline Perissodactyla & Hyracodontidae & Aprotodon fatehjangense & 21.2 \\
\hline Perissodactyla & Equidae & Calippus elachistus & 10.95 \\
\hline Perissodactyla & Equidae & Nannippus westoni & 10.95 \\
\hline Perissodactyla & Rhinocerotidae & Teleoceras proterum & 10.95 \\
\hline Artiodactyla & Palaeomerycidae & Yumaceras hamiltoni & 10.95 \\
\hline Perissodactyla & Equidae & Hippotherium plicatile & 10.95 \\
\hline Perissodactyla & Equidae & Calippus hondurensis & 11.6 \\
\hline Perissodactyla & Equidae & Protohippus gidleyi & 11.6 \\
\hline Perissodactyla & Equidae & Pseudhipparion skinneri & 11.7 \\
\hline Perissodactyla & Equidae & Neohipparion trampasense & 11.8 \\
\hline Perissodactyla & Equidae & Nannippus morgani & 9.05 \\
\hline Perissodactyla & Equidae & Hipparion tehonense & 12.25 \\
\hline Artiodactyla & Bovidae & Dorcadoryx orientalis & 8.725 \\
\hline Perissodactyla & Equidae & Hipparion chiai & 8.725 \\
\hline Perissodactyla & Equidae & Hipparion weihoense & 8.725 \\
\hline \multirow{2}{*}{ Perissodactyla } & Rhinocerotidae & Stephanorhinus orientalis & 8.725 \\
\hline & & Dinocrocuta gigantea & 8.725 \\
\hline Perissodactyla & Equidae & Calippus cerasinus & 11.6 \\
\hline Carnivora & & Borophagus orc & 9.05 \\
\hline Perissodactyla & Equidae & Calippus maccartyi & 9.05 \\
\hline Xenarthra & Megalonychidae & Pliometanastes protistus & 10.95 \\
\hline Artiodactyla & Camelidae & Hemiauchenia minima & 10.95 \\
\hline Perissodactyla & Tapiridae & Tapirus simpsoni & 11.1 \\
\hline Artiodactyla & Camelidae & Procamelus grandis & 12.75 \\
\hline Carnivora & Mustelidae & Cernictis hesperus & 9.15 \\
\hline Rodentia & Cricetidae & Goniodontomys disjunctus & 11.4 \\
\hline Artiodactyla & Capridae & Aragoral mudejar & 9.2 \\
\hline Artiodactyla & Bovidae & Tragoportax gaudryi & 9.2 \\
\hline Perissodactyla & Rhinocerotidae & Acerorhinus zernowi & 9.2 \\
\hline \multirow[t]{2}{*}{ Carnivora } & Ursidae & Indarctos nevadensis & 9.2 \\
\hline & & Magericyon anceps & 10.154 \\
\hline Artiodactyla & Moschidae & Micromeryx flourensianus & 15.804 \\
\hline Carnivora & Felidae & Pseudaelurus quadridentatus & 18.2 \\
\hline Artiodactyla & Palaeomerycidae & Yumaceras ruminalis & 9.25 \\
\hline Rodentia & Geomyidae & Pliosaccomys higginsensis & 9.25 \\
\hline Artiodactyla & Palaeomerycidae & Yumaceras figginsi & 11.2 \\
\hline Perissodactyla & Rhinocerotidae & Aphelops malacorhinus & 11.2 \\
\hline Perissodactyla & Equidae & Pliohippus nobilis & 11.3 \\
\hline Artiodactyla & Gelocidae & Pseudoceras skinneri & 11.9 \\
\hline Carnivora & Mustelidae & Promephitis maeotica & 9.427 \\
\hline Artiodactyla & Giraffidae & Samotherium maeoticum & 9.427 \\
\hline Artiodactyla & Bovidae & Procobus melania & 9.427 \\
\hline Artiodactyla & Bovidae & Tragoportax frolovi & 9.427 \\
\hline Artiodactyla & Bovidae & Tragoportax spectabilis & 9.427 \\
\hline
\end{tabular}

8.4711 .37937553 ground dwelling frugivore, grazer species 8.4710 .84978096 ground dwelling grazer, browser

8.4710 .79912409 ground dwelling grazer, browser

$8.47 \quad 12.25896304$ ground dwelling browser

8.4710 .49978802 ground dwelling omnivore

$8.47 \quad 11.6004237$ ground dwelling omnivore

8.4713 .5921598 ground dwelling browser

8.4714 .49937833 amphibious grazer

8.4713 .59906756 ground dwelling browser

8.510 .65670803 ground dwelling grazer

8.511 .26641425 ground dwelling grazer

8.514 .23063376 amphibious grazer

8.512 .36165435 ground dwelling browser

8.512 .15188927

8.511 .47263339 ground dwelling grazer

8.512 .11457561 ground dwelling grazer, browser

8.510 .93028989 ground dwelling grazer

8.511 .8329728 ground dwelling graze

8.610 .49057768 ground dwelling grazer

8.611 .71636273 ground dwelling browser

8.72510 .15670285 ground dwelling grazer, browser

8.72512 .22672684 ground dwelling browser

8.72512 .32343542 ground dwelling browser

$8.725 \quad 14.86548936$ ground dwelling browser

$\begin{array}{ll}8.725 & 12.84842482 \mathrm{NA}\end{array}$ NA

8.7511 .40054958 ground dwelling grazer

8.9510 .12155156 ground dwelling carnivore

8.9511 .19747131 ground dwelling grazer

8.9511 .75769067 arboreal

8.9511 .58211048 ground dwelling browser, grazer

9.0512 .61694106 amphibious browser

9.0513 .10627576 ground dwelling browser

5.18 scansorial carnivore, omnivore species

3.8005 ground dwelling herbivore species

9.211 .62344955 ground dwelling grazer, browser species

9.211 .28957471 ground dwelling grazer, browser genus

9.213 .70959164 ground dwelling browser species

9.212 .44604029 ground dwelling herbivore, carnivore species

$9.211 .91818044 \mathrm{NA}$

NA

$9.2 \quad 8.293911505$ ground dwelling browser, grazer

9.210 .30867346 scansoral

carnivore

$9.25 \quad 12.66$ ground dwelling browser

$9.25 \quad 2.903$ fossorial browser

9.2512 .628086 ground dwelling browser

9.2514 .38450352 ground dwelling browser

9.2512 .21060875 ground dwelling browser

9.258 .425733355 ground dwelling herbivore

9.4278 .006088368 scansorial carnivore, omnivore

9.42713 .38492715 ground dwelling grazer

9.42711 .98265282 ground dwelling grazer, browser

9.42711 .60733145 ground dwelling grazer, browser

9.42711 .77542017 ground dwelling grazer, browser herbivore

herbivore

herbivore

herbivore

omnivore

omnivore

herbivore

herbivore

herbivore

herbivore

herbivore

herbivore

herbivore

NA

herbivore

herbivore

herbivore

herbivore

herbivore

herbivore

herbivore

herbivore

herbivore

herbivore

herbivore

carnivore

herbivore

herbivore

herbivore

herbivore

herbivore

carnivore

herbivore

herbivore

herbivore

herbivore

omnivore

herbivore

carnivore

herbivore

herbivore

herbivore

herbivore

herbivore

herbivore

carnivore

herbivore

herbivore

herbivore

herbivore herbivore guild herbivore guild herbivore guild herbivore guild

herbivore guild herbivore guild herbivore guild herbivore guild herbivore guild

herbivore guild

herbivore guild NA

herbivore guild herbivore guild herbivore guild

herbivore guild herbivore guild herbivore guild herbivore guild herbivore guild herbivore guild herbivore guild NA

herbivore guild carnivore guild

herbivore guild

herbivore guild herbivore guild herbivore guild herbivore guild carnivore guild Rodentia

herbivore guild herbivore guild herbivore guild carnivore guild NA

herbivore guild carnivore guild herbivore guild Rodentia

herbivore guild herbivore guild herbivore guild herbivore guild carnivore guild herbivore guild herbivore guild herbivore guild herbivore guild 


\begin{tabular}{|c|c|c|c|}
\hline Artiodactyla & Bovidae & Tragoportax validus & 9.427 \\
\hline Perissodactyla & Equidae & Hipparion platigenis & 9.427 \\
\hline Creodonta & Hyaenodontidae & Dissopsalis carnifex & 9.427 \\
\hline Perissodactyla & Rhinocerotidae & Gaindatherium browni & 12.375 \\
\hline Artiodactyla & Suidae & Listriodon splendens & 18.2 \\
\hline Lagomorpha & Leporidae & Pronotolagus nevadensis & 9.45 \\
\hline Rodentia & Sciuridae & Marmota korthi & 9.45 \\
\hline Rodentia & Geomyidae & Parapliosaccomys oregonensis & 9.7 \\
\hline Xenarthra & Megalonychidae & Pliometanastes galushai & 9.5 \\
\hline Rodentia & Sciuridae & Marmota minor & 9.6 \\
\hline Rodentia & Cricetidae & Peromyscus antiquus & 10.85 \\
\hline Rodentia & Sciuridae & Spermophilus argonautus & 11.8 \\
\hline Rodentia & Heteromyidae & Diprionomys parvus & 16.05 \\
\hline Carnivora & Felidae & Lynx longignathus & 9.7 \\
\hline Rodentia & Sciuridae & Parapaenemarmota oregonensis & 9.7 \\
\hline Rodentia & Sciuridae & Spermophilus mckayensis & 9.7 \\
\hline Artiodactyla & Tayassuidae & Platygonus brachirostris & 9.7 \\
\hline Rodentia & Castoridae & Dipoides smithi & 9.7 \\
\hline Lipotyphla & Talpidae & Neurotrichus columbianus & 9.7 \\
\hline Lipotyphla & Talpidae & Gaillardia thomsoni & 10.45 \\
\hline Rodentia & Mylagaulidae & Hesperogaulus wilsoni & 11.4 \\
\hline Rodentia & Cricetidae & Basirepomys pliocenicus & 11.8 \\
\hline Rodentia & Cricetidae & Copemys esmeraldensis & 13.9 \\
\hline Carnivora & Mustelidae & Plionictis oregonensis & 9.8 \\
\hline Perissodactyla & Equidae & Calippus martini & 13.6 \\
\hline Carnivora & Felidae & Machairodus alberdiae & 10.154 \\
\hline Artiodactyla & Cervidae & Euprox dicranoceros & 10.154 \\
\hline Artiodactyla & Giraffidae & Decennatherium pachecoi & 10.154 \\
\hline \multirow[t]{2}{*}{ Perissodactyla } & Equidae & Hipparion catalaunicum & 10.154 \\
\hline & & Magericyon castellanus & 10.154 \\
\hline Artiodactyla & Suidae & Parachleuastochoerus crusafonti & 10.4 \\
\hline Perissodactyla & Chalicotheriidae & Chalicotherium grande & 14.81 \\
\hline Carnivora & Amphicyonidae & Amphicyon major & 18.2 \\
\hline Perissodactyla & Rhinocerotidae & Lartetotherium sansaniensis & 18.2 \\
\hline Perissodactyla & Rhinocerotidae & Alicornops simorrensis & 18.45 \\
\hline Proboscidea & Gomphotheriidae & Gomphotherium obscurum & 17.25 \\
\hline Carnivora & Mustelidae & Martes mellibulla & 10.4 \\
\hline Carnivora & Mustelidae & Mesomephitis medius & 10.4 \\
\hline Carnivora & Ailuridae & Simocyon diaphorus & 10.4 \\
\hline Carnivora & Mustelidae & Trochictis narcisoi & 10.4 \\
\hline Artiodactyla & Bovidae & Orygotherium escheri & 10.4 \\
\hline \multirow[t]{2}{*}{ Perissodactyla } & Equidae & Hippotherium sumegense & 10.4 \\
\hline & & Austroportax latifrons & 10.4 \\
\hline Artiodactyla & Bovidae & Miotragocerus monacensis & 11.925 \\
\hline Artiodactyla & Bovidae & Protragocerus chantrei & 11.925 \\
\hline Artiodactyla & Suidae & Parachleuastochoerus steinheimensis & 13.789 \\
\hline Carnivora & Mustelidae & Paralutra jaegeri & 15.804 \\
\hline Carnivora & Mustelidae & Martes munki & 16.435 \\
\hline Carnivora & Mustelidae & Trocharion albanense & 16.435 \\
\hline Carnivora & Ursidae & Ursavus brevirhinus & 17.95 \\
\hline Rodentia & Geomyidae & Pliogeomys russelli & 10.45 \\
\hline Artiodactyla & Merycoidodontidae & Merychyus major & 13.1 \\
\hline
\end{tabular}

9.42711 .51292546 ground dwelling grazer, browser genus $9.427 \quad 12.25435786$ ground dwelling browser

9.42711 .00209984 scansorial insectivore

9.42713 .59906756 ground dwelling browser

9.42711 .41851948 ground dwelling omnivore

$9.45 \quad 4.1$ ground dwelling grazer, browser

granivore, frugivore species

3.189 fossorial browser species

9.064 arboreal folivore species

7.0915 semifossorial granivore, frugivore species

3.2965 ground dwelling herbivore species

4.878 semifossorial granivore, frugivore species

2.404 ground dwelling granivore, browser species

9.79 .602554891 scansorial carnivore species

$9.7 \quad 7.68$ scansorial granivore, frugivore species

$9.7 \quad 5.151$ semifossorial granivore, frugivore species

$9.7 \quad 10.58$ ground dwelling browser, grazer species

9.76 .667248021 amphibious browser species

9.73 .265 fossorial insectivore species

$9.7 \quad 4.23$ fossorial insectivore species

9.76 .036632368 ground dwelling herbivore species

9.74 .095195306 ground dwelling herbivore species

$9.7 \quad 3.336$ ground dwelling herbivore species

9.87 .472 scansorial carnivore, omnivore species

9.911 .86726677 ground dwelling grazer species

10.15411 .51292546 scansorial carnivore genus

10.15411 .08234205 ground dwelling grazer, browser genus

10.15413 .70959164 ground dwelling browser genus

10.15412 .17606997 ground dwelling browser genus

10.15412 .19679324 NA

10.15410 .71392844 ground dwelling omnivore

10.15413 .75334076 ground dwelling browser

10.15412 .11672209 ground dwelling carnivore

10.15413 .27670565 ground dwelling browser

$10.154 \quad 13.38492715$ ground dwelling browser

10.25

14.99 ground dwelling browser

carnivore, omnivore genus

0.46 .214677166 scansorial carnivore, omnivore genus

10.410 .91425334 ground dwelling carnivore, omnivore genus

10.47 .313010255 scansorial carnivore, omnivore genus

10.49 .472835073 ground dwelling grazer, browser species

10.412 .34646127 ground dwelling grazer, browser genus

$10.4 \quad 10.91425334 \mathrm{NA}$

10.411 .40700655 ground dwelling grazer, browser genus

$10.4 \quad 10.91425334$ ground dwelling grazer, browser genus

10.411 .19747131 ground dwelling omnivore genus

10.48 .611668248 scansorial carnivore, omnivore species

10.47 .377482638 scansorial carnivore, omnivore genus

10.47 .17024998 scansorial carnivore, omnivore species

$10.4 \quad 9.58$ ground dwelling herbivore, carnivore species

10.452 .7175 fossorial browser species $10.45 \quad 11.79672574$ herbivore

herbivore

omnivore

herbivore

herbivore

herbivore

herbivore

herbivore

herbivore

herbivore

herbivore

carnivore

herbivore

herbivore

herbivore

herbivore

insectivore

insectivore

herbivore

herbivore

herbivore

carnivore

herbivore

carnivore

herbivore

herbivore

herbivore

NA

omnivore

herbivore

carnivore

herbivore

herbivore

herbivore

carnivore

carnivore

carnivore

carnivore

herbivore

herbivore

NA

herbivore

herbivore

omnivore

carnivore

carnivore

carnivore

omnivore

herbivore

NA herbivore guild

herbivore guild

herbivore guild

herbivore guild

Rodentia

Rodentia

herbivore guild

Rodentia

Rodentia

carnivore guild

Rodentia

Rodentia

herbivore guild

Rodentia

NA

NA

Rodentia

Rodentia

carnivore guild

herbivore guild

carnivore guild

herbivore guild

herbivore guild

herbivore guild

NA

herbivore guild

carnivore guild

herbivore guild

herbivore guild

herbivore guild

carnivore guild

carnivore guild

carnivore guild

carnivore guild

herbivore guild

herbivore guild

NA

herbivore guild

herbivore guild

NA

carnivore guild

carnivore guild

carnivore guild

carnivore guild

Rodentia 


\begin{tabular}{|c|c|c|}
\hline Rodentia & Castoridae & Eucastor tortus \\
\hline Carnivora & & Aelurodon taxoides \\
\hline Artiodactyla & Palaeomerycidae & Cranioceras unicornis \\
\hline Carnivora & Amphicyonidae & Amphicyon tairumensis \\
\hline Carnivora & Percrocutidae & Percrocuta tungurensis \\
\hline Artiodactyla & Giraffidae & Palaeotragus tungurensis \\
\hline Proboscidea & Gomphotheriidae & Platybelodon grangeri \\
\hline Perissodactyla & Equidae & Dinohippus leardi \\
\hline Rodentia & Geomyidae & Pliosaccomys dubius \\
\hline Rodentia & Zapodidae & Macrognathomys nanus \\
\hline Xenarthra & Mylodontidae & Thinobadistes segnis \\
\hline Artiodactyla & Antilocapridae & Plioceros dehlini \\
\hline Artiodactyla & Protoceratidae & Synthetoceras tricornatus \\
\hline Carnivora & Barbourofelidae & Barbourofelis fricki \\
\hline Rodentia & Cricetidae & Paramicrotoscoptes hibbardi \\
\hline Lipotyphla & Talpidae & Lemoynea biradicularis \\
\hline Eulipotyphla & Soricidae & Sorex edwardsi \\
\hline Carnivora & Barbourofelidae & Barbourofelis loveorum \\
\hline Eulipotyphla & Soricidae & Sorex yatkolai \\
\hline Eulipotyphla & Soricidae & Alluvisorex arcadentes \\
\hline Lagomorpha & Leporidae & Pronotolagus apachensis \\
\hline Rodentia & Sciuridae & Spermophilus fricki \\
\hline Perissodactyla & Rhinocerotidae & Teleoceras major \\
\hline Eulipotyphla & Soricidae & Limnoecus tricuspis \\
\hline Carnivora & Mustelidae & Plionictis ogygia \\
\hline Artiodactyla & Camelidae & Michenia yavapaiensis \\
\hline Carnivora & & Carpocyon robustus \\
\hline Artiodactyla & Moschidae & Parablastomeryx gregorii \\
\hline Perissodactyla & Equidae & Hippotherium occidentale \\
\hline Carnivora & & Borophagus littoralis \\
\hline Carnivora & Barbourofelidae & Barbourofelis morrisi \\
\hline Proboscidea & Gomphotheriidae & Serbelodon barbourensis \\
\hline Perissodactyla & Equidae & Pseudhipparion gratum \\
\hline Artiodactyla & Camelidae & Protolabis coartatus \\
\hline Carnivora & & Cynarctus crucidens \\
\hline Carnivora & & Paratomarctus euthos \\
\hline Perissodactyla & Equidae & Neohipparion affine \\
\hline Perissodactyla & Equidae & Calippus regulus \\
\hline Carnivora & & Carpocyon webbi \\
\hline Rodentia & Heteromyidae & Perognathus minutus \\
\hline Artiodactyla & Camelidae & Protolabis heterodontus \\
\hline Artiodactyla & Palaeomerycidae & Palaeomeryx magnus \\
\hline Carnivora & Ursidae & Plithocyon armagnacensis \\
\hline Artiodactyla & & Heteroprox larteti \\
\hline Artiodactyla & Cervidae & Dicrocerus elegans \\
\hline Artiodactyla & Bovidae & Eotragus sansaniensis \\
\hline Artiodactyla & Suidae & Conohyus simorrensis \\
\hline Perissodactyla & Rhinocerotidae & Brachypotherium brachypus \\
\hline Artiodactyla & Suidae & Hyotherium soemmeringi \\
\hline Artiodactyla & Tragulidae & Dorcatherium crassum \\
\hline Carnivora & Ursidae & Hemicyon sansaniensis \\
\hline Carnivora & Amphicyonidae & Pseudocyon sansaniensis \\
\hline
\end{tabular}

$\begin{array}{r}15.9 \\ 12.25 \\ 15.4 \\ 13.2 \\ 13.2 \\ 13.2 \\ 13.2 \\ 11.8 \\ 11.8 \\ 12.2 \\ 10.95 \\ 10.95 \\ 12.25 \\ 11.1 \\ 11.1 \\ 11.3 \\ 11.3 \\ 11.7 \\ 11.3 \\ 14.7 \\ 17.5 \\ 11.4 \\ 12.25 \\ 16.75 \\ 17.2 \\ 11.6 \\ 11.9 \\ 11.6 \\ 11.6 \\ 11.7 \\ 11.7 \\ 11.8 \\ 11.9 \\ 12.2 \\ 12.25 \\ 12.25 \\ 12.25 \\ 12.9 \\ 13.1 \\ 16.9 \\ 18.2 \\ 13.789 \\ 13.789 \\ 14.181 \\ 14.81 \\ 14.81 \\ 14.81 \\ 16.435 \\ 17.985 \\ 18.2 \\ 18.2 \\ \hline \\ \hline\end{array}$

10.556 .6035 amphibious browser species

10.6510 .85395891 ground dwelling carnivore, omnivore species 10.6511 .83382727 ground dwelling browser species $10.651 \quad 12.27277855$ ground dwelling carnivore genus 10.65111 .81226153 ground dwelling carnivore, omnivore genus 10.65112 .99348768 ground dwelling browser, grazer genus $10.651 \quad 14.73884718$ ground dwelling browser genus 10.7511 .74671509 ground dwelling browser species $\begin{array}{llll}10.85 & 3.3225 \text { fossorial browser species }\end{array}$ 10.851 .65 ground dwelling herbivore species 10.9513 .37838449 ground dwelling herbivore species 10.9510 .6099828 ground dwelling browser, grazer species 10.9512 .41144909 ground dwelling grazer species

11.112 .54876773 ground dwelling carnivore, omnivore species $\begin{array}{lll}11.1 & 3.97 \text { ground dwelling herbivore } & \text { species }\end{array}$ 11.13 .82 fossorial insectivore species 11.11 .945522902 ground dwelling omnivore species

11.212 .18728792 ground dwelling carnivore, omnivore species 11.31 .610063246 ground dwelling omnivore species $11.3 \quad 1.7445$ ground dwelling omnivore species $11.3 \quad 5.266$ ground dwelling grazer, browser species 11.45 .447 semifossorial granivore, frugivore species 11.414 .70643613 amphibious grazer species $11.4 \quad 1.6315$ ground dwelling omnivore species $\begin{array}{lll}11.4 & 6.022501652 \text { scansorial carnivore, omnivore species }\end{array}$ $\begin{array}{ll}11.5 & 11.46457118\end{array}$

11.5510 .1853941 ground dwelling carnivore, omnivore species 11.69 .892352009 ground dwelling browser, grazer species $\begin{array}{ll}11.6 & 12.25823063\end{array}$

11.610 .55881614 ground dwelling carnivore species

$11.6 \quad 11.265$ ground dwelling carnivore, omnivore species $11.6 \quad 14.827$ ground dwelling browser species

11.611 .62109325 ground dwelling grazer species

11.611 .92677988 ground dwelling browser, browser species

$11.6 \quad 8.613$ ground dwelling carnivore, omnivore species

11.69 .811685524 ground dwelling carnivore, omnivore species

11.612 .22934314 ground dwelling grazer species

$\begin{array}{lll}11.6 & 10.69290721 \text { ground dwelling grazer species }\end{array}$

11.610 .26768317 ground dwelling carnivore, omnivore species

$11.6 \quad 1.78$ ground dwelling granivore, browser species

$\begin{array}{ll}11.6 & 12.74734841 \text { ground dwelling browser, browser species }\end{array}$ 11.60812 .20600358 ground dwelling browser genus 11.60811 .66029091 ground dwelling herbivore, carnivore species 11.60810 .46294666 ground dwelling grazer, browser genus 11.60810 .71392844 ground dwelling grazer, browser species 11.60810 .04387618 ground dwelling grazer, browser genus 11.60811 .14220926 ground dwelling omnivore species 11.60814 .28523792 amphibious grazer species 11.60811 .05010586 ground dwelling omnivore species 11.6089 .951772772 ground dwelling frugivore, grazer species 11.60812 .32343542 ground dwelling herbivore, carnivore species $\begin{array}{lll}11.608 & 12.04155996 \text { ground dwelling carnivore } & \text { species }\end{array}$

\begin{tabular}{|c|c|}
\hline $\begin{array}{l}\text { herbivore } \\
\text { carnivore }\end{array}$ & $\begin{array}{l}\text { Rodentia } \\
\text { carnivore guild }\end{array}$ \\
\hline herbivore & herbivore guild \\
\hline carnivore & carnivore guild \\
\hline carnivore & carnivore guild \\
\hline herbivore & herbivore guild \\
\hline herbivore & herbivore guild \\
\hline herbivore & herbivore guild \\
\hline herbivore & Rodentia \\
\hline herbivore & Rodentia \\
\hline herbivore & herbivore guild \\
\hline herbivore & herbivore guild \\
\hline herbivore & herbivore guild \\
\hline carnivore & carnivore guild \\
\hline herbivore & Rodentia \\
\hline insectivore & NA \\
\hline omnivore & NA \\
\hline carnivore & carnivore guild \\
\hline omnivore & NA \\
\hline omnivore & NA \\
\hline herbivore & herbivore guild \\
\hline herbivore & Rodentia \\
\hline herbivore & herbivore guild \\
\hline omnivore & NA \\
\hline carnivore & carnivore guild \\
\hline NA & NA \\
\hline carnivore & carnivore guild \\
\hline herbivore & herbivore guild \\
\hline NA & NA \\
\hline carnivore & carnivore guild \\
\hline carnivore & carnivore guild \\
\hline herbivore & herbivore guild \\
\hline herbivore & herbivore guild \\
\hline herbivore & herbivore guild \\
\hline carnivore & carnivore guild \\
\hline carnivore & carnivore guild \\
\hline herbivore & herbivore guild \\
\hline herbivore & herbivore guild \\
\hline carnivore & carnivore guild \\
\hline herbivore & Rodentia \\
\hline herbivore & herbivore guild \\
\hline herbivore & herbivore guild \\
\hline omnivore & carnivore guild \\
\hline herbivore & herbivore guild \\
\hline herbivore & herbivore guild \\
\hline herbivore & herbivore guild \\
\hline omnivore & NA \\
\hline herbivore & herbivore guild \\
\hline omnivore & NA \\
\hline herbivore & herbivore guild \\
\hline omnivore & carnivore guild \\
\hline carnivore & carnivore guild \\
\hline
\end{tabular}




\begin{tabular}{|c|c|c|c|c|c|c|c|c|c|}
\hline Rodentia & Aplodontidae & Tardontia occidentale & 11.65 & 11.65 & 5.224 fossorial & herbivore & species & herbivore & Rodentia \\
\hline Carnivora & Felidae & Nimravides galiani & 11.8 & 11.65 & 11.76424836 scansorial & carnivore & species & carnivore & carnivore guild \\
\hline Rodentia & Sciuridae & Ammospermophilus junturensis & 11.85 & 11.65 & 3.98 semifossorial & granivore, frugivore & species & herbivore & Rodentia \\
\hline Lagomorpha & Ochotonidae & Hesperolagomys galbreathi & 12.2 & 11.65 & 4.847 ground dwelling & herbivore & species & herbivore & herbivore guild \\
\hline Lagomorpha & Ochotonidae & Oklahomalagus whisenhunti & 11.7 & 11.7 & 5.249 ground dwelling & herbivore & species & herbivore & herbivore guild \\
\hline Rodentia & Sciuridae & Miospermophilus lavertyi & 11.7 & 11.7 & 4.6685 scansorial & granivore, frugivore & species & herbivore & Rodentia \\
\hline Artiodactyla & Camelidae & Nothotylopus camptognathus & 11.7 & 11.7 & 12.72 ground dwelling & browser, browser & species & herbivore & herbivore guild \\
\hline Lipotyphla & Talpidae & Domninoides hessei & 11.7 & 11.7 & 5.008 fossorial & insectivore & species & insectivore & NA \\
\hline Lipotyphla & Talpidae & Domninoides knoxjonesi & 11.7 & 11.7 & 3.445 fossorial & insectivore & species & insectivore & NA \\
\hline Carnivora & Barbourofelidae & Barbourofelis osborni & 11.7 & 11.7 & 11.114 & & & NA & carnivore guild \\
\hline Rodentia & Cricetidae & Copemys mariae & 11.75 & 11.7 & 3.45 ground dwelling & herbivore & species & herbivore & Rodentia \\
\hline Artiodactyla & Camelidae & Megatylopus primaevus & 11.8 & 11.7 & 14.2394586 ground dwelling & browser & species & herbivore & herbivore guild \\
\hline Artiodactyla & Camelidae & Pliauchenia magnifontis & 11.8 & 11.7 & 12.37639487 ground dwelling & browser, browser & species & herbivore & herbivore guild \\
\hline Perissodactyla & Equidae & Hipparion forcei & 11.8 & 11.7 & 12.33163965 ground dwelling & browser & species & herbivore & herbivore guild \\
\hline Eulipotyphla & Soricidae & Tregosorex holmani & 11.8 & 11.7 & 3.2915 ground dwelling & omnivore & species & omnivore & NA \\
\hline Perissodactyla & Equidae & Pseudhipparion curtivallum & 11.9 & 11.7 & 11.77713553 ground dwelling & grazer & species & herbivore & herbivore guild \\
\hline Artiodactyla & Antilocapridae & Paracosoryx furlongi & 11.9 & 11.7 & 9.51 ground dwelling & browser, grazer & species & herbivore & herbivore guild \\
\hline Perissodactyla & Tapiridae & Tapirus johnsoni & 11.9 & 11.7 & 12.36421153 amphibious & browser & species & herbivore & herbivore guild \\
\hline Carnivora & Amphicyonidae & Ischyrocyon gidleyi & 12 & 11.7 & 12.29824209 ground dwelling & carnivore & species & carnivore & carnivore guild \\
\hline Perissodactyla & Equidae & Megahippus matthewi & 12.15 & 11.7 & 12.61776345 ground dwelling & browser & species & herbivore & herbivore guild \\
\hline Rodentia & Heteromyidae & Cupidinimus tertius & 12.2 & 11.7 & 2.802 ground dwelling & granivore, browser & species & herbivore & Rodentia \\
\hline Perissodactyla & Equidae & Pliohippus tantalus & 12.2 & 11.7 & 13.05565748 ground dwelling & browser & species & herbivore & herbivore guild \\
\hline Perissodactyla & Equidae & Protohippus supremus & 12.3 & 11.7 & 12.17651902 ground dwelling & grazer, browser & species & herbivore & herbivore guild \\
\hline Rodentia & Zapodidae & Macrognathomys gemmacolis & 12.45 & 11.7 & 1.897 ground dwelling & herbivore & species & herbivore & Rodentia \\
\hline Perissodactyla & Equidae & Pliohippus tehonensis & 12.6 & 11.7 & 12.1305 ground dwelling & browser & species & herbivore & herbivore guild \\
\hline Artiodactyla & Antilocapridae & Cosoryx cerroensis & 12.65 & 11.7 & 9.556857677 ground dwelling & grazer & species & herbivore & herbivore guild \\
\hline Artiodactyla & Tayassuidae & Prosthennops niobrarensis & 12.75 & 11.7 & 10.68071319 ground dwelling & herbivore, omnivore & species & omnivore & NA \\
\hline Rodentia & Castoridae & Nothodipoides planus & 12.8 & 11.7 & 6.49 amphibious & browser & species & herbivore & Rodentia \\
\hline Perissodactyla & Equidae & Pseudhipparion retrusum & 12.9 & 11.7 & 11.38622232 ground dwelling & grazer & species & herbivore & herbivore guild \\
\hline Artiodactyla & Merycoidodontidae & Merychyus novomexicanus & 13.1 & 11.7 & 11.72662251 ground dwelling & herbivore & species & herbivore & herbivore guild \\
\hline Perissodactyla & Equidae & Calippus placidus & 13.1 & 11.7 & 11.42314495 ground dwelling & grazer & species & herbivore & herbivore guild \\
\hline Perissodactyla & Equidae & Merychippus coloradense & 13.1 & 11.7 & 12.20850978 ground dwelling & grazer, browser & species & herbivore & herbivore guild \\
\hline Artiodactyla & Camelidae & Procamelus occidentalis & 13.1 & 11.7 & 12.5965993 ground dwelling & browser & species & herbivore & herbivore guild \\
\hline Perissodactyla & Equidae & Pliohippus pernix & 13.5 & 11.7 & 12.197 ground dwelling & browser & species & herbivore & herbivore guild \\
\hline Eulipotyphla & Erinaceidae & Untermannerix copiosus & 15.9 & 11.7 & 4.798 semifossorial & insectivore, carnivore & species & insectivore & NA \\
\hline Carnivora & Canidae & Leptocyon vafer & 16.5 & 11.7 & 8.576516717 ground dwelling & carnivore, omnivore & species & carnivore & carnivore guild \\
\hline Rodentia & Heteromyidae & Perognathus furlongi & 16.9 & 11.7 & 2.276 ground dwelling & granivore, browser & species & herbivore & Rodentia \\
\hline Perissodactyla & Equidae & Hypohippus affinis & 17.8 & 11.7 & 12.46367021 ground dwelling & browser & species & herbivore & herbivore guild \\
\hline Lagomorpha & Leporidae & Pronotolagus whitei & 11.75 & 11.75 & 6.35 ground dwelling & grazer, browser & species & herbivore & herbivore guild \\
\hline Rodentia & Castoridae & Dipoides tanneri & 11.75 & 11.75 & 7.582 amphibious & browser & species & herbivore & Rodentia \\
\hline Rodentia & Castoridae & Hystricops venustus & 11.75 & 11.75 & 9.2835 amphibious & browser & species & herbivore & Rodentia \\
\hline Rodentia & Sciuridae & Spermophilus cyanocittus & 11.85 & 11.75 & 4.55 semifossorial & granivore, frugivore & species & herbivore & Rodentia \\
\hline Rodentia & Cricetidae & Antecalomys phthanus & 11.85 & 11.75 & 3.1275 ground dwelling & herbivore & species & herbivore & Rodentia \\
\hline Rodentia & Heteromyidae & Cupidinimus prattensis & 11.9 & 11.75 & 3.0615 ground dwelling & granivore, browser & species & herbivore & Rodentia \\
\hline Rodentia & Geomyidae & Phelosaccomys hibbardi & 11.9 & 11.75 & 3.8415 & & & NA & Rodentia \\
\hline Lipotyphla & Talpidae & Domninoides mimicus & 11.95 & 11.75 & 4.851 fossorial & insectivore & species & insectivore & NA \\
\hline Lipotyphla & Talpidae & Scalopoides ripafodiator & 15.8 & 11.75 & 3.313 fossorial & insectivore & species & insectivore & NA \\
\hline Rodentia & Sciuridae & Protospermophilus quatalensis & 17.25 & 11.75 & 5.6115 scansorial & granivore, frugivore & species & herbivore & Rodentia \\
\hline Carnivora & Mustelidae & Hoplictis grangerensis & 11.8 & 11.8 & 9.862640802 scansorial & carnivore, omnivore & species & carnivore & carnivore guild \\
\hline Carnivora & Mustelidae & Martes stirtoni & 11.8 & 11.8 & 5.835076255 scansorial & carnivore, omnivore & species & carnivore & carnivore guild \\
\hline Carnivora & Mustelidae & Mionictis angustidens & 11.8 & 11.8 & 8.551 scansorial & carnivore, omnivore & species & carnivore & carnivore guild \\
\hline Rodentia & Sciuridae & Cynomyoides vatis & 11.8 & 11.8 & 5.7315 scansorial & granivore, frugivore & species & herbivore & Rodentia \\
\hline
\end{tabular}




\begin{tabular}{|c|c|c|}
\hline Rodentia & Mylagaulidae & Ceratogaulus minor \\
\hline Rodentia & Cricetidae & Pseudomyscus bathygnathus \\
\hline Proboscidea & Mammutidae & Mammut furlongi \\
\hline Rodentia & Castoridae & Eucastor phillisi \\
\hline Carnivora & Procyonidae & Protoprocyon savagei \\
\hline Eulipotyphla & Soricidae & Anchiblarinella wakeeneyensis \\
\hline Artiodactyla & Tayassuidae & Macrogenis crassigenis \\
\hline Carnivora & Barbourofelidae & Barbourofelis whitfordi \\
\hline Rodentia & Heteromyidae & Cupidinimus avawatzensis \\
\hline Artiodactyla & Moschidae & Longirostromeryx wellsi \\
\hline Eulipotyphla & Erinaceidae & Metechinus amplior \\
\hline Artiodactyla & Antilocapridae & Cosoryx furcatus \\
\hline Carnivora & Procyonidae & Bassariscus antiquus \\
\hline Rodentia & Heteromyidae & Mioheteromys amplissimus \\
\hline Perissodactyla & Equidae & Pliohippus fossulatus \\
\hline Carnivora & Mustelidae & Mionictis pristinus \\
\hline Rodentia & Castoridae & Prodipoides dividerus \\
\hline Artiodactyla & Antilocapridae & Ramoceros osborni \\
\hline Rodentia & Castoridae & Monosaulax pansus \\
\hline Rodentia & Cricetidae & Copemys russelli \\
\hline Rodentia & Heteromyidae & Perognathus trojectioansrum \\
\hline Rodentia & Cricetidae & Copemys longidens \\
\hline Perissodactyla & Equidae & Hypohippus osborni \\
\hline Perissodactyla & Equidae & Protohippus perditus \\
\hline Perissodactyla & Rhinocerotidae & Aphelops megalodus \\
\hline Carnivora & Hyaenidae & Thalassictis certa \\
\hline Proboscidea & Gomphotheriidae & Gomphotherium subtapiroideum \\
\hline Carnivora & Mustelidae & Trochotherium cyamoides \\
\hline Artiodactyla & & Taucanamo grandaevum \\
\hline Perissodactyla & Rhinocerotidae & $\begin{array}{l}\text { Hispanotherium grimmi } \\
\text { Hoploaceratherium tetradactylum }\end{array}$ \\
\hline Carnivora & Ursidae & Hemicyon goeriachensis \\
\hline Artiodactyla & Cervidae & Euprox furcatus \\
\hline Proboscidea & Gomphotheriidae & Gomphotherium steinheimense \\
\hline Artiodactyla & Bovidae & Eotragus clavatus \\
\hline Carnivora & Mustelidae & Martes delphinensis \\
\hline Carnivora & Viverridae & Viverrictis modica \\
\hline Carnivora & Mustelidae & Ischyrictis mustelinus \\
\hline Artiodactyla & Tayassuidae & Albanohyus pygmaeus \\
\hline Proboscidea & Deinotheriidae & Prodeinotherium bavaricum \\
\hline Lipotyphla & Talpidae & Domninoides riparensis \\
\hline Artiodactyla & Merycoidodontidae & Merychyus medius \\
\hline Carnivora & & Aelurodon stirtoni \\
\hline Carnivora & & Aelurodon ferox \\
\hline Perissodactyla & Equidae & Pseudhipparion hessei \\
\hline Artiodactyla & Antilocapridae & Merycodus necatus \\
\hline Eulipotyphla & Erinaceidae & Lanthanotherium sawini \\
\hline Rodentia & Sciuridae & Tamias ateles \\
\hline Rodentia & Sciuridae & Petauristodon mathewsi \\
\hline Artiodactyla & Camelidae & Aepycamelus robustus \\
\hline Eulipotyphla & Plesiosoricidae & Plesiosorex latidens \\
\hline Eulipotyphla & Erinaceidae & Metechinus nevadensis \\
\hline
\end{tabular}

\begin{tabular}{|c|c|c|c|c|}
\hline 11.8 & 11.8 & 5.256908672 ground dwelling & herbivore & species \\
\hline 11.8 & 11.8 & 3.269 ground dwelling & herbivore & species \\
\hline 11.8 & 11.8 & 14.694 ground dwelling & browser & species \\
\hline 11.8 & 11.8 & 7.132 & & \\
\hline 11.8 & 11.8 & 7.534 scansorial & frugivore, carnivore & species \\
\hline 11.8 & 11.8 & 1.9415 ground dwelling & omnivore & species \\
\hline 11.9 & 11.8 & 10.646 ground dwelling & herbivore, omnivore & species \\
\hline 12.25 & 11.8 & 11.0795 ground dwelling & carnivore, omnivore & species \\
\hline 12.65 & 11.8 & 3.003 ground dwelling & granivore, browser & species \\
\hline 12.8 & 11.8 & 9.621436468 ground dwelling & browser, grazer & species \\
\hline 15.85 & 11.8 & 5.259667146 semifossorial & insectivore, carnivore & species \\
\hline 16.4 & 11.8 & 9.831303254 ground dwelling & grazer & species \\
\hline 16.4 & 11.8 & 7.54 arboreal & frugivore, carnivore & species \\
\hline 14.8 & 11.85 & 3.7165 ground dwelling & granivore, browser & species \\
\hline 12.25 & 11.9 & 11.22920062 ground dwelling & browser & species \\
\hline 13 & 11.9 & 9.922240393 scansorial & carnivore, omnivore & species \\
\hline 13 & 11.9 & 7.213954928 amphibious & browser & species \\
\hline 13.1 & 11.9 & 9.826260485 ground dwelling & browser, grazer & species \\
\hline 14 & 11.9 & 6.93 amphibious & browser & species \\
\hline 14.9 & 11.9 & 3.184 ground dwelling & herbivore & species \\
\hline 16 & 11.9 & 1.293 ground dwelling & granivore, browser & species \\
\hline 16 & 11.9 & 3.346 ground dwelling & herbivore & species \\
\hline 16.4 & 11.9 & 12.47011204 ground dwelling & browser & species \\
\hline 17.25 & 11.9 & 12.05054741 ground dwelling & grazer, browser & species \\
\hline 17.8 & 11.9 & 14.091 grou & browser & species \\
\hline 11.925 & 1.925 & 9.781381475 ground dwelling & carnivore & genus \\
\hline 1.925 & 1.925 & 14.15629315 ground dwelling & browser & genus \\
\hline 13.789 & 11.925 & 8.922517235 scansorial & carnivore, omnivore & genus \\
\hline 13.789 & 11.925 & 9.304746361 ground dwelling & grazer, browser & genus \\
\hline 13.789 & 1.925 & 13.99741478 ground dwelling & browser & species \\
\hline 13.789 & 11.925 & 13.52999001 ground dwelling & browser & species \\
\hline 13.789 & 11.925 & 11.69482969 grou & herbivore, & genus \\
\hline 14.181 & 11.925 & 10.81984735 ground dwelling & grazer, browser & genus \\
\hline 14.181 & 11.925 & 15.75428721 ground dwelling & browser & genus \\
\hline 14.81 & 11.925 & 10.30867346 ground dwelling & grazer, browser & genus \\
\hline 15.804 & 11.925 & 5.991326412 scansorial & carnivore, omnivore & genus \\
\hline 15.804 & 11.925 & 6.684404525 NA & NA & genus \\
\hline 16.435 & 11.925 & 8.986989618 scansorial & carnivore, omnivore & genus \\
\hline 16.435 & .925 & 9.104421458 ground dwelling & herbivore, omnivore & genus \\
\hline 17.04 & 1.925 & 15.59334012 ground dwelling & & species \\
\hline 12.2 & 12 & 4.0395 fossorial & insectivore & species \\
\hline 13.75 & 12 & 11.63969489 & & \\
\hline 13.95 & 12 & 10.38515065 ground dwelling & carnivore, omnivore & species \\
\hline 17.05 & 12 & 10.68022331 ground dwelling & carnivore, omnivore & species \\
\hline 12.25 & 12.1 & 10.92836217 ground dwelling & grazer & species \\
\hline 16 & 12.1 & 9.072185266 ground dwelling & browser, grazer & species \\
\hline 16.25 & 12.1 & 4.5175 semifossorial & insectivore, carnivore & species \\
\hline 16.75 & 12.1 & 3.3875 scansorial & granivore, frugivore & species \\
\hline 16.9 & 12.1 & 5.368 scansorial & granivore, frugivore & species \\
\hline 13.8 & 12.15 & 12.93590052 ground dwelling & browser, browser & species \\
\hline 12.2 & 12.2 & 4.94 scansorial & insectivore & species \\
\hline 12.2 & 12.2 & 6.122 semifossorial & insectivore, carnivore & \\
\hline
\end{tabular}

\begin{tabular}{|c|c|}
\hline herbivore & Rodentia \\
\hline herbivore & Rodentia \\
\hline herbivore & herbivore guild \\
\hline NA & Rodentia \\
\hline omnivore & carnivore guild \\
\hline omnivore & NA \\
\hline omnivore & NA \\
\hline carnivore & carnivore guild \\
\hline herbivore & Rodentia \\
\hline herbivore & herbivore guild \\
\hline insectivore & NA \\
\hline herbivore & herbivore guild \\
\hline omnivore & carnivore guild \\
\hline herbivore & Rodentia \\
\hline herbivore & herbivore guild \\
\hline carnivore & carnivore guild \\
\hline herbivore & Rodentia \\
\hline herbivore & herbivore guild \\
\hline herbivore & Rodentia \\
\hline herbivore & Rodentia \\
\hline herbivore & Rodentia \\
\hline herbivore & Rodentia \\
\hline herbivore & herbivore guild \\
\hline herbivore & herbivore guild \\
\hline herbivore & herbivore guild \\
\hline carnivore & carnivore guild \\
\hline herbivore & herbivore guild \\
\hline carnivore & carnivore guild \\
\hline herbivore & herbivore guild \\
\hline herbivore & herbivore guild \\
\hline herbivore & herbivore guild \\
\hline omnivore & carnivore guild \\
\hline herbivore & herbivore guild \\
\hline herbivore & herbivore guild \\
\hline herbivore & herbivore guild \\
\hline carnivore & carnivore guild \\
\hline NA & carnivore guild \\
\hline carnivore & carnivore guild \\
\hline omnivore & NA \\
\hline herbivore & herbivore guild \\
\hline insectivore & NA \\
\hline NA & NA \\
\hline carnivore & carnivore guild \\
\hline carnivore & carnivore guild \\
\hline herbivore & herbivore guild \\
\hline herbivore & herbivore guild \\
\hline insectivore & NA \\
\hline herbivore & Rodentia \\
\hline herbivore & Rodentia \\
\hline herbivore & herbivore guild \\
\hline insectivore & NA \\
\hline insectivore & NA \\
\hline
\end{tabular}




\begin{tabular}{|c|c|c|c|c|c|c|c|c|c|}
\hline Lipotyphla & Talpidae & Mystipterus vespertilio & 12.2 & 12.2 & 2.25 fossorial & insectivore & species & insectivore & NA \\
\hline Rodentia & Cricetidae & Copemys loxodon & 13.1 & 12.2 & 3.3585 ground dwelling & herbivore & species & herbivore & Rodentia \\
\hline Artiodactyla & Moschidae & Longirostromeryx clarendonensis & 12.25 & 12.25 & 9.49 ground dwelling & browser, grazer & species & herbivore & herbivore guild \\
\hline Carnivora & & Cynarctus voorhiesi & 12.3 & 12.25 & 9.02099282 ground dwelling & carnivore, omnivore & species & carnivore & carnivore guild \\
\hline Rodentia & Castoridae & Prodipoides burgensis & 12.9 & 12.25 & 6.767729526 amphibious & browser & species & herbivore & Rodentia \\
\hline Perissodactyla & Rhinocerotidae & Peraceras superciliosum & 15.85 & 12.25 & 14.151 ground dwelling & browser & species & herbivore & herbivore guild \\
\hline Rodentia & Castoridae & Monosaulax skinneri & 16.4 & 12.3 & 6.72 amphibious & browser & species & herbivore & Rodentia \\
\hline Artiodactyla & Moschidae & Blastomeryx gemmifer & 18.45 & 12.3 & 9.230350826 ground dwelling & browser, grazer & species & herbivore & herbivore guild \\
\hline Carnivora & & Paratomarctus temerarius & 18.65 & 12.3 & 9.539064297 ground dwelling & carnivore, omnivore & species & carnivore & carnivore guild \\
\hline Artiodactyla & Bovidae & Protragocerus gluten & 13.789 & 12.375 & 10.99944899 ground dwelling & grazer, browser & genus & herbivore & herbivore guild \\
\hline Artiodactyla & Tragulidae & Dorcabune anthracotherioides & 14.81 & 12.375 & 10.79912409 ground dwelling & frugivore, grazer & genus & herbivore & herbivore guild \\
\hline Artiodactyla & Anthracotheriidae & Anthracotherium punjabiense & 14.81 & 12.375 & 10.29946312 ground dwelling & browser & genus & herbivore & herbivore guild \\
\hline Rodentia & Heteromyidae & Cupidinimus lindsayi & 16.75 & 12.4 & 2.0285 ground dwelling & granivore, browser & species & herbivore & Rodentia \\
\hline Carnivora & Mustelidae & Pliogale manka & 12.45 & 12.45 & 7.389 scansorial & carnivore, omnivore & species & carnivore & carnivore guild \\
\hline Rodentia & Zapodidae & Megasminthus tiheni & 16 & 12.45 & 4.026 ground dwelling & herbivore & species & herbivore & Rodentia \\
\hline Eulipotyphla & Soricidae & Limnoecus niobrarensis & 17.4 & 12.45 & 1.9755 ground dwelling & omnivore & species & omnivore & NA \\
\hline Carnivora & Mustelidae & Sthenictis junturensis & 12.5 & 12.5 & 7.402 scansorial & carnivore, omnivore & species & carnivore & carnivore guild \\
\hline Rodentia & Sciuridae & Ammospermophilus fossilis & 12.5 & 12.5 & 4.0405 semifossorial & granivore, frugivore & species & herbivore & Rodentia \\
\hline Rodentia & Geomyidae & Mojavemys wilsoni & 12.5 & 12.5 & 3.089 fossorial & browser & species & herbivore & Rodentia \\
\hline Rodentia & Sciuridae & Petauristodon jamesi & 18.6 & 12.6 & 5.7285 scansorial & granivore, frugivore & species & herbivore & Rodentia \\
\hline Artiodactyla & Bovidae & Sivoreas eremita & 12.629 & 12.629 & 10.99944899 ground dwelling & grazer, browser & genus & herbivore & herbivore guild \\
\hline Creodonta & Hyaenodontidae & Megistotherium osteothlastes & 12.629 & 12.629 & 13.507158 scansorial & insectivore & species & insectivore & NA \\
\hline Artiodactyla & Suidae & Kubanochoerus khinzikebirus & 12.629 & 12.629 & 13.65202702 ground dwelling & omnivore & genus & omnivore & NA \\
\hline Artiodactyla & Hippopotamidae & Kenyapotamus ternani & 14.81 & 12.629 & 12.20600358 amphibious & grazer, browser & species & herbivore & herbivore guild \\
\hline Creodonta & Hyaenodontidae & Dissopsalis pyroclasticus & 14.81 & 12.629 & 11.32659589 scansorial & insectivore & species & insectivore & NA \\
\hline Artiodactyla & Giraffidae & Progiraffa exigua & 16.435 & 12.629 & 13.62209341 ground dwelling & browser & genus & herbivore & herbivore guild \\
\hline Artiodactyla & Hippopotamidae & Morotochoerus ugandensis & 21.2 & 12.629 & 10.30867346 amphibious & grazer, browser & species & herbivore & herbivore guild \\
\hline Artiodactyla & Gelocidae & Walangania africanus & 21.2 & 12.629 & 9.799802156 ground dwelling & grazer, browser & genus & herbivore & herbivore guild \\
\hline Rodentia & Geomyidae & Phelosaccomys shotwelli & 12.65 & 12.65 & 3.017 fossorial & browser & species & herbivore & Rodentia \\
\hline Lagomorpha & Leporidae & Panolax sanctaefidei & 13.1 & 12.65 & 7.444257421 ground dwelling & grazer, browser & species & herbivore & herbivore guild \\
\hline Carnivora & & Aelurodon mcgrewi & 12.9 & 12.75 & 10.44440036 ground dwelling & carnivore, omnivore & species & carnivore & carnivore guild \\
\hline Perissodactyla & Equidae & Hippotherium quinni & 13 & 12.75 & 11.99 & & & NA & NA \\
\hline Carnivora & & Cynarctus saxatilis & 13.1 & 12.75 & 9.420113457 ground dwelling & carnivore, omnivore & species & carnivore & carnivore guild \\
\hline Artiodactyla & Palaeomerycidae & Procranioceras skinneri & 13.3 & 12.75 & 11.86599239 ground dwelling & browser & species & herbivore & herbivore guild \\
\hline Artiodactyla & Antilocapridae & Merycodus warreni & 13.75 & 12.75 & 9.542 ground dwelling & browser, grazer & species & herbivore & herbivore guild \\
\hline Perissodactyla & Equidae & Pliohippus mirabilis & 13.75 & 12.75 & 12.21521392 ground dwelling & browser & species & herbivore & herbivore guild \\
\hline Carnivora & & Carpocyon compressus & 16.4 & 12.75 & 9.905005343 ground dwelling & carnivore, omnivore & species & carnivore & carnivore guild \\
\hline Lagomorpha & Leporidae & Hypolagus parviplicatus & 16.4 & 12.75 & 6.489 ground dwelling & grazer, browser & species & herbivore & herbivore guild \\
\hline Perissodactyla & Equidae & Merychippus insignis & 16.5 & 12.75 & 11.92438015 ground dwelling & grazer, browser & species & herbivore & herbivore guild \\
\hline Perissodactyla & Equidae & Calippus proplacidus & 17.8 & 12.75 & 11.40400355 ground dwelling & grazer & species & herbivore & herbivore guild \\
\hline Artiodactyla & Merycoidodontidae & Ticholeptus zygomaticus & 17.95 & 12.75 & 11.67823366 ground dwelling & herbivore & species & herbivore & herbivore guild \\
\hline Artiodactyla & Tayassuidae & Prosthennops xiphodonticus & 18 & 12.75 & 10.5236064 ground dwelling & herbivore, omnivore & species & omnivore & NA \\
\hline Artiodactyla & Palaeomerycidae & Cranioceras teres & 13.1 & 12.8 & 11.48 ground dwelling & browser & species & herbivore & herbivore guild \\
\hline Artiodactyla & Antilocapridae & Ramoceros ramosus & 13.1 & 12.8 & 10.00468943 ground dwelling & browser, grazer & species & herbivore & herbivore guild \\
\hline Carnivora & Felidae & Pseudaelurus stouti & 12.9 & 12.9 & 8.2195 scansorial & carnivore & species & carnivore & carnivore guild \\
\hline Carnivora & Mustelidae & Brachypsalis modicus & 13 & 12.9 & 8.271483614 scansorial & carnivore, omnivore & species & carnivore & carnivore guild \\
\hline Artiodactyla & Leptomerycidae & Pseudoparablastomeryx francescita & 13.1 & 12.9 & 8.250620082 ground dwelling & frugivore & species & herbivore & herbivore guild \\
\hline Rodentia & Castoridae & Temperocastor valentinensis & 13.1 & 12.9 & 5.45757617 amphibious & browser & species & herbivore & Rodentia \\
\hline Artiodactyla & Moschidae & Longirostromeryx blicki & 13.45 & 12.9 & 9.581758898 ground dwelling & browser, grazer & species & herbivore & herbivore guild \\
\hline Perissodactyla & Equidae & Merychippus calamarius & 13.7 & 12.9 & 12.17363221 ground dwelling & grazer, browser & species & herbivore & herbivore guild \\
\hline Rodentia & Heteromyidae & Cupidinimus nebraskensis & 13.8 & 12.9 & 2.2345 ground dwelling & granivore, browser & species & herbivore & Rodentia \\
\hline Perissodactyla & Equidae & Parahippus cognatus & 13.8 & 12.9 & 11.62732082 ground dwelling & browser & species & herbivore & herbivore guild \\
\hline
\end{tabular}




\begin{tabular}{|c|c|}
\hline Perissodactyla & Equidae \\
\hline Rodentia & Mylagaulidae \\
\hline Eulipotyphla & Plesiosoricidae \\
\hline Lipotyphla & Talpidae \\
\hline Carnivora & Felidae \\
\hline Lagomorpha & Ochotonidae \\
\hline Carnivora & Mustelidae \\
\hline Artiodactyla & Antilocapridae \\
\hline Eulipotyphla & Erinaceidae \\
\hline Rodentia & Heteromyidae \\
\hline Rodentia & Zapodidae \\
\hline \multicolumn{2}{|l|}{ Carnivora } \\
\hline Carnivora & Mustelidae \\
\hline Artiodactyla & Antilocapridae \\
\hline Perissodactyla & Equidae \\
\hline Carnivora & Mustelidae \\
\hline Carnivora & Mustelidae \\
\hline Rodentia & Castoridae \\
\hline Rodentia & Geomyidae \\
\hline Lagomorpha & Ochotonidae \\
\hline Rodentia & Heteromyidae \\
\hline Rodentia & Heteromyidae \\
\hline Rodentia & Heteromyidae \\
\hline Artiodactyla & Palaeomerycidae \\
\hline Rodentia & Castoridae \\
\hline Rodentia & Zapodidae \\
\hline Rodentia & Geomyidae \\
\hline Rodentia & Geomyidae \\
\hline Carnivora & Mustelidae \\
\hline Carnivora & Procyonidae \\
\hline Carnivora & Ursidae \\
\hline Carnivora & Canidae \\
\hline Carnivora & Ursidae \\
\hline Perissodactyla & Rhinocerotidae \\
\hline Artiodactyla & Bovidae \\
\hline Artiodactyla & Cervidae \\
\hline Artiodactyla & Tayassuidae \\
\hline Artiodactyla & Cervidae \\
\hline Artiodactyla & Suidae \\
\hline Carnivora & Felidae \\
\hline Carnivora & Mustelidae \\
\hline Artiodactyla & Tragulidae \\
\hline Artiodactyla & Bovidae \\
\hline Artiodactyla & Suidae \\
\hline Carnivora & Mustelidae \\
\hline Artiodactyla & Tragulidae \\
\hline Artiodactyla & Moschidae \\
\hline Artiodactyla & Palaeomerycidae \\
\hline Perissodactyla & Equidae \\
\hline Artiodactyla & Suidae \\
\hline Artiodactyla & Antilocapridae \\
\hline Perissodactyla & Equidae \\
\hline
\end{tabular}

Megahippus mckennai

Plesiosorex donroosai

Domninoides valentinensis

Pseudaelurus marshi

Russellagus vonhof

Leptarctus primus

Submeryceros minor

Parvericius montanus

Mookomys altifluminis

Schaubeumys cartomylos

Tomarctus hippophaga

Martes parviloba

Merycodus nenzelensis

Merychippus republicanus

Miomustela madisonae

Brachypsalis pachycephalus

Anchitheriomys fluminis

Phelosaccomys annae

Hesperolagomys fluviatilis

Lignimus montis

Peridiomys borealis

Perognathus brevidens

Bouromeryx americanus

Monosaulax curtus

Miosicista angulus

Mojavemys galushai

Phelosaccomys neomexicanus

Leptarctus martini

Arctonasua gracilis

Plithocyon ursinus

Microtomarctus conferta

Ursavus pawniensis

Peraceras profectum

Paratragocerus caucasicus

Procervulus flerovi

Schizochoerus anatoliensis

Stehlinoceros elegantulus

Kubanochoerus gigas

Pseudaelurus aeluroides

Ischyrictis anatolicus

Dorcatherium vindobonense

Hypsodontus pronaticornis

Bunolistriodon meidamon

Sthenictis dolichops

Dorcatherium guntianum

Blastomeryx elegans

Lagomeryx parvulus

Acritohippus isonesus

Aureliachoerus aurelianensis

Merycodus sabulonis

Merychippus californicus
12.912 .62737665 ground dwelling browser

species

species

6.5315 scansorial insectivore

4.348 fossorial insectivore

10.1105 scansorial carnivore

5.0245 ground dwelling herbivore

12.96 .7025 scansorial omnivore species

species

species

species

12.98 .922517235 ground dwelling browser, grazer species

12.93 .7245 semifossorial insectivore, carnivore species

$12.95 \quad 2.57$ ground dwelling granivore, browser species

$12.95 \quad 3.04$ ground dwelling herbivore species

9.9085 ground dwelling carnivore, omnivore species

138.36722376 scansorial carnivore, omnivore species

1310.06459944 ground dwelling browser, grazer genus

1312.1714648 ground dwelling grazer, browser species

135.143171852 scansorial carnivore, omnivore species

138.254374135 scansorial carnivore, omnivore species

1310.217 amphibious browser species

$13 \quad 3.268$

134.4365 ground dwelling herbivore species

$13 \quad 3.657$ ground dwelling granivore, browser species

132.275305207 ground dwelling granivore, browser species

131.991 ground dwelling granivore, browser species

$13 \quad 10.95354077$ ground dwelling browser species

135.45757617 amphibious browser species

13.11 .692127666 ground dwelling herbivore species

13.13 .3435 fossorial browser species

13.12 .992 fossorial browser species

13.18 .17345941 scansorial omnivore species

13.1 9.1775 scansorial frugivore, carnivore species

13.111 .99265906 ground dwelling herbivore, carnivore species

13.19 .180798536 ground dwelling carnivore, omnivore species

$13.1 \quad 11.03$ ground dwelling herbivore, carnivore species

13.114 .29416861 ground dwelling browser species

13.210 .30867346 ground dwelling grazer, browser genus

13.2 9.392244594 ground dwelling grazer, browser genus

13.29 .680067731 ground dwelling grazer, browser species

$13.210 .30867346 \mathrm{NA}$ NA

13.213 .78787954 ground dwelling omnivore

13.29 .392244594 scansorial carnivore, omnivore genus

13.210 .20275455 ground dwelling frugivore, grazer species

13.211 .87673391 ground dwelling grazer, browser genus

13.211 .3839807 ground dwelling omnivore genus

13.27 .931 scansorial carnivore, omnivore species

13.29 .210340372 ground dwelling frugivore, grazer species

13.2 9.431241515 ground dwelling browser, grazer species

13.28 .517262259 ground dwelling browser

13.211 .99147847 ground dwelling grazer, browser species

$\begin{array}{ll}13.3 & 11.98755993 \text { ground dwelling grazer, browser }\end{array}$

\begin{tabular}{|c|c|}
\hline herbivore & herbivore guild \\
\hline herbivore & Rodentia \\
\hline insectivore & NA \\
\hline insectivore & NA \\
\hline carnivore & carnivore guild \\
\hline herbivore & herbivore guild \\
\hline omnivore & carnivore guild \\
\hline herbivore & herbivore guild \\
\hline insectivore & NA \\
\hline herbivore & Rodentia \\
\hline herbivore & Rodentia \\
\hline carnivore & carnivore guild \\
\hline carnivore & carnivore guild \\
\hline herbivore & herbivore guild \\
\hline herbivore & herbivore guild \\
\hline carnivore & carnivore guild \\
\hline carnivore & carnivore guild \\
\hline herbivore & Rodentia \\
\hline NA & Rodentia \\
\hline herbivore & herbivore guild \\
\hline herbivore & Rodentia \\
\hline herbivore & Rodentia \\
\hline herbivore & Rodentia \\
\hline herbivore & herbivore guild \\
\hline herbivore & Rodentia \\
\hline herbivore & Rodentia \\
\hline herbivore & Rodentia \\
\hline herbivore & Rodentia \\
\hline omnivore & carnivore guild \\
\hline omnivore & carnivore guild \\
\hline omnivore & carnivore guild \\
\hline carnivore & carnivore guild \\
\hline omnivore & carnivore guild \\
\hline herbivore & herbivore guild \\
\hline herbivore & herbivore guild \\
\hline herbivore & herbivore guild \\
\hline herbivore & herbivore guild \\
\hline NA & NA \\
\hline omnivore & NA \\
\hline carnivore & carnivore guild \\
\hline carnivore & carnivore guild \\
\hline herbivore & herbivore guild \\
\hline herbivore & herbivore guild \\
\hline omnivore & NA \\
\hline carnivore & carnivore guild \\
\hline herbivore & herbivore guild \\
\hline herbivore & herbivore guild \\
\hline herbivore & herbivore guild \\
\hline herbivore & herbivore guild \\
\hline omnivore & NA \\
\hline herbivore & herbivore guild \\
\hline herbivore & herbivore guild \\
\hline
\end{tabular}




\begin{tabular}{|c|c|c|c|c|c|c|c|c|c|}
\hline Artiodactyla & Protoceratidae & Prosynthetoceras francisi & 18.65 & 13.45 & 12.037 ground dwelling & browser & species & herbivore & herbivore guild \\
\hline Eulipotyphla & Erinaceidae & Brachyerix incertis & 17.3 & 13.5 & 4.379 semifossorial & insectivore, carnivore & species & insectivore & NA \\
\hline Carnivora & Amphicyonidae & Pliocyon robustus & 13.6 & 13.6 & 12.1105 ground dwelling & carnivore & species & carnivore & carnivore guild \\
\hline Artiodactyla & Camelidae & Aepycamelus stocki & 13.7 & 13.7 & 12.845 ground dwelling & browser, browser & species & herbivore & herbivore guild \\
\hline Carnivora & & Aelurodon asthenostylus & 15.55 & 13.7 & 10.41091818 ground dwelling & carnivore, omnivore & species & carnivore & carnivore guild \\
\hline Carnivora & & Paracynarctus kelloggi & 20.6 & 13.7 & 9.239443071 ground dwelling & carnivore, omnivore & species & carnivore & carnivore guild \\
\hline Carnivora & Procyonidae & Parapotos tedfordi & 13.75 & 13.75 & 8.17 scansorial & frugivore, carnivore & species & omnivore & carnivore guild \\
\hline Perissodactyla & Equidae & Merychippus sejunctus & 16.3 & 13.75 & 11.23 ground dwelling & grazer, browser & species & herbivore & herbivore guild \\
\hline Carnivora & Amphicyonidae & Amphicyon ingens & 16.65 & 13.75 & 12.93956003 ground dwelling & carnivore & species & carnivore & carnivore guild \\
\hline Artiodactyla & Palaeomerycidae & Dromomeryx borealis & 16.85 & 13.75 & 12.13030386 ground dwelling & browser & species & herbivore & herbivore guild \\
\hline Perissodactyla & Rhinocerotidae & Teleoceras medicornutum & 17.25 & 13.75 & 14.91444357 amphibious & grazer & species & herbivore & herbivore guild \\
\hline Carnivora & & Tomarctus brevirostris & 19.7 & 13.75 & 10.09162657 ground dwelling & carnivore, omnivore & species & carnivore & carnivore guild \\
\hline Carnivora & Amphicyonidae & Amphicyon confucianus & 13.789 & 13.789 & 12.42935433 ground dwelling & carnivore & genus & carnivore & carnivore guild \\
\hline Carnivora & Mustelidae & Mionictis dubia & 13.789 & 13.789 & 9.304746361 scansorial & carnivore, omnivore & species & carnivore & carnivore guild \\
\hline Artiodactyla & Bovidae & Protoryx enanus & 13.789 & 13.789 & 10.71392844 ground dwelling & grazer, browser & genus & herbivore & herbivore guild \\
\hline Artiodactyla & Giraffidae & Palaeotragus lavocati & 13.789 & 13.789 & 12.76553176 ground dwelling & browser, grazer & genus & herbivore & herbivore guild \\
\hline Artiodactyla & Cervidae & Stephanocemas thomsoni & 13.789 & 13.789 & $10.54814231 \mathrm{NA}$ & NA & genus & NA & NA \\
\hline Carnivora & Mustelidae & Ischyrictis zibethoides & 15.804 & 13.789 & 9.615595348 scansorial & carnivore, omnivore & genus & carnivore & carnivore guild \\
\hline Artiodactyla & & Taucanamo sansaniense & 15.804 & 13.789 & 9.304746361 ground dwelling & grazer, browser & species & herbivore & herbivore guild \\
\hline Carnivora & Felidae & Pseudaelurus lorteti & 16.435 & 13.789 & 9.615595348 scansorial & carnivore & species & carnivore & carnivore guild \\
\hline Rodentia & Eomyidae & Leptodontomys quartzi & 13.8 & 13.8 & 1.126 ground dwelling & herbivore & species & herbivore & Rodentia \\
\hline Rodentia & Zapodidae & Megasminthus gladiofex & 13.8 & 13.8 & 3.592 ground dwelling & herbivore & species & herbivore & Rodentia \\
\hline Rodentia & Castoridae & Monosaulax progressus & 13.8 & 13.8 & 6.06 amphibious & browser & species & herbivore & Rodentia \\
\hline Eulipotyphla & Heterosoricidae & Ingentisorex tumididens & 13.8 & 13.8 & 4.4915 scansorial & insectivore & species & insectivore & NA \\
\hline Rodentia & Cricetidae & Copemys barstowensis & 14 & 13.8 & 3.4715 ground dwelling & herbivore & species & herbivore & Rodentia \\
\hline Rodentia & Eomyidae & Pseudotheridomys pagei & 14.7 & 13.8 & 2.102 ground dwelling & herbivore & species & herbivore & Rodentia \\
\hline Lipotyphla & Talpidae & Achlyoscapter longirostris & 14.7 & 13.8 & 2.487 fossorial & insectivore & species & insectivore & NA \\
\hline Rodentia & Sciuridae & Spermophilus tephrus & 15.55 & 13.8 & 4.4895 semifossorial & granivore, frugivore & species & herbivore & Rodentia \\
\hline Lipotyphla & Talpidae & Mystipterus pacificus & 15.8 & 13.8 & 2.7 fossorial & insectivore & species & insectivore & NA \\
\hline Rodentia & Heteromyidae & Peridiomys oregonensis & 16.05 & 13.8 & 2.145484647 ground dwelling & granivore, browser & species & herbivore & Rodentia \\
\hline Rodentia & Cricetidae & Copemys pagei & 16.75 & 13.8 & 2.719 ground dwelling & herbivore & species & herbivore & Rodentia \\
\hline Rodentia & Heteromyidae & Peridiomys rusticus & 18.1 & 13.8 & 4.24 ground dwelling & granivore, browser & species & herbivore & Rodentia \\
\hline Rodentia & Heteromyidae & Cupidinimus halli & 16.9 & 13.9 & 2.456 ground dwelling & granivore, browser & species & herbivore & Rodentia \\
\hline Artiodactyla & Merycoidodontidae & Brachycrus siouense & 17.2 & 13.95 & 11.64957425 ground dwelling & herbivore & species & herbivore & herbivore guild \\
\hline Carnivora & Ursidae & Plithocyon barstowensis & 14 & 14 & 11.768 ground dwelling & herbivore, carnivore & species & omnivore & carnivore guild \\
\hline Artiodactyla & Tayassuidae & Dyseohyus fricki & 15.85 & 14 & 10.18317024 ground dwelling & herbivore, omnivore & species & omnivore & NA \\
\hline Rodentia & Heteromyidae & Mookomys formicarum & 16.75 & 14 & 2.4725 ground dwelling & granivore, browser & species & herbivore & Rodentia \\
\hline Rodentia & Cricetidae & Copemys tenuis & 16.9 & 14 & 3.1525 ground dwelling & herbivore & species & herbivore & Rodentia \\
\hline Rodentia & Eomyidae & Leptodontomys stirtoni & 16.9 & 14 & 1.991 ground dwelling & herbivore & species & herbivore & Rodentia \\
\hline Eulipotyphla & Heterosoricidae & Paradomnina relictus & 16.9 & 14 & 3.1725 scansorial & insectivore & species & insectivore & NA \\
\hline Rodentia & Heteromyidae & Mookomys subtilis & 17.4 & 14 & 1.68 ground dwelling & granivore, browser & species & herbivore & Rodentia \\
\hline \multirow[t]{2}{*}{ Carnivora } & & Protepicyon raki & 14.35 & 14.15 & 10.602 ground dwelling & carnivore, omnivore & species & carnivore & carnivore guild \\
\hline & Heteromyidae & Perognathoides eurekensis & 16.8 & 14.15 & 0.226285427 ground dwelling & granivore, browser & species & herbivore & herbivore guild \\
\hline Perissodactyla & Equidae & Heteropliohippus hulberti & 14.181 & 14.181 & 13.10262602 ground dwelling & grazer, browser & species & herbivore & herbivore guild \\
\hline Artiodactyla & Palaeomerycidae & Lagomeryx pumilio & 17.985 & 14.181 & 7.313010255 ground dwelling & browser & genus & herbivore & herbivore guild \\
\hline Artiodactyla & Cervidae & Procervulus dichotomus & 18.2 & 14.181 & 10.30867346 ground dwelling & grazer, browser & genus & herbivore & herbivore guild \\
\hline Carnivora & & Cynarctus galushai & 14.65 & 14.35 & 9.275096223 ground dwelling & carnivore, omnivore & species & carnivore & carnivore guild \\
\hline Artiodactyla & Antilocapridae & Merriamoceros coronatus & 15.05 & 14.35 & 9.529542978 ground dwelling & browser, grazer & species & herbivore & herbivore guild \\
\hline Carnivora & Amphicyonidae & Cynelos sinapius & 16.75 & 14.45 & 12.12109325 ground dwelling & carnivore & species & carnivore & carnivore guild \\
\hline Carnivora & Amphicyonidae & Pliocyon medius & 15.55 & 14.5 & 11.9633786 ground dwelling & carnivore & species & carnivore & carnivore guild \\
\hline Artiodactyla & Palaeomerycidae & Dromomeryx whitfordi & 16.5 & 14.5 & 11.94377457 ground dwelling & browser & species & herbivore & herbivore guild \\
\hline Artiodactyla & Merycoidodontidae & Merychyus relictus & 17.65 & 14.5 & 10.453 ground dwelling & herbivore & species & herbivore & herbivore guild \\
\hline
\end{tabular}




\begin{tabular}{|c|c|c|}
\hline Artiodactyla & Tayassuidae & Cynorca occidentale \\
\hline Rodentia & Castoridae & Monosaulax typicus \\
\hline Lagomorpha & Ochotonidae & Oreolagus colteri \\
\hline Rodentia & Heteromyidae & Cupidinimus whitlocki \\
\hline Rodentia & Geomyidae & Mojavemys magnumarcus \\
\hline Rodentia & Geomyidae & Parapliosaccomys transversus \\
\hline Carnivora & Canidae & Tephrocyon rurestris \\
\hline Rodentia & Sciuridae & Protospermophilus malheurensis \\
\hline Lipotyphla & Talpidae & Scapanoscapter simplicidens \\
\hline Perissodactyla & Equidae & Merychippus brevidontus \\
\hline Carnivora & Amphicyonidae & Amphicyon frendens \\
\hline Rodentia & Sciuridae & Spermophilus primitivus \\
\hline Artiodactyla & & Taucanamo inonuensis \\
\hline Artiodactyla & Suidae & Megalochoerus khinzikebirus \\
\hline Carnivora & Ursidae & Plithocyon antunesi \\
\hline Artiodactyla & Tragulidae & Dorcatherium peneckei \\
\hline Artiodactyla & Palaeomerycidae & Palaeomeryx kaupi \\
\hline Artiodactyla & Suidae & Bunolistriodon lockharti \\
\hline Primates & Proconsulidae & Proconsul major \\
\hline Perissodactyla & Equidae & Acritohippus quinni \\
\hline Rodentia & Aplodontidae & Ansomys hepburnensis \\
\hline Carnivora & Canidae & Euoplocyon brachygnathus \\
\hline Artiodactyla & Merycoidodontidae & Brachycrus buwaldi \\
\hline Carnivora & Canidae & Cynarctoides acridens \\
\hline Carnivora & & Paracynarctus sinclairi \\
\hline Artiodactyla & Leptomerycidae & Pseudoparablastomeryx scotti \\
\hline Carnivora & Canidae & Osbornodon fricki \\
\hline Artiodactyla & Camelidae & Rakomylus raki \\
\hline \multirow[t]{2}{*}{ Carnivora } & Procyonidae & Bassariscus parvus \\
\hline & Heteromyidae & Perognathoides kleinfelderi \\
\hline Carnivora & Canidae & Psalidocyon marianae \\
\hline Artiodactyla & Palaeomerycidae & Bouromeryx milleri \\
\hline Carnivora & Canidae & Protomarctus optatus \\
\hline Artiodactyla & Camelidae & Procamelus leptocolon \\
\hline Artiodactyla & Protoceratidae & Lambdoceras siouxensis \\
\hline Artiodactyla & Palaeomerycidae & Rakomeryx sinclairi \\
\hline Perissodactyla & Equidae & Merychippus relictus \\
\hline Rodentia & Aplodontidae & Liodontia alexandrae \\
\hline Perissodactyla & Equidae & Parahippus pawniensis \\
\hline Perissodactyla & Equidae & Desmatippus avus \\
\hline Perissodactyla & Equidae & Archaeohippus ultimus \\
\hline Perissodactyla & Equidae & Archaeohippus mourningi \\
\hline Lagomorpha & Ochotonidae & Oreolagus wallacei \\
\hline Eulipotyphla & Soricidae & Pseudotrimylus mawbyi \\
\hline Carnivora & Mustelidae & Martes cadeoti \\
\hline Carnivora & Mustelidae & Martes collongensis \\
\hline Carnivora & Mustelidae & Martes burdigaliensis \\
\hline Carnivora & & Aelurodon montanensis \\
\hline Carnivora & Mustelidae & Martes kinseyi \\
\hline Rodentia & Heteromyidae & Mookomys thrinax \\
\hline Rodentia & Heteromyidae & Peridiomys halis \\
\hline Rodentia & Heteromyidae & Perognathus ancenensis \\
\hline
\end{tabular}

\begin{tabular}{|c|c|c|c|c|}
\hline 22.2 & 14.65 & 9.961957361 ground dwelling & herbivore, omnivore & species \\
\hline 14.7 & 14.7 & 5.91 amphibious & browser & species \\
\hline 14.8 & 14.7 & 3.853 ground dwelling & herbivore & species \\
\hline 14.8 & 14.7 & 2.821 ground dwelling & granivore, browser & species \\
\hline 14.8 & 14.7 & 4.0285 fossorial & browser & species \\
\hline 14.8 & 14.7 & 3.819 fossorial & browser & species \\
\hline 15.65 & 14.7 & 9.751 ground dwelling & carnivore, omnivore & species \\
\hline 15.8 & 14.7 & 4.649 scansorial & granivore, frugivore & species \\
\hline 16 & 14.7 & 4.072 fossorial & insectivore & species \\
\hline 17.2 & 14.7 & 11.6435 ground dwelling & grazer, browser & species \\
\hline 17.6 & 14.7 & 12.64506029 ground dwelling & carnivore & species \\
\hline 16.85 & 14.8 & 4.736 semifossorial & granivore, frugivore & species \\
\hline 14.81 & 14.81 & 9.680067731 ground dwelling & grazer, browser & species \\
\hline 14.81 & 14.81 & 13.81551056 & & \\
\hline 14.81 & 14.81 & 11.87673391 ground dwelling & herbivore, carnivore & genus \\
\hline 5.275 & 14.81 & 11.00175157 ground dwelling & frugivore, grazer & species \\
\hline 18.2 & 14.81 & 11.51292546 ground dwelling & browser & species \\
\hline 18.2 & 14.81 & 11.67871159 ground dwelling & omnivore & genus \\
\hline 18.2 & 14.81 & 10.81977828 arboreal & omnivore & species \\
\hline 16.6 & 14.9 & 11.8038976 ground dwelling & grazer, browser & species \\
\hline 14.95 & 14.95 & 3.8 fossorial & herbivore & species \\
\hline 16.85 & 15.05 & 9.466893322 ground dwelling & carnivore, omnivore & species \\
\hline 17.65 & 15.05 & 12.43 ground dwelling & herbivore & species \\
\hline 21.55 & 15.05 & 7.867919977 ground dwelling & carnivore, omnivore & species \\
\hline 16.45 & 15.1 & 9.194864929 ground dwelling & carnivore, omnivore & species \\
\hline 17.3 & 15.1 & 8.576097913 ground dwelling & frugivore & species \\
\hline 17.65 & 15.1 & 10.59213342 ground dwelling & carnivore, omnivore & species \\
\hline 15.2 & 15.2 & 11.32 ground dwelling & browser, browser & species \\
\hline 15.85 & 15.2 & 7.43 arboreal & frugivore, carnivore & species \\
\hline 15.9 & 15.2 & 1.504719127 ground dwelling & granivore, browser & species \\
\hline 16.5 & 15.2 & 9.183 ground dwelling & carnivore, omnivore & species \\
\hline 17.95 & 15.3 & 10.963 ground dwelling & browser & species \\
\hline 20.15 & 15.3 & 9.33 ground dwelling & carnivore, omnivore & species \\
\hline 15.4 & 15.4 & 13.12236338 ground dwelling & browser & species \\
\hline 16.5 & 15.4 & 12.00150548 ground dwelling & browser & species \\
\hline 16.65 & 15.4 & 11.60906056 ground dwelling & browser & species \\
\hline 15.7 & 15.45 & 11.504 ground dwelling & grazer, browser & species \\
\hline 16.4 & 15.55 & 5.293 fossorial & herbivore & species \\
\hline 30.65 & 15.55 & 10.40337042 ground dwelling & browser & species \\
\hline 17.2 & 15.65 & 11.9955 ground dwelling & browser & species \\
\hline 16.85 & 15.7 & 10.156 ground dwelling & browser & species \\
\hline 16.95 & 15.7 & 11.6186978 ground dwelling & browser & species \\
\hline 15.8 & 15.8 & 4.9635 ground dwelling & herbivore & species \\
\hline 15.8 & 15.8 & 4.953 ground dwelling & omnivore & species \\
\hline 5.804 & 15.804 & 6.55085459 scansorial & carnivore, omnivore & genus \\
\hline 5.804 & 15.804 & 7.089659501 scansorial & carnivore, omnivore & genus \\
\hline 6.435 & 15.804 & 6.907755279 scansorial & carnivore, omnivore & genus \\
\hline 15.85 & 15.85 & 10.46851034 ground dwelling & carnivore, omnivore & species \\
\hline 15.85 & 15.85 & 8.492713832 scansorial & carnivore, omnivore & species \\
\hline 15.85 & 15.85 & 2.481 ground dwelling & granivore, browser & species \\
\hline 15.85 & 15.85 & 4.1505 ground dwelling & granivore, browser & species \\
\hline 15.85 & 15.85 & 2.441 ground dwelling & granivore, browser & species \\
\hline
\end{tabular}

$\begin{array}{ll}\text { omnivore } & \text { NA } \\ \text { herbivore } & \text { Rodentia } \\ \text { herbivore } & \text { herbivore guild } \\ \text { herbivore } & \text { Rodentia } \\ \text { herbivore } & \text { Rodentia } \\ \text { herbivore } & \text { Rodentia } \\ \text { carnivore } & \text { carnivore guild } \\ \text { herbivore } & \text { Rodentia } \\ \text { insectivore } & \text { NA } \\ \text { herbivore } & \text { herbivore guild } \\ \text { carnivore } & \text { carnivore guild } \\ \text { herbivore } & \text { Rodentia } \\ \text { herbivore } & \text { herbivore guild } \\ \text { NA } & \text { NA } \\ \text { omnivore } & \text { carnivore guild } \\ \text { herbivore } & \text { herbivore guild } \\ \text { herbivore } & \text { herbivore guild } \\ \text { omnivore } & \text { NA } \\ \text { omnivore } & \text { NA } \\ \text { herbivore } & \text { herbivore guild } \\ \text { herbivore } & \text { Rodentia } \\ \text { carnivore } & \text { carnivore guild } \\ \text { herbivore } & \text { herbivore guild } \\ \text { carnivore } & \text { carnivore guild } \\ \text { carnivore } & \text { carnivore guild } \\ \text { herbivore } & \text { herbivore guild } \\ \text { carnivore } & \text { carnivore guild } \\ \text { herbivore } & \text { herbivore guild } \\ \text { omnivore } & \text { carnivore guild } \\ \text { herbivore } & \text { herbivore guild } \\ \text { carnivore } & \text { carnivore guild } \\ \text { herbivore } & \text { herbivore guild } \\ \text { carnivore } & \text { carnivore guild } \\ \text { herbivore } & \text { herbivore guild } \\ \text { herbivore } & \text { herbivore guild } \\ \text { herbivore } & \text { herbivore guild } \\ \text { herbivore } & \text { herbivore guild } \\ \text { herbivore } & \text { Rodentia } \\ \text { herbivore } & \text { herbivore guild } \\ \text { herbivore } & \text { herbivore guild } \\ \text { herbivore } & \text { herbivore guild } \\ \text { herbivore } & \text { herbivore guild } \\ \text { herbivore } & \text { herbivore guild } \\ \text { omnivore } & \text { NA } \\ \text { carnivore } & \text { carnivore guild } \\ \text { carnivore } & \text { carnivore guild } \\ \text { carnivore } & \text { carnivore guild } \\ \text { carnivore } & \text { carnivore guild } \\ \text { carnivore } & \text { carnivore guild } \\ \text { herbivore } & \text { Rodentia } \\ \text { herbivore } & \text { Rerbivore }\end{array}$




\begin{tabular}{|c|c|c|c|c|c|c|c|c|c|}
\hline Rodentia & Sciuridae & Spermophilus jerae & 15.85 & 15.85 & 4.469 semifossorial & granivore, frugivore & species & herbivore & Rodentia \\
\hline Rodentia & Cricetidae & Copemys lindsayi & 15.85 & 15.85 & 2.702 ground dwelling & herbivore & species & herbivore & Rodentia \\
\hline Rodentia & Mylagaulidae & Galbreathia bettae & 15.85 & 15.85 & 5.1085 ground dwelling & herbivore & species & herbivore & Rodentia \\
\hline Rodentia & Castoridae & Euroxenomys inconnexus & 15.85 & 15.85 & 6.1005 amphibious & browser & species & herbivore & Rodentia \\
\hline Carnivora & Procyonidae & Arctonasua minima & 15.85 & 15.85 & 8.9595 scansorial & frugivore, carnivore & species & omnivore & carnivore guild \\
\hline Artiodactyla & Camelidae & Aepycamelus alexandrae & 16.9 & 15.85 & 12.95434373 ground dwelling & browser, browser & species & herbivore & herbivore guild \\
\hline \multirow[t]{2}{*}{ Artiodactyla } & Palaeomerycidae & Drepanomeryx falciformis & 17.2 & 15.85 & 11.41 ground dwelling & browser & species & herbivore & herbivore guild \\
\hline & & Jimomys labaughi & 17.25 & 15.85 & 4.242 ground dwelling & herbivore & species & herbivore & herbivore guild \\
\hline Artiodactyla & Protoceratidae & Lambdoceras trinitiensis & 17.3 & 15.85 & 11.94969542 ground dwelling & browser & species & herbivore & herbivore guild \\
\hline Eulipotyphla & Soricidae & Angustidens vireti & 17.375 & 15.85 & 2.9 ground dwelling & omnivore & species & omnivore & NA \\
\hline Perissodactyla & Equidae & Merychippus goorisi & 17.75 & 15.85 & 11.18462793 & & & NA & NA \\
\hline Perissodactyla & Rhinocerotidae & Teleoceras meridianum & 17.8 & 15.85 & 14.22890461 amphibious & grazer & species & herbivore & herbivore guild \\
\hline Perissodactyla & Rhinocerotidae & Peraceras hessei & 17.8 & 15.85 & 13.30699041 ground dwelling & browser & species & herbivore & herbivore guild \\
\hline Perissodactyla & Equidae & Merychippus primus & 18.1 & 15.85 & 11.42927049 ground dwelling & grazer, browser & species & herbivore & herbivore guild \\
\hline Rodentia & Mylagaulidae & Alphagaulus pristinus & 19.15 & 15.85 & 5.6411283 ground dwelling & herbivore & species & herbivore & Rodentia \\
\hline Rodentia & Mylagaulidae & Alphagaulus vetus & 19.6 & 15.85 & 6.26 ground dwelling & herbivore & species & herbivore & Rodentia \\
\hline Artiodactyla & Protoceratidae & Paratoceras wardi & 19.7 & 15.85 & 10.18433387 ground dwelling & browser & species & herbivore & herbivore guild \\
\hline Rodentia & Heteromyidae & Harrymys canadensis & 15.9 & 15.9 & 3.08 ground dwelling & granivore, browser & species & herbivore & Rodentia \\
\hline Rodentia & Eomyidae & Leptodontomys russelli & 15.9 & 15.9 & 1.033 ground dwelling & herbivore & species & herbivore & Rodentia \\
\hline Rodentia & Heteromyidae & Mioheteromys arcarius & 16 & 15.9 & 2.3 ground dwelling & granivore, browser & species & herbivore & Rodentia \\
\hline Rodentia & Heteromyidae & Lignimus austridakotensis & 16 & 16 & 2.6985 ground dwelling & granivore, browser & species & herbivore & Rodentia \\
\hline Eulipotyphla & Soricidae & Adeloblarina berklandi & 16 & 16 & 2.5405 ground dwelling & omnivore & species & omnivore & NA \\
\hline Artiodactyla & Palaeomerycidae & Subdromomeryx antilopinus & 16.85 & 16.05 & 11.00317837 ground dwelling & browser & species & herbivore & herbivore guild \\
\hline Rodentia & Heteromyidae & Proheteromys sulculus & 17.8 & 16.05 & 2.959 ground dwelling & granivore, browser & species & herbivore & Rodentia \\
\hline Artiodactyla & Camelidae & Paramiolabis taylori & 16.15 & 16.15 & 11.8125 ground dwelling & browser, browser & species & herbivore & herbivore guild \\
\hline Artiodactyla & Camelidae & Miolabis fricki & 16.65 & 16.15 & 12.4148451 ground dwelling & browser, browser & species & herbivore & herbivore guild \\
\hline Rodentia & Heteromyidae & Proheteromys maximus & 16.9 & 16.25 & 4.472 ground dwelling & granivore, browser & species & herbivore & Rodentia \\
\hline Rodentia & Aplodontidae & Tardontia nevadans & 16.4 & 16.4 & 5.0605 fossorial & herbivore & species & herbivore & Rodentia \\
\hline Carnivora & Amphicyonidae & Ysengrinia depereti & 16.435 & 16.435 & 11.34023158 ground dwelling & carnivore & species & carnivore & carnivore guild \\
\hline Carnivora & Mustelidae & Hoplictis florancei & 16.435 & 16.435 & 9.210340372 scansorial & carnivore, omnivore & genus & carnivore & carnivore guild \\
\hline Carnivora & Mustelidae & Martes sainjoni & 16.435 & 16.435 & 7.741291083 scansorial & carnivore, omnivore & genus & carnivore & carnivore guild \\
\hline Carnivora & Mustelidae & Mionictis artenensis & 16.435 & 16.435 & 8.293911505 scansorial & carnivore, omnivore & species & carnivore & carnivore guild \\
\hline Artiodactyla & & Amphimoschus artenensis & 16.435 & 16.435 & 10.12676924 ground dwelling & grazer, browser & genus & herbivore & herbivore guild \\
\hline Artiodactyla & & Amphimoschus ponteleviensis & 16.435 & 16.435 & 10.37314584 ground dwelling & grazer, browser & genus & herbivore & herbivore guild \\
\hline Proboscidea & Gomphotheriidae & Gomphotherium sylvaticum & 16.435 & 16.435 & 14.54543003 ground dwelling & browser & genus & herbivore & herbivore guild \\
\hline Proboscidea & Mammutidae & Zygolophodon metachijiensis & 16.435 & 16.435 & 14.90002814 ground dwelling & browser & genus & herbivore & herbivore guild \\
\hline Artiodactyla & Palaeomerycidae & Lagomeryx ruetimeyeri & 17.985 & 16.435 & 9.615595348 ground dwelling & browser & species & herbivore & herbivore guild \\
\hline Perissodactyla & Rhinocerotidae & Prosantorhinus germanicus & 17.985 & 16.435 & 13.12243244 ground dwelling & browser & genus & herbivore & herbivore guild \\
\hline Carnivora & Barbourofelidae & Prosansanosmilus peregrinus & 18.2 & 16.435 & 10.5964966 ground dwelling & carnivore, omnivore & species & carnivore & carnivore guild \\
\hline Artiodactyla & Bovidae & Eotragus artenensis & 18.2 & 16.435 & 10.12676924 ground dwelling & grazer, browser & genus & herbivore & herbivore guild \\
\hline Carnivora & Amphicyonidae & Amphicyon cooperi & 18.45 & 16.435 & 11.98265282 ground dwelling & carnivore & genus & carnivore & carnivore guild \\
\hline Artiodactyla & Cervidae & Procervulus praelucidus & 18.45 & 16.435 & 9.546517796 ground dwelling & grazer, browser & genus & herbivore & herbivore guild \\
\hline Proboscidea & Gomphotheriidae & Choerolophodon corrugatus & 18.45 & 16.435 & 14.90002814 ground dwelling & browser & genus & herbivore & herbivore guild \\
\hline Carnivora & Amphicyonidae & Ictiocyon socialis & 19.5 & 16.435 & 9.903418485 ground dwelling & carnivore & genus & carnivore & carnivore guild \\
\hline Carnivora & Amphicyonidae & Amphicyon giganteus & 21.2 & 16.435 & 12.61125855 ground dwelling & carnivore & species & carnivore & carnivore guild \\
\hline Artiodactyla & Anthracotheriidae & Brachyodus onoideus & 21.2 & 16.435 & 13.67735545 ground dwelling & browser & genus & herbivore & herbivore guild \\
\hline Carnivora & & Palaeogale minuta & 24.75 & 16.435 & 5.235 ground dwelling & carnivore, omnivore & species & carnivore & carnivore guild \\
\hline Artiodactyla & Antilocapridae & Paracosoryx wilsoni & 18.4 & 16.5 & 9.396849765 ground dwelling & browser, grazer & species & herbivore & herbivore guild \\
\hline Artiodactyla & Merycoidodontidae & Brachycrus laticeps & 16.85 & 16.6 & 12.49833049 ground dwelling & herbivore & species & herbivore & herbivore guild \\
\hline Perissodactyla & Equidae & Parapliohippus carrizoensis & 18.7 & 16.65 & 11.04134884 ground dwelling & grazer, browser & species & herbivore & herbivore guild \\
\hline Artiodactyla & Merycoidodontidae & Merychyus elegans & 20.2 & 16.75 & 10.54456154 ground dwelling & herbivore & species & herbivore & herbivore guild \\
\hline Artiodactyla & Tayassuidae & Hesperhys vagrans & 17.95 & 16.85 & 11.4321257 ground dwelling & herbivore, omnivore & species & omnivore & NA \\
\hline
\end{tabular}




\begin{tabular}{|c|c|c|}
\hline Artiodactyla & Merycoidodontidae & Merychyus smithi \\
\hline Rodentia & Dasyproctidae & Neoreomys australis \\
\hline Carnivora & Mustelidae & Leptarctus ancipidens \\
\hline Rodentia & Sciuridae & Miospermophilus wyomingensis \\
\hline Rodentia & Sciuridae & Protospermophilus oregonensis \\
\hline Perissodactyla & Equidae & Merychippus gunteri \\
\hline Carnivora & Amphicyonidae & Amphicyon longiramus \\
\hline Carnivora & Mustelidae & Mionictis letifer \\
\hline Perissodactyla & Equidae & $\begin{array}{l}\text { Texomys ritchiei } \\
\text { Archaeohippus penultimus } \\
\text { Namibictis senuti }\end{array}$ \\
\hline Carnivora & Viverridae & Orangictis gariepensis \\
\hline Carnivora & Canidae & Phlaocyon yatkolai \\
\hline Lagomorpha & Ochotonidae & Oreolagus wilsoni \\
\hline Rodentia & Sciuridae & Miospermophilus bryanti \\
\hline Artiodactyla & Protoceratidae & Lambdoceras hessei \\
\hline Artiodactyla & Moschidae & Parablastomeryx galushi \\
\hline Eulipotyphla & Erinaceidae & Brachyerix richi \\
\hline Perissodactyla & Rhinocerotidae & Brachypotherium americanum \\
\hline Eulipotyphla & Soricidae & Pseudotrimylus dakotensis \\
\hline Eulipotyphla & Soricidae & Pseudotrimylus roperi \\
\hline Eulipotyphla & Soricidae & Wilsonosorex bateslandensis \\
\hline Eulipotyphla & Soricidae & Wilsonosorex conulatus \\
\hline Artiodactyla & Palaeomerycidae & Aletomeryx gracilis \\
\hline Carnivora & Canidae & Desmocyon matthewi \\
\hline Carnivora & Canidae & Ectopocynus simplicidens \\
\hline Artiodactyla & Anthracotheriidae & Arretotherium fricki \\
\hline Rodentia & Heteromyidae & Schizodontomys amnicolus \\
\hline Rodentia & Florentiamyidae & Fanimus ultimus \\
\hline Insectivora & Proscalopidae & Mesoscalops scopelotemos \\
\hline Artiodactyla & Camelidae & Oxydactylus longipes \\
\hline Rodentia & Mylagaulidae & Galbreathia novellus \\
\hline Rodentia & Zapodidae & Schaubeumys galbreathi \\
\hline Eulipotyphla & Plesiosoricidae & Plesiosorex coloradensis \\
\hline Lipotyphla & Talpidae & Mystipterus martini \\
\hline Lipotyphla & Talpidae & Scalopoides isodens \\
\hline Perissodactyla & Equidae & Parahippus tyleri \\
\hline Artiodactyla & Merycoidodontidae & Merycochoerus magnus \\
\hline Eulipotyphla & Erinaceidae & Brachyerix macrotis \\
\hline Artiodactyla & Tayassuidae & Hesperhys pinensis \\
\hline Carnivora & Canidae & Metatomarctus canavus \\
\hline Perissodactyla & Equidae & Anchitherium clarencei \\
\hline Perissodactyla & Equidae & Archaeohippus blackbergi \\
\hline Perissodactyla & Rhinocerotidae & Menoceras barbouri \\
\hline Carnivora & Canidae & Phlaocyon leucosteus \\
\hline Carnivora & Mustelidae & Promartes lepidus \\
\hline Eulipotyphla & Soricidae & Limnoecus compressus \\
\hline Rodentia & Heteromyidae & Dikkomys matthewi \\
\hline Carnivora & Amphicyonidae & Daphoenodon superbus \\
\hline Artiodactyla & Camelidae & Stenomylus gracilis \\
\hline Carnivora & Canidae & Desmocyon thomsoni \\
\hline Lagomorpha & Ochotonidae & Oreolagus nebrascensis \\
\hline
\end{tabular}

\begin{tabular}{|c|c|c|c|c|}
\hline 19.15 & 16.85 & 10.61753619 ground dwelling & herbivore & species \\
\hline 16.9 & 16.9 & 8.216088099 ground dwelling & herbivore & species \\
\hline 21.7 & 16.9 & 7.856828497 scansorial & omnivore & species \\
\hline 22.9 & 16.9 & 4.421 scansorial & granivore, frugivore & species \\
\hline 17 & 17 & 6.1095 & & \\
\hline 18.85 & 17.05 & 10.50991173 ground dwelling & grazer, browser & species \\
\hline 21.7 & 17.05 & 11.62401942 ground dwelling & carnivore & species \\
\hline 17.1 & 17.1 & 6.471 scansorial & carnivore, omnivore & species \\
\hline 17.4 & 17.25 & 4.5825 ground dwelling & herbivore & species \\
\hline 17.65 & 17.3 & 11.015 ground dwelling & browser & species \\
\hline 17.319 & 17.319 & 9.233366223 scansorial & carnivore, omnivore & species \\
\hline 17.319 & 17.319 & 8.473513142 scansorial & carnivore, insectivore & species \\
\hline 17.375 & 17.375 & 9.533 ground dwelling & carnivore, omnivore & species \\
\hline 17.375 & 17.375 & 5.0885 ground dwelling & herbivore & species \\
\hline 17.375 & 17.375 & 4.0405 scansorial & granivore, frugivore & species \\
\hline 17.375 & 17.375 & 11.49272276 ground dwelling & browser & species \\
\hline 17.375 & 17.375 & 9.692943908 ground dwelling & browser & species \\
\hline 17.375 & 17.375 & 5.83 semifossorial & insectivore, carnivore & species \\
\hline 17.375 & 17.375 & 14.62 & & \\
\hline 17.375 & 17.375 & 3.198 ground dwelling & omnivore & species \\
\hline 17.375 & 7.375 & 4.408010307 ground dwelling & omnivore & species \\
\hline 17.375 & 17.375 & 2.919 gro & omnivore & species \\
\hline 17.375 & 17.375 & $3.2305 \mathrm{gr}$ & omnivore & species \\
\hline 19.55 & 17.375 & 10.13845101 gro & grazer & species \\
\hline 19.9 & 17.375 & 9.109329873 ground dwelling & carnivore, om & species \\
\hline 19.9 & 17.375 & 9.998098765 ground dwelling & carnivore, omnivore & species \\
\hline 19.9 & 17.375 & 11.89205271 ground dwelling & grazer & species \\
\hline 19.9 & 17.375 & 4.71 ground dwelling & granivore, browser & species \\
\hline 19.9 & 17.375 & 5.1 ground dwelling & herbivore & species \\
\hline 19.9 & 17.375 & $4.559 \mathrm{sc}$ & insect & species \\
\hline 20.2 & 17.375 & 12.00999512 ground dwelling & browser, brov & species \\
\hline 20.45 & 17.375 & 5.409 ground dwelling & herbivore & species \\
\hline 20.45 & 17.375 & 2.97 ground dwelling & herbivore & species \\
\hline 20.45 & 17.375 & 5.2575 scansorial & insectivore & species \\
\hline 20.45 & 17.375 & 3.379 fossorial & insectivore & species \\
\hline 20.45 & 17.375 & 3.4715 fossorial & insectivore & species \\
\hline 20.85 & .375 & 11.282 & & \\
\hline 21.1 & 17.375 & 12.7104033 ground dwelling & herbivore & species \\
\hline 21.1 & 17.375 & 4.892 semifossorial & insectivore, $\mathrm{c}$ & species \\
\hline 21.25 & 17.375 & 11.34067121 & & \\
\hline 21.7 & 17.375 & 9.487814592 ground dwelling & carnivore, omnivore & species \\
\hline 21.7 & 17.375 & 12.26994274 gro & browser & species \\
\hline 21.7 & 17.375 & 10.07201532 ground dwelling & browser & species \\
\hline 21.7 & 17.375 & 13.98177418 ground dwelling & browser & species \\
\hline 21.75 & 17.375 & 8.214696123 ground dwelling & carnivore, omnivore & species \\
\hline 21.95 & 17.375 & 8.246 scansorial & carnivore, omnivore & species \\
\hline 22.1 & .375 & 1.85 ground dwelling & omnivore & species \\
\hline 22.2 & 17.375 & 3.8665 ground dwelling & granivore, browser & species \\
\hline 22.5 & 17.375 & 11.29362949 ground dwelling & carnivore & species \\
\hline 22.7 & 17.375 & 10.9245 ground dwelling & browser, browser & species \\
\hline 22.75 & 17.375 & 8.923076447 ground dwellir & carnivore, omnivore & species \\
\hline 22.9 & 17.375 & 4.97 ground dwelling & herbivore & species \\
\hline
\end{tabular}

\begin{tabular}{|c|c|}
\hline herbivore & herbivore guild \\
\hline herbivore & Rodentia \\
\hline omnivore & carnivore guild \\
\hline herbivore & Rodentia \\
\hline NA & Rodentia \\
\hline herbivore & herbivore guild \\
\hline carnivore & carnivore guild \\
\hline carnivore & carnivore guild \\
\hline herbivore & herbivore guild \\
\hline herbivore & herbivore guild \\
\hline carnivore & carnivore guild \\
\hline insectivore & carnivore guild \\
\hline carnivore & carnivore guild \\
\hline herbivore & herbivore guild \\
\hline herbivore & Rodentia \\
\hline herbivore & herbivore guild \\
\hline herbivore & herbivore guild \\
\hline insectivore & NA \\
\hline NA & NA \\
\hline omnivore & NA \\
\hline omnivore & NA \\
\hline omnivore & NA \\
\hline omnivore & NA \\
\hline herbivore & herbivore guild \\
\hline carnivore & carnivore guild \\
\hline carnivore & carnivore guild \\
\hline herbivore & herbivore guild \\
\hline herbivore & Rodentia \\
\hline herbivore & Rodentia \\
\hline insectivore & NA \\
\hline herbivore & herbivore guild \\
\hline herbivore & Rodentia \\
\hline herbivore & Rodentia \\
\hline insectivore & NA \\
\hline insectivore & NA \\
\hline insectivore & NA \\
\hline NA & NA \\
\hline herbivore & herbivore guild \\
\hline insectivore & NA \\
\hline NA & NA \\
\hline carnivore & carnivore guild \\
\hline herbivore & herbivore guild \\
\hline herbivore & herbivore guild \\
\hline herbivore & herbivore guild \\
\hline carnivore & carnivore guild \\
\hline carnivore & carnivore guild \\
\hline omnivore & NA \\
\hline herbivore & Rodentia \\
\hline carnivore & carnivore guild \\
\hline herbivore & herbivore guild \\
\hline carnivore & carnivore guild \\
\hline herbivore & herbivore guild \\
\hline
\end{tabular}




\begin{tabular}{|c|c|c|c|c|c|c|c|c|c|}
\hline Rodentia & Heteromyidae & Stratimus strobeli & 22.9 & 17.375 & 3.0025 ground dwelling & granivore, browser & species & herbivore & Rodentia \\
\hline Rodentia & Mylagaulidae & Mesogaulus paniensis & 22.9 & 17.375 & 6.036632368 ground dwelling & herbivore & species & herbivore & Rodentia \\
\hline Rodentia & Cricetidae & Yatkolamys edwardsi & 22.9 & 17.375 & 3.9375 ground dwelling & herbivore & species & herbivore & Rodentia \\
\hline Rodentia & Heteromyidae & Harrymys magnus & 23.05 & 17.375 & 3.924 ground dwelling & granivore, browser & species & herbivore & Rodentia \\
\hline Rodentia & Zapodidae & Schaubeumys grangeri & 23.7 & 17.375 & 3.22 ground dwelling & herbivore & species & herbivore & Rodentia \\
\hline Artiodactyla & Merycoidodontidae & Merychyus minimus & 24.15 & 17.375 & 10.22430353 ground dwelling & herbivore & species & herbivore & herbivore guild \\
\hline Artiodactyla & Merycoidodontidae & Merychyus arenarum & 24.3 & 17.375 & 10.62147156 ground dwelling & herbivore & species & herbivore & herbivore guild \\
\hline Rodentia & Heteromyidae & Schizodontomys harkseni & 24.7 & 17.375 & 4.66 ground dwelling & granivore, browser & species & herbivore & Rodentia \\
\hline Rodentia & Eomyidae & Pseudotheridomys hesperus & 24.7 & 17.375 & 2.497 ground dwelling & herbivore & species & herbivore & Rodentia \\
\hline Insectivora & Proscalopidae & Proscalops secundus & 24.7 & 17.375 & 4.3175 scansorial & insectivore & species & insectivore & NA \\
\hline Artiodactyla & Moschidae & Pseudoblastomeryx advena & 25.2 & 17.375 & 9.22 ground dwelling & browser, grazer & species & herbivore & herbivore guild \\
\hline Rodentia & Zapodidae & Plesiosminthus clivosus & 26.55 & 17.375 & 2.235 & & & NA & Rodentia \\
\hline Carnivora & & Palaeogale sectoria & 35.85 & 17.375 & 6.208 ground dwelling & carnivore, omnivore & species & carnivore & carnivore guild \\
\hline Rodentia & Heteromyidae & Cupidinimus boronensis & 18.8 & 17.4 & 2.6805 ground dwelling & granivore, browser & species & herbivore & Rodentia \\
\hline Lagomorpha & Ochotonidae & Cuyamalagus dawsoni & 17.5 & 17.5 & 5.5335 ground dwelling & herbivore & species & herbivore & herbivore guild \\
\hline Rodentia & Eomyidae & Pseudotheridomys cuyamensis & 17.5 & 17.5 & 2.5225 ground dwelling & herbivore & species & herbivore & Rodentia \\
\hline Carnivora & Amphicyonidae & Cynelos idoneus & 17.6 & 17.6 & 11.57 ground dwelling & carnivore & species & carnivore & carnivore guild \\
\hline Carnivora & Mustelidae & Brachypsalis matutinus & 17.6 & 17.6 & 7.373 scansorial & carnivore, omnivore & species & carnivore & carnivore guild \\
\hline Carnivora & Mustelidae & Dinogale siouxensis & 17.6 & 17.6 & 6.6865 scansorial & carnivore, omnivore & species & carnivore & carnivore guild \\
\hline Artiodactyla & Palaeomerycidae & Sinclairomeryx riparius & 17.6 & 17.6 & 11.35423007 ground dwelling & browser & species & herbivore & herbivore guild \\
\hline Artiodactyla & Palaeomerycidae & Subdromomeryx scotti & 17.95 & 17.6 & 11.00635674 ground dwelling & browser & species & herbivore & herbivore guild \\
\hline Artiodactyla & Palaeomerycidae & Bouromeryx submilleri & 19.1 & 17.65 & 10.76851982 ground dwelling & browser & species & herbivore & herbivore guild \\
\hline Perissodactyla & Equidae & Protohippus vetus & 18.6 & 17.75 & 11.78659961 ground dwelling & grazer, browser & species & herbivore & herbivore guild \\
\hline Artiodactyla & Camelidae & Floridatragulus texanus & 17.8 & 17.8 & 10.9855 ground dwelling & browser, browser & species & herbivore & herbivore guild \\
\hline Artiodactyla & Protoceratidae & Prosynthetoceras texanus & 21.9 & 17.8 & 11.06746232 ground dwelling & browser & species & herbivore & herbivore guild \\
\hline Artiodactyla & Camelidae & Hesperocamelus stylodon & 17.9 & 17.9 & 12.78 ground dwelling & browser, browser & species & herbivore & herbivore guild \\
\hline Rodentia & Sciuridae & Protospermophilus angusticeps & 17.95 & 17.9 & 5.771 scansorial & granivore, frugivore & species & herbivore & Rodentia \\
\hline Rodentia & Aplodontidae & Ansomys nevadensis & 17.95 & 17.95 & 3.95 fossorial & herbivore & species & herbivore & Rodentia \\
\hline Rodentia & Eomyidae & Megapeomys bobwilsoni & 17.95 & 17.95 & 6.02 ground dwelling & herbivore & species & herbivore & Rodentia \\
\hline Artiodactyla & Tayassuidae & Cynorca sociale & 24.15 & 17.95 & 9.820636364 & & & NA & NA \\
\hline Perissodactyla & Rhinocerotidae & Plesiaceratherium fahlbuschi & 17.985 & 17.985 & 13.45860987 ground dwelling & browser & genus & herbivore & herbivore guild \\
\hline Artiodactyla & Suidae & Bunolistriodon adelli & 17.985 & 17.985 & 11.47147893 ground dwelling & omnivore & genus & omnivore & NA \\
\hline Artiodactyla & Hypertragulidae & Andegameryx serum & 18.45 & 17.985 & 8.411343345 ground dwelling & frugivore & genus & herbivore & herbivore guild \\
\hline Artiodactyla & Moschidae & Oriomeryx willii & 18.45 & 17.985 & 11.08234205 ground dwelling & browser, grazer & genus & herbivore & herbivore guild \\
\hline Artiodactyla & Suidae & Xenohyus venitor & 21.2 & 17.985 & 11.43924274 ground dwelling & omnivore & genus & omnivore & NA \\
\hline Artiodactyla & Camelidae & Aguascalientia wilsoni & 18 & 18 & 9.902045024 ground dwelling & browser, browser & species & herbivore & herbivore guild \\
\hline Rodentia & Zapodidae & Schaubeumys sabrae & 18.25 & 18.1 & 2.86 ground dwelling & herbivore & species & herbivore & Rodentia \\
\hline Rodentia & Sciuridae & Protospermophilus kelloggi & 22.9 & 18.1 & 5.311 scansorial & granivore, frugivore & species & herbivore & Rodentia \\
\hline Carnivora & Amphicyonidae & Amphicyon olisiponensis & 18.2 & 18.2 & 11.53595132 ground dwelling & carnivore & genus & carnivore & carnivore guild \\
\hline Artiodactyla & Merycoidodontidae & Brachycrus rusticus & 18.2 & 18.2 & 11.295 ground dwelling & herbivore & species & herbivore & herbivore guild \\
\hline Rodentia & Heteromyidae & Harrymys woodi & 18.2 & 18.2 & 3.773 ground dwelling & granivore, browser & species & herbivore & Rodentia \\
\hline Rodentia & Heteromyidae & Harrymys irvini & 18.25 & 18.2 & 4.43 ground dwelling & granivore, browser & species & herbivore & Rodentia \\
\hline Artiodactyla & Tragulidae & Dorcatherium parvum & 19.5 & 18.2 & 8.699166481 & & & NA & NA \\
\hline Primates & Proconsulidae & Afropithecus turkanensis & 21.2 & 18.2 & 10.81977828 arboreal & omnivore & species & omnivore & NA \\
\hline Lagomorpha & Ochotonidae & Desmatolagus schizopetrus & 22.9 & 18.2 & 6.1005 ground dwelling & grazer, browser & species & herbivore & herbivore guild \\
\hline \multirow[t]{2}{*}{ Artiodactyla } & Camelidae & Blickomylus galushai & 20.6 & 18.25 & 9.74 ground dwelling & browser, browser & species & herbivore & herbivore guild \\
\hline & Ischyromyidae & Ischyromys typus & 36.75 & 18.4 & 6.642 ground dwelling & herbivore & species & herbivore & herbivore guild \\
\hline \multirow[t]{3}{*}{ Artiodactyla } & Tragulidae & Siamotragulus bugtiensis & 18.45 & 18.45 & 9.482045413 ground dwelling & frugivore, grazer & genus & herbivore & herbivore guild \\
\hline & & Bugtimeryx pilgrimi & 18.45 & 18.45 & 9.977101208 ground dwelling & grazer, browser & genus & herbivore & herbivore guild \\
\hline & Rhinocerotidae & Caementodon oettingenae & 18.45 & 18.45 & 13.59906756 ground dwelling & browser & species & herbivore & herbivore guild \\
\hline Artiodactyla & Palaeomerycidae & Barbouromeryx trigonocorneus & 19.55 & 18.45 & 10.37279061 ground dwelling & browser & species & herbivore & herbivore guild \\
\hline Artiodactyla & Moschidae & Pomelomeryx boulangeri & 21.2 & 18.45 & 9.04685683 ground dwelling & browser, grazer & genus & herbivore & herbivore guild \\
\hline
\end{tabular}




\begin{tabular}{|c|c|c|}
\hline Artiodactyla & Moschidae & Pomelomeryx gracilis \\
\hline Carnivora & Ursidae & Phoberocyon hispanicus \\
\hline Carnivora & Canidae & Osbornodon iamonensis \\
\hline Rodentia & Geomyidae & Gregorymys larsoni \\
\hline Perissodactyla & Equidae & Anchitherium navasotae \\
\hline Perissodactyla & Equidae & Parahippus leonensis \\
\hline Artiodactyla & Camelidae & Australocamelus orarius \\
\hline Carnivora & Canidae & Osbornodon scitulus \\
\hline Artiodactyla & Anthracotheriidae & Arretotherium acridens \\
\hline Artiodactyla & Palaeomerycidae & Aletomeryx occidentalis \\
\hline Rodentia & Heteromyidae & Trogomys rupinimenthae \\
\hline Rodentia & Heteromyidae & Proheteromys floridanus \\
\hline Carnivora & Amphicyonidae & Daphoenodon robustum \\
\hline Perissodactyla & Equidae & Archaeohippus stenolophus \\
\hline Lagomorpha & Leporidae & Archaeolagus acaricolus \\
\hline Carnivora & Procyonidae & Bassaricyonoides phyllismillerae \\
\hline Carnivora & Canidae & Phlaocyon marslandensis \\
\hline Artiodactyla & Tragulidae & Dorcatherium libiensis \\
\hline Eulipotyphla & Soricidae & Pseudotrimylus blacki \\
\hline Primates & Cebidae & Dolichocebus gaimanensis \\
\hline Rodentia & Geomyidae & Pleurolicus sulcifrons \\
\hline Perissodactyla & Equidae & Archaeohippus mannulus \\
\hline \multirow[t]{2}{*}{ Carnivora } & Canidae & Cynarctoides gawnae \\
\hline & & Texomys stewarti \\
\hline Perissodactyla & Rhinocerotidae & Floridaceras whitei \\
\hline Carnivora & Amphicyonidae & Amphicyon galushai \\
\hline Artiodactyla & Merycoidodontidae & Merycochoerus proprius \\
\hline Carnivora & Canidae & Cynarctoides emryi \\
\hline Lagomorpha & Ochotonidae & Gripholagomys lavocati \\
\hline Artiodactyla & Camelidae & Michenia agatensis \\
\hline Artiodactyla & Merycoidodontidae & Merycoides harrisonensis \\
\hline Artiodactyla & Moschidae & Machaeromeryx tragulus \\
\hline Insectivora & Proscalopidae & Mesoscalops montanensis \\
\hline Carnivora & Amphicyonidae & Daphoenodon falkenbachi \\
\hline Perissodactyla & Equidae & Parahippus nebrascensis \\
\hline Carnivora & Amphicyonidae & Ysengrinia americana \\
\hline Artiodactyla & Protoceratidae & Syndyoceras cooki \\
\hline Artiodactyla & Camelidae & Tanymykter brachyodontus \\
\hline \multirow[t]{2}{*}{ Carnivora } & Mustelidae & Megalictis ferox \\
\hline & & Paciculus montanus \\
\hline Carnivora & Canidae & Cynarctoides luskensis \\
\hline Carnivora & Canidae & Phlaocyon minor \\
\hline Carnivora & Amphicyonidae & Adilophontes brachykolos \\
\hline Carnivora & Canidae & Phlaocyon annectens \\
\hline Carnivora & Amphicyonidae & Temnocyon percussor \\
\hline Perissodactyla & Rhinocerotidae & Menoceras arikarense \\
\hline Perissodactyla & Rhinocerotidae & Menoceras cooki \\
\hline Artiodactyla & Entelodontidae & Dinohyus hollandi \\
\hline Perissodactyla & Chalicotheriidae & Moropus elatus \\
\hline Carnivora & Amphicyonidae & Daphoenodon skinneri \\
\hline Carnivora & Canidae & Paraenhydrocyon wallovianus \\
\hline Carnivora & Canidae & Cynarctoides harlowi \\
\hline
\end{tabular}

\begin{tabular}{|c|c|c|c|c|}
\hline 21.2 & 18.45 & 8.411343345 ground dwelling & browser, grazer & genus \\
\hline 21.2 & 18.45 & 11.48989961 ground dwelling & herbivore, carnivore & genus \\
\hline 21.7 & 18.5 & 9.81240507 ground dwelling & carnivore, omnivore & species \\
\hline 18.6 & 18.6 & 3.55 fossorial & browser & species \\
\hline 18.65 & 18.6 & 11.7045 ground dwelling & browser & species \\
\hline 24.2 & 18.6 & 11.12303901 ground dwelling & browser & species \\
\hline 18.65 & 18.65 & 11.6865 ground dwelling & browser, browser & species \\
\hline 19.8 & 18.65 & 9.381517127 & & \\
\hline 22.3 & 18.65 & 12.12027421 ground dwelling & grazer & species \\
\hline 18.8 & 18.8 & 10.34 ground dwelling & grazer & species \\
\hline 19.2 & 18.8 & 2.53 ground dwelling & granivore, browser & species \\
\hline 21.8 & 18.85 & 1.6795 ground dwelling & granivore, browser & species \\
\hline 19 & 19 & 11.58891077 & & \\
\hline 20.5 & 19.15 & 11.20954586 ground dwelling & browser & species \\
\hline 21.05 & 19.2 & 5.5245 ground dwelling & grazer, browser & species \\
\hline 19.3 & 19.3 & 7.89 scansorial & frugivore, carnivore & species \\
\hline 19.5 & 19.5 & 8.676 ground dwelling & carnivore, omnivore & species \\
\hline 19.5 & 19.5 & 9.903418485 ground dwelling & frugivore, grazer & species \\
\hline 19.5 & 19.5 & 3.89 ground dwelling & omnivore & species \\
\hline 19.5 & 19.5 & 7.901007052 arboreal & frugivore, omnivore & species \\
\hline 26.05 & 19.525 & $4.4305 \mathrm{NA}$ & NA & species \\
\hline 19.55 & 19.55 & 12.93592305 ground dwelling & browser & species \\
\hline 19.6 & 19.6 & 7.685683786 ground dwelling & carnivore, omnivore & species \\
\hline 19.7 & 19.7 & 3.157 ground dwelling & herbivore & species \\
\hline 21.7 & 19.7 & 14.51 ground dwelling & browser & species \\
\hline 19.9 & 19.8 & 12.15387308 ground dwelling & carnivore & species \\
\hline 19.9 & 19.8 & 12.39164337 ground dwelling & herbivore & species \\
\hline 19.9 & 19.9 & 7.70975018 ground dwelling & carnivore, omnivore & species \\
\hline 24.7 & 19.9 & 5.391 ground dwelling & herbivore & species \\
\hline 20.2 & 20.15 & 11.57035157 ground dwelling & browser, browser & species \\
\hline 24.55 & 20.15 & 11.16901805 & & \\
\hline 20.2 & 20.2 & 9.16055795 ground dwelling & browser, grazer & species \\
\hline 20.2 & 20.2 & 4.45 scansorial & insectivore & species \\
\hline 20.8 & 20.2 & 11.9269489 ground dwelling & carnivore & species \\
\hline 21.4 & 20.2 & 12.046 & & \\
\hline 21.6 & 20.2 & 11.9800865 ground dwelling & carnivore & species \\
\hline 22.15 & 20.2 & 11.20615795 ground dwelling & browser & species \\
\hline 22.35 & 20.2 & 11.4065 ground dwelling & browser, browser & species \\
\hline 23.5 & 20.2 & 9.167071737 scansorial & carnivore, omnivore & species \\
\hline 24.1 & 20.45 & 4.398 ground dwelling & herbivore & species \\
\hline 20.8 & 20.7 & 7.598377481 ground dwelling & carnivore, omnivore & species \\
\hline 25.2 & 20.7 & 8.10078719 ground dwelling & carnivore, omnivore & species \\
\hline 20.9 & 20.8 & 11.69899216 ground dwelling & carnivore & species \\
\hline 21.05 & 20.85 & 8.075019661 ground dwelling & carnivore, omnivore & species \\
\hline 21.45 & 20.85 & 11.178 ground dwelling & carnivore & species \\
\hline 21.6 & 20.85 & 13.17782874 ground dwelling & browser & species \\
\hline 21.75 & 20.85 & 12.4339595 ground dwelling & browser & species \\
\hline 22.4 & 20.85 & 14.41227924 ground dwelling & omnivore & species \\
\hline 23 & 20.85 & 13.61076556 ground dwelling & browser & species \\
\hline 20.9 & 20.9 & 11.45948858 ground dwelling & carnivore & species \\
\hline 5.615 & 20.9 & 9.844909379 ground dwelling & carnivore, omnivore & species \\
\hline 20.95 & 20.95 & 7.124 ground dwelling & carnivore, omnivore & species \\
\hline
\end{tabular}

$\begin{array}{ll}\text { herbivore } & \text { herbivore guild } \\ \text { omnivore } & \text { carnivore guild } \\ \text { carnivore } & \text { carnivore guild } \\ \text { herbivore } & \text { Rodentia } \\ \text { herbivore } & \text { herbivore guild } \\ \text { herbivore } & \text { herbivore guild } \\ \text { herbivore } & \text { herbivore guild } \\ \text { NA } & \text { carnivore guild } \\ \text { herbivore } & \text { herbivore guild } \\ \text { herbivore } & \text { herbivore guild } \\ \text { herbivore } & \text { Rodentia } \\ \text { herbivore } & \text { Rodentia } \\ \text { NA } & \text { carnivore guild } \\ \text { herbivore } & \text { herbivore guild } \\ \text { herbivore } & \text { herbivore guild } \\ \text { omnivore } & \text { carnivore guild } \\ \text { carnivore } & \text { carnivore guild } \\ \text { herbivore } & \text { herbivore guild } \\ \text { omnivore } & \text { NA } \\ \text { omnivore } & \text { NA } \\ \text { NA } & \text { Rodentia } \\ \text { herbivore } & \text { herbivore guild } \\ \text { carnivore } & \text { carnivore guild } \\ \text { herbivore } & \text { herbivore guild } \\ \text { herbivore } & \text { herbivore guild } \\ \text { carnivore } & \text { carnivore guild } \\ \text { herbivore } & \text { herbivore guild } \\ \text { carnivore } & \text { carnivore guild } \\ \text { herbivore } & \text { herbivore guild } \\ \text { herbivore } & \text { herbivore guild } \\ \text { NA } & \text { NA } \\ \text { herbivore } & \text { herbivore guild } \\ \text { insectivore } & \text { NA } \\ \text { carnivore } & \text { carnivore guild } \\ \text { NA } & \text { NA } \\ \text { carnivore } & \text { carnivore guild } \\ \text { herbivore } & \text { herbivore guild } \\ \text { herbivore } & \text { herbivore guild } \\ \text { carnivore } & \text { carnivore guild } \\ \text { herbivore } & \text { herbivore guild } \\ \text { carnivore } & \text { carnivore guild } \\ \text { carnivore } & \text { carnivore guild } \\ \text { carnivore } & \text { carnivore guild } \\ \text { carnivore } & \text { carnivore guild } \\ \text { carnivore } & \text { carnivore guild } \\ \text { herbivore } & \text { herbivore guild } \\ \text { herbivore } & \text { herbivore guild } \\ \text { omnivore } & \text { hA } \\ \text { herbivore } & \text { herbivore guild } \\ \text { carnivore } & \text { carnivore guild } \\ \text { carnivore } & \text { carnivore guild } \\ & \end{array}$




\begin{tabular}{|c|c|c|c|}
\hline Artiodactyla & Camelidae & Priscocamelus wilsoni & 21.05 \\
\hline Artiodactyla & Camelidae & Michenia exilis & 21.95 \\
\hline Rodentia & Sciuridae & Similisciurus maxwelli & 21.05 \\
\hline Artiodactyla & & Delahomeryx browni & 21.05 \\
\hline Rodentia & Geomyidae & Gregorymys riograndensis & 21.05 \\
\hline Eulipotyphla & Erinaceidae & Brachyerix hibbardi & 21.05 \\
\hline Artiodactyla & Hypertragulidae & Nanotragulus ordinatus & 23.5 \\
\hline Eulipotyphla & Erinaceidae & Stenoechinus tantalus & 24.6 \\
\hline Carnivora & Amphicyonidae & Cynelos lemanensis & 21.2 \\
\hline Carnivora & Amphicyonidae & Ysengrinia gerandiana & 21.2 \\
\hline Carnivora & & Proailurus lemanensis & 21.2 \\
\hline Rodentia & Geomyidae & Entoptychus leptophrys & 21.2 \\
\hline \multirow[t]{3}{*}{ Artiodactyla } & Suidae & Hyotherium meisneri & 21.2 \\
\hline & & Phoberogale depereti & 21.2 \\
\hline & & Phoberogale ginesticus & 21.2 \\
\hline \multirow[t]{3}{*}{ Carnivora } & & Herpestides antiquus & 22.715 \\
\hline & Cainotheriidae & Cainotherium commune & 22.715 \\
\hline & Equidae & Anchippus texanus & 22.4 \\
\hline Rodentia & Geomyidae & Gregorymys curtus & 23.25 \\
\hline Lagomorpha & Leporidae & Archaeolagus macrocephalus & 24.7 \\
\hline Rodentia & Geomyidae & Gregorymys formosus & 24.35 \\
\hline Perissodactyla & Rhinocerotidae & Diceratherium niobrarense & 21.45 \\
\hline Perissodactyla & Tapiridae & Miotapirus harrisonensis & 22.15 \\
\hline Artiodactyla & Moschidae & Problastomeryx primus & 21.95 \\
\hline Perissodactyla & Rhinocerotidae & Diceratherium annectens & 27.5 \\
\hline Carnivora & Amphicyonidae & Cynelos caroniavorus & 21.7 \\
\hline Carnivora & Canidae & Euoplocyon spissidens & 21.7 \\
\hline Carnivora & Mustelidae & Oligobunis floridanus & 21.7 \\
\hline Carnivora & Mustelidae & Zodiolestes freundi & 21.7 \\
\hline Rodentia & Sciuridae & Nototamias hulberti & 21.7 \\
\hline Rodentia & Sciuridae & Petauristodon pattersoni & 21.7 \\
\hline Artiodactyla & Camelidae & Floridatragulus dolichanthereus & 21.7 \\
\hline Artiodactyla & Camelidae & Nothokemas floridanus & 21.7 \\
\hline Artiodactyla & Moschidae & Machaeromeryx gilchristensis & 21.7 \\
\hline Artiodactyla & Moschidae & Parablastomeryx floridanus & 21.7 \\
\hline Carnivora & Ursidae & Phoberocyon johnhenryi & 21.7 \\
\hline Artiodactyla & Tayassuidae & Floridachoerus olseni & 21.9 \\
\hline Rodentia & Castoridae & Pseudopalaeocastor barbouri & 22.6 \\
\hline Perissodactyla & Tapiridae & Nexuotapirus marslandensis & 23.5 \\
\hline Rodentia & Castoridae & Fossorcastor fossor & 24 \\
\hline Carnivora & Amphicyonidae & Mammacyon obtusidens & 21.75 \\
\hline Carnivora & Mustelidae & Megalictis frazieri & 21.75 \\
\hline Artiodactyla & Camelidae & Nothokemas waldropi & 25.55 \\
\hline Rodentia & Heteromyidae & Proheteromys toledoensis & 21.9 \\
\hline Artiodactyla & Protoceratidae & Prosynthetoceras orthrionanus & 21.9 \\
\hline Rodentia & Castoridae & Neatocastor hesperus & 22.3 \\
\hline Carnivora & Amphicyonidae & Daphoenodon notionastes & 23.05 \\
\hline Perissodactyla & Chalicotheriidae & Moropus oregonensis & 23.05 \\
\hline Perissodactyla & Rhinocerotidae & Diceratherium armatum & 32.7 \\
\hline Lagomorpha & Leporidae & Archaeolagus primigenius & 24.7 \\
\hline Eulipotyphla & Erinaceidae & Hibbarderix obfuscatus & 22.1 \\
\hline Rodentia & Mylagaulidae & Promylagaulus riggsi & 24.7 \\
\hline
\end{tabular}

2111.25594069 ground dwelling browser, browser species 2110.90654567 ground dwelling browser, browser

$21.05 \quad 10.5945$ ground dwelling browser, browser species

$21.05 \quad 3.781$ fossorial browser species

21.054 .359 semifossorial insectivore, carnivore species

21.058 .535051774 ground dwelling frugivore species

21.13 .879 semifossorial insectivore, carnivore species

$\begin{array}{ll}21.2 & 10.71392844 \text { ground dwelling carnivore species }\end{array}$

21.211 .45536084 ground dwelling carnivore species

21.29 .546517796 ground dwelling carnivore, omnivore species

21.23 .982 fossorial browser species

21.210 .71392844 ground dwelling omnivore genus

21.210 .47676217 ground dwelling herbivore, carnivore species

21.210 .61491728 ground dwelling herbivore, carnivore genus

$21.2 \quad 8.293911505$ ground dwelling carnivore, omnivore species

21.213 .81551056 ground dwelling grazer, browser species

$21.25 \quad 11.217$

$21.25 \quad 4.67$ fossorial browser

21.34 .3545 fossorial browser species

$21.45 \quad 14.56$ ground dwelling browser species

$21.55 \quad 11.301$ ground dwelling browser species

$21.6 \quad 9.61$ ground dwelling browser, grazer species

21.613 .72663718 ground dwelling browser species

$21.7 \quad 9.655$ ground dwelling carnivore species

21.79 .314205702 ground dwelling carnivore, omnivore species

21.710 .09553075 scansorial carnivore, omnivore species

21.710 .34666619 scansorial carnivore, omnivore species

$21.7 \quad 2.838$ scansorial granivore, frugivore species

21.75 .818 scansorial granivore, frugivore species

21.710 .49705723 ground dwelling browser, browser species

21.711 .42436627 ground dwelling browser, browser species

$21.7 \quad 8.42$ ground dwelling browser, grazer species

21.79 .944488279 ground dwelling browser, grazer species

21.711 .88401452 ground dwelling herbivore, carnivore species

21.710 .85755547 ground dwelling herbivore, omnivore species

$21.7 \quad 5.8305$

$21.7 \quad 12.283$ ground dwelling browser species

$21.7 \quad 6.923$

$21.75 \quad 11.16$ ground dwelling carnivore species

21.759 .689 scansorial carnivore, omnivore species

21.7510 .21866494 ground dwelling browser, browser species

$21.9 \quad 4.084$ ground dwelling granivore, browser species

21.910 .62947066 ground dwelling browser species

$21.9 \quad 6.943$

21.910 .90139329 ground dwelling carnivore species

$21.9 \quad 12.117$ ground dwelling browser species

21.914 .79137582 ground dwelling browser species

$21.95 \quad 6.4375$ ground dwelling grazer, browser species

22.13 .5285 semifossorial insectivore, carnivore species

22.14 .4475 ground dwelling herbivore species

$\begin{array}{ll}\text { herbivore } & \text { herbivore guild } \\ \text { herbivore } & \text { herbivore guild } \\ \text { herbivore } & \text { Rodentia } \\ \text { herbivore } & \text { herbivore guild } \\ \text { herbivore } & \text { Rodentia } \\ \text { insectivore } & \text { NA } \\ \text { herbivore } & \text { herbivore guild } \\ \text { insectivore } & \text { NA } \\ \text { carnivore } & \text { carnivore guild } \\ \text { carnivore } & \text { carnivore guild } \\ \text { carnivore } & \text { carnivore guild } \\ \text { herbivore } & \text { Rodentia } \\ \text { omnivore } & \text { NA } \\ \text { omnivore } & \text { NA } \\ \text { omnivore } & \text { NA } \\ \text { carnivore } & \text { carnivore guild } \\ \text { herbivore } & \text { herbivore guild } \\ \text { NA } & \text { NA } \\ \text { herbivore } & \text { Rodentia } \\ \text { herbivore } & \text { herbivore guild } \\ \text { herbivore } & \text { Rodentia } \\ \text { herbivore } & \text { herbivore guild } \\ \text { herbivore } & \text { herbivore guild } \\ \text { herbivore } & \text { herbivore guild } \\ \text { herbivore } & \text { herbivore guild } \\ \text { carnivore } & \text { carnivore guild } \\ \text { carnivore } & \text { carnivore guild } \\ \text { carnivore } & \text { carnivore guild } \\ \text { carnivore } & \text { carnivore guild } \\ \text { herbivore } & \text { Rodentia } \\ \text { herbivore } & \text { Rodentia } \\ \text { herbivore } & \text { herbivore guild } \\ \text { herbivore } & \text { herbivore guild } \\ \text { herbivore } & \text { herbivore guild } \\ \text { herbivore } & \text { herbivore guild } \\ \text { omnivore } & \text { carnivore guild } \\ \text { omnivore } & \text { NA } \\ \text { NA } & \text { Rodentia } \\ \text { herbivore } & \text { herbivore guild } \\ \text { NA } & \text { Rodentia } \\ \text { carnivore } & \text { carnivore guild } \\ \text { carnivore } & \text { carnivore guild } \\ \text { herbivore } & \text { herbivore guild } \\ \text { herbivore } & \text { Rodentia } \\ \text { herbivore } & \text { herbivore guild } \\ \text { NA } & \text { Rodentia } \\ \text { carnivore } & \text { carnivore guild } \\ \text { herbivore } & \text { herbivore guild } \\ \text { herbivore } & \text { herbive guild } \\ \text { herbivore } & \text { Rnsectivore } \\ \text { herbivore } & \end{array}$




\begin{tabular}{|c|c|c|}
\hline Rodentia & Florentiamyidae & Sanctimus falkenbachi \\
\hline Rodentia & Geomyidae & Entoptychus grandiplanus \\
\hline Didelphimorphia & Herpetotheriidae & Herpetotherium youngi \\
\hline Artiodactyla & Merycoidodontidae & Merycochoerus chelydra \\
\hline Artiodactyla & Merycoidodontidae & Hypsiops bannackensis \\
\hline Artiodactyla & Camelidae & Stenomylus hitchcocki \\
\hline Artiodactyla & Merycoidodontidae & Hypsiops breviceps \\
\hline Artiodactyla & Merycoidodontidae & Merycochoerus carrikeri \\
\hline Rodentia & Castoridae & Euhapsis breugerorum \\
\hline Rodentia & Castoridae & Fossorcastor greeni \\
\hline Carnivora & Canidae & Cormocyon haydeni \\
\hline Artiodactyla & Merycoidodontidae & Desmatochoerus megalodon \\
\hline Rodentia & Castoridae & Migmacastor procumbodens \\
\hline Rodentia & Geomyidae & Entoptychus sheppardi \\
\hline Rodentia & Heteromyidae & Schizodontomys sulcidens \\
\hline Carnivora & Canidae & Enhydrocyon crassidens \\
\hline Carnivora & Canidae & Paraenhydrocyon josephi \\
\hline Rodentia & Sciuridae & Sciurion campestre \\
\hline Perissodactyla & Equidae & Hypohippus equinus \\
\hline Rodentia & Cricetidae & Leidymys blacki \\
\hline Carnivora & Canidae & Cormocyon copei \\
\hline Carnivora & Canidae & Phlaocyon achoros \\
\hline Artiodactyla & Hypertragulidae & Nanotragulus loomisi \\
\hline Carnivora & Canidae & Cynarctoides lemur \\
\hline Lipotyphla & Geolabididae & Centetodon magnus \\
\hline Carnivora & Canidae & Enhydrocyon basilatus \\
\hline Carnivora & Canidae & Leptocyon vulpinus \\
\hline Carnivora & Canidae & Paraenhydrocyon robustus \\
\hline \multirow[t]{2}{*}{ Perissodactyla } & Equidae & Miohippus equinanus \\
\hline & Merycoidodontidae & Promerycochoerus superbus \\
\hline Artiodactyla & Camelidae & Miotylopus gibbi \\
\hline Artiodactyla & Anthracotheriidae & Arretotherium leptodus \\
\hline Rodentia & Castoridae & Fossorcastor brachyceps \\
\hline Perissodactyla & Rhinocerotidae & Diceratherium gregorii \\
\hline Artiodactyla & Merycoidodontidae & Oreodontoides oregonensis \\
\hline Artiodactyla & Merycoidodontidae & Mesoreodon minor \\
\hline Rodentia & Geomyidae & Pleurolicus dakotensis \\
\hline Rodentia & Allomyidae & Alwoodia harkseni \\
\hline Carnivora & Canidae & Enhydrocyon pahinsintewakpa \\
\hline Eulipotyphla & Erinaceidae & Amphechinus horncloudi \\
\hline Rodentia & Sciuridae & Nototamias quadratus \\
\hline Rodentia & Eutypomyidae & Eutypomys montanensis \\
\hline Rodentia & Florentiamyidae & Florentiamys tiptoni \\
\hline Rodentia & Mylagaulidae & Trilaccogaulus ovatus \\
\hline Lagomorpha & Leporidae & Palaeolagus philoi \\
\hline Artiodactyla & Merycoidodontidae & Phenacocoelus typus \\
\hline Perissodactyla & Equidae & Kalobatippus avus \\
\hline Artiodactyla & Camelidae & Miotylopus leonardi \\
\hline Artiodactyla & Merycoidodontidae & Leptauchenia decora \\
\hline \multirow[t]{3}{*}{ Rodentia } & Heteromyidae & Proheteromys ironcloudi \\
\hline & & Paciculus nebraskensis \\
\hline & & Heliscomys woodi \\
\hline
\end{tabular}

\begin{tabular}{|c|c|c|c|c|}
\hline 22.15 & 22.15 & 5.018 ground dwelling & herbivore & species \\
\hline 23.9 & 22.15 & 4.09 fossorial & browser & species \\
\hline 32.9 & 22.15 & 2.861 ground dwelling & omnivore & species \\
\hline 23.5 & 22.3 & 12.436 ground dwelling & herbivore & species \\
\hline 22.4 & 22.4 & 11.925 & & \\
\hline 24.8 & 22.4 & 10.68680263 ground dwelling & browser, browser & species \\
\hline 25.5 & 22.4 & 11.89454976 ground dwelling & herbivore & species \\
\hline 23.5 & 22.55 & 12.32733757 & & \\
\hline 22.6 & 22.6 & 5.7385 amphibious & browser & species \\
\hline 22.6 & 22.6 & 6.248 amphibious & browser & species \\
\hline 24.95 & 22.6 & 8.281970502 ground dwelling & carnivore, omnivore & species \\
\hline 26.25 & 22.6 & 12.17251471 ground dwelling & herbivore & species \\
\hline 22.615 & 22.615 & 6.51 amphibious & browser & species \\
\hline 22.65 & 22.65 & 4.5505 fossorial & browser & species \\
\hline 22.7 & 22.7 & 4.1805 ground dwelling & granivore, browser & species \\
\hline 24.35 & 22.75 & 10.26257251 ground dwelling & carnivore, omnivore & species \\
\hline 28.5 & 22.75 & 9.100724702 ground dwelling & carnivore, omnivore & species \\
\hline 22.9 & 22.9 & 3.126 scansorial & granivore, frugivore & species \\
\hline 22.9 & 22.9 & 12.4225 ground dwelling & browser & species \\
\hline 29.25 & 22.9 & 4.139 ground dwelling & herbivore & species \\
\hline 25.8 & 23 & 8.50608742 ground dwelling & carnivore, omnivore & species \\
\hline 23.05 & 23.05 & 7.8305 ground dwelling & carnivore, omnivore & species \\
\hline 27.5 & 23.05 & 7.562481876 ground dwelling & frugivore & species \\
\hline 28.5 & 23.05 & 7.574399613 ground dwelling & carnivore, omnivore & species \\
\hline 42.3 & 23.05 & 3.415291942 scansorial & insectivore & species \\
\hline 23.1 & 23.1 & 10.519 ground dwelling & carnivore, omnivore & species \\
\hline 23.1 & 23.1 & 8.57 ground dwelling & carnivore, omnivore & species \\
\hline 23.1 & 23.1 & 9.222847695 ground dwelling & carnivore, omnivore & species \\
\hline 25.55 & 23.1 & 10.49 ground dwelling & browser & species \\
\hline 25.7 & 23.1 & 12.522 ground dwelling & herbivore & species \\
\hline 30.75 & 23.2 & 10.778 ground dwelling & browser, browser & species \\
\hline 23.25 & 23.25 & 11.3839807 ground dwelling & grazer & species \\
\hline 23.65 & 23.25 & 5.9315 amphibious & browser & species \\
\hline 24.4 & 23.25 & 13.54094153 ground dwelling & browser & species \\
\hline 25.8 & 23.25 & 10.0230165 ground dwelling & herbivore & species \\
\hline 29.75 & 23.25 & 11.38120069 ground dwelling & herbivore & species \\
\hline 23.3 & 23.3 & $4.1155 \mathrm{NA}$ & NA & species \\
\hline 23.9 & 23.3 & 5.423 ground dwelling & herbivore & species \\
\hline 25.2 & 23.3 & 10.0885 ground dwelling & carnivore, omnivore & species \\
\hline 24.6 & 23.4 & 5.1685 semifossorial & insectivore, carnivore & species \\
\hline 24.7 & 23.4 & 3.6105 scansorial & granivore, frugivore & species \\
\hline 24.7 & 23.4 & 6.8545 ground dwelling & herbivore & species \\
\hline 24.7 & 23.4 & 4.8065 ground dwelling & herbivore & species \\
\hline 24.7 & 23.4 & 4.659 ground dwelling & herbivore & species \\
\hline 29.25 & 23.4 & 6.141 ground dwelling & grazer, browser & species \\
\hline 23.5 & 23.45 & 10.70927501 ground dwelling & herbivore & species \\
\hline 23.5 & 23.5 & 10.54123456 ground dwelling & browser & species \\
\hline 25.2 & 23.5 & 10.7735 ground dwelling & browser, browser & species \\
\hline 33.75 & 23.65 & 9.7995 ground dwelling & herbivore & species \\
\hline 26.25 & 23.7 & 2.223 ground dwelling & granivore, browser & species \\
\hline 26.25 & 23.8 & 4.3905 ground dwelling & herbivore & species \\
\hline 29.25 & 23.8 & 2.372 & & \\
\hline
\end{tabular}

$\begin{array}{ll}\text { herbivore } & \text { Rodentia } \\ \text { herbivore } & \text { Rodentia } \\ \text { omnivore } & \text { NA } \\ \text { herbivore } & \text { herbivore guild } \\ \text { NA } & \text { NA } \\ \text { herbivore } & \text { herbivore guild } \\ \text { herbivore } & \text { herbivore guild } \\ \text { NA } & \text { NA } \\ \text { herbivore } & \text { Rodentia } \\ \text { herbivore } & \text { Rodentia } \\ \text { carnivore } & \text { carnivore guild } \\ \text { herbivore } & \text { herbivore guild } \\ \text { herbivore } & \text { Rodentia } \\ \text { herbivore } & \text { Rodentia } \\ \text { herbivore } & \text { Rodentia } \\ \text { carnivore } & \text { carnivore guild } \\ \text { carnivore } & \text { carnivore guild } \\ \text { herbivore } & \text { Rodentia } \\ \text { herbivore } & \text { herbivore guild } \\ \text { herbivore } & \text { Rodentia } \\ \text { carnivore } & \text { carnivore guild } \\ \text { carnivore } & \text { carnivore guild } \\ \text { herbivore } & \text { herbivore guild } \\ \text { carnivore } & \text { carnivore guild } \\ \text { insectivore } & \text { NA } \\ \text { carnivore } & \text { carnivore guild } \\ \text { carnivore } & \text { carnivore guild } \\ \text { carnivore } & \text { carnivore guild } \\ \text { herbivore } & \text { herbivore guild } \\ \text { herbivore } & \text { herbivore guild } \\ \text { herbivore } & \text { herbivore guild } \\ \text { herbivore } & \text { herbivore guild } \\ \text { herbivore } & \text { Rodentia } \\ \text { herbivore } & \text { herbivore guild } \\ \text { herbivore } & \text { herbivore guild } \\ \text { herbivore } & \text { herbivore guild } \\ \text { NA } & \text { Rodentia } \\ \text { herbivore } & \text { Rodentia } \\ \text { carnivore } & \text { carnivore guild } \\ \text { insectivore } & \text { NA } \\ \text { herbivore } & \text { Rodentia } \\ \text { herbivore } & \text { Rodentia } \\ \text { herbivore } & \text { Rodentia } \\ \text { herbivore } & \text { Rodentia } \\ \text { herbivore } & \text { herbivore guild } \\ \text { herbivore } & \text { herbivore guild } \\ \text { herbivore } & \text { herbivore guild } \\ \text { herbivore } & \text { herbivore guild } \\ \text { herbivore } & \text { Rerbivore guild } \\ \text { herbivore } & \text { herbivore } \\ \text { NA } & \end{array}$




\begin{tabular}{|c|c|c|c|c|c|c|c|c|c|}
\hline Rodentia & Florentiamyidae & Hitonkala macdonaldtau & 24.7 & 23.85 & 3.2325 ground dwelling & herbivore & species & herbivore & Rodentia \\
\hline Rodentia & Cricetidae & Leidymys cerasus & 23.9 & 23.9 & 3.727 ground dwelling & herbivore & species & herbivore & Rodentia \\
\hline Eulipotyphla & Erinaceidae & Parvericius voorhiesi & 23.9 & 23.9 & 3.361 semifossorial & insectivore, carnivore & species & insectivore & NA \\
\hline Rodentia & Allomyidae & Parallomys americanus & 24.7 & 23.9 & 5.025 ground dwelling & herbivore & species & herbivore & Rodentia \\
\hline Lipotyphla & Geolabididae & Centetodon divaricatus & 24.7 & 23.9 & 3.4195 scansorial & insectivore & species & insectivore & NA \\
\hline Rodentia & Florentiamyidae & Sanctimus stuartae & 28 & 23.9 & 4.6145 ground dwelling & herbivore & species & herbivore & Rodentia \\
\hline Carnivora & Nimravidae & Nimravus sectator & 24 & 24 & 11.486 ground dwelling & carnivore & species & carnivore & carnivore guild \\
\hline Rodentia & Aplodontidae & Niglarodon petersonensis & 24.1 & 24.1 & 4.778 fossorial & herbivore & species & herbivore & Rodentia \\
\hline Rodentia & Mylagaulidae & Trilaccogaulus lemhiensis & 24.1 & 24.1 & 5.402 ground dwelling & herbivore & species & herbivore & Rodentia \\
\hline Rodentia & Geomyidae & Entoptychus fieldsi & 24.1 & 24.1 & 4.2995 fossorial & browser & species & herbivore & Rodentia \\
\hline Perissodactyla & Tapiridae & Nexuotapirus robustus & 24.3 & 24.2 & 12.3925 ground dwelling & browser & species & herbivore & herbivore guild \\
\hline Carnivora & Amphicyonidae & Daphoenus socialis & 24.3 & 24.3 & 10.027 ground dwelling & carnivore, omnivore & species & carnivore & carnivore guild \\
\hline Rodentia & Geomyidae & Entoptychus planifrons & 24.3 & 24.3 & 4.906 fossorial & browser & species & herbivore & Rodentia \\
\hline Rodentia & Florentiamyidae & Fanimus clasoni & 24.4 & 24.35 & 4.465 ground dwelling & herbivore & species & herbivore & Rodentia \\
\hline Rodentia & Mylagaulidae & Crucimys milleri & 24.7 & 24.35 & 3.536 ground dwelling & herbivore & species & herbivore & Rodentia \\
\hline Rodentia & Cricetidae & Geringia gloveri & 24.7 & 24.35 & 3.294 ground dwelling & herbivore & species & herbivore & Rodentia \\
\hline Rodentia & Heteromyidae & Proheteromys gremmelsi & 25.1 & 24.35 & 3.331 ground dwelling & granivore, browser & species & herbivore & Rodentia \\
\hline Lagomorpha & Leporidae & Archaeolagus ennisianus & 25.615 & 24.35 & 6.002 & & & NA & NA \\
\hline Lagomorpha & Leporidae & Palaeolagus hypsodus & 29.25 & 24.35 & 5.619 ground dwelling & grazer, browser & species & herbivore & herbivore guild \\
\hline Rodentia & Allomyidae & Campestrallomys dawsonae & 29.25 & 24.35 & 5.496 fossorial & herbivore & species & herbivore & Rodentia \\
\hline Rodentia & Florentiamyidae & Florentiamys agnewi & 24.4 & 24.4 & 4.4375 ground dwelling & herbivore & species & herbivore & Rodentia \\
\hline Rodentia & Geomyidae & Tenudomys macdonaldi & 24.4 & 24.4 & 4.378 fossorial & browser & species & herbivore & Rodentia \\
\hline \multirow[t]{2}{*}{ Rodentia } & Aplodontidae & Meniscomys hippodus & 25 & 24.4 & 4.246 fossorial & herbivore & species & herbivore & Rodentia \\
\hline & & Paciculus woodi & 25.1 & 24.4 & 3.498 ground dwelling & herbivore & species & herbivore & herbivore guild \\
\hline Lipotyphla & Talpidae & Quadrodens wilsoni & 25.1 & 24.4 & 3.8315 fossorial & insectivore & species & insectivore & NA \\
\hline Carnivora & & Palaeogale dorothiae & 26.25 & 24.4 & 6.099 ground dwelling & carnivore, omnivore & species & carnivore & carnivore guild \\
\hline Carnivora & Canidae & Sunkahetanka geringensis & 26.25 & 24.4 & 9.704 ground dwelling & carnivore, omnivore & species & carnivore & carnivore guild \\
\hline Primates & Omomyidae & Ekgmowechashala philotau & 26.55 & 24.4 & 7.64 arboreal & omnivore & species & omnivore & NA \\
\hline Carnivora & Canidae & Cynarctoides roii & 27.25 & 24.4 & 7.153 ground dwelling & carnivore, omnivore & species & carnivore & carnivore guild \\
\hline Rodentia & Allomyidae & Downsimus chadwicki & 29.25 & 24.4 & 4.0335 ground dwelling & herbivore & species & herbivore & Rodentia \\
\hline Rodentia & Cricetidae & Geringia mcgregori & 29.25 & 24.4 & 3.72 ground dwelling & herbivore & species & herbivore & Rodentia \\
\hline Rodentia & Heteromyidae & Proheteromys fedti & 29.25 & 24.4 & 2.867 & & & NA & Rodentia \\
\hline Carnivora & Nimravidae & Eusmilus cerebralis & 30.75 & 24.4 & 8.0295 & & & NA & carnivore guild \\
\hline Carnivora & Canidae & Archaeocyon leptodus & 30.9 & 24.4 & 8.051322247 ground dwelling & carnivore, omnivore & species & carnivore & carnivore guild \\
\hline Artiodactyla & Merycoidodontidae & Leptauchenia major & 31.25 & 24.4 & 10.22135727 ground dwelling & herbivore & species & herbivore & herbivore guild \\
\hline Lagomorpha & Leporidae & Megalagus primitivus & 31.4 & 24.4 & 6.941 ground dwelling & grazer, browser & species & herbivore & herbivore guild \\
\hline Carnivora & Nimravidae & Nimravus brachyops & 31.8 & 24.4 & 10.31926677 & & & NA & carnivore guild \\
\hline Carnivora & Canidae & Cynodesmus thooides & 33.1 & 24.4 & 9.370086429 ground dwelling & carnivore, omnivore & species & carnivore & carnivore guild \\
\hline Carnivora & Canidae & Mesocyon temnodon & 33.2 & 24.4 & 8.94491037 ground dwelling & carnivore, omnivore & species & carnivore & carnivore guild \\
\hline Artiodactyla & Merycoidodontidae & Desmatochoerus hesperus & 24.45 & 24.45 & 12.014 ground dwelling & herbivore & species & herbivore & herbivore guild \\
\hline Artiodactyla & & Archaeotherium trippensis & 24.5 & 24.5 & 15.048 ground dwelling & omnivore & species & omnivore & NA \\
\hline Rodentia & Cricetidae & Leidymys korthi & 24.7 & 24.7 & 3.774 ground dwelling & herbivore & species & herbivore & Rodentia \\
\hline Carnivora & Canidae & Philotrox condoni & 25.8 & 24.7 & 9.636305386 ground dwelling & carnivore, omnivore & species & carnivore & carnivore guild \\
\hline Eulipotyphla & Heterosoricidae & Domnina dakotensis & 26.55 & 24.7 & 3.4945 scansorial & insectivore & species & insectivore & NA \\
\hline Carnivora & Canidae & Phlaocyon taylori & 24.75 & 24.75 & 7.57 ground dwelling & carnivore, omnivore & species & carnivore & carnivore guild \\
\hline Lagomorpha & Leporidae & Megalagus abaconis & 24.75 & 24.75 & 7.02 ground dwelling & grazer, browser & species & herbivore & herbivore guild \\
\hline Rodentia & Florentiamyidae & Florentiamys loomisi & 25.2 & 24.8 & 5.025 ground dwelling & herbivore & species & herbivore & Rodentia \\
\hline Artiodactyla & Camelidae & Pseudolabis dakotensis & 25.2 & 24.8 & 10.87444109 ground dwelling & browser, browser & species & herbivore & herbivore guild \\
\hline Artiodactyla & Merycoidodontidae & Mesoreodon chelonyx & 29.75 & 24.8 & 11.59544804 ground dwelling & herbivore & species & herbivore & herbivore guild \\
\hline Eulipotyphla & Heterosoricidae & Domnina greeni & 24.9 & 24.9 & 3.521 scansorial & insectivore & species & insectivore & NA \\
\hline Carnivora & Canidae & Otarocyon cooki & 25.2 & 24.9 & 7.2 ground dwelling & carnivore, omnivore & species & carnivore & carnivore guild \\
\hline Rodentia & Florentiamyidae & Sanctimus stouti & 24.95 & 24.95 & 4.79 ground dwelling & herbivore & species & herbivore & Rodentia \\
\hline
\end{tabular}




\begin{tabular}{|c|c|c|c|c|c|c|c|}
\hline Artiodactyla & Camelidae & Miotylopus taylori & 24.95 & 24.95 & 11.163 ground dwelling & browser, browser & species \\
\hline Carnivora & Amphicyonidae & Paradaphoenus tooheyi & 25 & 25 & 9.51870599 ground dwelling & carnivore & species \\
\hline Carnivora & Nimravidae & Pogonodon eileenae & 25 & 25 & 10.97 ground dwelling & carnivore & species \\
\hline Rodentia & Aplodontidae & Niglarodon koerneri & 28.25 & 25 & 4.227 fossorial & herbivore & species \\
\hline Rodentia & Castoridae & Agnotocastor coloradensis & 33.3 & 25.15 & 7.052 amphibious & browser & species \\
\hline Carnivora & Canidae & Ectopocynus intermedius & 25.2 & 25.2 & 9.818053325 ground dwelling & carnivore, omnivore & species \\
\hline Rodentia & Florentiamyidae & Florentiamys kinseyi & 25.2 & 25.2 & 5.061 ground dwelling & herbivore & species \\
\hline Carnivora & Canidae & Ectopocynus antiquus & 27.75 & 25.2 & 9.165456585 ground dwelling & carnivore, omnivore & species \\
\hline Artiodactyla & Merycoidodontidae & Paroreodon parvus & 25.615 & 25.35 & 10.2 ground dwelling & herbivore & species \\
\hline Artiodactyla & Hypertragulidae & Hypertragulus calcaratus & 35.35 & 25.35 & 7.633414786 ground dwelling & frugivore & species \\
\hline Artiodactyla & Merycoidodontidae & Merycoides pariogonus & 25.45 & 25.45 & 10.44915782 ground dwelling & herbivore & species \\
\hline Artiodactyla & Merycoidodontidae & Mesoreodon floridensis & 25.55 & 25.55 & 11.53991661 ground dwelling & herbivore & species \\
\hline Carnivora & Amphicyonidae & Paradaphoenus cuspigerus & 25.615 & 25.55 & 8.29702482 ground dwelling & carnivore & species \\
\hline Carnivora & Canidae & Mesocyon brachyops & 30.75 & 25.55 & 9.1955 ground dwelling & carnivore, omnivore & species \\
\hline Insectivora & Micropternodontidae & Micropternodus morgani & 25.615 & 25.615 & 4.28 scansorial & insectivore & species \\
\hline Rodentia & Sciuridae & Miosciurus ballovianus & 25.615 & 25.615 & 3.6025 & & \\
\hline Artiodactyla & Hypertragulidae & Nanotragulus planiceps & 25.8 & 25.615 & 7.489970899 & & \\
\hline Carnivora & Canidae & Rhizocyon oregonensis & 28.5 & 25.615 & 7.946362789 ground dwelling & carnivore, omnivore & species \\
\hline Carnivora & Canidae & Phlaocyon latidens & 28.75 & 25.615 & 7.857933492 ground dwelling & carnivore, omnivore & species \\
\hline Artiodactyla & Merycoidodontidae & Eporeodon occidentalis & 28.75 & 25.615 & 11.11766042 ground dwelling & herbivore & species \\
\hline Carnivora & Canidae & Mesocyon coryphaeus & 28.95 & 25.615 & 9.437253684 ground dwelling & carnivore, omnivore & species \\
\hline Carnivora & Canidae & Osbornodon sesnoni & 30.45 & 25.615 & 9.175276993 ground dwelling & carnivore, omnivore & species \\
\hline Artiodactyla & Agriochoeridae & Agriochoerus guyotianus & 32.7 & 25.615 & 10.11100373 scansorial & herbivore & species \\
\hline Artiodactyla & Merycoidodontidae & Merycoidodon bullatus & 33.55 & 25.615 & 11.0486581 ground dwelling & herbivore & species \\
\hline Lagomorpha & Leporidae & Archaeolagus emeraldensis & 25.7 & 25.7 & 6.73 ground dwelling & grazer, browser & species \\
\hline Rodentia & Allomyidae & Allomys cristabrevis & 25.7 & 25.7 & 5.474 ground dwelling & herbivore & species \\
\hline Perissodactyla & Hyracodontidae & Aprotodon lanzhouensis & 25.715 & 25.715 & 13.90991655 ground dwelling & browser & species \\
\hline \multirow[t]{2}{*}{ Perissodactyla } & Indricotheriidae & Indricotherium transouralicum & 25.715 & 25.715 & 16.52806086 ground dwelling & browser & species \\
\hline & & Jimomys lulli & 25.8 & 25.8 & 3.321 ground dwelling & herbivore & species \\
\hline Rodentia & Sciuridae & Protospermophilus vortmani & 25.8 & 25.8 & 5.4385 & & \\
\hline Carnivora & Canidae & Enhydrocyon stenocephalus & 28.5 & 25.8 & 10.06475328 ground dwelling & carnivore, omnivore & species \\
\hline Perissodactyla & Equidae & Miohippus intermedius & 32.55 & 25.8 & 11.07596635 ground dwelling & browser & species \\
\hline Insectivora & Proscalopidae & Proscalops tertius & 33.1 & 26.05 & 4.572 scansorial & insectivore & species \\
\hline Carnivora & Canidae & Cynodesmus martini & 26.25 & 26.25 & 10.0245 ground dwelling & carnivore, omnivore & species \\
\hline Artiodactyla & Merycoidodontidae & Sespia californica & 28.95 & 27.05 & 7.871370042 ground dwelling & herbivore & species \\
\hline Artiodactyla & Protoceratidae & Protoceras skinneri & 30.25 & 27.25 & 10.67613883 ground dwelling & browser & species \\
\hline Carnivora & Amphicyonidae & Daphoenus ruber & 27.3 & 27.3 & 9.185 ground dwelling & carnivore, omnivore & species \\
\hline Rodentia & Sciuridae & Protosciurus tecuyensis & 27.3 & 27.3 & 6.17 scansorial & granivore, frugivore & species \\
\hline Carnivora & Canidae & Archaeocyon pavidus & 31.15 & 27.75 & 7.515026914 ground dwelling & carnivore, omnivore & species \\
\hline Rodentia & Eomyidae & Zetamys nebraskensis & 28 & 28 & 4.0995 ground dwelling & herbivore & species \\
\hline Rodentia & Cricetidae & Eumys brachyodus & 33.6 & 28 & 4.839 ground dwelling & herbivore & species \\
\hline Artiodactyla & Leptomerycidae & Pronodens silberlingi & 28.25 & 28.25 & 9.701436468 ground dwelling & frugivore & species \\
\hline Carnivora & Amphicyonidae & Temnocyon altigenis & 30.75 & 28.5 & 10.4225 ground dwelling & carnivore & species \\
\hline Artiodactyla & Merycoidodontidae & Eucrotaphus trigonocephalus & 28.75 & 28.75 & 10.902 & & \\
\hline Artiodactyla & Tayassuidae & Thinohyus lentus & 28.75 & 28.75 & 10.83997299 ground dwelling & herbivore, omnivore & species \\
\hline \multirow[t]{2}{*}{ Didelphimorphia } & Herpetotheriidae & Herpetotherium merriami & 28.75 & 28.75 & 3.211 ground dwelling & omnivore & species \\
\hline & & Perchoerus probus & 34.15 & 28.75 & 10.19580883 ground dwelling & herbivore, omnivore & species \\
\hline Rodentia & Eomyidae & Leptodontomys douglassi & 30.9 & 29.25 & 1.9925 ground dwelling & herbivore & species \\
\hline \multirow[t]{3}{*}{ Rodentia } & Eomyidae & Metadjidaumo hendryi & 32.9 & 29.25 & 2.34 ground dwelling & herbivore & species \\
\hline & & Ankylodon annectens & 33.3 & 29.25 & 3.4125 ground dwelling & insectivore & species \\
\hline & & Heliscomys vetus & 37.65 & 29.25 & 1.839 ground dwelling & herbivore & species \\
\hline Didelphimorphia & Herpetotheriidae & Herpetotherium fugax & 37.85 & 29.25 & 2.963 & & \\
\hline
\end{tabular}

\begin{tabular}{|c|c|}
\hline herbivore & herbivore guild \\
\hline carnivore & carnivore guild \\
\hline carnivore & carnivore guild \\
\hline herbivore & Rodentia \\
\hline herbivore & Rodentia \\
\hline carnivore & carnivore guild \\
\hline herbivore & Rodentia \\
\hline carnivore & carnivore guild \\
\hline herbivore & herbivore guild \\
\hline herbivore & herbivore guild \\
\hline herbivore & herbivore guild \\
\hline herbivore & herbivore guild \\
\hline carnivore & carnivore guild \\
\hline carnivore & carnivore guild \\
\hline insectivore & NA \\
\hline NA & Rodentia \\
\hline NA & NA \\
\hline carnivore & carnivore guild \\
\hline carnivore & carnivore guild \\
\hline herbivore & herbivore guild \\
\hline carnivore & carnivore guild \\
\hline carnivore & carnivore guild \\
\hline herbivore & herbivore guild \\
\hline herbivore & herbivore guild \\
\hline herbivore & herbivore guild \\
\hline herbivore & Rodentia \\
\hline herbivore & herbivore guild \\
\hline herbivore & herbivore guild \\
\hline herbivore & herbivore guild \\
\hline NA & Rodentia \\
\hline carnivore & carnivore guild \\
\hline herbivore & herbivore guild \\
\hline insectivore & NA \\
\hline carnivore & carnivore guild \\
\hline herbivore & herbivore guild \\
\hline herbivore & herbivore guild \\
\hline carnivore & carnivore guild \\
\hline herbivore & Rodentia \\
\hline carnivore & carnivore guild \\
\hline herbivore & Rodentia \\
\hline herbivore & Rodentia \\
\hline herbivore & herbivore guild \\
\hline carnivore & carnivore guild \\
\hline NA & NA \\
\hline omnivore & NA \\
\hline omnivore & NA \\
\hline omnivore & NA \\
\hline herbivore & Rodentia \\
\hline herbivore & Rodentia \\
\hline insectivore & NA \\
\hline herbivore & herbivore guild \\
\hline NA & NA \\
\hline
\end{tabular}




\begin{tabular}{|c|c|c|c|c|c|c|c|c|c|}
\hline Rodentia & Cricetidae & Eumys elegans & 35.1 & 29.45 & 5.093 ground dwelling & herbivore & species & herbivore & Rodentia \\
\hline Rodentia & Allomyidae & Campestrallomys siouxensis & 29.95 & 29.75 & 5.071 fossorial & herbivore & species & herbivore & Rodentia \\
\hline Rodentia & Geomyidae & Tenudomys bodei & 29.9 & 29.9 & 3.7715 fossorial & browser & species & herbivore & Rodentia \\
\hline Artiodactyla & Leptochoeridae & Leptochoerus spectabilis & 31.65 & 29.95 & 8.376163421 ground dwelling & grazer, browser & species & herbivore & herbivore guild \\
\hline Perissodactyla & Equidae & Miohippus obliquidens & 34.85 & 30.25 & 10.56157388 ground dwelling & browser & species & herbivore & herbivore guild \\
\hline Rodentia & Cylindrodontidae & Sespemys thurstoni & 30.75 & 30.75 & 5.671 ground dwelling & herbivore & species & herbivore & Rodentia \\
\hline Artiodactyla & Anthracotheriidae & Elomeryx armatus & 33.65 & 30.75 & 11.78834531 ground dwelling & browser & species & herbivore & herbivore guild \\
\hline Perissodactyla & Rhinocerotidae & Subhyracodon occidentalis & 33.85 & 30.75 & 13.41851909 ground dwelling & browser & species & herbivore & herbivore guild \\
\hline Artiodactyla & Hypertragulidae & Leptomeryx evansi & 37.4 & 30.9 & 8.244878293 ground dwelling & frugivore & species & herbivore & herbivore guild \\
\hline Perissodactyla & Rhinocerotidae & Diceratherium tridactylum & 32.55 & 31.05 & 13.88064834 ground dwelling & browser & species & herbivore & herbivore guild \\
\hline Carnivora & Canidae & Hesperocyon gregarius & 42.3 & 31.05 & 8.075 ground dwelling & carnivore, omnivore & species & carnivore & carnivore guild \\
\hline Carnivora & Nimravidae & Eusmilus bidentatus & 31.15 & 31.15 & 10.01624515 ground dwelling & carnivore & species & carnivore & carnivore guild \\
\hline Proboscidea & & Barytherium grave & 31.15 & 31.15 & 15.06827353 ground dwelling & grazer, browser & species & herbivore & herbivore guild \\
\hline Proboscidea & & Palaeomastodon beadnelli & 31.15 & 31.15 & 14.91412285 ground dwelling & grazer, browser & species & herbivore & herbivore guild \\
\hline Primates & Propliopithecidae & Aegyptopithecus zeuxis & 31.15 & 31.15 & 8.974618038 arboreal & omnivore & species & omnivore & NA \\
\hline Carnivora & Canidae & Oxetocyon cuspidatus & 31.25 & 31.15 & 7.636517708 ground dwelling & carnivore, omnivore & species & carnivore & carnivore guild \\
\hline Eulipotyphla & Erinaceidae & Proterix loomisi & 32.15 & 31.25 & 6.26 semifossorial & insectivore, carnivore & species & insectivore & NA \\
\hline Artiodactyla & Leptochoeridae & Leptochoerus supremus & 32.75 & 31.25 & 9.542 ground dwelling & grazer, browser & species & herbivore & herbivore guild \\
\hline Perissodactyla & Tapiridae & Protapirus simplex & 33.8 & 31.25 & 11.35115457 ground dwelling & browser & species & herbivore & herbivore guild \\
\hline Rodentia & Allomyidae & Oropyctis pediasius & 31.4 & 31.4 & 5.075 ground dwelling & herbivore & species & herbivore & Rodentia \\
\hline Rodentia & Aplodontidae & Prosciurus magnus & 32.8 & 31.4 & 4.685 fossorial & herbivore & species & herbivore & Rodentia \\
\hline Lagomorpha & Leporidae & Palaeolagus burkei & 34.45 & 31.4 & 5.73 ground dwelling & grazer, browser & species & herbivore & herbivore guild \\
\hline Rodentia & Aplodontidae & Prosciurus relictus & 37.65 & 31.4 & 4.119 & & & NA & Rodentia \\
\hline Artiodactyla & Camelidae & Paralabis cedrensis & 31.55 & 31.55 & 10.45635524 & & & NA & NA \\
\hline Perissodactyla & Hyracodontidae & Hyracodon leidyanus & 33.1 & 31.55 & 12.37623621 ground dwelling & browser & species & herbivore & herbivore guild \\
\hline Perissodactyla & Hyracodontidae & Hyracodon nebraskensis & 37.5 & 31.55 & 11.99489779 ground dwelling & browser & species & herbivore & herbivore guild \\
\hline Carnivora & Canidae & Osbornodon renjiei & 33.55 & 31.95 & 8.80626773 ground dwelling & carnivore, omnivore & species & carnivore & carnivore guild \\
\hline Rodentia & Allomyidae & Allomys storeri & 32.15 & 32.15 & 4.526 ground dwelling & herbivore & species & herbivore & Rodentia \\
\hline Rodentia & Aplodontidae & Leptoromys wilsoni & 32.35 & 32.15 & 4.695 fossorial & herbivore & species & herbivore & Rodentia \\
\hline \multirow[t]{2}{*}{ Eulipotyphla } & Heterosoricidae & Domnina gradata & 42.3 & 32.15 & 3.6005 scansorial & insectivore & species & insectivore & NA \\
\hline & & Heliscomys senex & 32.35 & 32.35 & 1.459 ground dwelling & herbivore & species & herbivore & herbivore guild \\
\hline Rodentia & Allomyidae & Haplomys galbreathi & 32.35 & 32.35 & 3.663 ground dwelling & herbivore & species & herbivore & Rodentia \\
\hline Rodentia & Allomyidae & Pelycomys placidus & 33.3 & 32.35 & 5.838 ground dwelling & herbivore & species & herbivore & Rodentia \\
\hline Lipotyphla & Geolabididae & Centetodon marginalis & 33.3 & 32.35 & 2.7 scansorial & insectivore & species & insectivore & NA \\
\hline Lagomorpha & Leporidae & Megalagus turgidus & 35.25 & 32.35 & 7.022 ground dwelling & grazer, browser & species & herbivore & herbivore guild \\
\hline Artiodactyla & Agriochoeridae & Agriochoerus gaudryi & 32.55 & 32.55 & 10.67613883 scansorial & herbivore & species & herbivore & herbivore guild \\
\hline Perissodactyla & Rhinocerotidae & Amphicaenopus platycephalus & 32.55 & 32.55 & 14.69 ground dwelling & browser & species & herbivore & herbivore guild \\
\hline Perissodactyla & Equidae & Miohippus assiniboiensis & 32.55 & 32.55 & 10.905 ground dwelling & browser & species & herbivore & herbivore guild \\
\hline Perissodactyla & Equidae & Miohippus gidleyi & 33.2 & 32.55 & 11.291 ground dwelling & browser & species & herbivore & herbivore guild \\
\hline Artiodactyla & Protoceratidae & Protoceras celer & 33.65 & 32.55 & 10.69669399 ground dwelling & browser & species & herbivore & herbivore guild \\
\hline Perissodactyla & Equidae & Mesohippus bairdi & 36.75 & 32.7 & 10.24707292 ground dwelling & browser & species & herbivore & herbivore guild \\
\hline Artiodactyla & Merycoidodontidae & Miniochoerus affinis & 35.1 & 32.75 & 10.367 ground dwelling & herbivore & species & herbivore & herbivore guild \\
\hline Artiodactyla & Merycoidodontidae & Merycoidodon culbertsoni & 37.6 & 32.75 & 10.61452527 ground dwelling & herbivore & genus & herbivore & herbivore guild \\
\hline Artiodactyla & & Archaeotherium lemleyi & 32.8 & 32.8 & 13.959 ground dwelling & omnivore & species & omnivore & NA \\
\hline Rodentia & Allomyidae & Campestrallomys annectens & 32.85 & 32.8 & 4.362 fossorial & herbivore & species & herbivore & Rodentia \\
\hline Rodentia & & Diplolophus insolens & 33.3 & 32.8 & 5.233 ground dwelling & herbivore & species & herbivore & Rodentia \\
\hline Artiodactyla & Leptochoeridae & Leptochoerus elegans & 33.9 & 32.8 & 8.254 ground dwelling & grazer, browser & species & herbivore & herbivore guild \\
\hline Rodentia & Sciuridae & Cedromus wardi & 36.05 & 32.8 & 5.502 scansorial & granivore, frugivore & species & herbivore & Rodentia \\
\hline Rodentia & Sciuridae & Protosciurus mengi & 36.05 & 32.8 & 5.793 scansorial & granivore, frugivore & species & herbivore & Rodentia \\
\hline Rodentia & Eomyidae & Paradjidaumo trilophus & 37.85 & 32.8 & 3.502 ground dwelling & herbivore & species & herbivore & Rodentia \\
\hline Rodentia & Aplodontidae & Prosciurus parvus & 33.6 & 32.85 & 3.809 fossorial & herbivore & species & herbivore & Rodentia \\
\hline Rodentia & Eomyidae & Adjidaumo minutus & 37.5 & 32.85 & 2.851 ground dwelling & herbivore & species & herbivore & Rodentia \\
\hline
\end{tabular}




\begin{tabular}{|c|c|c|c|c|c|c|c|c|c|}
\hline Perissodactyla & Tapiridae & Colodon cingulatus & 38.9 & 32.85 & 12.061 ground dwelling & browser & species & herbivore & herbivore guild \\
\hline Rodentia & Eomyidae & Paradjidaumo spokanensis & 32.9 & 32.9 & 3.94 ground dwelling & herbivore & species & herbivore & Rodentia \\
\hline Rodentia & Cricetidae & Scottimus ambiguus & 33.3 & 32.9 & 3.817 ground dwelling & herbivore & species & herbivore & Rodentia \\
\hline Rodentia & Cricetidae & Wilsoneumys planidens & 33.3 & 32.9 & 4.311 ground dwelling & herbivore & species & herbivore & Rodentia \\
\hline \multirow[t]{3}{*}{ Didelphimorphia } & Herpetotheriidae & Copedelphys stevensoni & 33.6 & 32.9 & 1.417 ground dwelling & omnivore & species & omnivore & NA \\
\hline & & Nanodelphys hunti & 42.45 & 32.9 & 1.707 scansorial & omnivore & species & omnivore & NA \\
\hline & & Epeiromys spanius & 32.95 & 32.95 & 4.62 ground dwelling & herbivore & species & herbivore & herbivore guild \\
\hline Rodentia & Eomyidae & Paradjidaumo validus & 32.95 & 32.95 & 4.105 ground dwelling & herbivore & species & herbivore & Rodentia \\
\hline Artiodactyla & & Stibarus obtusilobus & 36.25 & 32.95 & 7.332 ground dwelling & grazer, browser & species & herbivore & herbivore guild \\
\hline Rodentia & Cricetidae & Scottimus viduus & 37.65 & 32.95 & 3.952 ground dwelling & herbivore & species & herbivore & Rodentia \\
\hline Rodentia & Cricetidae & Scottimus exiguus & 33.1 & 33 & 4.234 ground dwelling & herbivore & species & herbivore & Rodentia \\
\hline Creodonta & Hyaenodontidae & Hyaenodon horridus & 36.9 & 33 & 10.48551769 ground dwelling & carnivore & species & carnivore & carnivore guild \\
\hline Insectivora & Apatemyidae & Sinclairella dakotensis & 37.5 & 33 & 5.415 arboreal & herbivore & species & herbivore & herbivore guild \\
\hline \multirow[t]{2}{*}{ Carnivora } & Amphicyonidae & Daphoenus hartshornianus & 33.4 & 33.2 & 9.269670186 ground dwelling & carnivore, omnivore & species & carnivore & carnivore guild \\
\hline & & Apletotomeus crassus & 33.4 & 33.2 & 2.153 ground dwelling & herbivore & species & herbivore & herbivore guild \\
\hline Carnivora & Nimravidae & Hoplophoneus primaevus & 34.55 & 33.2 & 10.15301945 ground dwelling & carnivore & species & carnivore & carnivore guild \\
\hline \multirow{2}{*}{ Carnivora } & Nimravidae & Dinictis felina & 35.5 & 33.2 & 10.03927101 ground dwelling & carnivore & species & carnivore & carnivore guild \\
\hline & & Poebrotherium wilsoni & 35.85 & 33.2 & 10.29967075 ground dwelling & browser, browser & species & herbivore & herbivore guild \\
\hline Artiodactyla & & Stibarus quadricuspis & 36.25 & 33.2 & 7.435 ground dwelling & grazer, browser & species & herbivore & herbivore guild \\
\hline Artiodactyla & Merycoidodontidae & Miniochoerus gracilis & 36.25 & 33.2 & 9.725148428 ground dwelling & herbivore & species & herbivore & herbivore guild \\
\hline Artiodactyla & & Archaeotherium mortoni & 36.6 & 33.2 & 11.99887837 ground dwelling & omnivore & species & omnivore & NA \\
\hline Perissodactyla & Tapiridae & Colodon occidentalis & 36.9 & 33.2 & 10.98 ground dwelling & browser & species & herbivore & herbivore guild \\
\hline Creodonta & Hyaenodontidae & Hyaenodon crucians & 37.2 & 33.2 & 9.134146382 ground dwelling & carnivore & species & carnivore & carnivore guild \\
\hline Carnivora & Amphicyonidae & Pseudocyonopsis antiquus & 33.289 & 33.289 & 10.29255537 ground dwelling & carnivore & genus & carnivore & carnivore guild \\
\hline Carnivora & Canidae & Hesperocyon coloradensis & 33.3 & 33.3 & 8.509453986 ground dwelling & carnivore, omnivore & species & carnivore & carnivore guild \\
\hline Artiodactyla & Hypertragulidae & Leptomeryx elissae & 33.3 & 33.3 & 7.075843991 ground dwelling & frugivore & species & herbivore & herbivore guild \\
\hline Carnivora & Amphicyonidae & Drassonax harpagops & 33.3 & 33.3 & 7.646 ground dwelling & herbivore, carnivore & species & omnivore & carnivore guild \\
\hline Eulipotyphla & Soricidae & Pseudotrimylus compressus & 33.3 & 33.3 & 3.362 ground dwelling & omnivore & species & omnivore & NA \\
\hline Carnivora & Amphicyonidae & Daphoenus vetus & 33.5 & 33.3 & 10.26654332 ground dwelling & carnivore, omnivore & species & carnivore & carnivore guild \\
\hline Artiodactyla & Camelidae & Paratylopus labiatus & 34.05 & 33.3 & 10.57806962 ground dwelling & browser, browser & species & herbivore & herbivore guild \\
\hline Creodonta & Hyaenodontidae & Hyaenodon mustelinus & 35.65 & 33.3 & 7.999 ground dwelling & carnivore & species & carnivore & carnivore guild \\
\hline Artiodactyla & Leptomerycidae & Hendryomeryx esulcatus & 36.9 & 33.3 & 8.507704869 & & & NA & NA \\
\hline Rodentia & Allomyidae & Pelycomys brulanus & 33.4 & 33.4 & 5.214 ground dwelling & herbivore & species & herbivore & Rodentia \\
\hline Rodentia & Castoridae & Agnotocastor readingi & 33.4 & 33.4 & 6.372 amphibious & browser & species & herbivore & Rodentia \\
\hline Lagomorpha & Leporidae & Palaeolagus hemirhizis & 34.7 & 33.4 & 4.845 ground dwelling & grazer, browser & species & herbivore & herbivore guild \\
\hline Rodentia & Eutypomyidae & Eutypomys thomsoni & 35.2 & 33.4 & 6.362 ground dwelling & herbivore & species & herbivore & Rodentia \\
\hline Carnivora & Amphicyonidae & Paradaphoenus minimus & 36.05 & 33.4 & 10.86820164 ground dwelling & carnivore & species & carnivore & carnivore guild \\
\hline \multirow[t]{2}{*}{ Rodentia } & Sciuridae & Oligospermophilus douglassi & 36.35 & 33.4 & 4.679 scansorial & granivore, frugivore & species & herbivore & Rodentia \\
\hline & Ischyromyidae & Ischyromys veterior & 37.75 & 33.4 & 6.206 ground dwelling & herbivore & species & herbivore & herbivore guild \\
\hline Artiodactyla & Agriochoeridae & Agriochoerus antiquus & 38.35 & 33.4 & 10.82352844 scansorial & herbivore & species & herbivore & herbivore guild \\
\hline Rodentia & Cricetidae & Scottimus longiquus & 33.55 & 33.55 & 4.7125 ground dwelling & herbivore & species & herbivore & Rodentia \\
\hline Rodentia & Eomyidae & Adjidaumo minimus & 37.75 & 33.6 & 1.895 ground dwelling & herbivore & species & herbivore & Rodentia \\
\hline Perissodactyla & Equidae & Miohippus grandis & 36.9 & 33.65 & 10.708 ground dwelling & browser & species & herbivore & herbivore guild \\
\hline \multirow[t]{2}{*}{ Lagomorpha } & Leporidae & Litolagus molidens & 33.75 & 33.75 & 5.084 ground dwelling & grazer, browser & species & herbivore & herbivore guild \\
\hline & & Poebrotherium eximium & 36.25 & 33.75 & 10.32084231 ground dwelling & browser, browser & species & herbivore & herbivore guild \\
\hline Artiodactyla & Hypertragulidae & Leptomeryx speciosus & 37.6 & 33.75 & 8.72745222 ground dwelling & frugivore & species & herbivore & herbivore guild \\
\hline Carnivora & Nimravidae & Hoplophoneus occidentalis & 33.8 & 33.8 & 11.11809552 ground dwelling & carnivore & species & carnivore & carnivore guild \\
\hline Artiodactyla & Anthracotheriidae & Aepinacodon americanus & 35.8 & 33.8 & 12.14748307 & & & NA & NA \\
\hline Perissodactyla & Rhinocerotidae & Subhyracodon mitis & 36.1 & 33.8 & 12.723 ground dwelling & browser & species & herbivore & herbivore guild \\
\hline Lagomorpha & Leporidae & Palaeolagus temnodon & 37.85 & 33.85 & 5.121 ground dwelling & grazer, browser & species & herbivore & herbivore guild \\
\hline Rodentia & Eomyidae & Adjidaumo intermedius & 35.9 & 33.9 & 2.357 ground dwelling & herbivore & species & herbivore & Rodentia \\
\hline Rodentia & Eomyidae & Adjidaumo maximus & 34.7 & 34.05 & 2.96 ground dwelling & herbivore & species & herbivore & Rodentia \\
\hline
\end{tabular}




\begin{tabular}{|c|c|c|}
\hline Perissodactyla & Brontotheriidae & Brontops brachycephalus \\
\hline Didelphimorphia & Herpetotheriidae & Copedelphys titanelix \\
\hline Didelphimorphia & Herpetotheriidae & Herpetotherium valens \\
\hline Lipotyphla & Geolabididae & Centetodon kuenzii \\
\hline Perissodactyla & Equidae & Mesohippus exoletus \\
\hline Perissodactyla & Amynodontidae & Metamynodon planifrons \\
\hline Rodentia & Cylindrodontidae & Pseudocylindrodon neglectus \\
\hline Rodentia & Eomyidae & Namatomys lloydi \\
\hline Lagomorpha & Leporidae & Megalagus brachyodon \\
\hline Perissodactyla & Rhinocerotidae & Trigonias osborni \\
\hline Carnivora & Amphicyonidae & Brachyrhynchocyon dodgei \\
\hline Insectivora & Micropternodontidae & Micropternodus borealis \\
\hline Artiodactyla & Anthracotheriidae & Bothriodon rostratus \\
\hline Artiodactyla & Protoceratidae & Pseudoprotoceras longinaris \\
\hline Perissodactyla & Hyracodontidae & Hyracodon priscidens \\
\hline Creodonta & Hyaenodontidae & Hyaenodon montanus \\
\hline Artiodactyla & Protoceratidae & Pseudoprotoceras minor \\
\hline Artiodactyla & & Krabitherium waileki \\
\hline Rodentia & Eomyidae & Aulolithomys vexilliames \\
\hline Rodentia & Castoridae & Agnotocastor galushai \\
\hline Lagomorpha & Leporidae & Chadrolagus emryi \\
\hline \multirow{2}{*}{ Rodentia } & & Nonomys simplicidens \\
\hline & Oligoryctidae & Oligoryctes cameronensis \\
\hline \multirow[t]{2}{*}{ Artiodactyla } & Hypertragulidae & Leptomeryx mammifer \\
\hline & & Heliscomys ostranderi \\
\hline Lipotyphla & Geolabididae & Centetodon chadronensis \\
\hline Artiodactyla & Oromerycidae & Eotylopus reedi \\
\hline Artiodactyla & Merycoidodontidae & Bathygenys alpha \\
\hline Perissodactyla & Rhinocerotidae & Penetrigonias dakotensis \\
\hline Rodentia & Eomyidae & Aulolithomys bounites \\
\hline Rodentia & Eomyidae & Zemiodontomys burkei \\
\hline Rodentia & Pipestoneomyidae & Pipestoneomys bisulcatus \\
\hline Carnivora & Amphicyonidae & Brachyrhynchocyon montanus \\
\hline Rodentia & Cylindrodontidae & Cylindrodon fontis \\
\hline Eulipotyphla & Heterosoricidae & Domnina thompsoni \\
\hline Artiodactyla & & Stibarus montanus \\
\hline Perissodactyla & Tapiridae & Colodon kayi \\
\hline Artiodactyla & Agriochoeridae & Agriochoerus maximus \\
\hline Rodentia & Eutypomyidae & Eutypomys parvus \\
\hline Carnivora & Ursidae & Parictis parvus \\
\hline Perissodactyla & Equidae & Mesohippus westoni \\
\hline Perissodactyla & Brontotheriidae & Brontops dispar \\
\hline Rodentia & Allomyidae & Pelycomys rugosus \\
\hline Eulipotyphla & Apternodontidae & Apternodus iliffensis \\
\hline Rodentia & Eomyidae & Centimanomys major \\
\hline Rodentia & Cylindrodontidae & Cylindrodon nebraskensis \\
\hline Perissodactyla & & Toxotherium hunteri \\
\hline Artiodactyla & Entelodontidae & Megachoerus latidens \\
\hline Artiodactyla & Protoceratidae & Heteromeryx dispar \\
\hline Perissodactyla & Rhinocerotidae & Trigonias yoderensis \\
\hline Artiodactyla & & Brachyhyops wyomingensis \\
\hline Carnivora & & Prohesperocyon wilsoni \\
\hline
\end{tabular}

\begin{tabular}{|c|c|c|c|c|}
\hline 38.6 & 34.15 & 15.17173318 ground dwelling & browser & species \\
\hline 37 & 34.3 & 1.778 ground dwelling & omnivore & species \\
\hline 37.85 & 34.3 & 3.379 ground dwelling & omnivore & species \\
\hline 42.55 & 34.3 & 2.781 scansorial & insectivore & species \\
\hline 35.5 & 34.55 & 10.312 ground dwelling & browser & species \\
\hline 36.5 & 34.55 & 13.98838807 amphibious & browser & species \\
\hline 37.5 & 34.7 & 4.483 ground dwelling & herbivore & species \\
\hline 37.75 & 34.7 & 3.23 ground dwelling & herbivore & species \\
\hline 37.85 & 34.85 & 5.624 ground dwelling & grazer, browser & species \\
\hline 38.15 & 34.85 & 12.82174003 ground dwelling & browser & species \\
\hline 37.1 & 34.95 & 10.45088518 ground dwelling & carnivore & species \\
\hline 37.5 & 34.95 & 3.728 scansorial & insectivore & species \\
\hline 35.1 & 35.1 & 11.665 ground dwelling & browser & species \\
\hline 37.5 & 35.35 & 9.530282583 ground dwelling & browser & species \\
\hline 37.75 & 35.5 & 11.77079498 ground dwelling & browser & species \\
\hline 38.35 & 35.5 & 9.29 ground dwelling & carnivore & species \\
\hline 38.35 & 35.5 & 8.888996575 ground dwelling & browser & species \\
\hline 35.55 & 35.55 & 10.04157359 ground dwelling & grazer, browser & species \\
\hline 35.65 & 35.65 & 2.96 ground dwelling & herbivore & species \\
\hline 35.65 & 35.65 & 6.267 amphibious & browser & species \\
\hline 36 & 35.65 & 4.15 ground dwelling & grazer, browser & species \\
\hline 36.55 & 35.65 & 2.777 ground dwelling & herbivore & species \\
\hline 37.5 & 35.65 & 1.498 scansorial & insectivore & species \\
\hline 37.6 & 35.65 & 9.420731004 ground dwelling & frugivore & species \\
\hline 37.75 & 35.65 & 1.397 ground dwelling & herbivore & species \\
\hline 37.85 & 35.65 & 2.609 scansorial & insectivore & species \\
\hline 38.05 & 35.65 & 10.19390691 ground dwelling & browser & species \\
\hline 38.6 & 35.65 & 7.795245132 ground dwelling & herbivore & species \\
\hline 37.75 & 35.7 & 12.25471771 ground dwelling & browser & species \\
\hline 37.6 & 35.8 & 4.249 ground dwelling & herbivore & species \\
\hline 37.6 & 35.8 & 4.778 ground dwelling & herbivore & species \\
\hline 37.65 & 35.8 & 3.853 ground dwelling & herbivore & species \\
\hline 35.85 & 35.85 & 8.645 ground dwelling & carnivore & species \\
\hline 36 & 35.85 & 4.476 ground dwelling & herbivore & species \\
\hline 37.5 & 35.85 & 2.98 scansorial & insectivore & species \\
\hline 37.85 & 35.85 & 7.507 ground dwelling & grazer, browser & species \\
\hline 43.05 & 35.85 & 10.32820218 ground dwelling & browser & species \\
\hline 39.35 & 35.9 & 10.67613883 scansorial & herbivore & species \\
\hline 36.9 & 36 & 5.6 ground dwelling & herbivore & species \\
\hline 37.75 & 36.1 & 7.178 ground dwelling & herbivore, carnivore & species \\
\hline 37.85 & 36.1 & 11.17252206 ground dwelling & browser & species \\
\hline 38.15 & 36.2 & 15.51432432 ground dwelling & browser & species \\
\hline 36.25 & 36.25 & 5.797 ground dwelling & herbivore & species \\
\hline 37.3 & 36.25 & 4.089 scansorial & insectivore & species \\
\hline 37.5 & 36.25 & 5.473 ground dwelling & herbivore & species \\
\hline 37.5 & 36.25 & 5.106 ground dwelling & herbivore & species \\
\hline 38.35 & 36.25 & 9.726 ground dwelling & browser & species \\
\hline 36.35 & 36.35 & 14.44263996 ground dwelling & omnivore & species \\
\hline 38.45 & 36.35 & 9.807197264 ground dwelling & browser & species \\
\hline 36.7 & 36.4 & 11.83085431 & & \\
\hline 38.75 & 36.5 & 11.485 ground dwelling & omnivore & species \\
\hline 36.55 & 36.55 & 8.123 ground dwelling & carnivore, omnivore & species \\
\hline
\end{tabular}

$\begin{array}{ll}\text { herbivore } & \text { herbivore guild } \\ \text { omnivore } & \text { NA } \\ \text { omnivore } & \text { NA } \\ \text { insectivore } & \text { NA } \\ \text { herbivore } & \text { herbivore guild } \\ \text { herbivore } & \text { herbivore guild } \\ \text { herbivore } & \text { Rodentia } \\ \text { herbivore } & \text { Rodentia } \\ \text { herbivore } & \text { herbivore guild } \\ \text { herbivore } & \text { herbivore guild } \\ \text { carnivore } & \text { carnivore guild } \\ \text { insectivore } & \text { NA } \\ \text { herbivore } & \text { herbivore guild } \\ \text { herbivore } & \text { herbivore guild } \\ \text { herbivore } & \text { herbivore guild } \\ \text { carnivore } & \text { carnivore guild } \\ \text { herbivore } & \text { herbivore guild } \\ \text { herbivore } & \text { herbivore guild } \\ \text { herbivore } & \text { Rodentia } \\ \text { herbivore } & \text { Rodentia } \\ \text { herbivore } & \text { herbivore guild } \\ \text { herbivore } & \text { Rodentia } \\ \text { insectivore } & \text { NA } \\ \text { herbivore } & \text { herbivore guild } \\ \text { herbivore } & \text { herbivore guild } \\ \text { insectivore } & \text { NA } \\ \text { herbivore } & \text { herbivore guild } \\ \text { herbivore } & \text { herbivore guild } \\ \text { herbivore } & \text { herbivore guild } \\ \text { herbivore } & \text { Rodentia } \\ \text { herbivore } & \text { Rodentia } \\ \text { herbivore } & \text { Rodentia } \\ \text { carnivore } & \text { carnivore guild } \\ \text { herbivore } & \text { Rodentia } \\ \text { insectivore } & \text { NA } \\ \text { herbivore } & \text { herbivore guild } \\ \text { herbivore } & \text { herbivore guild } \\ \text { herbivore } & \text { herbivore guild } \\ \text { herbivore } & \text { Rodentia } \\ \text { omnivore } & \text { carnivore guild } \\ \text { herbivore } & \text { herbivore guild } \\ \text { herbivore } & \text { herbivore guild } \\ \text { herbivore } & \text { Rodentia } \\ \text { insectivore } & \text { NA } \\ \text { herbivore } & \text { Rodentia } \\ \text { herbivore } & \text { Rodentia } \\ \text { herbivore } & \text { herbivore guild } \\ \text { omnivore } & \text { herbivore guild } \\ \text { herbivore } & \text { Narnivore } \\ \text { carnivore } & \end{array}$




\begin{tabular}{|c|c|c|}
\hline Creodonta & Hyaenodontidae & $\begin{array}{l}\text { Hyaenodon raineyi } \\
\text { Poebrotherium chadronense }\end{array}$ \\
\hline Artiodactyla & Merycoidodontidae & Limnenetes platyceps \\
\hline Artiodactyla & Merycoidodontidae & Bathygenys reevesi \\
\hline Rodentia & Cylindrodontidae & Ardynomys occidentalis \\
\hline Artiodactyla & Hypertragulidae & Parvitragulus priscus \\
\hline Rodentia & Cylindrodontidae & $\begin{array}{l}\text { Pseudocylindrodon pintoensis } \\
\text { Meliakrouniomys wilsoni }\end{array}$ \\
\hline Rodentia & Cylindrodontidae & Pseudocylindrodon texanus \\
\hline Perissodactyla & Hyracodontidae & Hyracodon petersoni \\
\hline Artiodactyla & Merycoidodontidae & Merycoidodon dunagani \\
\hline Artiodactyla & Merycoidodontidae & Merycoidodon presidioensis \\
\hline Perissodactyla & Equidae & Mesohippus texanus \\
\hline Artiodactyla & & Stibarus yoderensis \\
\hline Rodentia & Eomyidae & Yoderimys bumpi \\
\hline Perissodactyla & Brontotheriidae & Megacerops osborni \\
\hline Artiodactyla & & Brachyhyops viensis \\
\hline Artiodactyla & Hypertragulidae & Leptomeryx yoderi \\
\hline Creodonta & Hyaenodontidae & Hyaenodon venturae \\
\hline Rodentia & Eutypomyidae & Eutypomys inexpectatus \\
\hline Cimolodonta & Neoplagiaulacidae & Ectypodus lovei \\
\hline Creodonta & Hyaenodontidae & Hyaenodon microdon \\
\hline Rodentia & Eomyidae & Cupressimus barbarae \\
\hline Artiodactyla & Anthracotheriidae & $\begin{array}{l}\text { Bothriodon advena } \\
\text { Didelphidectes pumilis }\end{array}$ \\
\hline Artiodactyla & Protoceratidae & Pseudoprotoceras semicinctu \\
\hline Rodentia & Cylindrodontidae & Cylindrodon collinus \\
\hline Rodentia & Eomyidae & Paradjidaumo hansonorum \\
\hline \multirow[t]{2}{*}{ Rodentia } & Eomyidae & Yoderimys stewarti \\
\hline & Ischyromyidae & Leptotomus guildayi \\
\hline Artiodactyla & Oromerycidae & Montanatylopus matthewi \\
\hline Rodentia & Cylindrodontidae & Pseudocylindrodon medius \\
\hline Rodentia & Cylindrodontidae & Jaywilsonomys ojinagaensis \\
\hline Artiodactyla & Leptomerycidae & Hendryomeryx defordi \\
\hline Carnivora & Miacidae & Miacis cognitus \\
\hline \multirow[t]{2}{*}{ Perissodactyla } & Tapiridae & Colodon stovalli \\
\hline & Ischyromyidae & Pseudotomus johanniculi \\
\hline Artiodactyla & Anthracotheriidae & Anthracotherium pangan \\
\hline Insectivora & Micropternodontidae & Micropternodus montrosensis \\
\hline Primates & Amphipithecidae & Pondaungia cotteri \\
\hline Leptictida & Leptictidae & Leptictis douglassi \\
\hline Perissodactyla & Amynodontidae & Metamynodon chadronensis \\
\hline Perissodactyla & Brontotheriidae & Duchesneodus uintensis \\
\hline Carnivora & Miacidae & Miacis australis \\
\hline Perissodactyla & Hyracodontidae & Hyracodon medius \\
\hline \multirow[t]{2}{*}{ Artiodactyla } & Agriochoeridae & Protoreodon pumilus \\
\hline & Ischyromyidae & Quadratomus gigans \\
\hline \multirow[t]{2}{*}{ Rodentia } & Eomyidae & Viejadjidaumo magniscopuli \\
\hline & Ischyromyidae & Microparamys perfossus \\
\hline Rodentia & Eomyidae & Litoyoderimys lustrorum \\
\hline \multirow[t]{2}{*}{ Rodentia } & Eomyidae & Adjidaumo burkei \\
\hline & Plagiomenidae & Thylacaelurus montanus \\
\hline
\end{tabular}

$\begin{array}{r}36.55 \\ 36.55 \\ 36.95 \\ 37.1 \\ 37.1 \\ 37.75 \\ 38.6 \\ 36.6 \\ 36.6 \\ 36.7 \\ 37.1 \\ 37.75 \\ 38.45 \\ 36.7 \\ 36.7 \\ 36.7 \\ 37.75 \\ 37.85 \\ 39.75 \\ 38.35 \\ 42.55 \\ 36.9 \\ 36.9 \\ 36.9 \\ 36.9 \\ 37.75 \\ 37.85 \\ 37.85 \\ 37.85 \\ 42.45 \\ 36.95 \\ 37.65 \\ 38.6 \\ 38.35 \\ 37.1 \\ 38.35 \\ 42.7 \\ 37.15 \\ 37.5 \\ 42.9 \\ 37.2 \\ 38.35 \\ 40 \\ 42.3 \\ 43.05 \\ 45.25 \\ 37.3 \\ 37.3 \\ 37.35 \\ 37.35 \\ 37.75 \\ 42.2 \\ \hline\end{array}$

$36.55 \quad 6.998$ ground dwelling carnivore species

36.5510 .10356716 ground dwelling browser, browser - species

36.559 .737632358 ground dwelling herbivore

36.558 .151898471 ground dwelling herbivore species

$36.55 \quad 5.298$ ground dwelling herbivore species

36.557 .470151121 ground dwelling grazer, browser species

$36.55 \quad 5.272$ ground dwelling herbivore species

$36.6 \quad 5.198$ ground dwelling herbivore

36.611 .24405879 ground dwelling browser

36.610 .28104244 ground dwelling herbivore

$36.6 \quad 9.267$ ground dwelling herbivore

36.69 .193066782 ground dwelling browser

$36.7 \quad 7.867537137$ ground dwelling grazer, browser

$36.7 \quad 4.234$ ground dwelling herbivore

36.714 .55301026 ground dwelling browser

$36.7 \quad 12.264$ ground dwelling omnivore

36.78 .92593097 ground dwelling frugivore

$36.7 \quad 7.409$ ground dwelling carnivore

$36.75 \quad 6.594$ ground dwelling herbivore

82.82271949 arboreal omnivore

7.589 ground dwelling carnivore

36.92 .802 ground dwelling herbivore

36.912 .63424466 ground dwelling browser

$\begin{array}{lll}36.9 & 2.587 \text { scansorial omnivore }\end{array}$

10.4341134

4.756 ground dwelling herbivore

3.259 ground dwelling herbivore

4.612 ground dwelling herbivore

36.94 .987654606 ground dwelling herbivore

$\begin{array}{rr}36.9 & 4.987654606 \text { ground dwelling herbivore } \\ 36.95 & 11.3196132 \text { ground dwelling browser }\end{array}$

$36.95 \quad 3.788$ ground dwelling herbivore

$36.95 \quad 6.247$ ground dwelling herbivore

8.13783561 ground dwelling frugivore 7.882

37.1

37.110 .54020218 ground dwelling browser

37.19 .413 ground dwelling herbivore

37.1512 .80765263 ground dwelling browser

37.154 .641 scansorial insectivore

37.159 .104979856 arboreal omnivore

37.213 .59557256 amphibious browser species

37.214 .1426263 ground dwelling browser species

$37.2 \quad 7.633$

$37.2 \quad 10.958$ ground dwelling browser

37.29 .899396404 scansorial herbivore

$37.3 \quad 9.328$ ground dwelling herbivore

$37.3 \quad 3.031$ ground dwelling herbivore

3.93 ground dwelling herbivore

3.968 ground dwelling herbivore

1.592 ground dwelling herbivore

4.139 scansorial insectivore carnivore

herbivore

herbivore

herbivore

herbivore

herbivore

herbivore

herbivore

herbivore

herbivore

herbivore

herbivore

herbivore

herbivore

herbivore

herbivore

omnivore

herbivore

carnivore

herbivore

omnivore

carnivore

herbivore

herbivore

omnivore

NA

herbivore

herbivore

herbivore

herbivore

herbivore

herbivore

herbivore

herbivore

NA

herbivore

herbivore

herbivore

insectivore

omnivore

insectivore

herbivore

herbivore

herbivore

herbivore

herbivore

herbivore

herbivore

herbivore

herbivore

insectivore carnivore guild

herbivore guild

herbivore guild

herbivore guild

Rodentia

herbivore guild

Rodentia

herbivore guild

Rodentia

herbivore guild

herbivore guild

herbivore guild

herbivore guild

herbivore guild

Rodentia

herbivore guild

NA

herbivore guild

carnivore guild

Rodentia

NA

carnivore guild

Rodentia

herbivore guild

NA

Rodentia

Rodentia

herbivore guild

herbivore guild

Rodentia

Rodentia

herbivore guild

carnivore guild

herbivore guild

herbivore guild

herbivore guild

NA

herbivore guild

herbivore guild

carnivore guild

herbivore guild

herbivore guild

herbivore guild

Rodentia

herbivore guild

Rodentia

Rodentia

NA 


\begin{tabular}{|c|c|c|c|c|c|c|c|c|c|}
\hline Rodentia & Eomyidae & Paradjidaumo alberti & 46.75 & 37.4 & 2.804 ground dwelling & herbivore & species & herbivore & Rodentia \\
\hline Rodentia & Eomyidae & Paranamatomys storeri & 37.5 & 37.5 & 2.027 ground dwelling & herbivore & species & herbivore & Rodentia \\
\hline Lipotyphla & Geolabididae & Centetodon hendryi & 42.2 & 37.5 & 2.892 scansorial & insectivore & species & insectivore & NA \\
\hline Creodonta & Hyaenodontidae & Hyaenodon vetus & 39.85 & 37.6 & 9.143 ground dwelling & carnivore & species & carnivore & carnivore guild \\
\hline Lagomorpha & Leporidae & Palaeolagus primus & 37.75 & 37.75 & 4.691 ground dwelling & grazer, browser & species & herbivore & herbivore guild \\
\hline \multirow[t]{2}{*}{ Rodentia } & Eomyidae & Litoyoderimys auogoleus & 37.75 & 37.75 & 3.834 ground dwelling & herbivore & species & herbivore & Rodentia \\
\hline & Ischyromyidae & Paramys compressidens & 44.25 & 37.75 & 7.307 ground dwelling & herbivore & species & herbivore & herbivore guild \\
\hline Artiodactyla & Merycoidodontidae & Aclistomycter middletoni & 42.3 & 38.05 & 10.57116816 ground dwelling & herbivore & species & herbivore & herbivore guild \\
\hline Artiodactyla & Protoceratidae & Poabromylus kayi & 39.15 & 38.35 & 9.643087334 ground dwelling & browser & species & herbivore & herbivore guild \\
\hline Perissodactyla & Equidae & Haplohippus texanus & 39.6 & 38.35 & 9.532 ground dwelling & browser & species & herbivore & herbivore guild \\
\hline \multirow[t]{2}{*}{ Carnivora } & Amphicyonidae & Daphoenus lambei & 42.3 & 38.35 & 9.498290728 ground dwelling & carnivore, omnivore & species & carnivore & carnivore guild \\
\hline & Ischyromyidae & Pseudotomus petersoni & 44.75 & 38.45 & 8.345 ground dwelling & herbivore & species & herbivore & herbivore guild \\
\hline Artiodactyla & Hypertragulidae & Hypertragulus heikeni & 39.05 & 38.6 & 8.377931124 ground dwelling & frugivore & species & herbivore & herbivore guild \\
\hline Artiodactyla & Agriochoeridae & Protoreodon petersoni & 43.75 & 38.6 & 8.543394938 scansorial & herbivore & species & herbivore & herbivore guild \\
\hline Artiodactyla & Hypertragulidae & Simimeryx minutus & 38.75 & 38.75 & 7.09685816 ground dwelling & grazer, browser & species & herbivore & herbivore guild \\
\hline Perissodactyla & Lophiodontidae & Lophiodon rhinocerodes & 44.5 & 38.8 & 12.54254488 ground dwelling & folivore & genus & herbivore & herbivore guild \\
\hline \multirow[t]{2}{*}{ Artiodactyla } & & Antiacodon pygmaeus & 51.9 & 38.8 & 6.966 ground dwelling & grazer, browser & species & herbivore & herbivore guild \\
\hline & Equidae & Duchesnehippus intermedius & 39.35 & 38.9 & 9.43 ground dwelling & browser & species & herbivore & herbivore guild \\
\hline \multirow[t]{2}{*}{ Perissodactyla } & Amynodontidae & Amynodontopsis bodei & 40.5 & 38.9 & 13.14396534 ground dwelling & browser & species & herbivore & herbivore guild \\
\hline & Ischyromyidae & Mytonomys robustus & 43.3 & 39.05 & 7.625 ground dwelling & herbivore & species & herbivore & herbivore guild \\
\hline Rodentia & Cylindrodontidae & Pareumys guensburgi & 39.35 & 39.35 & 4.955 ground dwelling & herbivore & species & herbivore & Rodentia \\
\hline Rodentia & Eomyidae & Protadjidaumo typus & 49.25 & 39.35 & 2.509 ground dwelling & herbivore & species & herbivore & Rodentia \\
\hline Artiodactyla & Hypertragulidae & Leptomeryx blacki & 39.45 & 39.45 & 8.100642008 ground dwelling & frugivore & species & herbivore & herbivore guild \\
\hline Perissodactyla & Rhinocerotidae & Teletaceras mortivallis & 39.45 & 39.45 & 11.022 ground dwelling & browser & species & herbivore & herbivore guild \\
\hline Perissodactyla & Rhinocerotidae & Teletaceras radinskyi & 39.6 & 39.6 & 11.795 ground dwelling & browser & species & herbivore & herbivore guild \\
\hline Perissodactyla & Tapiridae & Plesiocolopirus hancocki & 42.7 & 39.6 & 10.2549419 ground dwelling & browser & species & herbivore & herbivore guild \\
\hline \multirow[t]{2}{*}{ Perissodactyla } & Equidae & Epihippus gracilis & 49.25 & 39.6 & 10.14289987 ground dwelling & browser & species & herbivore & herbivore guild \\
\hline & Protoptychidae & Presbymys lophatus & 39.75 & 39.75 & 4.378 ground dwelling & herbivore & species & herbivore & herbivore guild \\
\hline Rodentia & Eomyidae & Metanoiamys korthi & 39.75 & 39.75 & 2.635 ground dwelling & herbivore & species & herbivore & Rodentia \\
\hline Rodentia & Eomyidae & Paradjidaumo reynoldsi & 39.75 & 39.75 & 2.874 ground dwelling & herbivore & species & herbivore & Rodentia \\
\hline Rodentia & Simimyidae & Simimys landeri & 39.75 & 39.75 & 3.701 ground dwelling & herbivore & species & herbivore & Rodentia \\
\hline Artiodactyla & Agriochoeridae & Protoreodon pacificus & 40.1 & 39.75 & 9.386963659 scansorial & herbivore & species & herbivore & herbivore guild \\
\hline Artiodactyla & Oromerycidae & Protylopus pearsonensis & 40.1 & 39.75 & 9.746314413 ground dwelling & browser & species & herbivore & herbivore guild \\
\hline Artiodactyla & Hypertragulidae & Simimeryx hudsoni & 40.35 & 39.75 & 7.844 ground dwelling & grazer, browser & species & herbivore & herbivore guild \\
\hline Primates & Omomyidae & Chumashius balchi & 40.35 & 39.75 & 5.937 arboreal & insectivore & species & insectivore & NA \\
\hline \multirow[t]{2}{*}{ Acreodi } & & Simidectes merriami & 40.5 & 39.75 & 9.627 scansorial & carnivore & species & carnivore & carnivore guild \\
\hline & Ischyromyidae & Microparamys tricus & 40.5 & 39.75 & 3.993 ground dwelling & herbivore & species & herbivore & herbivore guild \\
\hline Artiodactyla & Protoceratidae & Leptoreodon stocki & 41.05 & 39.75 & 8.828587054 ground dwelling & browser & species & herbivore & herbivore guild \\
\hline Rodentia & Cylindrodontidae & Pareumys milleri & 41.55 & 39.75 & 4.346 ground dwelling & herbivore & species & herbivore & Rodentia \\
\hline \multirow[t]{6}{*}{ Rodentia } & Simimyidae & Simimys simplex & 43.05 & 39.75 & 2.723 ground dwelling & herbivore & species & herbivore & Rodentia \\
\hline & & Proterixoides davisi & 43.05 & 39.75 & 5.184 ground dwelling & insectivore & species & insectivore & NA \\
\hline & & Sespedectes singularis & 44.55 & 39.75 & 3.097 ground dwelling & insectivore & species & insectivore & NA \\
\hline & & Peradectes californicus & 45.15 & 39.75 & 1.85 scansorial & omnivore & species & omnivore & NA \\
\hline & & Griphomys alecer & 46.75 & 39.75 & 2.577 ground dwelling & herbivore & species & herbivore & herbivore guild \\
\hline & Ischyromyidae & Mytonomys coelumensis & 40 & 40 & 7.634 ground dwelling & herbivore & species & herbivore & herbivore guild \\
\hline Artiodactyla & Oromerycidae & Protylopus stocki & 42.85 & 40 & 8.868053857 ground dwelling & browser & species & herbivore & herbivore guild \\
\hline Perissodactyla & Hyracodontidae & Triplopus woodi & 42.85 & 40 & 10.783 ground dwelling & browser & species & herbivore & herbivore guild \\
\hline Acreodi & & Simidectes magnus & 43.55 & 40 & 8.919 scansorial & carnivore & species & carnivore & carnivore guild \\
\hline Artiodactyla & Protoceratidae & Leptoreodon leptolophus & 44.65 & 40 & 8.424778609 ground dwelling & browser & species & herbivore & herbivore guild \\
\hline Primates & Omomyidae & Dyseolemur pacificus & 43.05 & 40.1 & 5.458 arboreal & insectivore & species & insectivore & NA \\
\hline Artiodactyla & Protoceratidae & Leptoreodon pusillus & 46.85 & 40.1 & 7.781962818 ground dwelling & browser & species & herbivore & herbivore guild \\
\hline Perissodactyla & Amynodontidae & Megalamynodon regalis & 40.25 & 40.25 & 14.33994005 amphibious & browser & species & herbivore & herbivore guild \\
\hline
\end{tabular}




\begin{tabular}{|c|c|c|}
\hline Artiodactyla & Agriochoeridae & Protoreodon pearcei \\
\hline \multirow[t]{2}{*}{ Perissodactyla } & Tapiridae & Heteraletes leotanus \\
\hline & & Sespedectes stocki \\
\hline \multirow[t]{2}{*}{ Rodentia } & Eomyidae & Metanoiamys marinus \\
\hline & Ischyromyidae & Mytonomys mytonensis \\
\hline Artiodactyla & Agriochoeridae & Protoreodon walshi \\
\hline Artiodactyla & Oromerycidae & Protylopus petersoni \\
\hline Perissodactyla & Amynodontidae & Amynodon advenus \\
\hline Didelphimorphia & Herpetotheriidae & Herpetotherium knighti \\
\hline \multirow[t]{2}{*}{ Artiodactyla } & Oromerycidae & Protylopus robustus \\
\hline & Ischyromyidae & Eohaplomys tradux \\
\hline Insectivora & Apatemyidae & Apatemys uintensis \\
\hline \multirow[t]{3}{*}{ Lipotyphla } & Geolabididae & Centetodon aztecus \\
\hline & Ischyromyidae & Eohaplomys serus \\
\hline & Ischyromyidae & Mytonomys burkei \\
\hline Lagomorpha & Leporidae & Mytonolagus petersoni \\
\hline \multirow[t]{2}{*}{ Perissodactyla } & Hyracodontidae & Epitriplopus uintensis \\
\hline & Ischyromyidae & Eohaplomys matutinus \\
\hline \multirow[t]{2}{*}{ Carnivora } & & Procynodictis progressus \\
\hline & Ischyromyidae & Rapamys fricki \\
\hline \multirow[t]{4}{*}{ Artiodactyla } & Dichobunidae & Tapochoerus egressus \\
\hline & Ischyromyidae & Microparamys woodi \\
\hline & & Tapocyon robustus \\
\hline & Ischyromyidae & Tapomys tapensis \\
\hline Primates & Omomyidae & Yaquius travisi \\
\hline Primates & Microsyopidae & Craseops sylvestris \\
\hline Insectivora & Apatemyidae & Apatemys downsi \\
\hline Primates & Paromomyidae & Phenacolemur shifrae \\
\hline Carnivora & Miacidae & Miacis hookwayi \\
\hline \multirow[t]{3}{*}{ Artiodactyla } & Protoceratidae & Leptoreodon edwardsi \\
\hline & Ischyromyidae & Leptotomus leptodus \\
\hline & Ischyromyidae & Leptotomus caryophilus \\
\hline Lipotyphla & Geolabididae & Batodonoides powayensis \\
\hline Acreodi & Mesonychidae & Harpagolestes leotensis \\
\hline Artiodactyla & Dichobunidae & Pentacemylus progressus \\
\hline Artiodactyla & Protoceratidae & Leptotragulus clarki \\
\hline Artiodactyla & Dichobunidae & Bunomeryx elegans \\
\hline Artiodactyla & Agriochoeridae & Diplobunops matthewi \\
\hline Artiodactyla & Dichobunidae & Pentacemylus leotensis \\
\hline Artiodactyla & Protoceratidae & Leptotragulus medius \\
\hline Perissodactyla & Hyracodontidae & Triplopus obliquidens \\
\hline Perissodactyla & Hyracodontidae & Triplopus rhinocerinus \\
\hline Cetacea & & Pakicetus attocki \\
\hline Rodentia & Eomyidae & Metanoiamys fantasma \\
\hline Perissodactyla & Brontotheriidae & Protitanotherium superbum \\
\hline Perissodactyla & Brontotheriidae & Pseudodiplacodon progressum \\
\hline Artiodactyla & Anthracotheriidae & Heptacodon pellionis \\
\hline Creodonta & Hyaenodontidae & Proviverra longipes \\
\hline Artiodactyla & Dichobunidae & Auxontodon pattersoni \\
\hline Rodentia & Eutypomyidae & Janimus rhinophilus \\
\hline Artiodactyla & Oromerycidae & Protylopus annectens \\
\hline Artiodactyla & Camelidae & Poebrodon kayi \\
\hline
\end{tabular}

\begin{tabular}{|c|c|c|c|c|}
\hline 43.05 & 40.25 & 10.2805158 scansorial & herbivore & species \\
\hline 43.05 & 40.25 & 8.778182831 ground dwelling & browser & species \\
\hline 40.35 & 40.35 & 3.174 ground dwelling & insectivore & species \\
\hline 41.05 & 40.35 & 2.239 ground dwelling & herbivore & species \\
\hline 41.15 & 40.35 & 7.098 ground dwelling & herbivore & species \\
\hline 43.05 & 40.35 & 9.397293861 scansorial & herbivore & species \\
\hline 45.15 & 40.35 & 8.852527149 ground dwelling & browser & species \\
\hline 49.25 & 40.35 & 13.13165335 ground dwelling & browser & species \\
\hline 51.8 & 40.35 & 3.141 ground dwelling & omnivore & species \\
\hline 40.75 & 40.5 & 9.410701616 ground dwelling & browser & species \\
\hline 42.85 & 40.5 & 6.649 ground dwelling & herbivore & species \\
\hline 44.75 & 40.5 & 3.681334809 arboreal & herbivore & species \\
\hline 45.45 & 40.5 & 2.723974679 scansorial & insectivore & species \\
\hline 40.75 & 40.65 & 6.866 ground dwelling & herbivore & species \\
\hline 40.75 & 40.65 & 7.921 ground dwelling & herbivore & species \\
\hline 43.05 & 40.65 & 5.006 ground dwelling & grazer, browser & species \\
\hline 44.75 & 40.65 & 11.184 ground dwelling & browser & species \\
\hline 40.75 & 40.75 & 7.043 ground dwelling & herbivore & species \\
\hline 42.85 & 40.75 & 7.880862241 ground dwelling & carnivore, omnivore & species \\
\hline 42.85 & 40.75 & 6.873404432 ground dwelling & herbivore & species \\
\hline 43.05 & 40.75 & 9.052332734 ground dwelling & grazer, browser & species \\
\hline 43.05 & 40.75 & 3.448 ground dwelling & herbivore & species \\
\hline 43.05 & 40.75 & 9.467 scansorial & insectivore & species \\
\hline 40.9 & 40.9 & 7.536 ground dwelling & herbivore & species \\
\hline 40.9 & 40.9 & 7.739 arboreal & insectivore & species \\
\hline 40.9 & 40.9 & 6.707 arboreal & omnivore & species \\
\hline 42.85 & 40.9 & 4.388753852 arboreal & herbivore & species \\
\hline 42.85 & 40.9 & 4.532599493 arboreal & omnivore & species \\
\hline 43.05 & 40.9 & 7.2735 arboreal & carnivore & species \\
\hline 43.55 & 40.9 & 8.621168139 ground dwelling & browser & species \\
\hline 45.15 & 40.9 & 7.895620451 & & \\
\hline 45.45 & 40.9 & 6.624 ground dwelling & herbivore & species \\
\hline 45.45 & 41.05 & 1.153 scansorial & insectivore & species \\
\hline 41.55 & 41.15 & 11.914 ground dwelling & carnivore & species \\
\hline 41.55 & 41.15 & 8.615443786 ground dwelling & herbivore & species \\
\hline 41.55 & 41.15 & 8.149 ground dwelling & browser & species \\
\hline 41.65 & 41.15 & 7.97 ground dwelling & herbivore & species \\
\hline 42.05 & 41.15 & 10.23499074 scansorial & herbivore & species \\
\hline 43 & 41.15 & 8.794501965 ground dwelling & herbivore & species \\
\hline 43.15 & 41.15 & 8.664609092 ground dwelling & browser & species \\
\hline 44.95 & 41.15 & 10.45245306 ground dwelling & browser & species \\
\hline 43.05 & 41.25 & 11.063 ground dwelling & browser & species \\
\hline 52.2 & 41.25 & 10.29890232 amphibious & carnivore & species \\
\hline 41.3 & 41.3 & 2.929 ground dwelling & herbivore & species \\
\hline 41.4 & 41.4 & 7.887307993 ground dwelling & browser & species \\
\hline 41.4 & 41.4 & 14.20753053 & & \\
\hline 42.3 & 41.4 & 11.03928256 ground dwelling & browser & species \\
\hline 41.55 & 41.55 & 8.802636078 ground dwelling & carnivore & species \\
\hline 41.55 & 41.55 & 8.481 ground dwelling & grazer, browser & species \\
\hline 41.55 & 41.55 & 4.163808689 ground dwelling & herbivore & species \\
\hline 41.55 & 41.55 & 9.470286515 ground dwelling & browser & species \\
\hline 41.55 & 41.55 & 8.677311946 ground dwelling & browser, browser & species \\
\hline
\end{tabular}

$\begin{array}{ll}\text { herbivore } & \text { herbivore guild } \\ \text { herbivore } & \text { herbivore guild } \\ \text { insectivore } & \text { NA } \\ \text { herbivore } & \text { Rodentia } \\ \text { herbivore } & \text { herbivore guild } \\ \text { herbivore } & \text { herbivore guild } \\ \text { herbivore } & \text { herbivore guild } \\ \text { herbivore } & \text { herbivore guild } \\ \text { omnivore } & \text { NA } \\ \text { herbivore } & \text { herbivore guild } \\ \text { herbivore } & \text { herbivore guild } \\ \text { herbivore } & \text { herbivore guild } \\ \text { insectivore } & \text { NA } \\ \text { herbivore } & \text { herbivore guild } \\ \text { herbivore } & \text { herbivore guild } \\ \text { herbivore } & \text { herbivore guild } \\ \text { herbivore } & \text { herbivore guild } \\ \text { herbivore } & \text { herbivore guild } \\ \text { carnivore } & \text { carnivore guild } \\ \text { herbivore } & \text { herbivore guild } \\ \text { herbivore } & \text { herbivore guild } \\ \text { herbivore } & \text { herbivore guild } \\ \text { insectivore } & \text { NA } \\ \text { herbivore } & \text { herbivore guild } \\ \text { insectivore } & \text { NA } \\ \text { omnivore } & \text { NA } \\ \text { herbivore } & \text { herbivore guild } \\ \text { omnivore } & \text { NA } \\ \text { carnivore } & \text { carnivore guild } \\ \text { herbivore } & \text { herbivore guild } \\ \text { NA } & \text { NA } \\ \text { herbivore } & \text { herbivore guild } \\ \text { insectivore } & \text { NA } \\ \text { carnivore } & \text { carnivore guild } \\ \text { herbivore } & \text { herbivore guild } \\ \text { herbivore } & \text { herbivore guild } \\ \text { herbivore } & \text { herbivore guild } \\ \text { herbivore } & \text { herbivore guild } \\ \text { herbivore } & \text { herbivore guild } \\ \text { herbivore } & \text { herbivore guild } \\ \text { herbivore } & \text { herbivore guild } \\ \text { herbivore } & \text { herbivore guild } \\ \text { carnivore } & \text { NA } \\ \text { herbivore } & \text { Rodentia } \\ \text { herbivore } & \text { herbivore guild } \\ \text { NA } & \text { NA } \\ \text { herbivore } & \text { herbivore guild } \\ \text { carnivore } & \text { herbivore guild } \\ \text { herbivore } & \text { herbild } \\ \text { herbivore } & \end{array}$




\begin{tabular}{|c|c|c|}
\hline Artiodactyla & $\begin{array}{l}\text { Dichobunidae } \\
\text { Ischyromyidae }\end{array}$ & $\begin{array}{l}\text { Hylomeryx quadricuspis } \\
\text { Thisbemys medius }\end{array}$ \\
\hline Eulipotyphla & & Talpavus duplus \\
\hline \multirow[t]{2}{*}{ Artiodactyla } & Dichobunidae & Mytonomeryx scotti \\
\hline & Ischyromyidae & Pseudotomus eugenei \\
\hline Primates & Omomyidae & Ourayia hopsoni \\
\hline Artiodactyla & Protoceratidae & Leptotragulus proavus \\
\hline Perissodactyla & Rhinocerotidae & Uintaceras radinskyi \\
\hline Creodonta & Hyaenodontidae & Limnocyon potens \\
\hline Carnivora & Miacidae & Miocyon scotti \\
\hline Artiodactyla & Protoceratidae & Leptoreodon marshi \\
\hline Primates & Omomyidae & Ourayia uintensis \\
\hline Artiodactyla & Oromerycidae & Oromeryx plicatus \\
\hline Perissodactyla & & Isectolophus annectens \\
\hline Panameriungulata & Hyopsodontidae & Hyopsodus uintensis \\
\hline Carnivora & & Procynodictis vulpiceps \\
\hline Artiodactyla & Agriochoeridae & Protoreodon minor \\
\hline Rodentia & Reithroparamyidae & Reithroparamys sciuroides \\
\hline Perissodactyla & Brontotheriidae & Metatelmatherium ultimum \\
\hline Creodonta & Hyaenodontidae & Oxyaenodon dysodus \\
\hline \multirow[t]{2}{*}{ Artiodactyla } & Dichobunidae & Bunomeryx montanus \\
\hline & Protoptychidae & Protoptychus hatcheri \\
\hline Perissodactyla & Hyracodontidae & Triplopus implicatus \\
\hline Lagomorpha & Leporidae & Mytonolagus wyomingensis \\
\hline Artiodactyla & Protoceratidae & Poabromylus golzi \\
\hline Rodentia & Cylindrodontidae & Anomoemys lewisi \\
\hline Macroscelidea & Amphilemuridae & Macrocranion robinsoni \\
\hline Rodentia & Allomyidae & Spurimus selbyi \\
\hline Eulipotyphla & Nyctitheriidae & Nyctitherium christopheri \\
\hline Artiodactyla & Leptomerycidae & Hendryomeryx wilsoni \\
\hline Perissodactyla & Tapiridae & Colodon woodi \\
\hline \multirow[t]{2}{*}{ Primates } & Omomyidae & Macrotarsius siegerti \\
\hline & Ischyromyidae & Microparamys dubius \\
\hline Perissodactyla & Brontotheriidae & Telmatherium cultridens \\
\hline Primates & Paromomyidae & Phenacolemur mcgrewi \\
\hline Didelphimorphia & Herpetotheriidae & Peratherium marsupium \\
\hline Didelphimorphia & Herpetotheriidae & Copedelphys innominata \\
\hline \multirow[t]{2}{*}{ Artiodactyla } & Oromerycidae & Malaquiferus tourteloti \\
\hline & Ischyromyidae & Rapamys wilsoni \\
\hline Insectivora & Apatemyidae & Apatemys hendryi \\
\hline Primates & Microsyopidae & Uintasorex parvulus \\
\hline Insectivora & Apatemyidae & Apatemys bellus \\
\hline Carnivora & Miacidae & Miocyon magnus \\
\hline Cimolesta & Cimolestidae & Didelphodus rheos \\
\hline \multirow[t]{3}{*}{ Lagomorpha } & Leporidae & Tachylagus gawneae \\
\hline & Ischyromyidae & Churcheria baroni \\
\hline & Ischyromyidae & Microparamys nimius \\
\hline Rodentia & Eomyidae & Adjidaumo craigi \\
\hline Rodentia & Eutypomyidae & Eutypomys acares \\
\hline Rodentia & Eutypomyidae & Eutypomys obliquidens \\
\hline Rodentia & Eutypomyidae & Janimus dawsonae \\
\hline Rodentia & Eutypomyidae & Microeutypomys tilliei \\
\hline
\end{tabular}

$41.55 \quad 8.374$ ground dwelling herbivore

$41.55 \quad 9.933046355$ ground dwelling herbivore

$41.55-2.749$ scansoriat 2.55 insetivo

$41.55 \quad 8.263$ ground dwelling herbivore

41.559 .571 ground dwelling herbivore

$41.55 \quad 7.197$ arboreal insectivore

9.099 ground dwelling browser

$41.55 \quad 12.79377146$ ground dwelling browser

41.5510 .53235888 ground dwelling omnivore

41.559 .376 ground dwelling carnivore, omnivore

41.558 .735511267 ground dwelling browser

species

$41.55 \quad 8.921$ ground dwelling browser species

41.5510 .3238356 ground dwelling browser species

41.556 .806 scansorial omnivore species

41.557 .483258542 ground dwelling carnivore, omnivore species

41.658 .860273702 scansorial herbivore species

$41.65 \quad 6.185$ ground dwelling herbivore species

$41.65 \quad 14.26221207$ ground dwelling browser species

41.6510 .62315494 ground dwelling carnivore species

$41.65 \quad 7.714$ ground dwelling herbivore species

41.653 .513948796 ground dwelling herbivore species

$41.65 \quad 10.66$ ground dwelling browser species

$41.8 \quad 4.772$ ground dwelling grazer, browser species

$41.8 \quad 9.142$ ground dwelling browser species

41.84 .505348511 ground dwelling herbivore species

41.84 .094271983 ground dwelling insectivore species

41.84 .814919763 ground dwelling herbivore species

41.84 .071684419 scansorial insectivore species

41.87 .926049764 ground dwelling frugivore species

$41.8 \quad 10.10510789$ ground dwelling browser species

$41.8 \quad 7.67$ arboreal insectivore species

$41.8 \quad 2.958$ ground dwelling herbivore species

41.8 12.958 ground dwelling browser species

41.85 .475055686 arboreal omnivore species

$\begin{array}{lll}41.8 & 5.00636486 \text { ground dwelling omnivore } & \text { species }\end{array}$

$41.8 \quad 2.637$

41.958 .505402284 ground dwelling browser

10.57821197
ground dwelling carnivore, omnivore species

$42.3 \quad 4.95$ ground dwelling carnivore species

$42.3 \quad 5.137$ ground dwelling grazer, browser species

$42.3 \quad 2.93141388$ ground dwelling herbivore species

$\begin{array}{lll}42.3 & 3.348 \text { ground dwelling herbivore } & \text { species }\end{array}$

$42.3 \quad 2.1$ ground dwelling herbivore species

$42.3 \quad 4.483$ ground dwelling herbivore species

$42.3 \quad 5.308$ ground dwelling herbivore species

$42.3 \quad 2.754$ ground dwelling herbivore species

$\begin{array}{lll}42.3 & 3.024 \text { ground dwelling herbivore species }\end{array}$

\begin{tabular}{|c|c|}
\hline $\begin{array}{l}\text { herbivore } \\
\text { herbivore }\end{array}$ & $\begin{array}{l}\text { herbivore guild } \\
\text { herbivore guild }\end{array}$ \\
\hline $\begin{array}{l}\text { herbivore } \\
\text { insectivore }\end{array}$ & NA \\
\hline $\begin{array}{l}\text { Insectivore } \\
\text { herbivore }\end{array}$ & herbivore guild \\
\hline herbivore & herbivore guild \\
\hline insectivore & NA \\
\hline herbivore & herbivore guild \\
\hline herbivore & herbivore guild \\
\hline omnivore & NA \\
\hline carnivore & carnivore guild \\
\hline herbivore & herbivore guild \\
\hline insectivore & NA \\
\hline herbivore & herbivore guild \\
\hline herbivore & herbivore guild \\
\hline omnivore & NA \\
\hline carnivore & carnivore guild \\
\hline herbivore & herbivore guild \\
\hline herbivore & Rodentia \\
\hline herbivore & herbivore guild \\
\hline carnivore & carnivore guild \\
\hline herbivore & herbivore guild \\
\hline herbivore & herbivore guild \\
\hline herbivore & herbivore guild \\
\hline herbivore & herbivore guild \\
\hline herbivore & herbivore guild \\
\hline herbivore & Rodentia \\
\hline insectivore & NA \\
\hline herbivore & Rodentia \\
\hline insectivore & NA \\
\hline herbivore & herbivore guild \\
\hline herbivore & herbivore guild \\
\hline insectivore & NA \\
\hline herbivore & herbivore guild \\
\hline herbivore & herbivore guild \\
\hline omnivore & NA \\
\hline omnivore & NA \\
\hline NA & NA \\
\hline herbivore & herbivore guild \\
\hline herbivore & herbivore guild \\
\hline herbivore & herbivore guild \\
\hline omnivore & NA \\
\hline herbivore & herbivore guild \\
\hline carnivore & carnivore guild \\
\hline carnivore & carnivore guild \\
\hline herbivore & herbivore guild \\
\hline herbivore & herbivore guild \\
\hline herbivore & herbivore guild \\
\hline herbivore & Rodentia \\
\hline herbivore & Rodentia \\
\hline herbivore & Rodentia \\
\hline herbivore & Rodentia \\
\hline herbivore & Rodentia \\
\hline
\end{tabular}




\begin{tabular}{|c|c|c|c|c|c|c|c|c|c|}
\hline Rodentia & Eomyidae & Protadjidaumo pauli & 42.3 & 42.3 & 2.974 ground dwelling & herbivore & species & herbivore & Rodentia \\
\hline Rodentia & Cylindrodontidae & Pseudocylindrodon lateriviae & 42.3 & 42.3 & 4.639 ground dwelling & herbivore & species & herbivore & Rodentia \\
\hline Eulipotyphla & & Talpavus conjunctus & 42.3 & 42.3 & 3.401 scansorial & insectivore & species & insectivore & NA \\
\hline \multirow[t]{2}{*}{ Primates } & Omomyidae & Macrotarsius montanus & 42.3 & 42.3 & 7.848 arboreal & insectivore & species & insectivore & NA \\
\hline & Plagiomenidae & Thylacaelurus campester & 42.45 & 42.3 & 3.534 scansorial & insectivore & species & insectivore & NA \\
\hline Rodentia & Eomyidae & Metanoiamys lacus & 46.75 & 42.3 & 2.096 ground dwelling & herbivore & species & herbivore & Rodentia \\
\hline Cimolesta & Cimolestidae & Didelphodus serus & 42.45 & 42.45 & 4.884 ground dwelling & carnivore & species & carnivore & carnivore guild \\
\hline Panameriungulata & Hyopsodontidae & Hyopsodus fastigatus & 42.45 & 42.45 & 7.054 scansorial & omnivore & species & omnivore & NA \\
\hline Artiodactyla & & Ibarus ignotus & 42.85 & 42.45 & 7.714231145 ground dwelling & grazer, browser & species & herbivore & herbivore guild \\
\hline Perissodactyla & Amynodontidae & Metamynodon mckinneyi & 42.7 & 42.7 & 13.92685868 amphibious & browser & species & herbivore & herbivore guild \\
\hline Artiodactyla & Protoceratidae & Toromeryx marginensis & 43.3 & 42.7 & 9.348 ground dwelling & browser & species & herbivore & herbivore guild \\
\hline Acreodi & Mesonychidae & Harpagolestes uintensis & 44.95 & 42.7 & 11.961 ground dwelling & carnivore & species & carnivore & carnivore guild \\
\hline \multirow[t]{2}{*}{ Artiodactyla } & Dichobunidae & Tapochoerus mcmillini & 42.85 & 42.85 & 8.364042012 ground dwelling & grazer, browser & species & herbivore & herbivore guild \\
\hline & & Tapocyon dawsonae & 42.85 & 42.85 & 8.775512602 scansorial & insectivore & species & insectivore & NA \\
\hline Acreodi & & Simidectes medius & 43 & 42.85 & 8.58 scansorial & carnivore & species & carnivore & carnivore guild \\
\hline \multirow[t]{2}{*}{ Artiodactyla } & Agriochoeridae & Protoreodon parvus & 45.15 & 42.85 & 9.320577941 scansorial & herbivore & species & herbivore & herbivore guild \\
\hline & Sciuravidae & Sciuravus powayensis & 45.45 & 42.85 & 4.501 ground dwelling & herbivore & species & herbivore & herbivore guild \\
\hline Rodentia & Cylindrodontidae & Pareumys grangeri & 45.45 & 42.85 & 4.047 ground dwelling & herbivore & species & herbivore & Rodentia \\
\hline Primates & Microsyopidae & Uintasorex montezumicus & 45.45 & 42.85 & 2.698 arboreal & omnivore & species & omnivore & NA \\
\hline Trituberculata & & Aethomylos simplicidens & 50.45 & 42.85 & 3.429 ground dwelling & insectivore & species & insectivore & NA \\
\hline Artiodactyla & Dichobunidae & Hylomeryx annectens & 43 & 43 & 8.347 ground dwelling & herbivore & species & herbivore & herbivore guild \\
\hline Rodentia & Cylindrodontidae & Pareumys troxelli & 43 & 43 & 4.435 ground dwelling & herbivore & species & herbivore & Rodentia \\
\hline Primates & Omomyidae & Macrotarsius jepseni & 43.3 & 43 & 7.396 arboreal & insectivore & species & insectivore & NA \\
\hline Panameriungulata & Hyopsodontidae & Hyopsodus paulus & 52.1 & 43 & 6.528 scansorial & omnivore & species & omnivore & NA \\
\hline Primates & Omomyidae & Omomys lloydi & 51.65 & 43.05 & 5.542 arboreal & insectivore & species & insectivore & NA \\
\hline Primates & Omomyidae & Omomys carteri & 51.75 & 43.05 & 5.923 arboreal & insectivore & species & insectivore & NA \\
\hline Perissodactyla & & Grangeria anarsius & 44.95 & 43.15 & 10.761 & & & NA & NA \\
\hline \multirow[t]{3}{*}{ Artiodactyla } & & Laredochoerus edwardsi & 43.3 & 43.3 & 8.828 ground dwelling & grazer, browser & species & herbivore & herbivore guild \\
\hline & Laredomyidae & Laredomys riograndensis & 43.3 & 43.3 & 1.782 ground dwelling & herbivore & species & herbivore & herbivore guild \\
\hline & Sciuravidae & Pauromys simplex & 43.3 & 43.3 & 2.615 ground dwelling & herbivore & species & herbivore & herbivore guild \\
\hline Rodentia & Eutypomyidae & Microeutypomys karenae & 43.3 & 43.3 & 2.439 ground dwelling & herbivore & species & herbivore & Rodentia \\
\hline Perissodactyla & Brontotheriidae & Eotitanotherium osborni & 43.3 & 43.3 & 13.926 ground dwelling & browser & species & herbivore & herbivore guild \\
\hline Lipotyphla & Geolabididae & Centetodon pulcher & 50.45 & 43.3 & 3.402 scansorial & insectivore & species & insectivore & NA \\
\hline \multirow[t]{4}{*}{ Didelphimorphia } & Herpetotheriidae & Peratherium comstocki & 54.9 & 43.3 & 4.483 ground dwelling & omnivore & species & omnivore & NA \\
\hline & Sciuravidae & Pauromys texensis & 46.85 & 43.35 & 2.867 ground dwelling & herbivore & species & herbivore & herbivore guild \\
\hline & & Prolapsus junctionis & 46.85 & 43.35 & 4.284 ground dwelling & herbivore & species & herbivore & herbivore guild \\
\hline & & Prolapsus sibilatoris & 46.85 & 43.35 & 5.095 ground dwelling & herbivore & species & herbivore & herbivore guild \\
\hline Artiodactyla & Protoceratidae & Leptoreodon major & 45.65 & 43.55 & 9.337304923 ground dwelling & browser & species & herbivore & herbivore guild \\
\hline \multirow[t]{3}{*}{ Creodonta } & Hyaenodontidae & Sinopa major & 50.45 & 43.55 & 8.865 ground dwelling & carnivore & species & carnivore & carnivore guild \\
\hline & Ischyromyidae & Paramys delicatior & 50.65 & 43.55 & 6.572 ground dwelling & herbivore & species & herbivore & herbivore guild \\
\hline & Sciuravidae & Pauromys lillegraveni & 43.75 & 43.75 & 2.342 ground dwelling & herbivore & species & herbivore & herbivore guild \\
\hline Primates & Microsyopidae & Microsyops kratos & 43.75 & 43.75 & 8.041 arboreal & omnivore & species & omnivore & NA \\
\hline Primates & Omomyidae & Stockia powayensis & 44.05 & 43.75 & 6.242 arboreal & insectivore & species & insectivore & NA \\
\hline Rodentia & Eomyidae & Metanoiamys agorus & 45.45 & 43.75 & 2.593 ground dwelling & herbivore & species & herbivore & Rodentia \\
\hline \multirow[t]{3}{*}{ Primates } & Omomyidae & Washakius woodringi & 45.45 & 43.75 & 5.271 arboreal & insectivore & species & insectivore & NA \\
\hline & & Crypholestes vaughni & 45.45 & 43.75 & 2.927 ground dwelling & insectivore & species & insectivore & NA \\
\hline & & Patriolestes novaceki & 45.45 & 43.75 & 5.117 ground dwelling & insectivore & species & insectivore & NA \\
\hline \multirow[t]{2}{*}{ Perissodactyla } & Brontotheriidae & Metarhinus pater & 45.85 & 43.75 & 12.91363867 ground dwelling & browser & species & herbivore & herbivore guild \\
\hline & Ischyromyidae & Microparamys minutus & 50.65 & 43.75 & 3.229 ground dwelling & herbivore & species & herbivore & herbivore guild \\
\hline \multirow[t]{2}{*}{ Artiodactyla } & & Antiacodon venustus & 49.55 & 43.85 & 8.038 ground dwelling & grazer, browser & species & herbivore & herbivore guild \\
\hline & Ischyromyidae & Pseudotomus californicus & 44.05 & 44.05 & 6.914474875 ground dwelling & herbivore & species & herbivore & herbivore guild \\
\hline Rodentia & Reithroparamyidae & Uriscus californicus & 44.05 & 44.05 & 4.868 ground dwelling & herbivore & species & herbivore & Rodentia \\
\hline
\end{tabular}




\begin{tabular}{|c|c|c|c|c|c|c|c|c|c|}
\hline Artiodactyla & Dichobunidae & Mesomeryx grangeri & 44.75 & 44.25 & 8.01448353 ground dwelling & herbivore & species & herbivore & herbivore guild \\
\hline \multirow[t]{2}{*}{ Primates } & Omomyidae & Chipetaia lamporea & 44.5 & 44.5 & 7.02 arboreal & insectivore & species & insectivore & NA \\
\hline & & Scenopagus curtidens & 53.5 & 44.55 & 3.292 ground dwelling & insectivore & species & insectivore & NA \\
\hline Acreodi & Mesonychidae & Harpagolestes brevipes & 44.75 & 44.75 & 10.67047207 ground dwelling & carnivore & species & carnivore & carnivore guild \\
\hline \multirow[t]{4}{*}{ Creodonta } & Oxyaenidae & Apataelurus kayi & 44.75 & 44.75 & 9.29 ground dwelling & carnivore & species & carnivore & carnivore guild \\
\hline & Miacidae & Prodaphaenus uintensis & 44.75 & 44.75 & 8.519564844 arboreal & carnivore & species & carnivore & carnivore guild \\
\hline & Sciuravidae & Sciuravus altidens & 44.75 & 44.75 & 4.163808689 ground dwelling & herbivore & species & herbivore & herbivore guild \\
\hline & Ischyromyidae & Thisbemys uintensis & 44.75 & 44.75 & 7.487 ground dwelling & herbivore & species & herbivore & herbivore guild \\
\hline Perissodactyla & Amynodontidae & Amynodon reedi & 44.75 & 44.75 & 13.16958199 ground dwelling & browser & species & herbivore & herbivore guild \\
\hline Perissodactyla & Brontotheriidae & Sphenocoelus hyognathus & 44.75 & 44.75 & 12.94973856 ground dwelling & browser & species & herbivore & herbivore guild \\
\hline Perissodactyla & Brontotheriidae & Sthenodectes incisivum & 44.75 & 44.75 & 14.23634297 ground dwelling & browser & species & herbivore & herbivore guild \\
\hline Artiodactyla & & Achaenodon uintensis & 44.95 & 44.75 & 12.16002871 ground dwelling & grazer, browser & species & herbivore & herbivore guild \\
\hline Perissodactyla & Chalicotheriidae & Eomoropus amarorum & 45.15 & 44.75 & 10.761 ground dwelling & browser & species & herbivore & herbivore guild \\
\hline Acreodi & Mesonychidae & Mesonyx obtusidens & 51.75 & 44.75 & 10.915 ground dwelling & carnivore & species & carnivore & carnivore guild \\
\hline \multirow[t]{2}{*}{ Perissodactyla } & Brontotheriidae & Sthenodectes priscus & 44.95 & 44.95 & 13.479 ground dwelling & browser & species & herbivore & herbivore guild \\
\hline & & Stylinodon mirus & 52.3 & 44.95 & 10.776 ground dwelling & herbivore & species & herbivore & herbivore guild \\
\hline Perissodactyla & Brontotheriidae & Metarhinus parvus & 45.15 & 45.15 & $12.599 \mathrm{NA}$ & carnivore & genus & carnivore & carnivore guild \\
\hline \multirow[t]{2}{*}{ Artiodactyla } & & Achaenodon insolens & 45.15 & 45.15 & 12.68151635 ground dwelling & grazer, browser & species & herbivore & herbivore guild \\
\hline & Ischyromyidae & Pseudotomus littoralis & 45.15 & 45.15 & 6.59899818 ground dwelling & herbivore & species & herbivore & herbivore guild \\
\hline Perissodactyla & Hyracodontidae & Triplopus cubitalis & 45.15 & 45.15 & 9.991543258 ground dwelling & browser & species & herbivore & herbivore guild \\
\hline Cimolesta & Pantolestidae & Pantolestes natans & 47.7 & 45.15 & 9.093625872 amphibious & piscivore, durophage & species & carnivore & carnivore guild \\
\hline Perissodactyla & Equidae & Orohippus sylvaticus & 47.85 & 45.15 & 9.195316179 ground dwelling & browser & species & herbivore & herbivore guild \\
\hline Primates & Notharctidae & Notharctus robustior & 48.1 & 45.15 & 9.058761379 arboreal & omnivore & species & omnivore & NA \\
\hline Artiodactyla & & Achaenodon robustus & 48.25 & 45.15 & 12.57330238 ground dwelling & grazer, browser & species & herbivore & herbivore guild \\
\hline Perissodactyla & Tapiridae & Dilophodon minusculus & 49.25 & 45.15 & 9.026 ground dwelling & browser & species & herbivore & herbivore guild \\
\hline Primates & Omomyidae & Hemiacodon gracilis & 50.45 & 45.15 & 7.107 arboreal & insectivore & species & insectivore & NA \\
\hline \multirow[t]{2}{*}{ Primates } & Microsyopidae & Microsyops annectens & 51.3 & 45.15 & 7.708 arboreal & omnivore & species & omnivore & NA \\
\hline & Ischyromyidae & Leptotomus parvus & 51.45 & 45.15 & 6.843 ground dwelling & herbivore & species & herbivore & herbivore guild \\
\hline \multirow[t]{2}{*}{ Lipotyphla } & Geolabididae & Centetodon bembicophagus & 51.9 & 45.15 & 2.291612289 scansorial & insectivore & species & insectivore & NA \\
\hline & & Scenopagus priscus & 51.9 & 45.15 & 2.554314277 ground dwelling & insectivore & species & insectivore & NA \\
\hline \multirow[t]{2}{*}{ Perissodactyla } & Hyrachyidae & Hyrachyus eximius & 52.1 & 45.15 & 11.7229238 ground dwelling & browser & species & herbivore & herbivore guild \\
\hline & Viverravidae & Viverravus minutus & 52.4 & 45.15 & 6.166 ground dwelling & carnivore & species & carnivore & carnivore guild \\
\hline \multirow[t]{2}{*}{ Perissodactyla } & Brontotheriidae & Telmatherium altidens & 45.25 & 45.25 & 14.44671824 & & & NA & NA \\
\hline & Ischyromyidae & Thisbemys corrugatus & 51.15 & 45.25 & 8.134451964 ground dwelling & herbivore & species & herbivore & herbivore guild \\
\hline \multirow[t]{2}{*}{ Rodentia } & Reithroparamyidae & Reithroparamys delicatissimus & 51.8 & 45.25 & 5.934 ground dwelling & herbivore & species & herbivore & Rodentia \\
\hline & Ischyromyidae & Paramys copei & 55.65 & 45.25 & 6.347 ground dwelling & herbivore & species & herbivore & herbivore guild \\
\hline Primates & Omomyidae & Ageitodendron matthewi & 46.05 & 46.05 & 6.836 arboreal & insectivore & species & insectivore & NA \\
\hline Primates & Omomyidae & Sphacorhysis burntforkensis & 46.05 & 46.05 & 5.138 arboreal & insectivore & species & insectivore & NA \\
\hline Perissodactyla & Brontotheriidae & Palaeosyops laticeps & 46.3 & 46.15 & 12.91363867 ground dwelling & browser & species & herbivore & herbivore guild \\
\hline \multirow[t]{2}{*}{ Artiodactyla } & & Helohyus milleri & 46.3 & 46.15 & 9.862325776 amphibious & herbivore, omnivore & species & omnivore & NA \\
\hline & Metacheiromyidae & Metacheiromys dasypus & 46.45 & 46.15 & 8.566 scansorial & insectivore & species & insectivore & NA \\
\hline Artiodactyla & Dichobunidae & Homacodon vagans & 47.7 & 46.15 & 8.043205138 ground dwelling & herbivore & species & herbivore & herbivore guild \\
\hline Perissodactyla & Brontotheriidae & Palaeosyops robustus & 48.4 & 46.15 & 13.10684724 ground dwelling & browser & species & herbivore & herbivore guild \\
\hline Perissodactyla & Brontotheriidae & Telmatherium manteoceras & 48.55 & 46.15 & 13.08504847 ground dwelling & browser & species & herbivore & herbivore guild \\
\hline Artiodactyla & & Helohyus plicodon & 49.05 & 46.15 & 9.358223654 amphibious & herbivore, omnivore & species & omnivore & NA \\
\hline Creodonta & Oxyaenidae & Patriofelis ferox & 49.25 & 46.15 & 11.082 ground dwelling & carnivore & species & carnivore & carnivore guild \\
\hline Dinocerata & Uintatheriidae & Uintatherium anceps & 49.25 & 46.15 & 14.32539751 ground dwelling & herbivore & species & herbivore & herbivore guild \\
\hline Perissodactyla & & Isectolophus latidens & 49.75 & 46.15 & 10.08701808 ground dwelling & browser & species & herbivore & herbivore guild \\
\hline \multirow[t]{2}{*}{ Perissodactyla } & Hyrachyidae & Hyrachyus affinis & 49.75 & 46.15 & 10.94963225 & & & NA & NA \\
\hline & Sciuravidae & Pauromys schaubi & 50.1 & 46.15 & 2.268 ground dwelling & herbivore & species & herbivore & herbivore guild \\
\hline Primates & Omomyidae & Uintanius ameghini & 50.45 & 46.15 & 5.239 arboreal & insectivore & species & insectivore & NA \\
\hline Artiodactyla & Dichobunidae & Microsus cuspidatus & 50.95 & 46.15 & 7.24 ground dwelling & herbivore & species & herbivore & herbivore guild \\
\hline
\end{tabular}




\begin{tabular}{|c|c|c|c|c|c|c|c|c|c|}
\hline Perissodactyla & Brontotheriidae & Palaeosyops fontinalis & 51.8 & 46.15 & 12.20388927 ground dwelling & browser & species & herbivore & herbivore guild \\
\hline Primates & Omomyidae & Washakius insignis & 52 & 46.15 & 5.581338691 arboreal & insectivore & species & insectivore & NA \\
\hline \multirow[t]{4}{*}{ Primates } & Omomyidae & Trogolemur myodes & 52.5 & 46.15 & 4.958 arboreal & insectivore & species & insectivore & NA \\
\hline & Ischyromyidae & Paramys delicatus & 54.4 & 46.15 & 7.226278366 ground dwelling & herbivore & species & herbivore & herbivore guild \\
\hline & Viverravidae & Viverravus gracilis & 55.1 & 46.15 & 6.836 ground dwelling & carnivore & species & carnivore & carnivore guild \\
\hline & Metacheiromyidae & Metacheiromys marshi & 46.45 & 46.3 & 8.566 scansorial & insectivore & species & insectivore & NA \\
\hline Panameriungulata & Hyopsodontidae & Hyopsodus lepidus & 50 & 46.3 & 6.11 scansorial & omnivore & species & omnivore & NA \\
\hline Eulipotyphla & & Talpavus nitidus & 50.45 & 46.3 & 2.345 scansorial & insectivore & species & insectivore & NA \\
\hline Primates & & Anaptomorphus aemulus & 50.45 & 46.3 & 5.813 arboreal & insectivore & species & insectivore & NA \\
\hline \multirow[t]{2}{*}{ Creodonta } & Hyaenodontidae & Limnocyon verus & 50.45 & 46.3 & 8.853037473 ground dwelling & omnivore & species & omnivore & NA \\
\hline & & Scenopagus edenensis & 51.9 & 46.3 & 4.019 ground dwelling & insectivore & species & insectivore & NA \\
\hline Insectivora & Apatemyidae & Apatemys bellulus & 53.4875 & 46.3 & 3.247 arboreal & herbivore & species & herbivore & herbivore guild \\
\hline \multirow[t]{2}{*}{ Cimolesta } & Cimolestidae & Didelphodus altidens & 54.85 & 46.3 & 4.983 ground dwelling & carnivore & species & carnivore & carnivore guild \\
\hline & Ischyromyidae & Pseudotomus horribilis & 46.45 & 46.45 & 8.91 ground dwelling & herbivore & species & herbivore & herbivore guild \\
\hline Rodentia & Cylindrodontidae & Mysops minimus & 46.45 & 46.45 & 3.92 ground dwelling & herbivore & species & herbivore & Rodentia \\
\hline \multirow[t]{2}{*}{ Eulipotyphla } & Erinaceidae & Entomolestes grangeri & 46.45 & 46.45 & 2.276 semifossorial & insectivore, carnivore & species & insectivore & NA \\
\hline & & Vulpavus profectus & 47.4 & 46.45 & 7.24 arboreal & carnivore, omnivore & species & carnivore & carnivore guild \\
\hline \multirow[t]{2}{*}{ Rodentia } & Cylindrodontidae & Mysops parvus & 48.1 & 46.45 & 3.923 ground dwelling & herbivore & species & herbivore & Rodentia \\
\hline & Sciuravidae & Tillomys parvidens & 48.75 & 46.45 & 1.508 ground dwelling & herbivore & species & herbivore & herbivore guild \\
\hline Cimolesta & Esthonychidae & Trogosus castoridens & 48.95 & 46.45 & 11.693 ground dwelling & herbivore & species & herbivore & herbivore guild \\
\hline Perissodactyla & Brontotheriidae & Palaeosyops laevidens & 49.45 & 46.45 & 12.66191543 ground dwelling & browser & species & herbivore & herbivore guild \\
\hline Perissodactyla & Brontotheriidae & Palaeosyops paludosus & 49.45 & 46.45 & 12.8144486 ground dwelling & browser & species & herbivore & herbivore guild \\
\hline Carnivora & Miacidae & Miacis hargeri & 49.45 & 46.45 & 7.49 & & & NA & carnivore guild \\
\hline Primates & Notharctidae & Notharctus pugnax & 49.45 & 46.45 & 8.641 arboreal & omnivore & species & omnivore & NA \\
\hline Lipotyphla & Geolabididae & Marsholestes dasypelix & 49.55 & 46.45 & 2.416 scansorial & insectivore & species & insectivore & NA \\
\hline Creodonta & Hyaenodontidae & Tritemnodon agilis & 50.45 & 46.45 & 9.28632568 & & & NA & NA \\
\hline Primates & Notharctidae & Notharctus tenebrosus & 50.45 & 46.45 & 8.197 arboreal & omnivore & species & omnivore & NA \\
\hline \multirow[t]{2}{*}{ Creodonta } & Hyaenodontidae & Sinopa rapax & 50.65 & 46.45 & 7.996 ground dwelling & carnivore & species & carnivore & carnivore guild \\
\hline & & Vulpavus palustris & 50.65 & 46.45 & 7.452 arboreal & carnivore, omnivore & species & carnivore & carnivore guild \\
\hline Creodonta & Hyaenodontidae & Thinocyon velox & 50.65 & 46.45 & 7.389650841 & & & NA & NA \\
\hline \multirow[t]{2}{*}{ Perissodactyla } & Equidae & Orohippus pumilus & 50.75 & 46.45 & 10.25340192 ground dwelling & browser & species & herbivore & herbivore guild \\
\hline & Ischyromyidae & Pseudotomus robustus & 51.15 & 46.45 & 8.309 ground dwelling & herbivore & species & herbivore & herbivore guild \\
\hline Primates & Notharctidae & Smilodectes gracilis & 51.3 & 46.45 & 7.694 arboreal & omnivore & species & omnivore & NA \\
\hline \multirow[t]{2}{*}{ Creodonta } & Oxyaenidae & Patriofelis ulta & 51.45 & 46.45 & 10.785 ground dwelling & carnivore & species & carnivore & carnivore guild \\
\hline & & Oodectes herpestoides & 51.75 & 46.45 & 6.215 scansorial & insectivore & species & insectivore & NA \\
\hline Eulipotyphla & Nyctitheriidae & Nyctitherium serotinum & 51.9 & 46.45 & 2.522 scansorial & insectivore & species & insectivore & NA \\
\hline Primates & Microsyopidae & Microsyops elegans & 52 & 46.45 & 7.073 arboreal & omnivore & species & omnivore & NA \\
\hline Carnivora & Miacidae & Miacis parvivorus & 52.1 & 46.45 & 7.486 arboreal & carnivore & species & carnivore & carnivore guild \\
\hline \multirow{2}{*}{ Perissodactyla } & Hyrachyidae & Hyrachyus modestus & 52.2 & 46.45 & 11.33739811 ground dwelling & browser & species & herbivore & herbivore guild \\
\hline & Sciuravidae & Sciuravus nitidus & 52.4 & 46.45 & 4.866 ground dwelling & herbivore & species & herbivore & herbivore guild \\
\hline Rodentia & Cylindrodontidae & Pareumys boskeyi & 46.85 & 46.85 & 4.275 ground dwelling & herbivore & species & herbivore & Rodentia \\
\hline Artiodactyla & Achaenodontidae & Parahyus vagus & 47.15 & 47.15 & 12.166 ground dwelling & grazer, browser & species & herbivore & herbivore guild \\
\hline Panameriungulata & Hyopsodontidae & Hyopsodus minusculus & 52.1 & 47.4 & 5.722 scansorial & omnivore & species & omnivore & NA \\
\hline Acreodi & Mesonychidae & Harpagolestes macrocephalus & 47.55 & 47.55 & 11.9375 ground dwelling & carnivore & species & carnivore & carnivore guild \\
\hline \multirow[t]{2}{*}{ Cimolesta } & Esthonychidae & Tillodon fodiens & 47.55 & 47.55 & 12.014 ground dwelling & herbivore & species & herbivore & herbivore guild \\
\hline & Sciuravidae & Sciuravus bridgeri & 50.65 & 47.55 & 3.719 ground dwelling & herbivore & species & herbivore & herbivore guild \\
\hline Perissodactyla & Equidae & Xenicohippus craspedotum & 53.4875 & 47.55 & 9.36 ground dwelling & browser & species & herbivore & herbivore guild \\
\hline \multirow[t]{2}{*}{ Carnivora } & Miacidae & Miacis sylvestris & 47.7 & 47.7 & 8.358383888 & & & NA & carnivore guild \\
\hline & & Stylinodon inexplicatus & 50.65 & 47.7 & 10.08685282 ground dwelling & herbivore & species & herbivore & herbivore guild \\
\hline \multirow[t]{3}{*}{ Cimolesta } & Pantolestidae & Pantolestes longicaudus & 51.9 & 47.7 & 7.091 & & & NA & NA \\
\hline & Sciuravidae & Tillomys senex & 47.85 & 47.85 & 4.44 ground dwelling & herbivore & species & herbivore & herbivore guild \\
\hline & Sciuravidae & Sciuravus popi & 48.95 & 47.95 & 5.821 ground dwelling & herbivore & species & herbivore & herbivore guild \\
\hline
\end{tabular}




\begin{tabular}{|c|c|c|c|c|c|c|c|}
\hline & Ischyromyidae & Paramys excavatus & 56.8 & 48.1 & 5.547 ground dwelling & herbivore & species \\
\hline Primates & Notharctidae & Smilodectes mcgrewi & 51.75 & 48.25 & 7.86 arboreal & omnivore & species \\
\hline Creodonta & Oxyaenidae & Machaeroides eothen & 48.4 & 48.4 & 8.813550324 ground dwelling & carnivore & species \\
\hline \multirow[t]{3}{*}{ Artiodactyla } & Dichobunidae & Neodiacodexis emryi & 48.55 & 48.55 & 8.536995819 ground dwelling & grazer, browser & species \\
\hline & Sciuravidae & Taxymys lucaris & 49.55 & 48.55 & 3.25920775 ground dwelling & herbivore & species \\
\hline & Ischyromyidae & Acritoparamys wyomingensis & 50.25 & 48.55 & 5.613 ground dwelling & herbivore & species \\
\hline Eulipotyphla & Nyctitheriidae & Nyctitherium velox & 51.45 & 48.55 & 2.751 scansorial & insectivore & species \\
\hline \multirow[t]{3}{*}{ Leptictida } & Leptictidae & Palaeictops bridgeri & 51.9 & 48.75 & 5.424 ground dwelling & insectivore, carnivore & species \\
\hline & & Peradectes chesteri & 57.15 & 48.75 & 1.764 scansorial & omnivore & species \\
\hline & Ischyromyidae & Quadratomus sundelli & 48.95 & 48.95 & 8.832 ground dwelling & herbivore & species \\
\hline Panameriungulata & Hyopsodontidae & Hyopsodus tonksi & 50.25 & 48.95 & 6.142 scansorial & omnivore & species \\
\hline \multirow[t]{3}{*}{ Carnivora } & Miacidae & Miocyon major & 50.45 & 48.95 & 7.607 ground dwelling & carnivore, omnivore & species \\
\hline & Viverravidae & Viverravus sicarius & 51.75 & 48.95 & 7.383 ground dwelling & carnivore & species \\
\hline & Ischyromyidae & Thisbemys perditus & 55.65 & 48.95 & 6.09 ground dwelling & herbivore & species \\
\hline Primates & Omomyidae & Tarka stylifera & 49.25 & 49.25 & 7.475 arboreal & insectivore & species \\
\hline Perissodactyla & Brontotheriidae & Eotitanops borealis & 52.25 & 49.25 & 11.26981321 ground dwelling & browser & species \\
\hline Primates & & Anaptomorphus westi & 50.65 & 49.55 & 6.322 arboreal & insectivore & species \\
\hline \multirow[t]{3}{*}{ Rodentia } & Reithroparamyidae & Reithroparamys huerfanensis & 51.8 & 49.55 & 5.821 ground dwelling & herbivore & species \\
\hline & Ischyromyidae & Microparamys sambucus & 49.75 & 49.75 & 3.628 ground dwelling & herbivore & species \\
\hline & Sciuravidae & Pauromys exallos & 49.75 & 49.75 & 2.926 ground dwelling & herbivore & species \\
\hline Rodentia & Zapodidae & Elymys complexus & 49.75 & 49.75 & 1.656 ground dwelling & herbivore & species \\
\hline \multirow[t]{2}{*}{ Perissodactyla } & & Fouchia elyensis & 49.75 & 49.75 & 7.799 ground dwelling & browser & species \\
\hline & Epoicotheriidae & Tetrapassalus mckennai & 49.75 & 49.75 & 1.618 scansorial & insectivore & species \\
\hline \multirow[t]{2}{*}{ Artiodactyla } & Diacodexeidae & Diacodexis secans & 54.3 & 49.8 & 7.53 ground dwelling & herbivore & species \\
\hline & Ischyromyidae & Quadratomus grossus & 49.95 & 49.95 & 8.271 ground dwelling & herbivore & species \\
\hline \multirow[t]{4}{*}{ Rodentia } & Cylindrodontidae & Mysops fraternus & 50.1 & 50.1 & 3.068056481 ground dwelling & herbivore & species \\
\hline & Sciuravidae & Knightomys huerfanensis & 52.1 & 50.1 & 4.683 ground dwelling & herbivore & species \\
\hline & Sciuravidae & Knightomys depressus & 54.9 & 50.1 & 4.173 ground dwelling & herbivore & species \\
\hline & Sciuravidae & Guanajuatomys hibbardi & 50.15 & 50.15 & 5.116 ground dwelling & herbivore & species \\
\hline Primates & Notharctidae & Notharctus robinsoni & 52 & 50.25 & 8.479 arboreal & omnivore & species \\
\hline Creodonta & Hyaenodontidae & Proviverroides piercei & 50.45 & 50.45 & 8.902 ground dwelling & carnivore & species \\
\hline Primates & Omomyidae & Utahia kayi & 50.45 & 50.45 & 5.061 arboreal & insectivore & species \\
\hline \multirow[t]{2}{*}{ Perissodactyla } & Brontotheriidae & Eotitanops minimus & 51.8 & 50.45 & 11.06766097 ground dwelling & browser & species \\
\hline & & Armintodelphys dawsoni & 52.1 & 50.45 & 1.92554344 scansorial & omnivore & species \\
\hline \multirow[t]{2}{*}{ Perissodactyla } & Palaeotheriidae & Hyracotherium vasacciense & 53.7 & 50.45 & 8.805428415 ground dwelling & frugivore, folivore & species \\
\hline & Ischyromyidae & Quadratomus grandis & 51.75 & 50.65 & 7.429 ground dwelling & herbivore & species \\
\hline Cimolesta & Esthonychidae & Trogosus latidens & 51.8 & 50.65 & 12.629 ground dwelling & herbivore & species \\
\hline Primates & Omomyidae & Uintanius rutherfurdi & 51.9 & 50.65 & 5.31 arboreal & insectivore & species \\
\hline Primates & Omomyidae & Trogolemur amplior & 52.1 & 50.65 & 5.451 arboreal & insectivore & species \\
\hline Dinocerata & Uintatheriidae & Bathyopsis fissidens & 52.2 & 50.65 & 11.897 ground dwelling & herbivore & species \\
\hline Artiodactyla & Diacodexeidae & Diacodexis woltonensis & 50.9375 & 50.9375 & 7.356534544 ground dwelling & grazer, browser & species \\
\hline Primates & Omomyidae & Hemiacodon casamissus & 50.9375 & 50.9375 & 6.436 arboreal & insectivore & species \\
\hline Primates & Omomyidae & Loveina wapitiensis & 50.9375 & 50.9375 & 5.694 arboreal & insectivore & species \\
\hline \multirow[t]{2}{*}{ Primates } & Paromomyidae & Elwynella oreas & 51.8 & 50.9375 & 5.844 arboreal & omnivore & species \\
\hline & & Armintodelphys blacki & 51.9 & 50.9375 & 2.002 scansorial & omnivore & species \\
\hline Primates & Omomyidae & Artimonius witteri & 52 & 50.9375 & 5.939 arboreal & insectivore & species \\
\hline Creodonta & Oxyaenidae & Machaeroides simpsoni & 52.1 & 50.9375 & 8.335794895 ground dwelling & carnivore & species \\
\hline \multirow[t]{2}{*}{ Phenacodonta } & Phenacodontidae & Ectocion superstes & 52.1 & 50.9375 & 9.148 ground dwelling & herbivore & species \\
\hline & Epoicotheriidae & Dipassalus oryctes & 52.1 & 50.9375 & 4.317488114 scansorial & insectivore & species \\
\hline Primates & Omomyidae & Shoshonius cooperi & 52.2 & 50.9375 & 5.399 arboreal & insectivore & species \\
\hline Artiodactyla & Diacodexeidae & Diacodexis minutus & 52.3 & 50.9375 & 7.008 ground dwelling & herbivore & species \\
\hline Perissodactyla & Equidae & Protorohippus venticolus & 52.3 & 50.9375 & 9.039 & & \\
\hline
\end{tabular}

\begin{tabular}{|c|c|}
\hline herbivore & herbivore guild \\
\hline omnivore & NA \\
\hline carnivore & carnivore guild \\
\hline herbivore & herbivore guild \\
\hline herbivore & herbivore guild \\
\hline herbivore & herbivore guild \\
\hline insectivore & NA \\
\hline insectivore & NA \\
\hline omnivore & NA \\
\hline herbivore & herbivore guild \\
\hline omnivore & NA \\
\hline carnivore & carnivore guild \\
\hline carnivore & carnivore guild \\
\hline herbivore & herbivore guild \\
\hline insectivore & NA \\
\hline herbivore & herbivore guild \\
\hline insectivore & NA \\
\hline herbivore & Rodentia \\
\hline herbivore & herbivore guild \\
\hline herbivore & herbivore guild \\
\hline herbivore & Rodentia \\
\hline herbivore & herbivore guild \\
\hline insectivore & NA \\
\hline herbivore & herbivore guild \\
\hline herbivore & herbivore guild \\
\hline herbivore & Rodentia \\
\hline herbivore & herbivore guild \\
\hline herbivore & herbivore guild \\
\hline herbivore & herbivore guild \\
\hline omnivore & NA \\
\hline carnivore & carnivore guild \\
\hline insectivore & NA \\
\hline herbivore & herbivore guild \\
\hline omnivore & NA \\
\hline herbivore & herbivore guild \\
\hline herbivore & herbivore guild \\
\hline herbivore & herbivore guild \\
\hline insectivore & NA \\
\hline insectivore & NA \\
\hline herbivore & herbivore guild \\
\hline herbivore & herbivore guild \\
\hline insectivore & NA \\
\hline insectivore & NA \\
\hline omnivore & NA \\
\hline omnivore & NA \\
\hline insectivore & NA \\
\hline carnivore & carnivore guild \\
\hline herbivore & herbivore guild \\
\hline insectivore & NA \\
\hline insectivore & NA \\
\hline herbivore & herbivore guild \\
\hline NA & NA \\
\hline
\end{tabular}




\begin{tabular}{|c|c|c|}
\hline Panameriungulata & Hyopsodontidae & Hyopsodus walcottianus \\
\hline Artiodactyla & Dichobunidae & Bunophorus sinclairi \\
\hline Primates & Microsyopidae & Microsyops scottianus \\
\hline Primates & Notharctidae & Notharctus venticolus \\
\hline Leptictida & Leptictidae & Palaeictops multicuspis \\
\hline Creodonta & Hyaenodontidae & Prolimnocyon antiquus \\
\hline Perissodactyla & & Heptodon posticus \\
\hline \multirow[t]{2}{*}{ Primates } & Notharctidae & Cantius nunienus \\
\hline & Ischyromyidae & Mattimys kalicola \\
\hline Cimolesta & Esthonychidae & Esthonyx acutidens \\
\hline Perissodactyla & Brontotheriidae & Lambdotherium popoagicum \\
\hline Primates & Omomyidae & Loveina zephyri \\
\hline Panameriungulata & Hyopsodontidae & Hyopsodus wortmani \\
\hline Carnivora & Miacidae & Miacis latidens \\
\hline Perissodactyla & & Heptodon calciculus \\
\hline \multirow[t]{3}{*}{ Primates } & Omomyidae & Absarokius abbotti \\
\hline & & Vulpavus canavus \\
\hline & Viverravidae & Didymictis altidens \\
\hline Cimolodonta & Neoplagiaulacidae & Ectypodus childei \\
\hline \multirow{4}{*}{ Cimolesta } & Pantolestidae & Palaeosinopa veterrima \\
\hline & Ischyromyidae & Acritoparamys francesi \\
\hline & & Vulpavus australis \\
\hline & Viverravidae & Viverravus lutosus \\
\hline Carnivora & Miacidae & Miacis exiguus \\
\hline Macroscelidea & Amphilemuridae & Macrocranion nitens \\
\hline Phenacodonta & Phenacodontidae & Phenacodus intermedius \\
\hline Phenacodonta & Phenacodontidae & Phenacodus vortmani \\
\hline \multirow[t]{2}{*}{ Cimolesta } & Pantolestidae & Palaeosinopa didelphoides \\
\hline & Arctocyonidae & Thryptacodon antiquus \\
\hline Primates & Omomyidae & Aycrossia lovei \\
\hline Primates & Omomyidae & Strigorhysis huerfanensis \\
\hline Cimolesta & Esthonychidae & Trogosus grangeri \\
\hline Primates & Microsyopidae & Megadelphus lundeliusi \\
\hline Primates & Omomyidae & Artimonius australis \\
\hline Primates & Omomyidae & Absarokius metoecus \\
\hline Primates & Omomyidae & Washakius izetti \\
\hline Primates & Omomyidae & Artimonius nocerai \\
\hline \multirow[t]{2}{*}{ Primates } & Microsyopidae & Microsyops knightensis \\
\hline & Ischyromyidae & Thisbemys elachistos \\
\hline Carnivora & Miacidae & Uintacyon asodes \\
\hline Cimolesta & Coryphodontidae & Coryphodon armatus \\
\hline \multirow[t]{2}{*}{ Creodonta } & Hyaenodontidae & Tritemnodon strenuus \\
\hline & Sciuravidae & Knightomys cuspidatus \\
\hline Primates & Omomyidae & Shoshonius bowni \\
\hline Primates & Omomyidae & Strigorhysis bridgerensis \\
\hline \multirow[t]{2}{*}{ Primates } & Microsyopidae & Alveojunctus minutus \\
\hline & Sciuravidae & Sciuravus wilsoni \\
\hline Artiodactyla & & Hexacodus pelodes \\
\hline \multirow[t]{2}{*}{ Panameriungulata } & Hyopsodontidae & Hyopsodus powellianus \\
\hline & Sciuravidae & Knightomys senior \\
\hline \multirow[t]{2}{*}{ Cimolesta } & Esthonychidae & Megalesthonyx hopsoni \\
\hline & Ischyromyidae & Lophiparamys murinus \\
\hline
\end{tabular}

\begin{tabular}{|c|c|c|c|c|}
\hline 52.3 & 50.9375 & 7.99 scansorial & omnivore & species \\
\hline 52.4 & 50.9375 & 9.075025613 ground dwelling & herbivore & species \\
\hline 52.45 & 50.9375 & 7.281 arboreal & omnivore & species \\
\hline 52.5 & 50.9375 & 8.439 arboreal & omnivore & species \\
\hline 52.8 & 50.9375 & 5.491 ground dwelling & insectivore, carnivore & species \\
\hline 3.4875 & 50.9375 & 7.282 scansorial & carnivore & species \\
\hline 53.4875 & 50.9375 & 10.413 ground dwelling & browser & species \\
\hline 3.4875 & 50.9375 & 7.492 arboreal & omnivore & species \\
\hline 53.65 & 50.9375 & 3.448 ground dwelling & herbivore & species \\
\hline 53.7 & 50.9375 & 9.195 ground dwelling & herbivore & species \\
\hline 53.7 & 50.9375 & 9.726 ground dwelling & browser & species \\
\hline 53.7 & 50.9375 & 5.525 arboreal & insectivore & species \\
\hline 53.7 & 50.9375 & 6.31 scansorial & omnivore & species \\
\hline 53.8 & 50.9375 & 7.235 arboreal & carnivore & species \\
\hline 53.8 & 50.9375 & 9.652 ground dwelling & browser & species \\
\hline 53.8 & 50.9375 & 6.023 arboreal & insectivore & species \\
\hline 53.9 & 50.9375 & 7.24 arboreal & carnivore, omnivore & species \\
\hline 54.3 & 50.9375 & 8.519 ground dwelling & carnivore & species \\
\hline 54.65 & 50.9375 & 2.929437913 arboreal & omnivore & species \\
\hline 54.9 & 50.9375 & 8.03 amphibious & piscivore, durophage & species \\
\hline 55.25 & 50.9375 & 4.86 ground dwelling & herbivore & species \\
\hline 55.3 & 0.9375 & 7.027 arboreal & carnivore, omnivore & species \\
\hline 55.65 & 50.9375 & 6.231 ground dwelling & carnivore & species \\
\hline 55.7 & 50.9375 & 6.884 arboreal & carnivore & species \\
\hline 55.8 & 50.9375 & 3.365 ground dwelling & insecti & species \\
\hline 57.6 & 50.9375 & 10.62656204 ground dwelling & herbivore & species \\
\hline 58.1 & 50.9375 & 9.393233047 ground dwelling & herbivore & species \\
\hline 59.4 & 50.9375 & 7.049 amphibious & piscivore, durophage & species \\
\hline 59.8 & 50.9375 & 7.946 ground dwelling & omnivore & species \\
\hline 51.9 & 51.15 & 6.022 arboreal & insectivore & species \\
\hline 51.3 & 51.3 & $6.312 \mathrm{ar}$ & insect & species \\
\hline 51.75 & 51.3 & 11.925 ground dwelling & herbiv & species \\
\hline 52.1 & 51.3 & 7.011 arboreal & omnivore & species \\
\hline 51.45 & 51.45 & 6.113725948 arboreal & insectivore & species \\
\hline 54.25 & 51.45 & 5.957 arboreal & insectivore & species \\
\hline 51.75 & 51.65 & 5.435 arboreal & insectivore & species \\
\hline 51.95 & 51.65 & 4.655486644 arboreal & insectivore & species \\
\hline 52.6 & 51.65 & 6.949 arboreal & omniv & species \\
\hline 51.75 & 51.75 & 4.847 ground dwelling & herbiv & species \\
\hline 3.4875 & 51.75 & 8.148 arb & re, omnivore & species \\
\hline 54.45 & 51.75 & 13.07 ground dwelling & herbivore & species \\
\hline 54.6 & 51.75 & 8.697817666 ground dwelling & carnivore & species \\
\hline 51.8 & 51.8 & 3.877 ground dwelling & herbivore & species \\
\hline 51.8 & 51.8 & 5.619 arboreal & insectivore & species \\
\hline 51.8 & 51.8 & 5.769 arboreal & insectivore & species \\
\hline 51.8 & 51.8 & 4.743 arboreal & insectivore, omnivore & species \\
\hline 52.2 & 51.8 & 4.435 ground dwelling & herbivore & species \\
\hline 52.9 & 51.8 & 7.399035623 ground dwelling & herbivore & species \\
\hline 55.65 & 51.8 & 7.321 scansorial & omnivore & species \\
\hline 52.5 & 51.9 & 3.793 ground dwelling & herbivore & species \\
\hline 54 & 51.9 & 9.732030921 ground dwelling & herbivore & species \\
\hline 53.6 & 52 & 4.476 ground dwelling & herbivore & species \\
\hline
\end{tabular}

\begin{tabular}{|c|c|}
\hline omnivore & NA \\
\hline herbivore & herbivore guild \\
\hline omnivore & NA \\
\hline omnivore & NA \\
\hline insectivore & NA \\
\hline carnivore & carnivore guild \\
\hline herbivore & herbivore guild \\
\hline omnivore & NA \\
\hline herbivore & herbivore guild \\
\hline herbivore & herbivore guild \\
\hline herbivore & herbivore guild \\
\hline insectivore & NA \\
\hline omnivore & NA \\
\hline carnivore & carnivore guild \\
\hline herbivore & herbivore guild \\
\hline insectivore & NA \\
\hline carnivore & carnivore guild \\
\hline carnivore & carnivore guild \\
\hline omnivore & NA \\
\hline carnivore & carnivore guild \\
\hline herbivore & herbivore guild \\
\hline carnivore & carnivore guild \\
\hline carnivore & carnivore guild \\
\hline carnivore & carnivore guild \\
\hline insectivore & NA \\
\hline herbivore & herbivore guild \\
\hline herbivore & herbivore guild \\
\hline carnivore & carnivore guild \\
\hline omnivore & NA \\
\hline insectivore & NA \\
\hline insectivore & NA \\
\hline herbivore & herbivore guild \\
\hline omnivore & NA \\
\hline insectivore & NA \\
\hline insectivore & NA \\
\hline insectivore & NA \\
\hline insectivore & NA \\
\hline omnivore & NA \\
\hline herbivore & herbivore guild \\
\hline carnivore & carnivore guild \\
\hline herbivore & herbivore guild \\
\hline carnivore & carnivore guild \\
\hline herbivore & herbivore guild \\
\hline insectivore & NA \\
\hline insectivore & NA \\
\hline omnivore & NA \\
\hline herbivore & herbivore guild \\
\hline herbivore & herbivore guild \\
\hline omnivore & NA \\
\hline herbivore & herbivore guild \\
\hline herbivore & herbivore guild \\
\hline herbivore & herbivore guild \\
\hline
\end{tabular}




\begin{tabular}{|c|c|c|c|c|c|c|c|c|c|}
\hline Leptictida & Leptictidae & Palaeictops bicuspis & 55.65 & 52 & 6.346560649 ground dwelling & insectivore, carnivore & species & insectivore & NA \\
\hline Primates & Notharctidae & Cantius frugivorus & 55.65 & 52 & 7.67 arboreal & omnivore & species & omnivore & NA \\
\hline Primates & Microsyopidae & Microsyops angustidens & 55.7 & 52 & 6.693 arboreal & omnivore & species & omnivore & NA \\
\hline Cimolesta & Cimolestidae & Didelphodus absarokae & 55.95 & 52 & 5.327 ground dwelling & carnivore & species & carnivore & carnivore guild \\
\hline Phenacodonta & Phenacodontidae & Meniscotherium chamense & 56.05 & 52 & 8.503213982 ground dwelling & herbivore & species & herbivore & herbivore guild \\
\hline Creodonta & Oxyaenidae & Malfelis badwaterensis & 52.1 & 52.1 & 11.68175573 ground dwelling & carnivore & species & carnivore & carnivore guild \\
\hline Artiodactyla & & Antiacodon vanvaleni & 52.1 & 52.1 & 7.649692624 ground dwelling & grazer, browser & species & herbivore & herbivore guild \\
\hline Perissodactyla & & Selenaletes scopaeus & 52.1 & 52.1 & 8.518 ground dwelling & browser & species & herbivore & herbivore guild \\
\hline Primates & Omomyidae & Anemorhysis natronensis & 52.1 & 52.1 & 4.954 arboreal & insectivore & species & insectivore & NA \\
\hline \multirow[t]{3}{*}{ Primates } & Omomyidae & Trogolemur fragilis & 52.1 & 52.1 & 4.816724885 arboreal & insectivore & species & insectivore & NA \\
\hline & Epoicotheriidae & Tubulodon taylori & 52.1 & 52.1 & 5.078649596 scansorial & insectivore & species & insectivore & NA \\
\hline & Epoicotheriidae & Tubulodon woodi & 53.4875 & 52.1 & 6.717402703 scansorial & insectivore & species & insectivore & NA \\
\hline Proboscidea & Numidotheriidae & Numidotherium koholense & 52.2 & 52.2 & 13.23211424 ground dwelling & grazer, browser & species & herbivore & herbivore guild \\
\hline Carnivora & Miacidae & Miacis latouri & 52.2 & 52.2 & 5.475344102 & & & NA & carnivore guild \\
\hline Panameriungulata & Hyopsodontidae & Hyopsodus mentalis & 52.85 & 52.2 & 6.898 & & & NA & NA \\
\hline Creodonta & Oxyaenidae & Oxyaena lupina & 53.1 & 52.2 & 9.790364592 ground dwelling & carnivore & species & carnivore & carnivore guild \\
\hline Creodonta & Hyaenodontidae & Gazinocyon vulpeculus & 53.4875 & 52.2 & 7.573 ground dwelling & carnivore & species & carnivore & carnivore guild \\
\hline Acreodi & Hapalodectidae & Hapalodectes leptognathus & 53.8 & 52.2 & 5.415 ground dwelling & carnivore & species & carnivore & carnivore guild \\
\hline Artiodactyla & Dichobunidae & Bunophorus etsagicus & 54.2 & 52.2 & 8.978 ground dwelling & herbivore & species & herbivore & herbivore guild \\
\hline Creodonta & Oxyaenidae & Oxyaena forcipata & 54.3 & 52.2 & 11.057 ground dwelling & carnivore & species & carnivore & carnivore guild \\
\hline Phenacodonta & Phenacodontidae & Meniscotherium tapiacitum & 55.4 & 52.2 & 7.354 ground dwelling & herbivore & species & herbivore & herbivore guild \\
\hline \multirow[t]{4}{*}{ Macroscelidea } & Amphilemuridae & Macrocranion junnei & 55.6 & 52.2 & 3.889742938 ground dwelling & insectivore & species & insectivore & NA \\
\hline & Viverravidae & Didymictis leptomylus & 55.7 & 52.2 & 8.134 ground dwelling & carnivore & species & carnivore & carnivore guild \\
\hline & Ischyromyidae & Acritoparamys atwateri & 55.7 & 52.2 & 5.073 ground dwelling & herbivore & species & herbivore & herbivore guild \\
\hline & & Mimoperadectes labrus & 56.1 & 52.2 & 4.826 scansorial & omnivore & species & omnivore & NA \\
\hline Primates & Microsyopidae & Niptomomys doreenae & 57.25 & 52.2 & 4.249 arboreal & omnivore & species & omnivore & NA \\
\hline \multirow[t]{3}{*}{ Primates } & Paromomyidae & Phenacolemur praecox & 57.25 & 52.2 & 6.230467199 arboreal & omnivore & species & omnivore & NA \\
\hline & Viverravidae & Viverravus laytoni & 57.9 & 52.2 & 5.283 ground dwelling & carnivore & species & carnivore & carnivore guild \\
\hline & & Peradectes protinnominatus & 59.4 & 52.2 & 2.195 scansorial & omnivore & species & omnivore & NA \\
\hline Artiodactyla & Dichobunidae & Bunophorus macropternus & 53.4875 & 52.25 & 8.567 ground dwelling & herbivore & species & herbivore & herbivore guild \\
\hline Perissodactyla & Palaeotheriidae & Hyracotherium index & 56.05 & 52.25 & 8.329 & & & NA & NA \\
\hline Primates & Notharctidae & Pelycodus danielsae & 52.3 & 52.3 & 8.748304912 arboreal & herbivore & species & herbivore & herbivore guild \\
\hline Panameriungulata & Hyopsodontidae & Hyopsodus minor & 53.8 & 52.3 & 5.491 scansorial & omnivore & species & omnivore & NA \\
\hline Panameriungulata & Hyopsodontidae & Hyopsodus lysitensis & 55.65 & 52.3 & 6.422 scansorial & omnivore & species & omnivore & NA \\
\hline Primates & Paromomyidae & Simpsonlemur jepseni & 55.7 & 52.3 & 5.45859143 arboreal & omnivore & species & omnivore & NA \\
\hline Primates & Notharctidae & Pelycodus jarrovii & 57.3 & 52.3 & 8.505 arboreal & herbivore & species & herbivore & herbivore guild \\
\hline \multirow[t]{2}{*}{ Primates } & Omomyidae & Anemorhysis wortmani & 52.8 & 52.4 & 5.183 arboreal & insectivore & species & insectivore & NA \\
\hline & Ischyromyidae & Acritoparamys pattersoni & 53.85 & 52.4 & 6.072 ground dwelling & herbivore & species & herbivore & herbivore guild \\
\hline Cimolesta & Pantolestidae & Palaeosinopa lutreola & 55.3 & 52.4 & 5.792 amphibious & piscivore, durophage & species & carnivore & carnivore guild \\
\hline Primates & Microsyopidae & Microsyops latidens & 55.65 & 52.45 & 6.684 arboreal & omnivore & species & omnivore & NA \\
\hline Acreodi & & Wyolestes dioctes & 52.5 & 52.5 & 6.854 scansorial & carnivore & species & carnivore & carnivore guild \\
\hline Rodentia & Cylindrodontidae & Dawsonomys woodi & 52.5 & 52.5 & 4.522 ground dwelling & herbivore & species & herbivore & Rodentia \\
\hline Primates & Omomyidae & Anemorhysis sublettensis & 52.5 & 52.5 & 4.714 arboreal & insectivore & species & insectivore & NA \\
\hline \multirow[t]{2}{*}{ Primates } & & Chlororhysis knightensis & 52.5 & 52.5 & 4.682 arboreal & insectivore & species & insectivore & NA \\
\hline & Epoicotheriidae & Tubulodon pearcei & 52.5 & 52.5 & 5.992430974 scansorial & insectivore & species & insectivore & NA \\
\hline Didelphimorphia & Herpetotheriidae & Peratherium edwardi & 52.5 & 52.5 & 5.132753415 ground dwelling & omnivore & species & omnivore & NA \\
\hline Creodonta & Oxyaenidae & Ambloctonus major & 52.7 & 52.5 & 10.2619894 NA & NA & species & NA & NA \\
\hline Primates & Omomyidae & Loveina minuta & 53.4875 & 52.5 & 5.206 arboreal & insectivore & species & insectivore & NA \\
\hline Cimolesta & Coryphodontidae & Coryphodon lobatus & 54.2 & 52.5 & 13.5949178 ground dwelling & herbivore & species & herbivore & herbivore guild \\
\hline Cimolesta & Coryphodontidae & Coryphodon radians & 54.7 & 52.5 & 13.385 ground dwelling & herbivore & species & herbivore & herbivore guild \\
\hline Primates & Omomyidae & Jemezius szalayi & 52.6 & 52.6 & 5.672 arboreal & insectivore & species & insectivore & NA \\
\hline Creodonta & Oxyaenidae & Ambloctonus sinosus & 53.4875 & 52.6 & $11.37772016 \mathrm{NA}$ & NA & species & NA & NA \\
\hline
\end{tabular}




\begin{tabular}{|c|c|c|}
\hline Perissodactyla & Palaeotheriidae & Hyracotherium pernix \\
\hline Cimolesta & Esthonychidae & Esthonyx bisulcatus \\
\hline Perissodactyla & & Sifrhippus grangeri \\
\hline Perissodactyla & Equidae & Xenicohippus grangeri \\
\hline Primates & Notharctidae & Cantius abditus \\
\hline Artiodactyla & $\begin{array}{l}\text { Dichobunidae } \\
\text { Viverravidae }\end{array}$ & $\begin{array}{l}\text { Bunophorus robustus } \\
\text { Viverravus acutus }\end{array}$ \\
\hline Artiodactyla & Dichobunidae & Bunophorus pattersoni \\
\hline Primates & Notharctidae & Copelemur australotutus \\
\hline Creodonta & $\begin{array}{l}\text { Hyaenodontidae } \\
\text { Sciuravidae }\end{array}$ & $\begin{array}{l}\text { Prototomus secundarius } \\
\text { Knightomys minor }\end{array}$ \\
\hline Artiodactyla & $\begin{array}{l}\text { Diacodexeidae } \\
\text { Viverravidae }\end{array}$ & $\begin{array}{l}\text { Diacodexis metsiacus } \\
\text { Didymictis protenus } \\
\text { Ectoganus gliriformis }\end{array}$ \\
\hline Insectivora & Apatemyidae & Labidolemur kayi \\
\hline Phenacodonta & $\begin{array}{l}\text { Phenacodontidae } \\
\text { Ischyromyidae }\end{array}$ & $\begin{array}{l}\text { Copecion brachypternus } \\
\text { Paramys nini }\end{array}$ \\
\hline Perissodactyla & Isectolophidae & Systemodon tapirinus \\
\hline Primates & $\begin{array}{l}\text { Notharctidae } \\
\text { Sciuravidae }\end{array}$ & $\begin{array}{l}\text { Cantius angulatus } \\
\text { Knightomys reginensis }\end{array}$ \\
\hline Primates & Notharctidae & Copelemur tutus \\
\hline Artiodactyla & Dichobunidae & Bunophorus grangeri \\
\hline \multirow[t]{2}{*}{ Artiodactyla } & & Simpsonodus chacensis \\
\hline & Arctocyonidae & Chriacus gallinae \\
\hline Perissodactyla & & Homogalax protapirinus \\
\hline \multirow[t]{2}{*}{ Primates } & Notharctidae & Cantius mckennai \\
\hline & Plagiomenidae & Ellesmene eureka \\
\hline Primates & Paromomyidae & Phenacolemur fortior \\
\hline Primates & Omomyidae & Absarokius gazini \\
\hline Acreodi & Mesonychidae & Pachyaena gigantea \\
\hline Acreodi & Mesonychidae & Pachyaena gracilis \\
\hline Rodentia & Paramyidae & Franimys amherstensis \\
\hline Primates & Microsyopidae & Arctodontomys wilsoni \\
\hline Creodonta & Oxyaenidae & Palaeonictis occidentalis \\
\hline Primates & Notharctidae & Copelemur praetutus \\
\hline Primates & Omomyidae & Steinius vespertinus \\
\hline Acreodi & Mesonychidae & Pachyaena ossifraga \\
\hline Cimolesta & Pantolestidae & Palaeosinopa incerta \\
\hline Panameriungulata & Hyopsodontidae & Hyopsodus loomisi \\
\hline Macroscelidea & Apheliscidae & Haplomylus speirianus \\
\hline Leptictida & Leptictidae & Prodiacodon tauricinerei \\
\hline Lipotyphla & Geolabididae & Centetodon neashami \\
\hline \multirow[t]{2}{*}{ Primates } & Microsyopidae & Microsyops cardiorestes \\
\hline & Metacheiromyidae & Palaeanodon ignavus \\
\hline \multirow[t]{2}{*}{ Creodonta } & Hyaenodontidae & Prolimnocyon atavus \\
\hline & Viverravidae & Viverravus politus \\
\hline Carnivora & Miacidae & Vassacyon promicrodon \\
\hline Perissodactyla & Palaeotheriidae & Hyracotherium aemulor \\
\hline \multirow[t]{2}{*}{ Creodonta } & Hyaenodontidae & Prototomus robustus \\
\hline & Arctocyonidae & Anacodon ursidens \\
\hline Primates & Microsyopidae & Niptomomys thelmae \\
\hline Perissodacty & & Cardiolophus semihians \\
\hline
\end{tabular}

\begin{tabular}{|c|c|c|c|c|}
\hline 54.6 & 52.6 & 9.104979856 & & \\
\hline 55.65 & 52.6 & 8.818 ground dwelling & herbivore & species \\
\hline 55.7 & 52.6 & 8.845 ground dwelling & browser & species \\
\hline 55.65 & 52.7 & 8.866 ground dwelling & browser & species \\
\hline 55.65 & 52.7 & 8.015 arboreal & omnivore & species \\
\hline 55.7 & 52.7 & 8.149 ground dwelling & herbivore & species \\
\hline 56.8 & 52.7 & 5.989 ground dwelling & carnivore & species \\
\hline 58.55 & 52.7 & 8.16241514 ground dwelling & herbivore & species \\
\hline 52.75 & 52.75 & 7.796 arboreal & omnivore & species \\
\hline 55.7 & 52.75 & 7.787278609 ground dwelling & carnivore & species \\
\hline 55.2 & 52.8 & 3.539 ground dwelling & herbivore & species \\
\hline 55.65 & 52.8 & 7.22 ground dwelling & herbivore & species \\
\hline 57 & 52.8 & 9.076 ground dwelling & carnivore & species \\
\hline 58.1 & 52.8 & 10.844 ground dwelling & herbivore & species \\
\hline 59 & 52.8 & 3.267 arboreal & herbivore & species \\
\hline 59.8 & 52.8 & 8.396 ground dwelling & herbivore & species \\
\hline 52.85 & 52.85 & 5.408538237 ground dwelling & herbivore & species \\
\hline 52.85 & 52.85 & 9.659220954 ground dwelling & browser & species \\
\hline 52.85 & 52.85 & 7.096 arboreal & omnivore & species \\
\hline 53.65 & 52.85 & 2.285 ground dwelling & herbivore & species \\
\hline 53.65 & 52.85 & 8.258 arboreal & omnivore & species \\
\hline 54.5 & 52.85 & 9.106346283 ground dwelling & herbivore & species \\
\hline 55.65 & 52.85 & 7.844 ground dwelling & herbivore & species \\
\hline 55.7 & 52.9 & 7.295 scansorial & omnivore & species \\
\hline 55.8 & 52.9 & 9.395 ground dwelling & browser & species \\
\hline 55.95 & 52.9 & 7.474 arboreal & omnivore & species \\
\hline 53.05 & 53.05 & 6.726 scansorial & insectivore & species \\
\hline 53.05 & 53.05 & 6.325167525 arboreal & omnivore & species \\
\hline 53.1 & 53.05 & 5.735 arboreal & insectivore & species \\
\hline 55.1 & 53.05 & 11.31080928 ground dwelling & carnivore & species \\
\hline 55.25 & 53.05 & 10.427 ground dwelling & carnivore & species \\
\hline 56.1 & 53.05 & 4.276982106 ground dwelling & herbivore & species \\
\hline 56.1 & 53.05 & 6.192 arboreal & omnivore & species \\
\hline 58.1 & 53.05 & 10.53 ground dwelling & carnivore & species \\
\hline 53.1 & 53.1 & 7.414 arboreal & omnivore & species \\
\hline 53.25 & 53.1 & 5.952 arboreal & insectivore & species \\
\hline 55.45 & 53.1 & 11.08214255 ground dwelling & carnivore & species \\
\hline 55.7 & 53.1 & 6.723 amphibious & piscivore, durophage & species \\
\hline 55.7 & 53.1 & 5.942 scansorial & omnivore & species \\
\hline 55.95 & 53.1 & 4.897 ground dwelling & insectivore & species \\
\hline 56.9 & 53.1 & 4.817 ground dwelling & insectivore, carnivore & species \\
\hline 54 & 53.2 & 3.97 scansorial & insectivore & species \\
\hline 54.05 & 53.2 & 6.368 arboreal & omnivore & species \\
\hline 55.1 & 53.2 & 7.679740173 scansorial & insectivore & species \\
\hline 56.8 & 53.2 & 7.300905537 scansorial & carnivore & species \\
\hline 57.7 & 53.2 & 6.973 ground dwelling & carnivore & species \\
\hline 54.9 & 53.25 & 8.134 scansorial & carnivore, omnivore & species \\
\hline 55.1 & 53.25 & 9.286406304 & & \\
\hline 55.8 & 53.25 & 8.245129363 ground dwelling & carnivore & species \\
\hline 59.4 & 53.25 & 10.72335831 ground dwelling & omnivore & species \\
\hline .4875 & 53.3 & 4.543 arboreal & omnivore & species \\
\hline 54.2 & 53.3 & 9.395 ground dwelling & browser & species \\
\hline
\end{tabular}

$\begin{array}{ll}\text { NA } & \text { NA } \\ \text { herbivore } & \text { herbivore guild } \\ \text { herbivore } & \text { herbivore guild } \\ \text { herbivore } & \text { herbivore guild } \\ \text { omnivore } & \text { NA } \\ \text { herbivore } & \text { herbivore guild } \\ \text { carnivore } & \text { carnivore guild } \\ \text { herbivore } & \text { herbivore guild } \\ \text { omnivore } & \text { NA } \\ \text { carnivore } & \text { carnivore guild } \\ \text { herbivore } & \text { herbivore guild } \\ \text { herbivore } & \text { herbivore guild } \\ \text { carnivore } & \text { carnivore guild } \\ \text { herbivore } & \text { herbivore guild } \\ \text { herbivore } & \text { herbivore guild } \\ \text { herbivore } & \text { herbivore guild } \\ \text { herbivore } & \text { herbivore guild } \\ \text { herbivore } & \text { herbivore guild } \\ \text { omnivore } & \text { NA } \\ \text { herbivore } & \text { herbivore guild } \\ \text { omnivore } & \text { NA } \\ \text { herbivore } & \text { herbivore guild } \\ \text { herbivore } & \text { herbivore guild } \\ \text { omnivore } & \text { NA } \\ \text { herbivore } & \text { herbivore guild } \\ \text { omnivore } & \text { NA } \\ \text { insectivore } & \text { NA } \\ \text { omnivore } & \text { NA } \\ \text { insectivore } & \text { NA } \\ \text { carnivore } & \text { carnivore guild } \\ \text { carnivore } & \text { carnivore guild } \\ \text { herbivore } & \text { Rodentia } \\ \text { omnivore } & \text { NA } \\ \text { carnivore } & \text { carnivore guild } \\ \text { omnivore } & \text { NA } \\ \text { insectivore } & \text { NA } \\ \text { carnivore } & \text { carnivore guild } \\ \text { carnivore } & \text { carnivore guild } \\ \text { omnivore } & \text { NA } \\ \text { insectivore } & \text { NA } \\ \text { insectivore } & \text { NA } \\ \text { insectivore } & \text { NA } \\ \text { omnivore } & \text { NA } \\ \text { insectivore } & \text { NA } \\ \text { carnivore } & \text { carnivore guild } \\ \text { carnivore } \\ \text { carnivore } & \text { carnivore guild } \\ \text { NA } & \text { Narnivore guild } \\ \text { carnivore } & \\ \text { omnivore } & \text { Nornnivore } \\ \text { herbivore } & \end{array}$




\begin{tabular}{|c|c|c|}
\hline & Epoicotheriidae & Tubulodon atopum \\
\hline Perissodactyla & Palaeotheriidae & Hyracotherium cristatum \\
\hline \multirow[t]{2}{*}{ Macroscelidea } & Apheliscidae & Apheliscus insidiosus \\
\hline & & \\
\hline Panameriungulata & Hyopsodontidae & Hyopsodus simplex \\
\hline Creodonta & Oxyaenidae & Oxyaena intermedia \\
\hline Carnivora & Miacidae & Uintacyon rudis \\
\hline Primates & Omomyidae & Teilhardina demissa \\
\hline Carnivora & Miacidae & $\begin{array}{l}\text { Miacis petilus } \\
\text { Ectoganus gliriformes }\end{array}$ \\
\hline Panameriungulata & Hyopsodontidae & Hyopsodus pauxillus \\
\hline Primates & Omomyidae & Arapahovius advena \\
\hline Creodonta & Hyaenodontidae & Tritemnodon hians \\
\hline Dinocerata & Prodinoceratidae & Probathyopsis lysitensis \\
\hline Rodentia & Paramyidae & Franimys ambos \\
\hline Eulipotyphla & & $\begin{array}{l}\text { Talpavus sullivani } \\
\text { Oodectes jepseni }\end{array}$ \\
\hline Primates & Omomyidae & Anemorhysis pattersoni \\
\hline Artiodactyla & Diacodexeidae & Diacodexis kelleyi \\
\hline Primates & Paromomyidae & Simpsonlemur citatus \\
\hline Carnivora & $\begin{array}{l}\text { Miacidae } \\
\text { Ischyromyidae }\end{array}$ & $\begin{array}{l}\text { Uintacyon massetericus } \\
\text { Lophiparamys debequensis }\end{array}$ \\
\hline Primates & Notharctidae & Cantius ralstoni \\
\hline Eulipotyphla & Nyctitheriidae & Leptacodon catulus \\
\hline Primates & Picromomyidae & Picromomys petersonorum \\
\hline Lipotyphla & Geolabididae & Batodonoides vanhouteni \\
\hline Perissodactyla & & Homogalax aureus \\
\hline Creodonta & Hyaenodontidae & Prototomus phobos \\
\hline \multirow[t]{4}{*}{ Cimolodonta } & Neoplagiaulacidae & Parectypodus lunatus \\
\hline & Ischyromyidae & Apatosciuravus jacobsi \\
\hline & Ischyromyidae & Mytonomys wortmani \\
\hline & Ischyromyidae & Notoparamys costilloi \\
\hline Perissodactyla & Equidae & Xenicohippus osborni \\
\hline Creodonta & Hyaenodontidae & Galecyon mordax \\
\hline Creodonta & Hyaenodontidae & Prototomus martis \\
\hline Cimolesta & Coryphodontidae & Coryphodon eocaenus \\
\hline Rodentia & $\begin{array}{l}\text { Reithroparamyidae } \\
\text { Ischyromyidae }\end{array}$ & $\begin{array}{l}\text { Reithroparamys debequensis } \\
\text { Microparamys cheradius }\end{array}$ \\
\hline Cimolodonta & Neoplagiaulacidae & Parectypodus simpsoni \\
\hline Primates & Omomyidae & Teilhardina crassidens \\
\hline Dermoptera & & Plagiomene multicuspis \\
\hline Phenacodonta & Phenacodontidae & Phenacodus trilobatus \\
\hline Eulipotyphla & Erinaceidae & Leipsanolestes siegfriedti \\
\hline Primates & Omomyidae & Teilhardina americana \\
\hline \multirow[t]{2}{*}{ Primates } & Paromomyidae & Ignacius graybullianus \\
\hline & Omomyidae & Pseudotetonius ambiguus \\
\hline Creodonta & Hyaenodontidae & Arfia opisthotoma \\
\hline Primates & Omomyidae & Tetonius matthewi \\
\hline Macroscelidea & Apheliscidae & Haplomylus scottianus \\
\hline Primates & Paromomyidae & Phenacolemur simonsi \\
\hline Phenacodonta & Phenacodontidae & Ectocion osbornianus \\
\hline Perissodactyla & & Cardiolophus radinskyi \\
\hline
\end{tabular}

\begin{tabular}{|c|c|c|c|c|}
\hline 54.2 & 53.3 & 5.233 scansorial & insectivore & species \\
\hline 54.4 & 53.3 & 9.737534982 & & \\
\hline 55.1 & 53.3 & 4.304681012 ground dwelling & insectivore & species \\
\hline 55.7 & 53.3 & 5.633 ground dwelling & herbivore & species \\
\hline 55.7 & 53.3 & 6.226 scansorial & omnivore & species \\
\hline 55.8 & 53.3 & 10.582 ground dwelling & carnivore & species \\
\hline 57.25 & 53.3 & 7.103 arboreal & carnivore, omnivore & species \\
\hline 53.4 & 53.4 & 5.138 arboreal & insectivore & species \\
\hline 54.3 & 53.4 & 6.934 arboreal & carnivore & species \\
\hline 55.2 & 53.4 & 11.23903449 ground dwelling & herbivore & genus \\
\hline 55.2 & 53.4 & 5.207 scansorial & omnivore & species \\
\hline 53.45 & 53.45 & 5.165 arboreal & insectivore & species \\
\hline 53.4875 & 53.4875 & 9.422178201 ground dwelling & carnivore & species \\
\hline 53.4875 & 53.4875 & 10.487 ground dwelling & herbivore & species \\
\hline 53.4875 & 53.4875 & 4.304681012 ground dwelling & herbivore & species \\
\hline 53.4875 & 53.4875 & 1.933445645 scansorial & insectivore & species \\
\hline 53.4875 & 53.4875 & 6.180815 scansorial & insectivore & species \\
\hline 53.5 & 53.4875 & 5.295 arboreal & insectivore & species \\
\hline 53.7 & 53.4875 & 7.324 ground dwelling & herbivore & species \\
\hline 53.9 & 53.4875 & 6.006501352 arboreal & omnivore & species \\
\hline 55.4 & 53.4875 & 7.57 arboreal & carnivore, omnivore & species \\
\hline 55.65 & 53.4875 & 3.236 ground dwelling & herbivore & species \\
\hline 55.8 & 53.4875 & 7.219 arboreal & omnivore & species \\
\hline 53.5 & 53.5 & 1.982 scansorial & insectivore & species \\
\hline 53.5 & 53.5 & 3.29 arboreal & insectivore, omnivore & species \\
\hline 53.6 & 53.5 & 0.262364264 scansorial & insectivore & species \\
\hline 53.6 & 53.6 & 9.325 ground dwelling & browser & species \\
\hline 55.3 & 53.6 & 7.348117924 ground dwelling & carnivore & species \\
\hline 55.7 & 53.6 & 3.204102255 arboreal & omnivore & species \\
\hline 53.65 & 53.65 & 3.189 ground dwelling & herbivore & species \\
\hline 53.7 & 53.7 & 6.708 ground dwelling & herbivore & species \\
\hline 53.7 & 53.7 & 6.911 ground dwelling & herbivore & species \\
\hline 53.7 & 53.7 & 10.32406232 ground dwelling & browser & species \\
\hline 55.8 & 53.7 & 7.597 ground dwelling & carnivore & species \\
\hline 55.65 & 53.8 & 7.861166093 ground dwelling & carnivore & species \\
\hline 55.8 & 53.8 & 12.881 ground dwelling & herbivore & species \\
\hline 53.85 & 53.85 & 5.419 ground dwelling & herbivore & species \\
\hline 57.25 & 53.9 & 3.719 ground dwelling & herbivore & species \\
\hline 54.8 & 54 & 3.681834809 & & \\
\hline 55 & 54 & 5.223 arboreal & insectivore & species \\
\hline 55.8 & 54 & 6.473 arboreal & folivore & species \\
\hline 55.8 & 54 & 10.739 ground dwelling & herbivore & species \\
\hline 57.6 & 54 & 3.249 semifossorial & insectivore, carnivore & species \\
\hline 55.4 & 54.05 & 5.289 arboreal & insectivore & species \\
\hline 57.15 & 54.1 & 5.023880521 arboreal & omnivore & species \\
\hline 54.65 & 54.2 & 5.8 arboreal & insectivore & species \\
\hline 55.1 & 54.2 & 8.728493043 ground dwelling & carnivore & species \\
\hline 55.7 & 54.2 & 5.904 arboreal & insectivore & species \\
\hline 55.8 & 54.2 & 5.193 ground dwelling & insectivore & species \\
\hline 57.2 & 54.2 & 5.18693231 arboreal & omnivore & species \\
\hline 58.1 & 54.2 & 8.453577661 ground dwelling & herbivore & species \\
\hline 55.7 & 54.25 & 9.349233047 ground dwelling & browser & species \\
\hline
\end{tabular}

$\begin{array}{ll}\text { insectivore } & \text { NA } \\ \text { NA } & \text { NA } \\ \text { insectivore } & \text { NA } \\ \text { herbivore } & \text { herbivore guild } \\ \text { omnivore } & \text { NA } \\ \text { carnivore } & \text { carnivore guild } \\ \text { carnivore } & \text { carnivore guild } \\ \text { insectivore } & \text { NA } \\ \text { carnivore } & \text { carnivore guild } \\ \text { herbivore } & \text { herbivore guild } \\ \text { omnivore } & \text { NA } \\ \text { insectivore } & \text { NA } \\ \text { carnivore } & \text { carnivore guild } \\ \text { herbivore } & \text { herbivore guild } \\ \text { herbivore } & \text { Rodentia } \\ \text { insectivore } & \text { NA } \\ \text { insectivore } & \text { NA } \\ \text { insectivore } & \text { NA } \\ \text { herbivore } & \text { herbivore guild } \\ \text { omnivore } & \text { NA } \\ \text { carnivore } & \text { carnivore guild } \\ \text { herbivore } & \text { herbivore guild } \\ \text { omnivore } & \text { NA } \\ \text { insectivore } & \text { NA } \\ \text { omnivore } & \text { NA } \\ \text { insectivore } & \text { NA } \\ \text { herbivore } & \text { herbivore guild } \\ \text { carnivore } & \text { carnivore guild } \\ \text { omnivore } & \text { NA } \\ \text { herbivore } & \text { herbivore guild } \\ \text { herbivore } & \text { herbivore guild } \\ \text { herbivore } & \text { herbivore guild } \\ \text { herbivore } & \text { herbivore guild } \\ \text { carnivore } & \text { carnivore guild } \\ \text { carnivore } & \text { carnivore guild } \\ \text { herbivore } & \text { herbivore guild } \\ \text { herbivore } & \text { Rodentia } \\ \text { herbivore } & \text { herbivore guild } \\ \text { NA } & \text { NA } \\ \text { insectivore } & \text { NA } \\ \text { herbivore } & \text { herbivore guild } \\ \text { herbivore } & \text { herbivore guild } \\ \text { insectivore } & \text { NA guild } \\ \text { insectivore } & \text { NA } \\ \text { omnivore } & \text { NA } \\ \text { insectivore } & \text { NA } \\ \text { carnivore } & \text { carnivore guild } \\ \text { insectivore } & \text { NA } \\ \text { insectivore } & \text { NA } \\ \text { omnivore } & \text { NA } \\ \text { herbivore } & \text { herbivore }\end{array}$




\begin{tabular}{|c|c|c|c|c|c|c|c|c|c|}
\hline Creodonta & Oxyaenidae & Oxyaena gulo & 55.2 & 54.3 & 10.181 ground dwelling & carnivore & species & carnivore & carnivore guild \\
\hline \multirow[t]{2}{*}{ Cimolesta } & Esthonychidae & Esthonyx spatularius & 55.8 & 54.3 & 8.654 ground dwelling & herbivore & species & herbivore & herbivore guild \\
\hline & Sciuravidae & Knightomys cremneus & 54.4 & 54.4 & 3.297 ground dwelling & herbivore & species & herbivore & herbivore guild \\
\hline \multirow[t]{2}{*}{ Carnivora } & Miacidae & Gracilocyon winkleri & 55.5 & 54.4 & 6.173 scansorial & insectivore & species & insectivore & carnivore guild \\
\hline & Viverravidae & Viverravus rosei & 57.25 & 54.4 & 4.667 ground dwelling & carnivore & species & carnivore & carnivore guild \\
\hline \multirow[t]{2}{*}{ Creodonta } & Hyaenodontidae & Pyrocyon dioctetus & 54.45 & 54.45 & 7.870235848 ground dwelling & carnivore & species & carnivore & carnivore guild \\
\hline & Ischyromyidae & Notoparamys arctios & 54.5 & 54.5 & 5.919 ground dwelling & herbivore & species & herbivore & herbivore guild \\
\hline Primates & Omomyidae & Teilhardina tenuicula & 54.6 & 54.6 & 4.919 arboreal & insectivore & species & insectivore & NA \\
\hline Primates & Microsyopidae & Arctodontomys nuptus & 54.7 & 54.6 & 6.804 arboreal & omnivore & species & omnivore & NA \\
\hline Insectivora & Apatemyidae & Labidolemur serus & 54.8 & 54.6 & 3.869 arboreal & herbivore & species & herbivore & herbivore guild \\
\hline Eulipotyphla & Erinaceidae & Dartonius jepseni & 54.85 & 54.6 & 2.353 semifossorial & insectivore, carnivore & species & insectivore & NA \\
\hline Creodonta & Hyaenodontidae & Prototomus deimos & 55.5 & 54.65 & 6.227 ground dwelling & carnivore & species & carnivore & carnivore guild \\
\hline Artiodactyla & Diacodexeidae & Diacodexis primus & 55.8 & 54.65 & 7.186 ground dwelling & herbivore & species & herbivore & herbivore guild \\
\hline Creodonta & Hyaenodontidae & Prolimnocyon haematus & 55.95 & 54.65 & 6.377 scansorial & carnivore & species & carnivore & carnivore guild \\
\hline \multirow[t]{2}{*}{ Creodonta } & Oxyaenidae & Dipsalidictis transiens & 56.8 & 54.65 & 9.656 ground dwelling & carnivore & species & carnivore & carnivore guild \\
\hline & Ischyromyidae & Paramys pycnus & 55.2 & 54.7 & 4.016673355 ground dwelling & herbivore & species & herbivore & herbivore guild \\
\hline Macroscelidea & Apheliscidae & Apheliscus wapitiensis & 55.3 & 54.7 & 3.583270701 ground dwelling & insectivore & species & insectivore & NA \\
\hline Macroscelidea & Apheliscidae & Apheliscus chydaeus & 55.45 & 54.7 & 4.203 ground dwelling & insectivore & species & insectivore & NA \\
\hline Cimolodonta & Neoplagiaulacidae & Ectypodus tardus & 59 & 54.7 & 2.583953325 arboreal & omnivore & species & omnivore & NA \\
\hline Creodonta & Hyaenodontidae & Acarictis ryani & 55.6 & 54.8 & 5.737 ground dwelling & carnivore & species & carnivore & carnivore guild \\
\hline Creodonta & Hyaenodontidae & Arfia shoshoniensis & 56.1 & 54.8 & 8.396669906 ground dwelling & carnivore & species & carnivore & carnivore guild \\
\hline \multirow[t]{2}{*}{ Cimolodonta } & & Neoliotomus ultimus & 56.1 & 54.8 & 7.336120832 arboreal & omnivore & species & omnivore & NA \\
\hline & Ischyromyidae & Apatosciuravus bifax & 54.85 & 54.85 & 3.489 ground dwelling & herbivore & species & herbivore & herbivore guild \\
\hline Primates & Omomyidae & Tetonius mckennai & 54.85 & 54.85 & 5.391 arboreal & insectivore & species & insectivore & NA \\
\hline Lipotyphla & Geolabididae & Centetodon patratus & 54.9 & 54.85 & 3.226 scansorial & insectivore & species & insectivore & NA \\
\hline Acreodi & & Wyolestes apheles & 55.3 & 54.9 & 7.136 scansorial & carnivore & species & carnivore & carnivore guild \\
\hline Creodonta & Hyaenodontidae & Arfia zele & 55.5 & 54.9 & 7.713 ground dwelling & carnivore & species & carnivore & carnivore guild \\
\hline Carnivora & Miacidae & Miacis deutschi & 55.6 & 54.9 & 6.494 arboreal & carnivore & species & carnivore & carnivore guild \\
\hline Eulipotyphla & Nyctitheriidae & Plagioctenodon savagei & 55.6 & 54.9 & 3.388838433 scansorial & insectivore & species & insectivore & NA \\
\hline Cimolesta & & Azygonyx grangeri & 56.15 & 54.9 & 9.530826667 ground dwelling & herbivore & species & herbivore & herbivore guild \\
\hline Primates & Micromomyidae & Tinimomys graybulliensis & 56.75 & 54.9 & 3.747 arboreal & omnivore & species & omnivore & NA \\
\hline \multirow[t]{2}{*}{ Eulipotyphla } & & Talpavoides dartoni & 59.1 & 54.9 & 1.994 scansorial & insectivore & species & insectivore & NA \\
\hline & Palaeoryctidae & Eoryctes melanus & 55 & 55 & 4.003 scansorial & insectivore & species & insectivore & NA \\
\hline \multirow[t]{2}{*}{ Primates } & Micromomyidae & Micromomys antelucanus & 55.4 & 55.05 & 3.42 arboreal & omnivore & species & omnivore & NA \\
\hline & Ischyromyidae & Paramys atavus & 57.6 & 55.1 & 3.73 & & & NA & NA \\
\hline Acreodi & Mesonychidae & Dissacus praenuntius & 58.3 & 55.1 & 9.477 ground dwelling & carnivore & species & carnivore & carnivore guild \\
\hline \multirow[t]{2}{*}{ Insectivora } & Apatemyidae & Apatemys chardini & 55.2 & 55.2 & 2.843 arboreal & herbivore & species & herbivore & herbivore guild \\
\hline & & Scenopagus hewettensis & 55.25 & 55.2 & 3.459 ground dwelling & insectivore & species & insectivore & NA \\
\hline Eulipotyphla & Nyctitheriidae & Plagioctenoides microlestes & 55.25 & 55.25 & 1.791759469 scansorial & insectivore & species & insectivore & NA \\
\hline Acreodi & Hapalodectidae & Hapalodectes anthracinus & 55.4 & 55.25 & 5.123 ground dwelling & carnivore & species & carnivore & carnivore guild \\
\hline Macroscelidea & Apheliscidae & Haplomylus bozemanensis & 55.8 & 55.25 & 4.903 ground dwelling & insectivore & species & insectivore & NA \\
\hline Eulipotyphla & Nyctitheriidae & Plagioctenodon krausae & 57.15 & 55.25 & 1.485 scansorial & insectivore & species & insectivore & NA \\
\hline Dermoptera & & Plagiomene accola & 57.15 & 55.4 & 5.999 arboreal & folivore & species & herbivore & herbivore guild \\
\hline Creodonta & Hyaenodontidae & Arfia junnei & 55.6 & 55.45 & 7.083 ground dwelling & carnivore & species & carnivore & carnivore guild \\
\hline Phenacodonta & Phenacodontidae & Copecion davisi & 55.6 & 55.45 & 7.666 ground dwelling & herbivore & species & herbivore & herbivore guild \\
\hline Creodonta & Oxyaenidae & Dipsalidictis platypus & 56.8 & 55.45 & 8.757 ground dwelling & carnivore & species & carnivore & carnivore guild \\
\hline Artiodactyla & Diacodexeidae & Diacodexis ilicis & 55.5 & 55.5 & 7.058467111 ground dwelling & herbivore & species & herbivore & herbivore guild \\
\hline \multirow[t]{3}{*}{ Cimolesta } & Esthonychidae & Esthonyx gunnelli & 55.5 & 55.5 & 9.508 ground dwelling & herbivore & species & herbivore & herbivore guild \\
\hline & & Ectoganus bighornensis & 55.5 & 55.5 & 10.686 ground dwelling & herbivore & species & herbivore & herbivore guild \\
\hline & Arctocyonidae & Chriacus badgleyi & 55.6 & 55.5 & 7.093 scansorial & omnivore & species & omnivore & NA \\
\hline \multirow[t]{2}{*}{ Primates } & Notharctidae & Cantius torresi & 55.8 & 55.5 & 6.935 arboreal & omnivore & species & omnivore & NA \\
\hline & Arctocyonidae & Princetonia yalensis & 57.7 & 55.5 & 6.797503913 ground dwelling & omnivore & species & omnivore & NA \\
\hline
\end{tabular}




\begin{tabular}{|c|c|c|c|c|c|c|c|c|c|}
\hline \multirow[t]{3}{*}{ Phenacodonta } & Phenacodontidae & Ectocion parvus & 58.1 & 55.5 & 7.587 ground dwelling & herbivore & species & herbivore & herbivore guild \\
\hline & Viverravidae & Didymictis proteus & 61.9 & 55.5 & 8.46073105 ground dwelling & carnivore & species & carnivore & carnivore guild \\
\hline & Arctocyonidae & Thryptacodon barae & 55.6 & 55.6 & 7.397 ground dwelling & omnivore & species & omnivore & NA \\
\hline Eulipotyphla & & Diacodon alticuspis & 57.3 & 55.65 & 5.208 scansorial & insectivore & species & insectivore & NA \\
\hline Cimolesta & Barylambdidae & Barylambda faberi & 58.1 & 55.65 & 13.118 ground dwelling & herbivore & species & herbivore & herbivore guild \\
\hline \multirow[t]{2}{*}{ Macroscelidea } & Apheliscidae & Aletodon gunnelli & 57.85 & 55.7 & 7.084 ground dwelling & insectivore & species & insectivore & NA \\
\hline & & Peradectes elegans & 60.35 & 55.7 & 2.584 scansorial & omnivore & species & omnivore & NA \\
\hline Eulipotyphla & Parapternodontidae & Koniaryctes paulus & 55.8 & 55.8 & 1.992 scansorial & insectivore & species & insectivore & NA \\
\hline Primates & Micromomyidae & Tinimomys loomisi & 55.8 & 55.8 & 3.726 arboreal & omnivore & species & omnivore & NA \\
\hline Macroscelidea & Apheliscidae & Haplomylus simpsoni & 58.1 & 55.8 & 5.364 ground dwelling & insectivore & species & insectivore & NA \\
\hline \multirow[t]{2}{*}{ Primates } & Plesiadapidae & Plesiadapis cookei & 57.35 & 55.95 & 8.39 arboreal & herbivore & species & herbivore & herbivore guild \\
\hline & Palaeoryctidae & Palaeoryctes punctatus & 60.4 & 55.95 & 3.870168745 scansorial & insectivore & species & insectivore & NA \\
\hline Acreodi & & Wyolestes iglesius & 56.05 & 56.05 & 7.011 scansorial & carnivore & species & carnivore & carnivore guild \\
\hline Artiodactyla & Diacodexeidae & Diacodexis gracilis & 56.05 & 56.05 & 6.941 ground dwelling & herbivore & species & herbivore & herbivore guild \\
\hline Cimolesta & Barylambdidae & Barylambda jackwilsoni & 59.7 & 56.05 & 11.87 ground dwelling & herbivore & species & herbivore & herbivore guild \\
\hline Creodonta & Oxyaenidae & Palaeonictis peloria & 56.1 & 56.1 & 10.59286076 ground dwelling & carnivore & species & carnivore & carnivore guild \\
\hline Cimolesta & Coryphodontidae & Coryphodon proterus & 56.1 & 56.1 & 13.609 ground dwelling & herbivore & species & herbivore & herbivore guild \\
\hline Cimolesta & Titanoideidae & Titanoides nanus & 56.1 & 56.1 & 11.69 ground dwelling & herbivore & species & herbivore & herbivore guild \\
\hline Eulipotyphla & Nyctitheriidae & Limaconyssus habrus & 56.1 & 56.1 & 2.918 scansorial & insectivore & species & insectivore & NA \\
\hline Eulipotyphla & Nyctitheriidae & Ceutholestes dolosus & 56.3 & 56.1 & 3.66 scansorial & insectivore & species & insectivore & NA \\
\hline Cimolesta & & Azygonyx ancylion & 56.9 & 56.1 & 8.563788 ground dwelling & herbivore & species & herbivore & herbivore guild \\
\hline Creodonta & Oxyaenidae & Dipsalidictis aequidens & 57 & 56.1 & 10.03 ground dwelling & carnivore & species & carnivore & carnivore guild \\
\hline Creodonta & Oxyaenidae & Dipsalodon matthewi & 57 & 56.1 & 10.638 ground dwelling & carnivore & species & carnivore & carnivore guild \\
\hline Creodonta & Oxyaenidae & Dipsalidictis krausei & 57.05 & 56.1 & 9.522 ground dwelling & carnivore & species & carnivore & carnivore guild \\
\hline Dinocerata & Prodinoceratidae & Probathyopsis praecursor & 57.05 & 56.1 & 10.51 ground dwelling & herbivore & species & herbivore & herbivore guild \\
\hline Cimolodonta & Microcosmodontidae & Microcosmodon rosei & 57.15 & 56.1 & 3.417253781 arboreal & herbivore & species & herbivore & herbivore guild \\
\hline Dermoptera & & Worlandia inusitata & 57.15 & 56.1 & 3.974 arboreal & folivore & species & herbivore & herbivore guild \\
\hline Primates & Carpolestidae & Carpolestes simpsoni & 57.15 & 56.1 & 4.824 arboreal & herbivore & species & herbivore & herbivore guild \\
\hline Primates & Microsyopidae & Arctodontomys simplicidens & 57.2 & 56.1 & 6.5 arboreal & omnivore & species & omnivore & NA \\
\hline Eulipotyphla & Nyctitheriidae & Wyonycteris chalix & 57.25 & 56.1 & 1.825 scansorial & insectivore & species & insectivore & NA \\
\hline \multirow[t]{2}{*}{ Macroscelidea } & Apheliscidae & Apheliscus nitidus & 57.35 & 56.1 & 4.762 ground dwelling & insectivore & species & insectivore & NA \\
\hline & Arctostylopidae & Arctostylops steini & 57.7 & 56.1 & 5.618 scansorial & herbivore & species & herbivore & herbivore guild \\
\hline Dinocerata & Prodinoceratidae & Probathyopsis harrisorum & 58.1 & 56.1 & 11.036 ground dwelling & herbivore & species & herbivore & herbivore guild \\
\hline Primates & Plesiadapidae & Plesiadapis dubius & 58.1 & 56.1 & 6.589 arboreal & herbivore & species & herbivore & herbivore guild \\
\hline Eulipotyphla & & Diacocherus minutus & 59.1 & 56.1 & 3.157 scansorial & insectivore & species & insectivore & NA \\
\hline Primates & Paromomyidae & Dillerlemur pagei & 59.4 & 56.1 & 5.913 arboreal & omnivore & species & omnivore & NA \\
\hline Cimolodonta & Neoplagiaulacidae & Ectypodus powelli & 60.55 & 56.1 & 3.157937912 arboreal & omnivore & species & omnivore & NA \\
\hline Primates & Plesiadapidae & Plesiadapis gingerichi & 57.25 & 56.5665 & 7.998 arboreal & herbivore & species & herbivore & herbivore guild \\
\hline \multirow[t]{3}{*}{ Primates } & Carpolestidae & Carpolestes nigridens & 57.6 & 56.5665 & 4.465908119 arboreal & herbivore & species & herbivore & herbivore guild \\
\hline & & Ectoganus copei & 56.8 & 56.8 & 10.50232405 ground dwelling & herbivore & species & herbivore & herbivore guild \\
\hline & Arctocyonidae & Anacodon nexus & 57.35 & 56.8 & 9.676 ground dwelling & omnivore & species & omnivore & NA \\
\hline Dermoptera & & Planetetherium mirabile & 57.6 & 56.8 & 4.978 arboreal & folivore & species & herbivore & herbivore guild \\
\hline Creodonta & Oxyaenidae & Dipsalodon churchillorum & 57.65 & 56.8 & 10.19 ground dwelling & carnivore & species & carnivore & carnivore guild \\
\hline Primates & Plesiadapidae & Chiromyoides potior & 59.6 & 56.9 & 6.424 arboreal & herbivore & species & herbivore & herbivore guild \\
\hline \multirow[t]{2}{*}{ Cimolodonta } & Ptilodontidae & Prochetodon taxus & 57.15 & 57.15 & 5.126317647 arboreal & omnivore & species & omnivore & NA \\
\hline & Arctocyonidae & Thryptacodon pseudarctos & 57.6 & 57.15 & 8.71 ground dwelling & omnivore & species & omnivore & NA \\
\hline Cimolodonta & & Neoliotomus conventus & 58.1 & 57.15 & 6.906600275 arboreal & omnivore & species & omnivore & NA \\
\hline \multirow[t]{3}{*}{ Cimolodonta } & Neoplagiaulacidae & Parectypodus laytoni & 59.55 & 57.15 & 2.384947636 & & & NA & NA \\
\hline & Alagomyidae & Alagomys russelli & 57.2 & 57.2 & 1.618 ground dwelling & herbivore & species & herbivore & herbivore guild \\
\hline & Ischyromyidae & Paramys adamus & 57.2 & 57.2 & 3.581 ground dwelling & herbivore & species & herbivore & herbivore guild \\
\hline Macroscelidea & Apheliscidae & Aletodon conardae & 58.9 & 57.2 & 7.011 ground dwelling & insectivore & species & insectivore & NA \\
\hline Cimolodonta & Microcosmodontidae & Microcosmodon conus & 59 & 57.2 & 3.287094817 arboreal & herbivore & species & herbivore & herbivore guild \\
\hline
\end{tabular}




\begin{tabular}{|c|c|c|}
\hline Cimolodonta & Kogaionidae & Hainina vianeyae \\
\hline Cimolodonta & Taeniolabididae & Lambdopsalis bulla \\
\hline Cimolodonta & Taeniolabididae & Prionessus lucifer \\
\hline Cimolodonta & Taeniolabididae & Sphenopsalis nobilis \\
\hline Cimolodonta & Neoplagiaulacidae & Mesodmops tenuis \\
\hline Phenacodonta & Phenacodontidae & Ectocion mediotuber \\
\hline Macroscelidea & Apheliscidae & Haplomylus palustris \\
\hline Macroscelidea & Apheliscidae & Phenacodaptes sabulosus \\
\hline Primates & Carpolestidae & Carpolestes dubius \\
\hline Primates & Plesiadapidae & Plesiadapis fodinatus \\
\hline Phenacodonta & Phenacodontidae & Phenacodus magnus \\
\hline Acreodi & Mesonychidae & Dissacus argenteus \\
\hline Cimolodonta & Microcosmodontidae & $\begin{array}{l}\text { Pentacosmodon pronus } \\
\text { Propalaeanodon schaffi }\end{array}$ \\
\hline Cimolodonta & Neoplagiaulacidae & Mimetodon churchilli \\
\hline Primates & Micromomyidae & Micromomys silvercouleei \\
\hline \multirow[t]{2}{*}{ Cimolodonta } & Ptilodontidae & Prochetodon cavus \\
\hline & Palaeoryctidae & Aaptoryctes ivyi \\
\hline Eulipotyphla & Erinaceidae & Litolestes ignotus \\
\hline Cimolodonta & Neoplagiaulacidae & Neoplagiaulax hazeni \\
\hline Eulipotyphla & Nyctitheriidae & Leptacodon packi \\
\hline Leptictida & Leptictidae & Prodiacodon concordiarcensis \\
\hline Cimolesta & Barylambdidae & Barylambda churchilli \\
\hline Primates & Plesiadapidae & Plesiadapis churchilli \\
\hline Phenacodonta & Phenacodontidae & Ectocion major \\
\hline \multirow[t]{3}{*}{ Cimolesta } & Titanoideidae & Titanoides primaevus \\
\hline & Arctocyonidae & Thryptacodon australis \\
\hline & Arctocyonidae & Lambertocyon ischyrus \\
\hline Eulipotyphla & Erinaceidae & Oncocherus krishtalkai \\
\hline Primates & Carpolestidae & Carpocristes cygneus \\
\hline Eulipotyphla & & Diacocherus meizon \\
\hline Primates & Paromomyidae & Ignacius frugivorus \\
\hline Macroscelidea & & Litocherus lacunatus \\
\hline Cimolodonta & Neoplagiaulacidae & Neoplagiaulax hunteri \\
\hline Eulipotyphla & Nyctitheriidae & Leptacodon tener \\
\hline Cimolodonta & Ptilodontidae & Ptilodus kummae \\
\hline Cimolodonta & Ptilodontidae & Prochetodon foxi \\
\hline Cimolodonta & Neoplagiaulacidae & Mesodma pygmaea \\
\hline Primates & Micromomyidae & Micromomys vossae \\
\hline Cimolesta & Cyriacotheriidae & $\begin{array}{l}\text { Cyriacotherium argyreum } \\
\text { Raphictis gausion }\end{array}$ \\
\hline Phenacodonta & Phenacodontidae & Ectocion collinus \\
\hline Cimolodonta & Neoplagiaulacidae & Mimetodon silberlingi \\
\hline Primates & Carpolestidae & Carpodaptes stonleyi \\
\hline Cimolesta & Titanoideidae & Titanoides looki \\
\hline Primates & Plesiadapidae & Chiromyoides caesor \\
\hline \multirow[t]{2}{*}{ Phenacodonta } & Phenacodontidae & Phenacodus grangeri \\
\hline & Arctocyonidae & Thryptacodon demari \\
\hline \multirow[t]{2}{*}{ Cimolesta } & Pantolestidae & Bessoecetor septentrionalis \\
\hline & Arctocyonidae & Lambertocyon eximius \\
\hline \multirow[t]{2}{*}{ Acreodi } & Mesonychidae & Dissacus navajovius \\
\hline & & Paleotomus radagasti \\
\hline
\end{tabular}

\begin{tabular}{|c|c|c|c|c|}
\hline 57.25 & 57.25 & 4.317488114 arboreal & herbivore & species \\
\hline 57.25 & 57.25 & 6.65415252 arboreal & herbivore & species \\
\hline 57.25 & 57.25 & 5.910796644 arboreal & herbivore & species \\
\hline 57.25 & 57.25 & 9.210340372 arboreal & herbivore & species \\
\hline 57.25 & 57.25 & 3.295836866 arboreal & omnivore & species \\
\hline 59.1 & 57.35 & 8.187 ground dwelling & herbivore & species \\
\hline 57.6 & 57.6 & 4.688 ground dwelling & insectivore & species \\
\hline 59.1 & 57.6 & 5.168 ground dwelling & insectivore & species \\
\hline 57.7 & 57.65 & 5.246755465 arboreal & herbivore & species \\
\hline 58.9 & 57.65 & 6.911 arboreal & herbivore & species \\
\hline 59 & 57.65 & 11.104 ground dwelling & herbivore & species \\
\hline 57.7 & 57.7 & 8.97 ground dwelling & carnivore & species \\
\hline 57.7 & 57.7 & 4.007333185 arboreal & herbivore & species \\
\hline 57.7 & 57.7 & 3.123631689 scansorial & insectivore & species \\
\hline 57.7 & 57.7 & 4.50795406 arboreal & omnivore & species \\
\hline 57.7 & 57.7 & 2.692707424 arboreal & omnivore & species \\
\hline 57.8 & 57.7 & 4.821966064 arboreal & omnivore & species \\
\hline 58.2 & 57.7 & 4.835 scansorial & insectivore & species \\
\hline 59.2 & 57.7 & 3.007 semifossorial & insectivore, carnivore & species \\
\hline 59.2 & 57.7 & 4.50795406 & & \\
\hline 60.4 & 57.7 & 2.696 scansorial & insectivore & species \\
\hline 60.95 & 57.7 & 4.109 ground dwelling & insectivore, carnivore & species \\
\hline 57.8 & 57.8 & 12.718 ground dwelling & herbivore & species \\
\hline 61.9 & 57.8 & 7.03 arboreal & herbivore & species \\
\hline 58.1 & 57.85 & 9.428 ground dwelling & herbivore & species \\
\hline 58.7 & 57.85 & 12.846 ground dwelling & herbivore & species \\
\hline 59.9 & 57.85 & 7.958 ground dwelling & omnivore & species \\
\hline 61.8 & 57.85 & 7.606 & & \\
\hline 58 & 57.9 & 2.330755218 semifossorial & insectivore, carnivore & species \\
\hline 58.2165 & 57.9 & 4.384 & & \\
\hline 58.35 & 57.9 & 2.955 scansorial & insectivore & species \\
\hline 60.5 & 57.9 & 5.322 arboreal & omnivore & species \\
\hline 60.7 & 57.9 & 4.334 ground dwelling & insectivore & species \\
\hline 60.7165 & 57.9 & 3.817 arboreal & omnivore & species \\
\hline 60.95 & 57.9 & 2.21 scansorial & insectivore & species \\
\hline 61.9 & 57.9 & 4.745240183 arboreal & herbivore & species \\
\hline 61.9 & 57.9 & 5.197586908 arboreal & omnivore & species \\
\hline 64.25 & 57.9 & 2.143 arboreal & omnivore & species \\
\hline 58 & 58 & 3.359528276 arboreal & omnivore & species \\
\hline 58.25 & 58 & 8.34025345 ground dwelling & herbivore & species \\
\hline 58.8 & 58 & 5.561 ground dwelling & carnivore & species \\
\hline 60.4 & 58 & 8.485583241 ground dwelling & herbivore & species \\
\hline 60.9 & 58 & 3.233147915 arboreal & omnivore & species \\
\hline 61.9 & 58 & 5.342428733 arboreal & herbivore & species \\
\hline 58.1 & 58.1 & 12.063 ground dwelling & herbivore & species \\
\hline 59.5 & 58.1 & 6.02 arboreal & herbivore & species \\
\hline 60 & 58.1 & 10.427 ground dwelling & herbivore & species \\
\hline 60.35 & 58.1 & 7.706 ground dwelling & omnivore & species \\
\hline 60.95 & 58.1 & 4.602823448 amphibious & piscivore, durophage & species \\
\hline 61.9 & 58.1 & 8.46 ground dwelling & omnivore & species \\
\hline 63.7 & 58.1 & 8.33 ground dwelling & carnivore & species \\
\hline 58.3 & 58.2 & 6.065777431 scansorial & insectivore & species \\
\hline
\end{tabular}

\begin{tabular}{|c|c|}
\hline herbivore & herbivore guild \\
\hline herbivore & herbivore guild \\
\hline herbivore & herbivore guild \\
\hline herbivore & herbivore guild \\
\hline omnivore & NA \\
\hline herbivore & herbivore guild \\
\hline insectivore & NA \\
\hline insectivore & NA \\
\hline herbivore & herbivore guild \\
\hline herbivore & herbivore guild \\
\hline herbivore & herbivore guild \\
\hline carnivore & carnivore guild \\
\hline herbivore & herbivore guild \\
\hline insectivore & NA \\
\hline omnivore & NA \\
\hline omnivore & NA \\
\hline omnivore & NA \\
\hline insectivore & NA \\
\hline insectivore & NA \\
\hline NA & NA \\
\hline insectivore & NA \\
\hline insectivore & NA \\
\hline herbivore & herbivore guild \\
\hline herbivore & herbivore guild \\
\hline herbivore & herbivore guild \\
\hline herbivore & herbivore guild \\
\hline omnivore & NA \\
\hline NA & NA \\
\hline insectivore & NA \\
\hline NA & NA \\
\hline insectivore & NA \\
\hline omnivore & NA \\
\hline insectivore & NA \\
\hline omnivore & NA \\
\hline insectivore & NA \\
\hline herbivore & herbivore guild \\
\hline omnivore & NA \\
\hline omnivore & NA \\
\hline omnivore & NA \\
\hline herbivore & herbivore guild \\
\hline carnivore & carnivore guild \\
\hline herbivore & herbivore guild \\
\hline omnivore & NA \\
\hline herbivore & herbivore guild \\
\hline herbivore & herbivore guild \\
\hline herbivore & herbivore guild \\
\hline herbivore & herbivore guild \\
\hline omnivore & NA \\
\hline carnivore & carnivore guild \\
\hline omnivore & NA \\
\hline carnivore & carnivore guild \\
\hline insectivore & NA \\
\hline
\end{tabular}




\begin{tabular}{|c|c|c|c|c|c|c|c|c|c|}
\hline Macroscelidea & Apheliscidae & Dorraletes diminutivus & 59.4 & 58.2 & 3.943 ground dwelling & insectivore & species & insectivore & NA \\
\hline Cimolodonta & Ptilodontidae & Ptilodus fractus & 59.8 & 58.2 & 4.542980175 arboreal & herbivore & species & herbivore & herbivore guild \\
\hline Macroscelidea & & Litocherus zygeus & 59.2 & 58.2165 & 4.034 ground dwelling & insectivore & species & insectivore & NA \\
\hline Cimolesta & Titanoideidae & Titanoides gidleyi & 59.7 & 58.2165 & 12.38 ground dwelling & herbivore & species & herbivore & herbivore guild \\
\hline Primates & Plesiadapidae & Plesiadapis rex & 59.8 & 58.2165 & 6.862 arboreal & herbivore & species & herbivore & herbivore guild \\
\hline Macroscelidea & & Litocherus notissimus & 59.9 & 58.2165 & 3.62 ground dwelling & insectivore & species & insectivore & NA \\
\hline Dermoptera & & Elpidophorus elegans & 60.4 & 58.2165 & 6.317 arboreal & folivore & species & herbivore & herbivore guild \\
\hline Primates & Plesiadapidae & Plesiadapis anceps & 60.4 & 58.2165 & 6.716 arboreal & herbivore & species & herbivore & herbivore guild \\
\hline \multirow[t]{3}{*}{ Primates } & Carpolestidae & Carpodaptes hazelae & 60.5 & 58.2165 & 5.289 arboreal & herbivore & species & herbivore & herbivore guild \\
\hline & Arctocyonidae & Colpoclaenus keeferi & 60.5 & 58.2165 & 10.77292772 ground dwelling & omnivore & species & omnivore & NA \\
\hline & Arctocyonidae & Arctocyon ferox & 61.7 & 58.2165 & 10.98829553 ground dwelling & omnivore & species & omnivore & NA \\
\hline Phenacodonta & Phenacodontidae & Ectocion cedrus & 61.9 & 58.2165 & 7.811 ground dwelling & herbivore & species & herbivore & herbivore guild \\
\hline Eulipotyphla & Erinaceidae & Cedrocherus ryani & 58.3 & 58.3 & 3.62 semifossorial & insectivore, carnivore & species & insectivore & NA \\
\hline \multirow[t]{2}{*}{ Creodonta } & Oxyaenidae & Tytthaena parrisi & 58.35 & 58.3 & 8.013 ground dwelling & carnivore & species & carnivore & carnivore guild \\
\hline & Viverravidae & Protictis agastor & 58.35 & 58.3 & 7.721 ground dwelling & carnivore & species & carnivore & carnivore guild \\
\hline Macroscelidea & Apheliscidae & Utemylus serior & 58.4 & 58.3 & 5.934107048 ground dwelling & insectivore & species & insectivore & NA \\
\hline Cimolesta & Pentacodontidae & Bisonalveus holtzmani & 58.7 & 58.3 & 5.477 scansorial & insectivore & species & insectivore & NA \\
\hline \multirow[t]{3}{*}{ Macroscelidea } & Apheliscidae & Aletodon quadravus & 58.7 & 58.3 & 6.492 ground dwelling & insectivore & species & insectivore & NA \\
\hline & Viverravidae & Protictis paralus & 58.7835 & 58.3 & 6.08 ground dwelling & carnivore & species & carnivore & carnivore guild \\
\hline & Arctocyonidae & Chriacus oconostotae & 58.7835 & 58.3 & 8.635529488 scansorial & omnivore & species & omnivore & NA \\
\hline Cimolesta & Pantolambdidae & Caenolambda jepseni & 59.15 & 58.3 & 11.28560714 ground dwelling & herbivore & species & herbivore & herbivore guild \\
\hline \multirow[t]{3}{*}{ Insectivora } & Apatemyidae & Labidolemur soricoides & 59.5 & 58.3 & 2.861 arboreal & herbivore & species & herbivore & herbivore guild \\
\hline & Arctocyonidae & Mentoclaenodon acrogenius & 59.85 & 58.3 & 10.531 ground dwelling & omnivore & species & omnivore & NA \\
\hline & Arctocyonidae & Mimotricentes fremontensis & 60.35 & 58.3 & 7.891 ground dwelling & omnivore & species & omnivore & NA \\
\hline Eulipotyphla & Nyctitheriidae & Leptacodon munusculum & 60.9 & 58.3 & 2.08 scansorial & insectivore & species & insectivore & NA \\
\hline Primates & Picrodontidae & Picrodus silberlingi & 64.25 & 58.3 & 5.28 arboreal & herbivore & species & herbivore & herbivore guild \\
\hline \multirow[t]{3}{*}{ Eulipotyphla } & Erinaceidae & Cedrocherus aceratus & 58.35 & 58.35 & 1.708359943 semifossorial & insectivore, carnivore & species & insectivore & NA \\
\hline & Palaeoryctidae & Palaeoryctes cruoris & 58.35 & 58.35 & 4.122 scansorial & insectivore & species & insectivore & NA \\
\hline & Arctocyonidae & Lambertocyon gingerichi & 58.35 & 58.35 & 10.10677337 ground dwelling & omnivore & species & omnivore & NA \\
\hline Insectivora & Apatemyidae & Unuchinia dysmathes & 58.7 & 58.35 & 5.531156123 arboreal & herbivore & species & herbivore & herbivore guild \\
\hline Primates & Plesiadapidae & Chiromyoides minor & 59.7 & 58.35 & 5.566 arboreal & herbivore & species & herbivore & herbivore guild \\
\hline \multirow[t]{3}{*}{ Cimolodonta } & Ptilodontidae & Ptilodus wyomingensis & 60.9 & 58.65 & 4.990432587 arboreal & herbivore & species & herbivore & herbivore guild \\
\hline & & Paleotomus senior & 60.4 & 58.7 & 7.296578017 scansorial & insectivore & species & insectivore & NA \\
\hline & Palaeoryctidae & Pararyctes pattersoni & 60.4 & 58.7 & 2.887 ground dwelling & insectivore & species & insectivore & NA \\
\hline Cimolodonta & Ptilodontidae & Ptilodus montanus & 61.75 & 58.7 & 5.784972737 arboreal & herbivore & species & herbivore & herbivore guild \\
\hline Cimolodonta & Microcosmodontidae & Allocosmodon woodi & 58.7835 & 58.7835 & 3.828641396 arboreal & herbivore & species & herbivore & herbivore guild \\
\hline Cimolodonta & Neoplagiaulacidae & Neoplagiaulax paskapooensis & 58.7835 & 58.7835 & 3.871201011 arboreal & omnivore & species & omnivore & NA \\
\hline Cimolodonta & Neoplagiaulacidae & Neoplagiaulax serrator & 58.7835 & 58.7835 & 3.583518938 arboreal & omnivore & species & omnivore & NA \\
\hline Primates & Micromomyidae & Micromomys fremdi & 58.7835 & 58.7835 & 3.485 arboreal & omnivore & species & omnivore & NA \\
\hline Primates & Saxonellidae & Saxonella naylori & 58.8 & 58.7835 & 4.869 arboreal & herbivore & species & herbivore & herbivore guild \\
\hline Primates & Plesiadapidae & Nannodectes simpsoni & 59.4 & 58.7835 & 6.464 arboreal & herbivore & species & herbivore & herbivore guild \\
\hline Macroscelidea & Apheliscidae & Haplaletes pelicatus & 59.85 & 58.7835 & 5.339 ground dwelling & insectivore & species & insectivore & NA \\
\hline Primates & Palaechthonidae & Torrejonia sirokyi & 60.55 & 58.7835 & 6.958 arboreal & insectivore, omnivore & species & omnivore & NA \\
\hline Cimolesta & Cimolestidae & Gelastops parcus & 60.9 & 58.7835 & 4.964 scansorial & insectivore & species & insectivore & NA \\
\hline \multirow[t]{3}{*}{ Panameriungulata } & Hyopsodontidae & Promioclaenus acolytus & 61.6 & 58.7835 & 5.971 scansorial & omnivore & species & omnivore & NA \\
\hline & Arctocyonidae & Mimotricentes subtrigonus & 61.75 & 58.7835 & 7.606 & & & NA & NA \\
\hline & & Bryanictis microlestes & 60.9 & 58.85 & 6.266 ground dwelling & carnivore & species & carnivore & carnivore guild \\
\hline Cimolodonta & Neoplagiaulacidae & Neoplagiaulax mckennai & 61.9 & 58.85 & 4.107436932 arboreal & omnivore & species & omnivore & NA \\
\hline \multirow[t]{2}{*}{ Primates } & Plesiadapidae & Nannodectes gidleyi & 59.6 & 58.9 & 6.659 arboreal & herbivore & species & herbivore & herbivore guild \\
\hline & Viverravidae & Protictis haydenianus & 61.75 & 58.9 & 7.833 ground dwelling & carnivore & species & carnivore & carnivore guild \\
\hline \multirow[t]{2}{*}{ Cimolesta } & Pentacodontidae & Aphronorus fraudator & 60.8 & 59 & 5.169 scansorial & insectivore & species & insectivore & NA \\
\hline & Arctocyonidae & Chriacus pelvidens & 61.5 & 59 & 8.531 scansorial & omnivore & species & omnivore & NA \\
\hline
\end{tabular}




\begin{tabular}{|c|c|c|c|c|c|c|c|c|c|}
\hline & Arctocyonidae & Chriacus baldwini & 61.8 & 59.1 & 7.354 scansorial & omnivore & species & omnivore & NA \\
\hline Cimolesta & Pentacodontidae & Aphronorus orieli & 60.55 & 59.25 & 6.288 scansorial & insectivore & species & insectivore & NA \\
\hline Acreodi & Triisodontidae & Goniacodon levisanus & 61.5 & 59.25 & 7.692740528 scansorial & omnivore & species & omnivore & NA \\
\hline Cimolodonta & Neoplagiaulacidae & Parectypodus sinclairi & 61.75 & 59.35 & 2.763219489 arboreal & omnivore & species & omnivore & NA \\
\hline Cimolesta & Pantolambdidae & Caenolambda pattersoni & 59.85 & 59.4 & 11.9733541 ground dwelling & herbivore & species & herbivore & herbivore guild \\
\hline Panameriungulata & Hyopsodontidae & Promioclaenus pipiringosi & 59.85 & 59.4 & 6.624 scansorial & omnivore & species & omnivore & NA \\
\hline Phenacodonta & Phenacodontidae & Phenacodus bisonensis & 60.35 & 59.4 & 9.64455795 ground dwelling & herbivore & species & herbivore & herbivore guild \\
\hline Macroscelidea & Apheliscidae & Litomylus dissentaneus & 61.35 & 59.4 & 5.285 ground dwelling & insectivore & species & insectivore & NA \\
\hline Macroscelidea & Apheliscidae & Litomylus scaphicus & 61.7 & 59.4 & 5.629620522 ground dwelling & insectivore & species & insectivore & NA \\
\hline Primates & Carpolestidae & Carpodaptes aulacodon & 59.5 & 59.5 & 4.682 arboreal & herbivore & species & herbivore & herbivore guild \\
\hline Primates & Microsyopidae & Navajovius kohlhaasae & 59.7 & 59.5 & 4.134 arboreal & omnivore & species & omnivore & NA \\
\hline \multirow[t]{2}{*}{ Cimolodonta } & Neoplagiaulacidae & Ectypodus musculus & 60 & 59.5 & 3.517458956 arboreal & omnivore & species & omnivore & NA \\
\hline & Arctocyonidae & Colpoclaenus procyonoides & 61.2 & 59.55 & 8.843 ground dwelling & omnivore & species & omnivore & NA \\
\hline Macroscelidea & Apheliscidae & Haplaletes disceptatrix & 61.6 & 59.55 & 4.873 ground dwelling & insectivore & species & insectivore & NA \\
\hline \multirow[t]{2}{*}{ Phenacodonta } & Phenacodontidae & Phenacodus matthewi & 60.5 & 59.6 & 8.603 ground dwelling & herbivore & species & herbivore & herbivore guild \\
\hline & & Psittacotherium multifragum & 61.5 & 59.6 & 11.52951418 ground dwelling & herbivore & species & herbivore & herbivore guild \\
\hline Cimolodonta & Ptilodontidae & Ptilodus mediaevus & 61.75 & 59.6 & 5.418375101 arboreal & herbivore & species & herbivore & herbivore guild \\
\hline \multirow[t]{2}{*}{ Panameriungulata } & Hyopsodontidae & Protoselene opisthacus & 61.5 & 59.7 & 7.444 & & & NA & NA \\
\hline & Periptychidae & Periptychus carinidens & 63.8 & 59.7 & 10.238 ground dwelling & herbivore & species & herbivore & herbivore guild \\
\hline Macroscelidea & & Adunator ladae & 61.2 & 59.8 & 2.84 ground dwelling & insectivore & species & insectivore & NA \\
\hline Primates & Paromomyidae & Paromomys depressidens & 61.75 & 59.8 & 5.425 arboreal & omnivore & species & omnivore & NA \\
\hline Insectivora & Apatemyidae & Labidolemur major & 59.85 & 59.85 & 4.822 arboreal & herbivore & species & herbivore & herbivore guild \\
\hline Primates & Plesiadapidae & Nannodectes gazini & 59.85 & 59.85 & 5.948 arboreal & herbivore & species & herbivore & herbivore guild \\
\hline Panameriungulata & Hyopsodontidae & Protoselene novissimus & 59.85 & 59.85 & 7.298 scansorial & omnivore & species & omnivore & NA \\
\hline Cimolesta & Pentacodontidae & Bisonalveus browni & 60.4 & 59.85 & 4.526 scansorial & insectivore & species & insectivore & NA \\
\hline Primates & Picrodontidae & Picrodus canpacius & 60.5 & 59.85 & 4.94132766 arboreal & herbivore & species & herbivore & herbivore guild \\
\hline \multirow[t]{4}{*}{ Cimolodonta } & Ptilodontidae & Ptilodus gnomus & 60.7165 & 59.85 & 4.219507705 arboreal & herbivore & species & herbivore & herbivore guild \\
\hline & Viverravidae & Simpsonictis tenuis & 60.9 & 59.85 & 4.671 ground dwelling & carnivore & species & carnivore & carnivore guild \\
\hline & Arctocyonidae & Thryptacodon orthogonius & 60.9 & 59.85 & 7.045 ground dwelling & omnivore & species & omnivore & NA \\
\hline & Arctocyonidae & Arctocyon montanensis & 61.1 & 59.85 & 9.606 & & & NA & NA \\
\hline Cimolodonta & Cimolodontidae & Anconodon cochranensis & 61.1 & 59.85 & 4.027025634 arboreal & omnivore & species & omnivore & NA \\
\hline \multirow[t]{2}{*}{ Insectivora } & Apatemyidae & Unuchinia asaphes & 59.9 & 59.9 & 4.19 arboreal & herbivore & species & herbivore & herbivore guild \\
\hline & Periptychidae & Anisonchus sectorius & 61.8 & 59.9 & 7.392 ground dwelling & herbivore & species & herbivore & herbivore guild \\
\hline Cimolodonta & Ptilodontidae & Baiotomeus douglassi & 61.1 & 60 & 5.153976301 arboreal & omnivore & species & omnivore & NA \\
\hline Insectivora & Mixodectidae & Mixodectes malaris & 61.5 & 60 & 5.82 ground dwelling & insectivore & species & insectivore & NA \\
\hline Primates & & Palaechthon woodi & 61.75 & 60 & 4.802 arboreal & omnivore & species & omnivore & NA \\
\hline Primates & Carpolestidae & Elphidotarsius russelli & 60.4 & 60.1 & 4.568 arboreal & herbivore & species & herbivore & herbivore guild \\
\hline Cimolodonta & Neoplagiaulacidae & Neoplagiaulax donaldorum & 60.4 & 60.1 & 3.951243719 arboreal & omnivore & species & omnivore & NA \\
\hline \multirow[t]{2}{*}{ Primates } & Paromomyidae & Ignacius fremontensis & 60.9 & 60.1 & 4.639 arboreal & omnivore & species & omnivore & NA \\
\hline & Arctocyonidae & Mimotricentes tedfordi & 60.35 & 60.2 & 8.327246563 ground dwelling & omnivore & species & omnivore & NA \\
\hline Phenacodonta & Phenacodontidae & Tetraclaenodon puercensis & 64.25 & 60.2 & 8.936 ground dwelling & herbivore & species & herbivore & herbivore guild \\
\hline Cimolodonta & Neoplagiaulacidae & Neoplagiaulax nelsoni & 63.5 & 60.25 & 3.332 arboreal & omnivore & species & omnivore & NA \\
\hline Macroscelidea & Apheliscidae & Gingerichia geoteretes & 60.35 & 60.35 & 4.298902202 ground dwelling & insectivore & species & insectivore & NA \\
\hline Primates & Plesiadapidae & Nannodectes intermedius & 60.7 & 60.35 & 6.09 arboreal & herbivore & species & herbivore & herbivore guild \\
\hline Primates & Plesiadapidae & Plesiadapis praecursor & 60.7 & 60.35 & 6.406 arboreal & herbivore & species & herbivore & herbivore guild \\
\hline Leptictida & Leptictidae & Myrmecoboides montanensis & 60.95 & 60.35 & 4.15 ground dwelling & insectivore, carnivore & species & insectivore & NA \\
\hline \multirow[t]{3}{*}{ Macroscelidea } & Apheliscidae & Gingerichia hystrix & 60.4 & 60.4 & 3.850823495 ground dwelling & insectivore & species & insectivore & NA \\
\hline & & Paleotomus junior & 60.7165 & 60.4 & 4.625746356 scansorial & insectivore & species & insectivore & NA \\
\hline & Viverravidae & Simpsonictis pegus & 60.9 & 60.4 & 5.684 ground dwelling & carnivore & species & carnivore & carnivore guild \\
\hline Insectivora & Mixodectidae & Eudaemonema cuspidata & 60.9 & 60.4 & 5.704 ground dwelling & insectivore & species & insectivore & NA \\
\hline Insectivora & Apatemyidae & Jepsenella praepropera & 60.95 & 60.4 & 3.186 arboreal & herbivore & species & herbivore & herbivore guild \\
\hline Primates & Palaechthonidae & Plesiolestes problematicus & 60.95 & 60.4 & 5.669 arboreal & insectivore, omnivore & species & omnivore & NA \\
\hline
\end{tabular}




\begin{tabular}{|c|c|c|c|c|c|c|c|c|c|}
\hline \multirow[t]{2}{*}{ Cimolodonta } & Neoplagiaulacidae & Ectypodus szalayi & 61.45 & 60.4 & 2.973021227 arboreal & omnivore & species & omnivore & NA \\
\hline & Palaeoryctidae & Acmeodon secans & 61.7 & 60.4 & 5.202 scansorial & insectivore & species & insectivore & NA \\
\hline Cimolodonta & Neoplagiaulacidae & Parectypodus sylviae & 64.25 & 60.4 & 2.880021227 arboreal & omnivore & species & omnivore & NA \\
\hline Primates & Carpolestidae & Elphidotarsius shotgunensis & 60.5 & 60.5 & 4.668 arboreal & herbivore & species & herbivore & herbivore guild \\
\hline Acreodi & Triisodontidae & Stelocyon arctylos & 60.5 & 60.5 & 8.572 scansorial & omnivore & species & omnivore & NA \\
\hline Dermoptera & & Elpidophorus minor & 60.75 & 60.5 & 5.365 arboreal & folivore & species & herbivore & herbivore guild \\
\hline Cimolodonta & Neoplagiaulacidae & Ectypodus aphronorus & 60.9 & 60.5 & 2.890371758 arboreal & omnivore & species & omnivore & NA \\
\hline Primates & Palaechthonidae & Palenochtha minor & 60.95 & 60.5 & 3.949 arboreal & insectivore, omnivore & species & omnivore & NA \\
\hline Cimolodonta & Taeniolabididae & Catopsalis calgariensis & 61.1 & 60.5 & 10.13227811 arboreal & herbivore & species & herbivore & herbivore guild \\
\hline Primates & & Palaechthon alticuspis & 61.6 & 60.5 & 5.304 arboreal & omnivore & species & omnivore & NA \\
\hline Cimolesta & Pantolambdidae & Pantolambda cavirictus & 61.7 & 60.5 & 10.62518431 ground dwelling & herbivore & species & herbivore & herbivore guild \\
\hline Cimolodonta & Neoplagiaulacidae & Neoplagiaulax grangeri & 60.8 & 60.55 & 4.587799746 arboreal & omnivore & species & omnivore & NA \\
\hline Panameriungulata & Hyopsodontidae & Litaletes disjunctus & 61.05 & 60.55 & 6.545 scansorial & omnivore & species & omnivore & NA \\
\hline Panameriungulata & Hyopsodontidae & Promioclaenus lemuroides & 61.8 & 60.55 & 6.601 & & & NA & NA \\
\hline Cimolesta & Pentacodontidae & Aphronorus ratatoski & 60.7 & 60.7 & 5.671 scansorial & insectivore & species & insectivore & NA \\
\hline Primates & Picrodontidae & Picrodus calgariensis & 60.7165 & 60.7 & 4.114686504 arboreal & herbivore & species & herbivore & herbivore guild \\
\hline \multirow[t]{2}{*}{ Cimolesta } & Pentacodontidae & Coriphagus montanus & 60.8 & 60.7 & 5.345 scansorial & insectivore & species & insectivore & NA \\
\hline & Palaeoryctidae & Avunculus didelphodonti & 60.8 & 60.7 & 4.101 scansorial & insectivore & species & insectivore & NA \\
\hline Primates & Plesiadapidae & Pronothodectes jepi & 60.9 & 60.7 & 6.042 arboreal & herbivore & species & herbivore & herbivore guild \\
\hline Condylarthra & Chriacidae & Prothryptacodon hilli & 60.95 & 60.7 & 7.58 scansorial & insectivore & species & insectivore & NA \\
\hline Primates & Paromomyidae & Paromomys maturus & 60.95 & 60.7 & 6.409 arboreal & omnivore & species & omnivore & NA \\
\hline Cimolodonta & Ptilodontidae & Baiotomeus rhothonion & 60.7165 & 60.7165 & 2.995732274 arboreal & omnivore & species & omnivore & NA \\
\hline Cimolodonta & Neoplagiaulacidae & Parectypodus corystes & 60.7165 & 60.7165 & 3.871201011 arboreal & omnivore & species & omnivore & NA \\
\hline \multirow[t]{2}{*}{ Primates } & Plesiadapidae & Pronothodectes matthewi & 64.25 & 60.7165 & 5.759 arboreal & herbivore & species & herbivore & herbivore guild \\
\hline & Arctocyonidae & Chriacus punitor & 60.8 & 60.75 & 7.017 scansorial & omnivore & species & omnivore & NA \\
\hline Condylarthra & Chriacidae & Prothryptacodon furens & 60.95 & 60.75 & 7.212 scansorial & insectivore & species & insectivore & NA \\
\hline Cimolodonta & Eucosmodontidae & Stygimys jepseni & 61.05 & 60.75 & 4.461408399 arboreal & omnivore & species & omnivore & NA \\
\hline Carnivora & & Ictidopappus mustelinus & 60.8 & 60.8 & 5.606 ground dwelling & carnivore, omnivore & species & carnivore & carnivore guild \\
\hline Leptictida & Leptictidae & Leptonysson basiliscus & 60.8 & 60.8 & 5.024856237 ground dwelling & insectivore, carnivore & species & insectivore & NA \\
\hline Lipotyphla & Geolabididae & Stilpnodon simplicidens & 60.8 & 60.8 & 1.669150406 scansorial & insectivore & species & insectivore & NA \\
\hline \multirow[t]{2}{*}{ Cimolesta } & Pantolambdidae & Pantolambda intermedius & 61.5 & 60.8 & 10.938 ground dwelling & herbivore & species & herbivore & herbivore guild \\
\hline & Viverravidae & Protictis vanvaleni & 61.7 & 60.8 & 6.101433883 & & & NA & NA \\
\hline Primates & Carpolestidae & Elphidotarsius florencae & 64.25 & 60.8 & 4.12 arboreal & herbivore & species & herbivore & herbivore guild \\
\hline Panameriungulata & Hyopsodontidae & Litaletes ondolinde & 60.9 & 60.9 & 7.716666667 scansorial & omnivore & species & omnivore & NA \\
\hline \multirow[t]{3}{*}{ Primates } & Palaechthonidae & Premnoides douglassi & 60.9 & 60.9 & 5.094 arboreal & insectivore, omnivore & species & omnivore & NA \\
\hline & Arctocyonidae & Chriacus katrinae & 60.9 & 60.9 & 10.77292772 scansorial & omnivore & species & omnivore & NA \\
\hline & & Huerfanodon polecatensis & 60.9 & 60.9 & 10.301 scansorial & omnivore, herbivore & species & omnivore & NA \\
\hline \multirow[t]{2}{*}{ Cimolesta } & Pentacodontidae & Coriphagus encinensis & 61.5 & 60.9 & 6.081 scansorial & insectivore & species & insectivore & NA \\
\hline & & Microclaenodon assurgens & 61.5 & 60.9 & 7.22640016 scansorial & omnivore & species & omnivore & NA \\
\hline \multirow[t]{3}{*}{ Primates } & Palaechthonidae & Palenochtha weissae & 60.95 & 60.95 & 3.968 arboreal & insectivore, omnivore & species & omnivore & NA \\
\hline & Arctocyonidae & Loxolophus criswelli & 60.95 & 60.95 & 7.667 ground dwelling & omnivore & species & omnivore & NA \\
\hline & Palaeoryctidae & Palaeoryctes puercensis & 61.45 & 60.95 & 2.854 scansorial & insectivore & species & insectivore & NA \\
\hline Cimolodonta & Neoplagiaulacidae & Parectypodus clemensi & 61.45 & 60.95 & 3.524073801 arboreal & omnivore & species & omnivore & NA \\
\hline Cimolesta & Cimolestidae & Gelastops joni & 61.6 & 60.95 & 5.218 scansorial & insectivore & species & insectivore & NA \\
\hline \multirow[t]{2}{*}{ Insectivora } & Mixodectidae & Mixodectes pungens & 61.2 & 61.2 & 7.05 ground dwelling & insectivore & species & insectivore & NA \\
\hline & Escavadodontidae & Escavadodon zygus & 61.2 & 61.2 & 4.928 scansorial & insectivore & species & insectivore & NA \\
\hline \multirow[t]{2}{*}{ Cimolesta } & Pentacodontidae & Pentacodon occultus & 61.2 & 61.2 & 8.124 scansorial & omnivore & species & omnivore & NA \\
\hline & & Conoryctes comma & 61.2 & 61.2 & 9.726 scansorial & omnivore, herbivore & species & omnivore & NA \\
\hline Acreodi & Mesonychidae & Ankalagon saurognathus & 61.25 & 61.2 & 10.396 ground dwelling & carnivore & species & carnivore & carnivore guild \\
\hline Panameriungulata & Hyopsodontidae & Mioclaenus turgidus & 61.75 & 61.2 & 8.392 scansorial & omnivore & species & omnivore & NA \\
\hline \multirow[t]{2}{*}{ Cimolodonta } & Neoplagiaulacidae & Neoplagiaulax macrotomeus & 61.25 & 61.25 & 2.843366137 arboreal & omnivore & species & omnivore & NA \\
\hline & & Huerfanodon torrejonius & 61.25 & 61.25 & 9.945 scansorial & omnivore, herbivore & species & omnivore & NA \\
\hline
\end{tabular}




\begin{tabular}{|c|c|c|c|c|c|c|c|c|c|}
\hline Acreodi & Triisodontidae & Triisodon quivirensis & 61.5 & 61.25 & 11.045 scansorial & omnivore & species & omnivore & NA \\
\hline \multirow[t]{4}{*}{ Cimolesta } & Pentacodontidae & Pentacodon inversus & 61.5 & 61.25 & 7.057 scansorial & omnivore & species & omnivore & NA \\
\hline & Periptychidae & Haploconus angustus & 65.25 & 61.25 & 7.091 ground dwelling & herbivore & species & herbivore & herbivore guild \\
\hline & & Conoryctella pattersoni & 61.75 & 61.35 & 8.776 scansorial & omnivore, herbivore & species & omnivore & NA \\
\hline & Viverravidae & Protictis simpsoni & 61.45 & 61.45 & 7.8671055 ground dwelling & carnivore & species & carnivore & carnivore guild \\
\hline Cimolodonta & Neoplagiaulacidae & Parectypodus trovessartianus & 61.45 & 61.45 & 4.506894288 arboreal & omnivore & species & omnivore & NA \\
\hline Primates & Palaechthonidae & Plesiolestes nacimienti & 61.75 & 61.45 & 5.831 arboreal & insectivore, omnivore & species & omnivore & NA \\
\hline Cimolodonta & Taeniolabididae & Catopsalis fissidens & 61.8 & 61.5 & 9.319253976 arboreal & herbivore & species & herbivore & herbivore guild \\
\hline Cimolesta & Pentacodontidae & Aphronorus simpsoni & 61.75 & 61.75 & 5.173 scansorial & insectivore & species & insectivore & NA \\
\hline Panameriungulata & Hyopsodontidae & Litaletes sternbergi & 61.75 & 61.75 & 8.001819308 & & & NA & NA \\
\hline \multirow[t]{5}{*}{ Panameriungulata } & Hyopsodontidae & Protoselene griphus & 61.75 & 61.75 & 7.39 scansorial & omnivore & species & omnivore & NA \\
\hline & Periptychidae & Anisonchus onostus & 63 & 61.75 & 6.42 ground dwelling & herbivore & species & herbivore & herbivore guild \\
\hline & Arctocyonidae & Loxolophus spiekeri & 63 & 61.75 & 9.816 ground dwelling & omnivore & species & omnivore & NA \\
\hline & Arctocyonidae & Desmatoclaenus hermaeus & 63.2 & 61.75 & 9.218 ground dwelling & omnivore & species & omnivore & NA \\
\hline & Periptychidae & Anisonchus oligistus & 63.5 & 61.75 & 5.971 ground dwelling & herbivore & species & herbivore & herbivore guild \\
\hline \multirow[t]{3}{*}{ Acreodi } & & Oxyclaenus pugnax & 63.5 & 61.75 & 8.555 scansorial & omnivore & species & omnivore & NA \\
\hline & Arctocyonidae & Loxolophus pentacus & 64 & 61.75 & 9.615 ground dwelling & omnivore & species & omnivore & NA \\
\hline & Periptychidae & Periptychus coarctatus & 64.15 & 61.75 & 10.031 ground dwelling & herbivore & species & herbivore & herbivore guild \\
\hline \multirow[t]{3}{*}{ Cimolodonta } & Taeniolabididae & Taeniolabis taoensis & 64 & 62.8 & 10.62598023 arboreal & herbivore & species & herbivore & herbivore guild \\
\hline & Arctocyonidae & Loxolophus schizophrenus & 64.3 & 62.8 & 6.78 ground dwelling & omnivore & species & omnivore & NA \\
\hline & Arctocyonidae & Baioconodon denverensis & 64.9 & 62.8 & 8.344 ground dwelling & omnivore & species & omnivore & NA \\
\hline Cimolodonta & Neoplagiaulacidae & Mesodma thompsoni & 75 & 62.8 & 3.976645957 arboreal & omnivore & species & omnivore & NA \\
\hline \multirow[t]{8}{*}{ Cimolodonta } & Neoplagiaulacidae & Mesodma formosa & 82.85 & 62.8 & 3.49417403 arboreal & omnivore & species & omnivore & NA \\
\hline & Periptychidae & Haploconus elachistus & 63 & 62.9 & 6.13 ground dwelling & herbivore & species & herbivore & herbivore guild \\
\hline & Periptychidae & Ectoconus symbolus & 63.2 & 62.9 & 9.557 ground dwelling & herbivore & species & herbivore & herbivore guild \\
\hline & Periptychidae & Anisonchus athelas & 63.6 & 62.9 & 6.42 ground dwelling & herbivore & species & herbivore & herbivore guild \\
\hline & Periptychidae & Conacodon kohlbergeri & 64.05 & 62.9 & 6.418 ground dwelling & herbivore & species & herbivore & herbivore guild \\
\hline & Periptychidae & Ectoconus ditrigonus & 64.1 & 63 & 10.017 ground dwelling & herbivore & species & herbivore & herbivore guild \\
\hline & Periptychidae & Oxyacodon apiculatus & 64.3 & 63 & 6.5 ground dwelling & herbivore & species & herbivore & herbivore guild \\
\hline & Periptychidae & Oxyacodon ferronensis & 64.5 & 63.1 & 5.513 ground dwelling & herbivore & species & herbivore & herbivore guild \\
\hline Panameriungulata & Hyopsodontidae & Valenia wilsoni & 64.15 & 63.2 & 5.625 scansorial & omnivore & species & omnivore & NA \\
\hline Cimolodonta & Microcosmodontidae & Microcosmodon arcuatus & 63.3 & 63.3 & 3.538458957 arboreal & herbivore & species & herbivore & herbivore guild \\
\hline Acreodi & & Oxyclaenus corax & 63.3 & 63.3 & 7.134 scansorial & omnivore & species & omnivore & NA \\
\hline \multirow[t]{2}{*}{ Macroscelidea } & Apheliscidae & Litomylus orthronepius & 63.4 & 63.3 & 5.203 ground dwelling & insectivore & species & insectivore & NA \\
\hline & Periptychidae & Oxyacodon agapetillus & 64.75 & 63.3 & 5.196 ground dwelling & herbivore & species & herbivore & herbivore guild \\
\hline Cimolodonta & & Cimexomys minor & 66.8 & 63.3 & 3.631912816 arboreal & herbivore & species & herbivore & herbivore guild \\
\hline Cimolodonta & Taeniolabididae & Catopsalis waddleae & 63.4 & 63.4 & 10.27545696 arboreal & herbivore & species & herbivore & herbivore guild \\
\hline Acreodi & & Oxyclaenus simplex & 64.8 & 63.4 & 6.743 & & & NA & NA \\
\hline Cimolesta & Cimolestidae & Cimolestes incisus & 68.5 & 63.4 & 5.467 scansorial & insectivore & species & insectivore & NA \\
\hline Primates & & Pandemonium dis & 63.5 & 63.5 & 5.962 arboreal & insectivore, omnivore & species & omnivore & NA \\
\hline Primates & & Purgatorius unio & 63.5 & 63.5 & 5.287 arboreal & omnivore & species & omnivore & NA \\
\hline Acreodi & Triisodontidae & Eoconodon nidhoggi & 64.3 & 63.5 & 8.127 scansorial & omnivore & species & omnivore & NA \\
\hline \multirow[t]{2}{*}{ Cimolodonta } & Neoplagiaulacidae & Mesodma ambigua & 65.3 & 63.5 & 4.047721506 arboreal & omnivore & species & omnivore & NA \\
\hline & Arctocyonidae & Loxolophus hyattianus & 64.65 & 63.6 & 7.589 ground dwelling & omnivore & species & omnivore & NA \\
\hline \multirow[t]{3}{*}{ Cimolodonta } & Eucosmodontidae & Stygimys kuszmauli & 65.2 & 63.6 & 5.516793137 arboreal & omnivore & species & omnivore & NA \\
\hline & Arctocyonidae & Desmatoclaenus dianae & 63.7 & 63.7 & 7.987 ground dwelling & omnivore & species & omnivore & NA \\
\hline & Periptychidae & Conacodon entoconus & 64.05 & 63.7 & 7.728 & & & NA & NA \\
\hline \multirow[t]{4}{*}{ Acreodi } & & Oxyclaenus cuspidatus & 64.15 & 63.7 & 7.39 scansorial & omnivore & species & omnivore & NA \\
\hline & Arctocyonidae & Loxolophus priscus & 64.65 & 63.7 & 8.271 ground dwelling & omnivore & species & omnivore & NA \\
\hline & Periptychidae & Conacodon delphae & 64.75 & 63.7 & 8.105 ground dwelling & herbivore & species & herbivore & herbivore guild \\
\hline & Periptychidae & Mithrandir gillianus & 64.75 & 63.7 & 6.469 ground dwelling & herbivore & species & herbivore & herbivore guild \\
\hline Cimolodonta & Eucosmodontidae & Eucosmodon americanus & 64 & 64 & 6.401208927 arboreal & herbivore & species & herbivore & herbivore guild \\
\hline
\end{tabular}


Arctocyonidae

Panameriungulata Hyopsodontidae

Periptychidae

Periptychidae

Arctocyonidae

Taeniolabididae

Cimolestidae

Taeniolabididae

Arctocyonidae

Periptychidae

Periptychidae

Microcosmodontid

Taeniolabididae

Periptychidae

Periptychidae

Neoplagiaulacidae

Cimolodonta

Cimolodonta

Cimolodonta

Cimolodonta

Cimolodonta

Neoplagiaulacidae Ptilodontidae Taeniolabididae

Arctocyonidae

Periptychidae

Periptychidae

Periptychidae

Triisodontidae

Acreodi

Neoplagiaulacidae

Didelphimorphia Glasbiidae

Cimolodonta

Didelphimorphia Stagodontidae

Cimolesta Cimolestidae

Leptictida

Gypsonictopidae

Cimolodonta

Cimolomyidae

Cimolomyidae

Pediomyidae

Cimolodontida

Cimolodonta

Alphadontidae
Loxolophus stenognathus

Tiznatzinia vanderhoofi

Conacodon cophater

Oxyacodon priscilla

Protungulatum donnae

Catopsalis foliatus

Procerberus formicarum

Oxyprimus erikseni

Catopsalis joyneri

Baioconodon nordicus

Mimatuta morgoth

Taeniolabis lamberti

Ampliconus anton

Alticonus gazini

Mesodma garfieldensis

Cimexomys arapahoensis

Xyronomys robinsoni

Kimbetohia mziae

Catopsalis alexanderi

Baioconodon engdahli

Mimatuta minuial

Hemithlaeus josephi

Maiorana ferrisensis

Eoconodon copanus

ma hensleighi

Glasbius twitchelli

Essonodon browni

Didelphodon vorax

Cimolestes magnus

Gypsonictops illuminatus

Cimolomys gracilis

Meniscoessus robustus

Pediomys elegans

Cimolodon nitidus

Alphadon marshi

\begin{tabular}{|c|c|c|c|c|c|c|}
\hline 64 & 64 & 9.401 ground dwelling & omnivore & species & omnivore & NA \\
\hline 64.05 & 64 & 5.88 scansorial & omnivore & species & omnivore & NA \\
\hline 64.15 & 64 & 5.477 ground dwelling & herbivore & species & herbivore & herbivore guild \\
\hline 64.65 & 64 & 5.965 ground dwelling & herbivore & species & herbivore & herbivore guild \\
\hline 65.4 & 64 & 6.269 ground dwelling & omnivore & species & omnivore & NA \\
\hline 64.05 & 64.05 & 8.461833185 arboreal & herbivore & species & herbivore & herbivore guild \\
\hline 65.2 & 64.05 & 4.594 scansorial & carnivore, insectivore & species & insectivore & NA \\
\hline 65.2 & 64.05 & 4.874 ground dwelling & omnivore & species & omnivore & NA \\
\hline 6.775 & 64.05 & 7.797702036 & & & NA & NA \\
\hline 65.2 & 64.3 & 7.428 ground dwelling & omnivore & species & omnivore & NA \\
\hline 64.4 & 64.4 & 7.156 ground dwelling & herbivore & species & herbivore & herbivore guild \\
\hline 65.2 & 64.4 & 6.249 ground dwelling & herbivore & species & herbivore & herbivore guild \\
\hline 64.5 & 64.5 & 3.190418433 arboreal & herbivore & species & herbivore & herbivore guild \\
\hline 64.5 & 64.5 & 9.989005354 arboreal & herbivore & species & herbivore & herbivore guild \\
\hline 64.65 & 64.65 & 7.974 ground dwelling & herbivore & species & herbivore & herbivore guild \\
\hline 64.9 & 64.65 & 7.037 ground dwelling & herbivore & species & herbivore & herbivore guild \\
\hline 6.775 & 64.75 & 3.689834809 arboreal & omnivore & species & omnivore & NA \\
\hline 64.9 & 64.9 & 4.644390899 arboreal & herbivore & species & herbivore & herbivore guild \\
\hline 64.9 & 64.9 & 3.218875825 arboreal & omnivore & species & omnivore & NA \\
\hline 65.15 & 64.9 & 4.276666119 arboreal & omnivore & species & omnivore & NA \\
\hline 65.2 & 64.9 & 8.159828412 arboreal & herbivore & species & herbivore & herbivore guild \\
\hline 65.15 & 65.1 & 6.91 ground dwelling & omnivore & species & omnivore & NA \\
\hline 65.2 & 65.15 & 6.422 ground dwelling & herbivore & species & herbivore & herbivore guild \\
\hline 65.2 & 65.2 & 5.233 ground dwelling & herbivore & species & herbivore & herbivore guild \\
\hline 65.2 & 65.2 & 6.593 ground dwelling & herbivore & species & herbivore & herbivore guild \\
\hline 65.2 & 65.2 & 9.236 scansorial & omnivore & species & omnivore & NA \\
\hline 82.85 & 65.3 & 2.774685879 arboreal & omnivore & species & omnivore & NA \\
\hline 68 & 65.55 & 3.683 scansorial & herbivore, frugivore & species & omnivore & NA \\
\hline 68.45 & 65.55 & 7.539059428 arboreal & herbivore & species & herbivore & herbivore guild \\
\hline 68.5 & 65.55 & 6.635 aquatic & durophage & species & carnivore & carnivore guild \\
\hline 68.9 & 65.55 & 6.986 & & & NA & NA \\
\hline 68.9 & 65.55 & 4.565 ground dwelling & omnivore, frugivore & species & omnivore & NA \\
\hline 9.325 & 65.55 & 5.842741108 arboreal & herbivore & species & herbivore & herbivore guild \\
\hline 69.9 & 65.55 & 7.764466375 arboreal & herbivore & species & herbivore & herbivore guild \\
\hline 74.6 & 65.55 & 3.212 ground dwelling & insectivore & species & insectivore & NA \\
\hline 82.85 & 65.55 & 5.309692903 arboreal & omnivore & species & omnivore & NA \\
\hline 82.85 & 65.55 & 3.463 scansorial & omnivore & species & omnivore & NA \\
\hline
\end{tabular}




\section{References and Notes}

1. M. Cardillo, G. M. Mace, K. E. Jones, J. Bielby, O. R. Bininda-Emonds, W. Sechrest, C. D. Orme, A. Purvis, Multiple causes of high extinction risk in large mammal species. Science 309, 1239-1241 (2005). doi:10.1126/science.1116030 Medline

2. A. D. Barnosky, N. Matzke, S. Tomiya, G. O. U. Wogan, B. Swartz, T. B. Quental, C. Marshall, J. L. McGuire, E. L. Lindsey, K. C. Maguire, B. Mersey, E. A. Ferrer, Has the Earth's sixth mass extinction already arrived? Nature 471, 51-57 (2011). doi:10.1038/nature09678 Medline

3. J. A. Estes, J. Terborgh, J. S. Brashares, M. E. Power, J. Berger, W. J. Bond, S. R. Carpenter, T. E. Essington, R. D. Holt, J. B. C. Jackson, R. J. Marquis, L. Oksanen, T. Oksanen, R. T. Paine, E. K. Pikitch, W. J. Ripple, S. A. Sandin, M. Scheffer, T. W. Schoener, J. B. Shurin, A. R. E. Sinclair, M. E. Soulé, R. Virtanen, D. A. Wardle, Trophic downgrading of planet Earth. Science 333, 301-306 (2011). doi:10.1126/science.1205106 Medline

4. G. Ceballos, P. R. Ehrlich, A. D. Barnosky, A. García, R. M. Pringle, T. M. Palmer, Accelerated modern human-induced species losses: Entering the sixth mass extinction. Sci. Adv. 1, e1400253 (2015). doi:10.1126/sciadv.1400253 Medline

5. R. Dirzo, H. S. Young, M. Galetti, G. Ceballos, N. J. B. Isaac, B. Collen, Defaunation in the Anthropocene. Science 345, 401-406 (2014). doi:10.1126/science.1251817 Medline

6. World Wildlife Fund, Living Planet Report 2016. Risk and Resilience in a New Era (Gland, 2016).

7. F. A. Smith, C. E. Doughty, Y. Malhi, J.-C. Svenning, J. Terborgh, Megafauna in the Earth system. Ecography 39, 99-108 (2016). doi:10.1111/ecog.02156

8. C. N. Johnson, Ecological consequences of Late Quaternary extinctions of megafauna. Proc. Biol. Sci. 276, 2509-2519 (2009). doi:10.1098/rspb.2008.1921 Medline

9. C. E. Doughty, J. Roman, S. Faurby, A. Wolf, A. Haque, E. S. Bakker, Y. Malhi, J. B. Dunning Jr., J.-C. Svenning, Global nutrient transport in a world of giants. Proc. Natl. Acad. Sci. U.S.A. 113, 868-873 (2016). doi:10.1073/pnas.1502549112 Medline

10. F. A. Smith, J. I. Hammond, M. A. Balk, S. M. Elliott, S. K. Lyons, M. I. Pardi, C. P. Tomé, P. J. Wagner, M. L. Westover, Exploring the influence of ancient and historic megaherbivore extirpations on the global methane budget. Proc. Natl. Acad. Sci. U.S.A. 113, 874-879 (2016). doi:10.1073/pnas.1502547112 Medline

11. S. K. Lyons et al., Of mice, mastodons and men: Human-mediated extinctions on four continents. Evol. Ecol. Res. 6, 339-358 (2004).

12. P. L. Koch, A. D. Barnosky, Late Quaternary extinctions: State of the debate. Annu. Rev. Ecol. Syst. 37, 215-250 (2006). doi:10.1146/annurev.ecolsys.34.011802.132415

13. W. Zuo, F. A. Smith, E. L. Charnov, A life-history approach to the late Pleistocene megafaunal extinction. Am. Nat. 182, 524-531 (2013). doi:10.1086/671995 Medline

14. R. Nielsen, J. M. Akey, M. Jakobsson, J. K. Pritchard, S. Tishkoff, E. Willerslev, Tracing the peopling of the world through genomics. Nature 541, 302-310 (2017).

doi:10.1038/nature21347 Medline 
15. S. Oppenheimer, Out-of-Africa, the peopling of continents and islands: Tracing uniparental gene trees across the map. Philos. Trans. R. Soc. London B Biol. Sci. 367, 770-784 (2012). doi:10.1098/rstb.2011.0306 Medline

16. Q. D. Atkinson, R. D. Gray, A. J. Drummond, mtDNA variation predicts population size in humans and reveals a major Southern Asian chapter in human prehistory. Mol. Biol. Evol. 25, 468-474 (2008). doi:10.1093/molbev/msm277 Medline

17. A. Timmermann, T. Friedrich, Late Pleistocene climate drivers of early human migration. Nature 538, 92-95 (2016). doi:10.1038/nature19365 Medline

18. S. L. Carto, A. J. Weaver, R. Hetherington, Y. Lam, E. C. Wiebe, Out of Africa and into an ice age: On the role of global climate change in the late Pleistocene migration of early modern humans out of Africa. J. Hum. Evol. 56, 139-151 (2009). doi:10.1016/j.jhevol.2008.09.004 Medline

19. P. Mellars, J. C. French, Tenfold population increase in Western Europe at the Neandertal-tomodern human transition. Science 333, 623-627 (2011). doi:10.1126/science.1206930 $\underline{\text { Medline }}$

20. W. Roebroeks, P. Villa, On the earliest evidence for habitual use of fire in Europe. Proc. Natl. Acad. Sci. U.S.A. 108, 5209-5214 (2011). doi:10.1073/pnas.1018116108 Medline

21. Materials and methods are available as supplementary materials.

22. D. A. Burney, T. F. Flannery, Fifty millennia of catastrophic extinctions after human contact. Trends Ecol. Evol. 20, 395-401 (2005). doi:10.1016/j.tree.2005.04.022 Medline

23. S. T. Turvey, Ed., Holocene Extinctions (Oxford Univ. Press, 2009).

24. T. Surovell, N. M. Waguespack, "Human prey choice in the Late Pleistocene and its relation to megafaunal extinctions" in American Megafaunal Extinctions at the End of the Pleistocene, G. Hayes, Ed. (Springer, 2009), pp. 77-105.

25. J. Alroy, A multispecies overkill simulation of the end-Pleistocene megafaunal mass extinction. Science 292, 1893-1896 (2001). doi:10.1126/science.1059342 Medline

26. R. E. Plotnick, F. A. Smith, S. K. Lyons, The fossil record of the sixth extinction. Ecol. Lett. 19, 546-553 (2016). doi:10.1111/ele.12589 Medline

27. J. L. Payne, A. M. Bush, N. A. Heim, M. L. Knope, D. J. McCauley, Ecological selectivity of the emerging mass extinction in the oceans. Science 353, 1284-1286 (2016). doi:10.1126/science.aaf2416 Medline

28. F. A. Smith, A. G. Boyer, J. H. Brown, D. P. Costa, T. Dayan, S. K. M. Ernest, A. R. Evans, M. Fortelius, J. L. Gittleman, M. J. Hamilton, L. E. Harding, K. Lintulaakso, S. K. Lyons, C. McCain, J. G. Okie, J. J. Saarinen, R. M. Sibly, P. R. Stephens, J. Theodor, M. D. Uhen, The evolution of maximum body size of terrestrial mammals. Science 330, 12161219 (2010). doi:10.1126/science.1194830 Medline

29. T. Surovell, N. Waguespack, P. J. Brantingham, Global archaeological evidence for proboscidean overkill. Proc. Natl. Acad. Sci. U.S.A. 102, 6231-6236 (2005). doi:10.1073/pnas.0501947102 Medline

30. R. H. Peters, The Ecological Implications of Body Size (Cambridge Univ. Press, 1983). 
31. S. K. M. Ernest, J. H. Brown, Homeostasis and compensation: The role of species and resources in ecosystem stability. Ecology 82, 2118-2132 (2001). doi:10.2307/2680220

32. F. A. Smith, S. K. Lyons, S. K. M. Ernest, K. E. Jones, D. M. Kaufman, T. Dayan, P. A. Marquet, J. H. Brown, J. P. Haskell, Body mass of late Quaternary mammals. Ecology 84, 3403 (2003). doi:10.1890/02-9003

33. F. A. Smith, J. L. Payne, N. A. Heim, M. A. Balk, S. Finnegan, M. Kowalewski, S. K. Lyons, C. R. McClain, D. W. McShea, P. M. Novack-Gottshall, P. S. Anich, S. C. Wang, Body size across the Geozoic. Annu. Rev. Earth Planet. Sci. 44, 523-553 (2016). doi:10.1146/annurev-earth-060115-012147

34. D. E. Wilson, D. M. Reeder, Mammal Species of the World. A Taxonomic and Geographic Reference (John Hopkins, 2003); www.departments.bucknell.edu/biology/resources/msw3.

35. www.iucnredlist.org.

36. A. Azzaroli, Forest Bed elks and giant deer revisited. Zool. J. Linn. Soc. 112, 119-133 (1994). doi:10.1111/j.1096-3642.1994.tb00314.x

37. A. D. Rincón et al., Nuevo registro de Equus (Amerhippus) santaeelenae (Mammalia, Perissodactyla) del pozo de asfalto de Inciarte (Pleistoceno Superior), estado Zulia, Venezuela. Ameghiniana 43, 529-538 (2006).

38. A. D. Rincón, R. S. White, H. G. Mcdonald, Late Pleistocene cingulates (Mammalia: Xenarthra) from Mene de inciarte tar pits, Sierra de Perija, western Venezuela. J. Vertebr. Paleontol. 28, 197-207 (2008). doi:10.1671/0272-4634(2008)28[197:LPCMXF]2.0.CO;2

39. A. E. Zurita, A. A. Carlini, G. J. Scillato-Yané, E. P. Tonni, Mamíferos extintos del Cuaternario de la provincia del Chaco (Argentina) y su relación con aquellos del este de la región pampeana y de Chile. Rev. Geol. Chile 31, 65-87 (2004). doi:10.4067/S0716$\underline{02082004000100004}$

40. A. J. Stuart, A. M. Lister, Extinction chronology of the cave lion Panthera spelaea. Quat. Sci. Rev. 30, 2329-2340 (2011). doi:10.1016/j.quascirev.2010.04.023

41. A. J. Stuart, A. M. Lister, Extinction chronology of the woolly rhinoceros Coelodonta antiquitatis in the context of late Quaternary megafaunal extinctions in northern Eurasia. Quat. Sci. Rev. 51, 1-17 (2012). doi:10.1016/j.quascirev.2012.06.007

42. A. J. Stuart, A. M. Lister, New radiocarbon evidence on the extirpation of the spotted hyaena (Crocuta crocuta (Erxl.)) in northern Eurasia. Quat. Sci. Rev. 96, 108-116 (2014). doi:10.1016/j.quascirev.2013.10.010

43. A. J. Stuart, A. M. Lister, Patterns of Late Quaternary megafaunal extinctions in Europe and northern Asia. Cou. Forschungs. Senckenbg. 259, 289-299 (2007).

44. A. G. Kramarz, M. F. Tejedor, A. M. Forasiepi, A. C. Garrido, New early Miocene primate fossils from northern Patagonia, Argentina. J. Hum. Evol. 62, 186-189 (2012). doi:10.1016/j.jhevol.2011.11.002 Medline

45. A. Larramendi, Shoulder height, body mass, and shape of proboscideans. Acta Palaeontol. Pol. 61, 537-574 (2015). 
46. A. Mercerat, Caracteres diagnósticos de algunas especies de Creodonta conservadas en el Museo de La Plata. Rev. Mus. La Plata 2, 51-56 (1891).

47. A. R. Miño-Boilini, A. A. Carlini, J. O. Chiesa, N. P. Lucero, A. E. Zurita, First record of Scelidodon chiliense (Lydekker) (Phyllophaga, Scelidotheriinae) from the Lujanian stage (late Pleistocene-early Holocene) of Argentina. Neues Jahrbh.Geol. Paläon. 253, 373381 (2009). doi:10.1127/0077-7749/2009/0253-0373

48. A. Turner, The Big cats and Their Fossil Relatives: An Illustrated Guide to Their Evolution and Natural History (Columbia Univ. Press, 1997).

49. A. E. Sanders, Additions to the Pleistocene mammal faunas of South Carolina, North Carolina, and Georgia. Trans. Am. Philos. Soc. 2, 137-152 (2002).

50. B. B. Huckell, C. V. Haynes, The Ventana complex: New dates and new ideas on its place in Early Holocene western prehistory. Am. Antiq. 68, 353-371 (2003). doi:10.2307/3557084

51. B. D. Russell, A. H. Harris, A new leporine (Lagomorpha: Leporidae) from Wisconsinan deposits of the Chihuahuan Desert. J. Mammal. 67, 632-639 (1986). doi:10.2307/1381125

52. B. H. Slaughter, Platygonus compressus and associated fauna from the Laubach Cave of Texas. Am. Midl. Nat. 75, 475-494 (1966). doi:10.2307/2423406

53. B. H. Slaughter, The Moore Pit local fauna; Pleistocene of Texas. J. Paleontol. 40, 78-91 (1966).

54. B. J. MacFadden, Dispersal of Pleistocene Equus (Family Equidae) into South America and calibration of GABI 3 based on evidence from Tarija, Bolivia. PLOS ONE 8, e59277 (2013). doi:10.1371/journal.pone.0059277 Medline

55. B. Kurten, E. A. Anderson, Pleistocene Mammals of North America (Columbia Univ. Press, 1980).

56. B. Mackness, On the identity of Euowenia robusta De Vis, 1891 with a description of a new zygomaturine genus. Alcheringa 34, 455-469 (2010). doi:10.1080/03115511003793488

57. B. S. Ferrero, J. I. Noriega, A new upper Pleistocene tapir from Argentina: Remarks on the phylogenetics and diversification of Neotropical Tapiridae. J. Vertebr. Paleontol. 27, 504-511 (2007). doi:10.1671/0272-4634(2007)27[504:ANUPTF]2.0.CO;2

58. B. Shorrocks, The Giraffe: Biology, Ecology, Evolution and Behaviour (Wiley, 2016).

59. B. Van Valkenburgh, F. Grady, B. Kurtén, The Plio-Pleistocene Cheetah-Like Cat Miracinonyx inexpectatus of North America. J. Vertebr. Paleontol. 10, 434-454 (1990). doi:10.1080/02724634.1990.10011827

60. C. Cartelle, G. De Iuliis, F. Pujos, A new species of Megalonychidae (Mammalia, Xenarthra) from the Quaternary of Poço Azul (Bahia, Brazil). C. R. Palevol 7, 335-346 (2008). doi:10.1016/i.crpv.2008.05.006

61. C. Cartelle, G. De Iuliis, Eremotherium laurillardi: The Panamerican late Pleistocene Megatheriid sloth. J. Vertebr. Paleontol. 15, 830-841 (1995).

doi:10.1080/02724634.1995.10011265 
62. C. Cartelle, W. C. Hartwig, A new extinct primate among the Pleistocene megafauna of Bahia, Brazil. Proc. Natl. Acad. Sci. U.S.A. 93, 6405-6409 (1996). doi:10.1073/pnas.93.13.6405 Medline

63. C. Guerin, J. H. Winslow, M. Piboule, M. Faure, Le prétendu rhinocéros de Nouvelle Calédonie est un marsupial (Zygomaturus diahotensis nov. sp.): Solution d'une énigmeet conséquences paléogéographiques. Geobios 14, 201-217 (1981). doi:10.1016/S0016$\underline{6995(81) 80004-6}$

64. C. Guérin, Les rhinocéros (Mammalia, Perissodactyla) du Miocène terminal au Pléistocène supérieur en Europe occidentale. Comparaison avec les espèces actuelles. Doc. Lab. Géol. Lyon 79, 1-1185 (1980).

65. C. M. Deschamps, Late Cenozoic mammal bio-chronostratigraphy in southwestern Buenos Aires province, Argentina. Ameghiniana 42, 733-750 (2005).

66. C. Stock, The Pleistocene fauna of Hawver Cave. Univ. Calif. Publ. Bull. Dept. Geol. 10, 461-515 (1917).

67. C. N. Johnson, Australia's Mammal Extinctions: A 50,000-Year History (Cambridge Univ. Press, 2007).

68. D. B. Wright, "Tayassuidae” in C. M. Janis, K. M. Scott, L. L. Jacobs, Eds. Evolution of Tertiary mammals of North America (Cambridge Univ. Press, 1998), pp. 389-401.

69. D. Geraads et al., The large mammal fauna of the Oldowan sites of Melka Kunture. Studies on the Early Paleolithic Site of Melka Kunture, Ethiopia. Istit. Ital. Preist. Prot 2004, 169-192 (2004).

70. D. Geraads, Paléobiogéographie de l'Afrique du Nord depuis le Miocene terminal, d'après les grands Mammifères. Geobios 15, 473-481 (1982). doi:10.1016/S0016$\underline{6995(82) 80135-6}$

71. D. Mol et al., Extinct beaver Trogontherium cuvieri Fischer, 1809 (Mammalia, Rodentia, Castoridae) from the deep water channel between England and the Netherlands. Mededelingen Nederlands Instituut voor Toegepaste Geowetenschappen TNO 60, 193198 (1996).

72. D. Pate, Last recorded evidence for megafauna at Wet Cave, Naracoorte, South Australia 45,000 years ago. Aust. Arch 54, 53-55 (2002).

73. D. R. Ruez Jr., Earliest record of Palaeolama (Mammalia, Camelidae) with comments on "Palaeolama" guanajuatensis. J. Vertebr. Paleontol. 25, 741-744 (2005). doi:10.1671/0272-4634(2005)025[0741:EROPMC]2.0.CO;2

74. E. I. Rogaev, Y. K. Moliaka, B. A. Malyarchuk, F. A. Kondrashov, M. V. Derenko, I. Chumakov, A. P. Grigorenko, Complete mitochondrial genome and phylogeny of Pleistocene mammoth Mammuthus primigenius. PLOS Biol. 4, e73 (2006). doi:10.1371/journal.pbio.0040073 Medline

75. E. M. E. Billia, C. Petronio, Selected records of Stephanorhinus kirchbergensis (Jäger, 1839) (Mammalia, Rhinocerotidae) in Italy. Boll. Soc. Paleontol. Ital. 48, 21-32 (2009). 
76. E. Soibelzon et al., Los Xenarthra (Mammalia) del Ensenadense (Pleistoceno inferior a medio) de la Región Pampeana (Argentina). Rev. Mex. Cienc. Geol. 27, 449-469 (2010).

77. E. V. Oliveira, J. C. Pereira, Intertropical cingulates (Mammalia, Xenarthra) from the Quaternary of Southern Brazil: Systematics and paleobiogeographical aspects. Rev. Bras. Paleontol. 12, 167-178 (2009). doi:10.4072/rbp.2009.3.01

78. F. Ameghino, Notas sobre algunos mamiferos fosiles, nuevos ò poco conocidos del valle de Tarija. Anal. Mus. Buenos Aires 3, 225-261 (1902).

79. F. Ameghino, Notas sobre una pequeña coleccion de huesos de mamiferos procedentes de las grutas calcáreas de Iporangaen el Estado de Sao Paulo-Brazil. Cardozo 7, 59-124 (1907).

80. F. Ameghino, Observaciones generales sobre el orden de mamiferos estinguidos sudamericanos llamados toxodontes (Toxodontia) y sinopsis de los generos y especies hasta ahora conocidos. Anal. Mus. Plata 1, 1-66 (1887).

81. F. Pujos, G. De Iuliis, C. Cartelle, A Paleogeographic overview of tropical fossil sloths: Towards an understanding of the origin of extant suspensory sloths? J. Mamm. Evol. 24, 19-38 (2016). doi:10.1007/s10914-016-9330-4

82. F. Pujos, R. Salas, A new species of Megatherium (Mammalia: Xenarthra: Megatheriidae) from the Pleistocene of Sacaco and Tres Ventanas, Peru. Palaeontology 47, 579-604 (2004). doi:10.1111/j.0031-0239.2004.00376.x

83. G. Amori, M. Clout, Rodents on islands: A conservation challenge. ACIAR Mongr. Ser. 96, 63-68 (2003).

84. G. De Iuliis, C. Cartelle, A new giant megatheriine ground sloth (Mammalia: Xenarthra: Megatheriidae) from the late Blancan to early Irvingtonian of Florida. Zool. J. Linn. Soc. 127, 495-515 (1999). doi:10.1111/j.1096-3642.1999.tb01383.x

85. G. E. Jakway, Pleistocene Lagomorpha and Rodentia from the San Josecito Cave, Nuevo Leon, Mexico. Trans. Kans. Acad. Sci. 61, 313-327 (1958). doi:10.2307/3626451

86. G. G. Simpson, A new record of Euceratherium or Preptoceras (extinct Bovidae) in New Mexico. J. Mammal. 44, 583-584 (1963). doi:10.2307/1377152

87. G. G. Simpson, The principles of classification and a classification of mammals. Bull. Am. Mus. Nat. Hist. 85, 1-350 (1945).

88. G. Haynes, American Megafaunal Extinctions at the End of the Pleistocene (Springer, 2009).

89. G. J. Price, Taxonomy and palaeobiology of the largest-ever marsupial, Diprotodon Owen, 1838 (Diprotodontidae, Marsupialia). Zool. J. Linn. Soc. 153, 369-397 (2008). doi:10.1111/j.1096-3642.2008.00387.x

90. G. Lessa et al., Novos achados de mamíferos carnívoros do Pleistoceno final-Holoceno em grutas calcárias do Estado da Bahia. Acta Geol. Leop. 46, 157-169 (1998).

91. G. Prideaux, Systematics and Evolution of the Sthenurine Kangaroos (Univ. California Press, 2004).

92. H. A. Longman, Some Queensland fossil vertebrates. Mem. Queensl. Mus. 8, 16-28 (1924). 
93. H. G. McDonald, V. L. Naples, "Xenarthra," in Evolution of Tertiary Mammals of North America, C. M. Janis, G. F. Gunnell, M. D. Uhen, Eds. (Cambridge Univ. Press, 2007), pp.147-160.

94. H. Kirkland Jr., Some late Pleistocene fossils from Washita local fauna. Proc. Okla. Acad. Sci. 77, 113-115 (1997).

95. I. Agnarsson, L. J. May-Collado, The phylogeny of Cetartiodactyla: The importance of dense taxon sampling, missing data, and the remarkable promise of cytochrome b to provide reliable species-level phylogenies. Mol. Phylogenet. Evol. 48, 964-985 (2008). doi:10.1016/j.ympev.2008.05.046 Medline

96. J. Alroy, Synonymies and re-identifications of North American fossil mammals, in The Paleobiology Database (2002); http://paleodb.org.

97. J. G. Honey et al., Camelidae" in C. M. Janis, K. M. Scott, L. L. Jacobs, Eds., Evolution of Tertiary Mammals of North America (Cambridge UJniv. Press, 1998), pp. 439-462.

98. J. J. Austin, J. Soubrier, F. J. Prevosti, L. Prates, V. Trejo, F. Mena, A. Cooper, The origins of the enigmatic Falkland Islands wolf. Nat. Commun. 4, 1552 (2013). doi:10.1038/ncomms2570 Medline

99. J. L. Prado, M. T. Alberdi, B. Azanza, B. Sánchez, D. Frassinetti, The Pleistocene Gomphotheriidae (Proboscidea) from South America. Quat. Int. 126-128, 21-30 (2005). doi:10.1016/j.quaint.2004.04.012

100. J. Leidy, Notice of Remains of Extinct Vertebrata, From the Valley of the Niobrara River (Merrihew \& Thompson, 1858).

101. J. Meachen, A new species of Hemiauchenia (Artiodactyla, Camelidae) from the Late Blancan of Florida. Florida Mus. Nat. Hist. Bull. 45, 435-447 (2005).

102. J. Moojen, Nôvo gênero de Cricetidae do Brasil Central (Glires, Mammalia). Ver. Brasil. Biol. 25, 281-285 (1965).

103. J. N. McDonald, North American Bison: Their Classification and Evolution (Univ. California Press, 1981).

104. J. S. Brink et al., A new find of Megalotragus priscus (Alcelaphini, Bovidae) from the central Karoo, South Africa. Palaeont. Afr. 32, 17-22 (1995).

105. J. T. Faith, Late Pleistocene and Holocene mammal extinctions on continental Africa. Earth Sci. Rev. 128, 105-121 (2014). doi:10.1016/j.earscirev.2013.10.009

106. J. W. F. Reumer, L. Rook, K. Van Der Borg, K. Post, D. Mol, J. De Vos, Late Pleistocene survival of the saber-toothed cat Homotherium in northwestern Europe. J. Vertebr. Paleontol. 23, 260-262 (2003). doi:10.1671/0272-4634(2003)23[260:LPSOTS]2.0.CO;2

107. J. W. Gidley, Tooth characters and revision of the North American species of the genus Equus. Bull. Am. Mus. Nat. Hist. 14, 1-60 (1901).

108. J. Weinstock, E. Willerslev, A. Sher, W. Tong, S. Y. W. Ho, D. Rubenstein, J. Storer, J. Burns, L. Martin, C. Bravi, A. Prieto, D. Froese, E. Scott, L. Xulong, A. Cooper, Evolution, systematics, and phylogeography of pleistocene horses in the new world: A 
molecular perspective. PLOS Biol. 3, e241 (2005). doi:10.1371/journal.pbio.0030241 $\underline{\text { Medline }}$

109. J. H. Hope, Pleistocene mammal extinction: The problem of Mungo and Menindee, New South Wales. Alcheringa 2, 65-82 (1978). doi:10.1080/03115517808619080

110. K. J. Piper, A new species of Palorchestidae (Marsupialia) from the Pliocene and early Pleistocene of Victoria. Alcheringa 30 (sup1), 281-294 (2006). doi:10.1080/03115510609506867

111. K. M. Helgen, R. T. Wells, B. P. Kear, W. R. Gerdtz, T. F. Flannery, Ecological and evolutionary significance of sizes of giant extinct kangaroos. Aust. J. Zool. 54, 293-303 (2006). doi:10.1071/ZO05077

112. L. Dawson, On the uncertain generic status and phylogenetic relationships of the large extinct vombatid species Phascolomys medius Owen, 1872 (Marsupialia: Vombatidae). Aust. Mammal. 6, 5-13 (1983).

113. L. Dawson, T. Flannery, Taxonomic and phylogenetic status of living and fossil kangaroos and wallabies of the genus Macropus Shaw (Macropodidae: Marsupialia), with a new subgeneric name for the larger wallabies. Aust. J. Zool. 33, 473-498 (1985). doi:10.1071/ZO9850473

114. L. H. Soibelzon, E. P. Tonni, M. Bond, The fossil record of South American short-faced bears (Ursidae, Tremarctinae). J. S. Am. Earth Sci. 20, 105-113 (2005). doi:10.1016/j.jsames.2005.07.005

115. L. Pandolfi et al., The skull of Stephanorhinus hemitoechus (Mammalia, Rhinocerotidae) from the Middle Pleistocene of Campagna Romana (Rome, central Italy): Biochronological and paleobiogeographic implications. Bull. Geosci. 88, 1-12 (2012).

116. L. Pandolfi, A. Tagliacozzo, Stephanorhinus hemitoechus (Mammalia, Rhinocerotidae) from the Late Pleistocene of Valle Radice (Sora, Central Italy) and re-evaluation of the morphometric variability of the species in Europe. Geobios 48, 169-191 (2015). doi:10.1016/j.geobios.2015.02.002

117. L. Werdelin, Small Pleistocene felines of North America. J. Vertebr. Paleontol. 5, 194-210 (1985). doi:10.1080/02724634.1985.10011858

118. M. Arnal, G. Kramarz, First complete skull of an octodontoid (Rodentia, Caviomorpha) from the Early Miocene of South America and its bearing in the early evolution of Octodontoidea. Geobios 44, 435-444 (2011). doi:10.1016/j.geobios.2010.12.003

119. M. Benoit, What's the difference? A multiphasic allometric analysis of fossil and living lions. J. Vert. Paleontol. 27, 60 (2007).

120. M. Breda, R. Pini, C. Ravazzi, The palaeoenvironment of Cervalces latifrons (Johnson, 1874) from Fornaci di Ranica (late Early Pleistocene, Northern Italy). Palaeogeol. Paleoclim. Paleoecol. 216, 99-118 (2005). doi:10.1016/j.palaeo.2004.10.004

121. M. C. McKenna, S. K. Bell, Classification of Mammals: Above the Species Level (Columbia Univ. Press, 1997). 
122. M. Griffiths et al., Observations on the skulls of fossil and extant echidnas (Monotremata: Tachyglossidae). Aust. Mammal. 14, 87-101 (1991).

123. M. O. Woodburne et al., Madakoala, a new genus and two species of Miocene koalas (Marsupialia: Phascolarctidae) from South Australia and a new species of Perikoala" in Possums and Opossums: Studies in Evolution, M. Archer, Ed. (Surrey Beatty, 1987), pp. 293-317.

124. M. T. Alberdi et al., Study of cedral horses and their place in the Mexican quaternary. Rev. Mex. Cienc. Geol. 31, 221-237 (2014).

125. N. Johnson, G. J. Prideaux, Extinctions of herbivorous mammals in the late Pleistocene of Australia in relation to their feeding ecology: No evidence for environmental change as cause of extinction. Austral Ecol. 29, 553-557 (2004). doi:10.1111/j.14429993.2004.01389.x

126. N. P. Kalmykov, D. V. Kobylkin, M. A. Grigoryeva, V. N. Chernykh, Validity of the spiralhorned antelope species of the genus Spirocerus (Mammalia, Artiodactyla) in Central Asia. Dokl. Biol. Sci. 457, 233-235 (2014). Medline

127. P. Hay, A collection of Pleistocene vertebrates from southwestern Texas. Proc. U. S. Natl. Mus. 68, 1-18 (1926). doi:10.5479/si.00963801.68-2625.1

128. P. J. Mayhew, G. B. Jenkins, T. G. Benton, A long-term association between global temperature and biodiversity, origination and extinction in the fossil record. Proc. Biol. Sci. 275, 47-53 (2008). doi:10.1098/rspb.2007.1302 Medline

129. P. L. Koch, A. D. Barnosky, Late Quaternary extinctions: State of the debate. Annu. Rev. Ecol. Syst. 37, 215-250 (2006). doi:10.1146/annurev.ecolsys.34.011802.132415

130. P. Lopez-Mendoza et al., Mamiferos extintos del Pleistoceno de la Cuenca de Calama. Treb. Mus. Geol. Barcel. 17, 11-25 (2010).

131. P. M. Youngman, The extinct short-faced skunk Brachyprotoma obtusata (Mammalia, Carnivora): First records for Canada and Beringia. Can. J. Earth Sci. 23, 419-424 (1986). doi:10.1139/e86-043

132. P. O. Antoine, Pleistocene and Holocene rhinocerotids (Mammalia, Perissodactyla) from the Indochinese Peninsula. C. R. Palevol 11, 159-168 (2012). doi:10.1016/j.crpv.2011.03.002

133. P. S. Martin, H. E. Wright, Pleistocene Extinctions: The Search for a Cause (Yale Univ. Press, 1967).

134. P. S. Martin, R. G. Klein, Quaternary Extinctions: a Prehistoric Revolution (Univ. Arizona Press, 1984).

135. P. S. Martin, Twilight of the Mammoths: Ice Age Extinctions and the Rewilding of America (Univ. California Press, 2005).

136. R. A. Fariña et al., Megafauna: Giant Beasts of Pleistocene South America (Indiana Univ. Press, 2013). 
137. R. A. Fariña, A. Czerwonogora, M. di Giacomo, Splendid oddness: Revisiting the curious trophic relationships of South American Pleistocene mammals and their abundance. An. Acad. Bras. Cienc. 86, 311-331 (2014). doi:10.1590/0001-3765201420120010 Medline

138. R. Hoffstetter, Les gravigrades (Edentes Xenarthres) des cavernes de Lagoa Santa (Minas Gerais, Bresil). Ann. Sci. Natur. Zool. 11, 741-764 (1954).

139. R. Hoffstetter, Les Mammiferes Pleistocenes de la Republique de L'Equateur. Mem. Soc. Geol. France 66, 1-391 (1952).

140. R. K. McAfee, Reassessment of the cranial characters of Glossotherium and Paramylodon (Mammalia: Xenarthra: Mylodontidae). Zool. J. Linn. Soc. 155, 885-903 (2009). doi:10.1111/j.1096-3642.2008.00468.x

141. R. Lydekker, Contributions to a knowledge of the Fossil Vertebrates of Argentina. III - A study of extinct argentine ungulates. Anal. Mus. Plata Paleon. Argent. 2, 1-86 (1894).

142. R. Owen, On the fossil mammals of Australia. Part IX. Family Macropodidae; Genera Macropus, Pachysiagon, Leptosiagon, Procoptodon, and Palorchestes. Phil. Proc. R. Soc. 164, 783-803 (1874).

143. R. Van Frank, A fossil collection from northern Venezuela. 1. Toxodontidae (Mammalia, Notoungulata). Am. Mus. Novit. 1850, 1-38 (1957).

144. S. D. Webb, A cranium of Navahoceros and its phylogenetic place among New World Cervidae. Ann. Zool. Fenn. 28, 401-410 (1992).

145. S. D. Webb, "Late Cenozoic mammal dispersals between the Americas" in, The Great American Biotic Interchange, F. G. Stehli, S. D. Webb, Eds. (Plenum, 1985), pp. 357386.

146. S. T. Turvey, Holocene Extinctions (Oxford Univ. Press, 2009).

147. T. Deng, X. Wang, M. Fortelius, Q. Li, Y. Wang, Z. J. Tseng, G. T. Takeuchi, J. E. Saylor, L. K. Säilä, G. Xie, Out of Tibet: Pliocene woolly rhino suggests high-plateau origin of Ice Age megaherbivores. Science 333, 1285-1288 (2011). doi:10.1126/science.1206594 Medline

148. T. F. Flannery, L. Seri, The mammals of southern West Sepik Province, Papua New Guinea: Their distribution, abundance, human use, and zoogeography. Rec. Aust. Mus. 42, 173-208 (1990). doi:10.3853/j.0067-1975.42.1990.114

149. T. F. Flannery, Pleistocene faunal loss: Implications of the aftershock for Australia's past and future. Archaeol. Ocean. 25, 45-55 (1990). doi:10.1002/j.1834-4453.1990.tb00232.x

150. T. M. Flannery, P. Schouten, A Gap in Nature (Atlantic Monthly Press, 2001).

151. V. M. Bravo-Cuevas et al., Taxonomy and notes on the paleobiology of the late Pleistocene (Rancholabrean) antilocaprids (Mammalia, Artiodactyla, Antilocapridae) from the state of Hidalgo, central Mexico. Rev. Mex. Cienc. Geol. 30, 601-613 (2013).

152. V. V. Titov, Habitat conditions for Camelus knoblochi and factors in its extinction. Quat. Int. 179, 120-125 (2008). doi:10.1016/j.quaint.2007.10.022

153. W. C. Hartwig, C. Cartelle, A complete skeleton of the giant South American primate Protopithecus. Nature 381, 307-310 (1996). doi:10.1038/381307a0 Medline 
154. W. D. Lambert, J. Shoshani, "Proboscidea" in Evolution of Tertiary mammals of North America, C. M. Janis, K. M. Scott, L. L. Jacobs, Eds. (Cambridge Univ. Press, 1998), pp. 606-621.

155. W. E. Johnson, E. Eizirik, J. Pecon-Slattery, W. J. Murphy, A. Antunes, E. Teeling, S. J. O’Brien, The late Miocene radiation of modern Felidae: A genetic assessment. Science 311, 73-77 (2006). doi:10.1126/science.1122277 Medline

156. W. L. Puckette, J. A. Scholtz, The Hazen Mammoth (Mammuthus columbi), Prairie County, Arkansas. Arkansas Acad. Sci. Proc. 28, 53-56 (1974).

157. W. W. Dalquest, G. E. Schultz, Ice Age Mammals of Northwestern Texas (Midwest. State Univ. Press, 1992).

158. M. L. Merino, R. Vieira Rossi, in Encyclopedia of Quaternary Science; www.fcnym.unlp.edu.ar/catedras/proteccion/tp3/Neotropical01.pdf.

159. X. Wang, R. H. Tedford, Dogs: Their Fossil Relatives and Evolutionary History (Columbia Univ. Press, 2008).

160. https://paleobiodb.org/\#/.

161. R Core Team, R: A Language and Environment for Statistical Computing (R Found. Stat. Comp, 2015).

162. R Studio Team, $R$ Studio: Integrated Development for $R$ (R Studio, 2015).

163. M. Foote, D. M. Raup, Fossil preservation and the stratigraphic ranges of taxa. Paleobiology 22, 121-140 (1996). doi:10.1017/S0094837300016134 Medline

164. J. Alroy, New methods for quantifying macroevolutionary patterns and processes. Paleobiology 26, 707-733 (2000). doi:10.1666/00948373(2000)026<0707:NMFQMP>2.0.CO;2

165. E. S. Vrba, D. DeGusta, Do species populations really start small? New perspectives from the Late Neogene fossil record of African mammals. Philos. Trans. R. Soc. Lond. B Biol. Sci. 359, 285-292, discussion 292-293 (2004). doi:10.1098/rstb.2003.1397 Medline

166. O. Carranza-Castañeda et al., The early-late Hemphillian (Hh2) faunal assemblaged from Juchipila Basin, State of Zacatecas, Mexico and its biochronological correlation with other Hemphillian faunas in Central Mexico. Contrib. Sci. 521, 13-49 (2013).

167. P. Christiansen, Body size in Proboscidean, with notes on elephant metabolism. Zool. J. Linn. Soc. 140, 523-549 (2004). doi:10.1111/j.1096-3642.2004.00113.X

168. A. R. Evans, D. Jones, A. G. Boyer, J. H. Brown, D. P. Costa, S. K. M. Ernest, E. M. G. Fitzgerald, M. Fortelius, J. L. Gittleman, M. J. Hamilton, L. E. Harding, K. Lintulaakso, S. K. Lyons, J. G. Okie, J. J. Saarinen, R. M. Sibly, F. A. Smith, P. R. Stephens, J. M. Theodor, M. D. Uhen, The maximum rate of mammal evolution. Proc. Natl. Acad. Sci. U.S.A. 109, 4187-4190 (2012). doi:10.1073/pnas.1120774109 Medline

169. S. R. Fagan, Osteology of Mylagaulus laevis, a fossorial rodent from the upper Miocene of Colorado. Univ. Kansas Paleontol. Contrib. Vertebrata 9, 1-32 (1960).

170. M. Freudenthal, E. Martín-Suárez, Estimating body mass of fossil rodents. Scrip. Geo. 145, $1-130$ (2013). 
171. G. F. Gunnell et al., in Evolution of Tertiary Mammals of North America, C. M. Janis, G. F. Gunnell, M. D. Uhen, Eds. (Cambridge Univ. Press, 2007).

172. C. Hibbard, New rodents from the late Cenozoic of Kansas. Pap. Mich. Acad. Sci. Arts. Lett. 52, 115-131 (1967).

173. S. S. B. Hopkins, Reassessing the mass of exceptionally large rodents using toothrow length and area as proxies for body mass. J. Mammal. 89, 232-243 (2008). doi:10.1644/06MAMM-A-306.1

174. W. W. Korth, Miosicista angulus, A new Sicstine rodent (Zapodidae, Rodentia) from the Bartsovian (Miocene) of Nebraska. Trans. Nebr. Acad. Sci. 20, 97-101 (1983).

175. M. J. Orliac, E. Gilissen, Virtual endocranial cast of earliest Eocene Diacodexis (Artiodactyla, Mammalia) and morphological diversity of early artiodactyl brains. Proc. Biol. Sci. 279, 3670-3677 (2012). doi:10.1098/rspb.2012.1156 Medline

176. H. G. McDonald, Paleoecology of extinct Xenarthrans and the Great American Biotic Interchange. Bull. Fla. Mus. Nat. Hist. 45, 313-333 (2005).

177. R. T. McKenna, thesis, Portland State University, Portland, Oregan (2011).

178. S. Tomiya, thesis, University of California, Berkeley, Berkeley, California (2012).

179. C. F. Wellstead, Sedimentology of Norden Bridge and Egelhoff fossil quaries (Miocene) of north-central Nebraska. Trans. Nebr. Acad. Sci. 9, 67-85 (1981).

180. R. M. West, Geology and mammalian paleontology of the New Fork-Big Sandy Area, Sublette County, Wyoming. Field. Geol. 29, 1-193 (1973).

181. J. A. White, The Archaeolaginae (Mammalia, Lagomorpha) of North America, excluding Archaeolagus and Panolax. J. Vertebr. Paleontol. 7, 425-450 (1988). doi:10.1080/02724634.1988.10011674

182. R. W. Wilson, Early Miocene rodents and insectivores from northeastern Colorado. Univ. Kansas Paleontol. Contrib. Vertebrata. 8, 1-24 (1960).

183. K. L. Seymour, thesis, University of Toronto, Toronto (1999).

184. C. Hibbard, E. S. Riggs, Upper Pliocene vertebrates from Keefe Canyon, Meade County, Kansas. Bull. Geol. Soc. Am. 60, 829-860 (1949). doi:10.1130/00167606(1949)60[829:UPVFKC]2.0.CO;2

185. R. C. Hulbert Jr., B. J. MacFadden, Morphological transformation and cladogenesis at the base of the adaptive radiation of Miocene hypsodont horses. Am. Mus. Novit. 3000, 1-66 (1991).

186. C. Hibbard, Mammals of the Rexroad formation from Fox Canyon, Kansas. Contrib. Mus. Paleon. Univ. Mich. 8, 113-192 (1950).

187. L. N. Carraway, Fossil history of notiosorex (Soricomorpha: Soricidae) shrews with descriptions of new fossil species. West. N. Am. Nat. 70, 144-163 (2010). doi:10.3398/064.070.0202

188. C. W. Hibbard, The insectivores of the Rexroad fauna, Upper Pliocene of Kansas. $J$. Paleontol. 27, 21-32 (1953). 
189. R. H. Tedford, X. Wang, B. E. Taylor, Phylogenetic systematics of the North American Fossil Caninae (Carnivora: Canidae). Bull. Am. Mus. Nat. Hist. 325, 1-218 (2009). doi:10.1206/574.1

190. T. M. Bown, The fossil Insectivora of Lemoyne Quarry (Ash Hollow Formation, Hemphillian), Keith County, Nebraska. Trans. Nebr. Acad. Sci. 8, 99-122 (1980).

191. C. W. Hibbard, Pleistocene mammals from Kansas. State Geol. Surv. Kansas Bull. 41, 1-8 (1942).

192. M. F. Skinner, C. W. Hibbard, Early Pleistocene pre-glacial and glacial rocks and faunas of north-central Nebraska. Bull. Am. Mus. Nat. Hist. 148, 1-148 (1972).

193. E. Jiménez-Hidalgo, O. Carranza-Castañeda, Blacan camelids from San Miguel de Allende Guanajuanto, Central Mexico. J. Paleontol. 84, 51-65 (2010). doi:10.1666/08-154.1

194. G. S. Morgan, P. L. Sealey, S. G. Lucas, Pliocene and early Pleistocene (Blancan) vertebrates from the Palomas Formation $n$ the vicinity of Elephant Butte Lake and Caballo Lake, Sierra County, Southwestern New Mexico. New Mex. Mus. Nat. Hist. Sci. Bull. 53, 664-736 (2011).

195. J. Damuth, B. J. MacFadden, Body Size in Mammalian Paleobiology: Estimation and Biological Implications (Cambridge Univ. Press, 1990).

196. J. Alroy, "Putting North America's end-Pleistocene megafaunal extinction in context: large scale analyses of spatial patterns, extinction rates, and size distributions" in Extinctions in Near Time: Causes, Contexts, and Consequences, R. D. E. MacPhee, Ed. (Plenum, 1999), pp. 105-143.

197. J. Zachos, M. Pagani, L. Sloan, E. Thomas, K. Billups, Trends, rhythms, and aberrations in global climate 65 Ma to present. Science 292, 686-693 (2001).

doi:10.1126/science.1059412 Medline 